\title{
Berufsausbildung in Deutschland und den USA
}

\author{
- Institutionalisierung \\ des dualen Berufsbildungssystems in \\ vergleichender Perspektive -
}

\begin{abstract}
Dissertation
zur Erlangung des sozialwissenschaftlichen Doktorgrades der Sozialwissenschaftlichen Fakultät der Georg-August-Universität Göttingen
\end{abstract}

vorgelegt

von

Matthias Kreysing aus Recife/Brasilien

Göttingen 2003 
Betreuerin: Prof. Dr. Ilona Ostner

Gutachter: Prof. Dr. Horst Kern

Tag der mündlichen Prüfung: 26.05.2003 


\section{Inhaltsverzeichnis}

Einleitung

Teil I: Theoretische und methodische Grundlagen.

1. Berufsbildung als integraler Bestandteil von Wohlfahrtsstaatlichkeit.

2. Industrialisierungsansatz

3. Machtressourcenansatz

4. Historischer Institutionalismus .

4.1 Allgemeine Überlegungen ....

4.2 Skocpols historisch-institutionalistischer Ansatz

4.2.1 Staatliche Formation

4.2.2 Institutionelle Einflüsse .....

4.2.3 ,Policy Feedback`

4.3 Ideen, Interessen und Institutionen

4.3.1 Egalitarismus versus Beruflichkeit

4.3.2 Arbeitgeber, Gewerkschaften und der Staat

5. Methoden des Vergleichs.

6. Aufbau der Arbeit 50

Teil II: Die duale Berufsausbildung in Deutschland 
1.1 Staat und Bildungswesen 55

1.2 Die Lehrlingsausbildung bis zur Industrialisierung 59

1.3 Gewerbefreiheit, Industrialisierung und berufliche Bildung 65

1.4 Berufliche Bildung in der Weimarer Republik 77

2. Institutionen und Interessen 86

2.1 Ausbildungsmarkt und Beschäftigungssystem 86

2.2 Unternehmensverbände und Arbeitgeber 95

2.3 Gewerkschaften 110

2.4 Staatliche Institutionen 120

3. Staatliche Berufsbildungspolitik seit den 1970er Jahren 130

3.1 Strukturpolitische Konsolidierung des dualen Berufsbildungssystems 130

3.2 Stabilisierung der dualen Berufsausbildung 137

4. Aussichten für das duale Berufsausbildungssystem 144

Teil III: Duale Berufsausbildung in den USA?

1. Historische Grundlagen 148

1.1 Staatliche Institutionalisierung und Bildung. 148

1.2 Entstehung eines ,mass education'-Schulsystems 156

1.3 Berufliche Bildung - Von der Werkbank zur Schulbank 164

1.3.1 Niedergang der Lehrlingsausbildung. 164

1.3.2 Aufstieg des, vocationalism' im amerikanischen Schulsystem 172 


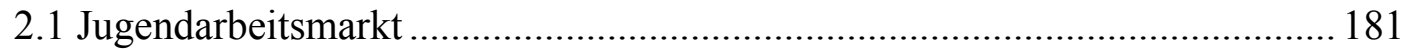

2.2 Unternehmensverbände und Arbeitgeber ...................................................... 191

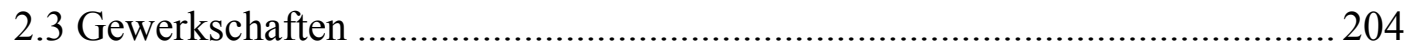

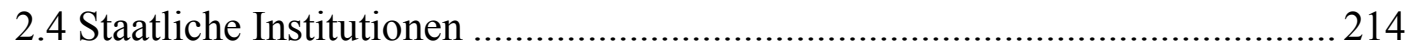

2.5 Lehrergewerkschaften und Eltern........................................................... 223

3. Bundesstaatliche Ausbildungs- und Berufsbildungspolitik ............................... 231

3.1 Ausbildungs- und Beschäftigungspolitik ................................................. 231

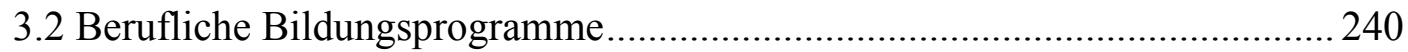

Teil IV: Clintons School-to-Work Opportunities Act …............................................. 248

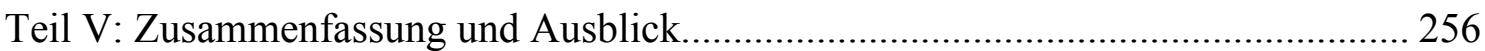

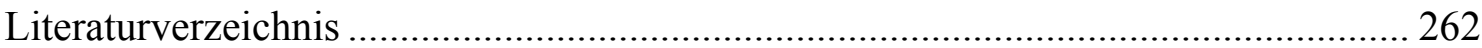




\title{
Einleitung
}

\begin{abstract}
„In studying foreign systems of education we should not forget that things outside the schools matter even more than things inside the schools, and govern and interpret the things inside. We cannot wander at pleasure among the educational systems of the world, like a child strolling through a garden, and pick off a flower from one bush and some leaves from another, and then expect that if we stick what we have gathered into the soil at home, we shall have a living plant. A national system of education is a living thing, the outcome of forgotten struggles and of battles long ago". It has in it some of the secret workings of national life" (Michael Sadler, Office of Special Inquiries and Reports of the English Board of Education 1895-1903, in: Phillips 1993, S. 15).
\end{abstract}

Bereits um die Jahrhundertwende gab es in den USA ein reges Interesse daran, das deutsche Berufsbildungssystem zu übernehmen. Dieses Interesse speiste sich aus zwei Quellen: Deutsche Immigranten, die in ihrer Heimat eine Lehrlingsausbildung durchlaufen hatten, setzten diese Tradition in den amerikanischen Regionen, in denen sie sich niederließen, fort. Außerdem stiegen mit wachsendem internationalen Handel der Austausch und das Wissen über verschiedene nationale Praktiken. Der Blick amerikanischer Geschäftsleute, Politiker und reformorientierter Pädagogen richtete sich nicht zufällig auf Deutschland. Denn diese junge Industrienation bedrohte die internationale Konkurrenzfähigkeit der amerikanischen Wirtschaft. Auf der Suche nach den Gründen für ihren Erfolg fand man ein nationales Berufsbildungssystem, das der aufstrebenden Industrie die benötigten Facharbeiter zuführte. Aber nicht nur die Qualifizierung interessierte die amerikanischen Reformer am deutschen System, sondern auch der sozialintegrative Aspekt. Jugendliche wurden nahtlos von der Schule ins Berufsleben überführt. In zyklischen Abständen wurde die Debatte um das deutsche Berufsbildungssystem immer wieder geführt. Zuletzt gab der 1983 veröffentlichte Bericht „A Nation at Risk“ den Startschuß für eine erneute Diskussion über die Schwächen des amerikanischen Schulsystems und ihre Auswirkungen auf die Wettbewerbsfähigkeit der amerikanischen Wirtschaft. Nach Meinung der Autoren hatte dies einen Mangel an qualifizierten Arbeitern zur Folge. Die Debatte kulminierte in den frühen 90er Jahren, als Bill Clinton in seinem Präsidentschaftswahlkampf die Schaffung eines ,national apprenticeship system ' propagierte. Im ersten Jahr seiner Präsidentschaft unterzeichnete Clinton den School-to-Work Opportunities Act, der jedoch weit von einem nationalen Berufsausbildungssystem entfernt ist. Seitdem ist es wieder leiser geworden um das Fehlen einer qualitativ-hochwertigen Alternative für ,non-college bound“ Schüler. 
Sinkendes Interesse am deutschen dualen Berufsausbildungssystem erklärt sich aus dem angeschlagenen Image des deutschen Modells. Dadurch haben die Befürworter eines solchen Systems ihr bestes Argument - den offensichtlichen Erfolg der deutschen Wirtschaft - verloren.

Clintons Versuch, das deutsche Berufsausbildungssystem in die USA zu übertragen, dient dieser Arbeit als Aufhänger für eine grundsätzliche Untersuchung, wieso es trotz zahlreicher Anläufe kein nationales duales Berufsausbildungssystem in den USA gibt. Theoretisches Fundament bilden dabei wohlfahrtsstaatliche Erklärungsansätze, da berufliche Bildung in den USA anders als in Deutschland als eine im weitesten Sinne sozialpolitische Maßnahme angesehen wird. Berufsbildungsprogramme sollen die Arbeitsplatz- und Verdienstchancen benachteiligter Bevölkerungsgruppen verbessern, damit diese ihren eigenen Lebensunterhalt verdienen können und keiner finanziellen Transferleistungen durch den Staat bedürfen. Diese Politik geht von der Idee der ,equality of opportunity“ aus, nach der allen Bürgern die gleichen Startchancen gewährt werden sollen. Sozial- und wirtschaftspolitische Aspekte liegen im amerikanischen Fall der Berufsbildung eng zusammen. Sie sind gewissermaßen eine Symbiose eingegangen, denn ,gute“ Wirtschaftspolitik schafft arbeitsproduktive Arbeitsplätze und reduziert insofern die Notwendigkeit staatlicher Sozialleistungen. Aber auch das deutsche Berufsbildungssystem enthält sozialpolitische Aspekte, die jedoch nur von Zeit zu Zeit nämlich bei hoher Jugendarbeitslosigkeit - betont werden. Und geht man in der Geschichte des deutschen Systems zurück, so stellt man fest, daß die Argumente, die zu seiner Wiederbelebung am Ende des 19. Jahrhunderts führten, sowohl gesellschafts- als auch wirtschaftspolitisch geprägt waren. Zudem scheint die Bezugnahme auf wohlfahrtsstaatliche Theorieansätze insofern gerechtfertigt, als der gewählte Ansatz des historischen Institutionalismus, in der spezifischen Ausprägung von Theda Skocpol, eine weitreichende Flexibilität aufweist, um auch berufsbildungspolitische Entwicklungen zu analysieren. Als Methode liegt dieser Arbeit eine qualitative, in Ansätzen komparatistische Untersuchung zugrunde, die mit Ausnahme einiger amerikanischer Regierungsquellen und Interviews mit dem Vizepräsidenten der amerikanischen ,non-profit'-Organisation ,Jobs for the Future' und Berufsschullehrern einer Vocational High School in Massachsusetts auf der Analyse von Sekundärliteratur basiert. 
Die Arbeit ist folgendermaßen gegliedert: Zunächst werden wichtige wohlfahrtsstaatliche Theorieansätze und die jeweiligen Gründe dargestellt, die zu ihrer Ablehnung oder auch Annahme geführt haben. Im darauffolgenden Teil findet sich eine Darstellung des deutschen dualen Berufsausbildungssystems, seiner historischen Entstehung und heutigen institutionellen Struktur. Dieser Teil bietet die Grundlage für die folgende Untersuchung des Scheiterns der dualen Berufsausbildung in den USA. Das deutsche duale Berufsausbildungssystem dient sozusagen als „Kontrastfolie“ der Arbeit, die sich der Leser immer wieder vor Augen halten sollte, um den amerikanischen Fall verstehen zu können. Der vierte Teil stellt den Hauptteil der Arbeit dar, in dem die ein duales Berufsausbildungssystem betreffenden Institutionen und Interessen für den amerikanischen Fall untersucht werden. Ausgangspunkt bildet dabei die Entstehung des amerikanischen Schulwesens und seine Bedeutung für die staatliche Institutionalisierung. Um den aktuellen Bezug herzustellen, setzt sich der fünfte Teil mit Clintons School-to-Work Opportunties Act auseinander. Den Abschluß der Arbeit bilden eine Zusammenfassung und ein Ausblick auf mögliche Vorteile des spezifisch amerikanischen Weges beruflicher Bildung.

Zum Schluß noch ein Bearbeitungshinweis: Bei Quellen aus dem Internet, die in HTML- und nicht in PDF-Format vorliegen, können keine exakten Seitenangaben gemacht werden. Um trotzdem einen möglichst genauen Verweis bieten zu können, hat der Verfasser in solchen Fällen auf die Angabe von Kapiteln zurückgegriffen. 


\section{Teil I: Theoretische und methodische Grundlagen}

Inwieweit ist Berufsbildung ein integraler Bestandteil des Wohlfahrtsstaates? Und wie entsteht wohlfahrtsstaatliche Politik? Verschiedene theoretische Ansätze sollen eine Antwort darauf geben: Die Industrialisierungsthese war einer der frühesten Versuche, das empirische Phänomen umfassender Wohlfahrtsstaaten $\mathrm{zu}$ erklären. Ihre Eindimensionalität ließ jedoch eine Analyse der Verschiedenartigkeit wohlfahrtsstaatlicher Programme nicht zu. Diese Schwachstelle wollte der Machtressourcenansatz beheben, indem er den Klassenkonflikt und später die politische Koalitionsbildung als ursächliche Kraft für die Ausgestaltung wohlfahrtsstaatlicher Programme konstatierte. Dabei konzentrierte er seine empirischen Untersuchungen auf die nordeuropäischen Wohlfahrtsstaaten, wodurch sich sein Blickfeld verengte. Gerade die USA fallen bei der Entstehung wohlfahrtsstaatlicher Programme aus dem klassisch-theoretischen Rahmen, weshalb ein flexiblerer und komplexerer Ansatz gewählt werden muß. Dieser findet sich im historischen Institutionalismus, wie ihn Theda Skocpol auf die USA anwendet.

\section{Berufsbildung als integraler Bestandteil von Wohlfahrtsstaatlichkeit}

Berufsbildung als eine sozialpolitische Aufgabe des Staates zu definieren, bedeutet gerade für den deutschen Beobachter eine Aufweichung bzw. Ausweitung des klassischen Verständnisses von Sozialpolitik. Neben der bedarfsorientierten Sozialhilfe sowie der steuerfinanzierten (Beamten-)Versorgung sind es die verschiedenen Sozialversicherungssysteme - wie Unfall-, Kranken- und Rentenversicherung -, die den Kern bundesdeutscher Sozialpolitik ausmachen. Auch in den USA gelten die beitragsfinanzierten Sozialversicherungen und unterschiedlichen ,public assistance'-Programme wie z.B. Aid for Dependent Children (AFDC) - als originäre sozialpolitische Betätigungsfelder. Der Begriff der, welfare’ umfaßt jedoch lediglich letztere.

Inwieweit läßt sich nun die Berufsbildung unter den sozialpolitischen Aufgabenkatalog des Staates subsumieren? Dazu ist zunächst eine idealtypische Bestimmung von Sozialpolitik bzw. Wohlfahrtsstaatlichkeit als umfassenderer Kategorie vorzunehmen. Anhand verschiedener Lebensrisiken, denen Jugendliche beim Übergang von der Schule ins Erwerbsleben begegnen, wird deutlich, wieso Berufsbildung ein integraler 
Bestandteil von Wohlfahrtsstaatlichkeit ist und damit in den sozialpolitischen Aufgabenbereich des Staates fällt.

Abgesehen von frühen Formen der privaten und kirchlichen Wohlfahrt ist Sozialpolitik und somit die Idee der Wohlfahrtsstaatlichkeit eine Erscheinung der modernen Nationalstaaten. „In modernen Gesellschaften gelten die Behandlung der Kranken, die Ausbildung der Unwissenden und die Unterstützung der Armen als ein kollektives Anliegen: Sie fallen in die Domäne der bürokratischen Verwaltung unter der Herrschaft des Nationalstaates.“ (Swaan 1993, S. 11) Die industrielle Modernisierung des 19. Jahrhunderts brachte auch die ,soziale Frage' mit sich: die Lohnabhängigkeit einer wachsenden Zahl von Erwerbstätigen, die durch Verdienstausfall aufgrund der Lebensrisiken Alter, Invalidität, Krankheit oder Arbeitslosigkeit besonders betroffen waren. Das neue Phänomen der Proletarisierung war eine offensichtliche Folge dieses sozioökonomischen Wandels. Das einzelne Individuum sah sich außerstande, diese Risiken alleine zu tragen. Die Antwort war eine Kollektivierung der Vorsorge in Form solidarischer Risikogemeinschaften: Anfangs in Gestalt von Arbeiterhilfskassen oder Versicherungsvereinen auf Gegenseitigkeit, die langfristig aber durch eine staatlich organisierte Zwangsvergemeinschaftung abgelöst wurden. In Deutschland erfolgte dies in den 1880er Jahren durch die Verabschiedung der Gesetze über die Unfall-, Krankensowie Invaliditäts- und Rentenversicherung. Die USA folgten erst 1935 mit dem Social Security Act nach.

Ein wesentliches legitimierendes Prinzip staatlicher Sozialpolitik ist die Gewährleistung sozioökonomischer Sicherheit: ,,...] understand the welfare state as an answer to the growing needs and demands for socioeconomic security in the context of an increasing division of labor, the expansion of markets, and the loss of 'security functions' by families and other communities." (Flora/Heidenheimer 1982, S. 8) Die institutionelle Ausgestaltung dieses Prinzips kann jedoch ganz unterschiedlich erfolgen - entweder in Form einer Minimalabsicherung oder der Statussicherung bzw. Besitzstandswahrung (vgl. Ostner 1997, S. 74 f.). Harold L. Wilensky umschreibt das Prinzip sozialer Sicherheit so: „The essence of the welfare state is government-protected minimum standards of income, nutrition, health, housing and education, assured to every citizen as a political right, not charity." (1975, S. 6) Daneben gibt es ein weiteres Grundprinzip wohlfahrtsstaatlicher Intervention, das im Gegensatz zum ersten aber umstrittener ist: die Verwirklichung von Gleichheit. $\mathrm{Zu}$ unterscheiden ist dabei zwischen Ergebnis- 
(equality of results) und Chancengleichheit (equality of opportunity). Während das Prinzip der Ergebnisgleichheit - also der Angleichung aller dem einzelnen zur Verfügung stehenden Ressourcen - sozialistischem Gedankengut entstammt, ist das Prinzip der Chancengleichheit eine wesentliche Komponente liberaler Ansätze. Durch die Betonung von Leistung legitimiert das Prinzip der Chancengleichheit die Entstehung von (Ergebnis-)Ungleichheit, z.B. in Form von Einkommens- und Statusunterschieden. So ist das deutsche Sozialversicherungssystem insofern meritokratisch, als seine Geldleistungen lohn- und beitragsbezogen sind. Vergleichbares findet sich im amerikanischen System der ,social security'. Beide Prinzipien - soziale Sicherheit und Gleichheit - interagieren miteinander, wobei sie sich ergänzen oder auch widersprechen können. Unabhängig von ihrer realtypischen Ausgestaltung tragen sie jedoch zur Verwirklichung sozialer Inklusion im Sinne sozialer Teilhaberechte (vgl. Marshall 1964) bei.

Handelt es sich bei den „klassischen“ sozialpolitischen Instrumenten - Sozialversicherung und Sozialhilfe - um reaktive Maßnahmen zur Umsetzung sozialer Sicherheit, so gilt für die Berufsbildung, daß sie bereits im Vorfeld- im Sinne des ,enabling' - aktiv zur langfristigen Absicherung sozioökonomischer Risiken, insbesondere von Erwerbslosigkeit, beiträgt. Darüber hinaus verwirklicht die Berufsbildung das Prinzip der Chancengleichheit, da sie den Jugendlichen gleiche „Startbedingungen“"verschafft.

Betrachtet man die Situation des Übergangs von der Schule in den Arbeitsmarkt - und somit die „Startbedingungen“ -, so zeichnet sich für die beiden Länder Deutschland und USA ein extrem konträres Bild ab. Folgende Fragen sind dabei zu berücksichtigen: Wieviele Jugendliche verlassen das Schulsystem ohne irgendeine Qualifikation? Wie groß ist das Arbeitslosigkeitsrisiko? Welche Karrierewege ergeben sich? Und welche langfristigen Konsequenzen haben schlechte „Startbedingungen“?

Jugendliche ohne einen schulischen Abschluß sind einem besonders hohen Risiko von Erwerbslosigkeit und sozialer Exklusion ausgesetzt. Dieses Risiko hat sich in den letzten Jahrzehnten unter den Bedingungen gestiegener Qualifikationserfordernisse sogar noch erhöht. In den USA waren ,high-school drop outs', die in den frühen 1980er Jahren auf den Arbeitsmarkt drängten, mit einer größeren Wahrscheinlichkeit arbeitslos und auf Teilzeit-Jobs angewiesen als diejenigen, die in den späten 1960er Jahren in den Arbeitsmarkt eintraten. Die Zahl der Schulabbrecher ergibt sich näherungsweise aus 
dem Anteil der Jugendlichen (16-19 Jahre), die nicht mehr die Schule besuchen: Für die USA betrug dieser Anteil im Jahr 1997 insgesamt ca. 22\%. Damit war er im Vergleich zum Jahr 1987 um wenige Prozentpunkte gefallen. In Deutschland waren im Jahr 1996 lediglich um die 11\% der Jugendlichen ohne Schulabschluß. Auch hier gab es im Vergleich zum Jahr 1987 eine geringe Absenkung. Diese Gruppe der Jugendlichen zeichnet sich häufig dadurch aus, daß ihnen nicht nur - für den Arbeitsmarkt wesentliche Basisqualifikationen fehlen, sondern daß sie auch durch ihren familiären Kontext benachteiligt sind. Langfristig haben sie ein erhöhtes Risiko, von sozialer Exklusion betroffen zu sein (vgl. OECD 1999, S. 10 f.).

Aber nicht nur die Gruppe der Schulabbrecher ist von Arbeitslosigkeit betroffen. Alle Jugendlichen stehen beim Übergang von der Schule in den Arbeitsmarkt einem hohen Arbeitslosigkeitsrisiko gegenüber. Auch hier hat sich in den letzten Jahrzehnten die Situation verschlechtert, wobei die Entwicklung in Deutschland und den USA unterschiedlich verlaufen ist. In Deutschland stieg die Arbeitslosenrate jugendlicher Männer (15-19 Jahre) von einem sehr niedrigen Niveau im Jahr 1979 (2,4\%) um über das Dreifache im Jahr 1997 (8,3\%). Damit lag die Arbeitslosenrate aber immer noch um die Hälfte unter der in den USA, wo sie zwischen 1979 und 1997 von 15,9\% auf 16,9\% angestiegen ist (vgl. OECD 1999, S. 74). In beiden Ländern war jedoch der Anteil der Jugendlichen, die 1997 länger als 12 Monate arbeitslos waren, sehr gering. Die Mehrzahl der Jugendlichen ist also nicht von Langzeitarbeitslosigkeit betroffen, sondern von einer Vielzahl kurzer Arbeitslosigkeitsphasen. In den USA sind Phasen der Arbeitslosigkeit für Jugendliche in den ersten fünf Jahren nach Verlassen der Schule sehr viel häufiger als für Jugendliche in Deutschland (vgl. ebd., S. 79-80).

Probleme des Übergangs von der Schule in den Arbeitsmarkt zeigen sich nicht nur in der Dauer der Arbeitslosigkeit, sondern auch in der Art der Beschäftigung, die sich an die (Schul-)Ausbildung anschließt: z.B. niedrig bezahlte Teilzeit-Jobs oder befristete Arbeitsverhältnisse. So befanden sich 1994 63\% aller vollbeschäftigten Jugendlichen (unter 25 Jahren) in den USA in niedrig bezahlten Beschäftigungsverhältnissen. In Deutschland lag der Anteil im selben Jahr bei 50,4\%, wobei zu berücksichtigen ist, daß es sich hier in der Mehrzahl um Lehrverhältnisse mit entsprechend niedrigerem Lohn handelt. Dies spiegelt sich auch in der Wahrscheinlichkeit wider, innerhalb einer fünfjährigen Periode (1986-1991) den Status des niedrig bezahlten Beschäftigten zu verlassen. Im Jahr 1986 lag die Wahrscheinlichkeit für Jugendliche in Deutschland, 
niedrig bezahlt zu sein, viermal so hoch wie für alle Beschäftigten. Über den gesamten Zeitraum betrachtet ergab sich aber eine beinahe gleich hohe Wahrscheinlichkeit für Jugendliche und alle anderen Beschäftigten. In den USA waren 1986 beinahe die Hälfte aller Jugendlichen in niedrig bezahlten Beschäftigungsverhältnissen; 61\% hatten innerhalb der fünf Jahre wenigstens einmal einen Niedriglohn-Job, und 14,4\% blieben den gesamten Zeitraum über in niedrig bezahlter Beschäftigung. In Deutschland traf dies nur auf 1,8\% der Jugendlichen zu (vgl. ebd., S. 16 f. und 75).

Zusammenfassend läßt sich also feststellen, daß der Anteil der Jugendlichen, die die Schule abbrechen, in Deutschland rund halb so groß ist wie in den USA. Ein ähnliches Bild zeigt sich bei der Jugendarbeitslosigkeit, die in den USA nicht nur doppelt so hoch ist, sondern im Zeitverlauf auch mehr Jugendliche betrifft. Darüber hinaus befinden sich in den USA mehr Jugendliche in niedrig bezahlten Beschäftigungsverhältnissen als in Deutschland. Durch die häufig wiederkehrenden Phasen von Arbeitslosigkeit und die damit verbundenen Arbeitgeberwechsel ${ }^{1}$ sind Jugendliche in den USA einem größeren Risiko ausgesetzt, ihr Humankapital zu verlieren. Es besteht die Gefahr eines ,lock in'Prozesses, an dessen Ende ein Ausstieg aus der Niedriglohnbeschäftigung nicht geschafft wird. Das duale Berufsbildungssystem in Deutschland scheint dagegen den Übergang von der Schule in den Arbeitsmarkt zu erleichtern: die niedrigere Arbeitslosenrate und insbesondere die geringere Anzahl von kurzen Arbeitslosigkeitsphasen deuten daraufhin, daß Jugendliche in Deutschland schneller in den Arbeitsmarkt integriert werden. Dadurch reduziert sich auch die Gefahr einer langfristigen Niedriglohnbeschäftigung.

Anhand der Daten wird deutlich, daß Jugendliche beim Übergang von der Schule in den Arbeitsmarkt besonderen Risiken ausgesetzt sind. Arbeitslosigkeit, Niedriglohnbeschäftigung und prekäre Beschäftigungsverhältnisse gefährden die soziale Sicherheit der Jugendlichen, denen die sozialen Sicherungssysteme aufgrund ihrer Beschäftigungssituation in der Regel verschlossen bleiben. Langfristig führt ein Scheitern der Integration in den Arbeitsmarkt häufig zu sozialer Exklusion. Berufsbildung soll die ,employability' der Jugendlichen erhöhen, um ihre Einstiegschancen in den Arbeitsmarkt zu verbessern und den Zugang zu stabilen, aufstiegsorientierten Beschäfti- 
gungsverhältnissen zu ermöglichen. Insofern dient Berufsbildung der Verwirklichung sozialer Sicherheit und Chancengleichheit und ist damit integraler Bestandteil von Wohlfahrtsstaatlichkeit. verschiedene Jobs zwischen seinem 18. und 32. Lebensjahr (vgl. Lynch 1999, S. 290). 


\section{Industrialisierungsansatz}

Der Versuch einer umfassenden Erklärung der Evolution von Industriegesellschaften führte in der Wohlfahrtsstaatsforschung zur Industrialisierungsthese. Harold Wilensky, ein bedeutender Vertreter dieses Ansatzes, faßte die grundlegende Idee folgendermaßen zusammen: „Over the long pull, economic level is the root cause of welfare state development...“ (Wilensky 1975, S. 47). Fortschreitende Industrialisierung und der daraus resultierende Wandel der Gesellschaften von Agrar- zu Industriegesellschaften erzeugen neue soziale Bedürfnisse, die durch bestehende, traditionelle Sicherungssysteme, wie Familie und Kirche, nicht aufgefangen werden können. Diese traditionellen Sicherungssysteme sind selber durch den Industrialisierungsproze $ß$ in Auflösung begriffen. Die den Industrialisierungsprozeß charakterisierenden sozioökonomischen Veränderungen sind das enorme Wachstum der Bevölkerung und die damit einhergehende Urbanisierung, effizienzsteigernde Arbeitsteilung, die Entstehung einer lohnabhängigen Arbeiterklasse, die konjunkturbedingter Arbeitslosigkeit ausgesetzt ist, und die Auflösung der Großfamilienstruktur (vgl. Pierson 1991, S. 15). Aufgrund dieser Veränderungen entstehen neue soziale Bedürfnisse, die wiederum eine kollektive Nachfrage nach staatlichen Maßnahmen hervorrufen, um die überforderten traditionellen Sicherungssysteme zu entlasten. So birgt die durch die Industrialisierung geschaffene Lohnabhängigkeit großer Bevölkerungsschichten neue Risiken in sich: Arbeitsunfälle, Krankheit und Arbeitslosigkeit bedrohen die Existenz derjenigen, die auf Lohn angewiesen sind, um sich und ihre Familie ernähren zu können, und denen andere Ressourcen verwehrt sind. Staatliche Maßnahmen werden eingefordert, um ein Minimum sozialer Sicherheit zu erhalten. Doch nicht nur individuelle Absicherung dient als Argument für staatliche Eingriffe, sondern auch die Nachfrage nach gesunden Arbeitern und deren Reproduktion im Prozeß der Industrialisierung (vgl. Weir/Orloff/Skocpol 1988, S. 10; Pierson 1991, S. 15). Auf der anderen Seite führt die Industrialisierung zu einem Anstieg der vorhandenen ökonomischen Ressourcen, die es dem Staat ermöglichen, den finanziellen Bedarf neuer sozialer Sicherungssysteme abzudecken. Der Wohlfahrtsstaat ist mithin sowohl ein Produkt neuer Bedürfnisse als auch neuer Ressourcen, beides generiert durch den Prozeß der Industrialisierung (vgl. Pierson 1991, S. 16; Skocpol 1995, S. 79). Vertreter der Industrialisierungsthese argumentieren im Sinne einer Konvergenz nationaler wohlfahrtsstaatlicher Systeme in Abhängigkeit vom Grad der erreichten Industrialisierung. „With economic growth all 
countries develop similar social security programs. Whatever their economic or political system, whatever the ideologies of elites or masses, the rich countries converge in types of health and welfare programs,...“ (Wilensky 1975, S. 86). Eine der Industrialisierung und dem ökonomischen Wachstum inhärente Logik läßt, trotz unterschiedlicher kultureller und politischer Traditionen, alle industriellen Gesellschaften konvergieren (vgl. Korpi 1983, S. 7). Dem Ansatz zufolge läßt sich sagen, daß die Entwicklung der Wohlfahrtsstaaten eine logische Folge der Industrialisierung darstellt, bei der staatliche Sozialpolitik eine Antwort auf neue soziale Bedürfnisse ist. Ermöglicht wird dies durch gestiegene finanzielle Ressourcen des Staates infolge wirtschaftlichen Wachstums.

Die Industrialisierungsthese wird insbesondere wegen ihrer Annahme einer deterministischen Beziehung zwischen wirtschaftlicher Entwicklung und staatlicher Sozialpolitik kritisiert. Die Identifikation neuer sozialer Bedürfnisse als Folge industrieller Entwicklung führe nicht notwendigerweise $\mathrm{zu}$ deren Befriedigung durch wohlfahrtsstaatliche Programme. Dies zeige sich beim Vergleich zweier Regionen mit ähnlicher industrieller Entwicklung, England und Massachusetts, die sich in ihrer wohlfahrtsstaatlichen Entwicklung wesentlich unterscheiden (vgl. Skocpol 1995, S. 79). Darüber hinaus bietet die Industrialisierungsthese keinen Erklärungsansatz für die tatsächliche Ausformung wohlfahrtsstaatlicher Programme. Andererseits liegt im qualitativen Vergleich wohlfahrtsstaatlicher Systeme die einzige Möglichkeit, Ursachen für die Schaffung spezifischer Programme und deren Fortentwicklung zu untersuchen (vgl. Pierson 1991, S. 18). Selbst Wilensky korrigierte seine These dahingehend, daß bei einer weitgehenden Angleichung der ökonomischen Entwicklungsstufe, wie sie in den westeuropäischen Industrienationen zu finden ist, die Wechselwirkung zwischen industrieller Entwicklung und Implementation einzelner wohlfahrtsstaatlicher Programme abgeschwächt wird: „Within Europe there was little connection between the level of industrialization and the initiation of welfare-state programs" (Wilensky et al. 1985, S. 79). Mit dieser Feststellung sinkt die Industrialisierung auf die Position einer beeinflussenden Variable ab, der der alleinige Erklärungsanspruch abhanden gekommen ist. Insbesondere die USA gelten als Ausnahmefall der Industrialisierungsthese, da sie trotz relativ früher Industrialisierung in der Schaffung staatlicher Sozialpolitik, wie Sozialversicherungsprogrammen, im Vergleich zu anderen Nationen zurückliegen. „This country was one of the world's industrial leaders, yet ,lagged“ far behind other nations..."(Skocpol 1995, S. 15). 
Bezogen auf die Berufsausbildung fällt dem Prozeß der Industrialisierung insofern eine bedeutende Rolle $\mathrm{zu}$, als wirtschaftliches und technologisches Wachstum eine steigende Nachfrage nach gut qualifizierten Arbeitskräften hervorbringt. Jedoch führt diese Nachfrage nicht zu einer Konvergenz der Berufsausbildungssysteme unterschiedlicher Industriegesellschaften, da Differenzen in vorindustriellen Ausbildungssystemen - wie handwerklicher Lehrlingsausbildung - die Anpassung an neue Bedürfnisse beeinflussen. So ist zwar die Industrialisierung Voraussetzung für die Ausdehnung oder Schaffung eines Berufsausbildungssystems, aber nicht für die Form, die es annimmt. 


\section{Machtressourcenansatz}

Im Gegensatz zur Industrialisierungsthese ist für den Machtressourcenansatzes der Prozeß der Industrialisierung lediglich eine notwendige, aber noch keine hinreichende Erklärung für die Entstehung von Wohlfahrtsstaaten und deren spezifische soziale Sicherungsformen. Mit der Industrialisierung und Etablierung einer kapitalistischen Wirtschaftsordnung entstehen neue Ungleichverteilungen sowohl ökonomischer als auch politischer Macht. Die kapitalistische Klasse besitzt mit ihrer Kontrolle über Produktionsmittel und Kapital umfangreiche Machtressourcen, die sie gegen die Interessen der lohnabhängigen Arbeiterklasse einsetzen kann. Diese zunächst ökonomischen Machtressourcen eröffnen auch politische Vorteile, da sie als Druckmittel für die Durchsetzung eigener Interessen verwendet werden können. Mit dem Prozeß der Demokratisierung bietet sich die Möglichkeit für die Arbeiterklasse, durch kollektive Organisierung bestehende Machtungleichgewichte zu korrigieren. Es ist dieser Machtkampf zwischen den beiden Klassen und die Spannung im Verhältnis der Logik der Politik zur Logik des Marktes, die in ihrer jeweiligen Konstellation die Entwicklung und Form sozialer Bürgerrechte bedingt (vgl. Pierson 1991, S. 29). Der Wohlfahrtsstaat ist für die Vertreter des Machtressourcenansatzes ein Produkt der kollektiven Organisation der Arbeiterklasse und der politischen Macht von Parteien, die für die Interessen der Arbeiterklasse eintreten. Entscheidend für die Durchsetzung von Arbeiterinteressen ist die Fähigkeit ihrer Vertretungsorgane, Machtressourcen zu mobilisieren. Korpi (1983) definiert Machtressourcen als „,..characteristics which provide actors - individuals or collectivities - with the ability to punish or reward other actors“ (S. 15). Machtressourcen lassen sich ferner in zwei Kategorien unterteilen: Als ,basic power resources` bezeichnet Korpi einerseits die Kontrolle über Produktionsmittel und Kapital und andererseits die Organisation von Humankapital. Die Kontrolle über Produktionsmittel zeichnet sich dabei durch geringe Mobilisierungskosten aus, wohingegen Organisation und kollektives Handeln des Faktors Arbeit mit hohen Mobilisierungskosten verbunden sind. „The ,latent ${ }^{\star}$ interests of citizens, derived from their positions in the class structure, cannot, in themselves generate collective action“" (Korpi 1978, S. 314). Auf der anderen Seite ergeben sich durch soziopolitische Strukturen, wie Gesetze und Bürokratien, die den Prozeß der gesellschaftlichen Entscheidungsfindung und Konfliktregulierung bestimmen, sogenannte ,derived power resources‘ (vgl. Korpi 1983, S. 16). Diese ändern sich zugunsten der Arbeiterklasse bei 
einer Ausdehnung der sozialen Rechte, da die Abhängigkeit der lohnabhängig Beschäftigten vom Markt und von den Arbeitgebern sinkt.

Der Grad der Machtressourcen der Arbeiterklasse wird von folgenden Faktoren beeinflußt: Erstens ist die Beziehung zwischen den Gewerkschaften und linken Parteien von entscheidender Bedeutung. Insbesondere wenn es sich um eine enge Kooperation zwischen einer linken Partei und den Gewerkschaften handelt, erhöht sich der Grad der Machtressourcen der Arbeiterbewegung. Andererseits sinkt der Umfang der Machtressourcen, wenn mehrere linke Parteien politische Macht im Interesse der Arbeiterbewegung anstreben. Ein weiterer Faktor ist der Grad der gewerkschaftlichen Organisation der Arbeiter und die Kooperation zwischen den Gewerkschaften. Die Form der Industriegewerkschaft übt dabei im Gegensatz zur Berufsgewerkschaft einen positiven Einfluß auf den Umfang der Machtressourcen aus, da die Berufsgewerkschaft das Prinzip der Exklusivität beinhaltet und mithin tendenziell zur Fragmentierung der Arbeiterbewegung beiträgt. Schließlich ist die Ausweitung des Wahlrechts und damit die Teilnahme der Arbeiterklasse an Wahlen ausschlaggebend für den Umfang ihrer Machtressourcen (vgl. Korpi 1983, S. 39). Die Verteilung der Machtressourcen in einer Gesellschaft bestimmt letztlich die Allokation gesellschaftlicher Ressourcen.

Die herausragende Rolle der Arbeiterbewegung in der wohlfahrtsstaatlichen Analyse des Machtressourcenansatzes trifft insbesondere für die Konsolidierungsphase westlicher Wohlfahrtsstaaten nach dem Zweiten Weltkrieg zu. Durch die wachsende Bedeutung sozialdemokratischer Parteien und ihrer Kooperation mit Gewerkschaften wurde die Arbeiterbewegung zum Subjekt der Sozialpolitik und beeinflußte aktiv die Ausgestaltung sozialer Sicherungsprogramme. Bis 1945 konnte die Arbeiterbewegung lediglich als Objekt staatlich-paternalistischer Sozialpolitik betrachtet werden (vgl. Esping-Andersen/Korpi 1984, S. 180). Aber gerade in jener Phase wurden die grundlegenden Institutionen der Wohlfahrtsstaaten geschaffen. Diese frühe Entwicklung der Wohlfahrtsstaaten fand fast ausschließlich unter liberaler oder konservativer politischer Führung statt, ohne direkte Beteiligung von Gewerkschaften. Indirekt übten sie durch ihre bloße Existenz Einfluß auf die grundsätzliche Institutionalisierung wohlfahrtsstaatlicher Programme aus, da die gewerkschaftliche Organisierung der Arbeiterklasse nach Ansicht der politischen Eliten eine Bedrohung der gewachsenen Sozialordnung darstellte. Deswegen widersetzten sich die Gewerkschaften zunächst staatlicher Sozialpolitik, die ihrer Ansicht nach gewerkschaftseigene Formen sozialer 
Unterstützung unterminierte und als Mittel zur Lohndrückung instrumentalisiert werden konnte.

Der Machtressourcenansatz basiert darüber hinaus auf der Annahme einer homogenen Arbeiterklasse, obgleich es bereits früh Unterschiede zwischen qualifizierten und unqualifizierten Arbeitern gab, die sich in der Organisation qualifizierter Arbeiter in sogenannten ,friendly societies“ manifestierten. Der niedrige Lohn unqualifizierter Arbeiter und deren beruflicher Status erlaubte es ihnen nicht, sich bei diesen Versicherungsvereinen gegen Einkommensausfälle und Krankheit abzusichern. Daraus ergaben sich unterschiedliche Interessen der Arbeiter (vgl. Pierson 1991, S. 35). Die Idee einer linearen Korrelation zwischen Machtressourcen der Arbeiterklasse und Institutionalisierung des Wohlfahrtsstaates wurde später von Esping-Andersen modifiziert, der die Bedeutung der politischen Koalitionsbildung zwischen einer starken Arbeiterbewegung, deren politische Parteien in den seltensten Fällen eine Mehrheit im Parlament bildeten, und anderen sozialen Gruppen, insbesondere Bauern, hervorhob. „In summary, we have to think in terms of social relations, not just social categories“ (Esping-Andersen 1990, S. 18).

Die Entstehung staatlich-organisierter Wohlfahrt in den USA verdeutlicht die analytische Schwäche des Machtressourcenansatzes, dessen Referenzmodell der schwedische Wohlfahrtsstaat ist. Der geringe Grad gewerkschaftlicher Organisation und das Fehlen einer Arbeiterpartei erklären möglicherweise die Tatsache, daß die USA kein umfassender Wohlfahrtsstaat mit Vollbeschäftigungsgarantie geworden sind. Gleichzeitig versagen diese Kriterien aber bei der Erklärung wohlfahrtsstaatlicher Entwicklungen in den USA zum Ende des 19. Jahrhunderts, wie den , civil war pensions'. Hierfür sind andere sozio-ökonomische Interessen als erklärende Variablen einzuführen (vgl. Skocpol 1995, S. 18 f.). Neben den früheren Entwicklungen lassen sich gerade auch die Reformen der 1930er und 1960er Jahre nicht ausreichend durch den Machtressourcenansatz erklären. Im Gegensatz zur Annahme eines eminenten Klassenkonflikts prägen regionale und ethnische Differenzen sozialpolitische Entwicklungen in den USA weitaus stärker. „The distinctive dynamics of American social politics have been rooted in shifting political coalitions that include, but necessarily go beyond, business and labor" (Weir/Orloff/Skocpol 1988, S. 16). Gewerkschaften und Arbeitgeber sind sicherlich entscheidende Kräfte hinter der Gestaltung eines Berufsausbildungssystems. Eine direkte Beziehung zwischen 
gewerkschaftlichen Machtressourcen und beruflicher Bildung zu bestimmen, wäre aber eine $\mathrm{zu}$ eindimensionale Betrachtung. Andere Faktoren, wie institutionelle Rahmenbedingungen und Entwicklungspfade, beeinflussen die Organisation beruflicher Bildung über die politische Stärke der Arbeiterbewegung hinaus. 


\section{Historischer Institutionalismus}

Die Schwächen der ersten beiden wohlfahrtsstaatstheoretischen Ansätze, gerade in der Analyse des amerikanischen Wohlfahrtsstaates, führten zur Etablierung eines umfassenderen Ansatzes, der neben der Rolle von Interessengruppen im politischen Entscheidungsprozeß den institutionellen Rahmen ihres Agierens und den politischen Lernprozeß hervorhebt. Anders als beim skandinavisch-sozialdemokratisch zentrierten Machtressourcenansatz greifen die Vertreter des historischen Institutionalismus nicht bloß auf die Organisierung der Arbeiterklasse zurück, sondern weiten ihre Perspektive auf verschiedene Interessengruppen und ihre institutionell bedingte Interessensartikulation als Einflußvariable auf wohlfahrtsstaatliche Entwicklungen aus. „In general, historical institutionalists work with a definition of institutions that includes both formal organizations and informal rules and procedures that structure conduct" (Thelen/Steinmo 1992, S. 2).

\subsection{Allgemeine Überlegungen}

Seit den 1980er Jahren erlebt der Institutionalismus eine Renaissance in der sozialwissenschaftlichen Forschung. Als sogenannter Neo-Institutionalismus greift er Ansätze des „alten“ Institutionalismus auf, den z.B. Emile Durkheim und Max Weber sowie auch Karl Polanyi und Thorstein Veblen vertraten. Während für Durkheim symbolische Systeme, wie Religion und Kollektivbewußtsein, soziale Institutionen ${ }^{2}$ darstellen, betont Weber die Bedeutung von Regeln und Normen, worunter er sowohl habitualisierte Sitten als auch legal definierte Regelsysteme versteht, für die Ordnung sozialer Strukturen und die Strukturierung sozialen Verhaltens (vgl. Scott 1995, S. 9 ff.). Einige Neo-Institutionalisten kritisieren den „alten“ Institutionalismus wegen seiner normativen Ausprägung, die lediglich zu einer Gegenüberstellung institutioneller Konfigurationen der administrativen, legalen und politischen Strukturen in verschiedenen Ländern geführt hätte. Dabei hätten diese Arbeiten für einen Vergleich entscheidende intermediäre Kategorien vernachlässigt (vgl. Thelen/Steinmo 1992, S. 3).

2 „Tatsächlich kann man, ohne den Sinn dieses Ausdrucks zu entstellen, alle Glaubensvorstellungen und durch die Gesellschaft festgesetzten Verhaltensweisen Institution nennen; die Soziologie kann also 
Als Gegenreaktion zum Behaviorismus in der Politikwissenschaft entwickelten sich zwei neo-institutionalistische Strömungen: der ,rational choice'-Ansatz und der historische Institutionalismus (vgl. ebd., S. 7). Verallgemeinernd gesagt, steht hier ein „kalkulatorischer“ Ansatz einem „kulturalistischen“ gegenüber. Tatsächlich sollten diese beiden Ansätze eher als Pole auf einem Kontinuum angesehen werden, zwischen denen verschiedene fachdisziplinäre Ausprägungen $\mathrm{zu}$ finden sind: ,[...] there are as many ,new institutionalisms' as there are social disciplines [...].“ (DiMaggio/Powell 1991, S. 1)

Der ,rational choice'-Ansatz basiert idealtypischerweise auf einer universellen, nicht kontextspezifischen Rationalitätsannahme. Danach handeln die (politischen) Akteure im Sinne einer interessenbezogenen Nutzenmaximierung. Institutionen bilden dabei den strategischen Kontext, innerhalb dessen der einzelne Akteur jeweils rationale - seinen Nutzen maximierende - Wahlentscheidungen trifft. Ändern sich die Spielregeln (Institutionen), so ändern sich bei gleichbleibenden Präferenzen die Entscheidungen ${ }^{3}$ (vgl. Thelen/Steinmo 1992, S. 7; Thelen 1999, S. 375). Während der ,rational choice'Ansatz also von einer exogenen Bestimmung der Präferenzen bzw. Interessen ausgeht, postuliert der historische Institutionalismus eine sozial-konstruktivistische (endogene) Perspektive von Interessen: ,the definition of interests and objectives is created in institutional contexts and is not separable from them.“ (Zysman 1994, in: Thelen 1999, S. 375)

Der historische Institutionalismus weitet seine Analyse über das wechselseitige Verhältnis von Institutionen und Interessen hinaus auf die Frage der Interaktion von Ideen, Interessen und Institutionen aus. Institutionen sind dabei der Transmissionsriemen zwischen Ideen und Interessen (vgl. Hall 1986, S. 280). Oder wie M. Rainer Lepsius es formuliert: „Ideen sind interessenbezogen, sie konkretisieren sich an Interessenlagen und erhalten durch diese Deutungsmacht. Institutionen formen Interessen und bieten Verfahrensweisen für ihre Durchsetzung, Institutionen geben Ideen Geltung in bestimmten Handlungskontexten.“ (Lepsius 1990, S. 7) Dabei interessieren sich

definiert werden als die Wissenschaft von den Institutionen, deren Entstehung und Wirkungsart.“ (Durkheim [1895]1961, S. 100)

${ }^{3}$ Dies läßt sich am klassischen Beispiel des ,rational choice'-Ansatzes, dem Gefangenendilemma, gut nachvollziehen. 
historische Institutionalisten insbesondere für das Zusammenspiel von Ideen, Interessen und Institutionen im Kontext institutioneller Formation: „It can be argued that when an idea becomes accepted and is embodied into a structural form then the institution has been created.“ (Peters 1999, S. 67) Einen vergleichbaren Erklärungsansatz wählte bereits Durkheim in seinem Werk „Physik der Sitten und des Rechts“, als er über die Entstehung des Konsensualvertrages folgendes schrieb: „Es reicht nicht aus, daß eine Institution nützlich ist, damit sie zur rechten Zeit aus dem Nichts auftaucht; es müssen auch die Mittel vorhanden sein, sie zu schaffen, das heißt, die vorhandenen Ideen müssen sie zulassen, und die bestehenden Institutionen dürfen sich ihrer Entstehung nicht widersetzen oder müssen sogar das für ihre Herausbildung erforderliche Material bereitstellen.“ (Durkheim 1991, S. 264) Diese „formative moments“ (Pierson 1993, S. 602) sind insofern von besonderer Bedeutung für historische Institutionalisten, als sie die weitere politische Entwicklung aufgrund der prägenden Kraft von Institutionen in eine bestimmte Richtung lenken. Hier kommt das Konzept der „Pfadabhängigkeit“ zum Tragen, das zum theoretischen Verständnis von ,policy continuities over time within countries and policy variation across countries“" (Thelen/Steinmo 1992, S. 10) beigetragen hat. Danach legen einerseits einmal geschaffene Institutionen (,formative moments") einen Pfad für das Handeln der Akteure fest. Dieser Pfad wird durch das institutionengeleitete Handeln der Akteure reproduziert. Andererseits beinhaltet das Konzept der „Pfadabhängigkeit“ auch die Idee, daß sich die Institutionen durch wandelnde Umwelteinflüsse und politische Konflikte - ,constrained by past trajectories“ (Thelen 1999, S. 387) - weiterentwickeln. „Path dependency in this view is not a mortmain on institutions and their policies. [...] There will be change and evolution, but the range of possibilities for that development will have been constrained by the formative period of the institution." (Peters 1999, S. 65)

Einige historische Institutionalisten konzentrieren sich dabei auf die institutionelle Eigendynamik und die positiven Rückkopplungsprozesse, die sie mit dem Begriff ,policy feedback' (vgl. Heclo 1974) umschreiben: ,[...] it [path dependency; Verf.] refers implicitly to the dynamics of self-reinforcing or positive feedback processes in a political system [...].“ (Pierson/Skocpol 2000, S. 10) Akteure passen ihre Strategien im Sinne der ,increasing returns“ an den durch die Institutionen geschaffenen Anreizstrukturen an und bestätigen damit die den Institutionen innewohnende Logik. Gleichzeitig beeinflussen Institutionen die politische Machtverteilung. Dadurch werden 
bestimmte Gruppen gestärkt, die wiederum zur institutionellen Reproduktion beitragen (vgl. Thelen 1999, S. 392 ff.).

Der historische Institutionalismus hat seine Stärken als ,a theory upon stability and continuity“ (Peters 1999, S. 68). Wie selbst Protagonisten des historischen Institutionalismus anmerken, mangelt es ihm aber an Erklärungsgehalt bezüglich Prozessen des institutionellen Wandels: ,[...] institutions explain everything until they explain nothing. Institutions are an independent variable and explain political outcomes in periods of stability, but when they break down, they become the dependent variable [...]." (Thelen/Steinmo 1992, S. 15) Die Idee der „critical junctures“ (vgl. Peters 1999, S. 69) versucht, diese Phasen der institutionellen Neuorientierung begrifflich zu fassen und zu erklären. Demnach kommen in solchen historisch kritischen Augenblicken verschiedene interne und/oder externe Faktoren zusammen, die das „,natürliche“ Beharrungsvermögen der Institutionen aus dem Gleichgewicht bringen und so „windows of opportunity“ schaffen, die die Möglichkeit eines Pfadwechsels eröffnen. Problematisch ist dabei jedoch, daß das Vorhandensein eines, critical juncture' lediglich ex post festgestellt werden kann. Dies läßt eine Prognostizierbarkeit institutionellen Wandels nicht zu (vgl. ebd., S. 68 f.).

Die vorliegende Arbeit untersucht den politischen Versuch, eine dem dualen Berufsbildungssystem vergleichbare Berufsausbildung in den USA zu institutionalisieren. Obgleich damit scheinbar die Frage des institutionellen Wandels im Mittelpunkt steht, liegt die Betonung der Arbeit - aufgrund des „negativen“ politisch-institutionellen Ergebnisses - auf der Analyse des institutionellen Beharrungsvermögens und der „policy continuities over time“. Damit nutzt sie die theoretische Stärke des historischen Institutionalismus in diesem Bereich und entgeht der Gefahr, ihren Erklärungsansatz auf „theoretischen Sand“ gebaut zu haben.

Im folgenden werden die Ausführungen zum historischen Institutionalismus insofern spezifiziert, als eine bestimmte Variante dieses theoretischen Ansatzes vorgestellt wird. Zur Untersuchung der wohlfahrtsstaatlichen Entwicklung der USA fügt Theda Skocpol einzelne Elemente des historischen Institutionalismus zu einem kohärenten Konzept zusammen. Diesen auf den amerikanischen Fall abgestimmten Ansatz nutzt die vorliegende Arbeit, um die Entwicklung der amerikanischen beruflichen Bildung nachzuzeichnen und Erklärungen anzubieten. 


\subsection{Skocpols historisch-institutionalistischer Ansatz}

Theda Skocpol, eine Vertreterin des historischen Institutionalismus, definiert die Entstehung des amerikanischen Wohlfahrtsstaates als Sonderweg. Dabei stützt sie ihre Argumentation auf drei Faktoren: ,state formation', also im engeren Sinne die historische Entwicklung eines staatlich-institutionellen Rahmens, ,institutional leverage` oder der politische Einfluß von Interessen in diesem institutionellen Rahmen und ,policy feedback' als Einfluß früherer Politiken und deren Erfolg oder auch Mißerfolg auf die Gestaltung neuer Politikvorhaben.

\subsubsection{Staatliche Formation}

Der Begriff der ,state formation“ umfaßt makropolitische, historische Prozesse, wie Teilnahme an Kriegen, politische Demokratisierung, Verfassungsgebung und Bürokratisierung, deren Form und zeitliche Abfolge in den industrialisierten, kapitalistischen Staaten höchst unterschiedlich waren (vgl. Skocpol 1995, S. 19; Weir/Orloff/Skocpol 1988, S. 17). Diese makropolitischen Prozesse etablieren und verändern staatliche Organisationen und Institutionen, durch die Akteure ihre politischen Ziele verfolgen können. Politische Organisationen werden in diesem Prozeß der staatlichen Formation mit Kapazitäten ausgestattet, die der Staat als Akteur für eigene Politikinitiativen nutzen kann. Staatliche Akteure, wie Politiker und Bürokraten, besitzen eigene Interessen, die einerseits zwar vom Lobbying verschiedener Interessengruppen beeinflußt werden, nicht aber nur deren Spiegelbild darstellen. Andererseits erweitert auch der internationale „Politikwettbewerb“ die Problemlösungsperspektive staatlicher Akteure (vgl. Skocpol 1992, S. 41 f.). Die Fähigkeit eines Staates, autonom agieren zu können, hängt dabei von folgenden Faktoren ab: Erstens von der Einbindung des Staates in transnationale Strukturen, die den Austausch von Politikkonzepten fördern und so Lösungen für nationale Probleme anbieten: ,,...constant awareness by administrators of foreign precedents and models of social policy“ (Skocpol 1993, S. 89). Darüber hinaus ist eine der Grundfunktionen jedes Staates die Aufrechterhaltung der inneren Ordnung, für deren Wahrung staatliche Akteure zwischen den unterschiedlichen Interessen autonom vermitteln müssen. Schließlich ist die Organisation einer umfassenden Bürokratie und die Schaffung einer von sozio-ökonomischen Interessen unabhängigen Beamtenschaft entscheidend für den Umfang staatlicher Kapazitäten zur Durchsetzung 
eigener Ziele gegen den Widerstand gesellschaftlicher Interessen: „,..civil service administrators...made more important contributions to social policy development than political parties or interest groups“ (ebd., S. 89). Die den staatlichen Akteuren zur Verfügung stehenden Kapazitäten beschränken die Umsetzung ihrer Interessen und mithin wohlfahrtsstaatliche Gestaltungsmöglichkeiten.

Am Beispiel der amerikanischen Entwicklung zeigt sich, welche Auswirkungen die staatliche Formation auf die Wohlfahrtsstaatsentstehung und die staatlichen Kapazitäten hat. Im Gegensatz zu vielen europäischen Staaten entstanden die USA aus der Revolution und dem Kampf gegen die Kolonialmacht Großbritannien. Diese Revolution war nicht bloß Ausdruck des Widerstandes gegen die Bevormundung durch eine äußere Gewalt, sondern auch Zeichen der Ablehnung zentralistisch-organisierter staatlicher Macht (vgl. Skocpol 1995, S. 20). Die kodifizierte Verfassung wurde gleichsam zum locus summae rerum potestatis und ersetzte so andere Formen der Identitätsstiftung: ,...the sovereign ideals of constitutionalism and the rule of law could reign impersonally above an economically expansionist and socially diverse country“ (ebd., S. 20). Aus dem Widerstand gegen die britische Kolonialmacht, deren politisches System und aus dem Fehlen einer absolutistisch-monarchischen Tradition entwickelten die USA eine über drei Ebenen (lokal, einzelstaatlich, bundesstaatlich) dezentralisierte, demokratische Staatsform. Die fehlende Institutionalisierung einer zentralisierten, bürokratischen Macht sollte wesentlich die Entwicklung wohlfahrtsstaatlicher Einrichtungen hemmen. So konnten die europäischen Staaten in ihren wohlfahrtsstaatlichen Bestrebungen auf eine gut funktionierende Bürokratie mit einer professionellen Beamtenschaft zurückgreifen. Diese bürokratischen Kapazitäten waren und sind von entscheidender Bedeutung für Planung, Implementation und Verwaltung der neu geschaffenen Sozialprogramme. „When state organizations adequate to the administrative tasks at hand are believed to be available, elites are more likely to respond to political incentives arising from popular mobilization...“ (Orloff 1988, S. 43). Darüber hinaus ist die zeitliche Abfolge von Bürokratisierung und Demokratisierung eines Staates von Bedeutung für die Form und Organisation der politischen Parteien. Während in Europa das allgemeine Wahlrecht dem Prozeß der Bürokratisierung folgte und daher politische Parteien eine geringe Chance hatten, ein Patronagesystem aufzubauen, führte die umgekehrte Abfolge der beiden Prozesse in den USA zur Institutionalisierung von Partei-Patronage. Stellenbesetzungen in der öffentlichen Verwaltung wurden von 
Parteien für parteipolitische Zwecke instrumentalisiert. Nicht Fachwissen zählte, sondern Loyalität $\mathrm{zu}$ einer Partei. Die frühe Institutionalisierung dieses Patronagesystems verhinderte Reformansätze zur Professionalisierung der Verwaltung, wie sie insbesondere in der ,Progressive Era' um die Jahrhundertwende gefordert wurden. Ein weiterer Grund für die späte Bürokratisierung der USA war die geopolitische Isolation, die die Unterhaltung einer umfangreichen stehenden Armee erübrigte (vgl. ebd., S. 43-45). Jedoch gab es Ausnahmen zu diesem Bild einer schwach ausgeprägten, mit geringen Kapazitäten ausgestatteten Bürokratie, wie die Landwirtschaftspolitik während des, New Deal ‘ zeigte. Das U.S. Department of Agriculture bezeichnet Skocpol als ,island of state strength in an ocean of weakness“ (Skocpol 1993, S. 91).

Neben der Partei-Patronage ist es die eminente Rolle der Gerichte, die die politische Entwicklung der USA im 19. Jahrhundert charakterisiert. Die Verfassung als Ort der Souveränität verlieh den Gerichten insofern politische Macht, als sie sich ein Entscheidungsmonopol über konstitutionelle Fragen aneigneten. Bereits Tocqueville erkannte die herausragende Stellung der Richter und ihren Einfluß auf die Politik: „Durch das Recht, Gesetze für verfassungswidrig zu erklären, dringt der amerikanische Richter in einem fort in die Tagespolitik ein“ (Tocqueville 1985, S. 170). So wurde die Schaffung neuer bundesstaatlicher Behörden häufig durch Gerichtsentscheidungen und Kompetenzstreitigkeiten behindert oder deren Befugnisse eingeschränkt. Reformorientierte Politiker adaptierten ihre politischen Strategien in der Arbeitsgesetzgebung entsprechend einer Gerichtsentscheidung des U.S. Supreme Court, die eine generelle Stundenbegrenzung der Arbeitszeit untersagte (Lochner Urteil von 1905). Um dieses Urteil zu umgehen, unterteilten sie die Arbeiterschaft in ,spezifische Klassen“. Für jede Klasse wurde anhand der jeweiligen spezifischen Kriterien ein Bedarf an öffentlicher Protektion festgelegt. Damit konnte die gerichtliche Ablehnung allgemeinverbindlicher Regelungen unterlaufen werden (vgl. Skocpol 1995, S. 99 f.). Zusammenfassend läßt sich sagen, daß die staatliche Formation der USA eine schwache bundesstaatliche Bürokratie hervorgebracht hat. Diese war insbesondere im 19. Jahrhundert durch Partei-Patronage gekennzeichnet. Darüber hinaus zeichnet sich die staatliche Kompetenzverteilung durch eine starke Fragmentierung aus, in der Bund, Einzelstaaten und Kommunen öffentliche Verantwortlichkeiten untereinander aufteilen. 


\subsubsection{Institutionelle Einflüsse}

Bestehende Institutionen definieren die Einflußmöglichkeiten von Interessengruppen auf den Politikprozeß und mithin die Entwicklung wohlfahrtsstaatlicher Programme. Inwieweit politisch-soziale Gruppen ihre Interessen im politischen Entscheidungsprozeß geltend machen können, wird durch den spezifischen institutionellen Rahmen, in dem sie agieren, bestimmt: ,...whole range of state and societal institutions that shape how political actors define their interests and that structure their relations of power to other groups“ (Thelen/Steinmo 1992, S. 2). Die Verbindung aus Interessengruppe und ihrem Zugang zu politischen Institutionen ermöglicht eine umfassende Analyse der Gründe des Erfolgs und des Scheiterns politischer Initiativen. Institutionelle Vorteile einzelner Gruppen werden deutlich und bieten Erklärungsansätze für sozialpolitische Entwicklungen. „The institutional arrangements of the state and political parties affect the capabilities of various groups to achieve self-consciousness, organize and make alliances“ (Skocpol 1992, S. 47). Die Fähigkeit, sich zu organisieren und eine Identität und politische Orientierung aufzubauen, ist die eine Seite, wie Institutionen und staatliche Strukturen Interessengruppen beeinflussen, die andere Seite ist die Kompatibilität zwischen Institution und Kapazität bzw. Identität einer Interessengruppe. Gerade die Kompatibilität definiert Zugang und Einfluß einer Interessengruppe auf den politischen Entscheidungsprozeß (vgl. ebd., S. 54). Dieser institutionell definierte Einfluß ist jedoch nicht statisch, sondern verändert sich mit dem politischen Prozeß und der Initiierung neuer Politiken: „...policies gradually change institutional constraints“ (Ashford 1986, S. 17). Der institutionelle Rahmen ist nicht bloß das Ergebnis des politischen Konflikts, sondern bestimmt wiederum die politischen Ziele und Kapazitäten der innerhalb seiner Grenzen agierenden Interessengruppen. Somit definieren Institutionen Machtverhältnisse und beeinflussen Interessen und Rollenwahrnehmungen der am politischen Diskurs beteiligten Akteure. „In this way, organizational factors affect both the degree of pressure an actor can bring to bear on policy and the likely direction of that pressure“" (Hall 1986, S. 19).

Die wohlfahrtsstaatliche Entwicklung der USA zeigt deutlich, wie Institutionen die Option kollektiver Aktion und politischer Koalitionsbildung beeinflussen und wie institutionell strukturierte Machtverhältnisse die Form des Wohlfahrtsstaates gestalten und dabei über die eindimensionale Perspektive des Antagonismus von Arbeit und Kapital hinausgehen. Das vor der Industrialisierung institutionalisierte Wahlrecht für 
alle weißen Männer und die daraus resultierende Etablierung eines Patronage-Systems führte zur Trennung von politischer Partizipation und von Arbeitsplatzkonflikten, so daß sich kein Klassenbewußtsein herausbilden konnte. Die Idee der Solidarität bezog sich daher häufig auf die ethnisch definierte Nachbarschaft, wo ein gemeinschaftliches Bewußtsein über soziale Statusgrenzen hinaus entstand. Das fehlende Klassenbewußtsein der Arbeiter wurde durch die fehlende Kooperation der Gewerkschaften mit einer spezifisch arbeiterorientierten politischen Partei verstärkt, die es aufgrund des frühen allgemeinen Wahlrechts und des Patronage-Systems in vergleichbarer Form zu europäischen Arbeiterparteien in den USA nicht gab. Darüber hinaus erschwerte die demokratisch-föderale Struktur der USA mit ihrer starken Position der Einzelstaaten die nationale Organisation sowohl kapitalistischer Interessen als auch der Arbeiterinteressen (vgl. Skocpol 1995, S. 25 f.; Weir/Orloff/Skocpol 1988, S. 21). „Nationally, American workers were left without organisational capacities to push for a social democratic programme..." (Skocpol 1995, S. 26).

Den Schwierigkeiten der Arbeiterinteressen, sich Zugang zu den politischen Institutionen und so Einfluß auf den politischen Entscheidungsprozeß zu verschaffen, standen andererseits die institutionell begünstigten Organisationen des Kapitals gegenüber. Diese hatten aufgrund des politisch-fragmentierten Systems der USA einen institutionellen Vorteil, da sie an vielen Punkten die Politik der Arbeiterinteressen blockieren konnten: „...during the early twentieth century business interests gained considerable negative leverage from the fact that forty-eight states, rather than one national government, were the loci for legislative struggles" (Skocpol 1992, S. 54-55). Die Arbeitgeberinteressen besitzen zwar weitreichenden negativen institutionellen Einfluß, aber um positive Politik betreiben zu können, fehlt ihnen mit Ausnahme der National Association of Manufacturers (NAM) eine umfassende Präsenz in den einzelnen Wahlbezirken. Interessengruppen, die über eine solche Verbreitung verfügen, nennt Skocpol „widespread federated interests“. Die amerikanischen Gewerkschaften fallen nicht in diese Kategorie, weil ihre Mitglieder auf einige Industriezentren konzentriert sind. Beispiele für „widespread federated interests“ sind Interessengruppen von Frauen und die Medical Association. Beide Gruppen organisieren sich entlang lokaler, einzelstaatlicher und bundesstaatlicher Hierarchien und sind daher in der Lage, institutionellen Einfluß auf allen Ebenen der politischen Struktur der USA auszuüben (vgl. ebd., S. 55). „In general, U.S. political structures allow unusual leverage to social 
groups that can, with a degree of discipline and consistency of purpose, associate many local political districts“ (ebd., S. 55). Gerade am Beispiel der Frauenverbände zeigt sich die Auswirkung des allgemeinen Wahlrechts für weiße Männer, das zu einer geschlechtsspezifischen Trennung der Gesellschaft führte und zwischen den Frauen ein gemeinsames politisches Bewußtsein entstehen ließ, vergleichbar dem Klassenbewußtsein der Arbeiter in Europa, die gleichermaßen vom politischen Wahlrecht ausgeschlossen waren. Hinzu kam ein allgemein höheres Bildungsniveau der Frauen in den USA als in anderen Nationen. Diese Kombination aus gemeinsamem politischen Bewußtsein und höherer Bildung begünstigte die Organisation in Verbänden, die aufgrund ihrer weiten Verbreitung die Initiierung sozialpolitischer Maßnahmen, insbesondere für Arbeiterinnen, Mütter und Kinder, positiv beeinflußten (vgl. Skocpol 1995, S. 27; Skocpol 1992, S. 50).

Auf der anderen Seite schafften es die amerikanischen Gewerkschaften nicht, die für sie ungünstig starke Position der Gerichte über eine Ausdehnung ihres Einflusses auf den Kongress zu überwinden, da sie regional konzentriert waren und das Patronage-System eine geschlossene Strategie verhinderte. „Leaders of local craft unions ... make deals with local political machines ... particularistic benefits as public jobs...“ (Skocpol 1995, S. 107). Mithin konnten die Gewerkschaften nur in Ausnahmesituationen (z.B. New Deal) Koalitionen mit anderen politischen Kräften, insbesondere der Demokratischen Partei, schließen, denen allerdings die nötige Stabilität für langfristige Strategien fehlten. Die politische Struktur der USA bietet sozialen Gruppen viele Ansatzpunkte, um einen negativen institutionellen Einfluß auszuüben. Andererseits benötigen Interessengruppen eine umfassende organisatorische Präsenz in den einzelnen Wahlbezirken, um positiven Einfluß geltend machen zu können. Diese „widespread federated interests“ besitzen dann umfangreiche institutionelle Einflußmöglichkeiten.

\subsection{3 ,Policy Feedback}

Die Dynamik dieses Erklärungsansatzes spiegelt sich gerade in der Idee des politischen Lernprozesses oder auch des ,policy feedback ${ }^{`}$ wider. Neben den Institutionen und ihrer Auswirkung auf die Einflußmöglichkeiten sozialer Gruppen generiert die Implementierung einer Politik Folgewirkungen auf zukünftige Politiken. Dabei ist jede Politik ein Produkt aus Lernprozessen, die durch die Erfahrungen bestehender Politiken in Gang gesetzt werden. Diese Erfahrungen können sowohl international als auch 
national begründet sein. Beim internationalen Austausch versuchen politische Reformer, sich die Erfahrungen ausländischer Politikansätze nutzbar zu machen und durch Politiktransfer Lösungen für eigene Probleme zu finden: ,,... contribution of reformers to adapting new policy responses consisted in percieving and transmitting foreign experience" (Heclo 1974, S. 310). Im nationalen Bereich gestaltet sich der politische Lernprozeß in zwei Bahnen: Einerseits werden politische Akteure durch implementierte Politiken dahingehend konditioniert, diese existierenden Lösungsansätze auf ähnlich gelagerte neue Probleme anzuwenden, wie sich in der Adaption der Sozialversicherung an neue Risiken feststellen läßt. ,,The incrementalism pervasive in policy making is one manifestation of the more general tendency to respond by analogizing“(ebd., S. 315).

Andererseits führt der politische Lernprozeß zur Nachfrage nach neuen Politiken, da jede implementierte Politik sogenannte, feedback effects' mit sich bringt. Dieses, policy feedback' übt seinen Einfluß in zweierlei Hinsicht aus: Erstens werden durch die Implementation einer neuen Politik und die dafür benötigten staatlichen Anstrengungen neue staatliche Kapazitäten geschaffen oder bereits bestehende transformiert. Veränderte staatliche Kapazitäten bieten wiederum die Option, auf dem einmal beschrittenen Pfad fortzufahren und neue Politikinitiativen einzuleiten. Zweitens beeinflußt eine neue Politik die Identität, Ziele und Fähigkeiten sozialer Gruppen, die sich auf die neuen Gegebenheiten in ihren politischen Strategien einstellen müssen (vgl. Skocpol 1992, S. 58). Darüber hinaus gibt es sowohl positive als auch negative Effekte, die eine Politik auf Politikvorschläge analoger Bereiche ausüben kann. So hatte die Einführung einer Rente für Bürgerkriegsveteranen in den USA positive Effekte auf die Organisation der Veteranen, die sich aufgrund ihrer kollektiven Identität zu einer gut organisierten und weitverbreiteten Interessengruppe entwickelten, und auf die Position der neugeschaffenen Administration, dem Bureau of Pensions, die in Kooperation mit dem Interessenverband der Veteranen eine aktive Politik in Richtung Verbesserung und Ausdehnung der Rente betrieb. Jedoch bedeutete die Tatsache, daß die Verwaltung der Rente in enger Verbindung zum Patronage-System stand, ein Hindernis für die Generalisierung der Rente, weil reformorientierte Akteure mit einer Ausdehnung der Veteranenrente eine Stärkung des Patronage-Systems befürchteten. Dieser negative Effekt der frühen Rentenpolitik verzögerte die Schaffung eines allgemeinen Rentensystems maßgeblich (vgl. ebd., S. 59 f.). 
Eine Politik ist dann erfolgreich, wenn sie die staatlichen Kapazitäten erweitert, um ihre Fortentwicklung zu gewährleisten, und wenn soziale Gruppen und politische Allianzen in die Lage versetzt werden, die Politik zu verteidigen oder sogar auszudehnen. Der Erfolg einer Politik kann somit gleichgesetzt werden mit ihrer prozessualen Weiterentwicklung. In der Dynamik des ,policy feedback‘ verliert Politik ihren Status als allein abhängige Variable und nimmt eine Doppelrolle als abhängige und unabhängige Variable ein: „,... what is normally considered the dependent variable (policy output) is also an independent variable..." (Heclo 1974, S. 315). Die Wahl einer Politik hat Auswirkungen auf die Optionen zukünftiger Entwicklungen und schafft neue Situationen, die die Nachfrage nach politischer Aktion stimulieren. Die Bedeutung der Effekte einer Politik auf nachfolgende Entscheidungen und Veränderungen erfordert bei der Analyse neuer sozialpolitischer Maßnahmen eine historische Betrachtungsweise, um den kontextuellen Zusammenhang erkennen zu können. Dabei wird deutlich, wie erfolgreich implementierte Politiken, aber auch Lernprozesse aufgrund gescheiterter Politik einen Rahmen für Reformen vorgeben.

Die Diskussion um die Entstehung der Wohlfahrtsstaaten hat gezeigt, daß sowohl der Industrialisierungs- als auch der Machtressourcenansatz Schwächen, insbesondere im Hinblick auf den amerikanischen Wohlfahrtsstaat, aufweisen. So bietet die Analyse von Sozialbudgets und Industrialisierungsniveaus wenig Aufschluß über qualitative Ausformungen des jeweiligen Wohlfahrtsstaates, die einen Vergleich hochindustrialisierter Nationen mit ähnlichem Sozialbudget erst sinnvoll machen. Andererseits beeinträchtigt die sozialdemokratisch-skandinavische Zentrierung des Machtressourcenansatzes die Beweiskraft dieses Ansatzes. Für die Analyse kontinentaleuropäischer Wohlfahrtsstaaten hat der Machtressourcenansatz eine neue Perspektive eröffnet, die Licht in die Erklärung wohlfahrtsstaatlicher Entwicklungen geworfen hat. Im Falle der USA bedeutet hingegen das Fehlen eines ausgeprägten Klassenbewußtseins und die extrem schwache gewerkschaftliche Organisierung, daß der Machtressourcenansatz die Entstehung früher Formen der Sozialpolitik (z.B. Veteranenrente) nicht erklären kann. Der von Skocpol gewählte Ansatz zur Analyse des amerikanischen Wohlfahrtsstaates versucht im Gegensatz dazu, durch das Zusammenspiel von drei Faktoren einen umfassenden Erklärungsrahmen zu schaffen. Innerhalb dieses Rahmens kann jede sozialpolitische Maßnahme entsprechend der drei Faktoren eingeordnet werden. 


\subsection{Ideen, Interessen und Institutionen}

Wie bereits oben ausgeführt, beansprucht der historische Institutionalismus für sich, daß er die Interaktion von Ideen, Interessen und Institutionen berücksichtigt. Danach finden Interessen und Ideen nur ihre Wirkung, wenn sie durch Institutionen transportiert werden. Gleichzeitig formen Institutionen nach Lepsius (1990) aber auch Interessen und verleihen Ideen Geltungsmacht.

Das Zusammenspiel und die Verflechtung von Ideen, Interessen und Institutionen scheint, den einmal erreichten, gleichgewichtigen Status quo zu perpetuieren. Ein Wandel der Institutionen kann daher nur erfolgen, wenn die Prozesse der Institutionalisierung mit Prozessen des Interessen- und Ideenwandels einhergehen. Im folgenden werden die für das jeweilige (Berufs-)Bildungssystem konstituierenden Ideen betrachtet: Für die USA ist das Prinzip des Egalitarismus eine verfassungsrechtlich normierte Idee, die sich in der institutionellen Struktur der ,comprehensive high school' widerspiegelt. Demgegenüber basiert das deutsche duale Berufsbildungssystem auf der Idee der Beruflichkeit, die mit einer Differenzierung des Bildungssystems korrespondiert.

Anschließend werden die für das duale Berufsbildungssystem relevanten Institutionen und Interessen in ihrer systemerhaltenden und reproduzierenden Interdependenz kurz eingeführt. Eine ausführliche Betrachtung ihrer historischen Entstehung und der spezifischen Interessen- und Institutionenverflechtung erfolgt in Teil II.

\subsubsection{Egalitarismus versus Beruflichkeit}

Bereits Max Weber hat das Verhältnis von Interessen und Ideen in seinen Arbeiten thematisiert: Wie entstehen Interessen und welche Konflikte gibt es zwischen ihnen? Und welche Ideen beeinflussen als Wertvorstellungen das Handeln von einzelnen und von Gruppen? In einem klassischen Zitat beschreibt Weber diese Interaktion: „Interessen (materielle und ideele), nicht: Ideen, beherrschen das Handeln der Menschen. Aber: die ,Weltbilder', welche durch ,Ideen' geschaffen wurden, haben sehr oft als Weichensteller die Bahnen bestimmt, in denen die Dynamik der Interessen das Handeln fortbewegte." (Weber 1978, S. 252) Zwischen Interessen und Ideen stehen als Vermittlungsinstanz Institutionen, die durch Ideen begründet und legitimiert werden. Damit besitzen Institutionen neben ihrer Regulierungsdimension auch eine symbolische Ordnungsdimension. In ihnen sind die handlungsleitenden Ideen gleichsam insti- 
tutionalisiert. Es bleibt jedoch die Frage, wie Ideen entstehen bzw. welche Ideen sich als handlungsleitend durchsetzen. Ideen sind nicht naturgegeben, sondern werden in gesellschaftlichen Prozessen der Interessendurchsetzung ,produziert“. Insofern sind sie selbst das Ergebnis von - national spezifischen - Institutionalisierungsprozessen: „Over time, each nation builds up a set of politically evocative concepts and collective reference points.“ (Hall 1989, S. 383 f.) Nachdem sie einmal entstanden und geltungsmächtig sind, wirken diese Ideen verhaltensstrukturierend. Ihre Wirksamkeit zeigt sich dabei am deutlichsten beim Wandel von Institutionen: In ihm tritt der Konflikt von Wertvorstellungen zutage - neue Ideen werden zur Legitimation sozialer Innovation herangezogen und müssen sich gegen ,alte“ Ideen durchsetzen bzw. ,alte“ Ideen sind noch so wirkungsmächtig, daß sie einem Wandel der sie verkörpernden institutionellen Ordnung entgegenstehen.

Das Prinzip des Egalitarismus - also der Verwirklichung von Gleichheit - ist fest in der politischen Tradition der USA verankert. Gleichheit kann sich jedoch auf unterschiedliche Tatbestände beziehen, z.B. materielle Ressourcen, Rechte, etc. Je nach Bezugspunkt ergibt sich deshalb eine spezifische Ausprägung des Egalitarismus: „Egalitarianism is a protean doctrine, because there are several different types of equality.“ (Stanford Encyclopedia of Philosophy) Für die Bestimmung der typisch amerikanischen Variante des Egalitarismus bedarf es einer historischen Analyse der politischen Inkorporation der Gleichheitsidee und deren Definition.

Mit der Verabschiedung der Declaration of Independence erhielt das Gleichheitsprinzip den Status einer politisch-konstituierenden Norm, auf deren Basis die Rebellion der Kolonien gegen das britische Mutterland legitimiert wurde: „All men are created equal“", schrieb Thomas Jefferson und griff damit die Forderungen seiner Landsleute nach gleichen politischen Rechten im Sinne der Repräsentation im britischen Parlament auf. Dieses Gleichheitsideal entstammte zwei gedanklichen Traditionen: Einerseits dem christlichen Menschenbild, andererseits der europäischen Aufklärung. Die amerikanischen Kolonisten beriefen sich dabei auf die zehn Gebote, die schließlich keinen Unterschied zwischen Status oder Person machten (vgl. Bailyn 1967, S. 32 f.). Unter den europäischen Autoren der Aufklärung war es insbesondere John Locke, dessen politische Ideen großen Einfluß auf die Diskussion in den amerikanischen Kolonien hatte. Nach Locke hat jeder Mensch die gleichen ,natürlichen Rechte“, wie das Recht auf Eigentum. In einem vorstaatlichen - quasi natürlichen - Zustand fehlen dem 
einzelnen aber die Mittel, um seine Rechte zu schützen. Der freiwillige Zusammenschluß einzelner zum Schutz ihrer „natürlichen Rechte“ führt zur Herausbildung des Staates, der über den Erlaß von Gesetzen (,civil law') die „,natürlichen Rechte“ garantiert. Da sich die Menschen freiwillig entschlossen haben, einen Staat zu gründen, sind sie auch insofern gleich, als sie den gleichen Willen hatten, diesem „Vertrag“ beizutreten (vgl. Pole 1993, S. 14 f.; Bailyn 1967, S. 27). Die politischen Ideen von John Locke bildeten für Thomas Jefferson die moralische Legitimation für seine eigenen Grundsätze: „In the Declaration of Independence, where Locke's ,Life, liberty and estate' became ,Life, liberty, and the pursuit of happiness', the whole position rested on the foundation of natural equality in human rights.“ (Pole 1993, S. 15)

Dem Prinzip der 'equality of rights' standen offensichtliche soziale Ungleichheiten gegenüber. Unter dem Eindruck ökonomischer Rezessionen und wachsender Immigration geriet das Gleichheitsideal unter legitimatorischen Druck. In der politischen Diskussion der 1850er Jahre kristallisierte sich ein „neues“, komplementäres Konzept von Gleichheit heraus, das sich auf Ideen aus der frühen Phase der amerikanischen Unabhängigkeit berufen konnte. Die Gleichheit der politischen und bürgerlichen Rechte sollte allen Bürgern den Weg ebnen, ihre legitimen Aspirationen verfolgen zu können. Alexander Hamilton schrieb in den Federalist Papers: „The door ought to be equally open to all.“ (zit. nach Pole 1993, S. 153) Basierend auf diesen Ideen entstand das Konzept der ,equality of opportunity' - also der Chancengleichheit -, das soziale Ungleichheiten dadurch rechtfertigt, daß jedem die Möglichkeit gegeben wird, durch individuelle Leistung zu Wohlstand und sozialem Ansehen zu kommen. Darin kommt auch die Idee des selbstverantwortlichen Handelns zum Tragen (vgl. Fogel 2000, S. 5 f.). Bei Rawls liest sich das später folgendermaßen: „Social and economic inequalities are to satisfy two conditions: first, they are to be attached to positions and offices open to all under conditions of fair equality of opportunity ${ }^{4}$; and second, they are to be to the greatest benefit of the least advantaged members of society." (Rawls 1993, S. 5-6)

Im Gegensatz zum Ideal der , equality of rights' bedarf es zur Verwirklichung der ,equality of opportunity’ einer aktiven staatlichen Politik, um gleiche Ausgangs- bzw.

\footnotetext{
${ }^{4}$ Das Prinzip der , fair equality of opportunity' geht über das Prinzip der 'equality of opportunity' hinaus, indem es jegliche mögliche Bevorteilung in den Ausgangsbedingungen, z.B. aufgrund familiären Wohlstands, ablehnt.
} 
Startbedingungen zu schaffen. Dies schlug sich in den USA in der öffentlich-finanzierten Bereitstellung von Schulen nieder, die in den 1820er Jahren mit der Einrichtung staatlicher Elementarschulen (,elementary school') begann. Im Verlauf des 19. Jahrhunderts entstand darüber hinaus ein System öffentlich-finanzierter Sekundarschulen - der sogenannten ,comprehensive high schools' -, die sich durch ihr Curriculum von den privaten Akademien unterschieden. Anstatt auf ein College-Studium vorzubereiten, boten die ,high schools' einen berufsqualifizierenden Unterricht für die Masse der Jugendlichen an. „At the start of the high school movement, secondary schools were distinctively American institutions: egalitarian, open, and, generally, publicly financed and provided.” (Goldin 1999, S. 69) Das öffentliche Schulsystem sollte allen - im Sinne der ,equality of opportunity' - die gleichen Chancen eröffnen.

Die Reformbewegung des, vocationalism' zu Beginn des 20. Jahrhunderts widersprach diesem Ideal. Der Begriff des ,vocationalism' beinhaltete die Idee einer generellen Ausrichtung der schulischen Ausbildung auf die Bedürfnisse der Wirtschaft. „Vocationalism as an educational ideal subsumes general education.” (Kliebard 1999, S. 120) Die Vertreter des 'vocationalism' befürworteten ein organisatorisch getrenntes Schulsystem, um ihr Ideal einer umfassenden schulischen Berufsbildung (,vocational education') umsetzen zu können. Dies hätte zu einer Aufspaltung des bestehenden Schulsystems der, comprehensive high school' in zwei voneinander unabhängige Schulformen geführt: einer der Allgemeinbildung im Sinne einer ,liberal education' verpflichteten ,high school' sowie einer auf die direkte Berufsqualifizierung ausgerichteten ,vocational high school'. Damit wäre die Idee der ,equality of opportunity' durchbrochen worden. Entsprechend vehement war auch der Widerstand gegen diese weitreichenden Reformvorstellungen. Statt einer organisatorisch separaten Berufsbildung blieb die ,vocational education' Teil des curricularen Programms der ,comprehensive high school' (vgl. ebd., S. 146): „Our system of free public education is legitimized by the assumption that acquiring positive abstract knowledge can be made interesting and appealing to everyone. Such knowledge not only gives each person a chance to social, economic, and political equality but further ensures the continuation of a democratic society where class and social lines are left open and fluid." (Cusick 1983, S. 109)

Im 19. Jahrhundert hat sich eine spezifisch amerikanische Variante des Egalitarismus konstituiert, die sich auf zwei wesentliche Prinzipien beruft: ,equality of rights' und 
,equality of opportunity'. Während von Beginn an die amerikanische Nation auf dem Prinzip gleicher Rechte basierte, entwickelte sich erst unter dem Eindruck wachsender sozialer Ungleichheiten das Konzept der Chancengleichheit, das diese Ungleichheiten auf der Basis individueller Leistung legitimiert. Die Institution der einheitlichen ,comprehensive high school' ist im amerikanischen Verständnis die idealtypische Verkörperung dieser Idee.

Dagegen ist das deutsche System der dualen Berufsbildung dem Berufsprinzip verpflichtet. Bereits die Lehrlingsausbildung der Zünfte war auf die Erlernung und Sozialisation in einem spezifischen Beruf ausgerichtet, den man sein Leben lang ausübte. Trotz der Gewerbeliberalisierung zu Beginn des 19. Jahrhunderts blieb die Ausbildung in einem Beruf entscheidende Grundlage für dessen spätere Ausübung. Einige gewerbliche Berufe durften auch weiterhin nur unter Nachweis der entsprechenden Qualifikation ausgeübt werden.

Der ursprüngliche Begriff des Berufs entstammt der christlich geprägten Ordnungsvorstellung, nach der im Beruf christliche Lebensführung und Arbeit im Sinne einer Berufung vereinigt werden. Die Reformation wertete den Begriff der Arbeit dahingehend auf, daß sich in ihr der Glaube zu bewähren habe. ,[...]: die Schätzung der Pflichterfüllung innerhalb der weltlichen Berufe als des höchsten Inhaltes, den die sittliche Selbstbetätigung überhaupt annehmen könne. Dies war es, was die Vorstellung von der religiösen Bedeutung der weltlichen Alltagsarbeit zur unvermeidlichen Folge hatte und den Berufsbegriff erzeugte.“ (Weber 1996 [1904/05], S. 40) Die Stellung des einzelnen in der Gesellschaft, also sein „Stand“, ergibt sich danach aus seiner göttlichen Berufung. Während Martin Luther eine statische Berufsauffassung ${ }^{5}$ vertrat, die jedem eine von gottgewollte Position bzw. Stellung zuweist, erfuhr die Berufsarbeit beim Calvinismus eine besondere Bedeutung als Mittel zur Erlangung des Gnadenstandes: „Und andererseits wurde, um jene Selbstgewißheit zu erlangen, als hervorragendstes Mittel rastlose Berufsarbeit eingeschärft. Sie und sie allein verscheuche den religiösen Zweifel und gebe die Sicherheit des Gnadenstandes.“ (ebd., S. 71) Mit der Aufklärung des 17. und 18. Jahrhunderts erfolgte eine Säkularisierung des Berufsbegriffs, wobei die

5 „So blieb also bei Luther der Berufsbegriff traditionalistisch gebunden. Der Beruf ist das, was der Mensch als göttliche Fügung hinzunehmen, worin er sich ,zu schicken’ hat [...].“ (Weber 1996 [1904/05], S. 45) 
Idee der Übertragung einer gesellschaftlichen Verpflichtung und Aufgabenerfüllung weiterhin ein konstituierendes Element darstellt. Im Zuge der industriellen Revolution treten ökonomisch-arbeitsorganisatorische Aspekte des Berufsbegriffs in den Vordergrund.

Danach sind Berufe verschiedenartige Qualifikationsbündel mit charakteristischen Ausprägungen und Anordnungen von Wissen, denen entsprechende Aufgabenfelder zugeordnet sind. Brater/Beck definieren Berufe als institutionalisierte „Muster der Zusammensetzung und Abgrenzung spezialisierter Arbeitsfähigkeiten“ (1982, S. 209), die gesetzlich reguliert und geschützt sind. Das (Berufs-)Bildungssystem dient der Vermittlung dieser standardisierten Qualifikationsmuster, wodurch sie auch einer kontinuierlichen Reproduktion unterliegen. In diesem Sinne sind Berufe eine idealtypische Vermittlungsinstanz zwischen dem einzelnen Individuum und der Gesellschaft. Für das Individuum stellt der Beruf eine standardisierte Form des Verkaufs der Arbeitskraft dar, über die der Zugang zum Arbeitsmarkt bzw. Arbeitsplatz geregelt wird. Damit ist er die „Schleuse“ zur Teilhabe am Erwerbssystem (vgl. Dostal 2002, S. 178 f.). Gesellschaftlich ist der Beruf eine Form der Statuszuweisung. Durch „Berufsvererbung“ und berufsförmige Schließungsprozesse reproduziert er zudem soziale Ungleichheiten (vgl. Voß 1997, S. 207): „Berufe sind damit also gesellschaftliche Instanzen, die zum einen regulieren, wie sich Personen auf die Gesellschaft beziehen und in ihr wirksam werden können - zum anderen steuern sie aber auch, wie gesellschaftliche Institutionen auf Individuen zugreifen, sie einordnen, Privilegien oder Nachteile zuweisen, sie als Personen prägen.“ (ebd., S. 207) Nach Schelsky ist der Beruf „eine der großen sozialen Sicherheiten, die der Mensch in der modernen Gesellschaft [...] noch besitzt" (1965, S. 238), weil er über die „Umwelt-“ und „Innenstabilisierung“ zur sozialen Integration des Individuums beiträgt.

In Deutschland hat sich das Prinzip der Beruflichkeit spätestens zum Ende des 19. Jahrhundert endgültig durchgesetzt, als Bismarck zur Stabilisierung des jungen Reichs eine Sozialpolitik des „,vorwärts gewandten Sozialkonservatismus“ (Ostner 1997, S. 76) verfolgte. Im Rückgriff auf die Tradition einer berufsständischen Organisation der Gesellschaft beschritt er damit einen dritten Weg der sozialen Inklusion zwischen Individualismus und Kollektivismus. „Berufe boten von nun an ihren Inhabern Verhandlungschancen, Statussicherheit sowie einen sozialen Ort im Betrieb und in der Gesellschaft.“ (ebd., S. 77) Der Absicherung der Beruflichkeit durch die Sozialpolitik, 
z.B. in Form eines Berufsschutzes in der Arbeitslosenversicherung, stand die Durchsetzung des Berufsprinzip in der Ausbildung und Betriebs- und Arbeitsorganisation komplementär gegenüber. Während das Berufsprinzip in den handwerklichen Arbeitsprozessen immer eine zentrale Rolle gespielt hatte, setzte es sich in der industriellen Produktion erst durch die Übernahme der handwerklich berufsförmigen Lehrlingsausbildung für die Facharbeiterqualifikation durch. Unternehmen der Maschinenbau- und Elektroindustrie formulierten als erste eigene, industriespezifische Berufsbilder mit standardisierten Ausbildungsplänen und Prüfungsanforderungen (vgl. Greinert 1993, S. 70 ff.). Diese neuen Industrieberufe fügten sich nahtlos in die entstehende funktions- und berufsbezogene Gestaltung der Betriebs- und Arbeitsorganisation ein. Idealtypischerweise ist die funktions- und berufsorientierte industrielle Organisation durch folgende Kennzeichen geprägt: Ein auf der Betriebsebene vertikal hochintegriertes Leistungsprofil mit allen Produktionsstufen und relevanten internen Dienstleistungen ermöglicht vielfältige interne Berufskarrieren. Die Aufbauorganisation ist nach dem Fachabteilungsprinzip gegliedert. Dementsprechend ist die Arbeitsteilung entlang berufstypischer Qualifikationsmuster geschnitten, die den Angehörigen verschiedener Berufsgruppen spezifische Einsatzfelder zuweist. Und schließlich basiert diese Form der Betriebs- und Arbeitsorganisation auf einer entlang formaler Kompetenzen vertikal differenzierten Statusorganisation mit unterschiedlichen Gratifikationen und beruflichen Entfaltungsmöglichkeiten (vgl. Baethge/Baethge-Kinsky 1998, S. 462 f.; Baethge-Kinsky 2001, S. 86 ff.). Die nach dem Berufsprinzip organisierte Arbeitsteilung eröffnete beiden Seiten - Unternehmen und Arbeitnehmern - die Chance, ihre Transaktionskosten zu senken: Für die Unternehmen entfällt z.B. die langwierige und kostenintensive Suche nach passend qualifizierten Beschäftigten, da sie auf dem externen (Berufs-)Arbeitsmarkt standardisierte Qualifikationen einkaufen können. Der dualen Berufsausbildung ist dadurch eine zentrale Funktion als Vermittlungsinstanz zwischen Schule und Betrieb bzw. Arbeitsmarkt zugekommen. Auf der einen Seite hat sie die Arbeitgeber mit qualifizierten Arbeitskräften versorgt. Über die in der Ausbildung erworbene Beruflichkeit sicherte sie auf der anderen Seite den Arbeitnehmern externe Arbeitsmarktchancen und schuf damit betriebliche Unabhängigkeit (vgl. Ostner 1997, S. 77).

Das Prinzip der Beruflichkeit war aber auch immer wieder von Tendenzen einer Entberuflichung gefährdet. In den 1960er Jahren gab es eine erste Flexibilisierungsdebatte, 
die die Realität des Berufswechsels und einer dynamischen Nutzung des erlernten beruflichen Arbeitsvermögens anerkannte. Dabei hielt man jedoch am Berufsprinzip mit seinen sozialen Verflechtungen fest. Die Forderung nach höherer Flexibilität richtete sich deshalb auf die vermittelten Qualifikationen, also die Berufsinhalte.

Seit den frühen 1990er Jahren scheint ein neuer, umfassenderer Trend in Richtung Entberuflichung eingesetzt zu haben. Diesmal stellt sich die Frage nach der Funktionalität des Berufsprinzips gleich auf drei Ebenen: der Vermittlung von Qualifikationen im Rahmen der dualen Berufsausbildung (vgl. Baethge 1996, S. 109 ff.), der betrieblichen Arbeitsorganisation (vgl. Kern/Sabel 1994, S. 605 ff.) sowie der Beschäftigungsstruktur und des Arbeitsmarktes (vgl. Wittwer 1996, S. 11 ff.). Bezüglich des individuellen Qualifikationsprofils zeichnet sich ein Wandel in den Anforderungen von einer berufsfachlichen Erfahrungsqualifikation zu Wissens- und Analysequalifikationen ab. Robert Reich (1992) bezeichnet diesen neuen Beschäftigtentypus als Symbolanalytiker. Gleichzeitig erweist sich nach Kern/Sabel (1994) das mit dem Arbeitskräftetypus Facharbeiter verbundene Berufsprinzip als dysfunktional für die Umsetzung einer prozeßorientierten Arbeitsorganisation, die über die Ausnutzung querfunktionaler Kooperationspotentiale ihre Innovationsfähigkeit und Reaktionsgeschwindigkeit erhöht. Und schließlich geht Wittwer (1996) davon aus, daß sich aufgrund des beschleunigten ökonomischen Strukturwandels die Halbwertzeit von erworbenen Berufsqualifikationen verringert, was wiederum zu Anpassungen im Beschäftigungssystem führt. Trotz dieser Entberuflichungstendenzen ${ }^{6}$ hält die duale Berufsausbildung am Berufsprinzip als zentraler Qualifikationskategorie fest, wie sich in der Neuregelung und Neuschaffung verschiedener Berufsbilder zeigt. Ende der 1990er Jahre wurden neue Berufe im Bereich der Informations- und Kommunikationstechnologie ${ }^{7}$ sowie der Medienwirtschaft entwickelt, die in ihren Ausbildungsordnungen die neuen Qualifikationsanforderungen berücksichtigen, ohne das Konzept eines berufsfachlich definierten Profils („Kernberuf“) aufzugeben (vgl. iwd 2002, S. 7). Und in diesem Jahr hat die Investmentbranche mit der Schaffung eines Ausbildungsberufs „Investmentfonds-

\footnotetext{
${ }^{6}$ In der Sozialpolitik kann ebenfalls eine Erosion des Berufsprinzips festgestellt werden: So hat z.B. die gesetzliche Neuregelung der Zumutbarkeitsbestimmungen für Arbeitslose im AFG den besonderen Berufsschutz aufgehoben.

${ }^{7}$ Eine erste Evaluation der Akzeptanz und Umsetzung der neuen IT-Berufe in der Praxis hat bereits stattgefunden: vgl. dazu Borch, H./Weißmann, H. (2002): IT-Berufe machen Karriere, Bielefeld: W. Bertelsmann.
} 
Kaufmann" begonnen, um ihren steigenden Bedarf an qualifizierten Nachwuchskräften decken zu können (vgl. Frankfurter Allgemeine Zeitung 2002b, S. 71). Vielleicht sind dies Beispiele einer „,neuen“ Beruflichkeit.

\subsubsection{Arbeitgeber, Gewerkschaften und der Staat}

Im vorangegangenen Kapital stand die Beziehung zwischen Ideen und Institutionen im Mittelpunkt der Überlegungen. Im folgenden soll nun das Verhältnis zwischen Interessen und Institutionen am Untersuchungsgegenstand der Berufsausbildung in Grundzügen aufgezeigt werden. Dabei wird auf das spezifische institutionelle ,Setting' der deutschen dualen Berufsbildung abgestellt, da diese dem amerikanischen Politikansatz als Vorbild diente und insofern die Kontrastfolie für die gesamte Untersuchung darstellt.

Im Sinne des historischen Institutionalismus sind Interessen sozial konstruiert. Sie stehen damit im Kontext sozialer, politischer und ökonomischer Bedingungen. Institutionen kommt daher nach Hall (1986) eine entscheidende Rolle bei der Definition und Artikulation von Interessen zu. Die spezifischen institutionellen Bedingungen definieren somit den - politischen - Rahmen, innerhalb dessen eine Strukturierung der Problembearbeitung und Lösungssuche erfolgt. Gleichzeitig dienen Institutionen als organisatorische Mittel für die Durchsetzung von Interessen. Dieses Wechselspiel darf jedoch nicht als monokausale Beziehung in dem Sinne verstanden werden, daß Institutionen als unabhängige Variable immer die abhängige Variable Interessen determinieren. Gerade in Zeiten des - institutionellen - Umbruchs (,critical junctures') kann sich dieses Verhältnis umkehren: „, [...] when the institutions break down, they become the dependent variable, whose shape is determined by the political conflicts that such institutional breakdown unleashes." (Thelen/Steinmo 1992, S. 15)

Das deutsche duale Berufsbildungssystem ist eingebunden in ein komplexes Institutionengeflecht, das für die beteiligten Akteure die notwendigen Anreize schafft, um die langfristige Stabilität dieses Systems zu sichern. Die Bereitstellung einer ausreichenden Anzahl qualifizierter Arbeitskräfte ist für Firmen, die auf einem freien Arbeitsmarkt konkurrieren, ein (quasi-) Kollektivgut: der einzelne Arbeitgeber kann -unabhängig davon, ob er selber zur Erstellung des Kollektivgutes beigetragen hat oder nicht - von der Nutzung (dem Konsum) nicht ausgeschlossen werden. Unter der Annahme der individuellen Nutzenmaximierung verhält sich der einzelne Arbeitgeber dann rational, 
wenn er als „Trittbrettfahrer“ die Ausbildungsanstrengungen der anderen ausnutzt. Damit unterliegt eine Berufsausbildung, die betriebsunabhängige, marktgängige Qualifikationen $^{8}$ vermittelt, dem klassischen Problem kollektiven Handelns: dem ,freeriding' (vgl. Olson 1992, S. 8 ff.).

Um dieses Dilemma aufzuheben, bedarf es also - wie bereits angedeutet - eines spezifischen ,institutional settings', das die Interessenkonstellation der Arbeitgeber im Sinne der Bereitstellung des Kollektivguts positiv verändert. Im Fall der deutschen dualen Berufsausbildung ist dies durch das Zusammenwirken von Arbeitgeber- bzw. Wirtschaftsverbänden - insbesondere den ,quasi-öffentlichen', selbstverwalteten Industrie- und Handelskammern sowie den Handwerkskammern -, Gewerkschaften und dem Staat gegeben. Aufgrund ihrer Organisationsform als Industriegewerkschaften stehen die deutschen Gewerkschaften einem allgemeinen und umfassenden Ausbildungssystem positiv gegenüber. Sie nutzen ihre Organisationsmacht also nicht, um die Ausbildungstätigkeit der Firmen einzuschränken und so die Löhne für qualifizierte Arbeitskräfte hoch zu halten, wie es z.B. berufsspezifisch organisierte Gewerkschaften in den USA handhaben. Über die sektoral organisierten Tarifverhandlungen unterstützen sie die Ausbildungsanstrengungen der Arbeitgeber insofern, als sie über eine niedrige Lehrlingsvergütung die Ausbildungskosten gering halten. Darüber hinaus haben sie über die Betriebsräte die Möglichkeit, im einzelnen Betrieb bei der Organisation der Ausbildung mitzuwirken und die Ausbildungsaktivitäten zu kontrollieren. Das ermöglicht es ihnen, auf der Ebene der Definition von Ausbildungsordnungen Handlungsspielräume für die innerbetriebliche Ausgestaltung zu zulassen (vgl. Streeck et al. 1987, S. 3 ff.).

Eine besondere Rolle in der Organisation der Berufsausbildung kommt den Arbeitgeberverbänden und den öffentlich-rechtlichen Kammern zu. Sie haben eine entscheidende Koordinationsfunktion inne: Hall und Soskice sprechen in diesem Zusammenhang von ,Coordinated Market Economy’. Die Koordination zwischen den Firmen

\footnotetext{
8 Zum selben Ergebnis des Marktversagens kommt Becker mit seiner Unterscheidung zwischen betriebsspezifischen und marktgängigen Qualifikationen: Für eine Firma ist es wirtschaftlich nicht rational, in marktgängige Qualifikationen seiner Arbeitskräfte zu investieren, weil sie nach der Ausbildung dem Arbeitnehmer entweder den der Qualifikation entsprechenden Marktlohn zahlen müßte, was für sie teurer wäre als die Qualifikation auf dem Markt einzukaufen, oder einen Lohn unterhalb des Marktniveaus, was zum Arbeitsplatzwechsels des Arbeitnehmers führen würde (vgl. Becker 1993).
} 
erlaubt es den Unternehmen, eine Strategie der ,high-quality production' zu verfolgen, die unter anderem die Bereitstellung einer ausreichenden Anzahl qualifizierter Arbeitskräfte erfordert (vgl. Hall/Soskice 2001, S. 24 ff.; Wood 2001, S. 249 f.). Die Koordinationsfunktion umfaßt die vier folgenden Aktivitäten: Informationsaustausch, Beratung, Überwachung und Sanktionierung (vgl. Culpepper 2001, S. 279). Im deutschen Fall bedeutet dies z.B. die Ausarbeitung von Ausbildungsordnungen auf Bundesebene, die Beratung der Firmen bei der Ausbildungsorganisation sowie die Verwaltung und Überwachung der Ausbildung. Dabei ist entscheidend, daß diese originär staatlichen Aufgaben von wirtschaftsnahen Institutionen wahrgenommen werden. Der Staat hat seine Regelungskompetenz daher an die Arbeitgeberverbände und Kammern delegiert. Aufgrund ihrer größeren Nähe zu den Unternehmen haben die Wirtschaftsverbände einen Informationsvorsprung gegenüber dem Staat. Zudem genießen sie mehr Vertrauen, was es ihnen erleichtert, die für eine effektive Kontrolle notwendigen Information zu erhalten. Für die Unternehmen reduzieren sich sozusagen die ,distrust'Transaktionskosten, die ansonsten mit der Organisation eines - staatlich verwalteten Berufsbildungssystems verbunden sind (vgl. Soskice 1994a, S. 42). Darüber hinaus bedeutet die Standardisierung der Berufsbildung in Ausbildungsberufe mit entsprechenden Ausbildungsordnungen eine Absicherung sowohl für die Arbeitgeber als auch für die Arbeitnehmer. Die Ausbildungsordnungen orientieren sich aufgrund der konzeptionellen Beteiligung der Wirtschaftsverbände an den Bedürfnissen der Unternehmen. Über externe (Berufs-) Arbeitsmärkte können die Unternehmen standardisierte Qualifikationen einkaufen, wodurch sich bei einer Abwerbung selbst ausgebildeter Arbeitnehmer das Risiko eines Verlusts der Ausbildungsinvestitionen minimiert. Für die Arbeitnehmer stellt die Standardisierung eine Absicherung ihrer Beschäftigungsposition dar, da sie gegenüber dem einzelnen Arbeitgeber an Unabhängigkeit gewinnen.

Hinsichtlich ihrer Fähigkeit, abweichendes Verhalten von einzelnen Firmen zu sanktionieren, sind die Arbeitgeberverbände auf das Drohpotential des Staates angewiesen. Nur über den Hinweis, daß der Staat bei fehlendem kollektiven Verhalten, in die selbstverwaltete Autonomie der Wirtschaft - z.B. über die Erhebung einer Ausbildungssteuer - eingreifen könnte, erwächst den Verbänden eine „geliehene“ Autorität. In Ausnahmefällen, wie im Baugewerbe in den 1980er Jahren, können die Arbeitgeberverbände in Kooperation mit den Gewerkschaften dies auch über Tarifverhand- 
lungen erreichen, indem sie für ihre Branche eine Umlagefinanzierung der Ausbildung vereinbaren (vgl. Streeck 1983). Anders als die Arbeitgeberverbände haben die Industrie- und Handelskammern sowie die Handwerkskammern aufgrund der Zwangsmitgliedschaft der Firmen ein größeres Sanktionspotential, das sie z.B. dazu nutzen, überbetriebliche Ausbildungsstätten über Umlagen zu finanzieren. Insgesamt läßt sich festhalten, daß die Koordinationsfunktion der Arbeitgeberverbände und Kammern eine entscheidende Voraussetzung für die Bereitstellung des Kollektivgutes Berufsbildung darstellt. Wie im folgenden ausführlich gezeigt wird, fehlt gerade diese Voraussetzung in den $\mathrm{USA}^{9}$.

Der Staat hat in der ,coordinated market economy' nach Stewart Wood die Aufgabe, durch seine (Rahmen-)Gesetzgebung das institutionelle Fundament der Koordination zu schützen: ,[...] business sees the state's function as one of ensuring that the rich networks of business coordination are protected.“ (Wood 2001, S. 251) Im deutschen Fall fällt die Gesetzgebungszuständigkeit für die Berufsbildung in den Kompetenzbereich der Bundesregierung. Diese übt geradezu idealtypisch für eine ,coordinated market economy' ihre Funktion als Rahmengesetzgeberin aus. Das Berufsbildungsgesetz $(\mathrm{BBiG})$ regelt die wesentlichen Eckpfeiler der Berufsausbildung, überträgt deren Ausführung und konkrete Ausgestaltung aber den Arbeitgeberverbänden, Gewerkschaften und Kammern. Wie bereits angedeutet, delegiert der Staat z.B. seine Regelungskompetenz im Bereich der Ausbildungsordnungen an die Arbeitgeberverbände und Gewerkschaften. Ihm bleibt dabei nur noch eine „Notariatsfunktion“ (vgl. Streeck et al. 1987, S. 15). Durch die Delegation staatlicher Verantwortung stärkt der Staat die Position der Verbände und damit deren Koordinationsfähigkeit. Gleichzeitig ist die Macht der Bundesregierung verfassungsrechtlich soweit beschränkt, daß ihre Fähigkeit, die institutionellen Voraussetzungen der Koordination durch die Verabschiedung von Gesetzen zu unterlaufen, gering ist. Dies stellt wiederum eine Garantie für die Unternehmen dar, daß die Institutionen der Koordination vor negativen staatlichen Interventionen weitgehend geschützt sind und somit auch die langfristigen Investitionen in kollektive Güter, wie z.B. betriebsunabhängige Qualifikationen (vgl. Wood 2001, S. 256 f.). Die Kombination einer in ihrer Macht beschränkten Regierung

\footnotetext{
${ }^{9}$ Hall und Soskice führen neben Großbritannien die USA als Beispiel einer ,liberal market economy' an, die sich gerade durch das Fehlen von Institutionen der (Unternehmens-)Koordination auszeichnet.
} 
mit starken Arbeitgeberverbänden scheint also eine wesentliche Voraussetzung für die langfristige Stabilität des institutionellen Gerüsts unternehmerischer Koordination zu sein.

Der institutionelle Rahmen (,institutional setting'), in den das duale Berufsausbildungssystem eingebettet ist, schafft also die notwendigen Anreize für die Akteure, sich systemkonform zu verhalten und so zur langfristigen Stabilität beizutragen. Im folgenden wird dieser Zusammenhang zwischen Institutionen und Interessen ausführlich analysiert, wenn es um die Entstehung und strukturelle Ausformung der deutschen dualen Berufsausbildung geht. 


\section{Methoden des Vergleichs}

Eine auf den theoretischen Ansatz des historischen Institutionalismus rekurrierende Herangehensweise steht in einer engen Beziehung zur induktiv-orientierten Methode des historisch interpretativen, makro-kausalen Vergleichs. Empirische Sozialforschung beinhaltet in der Regel immer irgendeine Form des Vergleichs. Durch einen Vergleich wird z.B. erst die Möglichkeit geschaffen, Aussagen über empirische Regelmäßigkeiten zu treffen. Gegenüber diesem sehr weit gefaßten Verständnis des Vergleichs grenzt sich die komparative Methode im engeren Sinne dadurch ab, daß ihr Gegenstand des Vergleichs makrosoziale Einheiten sind. Auch bei der hier vorliegenden Arbeit findet die komparative Methode ihre Anwendung im Vergleich makrosozialer Einheiten, nämlich der spezifischen Berufsbildungssysteme zweier Länder.

In der vergleichenden Sozialforschung lassen sich zwei konkurrierende Paradigmen definieren: fallorientiertes versus variablenorientiertes Forschungsdesign (vgl. Ragin 1987, S. 12-13, 31-32). Beide Ansätze unterscheiden sich hinsichtlich verschiedener Merkmale. Dabei leitet sich die spezifische Form vom unterschiedlichen Erkenntnisinteresse ab. In ihrer im folgenden dargestellten Konzeption bilden die beiden Ansätze die idealtypischen Pole auf einem Kontinuum, das in der Praxis auch Mischformen der beiden Paradigmen aufweist.

Der variablenorientierten Ansatz verfolgt das Ziel, allgemeingültige Aussagen über Beziehungen zwischen bestimmten Variablen in einer großen Zahl von Fällen herzustellen bzw. von allgemeinen Theorien abgeleitete Hypothesen über die Beziehung von Variablen zueinander zu testen. Da sich das Erkenntnisinteresse auf allgemeingültige Erklärungen richtet, muß eine variablenorientierte Forschung auf einer möglichst großen Fallzahl basieren. Nur so kann eine angemessene Validität garantiert werden. Der einzelne Fall spiegelt sich lediglich in den Werten der forschungsrelevanten Variablen wider. Seine spezifisch historischen Konfigurationen finden dabei keine Beachtung. Die Untersuchung der Beziehung einer begrenzten Anzahl theoretisch abgeleiteter unabhängiger Variablen zur abhängigen Variablen erfolgt schließlich anhand statistischer (multivariater) Analysemethoden. Insofern liegt diesem Ansatz eine extensive Forschungslogik zugrunde (vgl. Ragin 1994, S. 299-301; Ragin 1987, S. 31-32 und 53; Borchert 1995, S. 85). 
Im Gegensatz dazu zielt der fallorientierte Ansatz auf eine historisch interpretative und - in der Regel auch - kausal analytische Untersuchung weniger Fälle. Allein die geringe Fallzahl ermöglicht eine holistische Betrachtung der komplexen Konfiguration von historischen Ereignissen und Strukturen des jeweils spezifischen Einzelfalls. Kausale Beziehungsmuster zwischen einer großen Bandbreite von Variablen sind jedoch eingebettet in kontextuale Erklärungen. Nach Charles Ragin weist der fallorientierte Ansatz mehrere Vorzüge gegenüber einer variablenorientierten Forschung auf: So ist dieser geeigneter, Kombinationen verschiedener Ursachen und ihrer Wirkungen zu erfassen. Statistische Methoden beschränken sich typischerweise auf einförmige Beziehungsmuster. Darüber hinaus sucht der fallorientierte Ansatz nach Erklärungen für jeden Einzelfall, wodurch empirische Unregelmäßigkeiten nicht unerklärt bleiben. Der fallorientierte Ansatz geht zudem nicht davon aus, daß die untersuchten Fälle repräsentativ sind, und entzieht sich damit der Gefahr, die Ergebnisse zu generalisieren. Und schließlich setzt er eine intensive Auseinandersetzung mit den Fällen voraus, um jeden einzelnen Fall mit allen anderen vergleichen zu können (vgl. Ragin 1987, S. 1516). Für eine solchermaßen intensive Forschung greift der fallorientierte Ansatz auf die (qualitativ) komparative Methode zurück.

Die komparative Methode basiert im Kern auf zwei unterschiedlichen methodischen Logiken induktiver Analyse, die John Stuart Mill in seinem Werk „A System of Logic“ Ende des 19. Jahrhunderts beschreibt. Danach gibt es einerseits die Konkordanzmethode (method of agreement), andererseits die Differenzmethode (method of difference). Aus einer Kombination beider Methoden ergibt sich die indirekte Differenzmethode (indirect method of difference bzw. joint method of agreement and difference) (vgl. Mill 1874, S. 278-284).

Die Konkordanzmethode stellt Fälle mit Ähnlichkeiten im zu erklärenden Phänomen (explanandum) gegenüber, um gemeinsame Bedingungen in den erklärenden Faktoren (unabhängigen Variablen) zu finden: „In the Method of Agreement, we endeavored to obtain instances which agreed in the given circumstances but differed in every other [...].“ (Mill 1874, S. 280) Um kausale Beziehungen zwischen unabhängigen Variablen und Explanandum herstellen zu können, bedarf es bei der Konkordanzmethode einer Fallauswahl, die dem ,most different cases design' entspricht. Andernfalls sind die kausal relevanten unabhängigen Variable schwer zu bestimmen. Gleichzeitig findet diese Methode häufig Anwendung bei Untersuchungen, die durch die Nebeneinander- 
stellung von Fällen eine Theorie belegen wollen: „Theory dominates in this approach, with each case being presented to support an overarching and deductive argument.“ (Janoski/Hicks 1994, S. 15) Damit steht sie dem von Skocpol und Somers definierten Ansatz einer 'parallel demonstration of theory' sehr nahe (vgl. Skocpol/Somers 1980, S. 175-178). Hier besteht jedoch die Gefahr einer Überbetonung der Theorie auf Kosten der Erklärung von Varianzen zwischen den Fällen.

\section{Mills Methoden induktiver Untersuchung}

1. Konkordanzmethode (method of agreement):

2. Differenzmethode (method of difference):

$\begin{array}{llll}\text { Fall } 1 & \text { Fall } 2 & \text { Fall 1 } & \text { Fall } 2\end{array}$

Unabhängige

Variablen

$\begin{array}{lll}X 1 & \text { ja } & \text { ja } \\ X 2 & \text { ja } & \text { ja } \\ X 3 & \text { ja } & \text { ja }\end{array}$

ja

ja

ja

ja

ja

Abhängige

Variable

Y ja

ja

ja

ja

nein

3. Indirekte Differenzmethode (indirect method of difference):

\section{Unabhängige}

Variablen

Fall 1

Fall 2

Fall 3

Fall 4

$\mathrm{X} 2$
$\mathrm{X} 3$

Abhängige

Variable

Y ja

ja
ja
ja

ja

nein

ja

nein

nein

nein

ja

nein

nein

Quelle: Mill 1874, S. 278-284 und Janoski/Hicks 1994, S. 14

Die Differenzmethode geht dagegen von Unterschieden im Explanandum aus und sucht dementsprechend nach Abweichungen bei den unabhängigen Variablen. Der Anspruch einer kausal analytischen Untersuchung setzt hierbei voraus, daß sich die Fälle in grundlegenden Kriterien ähneln: ,[...] in the present method we require, on the contrary, two instances resembling one another in every other respect, but differing in the presence or absence of the phenomenon we wish to study." (Mill 1874, S. 280) Die Differenzmethode erfordert also die Auswahl möglichst ähnlicher Fälle. Sie entspricht damit dem ,most similar cases design'. In der Methodologie von Skocpol und Somers bestehen gewisse Überschneidungen zwischen der Differenzmethode und dem Ansatz 
des ,contrast of context', wobei dieser insbesondere die Grenzen generalisierter Theorien aufzeigen möchte (vgl. Skocpol/Somers 1980, S. 178-181). Da die Konkordanzmethode eine wirkliche kausal analytische Beziehung zwischen unabhängiger Variable und Explanandum nicht feststellen kann, schlägt John Stuart Mill vor, daß ihre Ergebnisse als Grundlage für die Anwendung der Differenzmethode dienen sollten (vgl. Mill 1874, S. 282).

Die Kombination beider Methoden, die Mill als indirekte Differenzmethode bezeichnet, führt insofern zu einer methodologischen Verbesserung, als zwei Gruppen von Fällen eine mit dem zu erklärenden Phänomen (,positive cases'), die andere ohne (,negative cases') - miteinander verglichen werden. Die negativen Fälle dienen dabei sozusagen als „Kontrastfolie“. In beiden Gruppen kommt zunächst die Konkordanzmethode zur Anwendung. Die Auswahl der positiven Fälle erfolgt entsprechend der Konkordanzmethode nach den Ähnlichkeiten im Explanandum und den unabhängigen Variablen. Bei den negativen Fällen besteht die Konkordanz gerade darin, daß sie das Explanandum und die - kausal relevanten - unabhängigen Variablen nicht aufweisen. Auf der Ebene darüber setzt die Differenzmethode an, indem sie die beiden Fallgruppen einander gegenüberstellt und so die kausalen Beziehungen deutlich macht: „One may then attribute causal force to the variables that do not have shared values." (Janoski/Hicks 1994, S. 15) Den Einsatz der indirekten Differenzmethode favorisieren Skocpol und Somers im Rahmen ihres dritten komparativ historischen Ansatzes, der ,macro-causal analysis'. Sie versuchen, diese bewußt an der Logik der statistischen Methode auszurichten: „This third variant of comparative history is, indeed, a kind of multivariate analysis [...].“ (Skocpol/Somers 1980, S. 182) Dabei müssen sie aber gleichzeitig konstatieren, daß eine stringente Kausalanalyse beim Vergleich historischer Ereignisse nicht möglich ist (vgl. ebd., S. 193).

Diese Arbeit ist eindeutig dem fallorientierten Paradigma vergleichender Sozialforschung zuzuordnen. Die Anwendung der komparativen Methode entspricht dem Ziel, die einzelnen Fällen in ihrem historisch spezifischen Kontext zu verstehen und über die Entschlüsselung der komplexen Ursachenkonfigurationen kausal analytische Erklärungen anzubieten. Die vorliegende Untersuchung folgt dabei der Millschen Logik der Differenzmethode, indem sie zwei Fälle vergleicht, die sich im Explanandum unterscheiden und deren Differenz anhand unterschiedlicher Ausprägungen der unabhängigen Variablen erklärt werden soll. Das zu erklärende Phänomen ist hierbei die Entste- 
hung eines dualen Berufsausbildungssystems. Die Suche nach den Bedingungen (unabhängigen Variablen) dieser Form der Berufsbildung richtet entsprechend dem Ansatz des historischen Institutionalismus auf das fallspezifische Institutionengeflecht. Im Gegensatz zur idealtypischen Form der Differenzmethode steht hier jedoch der „negative“ Fall, die USA, im Mittelpunkt der Analyse, während der ,positive“ Fall, Deutschland, als Kontrastfolie dient. Die USA versuchten in ihrer Geschichte mehrmals, eine dem deutschen dualen Berufsausbildungssystem vergleichbare Berufsbildung zu schaffen. Dieser explizite Blick nach Deutschland legt es nahe, die institutionellen Bedingungen der deutschen Berufsausbildung als kontrastierenden Bezugspunkt bei der Analyse des amerikanischen Falls heranzuziehen, um Erklärungen für das wiederholte Scheitern dieser berufsbildungspolitischen Idee aufzuspüren. 


\section{Aufbau der Arbeit}

Die folgende Untersuchung soll die Frage beantworten, wieso die Einführung einer dem deutschen dualen Berufsbildungssystem vergleichbaren Berufsausbildung trotz des hohen politischen Stellenwertes unter Präsident Clinton scheiterte. Die deutsche duale Berufsausbildung galt den Reformern als nachahmenswertes Vorbild, und der Besuch verschiedenster amerikanischer Politiker, darunter auch Hillary Clinton, in Deutschland stellen diese Bedeutung für die amerikanische Reformdebatte in den frühen 1990er Jahren exemplarisch heraus. Aufgrund des modellhaften Charakters der deutschen Berufsausbildung für die USA liegt es für die Beantwortung der Forschungsfrage nahe, zuerst den deutschen Fall eingehend zu analysieren, um ihn anschließend sozusagen als „Kontrastfolie“ dem amerikanischen Fall gegenüberzustellen. Zudem können nur anhand des deutschen Falls die spezifischen institutionellen Voraussetzungen herausgearbeitet werden, deren Vorhandensein für die USA zu untersuchen ist. Da die Forschungsfrage unter Heranziehung des historisch-institutionalistischen Ansatzes von T. Skocpol beantwortet werden soll, ergibt sich daraus die Notwendigkeit, diesen Ansatz auch auf den deutschen Fall anzuwenden, um die Vergleichbarkeit der beiden Fälle zu garantieren.

Die Analyse der dualen Berufsausbildung in Deutschland (Teil II) gliedert sich dementsprechend in die drei Abschnitte: Historische Grundlagen, Institutionen und Interessen sowie staatliche Berufsbildungspolitik seit den 1970er Jahren. Im ersten Abschnitt wird die historische Entwicklung und strukturelle Ausformung der Lehrlingsausbildung im 19. und frühen 20. Jahrhundert nachgezeichnet. Besonderes Augenmerk gilt dabei der Frage des spezifischen Verhältnisses zwischen Staat - in diesem Fall wird auf Preußen als für die gesamtdeutsche Entwicklung wichtigstem deutschen Staat Bezug genommen - und (Schul-)Bildung bzw. beruflicher Bildung: Inwieweit hat der Staat regulativ in den Bildung- bzw. Berufsbildungsbereich eingegriffen? Welche anderen Akteure konnten sich in diesem Bereich etablieren bzw. verloren durch die Intervention des Staates an Einflußmöglichkeiten? Welche Struktur liegt der Berufsausbildung am Ende dieser Zeitperiode zugrunde?

Der Abschnitt „Institutionen und Interessen“ untergliedert sich in die Teile: Ausbildungsmarkt und Beschäftigungssystem, Unternehmensverbände und Arbeitgeber, Gewerkschaften und staatliche Institutionen. Diese Unterteilung ergibt sich aus den Er- 
gebnissen der historischen Analyse, die die für das duale Berufsbildungssystem entscheidenden Akteure Arbeitgeber und deren Verbände, Gewerkschaften und Staat herausstellt. Aber auch die spezifische institutionelle Ausformung des Ausbildungsmarktes und des gesamten Beschäftigungssystems - inklusive der Betriebs- und Arbeitsorganisation sowie des Arbeitsmarktes - hängen mit der besonderen Form der Berufsausbildung zusammen bzw. interagieren mit ihr. In diesem Abschnitt richtet sich das Augenmerk auf die Frage, inwiefern die institutionellen Strukturen Anreize schaffen, so daß sich die Akteure systemstabilisierend verhalten: Welche Bedeutung haben Unternehmensverbände für die Interessen der Arbeitgeber? Wie beeinflußt der Ausbildungsmarkt das Verhalten der Jugendlichen? Welche Auswirkung hat die Organisationsform auf das Verhalten der Gewerkschaften? Und welche Rolle kommt den staatlichen Institutionen zu?

Im letzten Abschnitt steht im Sinne des ,policy feedback' die Berufsbildungspolitik der vergangenen drei Jahrzehnte im Mittelpunkt der Untersuchung. Hier zeigt sich, wie Reformbestrebungen - z.B. die Einrichtung eines Berufsgrundbildungsjahres - aufgrund der historisch gewachsenen institutionellen Strukturen und den sich daraus ergebenen Interessen der Akteure gescheitert sind. Aus solchen Erfahrungen haben die beteiligten Akteure gelernt und ihre Problemlösungsstrategien entsprechend angepaßt. Dies hat zur Folge, daß Reformen bzw. Problemlösungen sich innerhalb des durch die institutionelle Struktur gesetzten Rahmens bewegen. Damit bestätigt sich für das deutsche duale Berufsbildungssystem die These der Pfadabhängigkeit.

Vor dem Hintergrund des deutschen Falls steht im Teil III die Analyse des amerikanischen Falls, auf den sich die allgemeine Forschungsfrage im wesentlichen bezieht. Wie Teil II ist auch dieser Teil in drei Abschnitte untergliedert: Historische Grundlagen, Institutionen und Interessen sowie bundesstaatliche Ausbildungs- und Berufsbildungspolitik. Auch hier konzentriert sich der erste Abschnitt auf die Untersuchung der institutionellen Ausformung und strukturellen Entwicklung der beruflichen Bildung in den USA im 19. und frühen 20. Jahrhundert. Dabei gilt es, die Entstehung eines allgemeinen (Schul-)Bildungssystems und das Verhältnis des Staates zu diesem darzustellen, da sich daraus Konsequenzen für die Struktur der Berufsbildung ergeben.

Der zweite Abschnitt unterteilt sich in die Kapitel: Jugendarbeitsmarkt, Unternehmensverbände und Arbeitgeber, Gewerkschaften, staatliche Institutionen sowie Leh- 
rergewerkschaften und Eltern. Diese Unterteilung basiert auf der Analyse des deutschen Falls, anhand dessen die für die duale Berufsausbildung wesentlichen Institutionen und Akteure herausgearbeitet wurden. Anders als im deutschen Fall ergibt sich die Notwendigkeit, die Rolle der Lehrergewerkschaften und Eltern im Zusammenhang mit der Reform der Berufsausbildung zu untersuchen, da sich beide als wichtige Akteure im Politikfeld der US-amerikanischen Schulreform herausgestellt haben. Wie im deutschen Fall geht es auch hier um die Frage der institutionellen Strukturen und der durch sie geschaffenen Anreizstruktur, wobei im Hintergrund die Frage nach der Kompatibilität mit der institutionellen Struktur einer dualen Berufsausbildung steht: Welchen Einfluß hat die Funktionsweise des Jugendarbeitsmarktes auf das Verhalten der Jugendlichen und der Arbeitgeber? Inwieweit können die amerikanischen Unternehmensverbände die zur Erstellung des Kollektivgutes Berufsqualifikation notwendige Regulationsfunktion übernehmen? Wie wirkt sich die Organisationsform der Berufsgewerkschaft auf das Verhalten der Gewerkschaften aus? Welche Steuerungskapazität besitzt der Staat und kann er möglicherweise funktionale Äquivalente schaffen, die fehlende institutionelle Voraussetzungen ausgleichen?

Im dritten Abschnitt steht wieder im Sinne des ,policy feedback' die Berufsbildungspolitik der letzten Jahrzehnte im Mittelpunkt der Untersuchung. Auch hier gilt es aufzuzeigen, wie die beteiligten Akteure aus den Erfahrungen erfolgreicher bzw. gescheiterter Reformen gelernt haben und entsprechend ihre zukünftigen Problemlösungsoptionen angepaßt haben. Wie im deutschen Fall läßt sich dabei die These einer pfadabhängigen Entwicklung bestätigen.

Der Teil IV greift die aktuelle Reformdebatte unter Präsident Clinton auf und untersucht das Ergebnis dieser Debatte, den School-to-Work Opportunities Act (STWOA). Dabei wird deutlich, daß sich das erklärte Ziel einer Einführung der dualen Berufsausbildung nicht realisieren ließ. Vielmehr setzt das neue Gesetz die Entwicklung einer dezentral organisierten und schulisch geprägten Berufsbildung fort. Mit dem Auslaufen der finanziellen Förderung durch die amerikanische Bundesregierung hat es zudem seine begrenzte Bedeutung weitgehend verloren. Im abschließenden Kapitel bleibt zu fragen, welche berufsbildungspolitische Option eine - mit Blick auf das ,institutional setting' gangbarere Alternative für die USA ist, um sein sozialpolitisches und qualifikatorisches Defizit im Bereich der ,non college-bound' Jugendlichen auszugleichen. 
Zusammenfassend ist zu sagen, daß es sich bei der vorliegenden Arbeit nicht um eine narrative Analyse zweier unterschiedlicher Entwicklungspfade beruflicher Bildungssysteme handelt. Es soll vielmehr anhand des strukturellen institutionellen Gefüges der USA das Scheitern des berufsbildungspolitischen Reformansatzes der Clinton Regierung erklärt werden. Dabei greift die Untersuchung auf den historisch institutionalistischen Theorieansatz zurück, der die gewachsene institutionelle Infrastruktur in den Mittelpunkt seines Erklärungsmodells stellt. Die Analyse der deutschen dualen Berufsausbildung ergibt sich aus der amerikanischen Reformdebatte und dient - wie bereits erwähnt - als „Kontrastfolie“ für den amerikanischen Fall.

Grundlage der vorliegenden Arbeit bildet eine Sekundäranalyse. Sie bezieht ihre empirischen Daten folglich aus verschiedenen Monographien, Sammelbeiträgen und Artikeln zu den beiden Fällen. Darüber hinaus wurde dieses Material durch offizielle Dokumente - wie Stellungnahmen oder Protokolle des U.S. Kongresses - und Expertengespräche mit Richard Kazis (Vize-Präsident von Jobs for the Future) sowie Lehrern der Vocational High School in Lowell, Massachusetts, ergänzt. 


\section{Teil II: Die duale Berufsausbildung in Deutschland}

Um die amerikanischen Versuche einer Übernahme des deutschen Berufsbildungssystems verstehen zu können, ist es wichtig, dessen institutionelle Struktur und die Einbettung in das überlagernde institutionelle Dach des deutschen Sozialmodells zu erkennen. Unter Heranziehung des historisch-institutionalistischen Ansatz von $\mathrm{T}$. Skocpol soll im folgenden der Frage nachgegangen werden, wie sich die institutionelle Struktur der dualen Berufsausbildung historisch entwickelt hat und welche Institutionen und Interessen zu dessen Funktionsfähigkeit sowie Stabilität beitragen. Anhand der Berufsbildungspolitik der letzten Jahrzehnte zeigt sich dabei die strukturelle Kontinuität der dualen Berufsausbildung, die sich aus dem historisch gewachsenen institutionellen Rahmen und den sich darauf beziehenden Interessen erklärt.

\section{Historische Grundlagen}

Die Geschichte der Berufsausbildung in Deutschland geht auf die Lehrlingsausbildung der Gilden und Zünfte bis weit in das 13. Jahrhundert zurück. Entscheidende institutionelle Elemente, wie die hierarchisch-qualifikatorische Dreiteilung Lehrling - Geselle - Meister und die Selbstverwaltung der handwerklichen Korporationen fanden sich noch zu Beginn des 19. Jahrhunderts. Der absolutistische Staat hatte aber spätestens zum Ende des 18. Jahrhunderts damit begonnen, seine regulatorische Kompetenz auch auf diesen gesellschaftlichen Bereich auszudehnen. Das Allgemeine Landrecht für die Preußischen Staaten von 1794 ist ein Beispiel für diese Entwicklung.

Unter der Eindruck der französischen Revolution und der militärischen Niederlage von 1806 sah sich Preußen in seiner Existenz bedroht. Nur durch eine Revolution von oben glaubte die militärisch-politische Elite den preußischen Staat retten zu können. Die Einführung der Gewerbefreiheit war ein wichtiger Reformbestandteil. Gleichzeitig stellte sie eine der größten Bedrohungen für die handwerkliche Selbstverwaltung durch die Gilden und Zünfte dar. Das hatte auch Auswirkungen auf die Lehrlingsausbildung, deren Niedergang im frühen 19. Jahrhundert unausweichlich schien. Dieser historische Augenblick kann im Sinne des historischen Institutionalismus als ,critical juncture' 
bezeichnet werden, da sich hier das Fundament einer Institution aufzulösen begann und sich die Option einer Neuorientierung eröffnete.

Die folgenden Kapitel zeichnen die Entwicklung der Lehrlingsausbildung vom frühen 19. Jahrhundert bis zur Weimarer Republik nach. Diesen weiten Bogen zu spannen, ist notwendig, um den historisch-institutionellen Verlauf der deutschen Berufsausbildung und seine endgültige institutionelle Ausformung im frühen 20. Jahrhundert verstehen zu können.

\subsection{Staat und Bildungswesen}

Anhand der Entwicklung des preußischen Bildungswesens, insbesondere des Volksschulwesens, soll im folgenden das Verhältnis des Staates zur allgemeinen (Schul)Bildung und die Herausbildung institutioneller Charakteristika des Schulwesens im 19. Jahrhundert dargestellt werden. Die preußische Schulorganisation beeinflußte nach der Reichsgründung 1871 das Schulwesen in den anderen deutschen Staaten trotz der fortbestehenden Kulturhoheit der Länder in besonderem Maße, so daß die exemplarische Analyse des preußischen Falles hier gerechtfertigt ist.

Ende des 18. Jahrhunderts hatte im preußischen Schulwesen ein Modernisierungsprozeß eingesetzt, der sich in der Reformära des frühen 19. Jahrhunderts unter dem allgemeinen politischen und sozio-ökonomischen Veränderungsdruck noch beschleunigen sollte. Unter dem Eindruck der Aufklärung gewann die Diskussion über eine Ausweitung und Reform der Schulbildung an Bedeutung. Dabei setzte sich die Auffassung durch, daß der Staat im Zentrum einer umfassenden Schulreform stehen müsse. In diesem Sinne ist auch der politische Aspekt der Aufklärungspädagogigk zu verstehen, der eine über die Schule vermittelte Integration der Bevölkerung in den sich entwickelnden modernen Staat vorsah. Von einer Steigerung der Volksbildung versprachen sich die Schulreformer einen ökonomischen Vorteil für den Staat: Die Bildungspolitik sollte ein wesentlicher Bestandteil einer staatlich-merkantilistischen Wirtschaftsförderung sein. Der preußische Justizminister von Zedlitz faßte diese Überlegungen 1787 in seinen „Vorschlägen zur Verbesserung des Schulwesens in den Königlichen Landen“ zu einem pragmatischen Programm zusammen. Es beinhaltete die Idee einer Ausweitung der schulischen Erziehung unter gleichzeitiger Begrenzung 
derselben nach sozialen Kriterien in Form eines ständisch differenzierten Schulwesens mit Bauern-, Bürger- und Gelehrtenschulen. Darüber hinaus sollte eine direkt dem König unterstellte Schulbehörde, das sogenannte Oberschulkollegium, geschaffen werden, um die staatliche Kontrolle über alle Schulen zu gewährleisten. Wegen der geringen finanziellen Ausstattung dieser Behörde und dem Widerstand partikularer, traditionaler Machtinteressen, die ihre überkommenen Patronatsrechte verletzt sahen, konnte das Oberschulkollegium die ihm zugedachte Funktion nicht ausfüllen. Der Konflikt zwischen staatlicher Schulorganisation und partikularen Machtzentren mit feudalen Sonderrechten setzte sich bis ins 19. Jahrhundert fort (vgl. Baumgart 1990, S 13 ff.; Kuhlemann 1992, S. 52 ff.; Neugebauer 1989, S. 25 ff.). Mit dem Erlaß des „Allgemeinen Landrechts für die Preußischen Staaten“ (ALR) im Jahre 1794 wurde dem Staat die Verantwortung für das Bildungswesen zugesprochen: „Schulen und Unterricht sind Veranstaltungen des Staates, welche den Unterricht in nützlichen Kenntnissen und Wissenschaften zur Absicht haben." (§ 1, 12. Teil des ALR, zit. nach: Baumgart 1990, S. 26) Die Regelungen des ALR bildeten bis zum Ende des 19. Jahrhunderts die gesetzliche Grundlage für die Organisation des Schulwesens.

Die preußische Niederlage gegen Napoleon 1806/7 stellte eine existentielle Krise für den ständisch-absolutistischen Staat dar, die eine weitreichende gesellschaftliche und staatliche Neuordnung erforderte. Die Stein-Hardenbergschen Reformen umfaßten neben der Konstituierung einer liberalen Agrar- und Gewerbeverfassung sowie einer die Selbstverwaltungsrechte ausweitenden Städteordnung auch Überlegungen zu einer grundlegenden Bildungsreform. Wilhelm von Humboldt übernahm 1808 die Leitung der neu eingerichteten Sektion für Kultur und öffentlichen Unterricht im preußischen Innenministerium, die nach Auflösung des Oberschulkollegiums entstanden war. Unter seiner Ägide fanden die pädagogischen Ideen des Neuhumanismus Eingang in die Planungen zur Schulreform. Der Neuhumanismus kritisierte die utilitaristische Aufklärungspädagogik des 18. Jahrhunderts. In seinem Mittelpunkt stand das Ideal einer allgemeinen Menschenbildung, das sich gegen eine utilitaristisch begründete gesellschaftliche Verwertbarkeit des Individuums und eine frühzeitige ständisch-berufliche Orientierung der Schulerziehung richtete. Allen Schülern sollten gemeinsame Begriffe, Kenntnisse und Wertvorstellungen vermittelt werden. Auf der Basis dieser Allgemeinbildung sollte ein Wechsel zwischen verschiedenen beruflichen Tätigkeiten ermöglicht und die spätere Aneignung beruflichen Fachwissens erleichtert werden. In 
dieser Hinsicht stand der Neuhumanismus im Zusammenhang mit den wirtschaftlichen Reformen, insbesondere der Einführung der Gewerbefreiheit. Primär beinhaltete er aber die Idee einer auf einer allgemeinen Menschenbildung basierenden Nationalerziehung zur politisch-sozialen Reorganisation des preußischen Staates (vgl. Schleunes 1989, S. 66 f. und 73 ff.; Kuhlemann 1992, S. 60 ff.).

Die praktische Umsetzung der neuhumanistischen Reformideen erforderte eine umfassende Neuordnung des gesamten Schulwesens: So sollte das Bildungswesen vom Elementarunterricht bis zum Universitätsstudium eine durchgängige organisatorische Einheit bilden, die allen Staatsbürgern die gleichen Bildungschancen einräumt. Aus den Volksschulen sollten Elementarschulen werden, die alle schulpflichtigen Kinder durchlaufen und auf denen die höheren Schulen, insbesondere die Gymnasien, aufbauen. Die Schaffung eines einheitlichen nationalen Bildungswesens scheiterte aber an der desolaten öffentlichen Finanzsituation. Die Reform des Volksschulwesens konnte aufgrund des Widerstandes der Gutsbesitzer nicht durchgesetzt werden. Diese sahen ihre traditionalen Patronatsrechte bedroht und waren nicht bereit die zusätzlichen finanziellen Belastungen, die durch den notwendigen Ausbau der Volksschulen auf sie zugekommen wären, zu tragen. Nur im Bereich des höheren Schulwesens konnten aufgrund günstigerer Ausgangsbedingungen Teile der neuhumanistischen Bildungsreform umgesetzt werden. Ein Resultat war die Herausbildung des altsprachlichen Gymnasiums als zentraler Bildungsinstitution des höheren Schulwesens und dessen enge Verkoppelung mit der staatlichen Bürokratie (vgl. Herrlitz et al. 1998, S. 37 f.; Becker/Kluchert 1993, S. 3 f.).

Die Bildungsreform gewann durch das Auseinanderdriften ihrer Bewegungen Scheitern im Volksschulwesen, partieller Erfolg im höheren Schulwesen - eine Eigendynamik, die dem Ziel der Bildungsreformer, ein einheitliches nationales Bildungswesen zu schaffen, zuwiderlief. Mit dem Scheitern des Süvernschen Unterrichtsgesetzentwurfs von 1819, der im Geist der neuhumanistischen Bildungsidee jede Form der beruflich orientierten Bildungsorganisation ablehnte, setzte eine Phase der Revision der liberalen Schulpolitik ein. Die konservative Kritik richtete sich gegen die Idee einer ständisch-indifferenten Schulorganisation. Die curriculare Festlegung des Volksschulunterrichts auf die Vermittlung christlicher Wertvorstellungen sollte zu einer Stabilisierung der traditionalen gesellschaftlichen Ordnung beitragen. Statt gleicher 
Bildungschancen für alle galt nun das Ideal der ständischen Bildungsbeschränkung (vgl. Schleunes 1989, S. 96 ff.; Baumgart 1990, S. 93 ff.).

Die Verordnung zur Regelung der Abiturienten von 1834 bestimmte, daß ,untüchtige“ Absolventen nicht mehr zum höheren Staatsdienst und zum Universitätsstudium zugelassen wurden. Darüber hinaus erließ das inzwischen gegründete Kultusministerium 1837 einen für alle Gymnasien verbindlichen Lehrplan, der im Sinne der neuhumanistischen Bildungsidee die Bedeutung des altsprachlichen Unterrichts hervorhob: „Die Bedürfnisse des modernen Wirtschaftslebens blieben unerfüllt und sie sollten es bleiben.“ (Mast 1989, S. 139) Diese Maßnahmen bedeuteten eine Durchsetzung des im Neuhumanismus impliziten Leistungsprinzips und richteten sich insofern gegen überkommene Privilegien des Adels. Die Monopolstellung des Gymnasiums als vorbereitender Anstalt für Universität und Staatsdienst wurde dadurch weiter gefestigt. Entsprechend unterlagen die Prüfungen einer besonderen staatlichen Aufsicht. Vergleichbare Normierungstendenzen beinhalteten die „Stiehlschen Regulative“ von 1854, so benannt nach dem Referenten für das Volksschulwesen im Ministerium. Darin wurden detaillierte Vorschriften zu Lehrplan und -methoden in den Volksschulen sowie zur Ausbildung der Volksschullehrer in den Lehrerseminaren niedergelegt. Eine zentrale Rolle nahm auch hier der christliche Religionsunterricht ein. Die Idee einer die traditionalen Gesellschaftsstrukturen stabilisierenden Schulerziehung ließ sogar utilitaristische Überlegungen, wie die Vermittlung praktischer Fertigkeiten, in den Hintergrund treten (vgl. Baumgart 1990, S. 192 ff.; Herrlitz et al. 1998, S. 61 f.; Kuhlemann 1992, S. 237; Mast 1989, S. 137 f.). Damit hatte sich ein staatlich normierter Standardisierungsprozeß in allen Bereichen des Schulwesens durchgesetzt.

Die zur Umsetzung und staatlichen Kontrolle notwendige Verwaltung wurde in den ersten Jahrzehnten des 19. Jahrhunderts geschaffen. Aus der Sektion für Kultus und Unterricht des Innenministeriums entstand 1817 das Ministerium der geistlichen, Unterrichts- und Medizinalangelegenheiten mit unterschiedlichen Abteilungen für die Universitäten, das höhere Schulwesen und das Volksschulwesen. In den unteren Instanzen setzte sich diese Trennung fort: Für die höheren Schulen waren die Provinzialschulkollegien zuständig. Die Aufsicht der Volksschulen oblag den Bezirksregierungen sowie den Kreis- bzw. Lokalschulinspektionen, die bis zum Schulaufsichtsgesetz von 1872 in den Amtsbereich der Geistlichen fielen. Das Selbstverwaltungsrecht der Städte erstreckte sich auch auf die Verwaltung des Schulwesens und erlaubte es 
ihnen, eigene Schuldeputationen zu bilden, die sich aus Mitgliedern des Magistrats, der Stadtverordnetenversammlung und sachverständigen Bürgern zusammensetzten. Ihre Kompetenz beschränkte sich jedoch auf die niederen Schulen und auf die ,äußeren Angelegenheiten', d.h. Errichtung und Unterhaltung der Schulen. Für die Regulierung der ,inneren Angelegenheiten' - Gegenstand und Form des Unterrichts - blieb weiterhin der Staat zuständig (vgl. Becker/Kluchert 1993, S. 29 ff.; Kuhlemann 1992, S. 63 f.; Meyer 1976, S. 47 ff.).

Bereits mit dem preußischen Allgemeinen Landrecht wurde wenigstens normativ die Verantwortung des Staates für das Schulwesen festgeschrieben. In der Reformära des frühen 19. Jahrhunderts hatte der Staat die Funktion des ,Impulsgebers' in allen gesellschaftlichen Bereichen. Die Schulreformer sahen in der Idee des Neuhumanismus die Chance, durch die Schaffung eines auf der allgemeinen Menschenbildung basierenden einheitlichen Systems der Nationalerziehung die geistige und gesellschaftliche Erneuerung des preußischen Staates voranzutreiben. Ihre Kritik an einer utilitaristischen Ausrichtung des Schulwesens blieb in der Folgezeit richtungsweisend für die inhaltliche Gestaltung der Schulerziehung sowohl in den höheren Schulen, insbesondere den Gymnasien, als auch in den Volksschulen. Die staatliche Aufsicht und Reglementierung der Schulen führte zur Vereinheitlichung und Standardisierung der Lehrinhalte sowie zur Formalisierung der Zugangsberechtigungen zur Universität und zum höheren Staatsdienst. Den Gymnasien kam insofern eine staatsfunktionale Rolle als Institution zur Selbstreproduktion der Beamtenschaft zu. Für die Entwicklung der beruflichen Bildung war die Ablehnung aller utilitaristischen Tendenzen im allgemeinen Schulwesen entscheidend.

\subsection{Die Lehrlingsausbildung bis zur Industrialisierung}

Nach dem Ende des dreißigjährigen Krieges 1648 lagen die deutschen Staaten wirtschaftlich darnieder. Weite Landstriche waren durch die Kriegsfolgen völlig entvölkert. Die mittelalterlich-ständische Gesellschaftsordnung stand an einem Wendepunkt. In dieser wirtschaftlichen Notsituation häuften sich die Stimmen, die für eine Abschaffung der handwerklichen Zunftordnungen plädierten, um deren Ausbildungsmonopol zu 
brechen. Die partikularistischen Interessen der Zünfte schienen einer wirtschaftlichen Erholung entgegenzustehen. Das sich entwickelnde absolutistische Staatsverständnis stand den autonomen Korporationen ebenfalls kritisch gegenüber. Andererseits dürfen die Zünfte nicht nur unter ökonomischen Bedingungen betrachtet werden, sondern in ihrer gesamtgesellschaftlichen Bedeutung. Sie waren ein wichtiger Stabilisator der sozialen Ordnung. In ihnen vollzog sich die ständische Sozialisation und soziale Normierung. Deshalb konnten und wollten die absolutistisch regierten Territorialstaaten auch nicht vollends auf sie verzichten. Die weitreichenden Privilegien der Zünfte widersprachen aber ihrem Staatsverständnis. Der Konflikt zwischen staatlichem Absolutheitsanspruch und korporativer Autonomie zieht sich daher bis zum Beginn des 19. Jahrhunderts wie ein roter Faden durch die Berufsbildungspolitik. Im folgenden soll ein Überblick über den Wandel des Verhältnisses des Staates zu den Zünften und dessen Auswirkungen auf die Form der Lehrlingsausbildung bis zu den Anfängen der Industrialisierung gegeben werden.

Die Zünfte waren seit ihrem Bestehen nicht nur eine ökonomische, sondern auch eine politische und soziale Institution. Die Regulierung der Arbeitsbedingungen und Festlegung der Qualität und Preise der Produkte oblag ihnen ebenso wie die politische Korporierung der Bürger der Stadt für die Zwecke des Stadtregiments oder der Stadtwehr. Im wesentlichen waren sie aber ein Sozialgebilde, daß nicht nur alle Lebensbereiche seiner Mitglieder regelte, sondern die gesamte Sozialstruktur der Stadt bestimmte. Niedergelegt waren diese Bestimmungen in den Zunftverfassungen, die keinesfalls einheitlich waren, sondern nach Region, Stadt und Handwerk variierten. Die Versammlung aller Meister einer Zunft (sog. Morgensprache) beschloß die Regeln, die für alle Mitglieder bindend waren. Als autoritas privata stand sie damit der autoritas publica, der städtischen oder staatlichen Obrigkeit, gegenüber. Während in der Entstehungszeit die städtische Obrigkeit die Handwerker priviligierte, sich zu korporieren, nahm in der Folgezeit die Bedeutung des Selbstbestimmungsrechts der Zünfte und damit ihre Autonomie zu. Dies wurde durch die politische Bedeutung der Zünfte in den Städten noch gefördert. Die korporative Autonomie und obrigkeitliche Aufsichtsansprüche befanden sich aber in einem ständigen Spannungs- und Wechselverhältnis (vgl. Stadelmann/Fischer 1955, S. 96 f.; Stratmann 1993, S. 95 f.).

Mit der Herausbildung des absolutistischen Territorialstaates im 17. und 18. Jahrhundert geriet die Autonomie und das Selbstbestimmungsrecht der Zünfte unter Druck. 
Ausgehend vom absolutistischen Staatsverständnis, daß der Staat die einzige und oberste Rechtsquelle darstellt, mußte sich der Konflikt mit den Zünften zuspitzen. Als aufgrund der Aufhebung des Edikt von Nantes ${ }^{10}$ durch Ludwig XIV. die französischen Hugenotten zu Tausenden nach Brandenburg und Hessen flohen, wo sie als qualifzierte Fachkräfte von den Landesherren willkommen geheißen wurden, versuchten die Zünfte wegen fehlender deutscher Meisterbriefe deren Ansiedlung und Arbeitsaufnahme zu verhindern. Der brandenburgische Kurfürst Friedrich Wilhelm I. (1620-1688) beantragte daraufhin im Reichstag des Heiligen Römischen Reiches deutscher Nation die Verabschiedung eines Gesetzes zur Abschaffung der Zünfte. Auch wenn dieser Gesetzesvorschlag am Widerstand insbesondere der Reichsstädte scheiterte, macht er deutlich, daß die Akzeptanz der korporativen Autonomie der Zünfte schwand (vgl. Stratmann 1993, S. 58).

Der Reichsabschied von 1731 markiert einen Höhepunkt in der Handwerksgesetzgebung des Reichstages. Die Zuständigkeit des Reichstages ergab sich aus dem territorial übergreifenden Zusammenhang der Handwerke, insbesondere der Gesellenverbände. Durch diesen Reichsabschied wurde de jure eine einheitliche Zunftordnung festgelegt, die bis zur Auflösung des Heiligen Römischen Reiches im Jahre 1806 bestehen blieb. Wesentliche Elemente dieser Zunftordnung waren die Hervorhebung der heteronomen Zwecke der Zünfte, ihre berufsordnende Funktion und das Kartellverbot. Eine Ausnahme bildeten staatlich angeordnete Zwangskartelle. Anstatt die korporative Autonomie der Zünfte abzuschaffen, beschränkte sich das Reichsgesetz darauf, diese zu beschneiden und alle gewohnheitsrechtlichen Sitten und Gebräuche der staatlichen Aufsicht bzw. Zustimmung zu unterwerfen. Mit dem Reichsabschied „sollen im Heil. Röm. Reich die Handwerker unter sich keine Zusammenkünfte, ohne Vorwissen ihrer ordentlichen Obrigkeit, [...] anzustellen Macht haben [...].“ (zit. nach: Stratmann 1993, S. 103) Darüber hinaus regulierte die Reichszunftordnung die handwerkliche Berufsausbildung, indem sie Art und Weise der Zulassung als Lehrling, des Lehrbriefs, der Wanderjahre und des Meisterstücks festsetzte. Einige auf langer Tradition basierende Zunftbräuche wurden als Mißbräuche benannt und verboten, z.B. Preisabsprachen. Während die Zuweisung einer politischen Funktion der Zünfte

\footnotetext{
${ }^{10}$ Das Edikt von Nantes (1598) sicherte den Protestanten in Frankreich die freie Religionsausbübung zu.
} 
unterblieb, hielt das Reichsgesetz an ihrer sozialen Funktion fest (vgl. Stadelmann/Fischer 1955, S. 101).

Preußen und Sachsen gehörten zu den ersten Staaten, die die Regelungen des Reichsabschieds übernahmen und in eigene Verordnungen überführten. So erließ Preußen 1733 eine neue „Handwerksordnung“, auf deren Grundlage eine Reihe von „GeneralPrivilegien“ für die einzelnen Handwerke formuliert wurden. Diese legten die Vorgaben des Reichsgesetzes eng aus und bestimmten, daß die Zünfte nur mit Kenntnis und Erlaubnis des Magistratsbeisitzers zusammentreten durften. 1772 wurde ein kaiserliches Dekret erlassen, das im Rahmen der Reichszunftordnung den Zünften eine Beschränkung der Lehrlings- und Gesellenzahl verbot. Tatsächlich war aber der Reichsabschied in vielen Reichsstädten aufgrund der dortigen Stellung der Zünfte zu diesem Zeitpunkt noch gar nicht umgesetzt worden. De jure markierte der Reichsabschied von 1731 einen einschneidenden Wandel im Verhältnis des Staates zu den Zünften. De facto mangelte es ihm jedoch in weiten Teilen Deutschlands an Durchsetzungskraft (vgl. Stadelmann/Fischer 1955, S. 101 f.; Stratmann 1993, S. 103 f.). Preußen nahm mit seinen „General-Privilegien“, die die lokale Autonomie der Zünfte weitgehend beschränkten und sie der Kontrolle des Staates unterstellten, eine Sonderrolle ein. Mit dem Allgemeinen Landrecht für die Preußischen Staaten von 1794 setzte sich die Kodifizierung des staatlichen Einflusses auf die Zünfte fort: „Der Landesherr allein hat das Recht, eine bisher ungeschlossen gewesene Zunft in eine geschlossene zu verwandeln; d.h. die Zahl der Mitglieder, aus welchen die Zunft an einem Ort bestehen soll, zu bestimmen.“ (§ 183 ALR, in: Stratmann/Schlüter 1982, Dok. 1) Das Allgemeine Landrecht umfaßte detaillierte Regelungen für alle Bereiche des Zunftlebens - von der Zunftverfassung über das Meisterrecht bis hin zur Lehrlingsausbildung.

Durch die Kodifizierung der Rechte und Pflichten der Zünfte bzw. ihrer Mitglieder schränkte die staatliche Verwaltung zwar die korporative Autonomie ein. Andererseits legitimierten diese Bestimmungen die grundsätzliche Existenz von Zünften und förderten deren Fortbestehen. Statt ihre Abschaffung voranzutreiben, beschritt man den Weg der staatlich gesetzten Reform - sowohl aufgrund ordnungspolitischer Überlegungen als auch aufgrund des starken Widerstandes der machtpolitisch etablierten und wohl organisierten Handwerkskorporationen. Unter die staatliche Regulierung fiel auch die berufsausbildende Funktion der Zünfte. Die Berufserziehung verlor dadurch ihre korporativ-kollegiale Beschränkung und rückte ins staatlich-öffentliche Interesse. 
Die Regelung und Beaufsichtigung der Lehrlingsausbildung gehörte zu den wesentlichen Aufgaben der Zünfte. Da nur die in der Zunft ausgebildeten Lehrlinge auch eine Meisterschaft anstreben konnten, bot sich den Zünften mit der Kontrolle und Steuerung der Zahl der Lehrlinge ein wesentliches Instrument zur Marktregulation. Deshalb gab es bereits bei der Zulassung als Lehrling durch die Zunft selektive Auswahlkriterien: Der Meister hatte vor der Zunftversammlung die ehrliche, eheliche, christliche und deutsche Geburt seines Lehrlings nachzuweisen. Gleichzeitig prüfte diese, ob der Meister überhaupt berechtigt war, einen Lehrling anzunehmen. Dieser Akt nannte sich „Aufdingung vor offener Lade“ (vgl. Stratmann 1993, S. 197 f.). Das Lehrverhältnis zwischen Meister und Lehrling basierte somit auf einem kollektiv-rechtlichen Vertrag. Ohne Zustimmung der Zunft konnte der Meister z.B. den Lehrling nicht entlassen. Im Lehrvertrag wurden die Lehrzeit, die Höhe des zu zahlenden Lehrgeldes, die Kleidung und Unterbringung etc. festgelegt (vgl. Grießinger/Reith 1986, S. 152; Stratmann 1993, S. 197 f.).

Mit Beginn der Lehre wechselte der Junge aus dem elterlichen Haushalt in den des Meisters. Eine vereinzelte Ausnahme bildeten die Handwerke des Baugewerbes, wo aufgrund des Arbeitsplatzes die Unterbringung des Lehrlings im Haushalt des Meisters nicht notwendig war. Der Eintritt in den Haushalt des Meisters bedeutete auch, daß die bisher bei den Eltern liegende Erziehungsgewalt auf den Meister als Vertreter der Zunft überging. In verschiedenen Gesetzen wurde dieser Erziehungsauftrag kodifiziert, so z.B. auch in der preußischen Handwerksordnung von 1733, die explizit auf die Erziehungsund Ausbildungsfunktion des Meisters verwies. Der Sozialisation und Erziehung des Lehrlings im Sinne des zünftigen Ethos wurde häufig mehr Bedeutung beigemessen als seiner Ausbildung in den Fertigkeiten des Handwerks. Die enge Verknüpfung dieser beiden Elemente - Erziehung und Ausbildung - entwickelte sich im Laufe des 18. Jahrhunderts zur Achillesferse der Lehrlingsausbildung (vgl. Stratmann 1993, S. 43). Unter den Aspekt „Erziehung“ fiel auch die Übertragung von Haus- und Hilfsdiensten auf den Lehrling als Familienmitglied. Je mehr die Meister ihre Lehrlinge als Hausknechte mißbrauchten und dabei die handwerkliche Ausbildung vernachlässigten, desto stärker wurde die allgemeine Kritik an der Handwerkslehre. Karl F. von Klöden (1786-1856), späterer Gewerbeschuldirektor in Berlin, machte diese Situation in einem Bericht über seine Lehrzeit deutlich: ,,...̈̈berall, besonders in den kleinen Werkstätten, wurden die Lehrburschen $\mathrm{zu}$ allem möglichen gebraucht, und ich wußte, daß sie 
eigentlich nur die europäischen Sklaven waren.“ (zit. nach: ebd., S. 218) Da die Zünfte nicht in der Lage waren, diesem weit verbreitetem Mißstand ein Ende zu setzen, griff zunehmend der Staat durch Erlaß von Handwerksordnungen ein. In den kursächsischen General-Innungsartikeln von 1780 war festgelegt, daß die Zahlung des Lehrgeldes an den Meister von einem Nachweis über eine angemessene handwerkliche Ausbildung abhing (vgl. Stratmann 1967, S. 118).

Die Eltern des Lehrlings zahlten an den Meister ein im Lehrvertrag festgelegtes Lehrgeld. Begründet wurde die Zahlung eines Lehrgeldes mit Aufwendungen des Meisters für Verpflegung und Unterbringung des Lehrlings. Dies wird z.B. daran deutlich, daß im Bauhandwerk kein Lehrgeld verlangt wurde, da hier die Lehrlinge nicht bei den Meistern wohnten. Die Höhe des Lehrgeldes war jedoch nicht vorgeschrieben, sondern ergab sich aus der Dauer der Lehre. Dieser Zusammenhang läßt sich grob mit folgendener Formel beschreiben: Je höher das Lehrgeld, desto kürzer die Lehrzeit. Wohlhabende Eltern nutzten dies, um durch Zahlung eines entsprechend hohen Lehrgeldes die Lehrzeit auf ein Minimum zu reduzieren, sog. „Abkaufen der Lehre“. Weitaus verbreiteter war aber die Praxis, daß die Lehrzeit verlängert wurde, weil kein Lehrgeld gezahlt werden konnte. Hier mußte der Lehrling seine Lehre gewissermaßen abverdienen. Das Recht der Meister, die Lehrzeit je nach Höhe des Lehrgeldes festzusetzen, beinhaltete die Gefahr des Mißbrauchs. Um dies zu vermeiden, schrieben die preußischen General-Privilegien eine einheitliche Lehrzeit von drei Jahren vor. Für Lehrlinge, die kein Lehrgeld zahlen konnten, bestand die Ausnahmeregelung einer Lehrzeitverlängerung, die unter Aufsicht des städtischen Magistrats ausgehandelt werden mußte (vgl. Grießinger/Reith 1986, S. 152 ff.; Stratmann 1993, S. 197 ff.). Lehrgeldzahlung, Lehrzeit und Ausbildungspflichten standen in einem reziproken Verhältnis, das zunehmend gesetzlich geregelt wurde, um die Zunftwillkür einzuschränken.

Zusammenfassend läßt sich sagen, daß die wachsende Kritik an den Zünften im 17. und 18. Jahrhundert die staatliche Obrigkeit in verschiedenen deutschen Territorialstaaten zum Anlaß nahm, regulierend in die durch die Zünfte geprägte ständische Ordnung einzugreifen. Die damit einhergehende Einschränkung der Zunftautonomie fügte sich nahtlos in das sich durchsetzende absolutistische Staatsverständnis. Anstatt die Zünfte 
abzuschaffen, was aufgrund ihrer starken Stellung nicht ohne weiteres möglich gewesen wäre, nutzte die staatliche Obrigkeit deren gesellschafts- und sozialpolitisch stabilisierende Wirkung für ihre eigenen machtpolitischen Interessen. Die doppelte Funktion der Lehrlingsausbildung - (gesellschaftspolitische) Erziehung und (ökonomische) Ausbildung - wurde durch die staatliche Regulation bestätigt und gefördert.

\subsection{Gewerbefreiheit, Industrialisierung und berufliche Bildung}

Gegen Ende des 18. Jahrhunderts hatte sich die Situtation der Zünfte und Lehrlingsausbildung insoweit stabilisiert, als die staatlichen Obrigkeiten die Zünfte als gesellschaftspolitischen Ordnungsstabilisator bestätigt hatten. Statt sie abzuschaffen, hatte man ihre umfassende Autonomie durch gesetzliche Regulation beschränkt und der staatlichen Kontrolle in weiten Teilen unterworfen, wie es im preußischen Allgemeinen Landrecht deutlich wird. Die französische Revolution und die darauf folgende Herrschaft Napoleons stellte diese gesellschaftliche Institution endgültig in Frage. In Frankreich etablierte sich ein äußerst restriktives Berufsvereinsrecht. Jegliche nichtstaatlichen Korporationen und ihre Privilegien wurden durch die revolutionäre Gesetzgebung aufgehoben, um eine uneingeschränkte Gewerbefreiheit zu institutionalisieren. Darüber hinaus waren alle beruflichen Vereinigungen und Koalitionen - auch privatrechtlicher Art - verfassungswidrig, weil sie eine Beschränkung der Gewerbefreiheit darstellten. Diesen Rechtsnormen lag die staatstheoretische Idee zugrunde, daß es zwischen den Einzelinteressen des Individuums und dem durch den Staat repräsentierten Allgemeininteresse keine weiteren intermediären Instanzen geben dürfe.

Mit den napoleonischen Feldzügen und der Gründung des Rheinbundes (1806) kam es zur Einführung des französischen Rechts (Code Civil) auf deutschem Boden. In den französisch besetzten linksrheinischen Gebieten, dem Königreich Westfalen, dem Herzogtum Berg und den hanseatischen Departements galt nun die uneingeschränkte Gewerbefreiheit, die ein generelles Zunftverbot bedeutete. Eine ähnliche Entwicklung hin zur Gewerbefreiheit gab es auch in Preußen. Nach der militärischen Niederlage Preußens in der Doppelschlacht von Jena und Auerstädt (1806) kam es zu einer umfangreichen Reformdynamik in allen gesellschaftspolitischen Bereichen. Die SteinHardenbergschen Reformen sind zum Inbegriff der politisch-ökonomischen Moderni- 
sierung Preußens geworden. Als preußischer Staatskanzler bereitete Hardenberg ein Edikt zur Einführung einer allgemeinen Gewerbesteuer vor, das am 2. November 1810 erlassen wurde und als Basis für das Gesetz zur Einführung der Gewerbefreiheit vom 7. September 1811 diente. Während die Einführung einer Gewerbesteuer dem Staat mehr politische Handlungsfreiräume schaffen sollte, erhoffte sich die preußische Ministerialbürokratie durch die Aufhebung des Zunftzwanges und die Einführung der Gewerbefreiheit, die wirtschaftliche Entwicklung zu beschleunigen und den allgemeinen Wohlstand zu fördern (vgl. Stadelmann/Fischer 1955, S. 107).

Das Gesetz über die „polizeilichen Verhältnisse der Gewerbe“ von 1811 schuf mit den folgenden Paragraphen die Grundlage für die Einführung der Gewerbefreiheit in allen preußischen Provinzen und entzog der Lehrlingsausbildung ihren kollektiv-rechtlichen Charakter:

„6. Wer bisher nicht zünftig war, kann unter Beachtung der Vorschriften $§ .1$ bis 5. [Gewerbeschein, Bürgerrecht, Militärpflicht] auf den Grund seines Gewerbescheins jedes Gewerbe treiben, ohne deshalb genöthigt zu seyn, irgend einer Zunft beizutreten.

7. Er ist demohnerachtet auch berechtigt, Lehrlinge und Gehülfen anzunehmen.

8. In diesem Falle wird die Lehrzeit oder die Dauer des Dienstes, das etwanige Lehrgeld, Lohn, Kost und Behandlung bloß durch freien Vertrag bestimmt.“ (zit. nach: Stratmann/Schlüter 1982, S. 72-73)

Auf der Grundlage des Gesetzes konnte jeder der bestimmte Voraussetzungen erfüllte, ein Gewerbe ausüben, ohne eine Meisterlehre in dem entsprechenden Gewerbe durchlaufen zu haben und ohne einer Zunft beitreten zu müssen. Die Aufhebung des Zunftzwanges bedeutete jedoch nicht, daß die Zünfte verboten wurden. Bestehende Zünfte und Handwerksinnungen, deren öffentlicher Charakter im Allgemeinen Preußischen Landrecht noch bestätigt wurde, existierten als freiwillige, privatrechtliche Vereine fort, die auch weiterhin das gemeinschaftliche Interesse ihrer Mitglieder vertreten sollten. Das Gesetz löste aber mit $\S 6$ das rechtliche Ausbildungsmonopol der Zünfte auf, indem es die Aufnahme eines Lehrlings jedem Gewerbetreibenden zugestand unabhängig von seiner Mitgliedschaft in einer Zunft und dem Besitz eines Meisterbriefs. Die Lehrlingsausbildung wurde zu einer privatrechtlichen Angelegenheit zwischen Lehrling und Lehrherr. 
Preußen nahm mit seiner Reformgesetzgebung eine Vorreiterrolle unter den deutschen Staaten ein. In Sachsen wurden die Privilegien der Zünfte zwar beschränkt, der Zunftzwang wurde aber erst 1861 aufgehoben. Württemberg und Baden behielten ihre Zunftverfassung bis in die Mitte des 19. Jahrhunderts bei. Andere Staaten, wie z.B. Hannover, hoben die unter der napoleonischen Herrschaft erlassenen Gesetze zur Gewerbefreiheit in der Zeit der Restauration wieder auf (vgl. Stratmann/Schlüter 1982, S. 12 ff.).

Die Auswirkungen des Gesetzes zur Gewerbefreiheit von 1811 auf die tatsächliche Situation des Handwerks in Preußen waren in den folgenden Jahren relativ gering. Von den Antragstellern für einen Gewerbeschein besaß eine große Mehrheit die Meisterwürde. Dies hing auch damit zusammen, daß viele Konsumenten Vorbehalte gegenüber Handwerkern hatten, die ihre Befähigung zum Gewerbe nicht durch eine Meisterlehre erworben hatten. Darüber hinaus führte die Gewerbefreiheit in den Jahren zwischen 1816 und 1831 zu keinem relativen Anstieg des Handwerks im Vergleich zur Gesamtbevölkerung. Erst mit der Gründung des Zollvereins (1834) und der damit einhergehenden wirtschaftlichen Expansionsphase erhöhte sich die Zahl der Meister, Gesellen und Lehrlinge innerhalb von drei Jahren um ca. 10 \% (vgl. Rinneberg 1985, S. 20 ff.; Kaufhold 1971, S. 176 f. und 186).

Die Aufhebung des Ausbildungsmonopols der Zünfte durch das Gesetz von 1811 zeigte bereits in den ersten Jahren nachteilige Wirkungen auf den Bestand der Lehrlingsausbildung. Mit der Substitution des kollektiv-rechtlichen Charakters der Lehrlingsausbildung durch einen privatrechtlichen Lehrvertrag verloren die Zünfte ihre Kontrollfunktion. Im Lehrvertrag sollten die beiden Parteien - Lehrherr und Lehrling bzw. Eltern - die Dauer der Lehrzeit, die Höhe des Lehrgeldes und die Unterbringung des Lehrlings festlegen. Häufig wurden diese Lehrverträge nicht schriftlich abgefasst, wodurch eine Klärung späterer Streitigkeiten erschwert wurde. Außerdem sank die Verbindlichkeit des Lehrvertrages. Bei Kontraktbrüchigkeit hatte der Lehrling mit keinen Sanktionen zu rechnen, da die Zünfte ihre sanktionierenden Institutionen, wie z.B. das Weiterbeschäftigungsverbot eines einem anderen Meister entlaufenen Lehrlings, nicht mehr durchsetzen konnten. Das neue Gesetz ermöglichte es jedem Lehrling, die Lehre abzubrechen und einen Gewerbeschein zu lösen. Dadurch sahen sich die Meister dem Risiko ausgesetzt, daß sie in den Lehrling investierten und, bevor sich diese Investition durch die billige Arbeitskraft des Lehrlings amortisieren konnte, dieser 
die Lehre beendete. Die Konsequenz war, daß viele Meister ihre Qualifizierungsfunktion vernachlässigten und Lehrlinge zunehmend als billige Arbeitskräfte einsetzten. Der Berliner Stadtrat Dracke (1819) faßte in seiner Denkschrift „Über den Vorzug einer geregelten Gewerbe-Verfassung und über die Nachtheile einer allgemeinen Gewerbefreiheit“ diesen Umstand folgendermaßen zusammen: „Um den Lehrling, welcher bei einem zu keiner Gewerksverbindung gehörenden Mann zur Lehre tritt, bekümmert sich Keiner; nur der, welcher solchen angenommen hat, soll es thun, solches wird aber aus Liebe zum Gewinn fast immer vernachlässigt.“ (abgedruckt in: Stratmann/Schlüter 1982, S. 198) Kritik an der Gewerbefreiheit wurde an verschiedenen Stellen laut. Einige sprachen sogar von, gewerblicher Anarchie'. Handwerksvereinigungen forderten die Festschreibung der Meisterwürde als Befähigungsnachweis sowohl für die Ausbildung von Lehrlingen als auch für den Betrieb eines Gewerbes und den schriftlichen Lehrvertrag.

Mit der Gewerbeordnung von 1845 bestätigte Preußen seine liberale Gewerbepolitik. Das neue Gesetz änderte nur in wenigen Punkten die Bestimmungen der Gewerbeordnung von 1811. So war der Beginn eines Gewerbebetriebes nicht mehr an die Bürgerrechte gebunden, sondern an die Anmeldung bei der Kommunalbehörde. Auch der privatrechtliche, nicht zwingend schriftliche Lehrvertrag blieb erhalten (§ 134). Nur in einigen Handwerksberufen hing die Berechtigung zur Lehrlingsausbildung nun von der Meisterprüfung ab. Den Forderungen der Handwerker nach Einführung des großen Befähigungsnachweises, d.h. der Koppelung des Niederlassungs- und Ausbildungsrechts an die Meisterwürde, kam dieses Gesetz nicht nach. Andererseits schaffte es aber auch die Zünfte bzw. Innungen nicht ab, sondern bestätigte deren Existenz und Funktion als gewerbliche Interessenvertretung.

Im Zuge der Revolution von 1848/49 fand eine Revision der bestehenden Gewerbeordnungen in Deutschland statt. Auf dem deutschen Handwerker- und Gewerbe-Kongreß in Frankfurt 1848 verabschiedeten die Vertreter der verschiedenen Handwerksvereinigungen einen Entwurf einer allgemeinen Handwerker- und Gewerbeordnung. Wesentlicher Punkt war die Abschaffung der Gewerbefreiheit und die Wiedereinführung der Zwangsinnungen. Als Reaktion auf die Revolutionsereignisse erließ der preußische Staat im Februar 1849 eine neue Gewerbeordnung, die das Gesetz von 1845 in wesentlichen Punkten modifizierte. Zwar wurden keine Zwangsinnungen eingeführt, aber die bestehenden Innungen erhielten ein Vorrecht in der Lehrlingsausbildung. So 
konnte durch Ortsstatut bestimmt werden, daß die Aufnahme und Entlassung aller Lehrlinge vor der jeweiligen Innung erfolgt (§ 45). Darüber hinaus wurde den Innungen eine Aufsichtsfunktion über die Ausbildung zugesprochen, die sich auch auf die NichtInnungsmitglieder erstreckte. Bei einer Vielzahl von Handwerksberufen - z.B. Müller, Bäcker und Schlosser - galt die Meisterprüfung nicht nur als Voraussetzung für die Ausbildungsbefugnis, sondern auch für die Aufnahme eines selbständigen Gewerbebetriebes (§§ 23-25) (vgl. Stratmann/Schlüter 1982, S. 17 ff.; Rinneberg 1985, S. 43 ff.). Das Gesetz stärkte mit diesen Bestimmungen die Stellung der Innungen entscheidend, wie dies auch in dem Gutachten der Regierung Minden von 1860 deutlich wurde: „Erst nach der Emanation der Gewerbe-Novelle von 1849 haben sich Innungen in unserem Bezirk wieder gebildet.“ (abgedruckt in: Stratmann/Schlüter (Hg.) 1982, S. 150)

Unter dem Eindruck der Revolutionsjahre 1848/49 suchte die preußische Regierung durch die Revision der Gewerbeordnung den Forderungen der Handwerker entgegenzukommen, um diese aus der unzufriedenen Masse herauszulösen. Der Handwerkerstand sollte durch Schutzbestimmungen in seiner ökonomischen und gesellschaftlichen Existenz gestärkt werden. Hierdurch versprach sich der Staat, ein die gesellschaftliche Ordnung stabilisierendes Element zu erhalten. Die Gewerbeordnung von 1849 kennzeichnet deshalb auch den Beginn einer staatlichen Mittelstandspolitik (vgl. Kaufhold 1979, S. 127; Winkler 1971, S. 164 ff.). Mit der wachsenden Industrialisierung und dem wirtschaftlichen Aufschwung in den 1850er Jahren lebte die Debatte über eine liberale Gewerbeverfassung in vielen deutschen Staaten wieder auf. Die Gründung des Norddeutschen Bundes 1866 trieb diese Entwicklung weiter voran. Eine Vereinheitlichung der unterschiedlichen Gewerbeordnungen gehörte zu den ersten Aufgaben, die sich die parlamentarischen Abgeordneten stellten (vgl. Schlüter/Stratmann 1985, S. 22 f.).

Die am 21. Juni 1869 verabschiedete Gewerbeordnung des Norddeutschen Bundes stellte einen Kompromiß der verschiedenen Gewerbegesetze dar. Als grundsätzliches Prinzip galt die Förderung der Gewerbefreiheit. Neben der Abschaffung des Befähigungsnachweises für diejenigen Handwerksberufe, bei denen er noch obligatorisch war, setzte sich auch in der Regelung der Lehrlingsausbildung der liberale Grundgedanke durch. In $\S 105$ lautete es: „Die Festsetzung der Verhältnisse zwischen den selbständigen Gewerbetreibenden und ihren Gesellen, Gehülfen und Lehrlingen ist 
Gegenstand freier Uebereinkunft.“ (abgedruckt in: Schlüter/Stratmann 1985, S. 149) Den Forderungen des Handwerks nach Schriftlichkeit des Lehrvertrages sowie nach Ein- und Austragung der Lehrverhältnisse in eine Lehrlingsrolle wurde nicht entsprochen. Der Lehrvertrag war - wie bereits in den Gewerbeordnungen von 1811 und 1845 - ein privatrechtlicher Vertrag, für den die Regelungen des allgemeinen Vertragsrechts galten (vgl. Rinneberg 1985, S. 54). Nach der Reichsgründung im Jahre 1871 gewann die Gewerbeordnung Gültigkeit für das gesamte Deutsche Reich. Damit waren Gewerbeangelegenheiten sowie Regelungen der Lehrlingsausbildung Teil der Reichsgesetzgebung (vgl. Greinert 1993, S. 42).

In Folge der umfangreichen Liberalisierung des Innungs- und Ausbildungsrechts stieg erneut - wie bereits nach dem Erlaß der Gewerbeordnung von 1811 - das Risiko des Meisters, Ausbildungsinvestitionen zu verlieren, da sowohl der Meister als auch der Lehrling sich von dem Vertrag ohne weitreichende Konsequenzen lösen konnten. Dementsprechend verfolgten viele Meister die Strategie, das potentielle Risiko eines Verlusts ihrer Investitionen zu minimieren, indem sie Lehrlinge zunehmend als billige Arbeitskräfte im Produktionsprozeß einsetzten und mithin keinen Nachteil bei einer frühzeitigen Auflösung des Ausbildungsvertrages erlitten. Lujo Brentano schrieb in einem Gutachten zur Reform des Lehrlingswesens im Jahre 1875: „Das häufige Vorkommen solchen Vertragsbruchs hat wiederum schlimme Folgen für die Lehrlinge gehabt. Es rentirt sich nämlich für tüchtige Meister nicht mehr, Lehrlinge anzunehmen, und diejenigen, die noch Lehrlinge annehmen, denken nicht mehr daran, sie zu lehren.“ (wiederabgedruckt in: Schlüter/Stratmann 1985, S. 94) Zeitgenossen bezeichneten diese Form der Ausbeutung von Lehrlingen als „Lehrlingszüchterei“, da sie häufig mit der Beschäftigung einer Vielzahl von „Lehrlingen“ bei einem Meister verbunden war (vgl. Körzel 1996, S. 233 f.). Infolgedessen kam es zu einem drastischen Absinken der Qualität der Berufsausbildung.

Um ihrem Widerstand gegen die Gewerbeliberalisierung Nachdruck zu verleihen, gründeten Handwerksvertreter 1873 in Leipzig den „,Verein selbständiger Handwerker und Fabrikanten“. In seinem Programm forderte der Verein die Einführung von Gewerbe- und Handwerkskammern sowie fachgewerbliche Korporationen und obligatorische Fortbildungsschulen. Auf der „Allgemeinen Deutschen Handwerkerversammlung“ im Jahre 1882 setzten sich die Delegierten für die Einführung der Zwangsinnung und des „großen Befähigungsnachweises“, d.h. der Koppelung der Ausbildungs- und 
Gewerbeausübungsbefugnis an die Meisterprüfung, ein. Gleichzeitig wurde die Gründung des „Allgemeinen Deutschen Handwerkerbundes“ beschlossen, in dem der 1873 gegründete Verein aufging. Politische Unterstützung erfuhr die Handwerkerbewegung durch konservative Parteien und das Zentrum (vgl. Winkler 1971, S. 164 ff.; Kaufhold 1979, S. 127 f.).

Bei ihren Protesten gegen die Gewerbeliberalisierung nutzten die Handwerksvertreter den augenscheinlichen Verfall der Lehrlingsausbildung, um auf die gesellschaftspolitischen Folgen der wirtschaftlichen Liberalisierung und die Bedeutung des Handwerks als gesellschaftlichem Stabilisierungsfaktor hinzuweisen. Im Kontext der damaligen Debatte um gesellschaftliche Auflösungstendenzen bot sich die Handwerksausbildung mit ihrer klassischen Hierarchie, Lehrling - Geselle - Meister, als eine Problemlösungsoption an. Interessenvertreter des Handwerks stellten die Bedeutung der Lehrlingsausbildung als Erziehungsverhältnis mit sozialintegrativen Momenten heraus. Dabei spielte das Konzept des Berufs als Kombination aus den für die Ausübung eines spezifischen Gewerbes benötigten Fähigkeiten und Kenntnissen und den dazugehörigen Wertanschauungen im Sinne einer inneren Berufung eine zentrale Rolle für die sozialintegrative Funktionalität der Berufsausbildung. Dem gelernten Arbeiter und Handwerker, der sozial integriert, ökonomisch abgesichert und aufgrund der Statusdifferenzierung einer „organisch“ aufgebauten Gesellschaft kollektiv organisierbar war, wurde der ungelernte jugendliche Arbeiter gegenübergestellt, der sich durch frühe wirtschaftliche Unabhängigkeit dem Erziehungseinfluß der Eltern entzog und ein unstetes Leben in den Arbeitermilieus der industriellen Großstädte führte. Die Reproduktion der sozialen Ordnung schien durch die wachsende Zahl ungelernter Arbeiter gefährdet (vgl. Körzel 1996, S. 130 f.; Stratmann 1992, S. 2 f.).

Die Gewerberechtsnovelle von 1878 brachte der Lehrlingsausbildung eine wesentliche Änderung. Obgleich ein Zwang zur schriftlichen Abfassung des Lehrvertrages nicht festgelegt wurde, so wurde doch der Rückkehranspruch des Meisters bei Kontraktbrüchigkeit des Lehrlings an die Schriftlichkeit des Vertrages gebunden. Darüber hinaus war der Lehrmeister verpflichtet, dem Lehrling nach Abschluß der Lehre ein Zeugnis auszustellen (vgl. Greinert 1993, S. 43; Hoffmann 1962, S. 10 f.). Diese Maßnahmen führten zu einer stärkeren rechtlichen Formalisierung der Lehrlingsausbildung. Andererseits beklagten Handwerksvertreter und konservative Parlamentarier, daß es kein entsprechend legitimiertes Organ zur Kontrolle der Lehrlingsverhältnisse gebe. Sie 
schlugen deshalb eine Neugestaltung des Innungswesens vor, bei der die Innungen eine zentrale Aufsichts- und Kontrollfunktion wahrnehmen sollten.

In der Gewerbeordnungsnovelle von 1881 fanden diese konservativen Forderungen eine weitgehende Entsprechung. Sie beinhaltete die Rückübertragung einiger öffentlicher Rechte und Pflichten auf die Innungen, obgleich das Ziel der Zwangsmitgliedschaft nicht erreicht wurde. Die Innungen erhielten das Recht, die Ausgestaltung des Lehrverhältnisses zu regeln, z.B. die Dauer der Lehrzeit und den Besuch der Fortbildungsschulen: „Aufgabe der Innungen ist: [...] die nähere Regelung des Lehrlingswesens und der Fürsorge für die technische, gewerbliche und sittliche Ausbildung der Lehrlinge [...] zu entscheiden.“ (§ 97 der Gewerbeordnung vom 18. Juli 1881, wiederabgedruckt in: Schlüter/Stratmann (Hg.) 1985, S. 182) Die Übertragung öffentlicher Verantwortungsbereiche stärkte die Innungen als intermediäre Instanzen in der Regelung der Lehrlingsausbildung. Dies stellte den Beginn einer Steuerung der Berufsbildung in Form staatlich legitimierter Selbstverwaltung der Wirtschaft dar. Die Gewerberechtsnovellen von 1884, 1886 und 1887 festigten die Stellung der Innungen weiter (vgl. Greinert 1993, S. 44; Hoffmann 1962, S. 11; Schlüter/Stratmann 1985, S. 27).

Eine Verkoppelung der beiden Bereiche - korporative Handwerksorganisation und Lehrlingswesen - kam erst 1897 mit dem sogenannten „Handwerkerschutzgesetz“ zustande. Das Gesetz ermöglichte die Errichtung fakultativer Zwangsinnungen in einem Bezirk, falls die Mehrheit der beteiligten Gewerbetreibenden dem zustimmten. Ferner regelte das Gesetz die Einrichtung von Handwerkskammern als Selbstverwaltungskörperschaften des öffentlichen Rechts ( $\S \S 103$ ff.), die eine über das beruflich-fachliche Interesse der Innungen hinausgehende Instanz bilden sollten. Ihre Aufgaben lagen in der Organisation der Ausbildung und Prüfung von Lehrlingen sowie der Festlegung von Lehrlingshöchstzahlen je Betrieb, der Dauer der Lehrzeit und der Lehrverträge (vgl. Körzel 1996, S. 238 f.; Greinert 1993, S. 45; Kaufhold 1979, S. 129; Schlüter/Stratmann 1985; S. ; Winkler 1971, S. 175 ff.). Dem Gesetz ging die Debatte um die Arbeiterjugendfrage voraus, die ihren Höhepunkt während der Bergarbeiteraufstände von 1889 erreichte. Bismarck und die staatliche Bürokratie machten die ungelernten jugendlichen Arbeiter, die sich in der Bergbauindustrie besonders zahlreich fanden, als treibende Kraft hinter den Aufständen aus und deuteten die fehlende Erziehung und Disziplin als Ursache ihres Verhaltens (vgl. Stratmann 1992, S. 
156 ff.). Die Forderung des Handwerks nach einer Reinstitutionalisierung der Lehrlingsausbildung eröffnete der staatlichen Bürokratie die Möglichkeit zur Umsetzung ihrer Idee einer Pädagogisierung dieser sozialen Gruppe (vgl. Körzel 1996, S. 120 f.). Darüber hinaus sollte der Proletarisierung des Mittelstandes durch Stärkung des Handwerks begegnet werden, um so ein „Bollwerk gegen die Sozialdemokratie“ (Greinert 1995, S. 21) zu schaffen.

Am Vorabend des ersten Weltkrieges markierte das Gesetz zum sogenannten „kleinen Befähigungsnachweis“ von 1908, das die Berechtigung zur Ausbildung von Lehrlingen an den Besitz eines Meistertitels knüpfte, die finale Konsolidierung des Handwerksrechts im deutschen Kaiserreich (vgl. Körzel 1996, S. 22; Hoffmann 1962, S. 12). Die Einführung des „kleinen Befähigungsnachweises“ schränkte indirekt die Gewerbefreiheit der nichtgeprüften Handwerker sowie derjenigen ohne Meistertitel ein, da eine rechtliche Chancengleichheit im Wettbewerb nicht mehr gegeben war. In den folgenden Jahren wuchs die Zahl der geprüften Meister. Faktisch setzte sich damit die idealtypische Strukturierung der Lehrlingsausbildung in die Stufen Lehrling - Geselle Meister als Norm durch (vgl. Kaufhold 1979, S. 130 f.). Das Gesetz legte insofern den Grundstein für eine spätere rechtliche Festschreibung des „großen Befähigungsnachweises“.

Die Mittelstandspolitik in Form der Handwerkerschutzpolitik zur Stabilisierung der gesellschaftlichen Ordnung stellte eine Seite der Medaille dar. Auf der anderen fand sich ein „,modernes“ Element der Berufsausbildung in Gestalt der Fortbildungsschulen, die die klassische Lehrlingsausbildung im theoretischen und praktischen Bereich ergänzen sollten. Häufig wurde die Qualität der Handwerksausbildung aufgrund der Spezialisierung der Handwerksbetriebe sowie des Mißbrauchs der Lehrlinge als billige Arbeitskräfte kritisiert. Die Protagonisten der Fortbildungsschulen, deren Ausweitung und Integration in die Berufsausbildung sie forderten, führten deshalb neben erzieherischen und gesellschaftspolitischen Argumenten, die im öffentlichen Diskurs eine zentrale Bedeutung besaßen, ökonomische Überlegungen an, die sich in der Diskussion über die internationale Wettbewerbsfähigkeit der deutschen Wirtschaft manifestierten (vgl. Körzel 1996, S. 359 und 369-371; Greinert 1995, S. 23 f.).

Die Idee der Fortbildungsschulen geht auf die Einrichtung von Gewerbeschulen in Preußen in den 1820er Jahren zurück. Anfangs wurden diese schulischen Angebote 
überwiegend von Privatleuten und Vereinen organisiert. Unterstützungen durch den Staat und städtische Gründungen erfolgten erst später. Die Möglichkeit, die betriebliche Ausbildung durch den Besuch einer Gewerbeschule zu verkürzen, eröffnete die Gewerbeordnung von 1849 (§ 36). Damit erkannte das Gesetz zum ersten Mal einen weiteren Lernort neben der handwerklichen Werkstatt an. Eine ortsstatutorische Fortbildungsschulpflicht ermöglichte die Gewerbeordnung von 1869 (§ 106). Danach konnten Gesellen, Gehilfen und Lehrlinge unter achtzehn Jahren verpflichtet werden, eine örtliche Fortbildungsschule zu besuchen. Der Arbeitgeber bzw. Lehrherr mußte sie dazu von der Arbeit freistellen. Fortbildungsschulen konnten sich aber in der Regel nur finanziell wohlhabende Gemeinden und Großstädte leisten. Um die Entwicklung der Fortbildungsschulen in Preußen zu fördern, sah der Fortbildungsschulerlaß von 1874 eine staatliche Subventionierung vor. Die Bewilligung war jedoch von der Voraussetzung abhängig, daß eine ortsstatutorische Fortbildungsschulpflicht bestand. Im Jahre 1882 waren von 623 gewerblichen Fortbildungsschulen in Preußen 335 obligatorisch. Um die Jahrhundertwende sollte sich das Verhältnis auf beinahe $90 \%$ erhöht haben (vgl. Harney 1980, S. 77 und 87). Mit dem Übergang der Zuständigkeit für das gewerbliche Schulwesen vom Kultus- auf das Handelsministerium im Jahre 1884 verschob sich auch die inhaltliche Profilierung dieses Schultyps. Bis dahin galt die Fortbildungsschule als Fortsetzung der Volksschule mit einem deutlich allgemeinbildenden Charakter. Sie sollte gehobenen Elementarbildungsansprüchen gerecht werden. Eine vom Unterrichtsministerium 1872 eingesetzte Kommission sah eine wesentliche Aufgabe der Fortbildungsschule in der Integration allgemeiner Bildung, die sich in der „Vertiefung und praktischen Einübung des in der Elementarschule Gelernten“ (zit. nach: Harney 1980, S. 71) manifestierte. Die gewerbefachliche Ausgestaltung des Unterrichts blieb den Kommunen überlassen, die sich dabei an den örtlichen Gegebenheiten orientieren sollten. Im Handelsministerium wurde das gewerbliche Unterrichtswesen als ein Bestandteil der Wirtschaftspolitik gesehen. Die öffentliche Kritik an der mangelnden Konkurrenzfähigkeit der deutschen Wirtschaft meinte, eine Ursache dafür im Zustand der gewerblichen Ausbildung erkannt zu haben. Durch eine klare Ausrichtung der Fortbildungsschulen auf die gewerbliche Fachausbildung sollte die praktische Lehre ergänzt und so die fachliche Qualifizierung der Handwerker bzw. Arbeiter gesteigert werden (vgl. Körzel 1996, S. 366 ff.). Zur Begründung seines Entwurfs für ein Fortbildungsschulgesetz sagte der preußische Minister für Handel und Gewerbe R. Sydow 1911 vor dem Abgeordnetenhaus: „Hier 
setzt nun die Fortbildungsschule ergänzend ein. Sie gibt dem jugendlichen Arbeiter, dem Lehrling die Kenntnis aller Zweige seines Berufs in praktischer Anschauung, und sie gewährt ihm darüber hinaus eine Kenntnis der theoretischen Grundlagen [...].“ (abgedruckt in: Stütz (Hg.) 1969, S. 98) Der Gesetzentwurf sah zudem eine allgemeine Fortbildungsschulpflicht vor. Sein Scheitern führte dazu, daß durch die Gewerberechtsnovelle von 1912 die ortsstatutorische Fortbildungsschulpflicht auf Anordnung der höheren Verwaltungsbehörde erfolgen konnte (vgl. Greinert 1993, S. 57).

Ideen zur Profilierung der Fortbildungsschule fanden sich bei verschiedenen reformorientierten Pädagogen. Oskar W. Pache aus Leipzig, der dem „Verein der Lehrer und Freunde der deutschen Fortbildungsschule“ vorstand, forderte die gewerbefachliche Ausrichtung der Fortbildungsschule ebenso wie der badische Gewerbeschuldirektor Friedrich Rücklin. Der bekannteste unter ihnen war Georg Kerschensteiner, der als Stadtschulrat in München das Fortbildungsschulwesen in Richtung auf ein Berufsschulmodell reorganisierte. In seiner 1901 veröffentlichten Preisschrift „Die staatsbürgerliche Erziehung der deutschen Jugend“" stellte er seine Idee der Berufsbildung als Grundlage für die staatsbürgerliche Erziehung einem größeren Publikum vor. Die Bildung der Jugendlichen sollte sich am Berufskonzept ausrichten. Er kritisierte die auf dem neuhumanistischen Bildungsideal basierende allgemeinbildende Funktion der Fortbildungsschule, die für die spätere Berufsausübung von geringem Nutzen sei. Das Problem der sozialen Integration der Arbeiterjugend sollte durch eine beruflich organisierte Erziehung gelöst werden, die die für die staatsbürgerliche Erziehung wichtigen Tugenden - Gewissenhaftigkeit, Fleiß, Beharrlichkeit und Selbstüberwindung - vermittelte. In einer späteren Schrift schrieb Kerschensteiner: „Wir müssen die Aufgabe der staatsbürgerlichen Erziehung an der Wurzel fassen. Wir fassen sie aber nur an der Wurzel, wenn wir uns entschließen, unsere Schulen zu Arbeitsgemeinschaften im kleinen zu machen [...].“ (Kerschensteiner 1914, S. 84-85) Die Herstellung gemeinsamer Arbeiten sollte den Gemeinschaftscharakter fördern und die Bedeutung des Einzelnen als Teil eines „organischen“ Ganzen verdeutlichen. Kritik wurde am Kerschensteinerschen Berufsbegriff geübt, der sich eng an den vom Handwerk überkommenen Traditionen orientierte und die veränderten Bedingungen der industriellen Arbeit nicht miteinbezog (vgl. Gonon 1992, S. 169 ff.; Muth 1985, S. 42 ff.; Bruchhäuser/Lipsmeier 1985, S. 10 f.; Blankertz 1963, S. 17 f.). 
Dieses Kapitel hat gezeigt, daß die liberalen Reformen der Gewerbeordnungen in Deutschland zu Beginn des 19. Jahrhunderts zu Auflösungserscheinungen in der Lehrlingsausbildung führten. Bedingt durch die geschwächte Position der Innungen kam es zu Mißbräuchen der Lehrlinge, die von ihren Lehrherren als billige Arbeitskräfte eingesetzt wurden. Das zunehmende Problem der Kontraktbrüche vonseiten der Lehrlinge aufgrund fehlender Sanktionen verstärkte diese Situation noch. Gegen Mitte des 19. Jahrhunderts kam der Staat den Forderungen der Handwerker nach Einschränkung der Gewerbefreiheit entgegen, um den handwerklichen Mittelstand als gesellschaftsstabilisierende Kraft im Kampf gegen sozial-revolutionäre Strömungen zu gewinnen. Wachsende Industrialisierung und wirtschaftsfördernde staatliche Zusammenschlüsse - wie der Norddeutsche Bund - erforderten eine liberale Gewerbepolitik. Die Innungen erhielten den Status privatrechtlicher Vereine, während die Lehrlingsausbildung allein der „freien Übereinkunft“ zwischen Meister und Lehrling unterlag. Die durch die Industrialisierung hervorgerufenen sozialen Probleme, wie z.B. die Proletarisierung jugendlicher Arbeiter, zwangen den Staat, gesellschaftspolitische Maßnahmen zur Erhaltung der sozialen Ordnung zu ergreifen. Die Stärkung der beiden zentralen handwerklichen Institutionen - Innung und Lehrlingsausbildung - bildeten den Kern einer gesellschaftsstabilisierenden Mittelstandspolitik. Durch die Übertragung staatlicher Kompetenzen auf private Korporationen war eine gesellschaftsnähere Aufsicht und Kontrolle möglich. Gleichzeitig sicherte sich dadurch der Staat die Loyalität einer breiten gesellschaftlichen Schicht, des Handwerks. Der erzieherische und sozial-integrative Charakter der Lehrlingsausbildung ermöglichte eine staatsbegünstigende Einflußnahme auf Jugendliche in der kritischen Phase zwischen Volksschule und Militärdienst. Aber auch unter qualifikatorischen Gesichtspunkten kam der Lehrlingsausbildung in Zeiten wachsender internationaler Konkurrenz eine wichtige Bedeutung $\mathrm{zu}$, die durch den Ausbau des Fortbildungsschulwesens als ergänzende Komponente der berufspraktischen Ausbildung gestärkt wurde. Zum Ende des Kaiserreichs war die Reinstitutionalisierung des Handwerks mit Innungswesen und Handwerkskammern sowie Lehrlingsausbildung weitgehend abgeschlossen. 


\subsection{Berufliche Bildung in der Weimarer Republik}

Am Vorabend des Ersten Weltkrieges hatte sich die traditionelle Handwerksausbildung wieder etabliert. Die Gewerbenovelle von 1908 räumte dem Handwerk eine ordnungspolitische Monopolstellung im gewerblichen Ausbildungswesen ein. Wachsende Ausbildungsanstrengungen industrieller Betriebe stellten diese Monopolstellung aber in Frage. Der sich daraus ergebende Konflikt zwischen Handwerk und Industrie sollte die Berufsbildungspolitik der Weimarer Republik in weiten Teilen bestimmen. In dem Ausnahmezustand des Krieges geriet die handwerkliche Lehrlingsausbildung in die Krise. Die Einberufung zum Militärdienst zwang viele Meister ihre Betriebe einzustellen. Dadurch sanken die Ausbildungskapazitäten im Handwerk. Gleichzeitig bot sich den Jugendlichen die Alternative, eine besser entlohnte Arbeit in der unter Arbeitskräftemangel leidenden Industrie anzunehmen, die zudem mit einem höheren Maß an persönlicher Freiheit verbunden war. Dies führte zu Forderungen der Interessenverbände des Handwerks, durch staatliche Unterstützung, z.B. die Befreiung ausbildender Meister vom Kriegseinsatz, die Situation des Handwerks zu verbessern, um die Lehrlingsausbildung zu sichern. Eine anderes Bild zeigte sich in der Industrie: Die starke Einbindung in die Kriegswirtschaft und die damit verbundene geringere Krisenanfälligkeit erlaubte es den Betrieben ihre Ausbildungsaktivitäten auszubreiten. Jedoch ist davon auszugehen, daß die Qualität der Ausbildung unter den kriegswirtschaftlichen Bedingungen litt, wie sich in der generellen Einschränkung des Fortbildungsschulunterrichts zeigte. Nach Kriegsende normalisierte sich die Ausbildungssituation sowohl im Handwerk als auch in der Industrie, so daß das vom Handwerk geforderte Notprogramm obsolet wurde. Bereits 1919 lag die Einstellungsquote von Lehrlingen im Handwerk um bis zu 25 \% über dem Niveau von 1914 (vgl. Muth 1985, S. 39 f.; Schütte 1992, S. 24 ff.).

Ein zentrales Problem des gewerblichen Ausbildungssystems zu Beginn der Weimarer Republik war die Diskrepanz zwischen handwerklicher Ausbildung und Qualifikationsbedürfnissen der Industrie. Im Januar 1918, d.h. noch vor Kriegsende, unterbreitete der Verein deutscher Maschinenbauanstalten (VdMA), der sich als Gründungsmitglied des bereits 1908 eingerichteten Deutschen Ausschusses für technisches Schulwesen (DATSCH) als besonders reformorientiert gezeigt hatte, einen Vorschlag zur Neugestaltung der Berufserziehung in der Industrie: Die beiden wesentlichen Punkte betrafen die Reform des Prüfungswesens und die Abhängigkeit vom Handwerk. Der 
DATSCH konkretisierte im selben Jahr die Überlegungen der Industrie zur Reform der gewerblichen Ausbildung in einem Acht-Punkte-Programm: Neben der Forderung nach einer systematisch organisierten Berufsberatung zur Verbesserung der Berufssteuerung sollte durch die Einführung standardisierter Lehrverträge die Professionalisierung der industriellen Lehrlingsausbildung vorangetrieben werden. Die Durchführung der praktischen Ausbildung in Lehrwerkstätten galt es durch schulischen Unterricht in Werkschulen zu ergänzen. Darüber hinaus sollte ein von der Industrie kontrolliertes Prüfungswesen etabliert werden (vgl. Schütte 1992, S. 29 f.).

Auf Initiative des Vereins Deutscher Ingenieure (VDI) war 1908 in Kooperation mit dem Verein deutscher Maschinenbauanstalten (VdMA) der Deutsche Ausschuß für Technisches Schulwesen (DATSCH) entstanden, dessen Ziele die Bündelung der berufsbildungspolitischen Interessen der Industrie und die konzeptionelle Weiterentwicklung der industriellen Lehrlingsausbildung waren. Im Jahre 1919 stellte der DATSCH seinen ersten inhaltlich systematisierten (Muster-) Lehrgang für Maschinenschlosser vor. Weitere Lehrgänge folgten in den 20er Jahren. Darüber hinaus erarbeitete er auch Lehrmaterialien für die verschiedenen Lehrgänge. Mit der Gründung des Arbeitsausschusses für Berufsausbildung (AfB), dessen Gründungsmitglieder neben dem DATSCH der Reichsverband der Deutschen Industrie (RDI) und die Vereinigung der Deutschen Arbeitgeberverbände (VDA) waren, kam es zu einer Aufgabenteilung. Der DATSCH sollte eine Abgrenzung und Systematisierung von Industrieberufen vornehmen. Dies bedeutete, daß sich die inhaltliche Gestaltung von Lehrgängen an einem definierten Berufsbild auszurichten hatte. Erste Berufsabgrenzungen fanden in der Metallindustrie sowie dem Schiffbau und der Chemischen Industrie statt. Ziel der Berufsabgrenzungen war eine klare Hierarchisierung der Tätigkeitsprofile von ungelernten und angelernten Arbeitern sowie von Facharbeitern. Damit übernahm die Industrie die Berufskonzeption des Handwerks zur Organisation ihrer Arbeitsprozesse. In den 20er Jahren war der DATSCH der ausbildungspolitische, think tank' der deutschen Industrie (vgl. Greinert 1993, S. 66 ff.; Muth 1985, S. 348 ff.; Hoffmann 1962, S. 36 f.).

Ein wesentliches Merkmal der industriellen Lehrlingsausbildung, durch das sie sich von der Handwerkslehre abgrenzte, war die Lehrwerkstatt. Während es „Fabriklehrlinge“ bereits zu Beginn des 19. Jahrhunderts gab, deren Ausbildung aber unsystematisch im laufenden Produktionsbetrieb erfolgte, setzte sich bei den deutschen Staatseisenbahnen 
in den 1870er Jahren das Modell der Lehrwerkstatt durch. Die Industrie nutzte dieses Modell der Ausbildung erst später: 1890 richtete der Elektro-Großbetrieb Schuckert \& Co. in Nürnberg die erste Lehrwerkstatt ein. Der Maschinenbaubetrieb MAN in Augsburg folgte zwei Jahre später nach. Bis zum Ersten Weltkrieg stieg die Zahl nur langsam an. So gab es 1912 gerade 11 Lehrwerkstätten. Im Jahre 1926 hatte sich die Zahl jedoch auf 175 erhöht (vgl. Rinneberg 1985, S. 33 ff.; Greinert 1993, S. 70 ff.). Die Idee der Lehrwerkstatt war es, einen vom laufenden Produktionsprozeß unabhängigen Lernort zu etablieren. Damit wurde die Voraussetzung für eine systematisch aufgebaute Ausbildung geschaffen. Lehrlinge konnten je nach Schwierigkeitsgrad aufeinander abgestimmte Arbeitsoperationen erlernen, ohne durch den Druck des eigentlichen Arbeitsprozesses abgelenkt zu sein. Eine Konsequenz der Lehrwerkstätten war die Einrichtung von standardisierten, berufsspezifischen Lehrgängen, die vom DATSCH konzipiert wurden. Das Modell der Lehrwerkstatt setzte sich insbesondere in der Metall- und Elektroindustrie sowie dem Maschinenbau durch. In diesen Bereichen war der Bedarf an qualifizierten Facharbeitern aufgrund moderner Fertigungsverfahren und einer auf Qualitätsarbeit basierenden Produktpolitik besonders hoch. Dies spiegelt sich auch in den Lehrlingsquotienten (Verhältnis der Anzahl der Lehrlinge bezogen auf die Gesamtzahl der Beschäftigten) für die einzelnen Industriebranchen wider: So ergab die 1925 durchgeführte Volks-, Berufs- und Betriebszählung für die Eisen-, Stahl- und Metallindustrie einen Lehrlingsquotienten von 14,6 \%, für den Maschinen-, Apparateund Fahrzeugbau 11,2 \% und für die elektrotechnische, feinmechanische und optische Industrie 8,5\%. In der Chemie und dem Bergbau lag der Lehrlingsquotient unter einem Prozent. Für das Handwerk ergab sich ein durchschnittlicher Lehrlingsquotient von 14,4 \% (vgl. Schütte 1992, S. 65 und 67). Während die Metallindustrie und der Maschinenbau ihren Facharbeiterbedarf weitgehend selber ausbildete, war die Mehrzahl der industriellen Betriebe hinsichtlich ihres Fachkräftebedarfs auf die Ausbildungsleistungen des Handwerks angewiesen (vgl. Muth 1985, S. 321 ff.).

In der Berliner Metallindustrie entwickelte sich ein Ausbildungsmodell, das die praktische Ausbildung in der Lehrwerkstatt durch theoretischen Unterricht in werkseigenen Schulen („Werkschulen“) ergänzte. Dieses sogenannte „Berliner Modell“ hatte den Vorteil, daß die praktische und theoretische Ausbildung inhaltlich eng aufeinander abgestimmt werden konnte. Dadurch war eine optimale Umsetzung der vom DATSCH konzipierten Lehrgänge möglich. Die Industriebetriebe hatten darüber hinaus mehr 
Unabhängigkeit in der Ausgestaltung ihrer Berufsausbildung als die öffentlichen Fortbildungsschulen, die sich nach staatlichen Vorgaben richten mußten. Die in den Werkschulen eingesetzten Lehrer entstammten in der Regel dem eigenen Betriebspersonal und besaßen deshalb detaillierte Kenntnisse des spezifischen Produktionsprozesses, die sie für einen anwendungsorientierten theoretischen Unterricht nutzen konnten. Den Vorteilen der Werkschulen standen jedoch hohe finanzielle Kosten gegenüber, die viele - gerade kleinere und mittlere Betriebe - nicht tragen konnten. Spätestens mit der Weltwirtschaftskrise verlor die Industrie ihr Interesse an Werkschulen, da mit den öffentlichen Fortbildungsschulen zudem eine kostengünstigere Alternative zur Verfügung stand (vgl. Greinert 1993, S. 73; Muth 1985, S. 550 ff.).

Wie bereits gezeigt, gab es seit der Jahrhundertwende deutliche Bestrebungen, die allgemeinen Fortbildungsschulen in Richtung von Berufsschulen weiterzuentwickeln, um sowohl erzieherische als auch qualifikatorische Ziele zu erreichen. Während im Kaiserreich die sozialintegrative und disziplinierende Funktion der Fortbildungsschule im Vordergrund stand, sollte durch die Betonung ihrer beruflichen Qualifikationsfunktion die Legitimation der Fortbildungsschule auf eine neue Basis gestellt werden. Die Festschreibung der Fortbildungsschulpflicht bis zum vollendeten 18. Lebensjahr in Artikel 145 der Weimarer Reichsverfassung machte den neuen Stellenwert dieser Schulform deutlich. Diese Bestimmung hatte jedoch eher symbolischen Charakter, da die konkrete Ausführung einem Reichsgesetz vorbehalten war. Auf der Reichsschulkonferenz im Sommer 1920 wurden „Leitsätze zum Berufsschulwesen“ verabschiedet, die als Ziel der Berufsschule die Ergänzung und Vertiefung der beruflichen Ausbildung definierten. Darüber hinaus einigten sich die Teilnehmer auf ein berufsschulpolitisches Programm, das den Ausbau der Berufsschule im wesentlichen befürwortete, diesen aber von den finanziellen Ressourcen abhängig machte. Für die Umsetzung einer gesetzlich festgeschriebenen Berufsschulpflicht wären die Länder und Kommunen unter finanzieller Beteiligung des Reiches verantwortlich gewesen, was zur Blockade einer reichsgesetzlichen Regelung durch die finanziell überforderten Länder führte. So kam es nur in einzelnen Ländern zu gesetzlichen Regelungen. Preußen erließ 1923 ein Gesetz zur „Erweiterung der Berufsschulpflicht“, das zwar keine allgemeine Berufsschulpflicht vorsah, aber deren Einführung durch Orts- oder Kreissatzung erlaubte. Generell dauerte die Schulpflicht bis zur Vollendung des 18. Lebensjahres, bei Lehrlingen konnte eine Verlängerung bis zum Ende der Lehrzeit vorgeschrieben 
werden. Die Kommunen erhielten zur Finanzierung der Berufsschulen in der Regel neben staatlicher Unterstützung auch Arbeitgeberbeiträge in Form von Schulgeld. Aufgrund des Widerstandes der Arbeitgeber fand der Berufsschulunterricht meistens außerhalb der Arbeitszeit statt. Lediglich bei den Lehrlingen wurden Ausnahmen gemacht, da der Berufsschulunterricht als Teil der Ausbildung anerkannt war. Den ungelernten jugendlichen Arbeitern drohte beim Besuch des Unterrichts während der Arbeitszeit entsprechender Lohnentzug (vgl. Schütte 1992, S. 141 ff.; Hoffmann 1962, S. 78 f.).

In den Jahren der sogenannten „Stabilisierungskrise“ von 1923 bis 1926 kam es zu einer Ausweitung des Berufsschulwesens aus arbeitspolitischen Motiven. Die Jugendarbeitslosigkeit hatte dramatische Ausmaße angenommen. Von den Jugendlichen in Westdeutschland, die nach ihrer Schulentlassung entweder eine Arbeits- oder Lehrstelle suchten, blieben zu Beginn des Jahres 1924 zwischen 25 und $70 \%$ erfolglos. Als Reaktion auf diese Krise des Jugendarbeitsmarktes erließ die Reichsregierung mehrere Verordnungen, die die Bedingungen der staatlichen Fürsorge für Jugendliche neu regelten. So konnten jugendliche Arbeitslose über 16 Jahren zum Besuch der Berufsschule gezwungen werden. Staatliche Leistungen der Erwerbslosenfürsorge waren an die Teilnahme an beruflichen Bildungsmaßnahmen gekoppelt. Darüber hinaus konnten Kommunen jugendliche Arbeitslose zu „Pflichtarbeiten“ heranziehen. Die verschiedenen arbeitspolitischen Maßnahmen der Länder setzten auf eine Kombination von Berufsschule und öffentlichen Arbeiten, um die „Arbeitsmoral“ der Jugendlichen $\mathrm{zu}$ erhalten. Unter der arbeits- und sozialpolitischen Instrumentalisierung der Berufsschule litt allerdings die Qualität des Berufsschulunterrichts. Die Berufsschule war zu einem bloßen „Verwahrungsort“ für jugendliche Arbeitslose geworden (vgl. Stachura 1989, S. 101 ff.; Schütte 1992, S. 48 ff.).

Ein berufsbildungspolitisches Ziel der Reichsregierung war die Konsolidierung der verschiedenen gesetzlichen Regelungen zum Lehrlingswesen in einem nach einheitlichen Grundsätzen aufgebauten Berufsausbildungsgesetzes. Bereits 1921 legten die Reichsministerien für Arbeit und Wirtschaft einen ersten gemeinsamen Referentenentwurf vor. Das neugegründete Reichsarbeitsministerium sah darin die Chance, seine im Gegensatz zum Reichswirtschaftsministerium vornehmlich sozialpolitischen Interessen in der Berufsausbildung durchsetzen zu können. Ein umstrittener Punkt betraf die Kontrolle des Lehrlingswesens: Staatliche Landeszentralbehörden, paritätisch mit 
Vertretern der Arbeitgeber und Arbeitnehmer besetzt und verwaltungstechnisch den Landesarbeitsämtern unterstellt, sollten innerhalb der Länder die allgemeine Aufsicht innehaben. Den Kammern und Innungen kam die Aufgabe zu, lokale berufsbezogene Entscheidungen zu treffen und zu überwachen. Hinsichtlich des Prüfungswesens schrieb der Entwurf gemeinsame Prüfungsausschüsse von Handwerkskammern und Industrieund Handelskammern ${ }^{11}$ vor. Während die Gewerkschaften ihre Forderung nach paritätischer Mitbestimmung im Entwurf wiederfanden, protestierten die Handwerksvertreter gegen eine Einschränkung der Kontrollbefugnisse der Handwerkskammern und Innungen sowie gegen die rechtliche Gleichstellung von Industrie und Handwerk. Nach langjährigen Beratungen verabschiedete das Kabinett 1927 den ersten „Entwurf eines Berufsausbildungsgesetzes“. Kritik kam vom Handwerk, das in der Einführung paritätischer (Prüfungs-)Ausschüsse ( $\S$ 42-56 und $\S \S$ 69-86) einen schweren Eingriff in das Selbstverwaltungsrecht der Handwerkskammern sah. Den Gewerkschaften dagegen gingen die Mitbestimmungsregelungen nicht weit genug, und die Industrie lehnte den weiten Geltungsbereich des Gesetzes ( $\S 1$ 1-4), der alle erwerbstätigen 14- bis 18-Jährigen umfaßte, grundsätzlich ab. Durch eine interessenpolitische Kooperation von Handwerk und Industrie verlängerten sich die parlamentarischen und ministeriellen Beratungen des Gesetzentwurfes, so daß eine Verabschiedung in der Weimarer Republik nicht mehr zustandekam (vgl. Muth 1985, S. 444 ff.; Hoffmann 1962, S. 93 ff.).

Um hinsichtlich der Ablehnung des Gesetzentwurfes miteinander kooperieren zu können, mußten Industrie und Handwerk den zwischen ihnen strittigen Punkt des Prüfungswesens durch eine Sondervereinbarung aus dem Weg räumen. Aufgrund des in der Reichsgewerbeordnung verankerten Prüfungsmonopols des Handwerks besaßen die in der Industrie durchgeführten „Gesellenprüfungen“ lediglich eine berufliche Bedeutung, d.h. die mit einer Abschlußprüfung vor der Handwerkskammer verbundenen gesetzlichen Vorteile - wie die Möglichkeit einer späteren Meisterprüfung blieben den „Gesellen“ bzw. Facharbeitern der Industrie vorenthalten. Die Industrie

\footnotetext{
11 Mit dem preußischen Kammergesetz von 1870 wurde die Tätigkeit der Handelskammern, die eine Mischung aus den unter der französischen Besatzung errichteten, chambres de commerce' und kaufmännischen Korporationen waren, auf eine gesetzliche Grundlage gestellt. Im Jahre 1897 erhielten die Kammern den Status einer juristischen Person. Seit 1924 heißen die Handelskammern offiziell Industrie- und Handelskammern (vgl. Adam 1979, S. 13 ff.; König 1981, S. 17 ff.).
} 
forderte deshalb eine gesetzliche Neuordnung des Prüfungswesens unter organisatorischer Einbindung der Industrie- und Handelskammern, wie sie teilweise schon üblich war. Von der rechtlichen Anerkennung der industriellen „Gesellenprüfung“ versprachen sich die Industrievertreter eine Anhebung des Standesbewußtseins und der Attraktivität der industriellen Lehrlingsausbildung sowie eine Vereinheitlichung und Systematisierung der Ausbildung aufgrund definierter Qualifikationsstandards. Die Handwerksverbände lehnten eine Aufhebung ihres Prüfungsmonopols und eine Verselbständigung der industriellen Lehre ab. Als Kompromiß zeichnete sich die Einrichtung gemeinsamer Prüfungsausschüsse von Handwerkskammern und Industrie- und Handelskammern ab. Beide Interessengruppen empfahlen ihren jeweiligen Kammern solche gemeinsamen Prüfungen für Lehrlinge aus Industriebetrieben in ihrem Bezirk durchzuführen. Eine Studie von 1930 zeigte jedoch nur in wenigen Fällen eine erfolgreich Umsetzung der Kooperationsvereinbarung. Damit war die zentrale Forderung der Industrie nach einem von der Industrie kontrollierten eigenständigen Prüfungswesen gescheitert (vgl. Schütte 1992, S. 82 ff.).

Als neuer Akteur auf dem Berufsbildungsparkett suchten die Gewerkschaften auf zweierlei Weise Einfluß auf die Berufserziehung zu nehmen: Einerseits engagierten sie sich in den Debatten um ein Berufsausbildungsgesetz, indem sie ihre Mitbestimmungsrechte gesetzlich verankert sehen wollten, und andererseits nutzten sie ihre seit 1918 rechtlich festgelegten tarifvertraglichen Einflußmöglichkeiten auf die Ausgestaltung der Berufsausbildung. Wie bei der Einrichtung paritätischer Ausschüsse sahen die Handwerksverbände in der Ausweitung des kollektiven Arbeitsrechts auf den Bereich des Lehrlingswesens einen Eingriff in das Selbstverwaltungsrecht der Handwerkskammern und Innungen. Gegen die Einbeziehung der Lehrlingsausbildung sprach ihrer Meinung nach das rechtliche Verständnis des Lehrvertrages als Ausdruck eines Erziehungsverhältnisses. Die Gewerkschaften betonten hingegen den arbeitsrechtlichen Charakter des Lehrvertrages. Eine Entscheidung des Reichsarbeitsgerichtes von 1928 schaffte endgültige Klarheit, indem es den Lehrvertrag als Arbeitsvertrag definierte und damit seine tarifrechtliche Regelung bestätigte (vgl. Muth 1985, S. 425 ff.; Schütte 1992, S. 120 ff.; Stachura 1989, S. 108 f.). Für das Handwerk bedeutete dies eine Einschränkung seiner lohnpolitischen Gestaltungsmöglichkeiten: „Billige“ Lehrlinge waren ein wesentliches Element der Existenzsicherung des Handwerks. 
Nach dem Ende des Ersten Weltkrieges und der Rückkehr vieler Handwerksmeister in ihre Betriebe konnte die Handwerksausbildung ihre kriegsbedingte Krise schnell überwinden. Jetzt stellte sich dem Handwerk aber eine neue Herausforderung in Gestalt der sich entwickelnden rationalisierten Industrielehre, die das handwerkliche Ausbildungsmonopol - wie es sich seit dem Ende des 19. Jahrhunderts herausgebildet hatte - in Frage stellte. Technisch moderne Branchen der Industrie, wie z.B. der Maschinenbau, hatten die Notwendigkeit einer eigenen Qualifikation ihres „Facharbeiternachwuchses“ erkannt. Durch die Einrichtung des DATSCH schuf sich die Industrie eine unternehmensübergreifende Institution, die die Konzeptionierung und Standardisierung der industriellen Ausbildung vorantrieb. Anhand von Modell Lehrgängen sollten die Unternehmen ihre Ausbildungsanstrengungen neu organisieren. Einige Unternehmen gründeten Lehrwerkstätten und eigene Werkschulen, um die Ausbildung der Lehrlinge vom laufenden Produktionsbetrieb unabhängiger zu machen. Die Ausbildung im eigentlichen Betrieb verlor dadurch jedoch nicht seine Bedeutung. Bei der Konzeption seiner Lehrgänge orientierte sich der DATSCH an der traditionellen Berufsidee des Handwerks, d.h. in den Lehrgängen sollten die Lehrlinge einen spezifischen industriellen Beruf erlernen. Die Beibehaltung des handwerklichen Berufskonzepts sollte weitreichende Konsequenzen für die industrielle Arbeitsorganisation haben.

Der Konflikt zwischen Industrie und Handwerk kulminierte in der Frage der Gesellenprüfung. Zur Professionalisierung der Industrielehre gehörte auch eine von der Industrie bestimmte Prüfung vor den Industrie- und Handelskammern. Das Handwerk dagegen lehnte eine Aufweichung des Prüfungsmonopols der Handwerkskammern und Innungen $\mathrm{ab}$. Unter dem Druck des von beiden Parteien abgelehnten Berufsausbildungsgesetzes entschloß man sich, gemeinsame Prüfungsausschüsse von Handwerkskammern und Industrie- und Handelskammern für die Industrielehrlinge einzurichten. Die Industrie konnte sich mit ihrer Forderung nach eigenen Prüfungsorganen nicht durchsetzen.

Durch die verfassungsrechtliche Verankerung der Fortbildungsschulpflicht wurde die Rolle der Berufsschule als komplementärem Lernort zum Betrieb weiter gefestigt, auch wenn sich diese aus finanzpolitischen Gründen nicht flächendeckend durchsetzen ließ. In diesem Bereich hatte der Staat einen direkten Zugriff auf die inhaltliche und organisatorische Gestaltung der beruflichen Bildung. Neben Staat und Arbeitgeber trat 
mit den Gewerkschaften einer weiterer Akteur auf die Bühne der Berufsausbildung. Über die Tarifpolitik versuchten diese ihren Einfluß auf die Lehrlingsausbildung auszuweiten, da eine gesetzliche Festlegung ihres Mitbestimmungsanspruches durch das Scheitern des Berufsausbildungsgesetzes unerreichbar schien. 


\section{Institutionen und Interessen}

Die institutionellen Strukturen und ,institutional actors“ (Immergut 1992, S. 21) formen die Interessen der einzelnen Akteure - ohne diese zu determinieren - und bieten Verfahrensweisen für ihre Durchsetzung. Sie stabilisieren das Verhalten der Akteure und bilden somit die Grundlage sozio-politischer Kontinuität. Wie bereits dargestellt, sprechen Vertreter des historischen Institutionalismus in diesem Zusammenhang von ,path dependency’: Ein einmal eingeschlagener Weg kann (bzw. wird) so einfach nicht wieder verlassen werden, wobei der Fortschritt manchmal in Schlangenlinien erfolgt.

Das duale Berufsbildungssystem ist eine komplexe Konfiguration verschiedener institutioneller Strukturen und Akteure, deren Interessen wiederum auf die Institutionen bezogen sind. So streben z.B. Jugendliche eine Ausbildung in einem - staatlich anerkannten - Beruf an, weil ihnen die zentrale Bedeutung des Berufskonzepts für die Steuerung des Beschäftigungssystems bewußt ist. Durch das Wechselspiel und Ineinandergreifen der Institutionen - wie Beruf, Selbstverwaltung durch (Wirtschafts-) Kammern, Industriegewerkschaften, etc. - gewinnt das System der Berufsausbildung an Stabilität. Im folgenden werden die relevanten stabilitätserhaltenden institutionellen Strukturen und ,institutional actors' analysiert.

\subsection{Ausbildungsmarkt und Beschäftigungssystem}

Der Übergang von der Schule in das Beschäftigungssystem ist in Deutschland für die Mehrzahl der Jugendlichen durch das duale Berufsbildungssystem geprägt. Dabei lassen sich zwei Schwellen feststellen, die von den Jugendlichen überschritten werden müssen: Erstens ist der Wechsel von der allgemeinbildenden Schule in die berufliche Ausbildung zu bewältigen, zweitens der Einstieg in ein Beschäftigungsverhältnis. Die Vermittlungs- und ,matching'-Prozesse finden im ersten Fall auf dem Ausbildungsmarkt und im zweiten Fall auf dem Arbeitsmarkt statt. Als zentraler Regulationsinstitution kommt dem Berufskonzept auf beiden Märkten eine entscheidende stabilisierende Funktion zu. Diese setzt sich in der spezifischen Ausgestaltung der betrieblichen Arbeitsorganisation fort. Im folgenden soll die institutionelle Ausformung dieses Übergangsprozesses und seine interessensteuernde Wirkung analysiert werden. 
An der Schwelle vom allgemeinbildenden Schulsystem zur Berufsausbildung treten verschiedene Abstimmungsprobleme auf, die die Verteilung der Ausbildungsplatzchancen unter den Jugendlichen beeinflussen. Anhand von vier Kriterien lassen sich diese Verteilungschancen näher beleuchten: schulische Qualifikation, Region, Geschlecht und Nationalität. Hinsichtlich der schulischen Qualifikation ist auf das differenzierte allgemeinbildende Schulsystem in Deutschland hinzuweisen. Dabei ist zwischen drei Schulabschlüssen zu unterscheiden: dem Hauptschulabschluß (nach 9 Jahren), dem Realschulabschluß (nach 10 Jahren) und dem Abitur als gymnasialer Abschluß (nach 13 Jahren). Während das Abitur zur Aufnahme eines Universitätsstudiums berechtigt, gilt dies für die beiden anderen Abschlüsse nicht. Um Ausbildungsplätze im dualen Berufsbildungssystem konkurrieren jedoch Bewerber aus allen drei Schulkategorien. Dies führt zu Ausdifferenzierungen in der Verteilung auf die verschiedenen Ausbildungsberufe und einem je nach Ausbildungsplatzangebot verschärften Verdrängungswettbewerb.

In den 1950er und 1960er Jahren existierte ein Ausbildungsplatzüberangebot, so daß auch Jugendliche ohne Schulabschluß eine Lehrstelle erhielten. Die Konkurrenz zwischen den ausbildenden Betrieben führte aber schon damals $\mathrm{zu}$ einer horizontalen Differenzierung, die ein qualitatives Gefälle von Großunternehmen zum Handwerk beinhaltete. In den 1970er Jahren überstieg die Nachfrage nach Ausbildungsplätzen das Angebot. In der Folge paßten die Betriebe ihr Auswahlverhalten an und erhöhten die Anforderungen. Die steigende Zahl von Bewerbern mit Abitur verstärkte diese Entwicklung noch. Obgleich nicht alle Betriebe für alle Ausbildungsberufe Abiturienten anderen Bewerbern vorzogen, konnte doch - gerade in kaufmännisch-administrativen Ausbildungsberufen - eine Substitution von Realschulabsolventen festgestellt werden (vgl. Sinnhold 1990, S. 73 f.).

Insgesamt zeigt sich ein nach Schulabschlüssen differenziertes Bild: Jugendliche ohne Hauptschulabschluß haben durchgängig unterdurchschnittliche Vermittlungschancen auf dem Ausbildungsmarkt. Die Situation für Jugendliche mit Hauptschulabschluß hat sich in Zeiten der Ausbildungsknappheit verschlechtert. Darüber hinaus konkurrieren sie mit einer wachsenden Zahl von Jugendlichen mit Realschulabschluß um eine begrenzte Anzahl von Lehrstellen. In ihrer Berufswahl stellen sich die Hauptschüler auf diese - für sie - externen Bedingungen entsprechend ein: Je näher das Verlassen der Schule rückt, desto stärker treten die persönlichen Interessen hinsichtlich der Berufs- 
wahl zurück. Um eine mögliche Arbeitslosigkeit zu vermeiden, orientieren sich die Bewerbungen am jeweiligen Ausbildungsplatzangebot und an den für Hauptschüler als realistisch erachteten Ausbildungsberufen. So stimmen die Berufswünsche ausbildungsplatzsuchender männlicher Hauptschüler mit den prozentualen Verteilungen der männlichen Auszubildenden im Bereich der ersten drei Ausbildungsberufe überein. Es handelt sich dabei ausschließlich um gewerbliche Ausbildungsberufe mit dem Berufsfeld KFZ-Mechanik an erster Stelle (vgl. Heinz/Krüger/Rettke et al. 1987, S. 113 ff.; Bundesministerium für Bildung und Forschung 2001).

Jugendliche mit Realschulabschluß haben im Vergleich zu Hauptschülern weitaus bessere Vermittlungschancen. Die Quote der unvermittelten Bewerber mit Realschulabschluß lag in den 1980er Jahren immer unter derjenigen der Hauptschulabsolventen. Mit der Zeit verschob sich die berufsfachliche Spezialisierung der Realschulabsolventen in Richtung auf Fertigungsberufe. Insbesondere im Elektrobereich ließen sich deutliche Zuwächse feststellen. Aber auch die Berufsbereiche Metall und Mechanik wiesen einen wachsenden Anteil von Realschulabsolventen auf. Andererseits war im gesamten Dienstleistungsbereich ein Rückgang von Auszubildenden mit Realschulabschluß zu verzeichnen. Diese Entwicklung ist auf die bereits erwähnte Zunahme an Abiturienten unter den Auszubildenden zurückzuführen (vgl. Strikker 1990, S. 41; Steinmann 2000, S. 143 ff.; Timmermann 1988, S. 51).

Während die Abiturienten zu Beginn der 1980er Jahre - wie die Hauptschüler - einen überproportionalen Anteil an den unvermittelten Bewerbern darstellten, verbesserten sich ihre Vermittlungschancen in den darauffolgenden Jahren. Die Mehrzahl der Abiturienten (ca. 60\%) findet sich seitdem in den Ausbildungsberufen des Dienstleistungsbereichs. Hier konzentrieren sich die Fälle auf den kaufmännsichen Büro- und Verwaltungsbereich sowie das Banken- und Versicherungswesen (vgl. Steinmann 2000, S. 147). Die Gruppe der Abiturienten nimmt aber insoweit eine Sonderrolle ein, als ihr mit dem Abitur vielfältige Bildungsoptionen, wie z.B. ein Hochschulstudium oder eine Ausbildung an einer Berufsakademie, offenstehen. Gegen Ende der 1980er Jahre nahmen Abiturienten nach der Lehre in zunehmendem Maße ein Studium auf. Dadurch veränderte sich das Einstellungsverhalten einiger Betriebe gegenüber Abiturienten. Das Risiko eines Verlusts der Ausbildungsinvestitionen ist bei Abiturienten im Vergleich zu Realschülern höher (vgl. Windolf/Hohn 1984, S. 100 ff.). 
Die Vermittlungschancen der Jugendlichen auf dem Ausbildungsmarkt hängen also wesentlich von ihrer schulischen Qualifikation ab. In Zeiten von Ausbildungsplatzknappheit und gestiegenen allgemeinbildenden Schulqualifikationen passten die Unternehmen ihr Rekrutierungsverhalten dahingehend an, daß sie höhere Einstellungsvoraussetzungen definierten. Neben der schulischen Leistung verwendeten immer mehr Unternehmen auch psychologische Tests in ihren Auswahlverfahren. Dadurch verstärkten sich bereits in Ansätzen vorhandene Tendenzen einer vertikalen berufsfachlichen Strukturierung des Ausbildungsmarktes entlang allgemeinbildender Schulabschlüsse. So verschob sich die berufsfachliche Ausrichtung der Realschulabsolventen zunehmend in den Fertigungsbereich, wo sie in Konkurrenz zu den Hauptschülern traten. Bei den Abiturienten verfestigte sich demgegenüber die berufsfachliche Spezialisierung auf den Dienstleistungsbereich.

Darüber hinaus beeinflußt die unterschiedliche regionale Verteilung der Ausbildungsplätze die Vermittlungschancen der Jugendlichen, die sich in ihren Bewerbungen auf Unternehmen und Betriebe im regionalen Umfeld konzentrieren (vgl. Timmermann 1988, S. 50). Die Mobilitätsbereitschaft der Jugendlichen aus den neuen Bundesländern ist dabei deutlich höher als die der Jugendlichen aus den alten Bundesländer (vgl. Bundesministerium für Bildung und Forschung 2001). Zwischen den Bundesländern zeigen sich erhebliche Differenzen. Im Jahr 2000 konnte folgende Situation festgestellt werden: Während im Süden eine Angebots-Nachfrage-Relation von 105 Ausbildungsplätzen pro 100 Bewerbern bestand, verkehrte sich die Relation in den Stadtstaaten in ihr Gegenteil und lag im Durchschnitt bei $97 \mathrm{zu} 100$ (vgl. Bundesministerium für Bildung und Forschung 2001). Aber auch innerhalb der Bundesländer kommt es zu Diskrepanzen zwischen Ausbildungsplatzangebot und -nachfrage, die auf einen fehlenden Austausch zwischen ländlichen und städtischen Regionen hinweisen. Durch den „Altbestand“ an unvermittelten Bewerbern reproduzieren sich zudem die regionalen Ungleichgewichte (vgl. Strikker 1990, S. 43).

Die beiden Merkmale Geschlecht und Nationalität sind weitere Einflußgrößen, die die Übergangschancen von der Schule in eine Ausbildung bestimmen. Junge Frauen haben dabei deutlich geringere Aussichten auf einen Ausbildungsplatz als männliche Bewerber. Darüber hinaus ziehen sie sich schneller vom Ausbildungsmarkt zurück und konzentrieren sich auf wenige Ausbildungsberufe (vgl. Lemmermöhle-Thüsing 1990, S. 70 f.). Die weitaus schlechtesten Vermittlungschancen haben ausländische Bewerber. In 
den 1980er Jahren lag der Anteil der unvermittelten Bewerbern an allen ausländischen Bewerbern bei $10 \%$ und war damit im Vergleich zu den deutschen Bewerbern doppelt so hoch (vgl. Strikker 1990, S. 42; Sinnhold 1990, S. 76 f.). Trotz einer entspannteren Ausbildungsmarktsituation lag 1998 der Anteil der unvermittelten ausländischen Jugendlichen mit 21,9\% aller unvermittelten Bewerber deutlich über dem Anteil junger Ausländer an allen gemeldeten Bewerbern (14,4\%) (vgl. Bundesministerium für Bildung und Forschung 1999, S. 37).

Die Gesamtabstimmung zwischen Ausbildungsplatzangebot und -nachfrage beeinflußt den Grad der Ausprägung der vier Merkmale auf die Vermittlungschancen der Jugendlichen. Je angespannter die Ausbildungsmarktsituation ist, desto deutlicher treten die Unterschiede in den Ausbildungschancen zutage. Während in den frühen 1980er Jahren aufgrund demographischer Veränderungen die Nachfrage an Ausbildungsplätzen das Angebot überstieg, konnte für die späten 1980er und frühen 1990er Jahren ein Ausbildungsplatzüberangebot verzeichnet werden. Durch die konjunkturbedingte Abnahme an Ausbildungsstellen kam es Mitte der 1990er Jahren erneut zu einem Ausbildungsplatzdefizit (vgl. Institut für Arbeitsmarkt- und Berufsforschung 1998a). Im Jahr 2000 befand sich der Ausbildungsmarkt erstmals wieder in einem rechnerischen Gleichgewicht (vgl. Bundesministerium für Bildung und Forschung 2001, S. 11).

Nach Abschluß ihrer Ausbildung haben die Jugendlichen den Übergang in ein Beschäftigungsverhältnis zu bewältigen. Der, matching’-Prozeß zwischen Ausbildung und Erwerbstätigkeit erfolgt über den Arbeitsmarkt, wo die Qualifikationsprofile der Absolventen auf die Qualifikationsanforderungen der Arbeitgeber treffen. Dabei ist die Vorstellung eines einheitlichen Arbeitsmarktes insofern zu revidieren, als sich einzelne Teilarbeitsmärkte definieren lassen, die relativ autonom voneinander sind. Bereits in den frühen 1970er Jahren entwickelten Doeringer und Piore ihre duale Arbeitsmarkttheorie: Danach kann einerseits zwischen internen und externen sowie primären und sekundären Arbeitsmärkten unterschieden werden. Interne Arbeitsmärkte stellen gegenüber externen Arbeitsmärkten keine kompetitiven Märkte dar. In ihnen ersetzen bürokratische Zuweisungsregeln den Marktmechanismus. Durch besondere Beförderungsregelungen - z.B. nach dem Senioritätsprinzip - und Betriebsrenten sollen qualifizierte Arbeitskräfte an das Unternehmen gebunden werden, um ein betriebsspezifisches Qualifikationspotential zu schaffen. Der Austausch zwischen externem und internem Arbeitsmarkt findet über sogenannte ,ports of entry' statt, die 
begrenzte Eintrittsmöglichkeiten bieten (vgl. Doeringer/Piore 1971, S. 13 ff.; Piore 1978, S. 69 ff.). Der Unterteilung in primäre und sekundäre Arbeitsmärkte liegt demgegenüber das Konzept qualitativ unterschiedlicher Arbeitsplätze zugrunde. Arbeitsplätze im primären Arbeitsmarkt zeichnen sich durch eine relativ hohe Beschäftigungsstabilität, gute Einkommensbedingungen sowie institutionalisierte Karriereleitern aus. Der sekundäre Arbeitsmarkt ist dagegen durch niedrige Arbeitseinkommen, geringe Aufstiegsmöglichkeiten und fehlende Arbeitsplatzsicherheit charakterisiert. Insbesondere das Qualifikationsprofil des Arbeitnehmers entscheidet über die berufliche Plazierung in einem der beiden Segmente. Zwischen internem und primärem Arbeitsmarkt besteht dabei eine weitgehende Übereinstimmung.

Für den deutschen Arbeitsmarkt entwarfen Lutz und Sengenberger ein auf der dualen Arbeitsmarkttheorie basierendes eigenes Konzept, das von einem dreigeteilten Arbeitsmarkt ausgeht. Ursächlich für die Dreiteilung des Arbeitsmarktes in einen betriebsinternen, einen berufsfachlichen und einen unstrukturierten Sektor ist die Personalpolitik der Betriebe. Die einzelbetrieblichen Beschäftigungsstrategien bestimmen die nachgefragten Qualifikationen, die im Interesse des Unternehmens möglichst effizient im Produktionsprozeß eingesetzt werden sollen (vgl. Lutz/Sengenberger 1974, S. 43 ff.; Sengenberger 1987, S. 117 f.).

Der unstrukturierte Teilarbeitsmarkt beinhaltet diejenigen Arbeitskräfte, die aufgrund ihrer unspezifischen Qualifikation keinen Zugang zu den anderen Teilarbeitsmärkten gefunden haben. Aufgrund der geringen Qualifikationsanforderungen der angebotenen Arbeitsplätze müssen die Arbeitskräfte nicht angelernt werden und sind sofort produktiv. Bei einem Arbeitsplatzwechsel gehen keine betriebsspezifischen Qualifikationen verloren, weshalb beide Seiten ein geringes Interesse an einer dauerhaften Bindung haben. Diese Bindungslosigkeit drückt sich in hohen Fluktuationen und instabilen Beschäftigungsverhältnissen aus. Wesentliche Voraussetzungen für die Existenz eines unstrukturierten Arbeitsmarktes sind eine arbeitsintensive Produktion, eine große Zahl von verfügbaren Arbeitskräften und fehlende regulierende Institutionen, wie z.B. Kündigungsschutzgesetze. In Deutschland findet sich dieser Arbeitsmarkttypus annäherungsweise in der Beschäftigung von Frauen in der Textil- und Bekleidungsindustrie mit der dort vorherrschenden tayloristischen Arbeitsorganisation. Daneben gehören auch Ausländer und Jugendliche ohne Berufsausbildung $\mathrm{zu}$ den überproportional 
vertretenen Beschäftigtengruppen in diesem Segment (vgl. Sengenberger 1987, S. 119 ff.; Lutz/Sengenberger 1974, S. 57 f.).

Im Gegensatz dazu handelt es sich beim berufsfachlichen Teilarbeitsmarkt um einen strukturierten Markt. Die strukturierende Grundlage bilden standardisierte, berufsspezifische sowie überbetrieblich einsetzbare Qualifikationen. Über den Nachweis solcher Qualifikationen, z.B. in Form eines Gesellenbriefs, können sich Arbeitskräfte Zutritt zu diesem Arbeitsmarktsegment verschaffen. Dadurch entstehen Mobilitätsbarrieren, die Arbeitskräfte ohne entsprechenden Qualifikationsnachweis vom berufsfachlichen Teilarbeitsmarkt ausschließen. Die Ausbildungsinhalte orientieren sich an überbetrieblich definierten Berufsbildern, die sich in der Struktur der Arbeitsplätze und den betrieblichen Qualifikationsanforderungen widerspiegeln. Für die Betriebe hat eine berufsfachliche Arbeitsorganisation den Vorteil, daß aufgrund der Standardisierung der Austausch von Arbeitskräften weitgehend transaktionskostenneutral ist. Die Arbeitskräfte sind sofort einsatzfähig und produktiv. Während das Beschäftigungsniveau im Sinne einer gleichbleibenden Tätigkeit im erlernten Beruf relativ stabil ist, erweisen sich die Beschäftigungsverhältnisse aufgrund zwischenbetrieblicher Mobilität als instabil. Die Bindung der Arbeitskraft gilt nicht dem einzelnen Betrieb, sondern dem Beruf. Wesentliche Funktionsvoraussetzung für den berufsfachlichen Teilarbeitsmarkt ist die Kongruenz von Arbeitsplatzanforderungen und Qualifikationsprofil der Arbeitskräfte. Veränderungen in den qualifikatorischen Determinanten der Arbeitsplätze z.B. aufgrund neuer Technologien - müssen sich dementsprechend in Anpassungen der überbetrieblichen Ausbildungsnormen niederschlagen (vgl. Sengenberger 1987, S. 128 ff.).

Die Entstehung und Fortexistenz des berufsfachlichen Teilarbeitsmarktes bedarf einer überbetrieblichen Instanz, die für die Festlegung und Überwachung der standardisierten Qualifikationsprofile verantwortlich ist. Andernfalls besteht die Gefahr, daß das berufsfachliche Segment in den beiden anderen Teilarbeitsmärkten aufgeht. Ein klassisches Beispiel für diese Form der kollektiven Arbeitsmarktorganisation findet sich im Handwerk. Wie bereits oben dargestellt, regelten die jeweiligen Zünfte den Zugang zum berufsfachlichen Arbeitsmarkt und kontrollierten die Einhaltung der Qualifikationsstandards. Nur Meistern war die selbständige Ausübung des Gewerbes erlaubt. Ihnen oblag auch die Lehrlingsausbildung. Die Zünfte konnten z.B. über die Festlegung der Zahl der Lehrlinge pro Meister den Arbeitsmarkt regulieren. Trotz der Aufhebung 
des Zunftzwangs im 19. Jahrhundert blieben vielerorts die Zünfte als freiwillige Vereinigungen bestehen. Außerdem übernahm der Staat durch gesetzliche Regelungen - z.B. die Durchsetzung des „kleinen Befähigungsnachweises“ - eine Ersatzfunktion. Mit der Einrichtung von öffentlich-rechtlichen Handwerkskammern schuf er zudem eine intermediäre Instanz, die als Selbstverwaltungsorgan staatliche Aufgaben eigenverantwortlich ausübt. Das Prinzip der berufsfachlichen Arbeitsmarktorganisation setzte sich auch in der Industrie durch, die bei der Besetzung ihrer Facharbeiterpositionen auf die im Handwerk qualifizierten Fachkräfte zurückgriff.

Betriebsinterne Teilarbeitsmärkte bildeten sich im Prozeß einer durch großbetriebliche Unternehmen getragenen betriebszentrierten Arbeitskräftestrategie in den frühen 1960er Jahren heraus (vgl. Lutz 1987, S. 185 ff.). Im Vordergrund steht die Sicherung der betrieblichen Autonomie gegenüber dem externen Arbeitsmarkt. Durch eine konsequente Förderung betriebsspezifischer Qualifikationen wird die zwischenbetriebliche Mobilität beschränkt. Für Arbeitskräfte ist der Wechsel des Arbeitsplatzes mit dem Verlust von Qualifikationen und somit von zukünftig realisierbarem Einkommen verbunden. Dadurch erhöht sich die individuelle Betriebsbindung und Loyalität gegenüber dem Betrieb. Umgekehrt hat auch das Unternehmen ein Interesse an einer langfristigen Bindung betriebsspezifischer Qualifikationen und bietet deshalb als Gegenleistung eine implizite Beschäftigungssicherheit. In Zeiten konjunkturellen Abschwungs verzichten die Unternehmen auf Entlassungen. Institutionelle Regelungen, wie z.B. das Kurzarbeitergeld, haben dabei eine unterstützende Wirkung. Ein weiterer Anreiz zur betrieblichen Bindung liegt in der nach dem Senioritätsprinzip organisierten vertikalen Mobilität innerhalb des Betriebes (vgl. Sengenberger 1987, S. 150 ff.). Der Zugang zum betriebsinternen Arbeitsmarkt eröffnet sich in der Regel über die berufliche Ausbildung. Dies spiegelt sich auch im Rekrutierungsverhalten der Betriebe wider, die z.B. Mitarbeiter- und Kundenkinder aufgrund ihrer familiär-emotionalen Beziehung zum Unternehmen bei der Vergabe von Ausbildungsplätzen bevorzugen (vgl. Windolf/Hohn 1984, S. 109). Durch die Einbeziehung der Berufsausbildung treffen im betriebsinternen Teilarbeitsmarkt berufsfachliche und betriebsspezifische Qualifikationen zusammen.

Den Teilarbeitsmarktsegmenten steht auf der anderen Seite eine nach dem Berufsprinzip strukturierte Arbeits- und Betriebsorganisation gegenüber. Diese sich bereits in den Anfängen der Industrialisierung herausbildende arbeitsorganisatorische Struktur stellte 
bis in die 1980er Jahre ein wesentliches Element des deutschen Produktionsmodells dar. Folgende Merkmale kennzeichnen in idealtyischer Weise eine berufsbezogene Arbeitsorganisation: Die Betriebsstruktur beinhaltet ein alle Produktionsstufen umfassendes Leistungsprofil mit entsprechend vielfältigen beruflichen Optionen. Die Aufbauorganisation orientiert sich am Fachabteilungsprinzip mit funktional unterteilten Stabsabteilungen und produzierenden Bereichen. Auf der Ebene des einzelnen Arbeitsplatzes bestimmen berufliche Qualifikationen die Aufgabenverteilung und die Kompetenzabgrenzung. Die Kooperation zwischen Fachabteilungen und Beschäftigten ist aufgrund hierarchisch gestufter Befugnisse durch Über- und Unterordnungen charakterisiert. Zudem findet sich eine nach formalen Kompetenzen gegliederte Statusorganisation (vgl. Baethge/Baethge-Kinsky 1998, S. 462 f.). Über die beruflich normierte Aufgabenzuordnung gewinnen sowohl die berufsfachlich qualifizierte Arbeitskraft als auch der Betrieb an relativer Autonomie. Während der Facharbeiter nicht an einen betriebsspezifischen Arbeitsplatz gebunden ist, schafft die berufsfachliche Standardisierung Handlungssicherheit für den Betrieb im Arbeitskräfteeinsatz (vgl. Allmendinger 1989, S. 239).

Die Ausnutzung der berufsfachlichen Qualifikationen eröffnete den Betrieben ein konstantes Leistungssteigerungspotential, das in einer Optimierung der Arbeitstechniken und -prozesse sowie in geringfügigen Produktverbesserungen lag. Die darauf basierende Geschäftspolitik der Unternehmen richtete sich auf die Erschließung vorhandener Märkte mit qualitativ hochwertigen Produkten. Seit den frühen 1990er Jahren stehen die Unternehmen aufgrund einer zunehmenden Globalisierung unter wachsendem Wettbewerbsdruck, der von ihnen schnellere Produktinnovationen verlangt. Die berufsbezogene Arbeitsorganisation scheint aber dieser Herausforderung nicht gewachsen zu sein, da berufliche Kompetenzabgrenzungen ein „Denken in Kategorien einer prioritären Zuständigkeit“ (Kern/Sabel 1994, S. 613) erzeugen und damit einer innovationsfördernden Wissensintegration entgegenstehen (vgl. Kern 1997, S. 276 ff.).

Trotz potentieller Erosionstendenzen stellt die Berufskategorie immer noch eine entscheidende Größe im Übergang von der Ausbildung in ein Beschäftigungsverhältnis dar. Empirische Untersuchungen zeigen, daß über verschiedene Geburtskohorten hinweg die Übereinstimmung zwischen Ausbildungsberuf und ausgeübtem Beruf beim Beschäftigungseinstieg weit über 50\% lag (vgl. Konietzka 1999, S. 388 f.; Westhoff 1991, S. 56; Hillmert 2001, S. 233 ff.). Nach einer Erhebung des Bundesinstituts für 
Berufsbildung arbeiteten über zwei Drittel der betrieblich ausgebildeten Absolventen des Jahres 1999 fünf Monate nach ihrem Ausbildungsabschluß im erlernten Beruf (vgl. Bundesministerium für Bildung und Forschung 2001).

Die Übergänge von der Schule in die Ausbildung und danach in die Beschäftigung sind beruflich strukturiert. Obgleich der Zugang zum beruflichen Bildungssystem für alle Jugendliche unabhängig vom schulischen Abschluß offen ist, lassen sich nach Ausbildungsberufen differenzierte Strukturen feststellen. Der wachsende Anteile an Bewerbern mit Abitur hat $\mathrm{zu}$ einer Verdrängung von Realschulabsolventen aus Dienstleistungsberufen geführt. Dies hatte wiederum Konsequenzen für die Position der Hauptschüler und ihre Vermittlungschancen im gewerblichen Berufsbereich. Je nach Ausbildungsmarktsituation verschärfen oder entspannen sich die Strukturausdifferenzierungen. Nach Abschluß ihrer Ausbildung treffen die Jugendlichen auf einen berufsfachlich-qualifikatorisch segmentierten Arbeitsmarkt. Die Vermittlung in ein Beschäftigungsverhältnis findet entweder über die nach formalen Berufszertifikaten organisierten berufsfachlichen Teilarbeitsmärkten statt oder - für die Mehrzahl der Jugendlichen - über betriebsinterne Arbeitsmärkte, deren ,port of entry' häufig in der betrieblichen Ausbildung liegt. Eine berufsbezogene Arbeitsorganisation stellt den Endpunkt dieses sich selbst reproduzierenden Übergangsprozesses dar.

\subsection{Unternehmensverbände und Arbeitgeber}

Wie alle betrieblich orientierten Berufsbildungssysteme, so hängt auch das deutsche duale Berufsausbildungssystem wesentlich von der Beteiligung der einzelnen Unternehmen und Arbeitgeber ab. Sie sind in ihrer Entscheidung grundsätzlich frei, Ausbildungsplätze anzubieten oder ihren Fachkräftebedarf über den externen Arbeitsmarkt abzudecken. Dadurch kann es zu kollektiv dysfunktionalen Effekten kommen. In diesem Zusammenhang ist die Bedeutung von Verbänden, insbesondere Unternehmensverbänden, hervorzuheben. Nach einer kurzen Erläuterung des theoretischen Problems des kollektiven Handels und der Bereitstellung von öffentlichen Gütern wird im folgenden die Rolle der Unternehmensverbände im Berufsbildungssystem darge- 
stellt. Anschließend folgt eine Analyse der einzelbetrieblichen Interessen an der betrieblichen Berufsausbildung.

Öffentliche Güter zeichnen sich idealtypisch dadurch aus, daß niemand von ihrem Konsum ausgeschlossen werden kann (Nicht-Ausschlußfähigkeit) und der einzelne Konsum den Nutzen für die Gesamtheit nicht verringert (Nicht-Rivalitätsprinzip) (vgl. Olson 1992, S. 13 f.). Für den einzelnen ist es deshalb aus ökonomischer Sicht rational nicht sinnvoll, sich an der Bereitstellung eines öffentlichen Gutes $\mathrm{zu}$ beteiligen. Bezogen auf die berufliche Bildung ergibt sich daraus folgendes Problem: Berufliche Bildung, die überbetriebliche, allgemeine Qualifikationen vermittelt, stellt für den einzelnen Betrieb ein ,quasi-öffentliches' Gut dar. Ohne sich an der Ausbildung beruflich qualifizierter Arbeitskräfte beteiligt zu haben, kann der einzelne Betrieb qualifizierte Fachkräfte über den externen Arbeitsmarkt anwerben. Dies trifft jedoch nicht auf betriebsspezifische Qualifikationen zu, da diese für andere Betriebe nur von geringem Nutzen sind. Für den einzelnen Betrieb kann es also ökonomisch rational sein, im Rahmen einer allgemeinen Berufsausbildung nicht selber auszubilden, sondern ausgebildete Fachkräfte abzuwerben. Eine solche für den einzelnen Betrieb ökonomisch rationale Entscheidung ist aus Sicht der Gesellschaft höchst irrational und führt zum Versagen des marktwirtschaftlichen Allokationsmechanismus. In der Theorie des kollektiven Handelns bezeichnet man dieses Phänomen als ,free-riding' oder ,Trittbrettfahren' (vgl. Streeck 1983, S. 1 ff.; Streeck et al. 1987, S. 1; Olson 1992, S. 8 ff.; Crouch 1993, S. 10). Zur Lösung dieses Problems lassen sich drei Ansätze nennen: Erstens kann der Staat die Bereitstellung qualifizierter Fachkräfte über ein steuerfinanziertes Berufsbildungssystem sicherstellen. Diese Option birgt jedoch die Gefahr in sich, daß am betrieblichen Bedarf vorbei ausgebildet wird. Eine weitere Möglichkeit stellt die staatliche Nicht-Intervention in den Markt dar. Die Unternehmen richten ihre Ausbildungsinvestitionen ausschließlich an betriebsspezifischen Erfordernissen aus und passen ihre Arbeitsorganisation entsprechend an. Darüber hinaus wird die Investition in Humankapital dem einzelnen Individuum überlassen. Eine dritte Option ist die Übertragung para-staatlicher Kompetenzen auf freiwillige oder zwangsverpflichtete Verbände - im Sinne eines, private interest government' - , die ein vermittelndes Bindeglied zwischen kollektiven und individuellen Interessen darstellen (vgl. Streeck/Schmitter 1985, S. 14 ff.; Greinert 1988, S. 145 ff.). Das deutsche duale Berufsbildungssystem verbindet Elemente einer steuerfinanzierten staatlichen Organi- 
sation der beruflichen Bildung, insbesondere in Form der Berufsschulen, mit verbandlicher Selbstregulation, die sich durch ihre Praxisnähe und staatsentlastende Funktion auszeichnet.

Die Verbände der Unternehmen und Arbeitgeber sind für die Regulation der Berufsbildung in Deutschland von besonderer Bedeutung. Ihre Einbindung in staatliche Entscheidungsprozesse und die ihnen übertragenen, para-staatlichen' Kompetenzen schaffen die Grundlage für die Überwindung individueller ökonomischer Rationalitäten und ermöglichen damit die Bereitstellung des ,quasi-öffentlichen' Gutes beruflicher Bildung. Die organisierten Interessen der Arbeitgeber lassen sich in drei Kategorien unterteilen: Wirtschaftsverbände, die die allgemeinen wirtschaftspolitischen Interessen einer Branche vertreten, Arbeitgeberverbände, die die sozial- und tarifpolitischen Interessen der Arbeitgeber gegenüber den Gewerkschaften im Rahmen der Tarifverhandlungen wahrnehmen, und Industrie- und Handelskammern bzw. Handwerkskammern, die die regionalen Interessen aller Gewerbetreibenden repräsentieren. Auf nationaler Ebene ist der Bundesverband der Deutschen Industrie (BDI) der einflußreichste Vertreter unter den Wirtschaftsverbänden. Das Handwerk ist dagegen im Zentralverband des Deutschen Handwerks (ZDH) zusammengeschlossen. Die Arbeitgeberverbände der privatwirtschaftlichen Unternehmen sind in der Bundesvereinigung der Deutschen Arbeitgeberverbände (BDA) organisiert. Und auch die Kammern haben ihre eigenen Spitzenverbände: den Deutschen Industrie- und Handelskammertag (DIHK) und den Deutschen Handwerkskammertag (DHKT). Um die Interessen der Unternehmen an der Berufsbildung besser koordinieren $\mathrm{zu}$ können, haben die Spitzenverbände der Wirtschaft in den 1970er Jahren das Kuratorium der deutschen Wirtschaft für Berufsbildung (KWB) gegründet (vgl. Müller-Jentsch 1997, S. 175; Streeck et al. 1987, S. 10 f.).

Die Entstehung der deutschen Wirtschaftsverbände als Interessenorganisationen einzelner Industriezweige oder Branchen geht zurück auf die erste Hälfte des 19. Jahrhunderts. Im Zuge der industriellen Revolution suchten die Unternehmer nach Formen der organisierten Interessenvertretung, die von den traditionellen korporativen Verbänden unabhängig sein und über diese hinausgehen sollten. Als rechtliche Grundlage bot sich hier der private Verein an, der im Gegensatz zur Korporation auf dem Prinzip der Freiwilligkeit beruhte. In der Frühphase der Interessenorganisierung zeichneten sich bereits zwei Typen der Organisation ab, die noch heute Bestand haben: Einerseits 
entwickelten sich eher staatsnahe Interessenvertretungen in Form der Handelskammern, andererseits entstanden „staatsfreie“ Verbände, wie z.B. der 1819 gegründete Deutsche Handels- und Gewerbeverein, der eine schutzzöllnerische Interessenpolitik vertrat.

Anfangs waren diese Interessenvertretungen nur schwach organisiert und dem bürgerlichen Verein näher als dem Wirtschaftsverband. In den 1860er Jahren wurde die Gründung freier Interessenverbände von der preußischen Schwerindustrie dominiert. Ihr politisches Gewicht war aber noch gering. Erst nach der Konstituierung eines Nationalstaates mit zentralen Entscheidungsorganen im Jahre 1871 nahm die Bedeutung der Interessenvertretung durch Wirtschaftsverbände zu. Gefördert wurde die Entstehung neuer Verbände zudem durch die Zunahme staatlicher Interventionen in die Wirtschaft. Zwischen 1870 und 1907 kam es zur Gründung von 495 neuen Verbänden. Die Vielzahl der unterschiedlichen Wirtschaftsverbände erforderten wiederum eine verbandliche Konzentration, die zu einem gestuften Verbandsystem mit Dachverbänden führte. Der erste Dachverband, der Centralverband Deutscher Industrieller (CDI), war ein Zusammenschluß schutzzöllnerisch eingestellter Industrieverbände, insbesondere der Textil- und Schwerindustrie (vgl. Kessler 1907, S. 6). Infolge der Dominanz der Großunternehmen kam es 1895 zur Abspaltung kleinerer und mittlerer Unternehmen und zur Gründung des Bundes der Industriellen als zweitem industriellen Spitzenverband. Während des Kaiserreichs nahm die Bürokratisierung der Verbände zu, die die Position der professionalisierten Verbandsfunktionäre gegenüber den ehrenamtlichen Honoratioren stärkte. Darüber hinaus fand eine schrittweise Einbeziehung der Wirtschaftsverbände in den politischen Entscheidungsprozeß statt, um die Sachkenntnis der Verbände zu nutzen sowie die Umsetzung von Entscheidungen zu effektivieren.

Die Kriegswirtschaft im Ersten Weltkrieg hatte eine weitreichende Verflechtung von staatlicher Verwaltung und Wirtschaftsverbänden zur Folge, die für die weitere Entwicklung der Verbände in Deutschland von besonderer Bedeutung sein sollte. Erstmals wurden den Verbänden quasi-staatliche Aufgaben und Kompetenzen - wie sie bereits bei den Kammern bekannt waren - übertragen, wodurch sie eine Doppelfunktion erhielten: Einerseits vertraten sie die Interessen ihrer Mitglieder gegenüber dem Staat, andererseits waren sie selber an der Formulierung, Umsetzung und Legitimierung staatlicher Politik beteiligt. In der Weimarer Republik setzte sich der Prozeß der verbandlichen Konzentration fort. Der Centralverband Deutscher Industrieller und der 
Bund der Industriellen schlossen sich 1919 zum Reichsverband der Deutschen Industrie (RDI) zusammen. Daneben traten immer mehr Einzelverbände den Dachverbänden bei. So lag der Organisationsgrad des RDI bei ca. $80 \%$ aller industriellen Unternehmen. Nach 1945 konnten die Allierten eine Dezentralisierung und Demokratisierung der Verbandsstrukturen nicht durchsetzten, so daß beim Wiederaufbau der Spitzenverbände eine Anknüpfung an die historisch gewachsenen Strukturen der Weimarer Republik erfolgte (vgl. Ullmann 1990, S. 95 ff.; Erdmann 1966, S. 227 ff.). Der Bundesverband der Deutschen Industrie (BDI) mit seinen 34 Mitgliederverbänden ist die Nachfolgeorganisation des Reichsverbands der Deutschen Industrie. Sein Aufgabenspektrum umfaßt alle wirtschaftspolitischen Themenbereich darunter auch die Berufsbildung, die durch eine eigene Abteilung vertreten ist.

Arbeitgeberverbände entstanden in Deutschland erst gegen Ende des 19. Jahrhunderts als Reaktion auf die wachsende Gewerkschaftsbewegung. Die vor 1904 entstandenen nationalen Arbeitgeberverbände waren in der Regel Zusammenschlüsse kleingewerblicher und handwerklicher Betriebe, wie z.B. der 1869 gegründete Deutsche Buchdruckerverein. Mit dem 1890 gegründeten Gesamtverband Deutscher Metallindustrieller entstand erstmals ein Arbeitgeberverband der großen Unternehmen, der mit seinen Bezirks- und Ortsgruppen zum Vorbild für alle auf nationaler Ebene zentralisierten Branchenverbände wurde (vgl. Knips 1996, S. 89 ff.). Der Zweck der neugegründeteten Arbeitgeberverbände lag ausschließlich in der kollektiven Abwehr gewerkschaftlicher Aktivitäten. Die Arbeitgeberverbände lehnten die Gewerkschaften als Interessenvertreter der Arbeiter grundsätzlich ab. Für die Entwicklung der Arbeitgeberverbände war der sogenannte „Crimmitschauer Streik“ im Winter 1903/04 von besonderer Bedeutung. In der sächsischen Stadt Crimmitschau streikten mehrere Tausend Weber über fünf Monate für die Einführung des 10-Stundentages. Im Verlauf des Streiks erhielten die Streikenden finanzielle Unterstützung von gewerkschaftlicher Seite aus ganz Deutschland. Daraufhin entschloß sich der Centralverband Deutscher Industrieller, eine allgemeine Hilfsaktion für die bestreikten Textilunternehmen zu starten. Außerdem sollte die Zusammenarbeit zwischen den einzelnen Arbeitgeberverbänden durch Errichtung der Hauptstelle deutscher Arbeitgeberverbände gestärkt werden. Gleichzeitig wurde auf Initiative des Bundes der Industriellen der Verein deutscher Arbeitgeberverbände gegründet. Beide Spitzenvereinigungen schlossen sich 1913 zur Vereinigung der Deutschen Arbeitgeberverbände zusammen (vgl. Erdmann 
1966, S. 53 ff.; Kessler 1907, S. 47 ff.; Bunn 1989, S. 169 ff.). Erst unter den Bedingungen der Kriegswirtschaft und der Weimarer Republik wandelte sich die Einstellung der Arbeitgeberverbände gegenüber den Gewerkschaften, die seitdem als Tarifvertragsparteien anerkannt werden. Nach dem Zweiten Weltkrieg erfolgte die Gründung der Bundesvereinigung der Deutschen Arbeitgeberverbände als Nachfolgeorganisation der Vereinigung der Deutschen Arbeitgeberverbände. Sie umfaßt 54 Fachspitzenverbände und 14 überfachliche Landesverbände. Der Organisationsgrad liegt bei ca. 80\% aller Beschäftigten der Privatwirtschaft (vgl. Müller-Jentsch 1997, S. 176-178). Wie der BDI, verfügt auch die BDA über eine Fachabteilung zur Berufsbildung, die neben der Tarifpolitik ein wichtiges Tätigkeitsfeld darstellt (vgl. Bunn 1989, S. 176 f.).

Der Ursprung der Industrie- und Handelskammern geht auf zwei unterschiedliche Entwicklungen zu Beginn des 19. Jahrhunderts zurück. Unter der napoleonischen Besatzung waren im Rheinland Handelskammern nach französischem Vorbild gegründet worden, die von Preußen nach 1814/15 unverändert übernommen wurden. Daneben gab es in Preußen die Tradition kaufmännischer Korporationen, die sich in wichtigen Handelsstädten wie Berlin, Danzig und Königsberg bereits früh gebildet hatten. Während in die Handelskammern einige wenige „,notabelne Kaufleute“ gewählt wurden, die als beratendes Gremium die Rolle eines staatlichen Hilfsorgans hatten, handelte es sich bei den kaufmännischen Korporationen um einen Zusammenschluß der örtlichen Kaufleute. Entgegen der liberalen Grundkonzeption der preußischen Gewerbepolitik waren die Statuten der kaufmännischen Korporationen staatlich sanktioniert. Darüber hinaus sollten sie nach einem ministeriellen Bericht die „Staatsorgane bei der Ausführung von finanziellen und sonstigen allgemeinen Staatszwecken“ (Fischer 1964, S. 21) unterstützen. Die kaufmännische Ausbildung und die Abnahme von Gesellenprüfungen gehörten ebenfalls zu ihren wesentlichen Aufgaben.

In den 1830er Jahren entstand aus diesen beiden Traditionen die Handelskammerstruktur, wie sie sich gegen Ende des 19. Jahrhunderts durchsetzen sollte: Alle Gewerbetreibenden unterlagen einem Beitrittszwang und mußten sich durch eine Umlage an den Kosten der Kammer beteiligen. Darin spiegelte sich das korporative Element der kaufmännischen Korporationen wider. Andererseits hatte die Handelskammer primär nicht die Funktion der Interessenvertretung, sondern sollte die staatliche Verwaltung bei ihren Aufgaben unterstützen, indem sie die Behörden informierte und zu 
gewerbepolitischen Fragen Stellung nahm. Darüber hinaus konnte die staatliche Verwaltung ihr ,quasi-staatliche' Kompetenzen übertragen. Die Handelskammern nach französischem Vorbild hatten sich somit von reinen Beiräten zu öffentlich-rechtlichen Selbstverwaltungskörperschaften gewandelt. Das preußische Handelskammergesetz von 1870 und die Gesetzesnovelle von 1897, die die Einrichtung von Handwerkskammern vorschrieb, bestätigten den öffentlich-rechtlichen Charakter der Handelskammern. Neben der staatlichen ,Hilfsorganfunktion' betonte das Gesetz aber auch die Interessenwahrnehmung aller Gewerbetreibenden durch die Kammern. So sollten die Ansprüche beider Seiten Berücksichtigung finden (vgl. Fischer 1964, S. 26 ff. und 7375; Lichter 1996, S. 8 ff.). Die Gesetzesnovelle von 1897 ermöglichte den Kammern zudem die Schaffung von Einrichtungen zur Förderung der beruflichen Ausbildung (vgl. Thalmann 1996, S. 252). Da in den Handelskammern nicht nur Kaufleute sondern auch Industrielle vertreten waren, gab es enge Verflechtungen zwischen den industriellen Interessenverbänden, wie z.B. dem Centralverband Deutscher Industrieller, und den Handelskammern. Auf personeller Ebene zeigte sich das in der Rekrutierung der hauptberuflichen Verbandsfunktionäre aus dem Potential der Handelskammersyndici (vgl. Kaelble 1971, S. 185). Die wachsende Bedeutung der Industrie in den Handelskammern führte nach dem Ersten Weltkrieg zur Umbenennung in Industrie- und Handelskammern.

Nach 1945 etablierten sich die Industrie- und Handelskammern sowie die Handwerkskammern wieder in ihrer, alten' Verfassung, trotz Widerständen der alliierten Besatzungsmächte. Diese wollten die Kammern als freiwillige Vereinigungen - angelehnt an die Struktur der amerikanischen Chamber of Commerce - konstituieren. Die Kammern setzten aber ihren auf Beitrittszwang basierenden öffentlich-rechtlichen Status durch (vgl. Herrigel 1996, S. 169 f.). Das Industrie- und Handelskammergesetz von 1956 legte den öffentlich-rechtlichen Status der Kammern für die gesamte Bundesrepublik fest (vgl. DIHT 1987, S. 6). Widerstände von Unternehmen gegen die Zwangsmitgliedschaft konnten bisher von den Kammern gerichtlich überwunden werden. Das Bundesverfassungsgericht hat vor kurzem in einem Urteil die Beitrittspflicht für Unternehmen mit dem Argument gerechtfertigt, daß für die wirtschaftliche Selbstverwaltung die Mitwirkung aller Unternehmen erforderlich sei (vgl. Frankfurter Allgemeine Zeitung 2002a, S. 13). Die 82 Industrie- und Handelskammern sind im Deutschen Industrie- und Handelskammertag (DIHK) zusammengeschlossen, der sich 
als Interessenvertreter aller gewerblichen Unternehmen auf nationaler Ebene versteht. Das Handwerk wird vom Deutschen Handwerkskammertag (DHKT) vertreten.

Die verschiedenen Unternehmensverbände übernehmen auf den einzelnen Ebenen national, regional und sektoral - des Berufsbildungssystems unterschiedliche Funktionen. Besonders eingebunden in die Regulation der beruflichen Bildung sind dabei die Arbeitgeberverbände und die Industrie- und Handelskammern bzw. die Handwerkskammern. Auf nationaler Ebene entsenden die Arbeitgeber auf Vorschlag des Kuratoriums der Deutschen Wirtschaft für Berufsbildung Vertreter in den Hauptausschuß des Bundesinstituts für Berufsbildung (BIBB), dem neben den Arbeitgebern Beauftragte der Gewerkschaften, der Länder und des Bundes angehören. Hier werden grundlegende Aspekte der Berufsbildungspolitik diskutiert und Ausbildungsordnungen sowie Rahmenlehrpläne für einzelne Berufe erarbeitet. Ausbildungsordnungen setzen Mindestanforderungen für die betriebliche Ausbildungspraxis, die von den Unternehmen aber überschritten werden können. Die Einführung neuer Ausbildungsordnungen, wie z.B. in den 1980er Jahren in der Metallindustrie, basiert auf einem Abstimmungsprozeß zwischen Arbeitgebern und Gewerkschaften. Für die Arbeitgeber übernehmen Vertreter des jeweiligen Fachverbandes aufgrund ihres Sachverstandes die wesentliche Entwicklungsarbeit (vgl. Müller-Jentsch 1997, S. 190). Der Staat übt in diesem Prozeß eine Art „Notariatsfunktion“ aus (vgl. Streeck et al. 1987, S. 15 ff.).

Auf regionaler Ebene sind Beauftragte der Arbeitgeberverbände und Kammern in den Landesausschüssen für Berufsbildung vertreten, wo sie an der Erarbeitung und Durchführung landesspezifischer Maßnahmen zur Förderung der beruflichen Bildung mitwirken (vgl. Weber 1987, S. 139 ff.). Darüber hinaus sitzen Arbeitgebervertreter in den Berufsbildungsausschüssen der Kammern. Diese sind im Gegensatz zur allgemeinen Organisation und Konzeption der Kammern als öffentlich-rechtlicher Selbstverwaltungskörperschaften der Wirtschaft paritätisch mit Arbeitgeber- und Arbeitnehmervertretern besetzt. Durch das Berufsbildungsgesetz von 1969 wurden die Kompetenzen der Kammern im Bereich der Ausbildungsordnungen, für die sie bis dahin allein zuständig waren, stark eingeschränkt. Ihre Aufgaben liegen seitdem in der Verwaltung und Überwachung der Berufsbildung sowie der Information der Betriebe und Unternehmen in allen berufsbildungspolitischen Aspekten. Eine traditionell wichtige Aufgabe ist ferner die Durchführung von Prüfungen. In den letzten Jahren haben die Kammern eine entscheidende Rolle in der Koordination und Finanzierung überbe- 
trieblicher Ausbildungsstätten - teilweise durch die Einführung einer allgemeinen Kammerumlage - übernommen (vgl. Streeck et al. 1987, S. 20 ff.). Durch den Einsatz von „Ausbildungsplatzentwicklern“, die nicht ausbildende Betriebe direkt ansprechen sowie praktische Beratung und Unterstützung für kleine und mittlere Betriebe anbieten, tragen die Kammern auch zur Ausweitung des betrieblichen Ausbildungsplatzangebotes bei (vgl. Bundesregierung 1999, S. 43 f.). Die Stellung der Kammern als Interessenvertretungsorgane der regionalen Wirtschaft schafft eine Vertrauensbasis, die es ihnen ermöglicht, den Austausch berufsbildungsbezogener Informationen zwischen den Unternehmen zu fördern. So können ,best practice'-Modelle leicht weitergegeben werden (vgl. Soskice 1994a, S. 42 f.; Culpepper 1996, S. 27 ff.). Ihre besondere Funktion in der Regulation der Berufsbildung ist ein wesentliches Argument in der Diskussion über die Legitimation des öffentlich-rechtlichen Status der Kammern. Deshalb haben die Kammern ein immanentes Interesse am Erhalt der betrieblich orientierten Berufsbildung.

Die Arbeitgeberverbände - und Gewerkschaften - haben über die kollektiven Tarifverhandlungen einen wichtigen Hebel in der Hand, die Zahl der Ausbildungsplätze zu beeinflussen. In den auf sektoraler Ebene stattfindenden Tarifverhandlungen entscheiden die Vertragspartner über die Höhe der Ausbildungsvergütung für Lehrlinge, die den größten Kostenanteil für die Unternehmen in der Lehrlingsausbildung darstellt und damit einen maßgeblichen Einfluß auf deren Ausbildungsbereitschaft hat (vgl. Franz/Soskice 1994, S. 10 ff.). Ferner gibt es Fälle, in denen Arbeitgeber und Gewerkschaften tarifvertragliche Regelungen vereinbart haben, durch die nicht ausbildende Betriebe gezwungen wurden, sich an der Finanzierung der beruflichen Bildung zu beteiligen. Ein Beispiel findet sich in der Bauwirtschaft (vgl. Streeck 1983, S. 7 ff.). In anderen Fällen haben die Verhandlungspartner eine Senkung der Ausbildungsvergütung im Tausch gegen mehr Ausbildungsplätze vereinbart. So konnten 1999 in der holzverarbeitenden Industrie Baden-Württembergs die Betriebe den Auszubildenden niedrigere Löhne zahlen, wenn sie im Gegenzug 10 \% mehr Lehrlinge als 1997 einstellten (vgl. IWD 2000). Über die Tarifverhandlungen können die Arbeitgeberverbände also Anreize schaffen, Unternehmen zur Bereitstellung von Ausbildungsplätzen zu bewegen.

Während der BDI in erster Linie auf nationaler Ebene und bei allgemeinen berufsbildungspolitischen Themen in Erscheinung tritt, leisten die Arbeitgeberverbände über die 
Tarifverhandlungen einen entscheidenden Beitrag zur Akzeptanz der Berufsbildung. Die wichtigste Rolle in der Regulation der Berufsbildung haben jedoch die Kammern, denen die Verwaltung und Kontrolle der beruflichen Bildung in den Betrieben obliegt und die den Informationsaustausch zwischen den Betrieben fördern. Die Betriebe greifen dabei bereitwillig auf die Unterstützung der Kammern zurück, weil diese als Selbstverwaltungskörperschaften der regionalen Wirtschaft besonderes Vertrauen genießen. Alle Arbeitgebervertreter sehen in der dualen Berufsbildung einen entscheidenden Beitrag zur Sicherung der Wettbewerbsfähigkeit der deutschen Wirtschaft und wollen deshalb an diesem System festhalten. Auch wenn sie die grundsätzliche Struktur der Berufsbildung mit der starken Stellung der Betriebe und dem Berufskonzept beibehalten wollen, so fordern sie doch eine stärkere Flexibilisierung der Ausbildungsgänge, um den Betrieben größere Spielräume in der Anpassung der Ausbildung an betriebsspezifische Anforderungen $\mathrm{zu}$ geben (vgl. BMBF/Berufsbildungsbericht 2001, Anlage 3, S. 8 ff.).

Die Unternehmensverbände und ihre Einbindung in die Organisation der Berufsbildung bieten den institutionellen Rahmen, innerhalb dessen die einzelnen Unternehmen und Betriebe ihre individuell-rationale Entscheidung treffen, selber auszubilden oder qualifizierte Fachkräfte auf dem externen Arbeitsmarkt anzuwerben. Anhand der ökonomischen Humankapitaltheorie sollen einige grundsätzliche Überlegungen zur Bereitstellung betrieblicher Ausbildung angestellt werden, um anschließend die Besonderheiten des dualen Berufsbildungssystems in Deutschland besser verstehen zu können.

Humankapitaltheoretische Ansätze ${ }^{12}$ gehen grundsätzlich davon aus, daß der einzelne Unternehmer gewinnorientiert denkt und sein finanzielles Engagement in der betrieblichen Ausbildung an dieser Maxime ausrichtet. Es stellt sich dann die Frage, wieso Betriebe in das Humankapital ihrer Belegschaft investieren? Unternehmen verwenden für die Produktion ihrer Güter spezifische Produktionstechnologien und -prozesse, die eine bestimmte Qualifikationsstruktur der Beschäftigten voraussetzen. Diese Qualifikationen können entweder durch die Ausbildung im Betrieb erworben werden oder über den Arbeitsmarkt beschafft werden. Grundsätzlich gilt für den Einsatz von Ar-

\footnotetext{
${ }^{12}$ Vgl. ausführlich Becker (1993).
} 
beitskräften, daß der Lohnsatz gleich dem Grenzprodukt der Arbeit ist. Bei der betrieblichen Ausbildung stehen den Kosten zum Zeitpunkt der Einstellung jedoch niedrigere Erträge aufgrund geringer Grenzproduktivität gegenüber, so daß für die Entscheidung eine Betrachtung der gesamten Ausbildungs- und Beschäftigungszeit des Auszubildenden notwendig ist.

Die Entscheidung des Unternehmens über Investitionen in Humankapital hängt mithin entscheidend davon ab, ob Grenzerträge, also eine Abweichung des Lohnsatzes vom Grenzprodukt, überhaupt realisierbar sind. Da von einem idealtypischen Arbeitsmarkt ausgegangen wird, ist für die Beantwortung der Frage der Realisierbarkeit von Grenzerträgen eine Unterscheidung zwischen betriebsspezifischem und allgemein verwertbarem Humankapital vorzunehmen (vgl. Becker 1993, S. 33 ff.). Betriebsspezifisches Humankapital verliert außerhalb des Unternehmens seinen Wert und unterliegt deshalb auf dem Arbeitsmarkt keinem Wettbewerb. "Betriebsspezifische Ausbildung führt mithin dazu, daß das Humankapital der Beschäftigten nicht mehr mobil ist." (Kempf 1985, S. 55) Das Unternehmen ist somit in der Lage, den Lohn des betriebsspezifisch qualifizierten Arbeitnehmers unter seine Grenzproduktivität zu drücken. Allgemein verwertbares Humankapital erhöht hingegen die Mobilität der qualifizierten Fachkraft, da diese Form des Humankapitals in allen Unternehmen gleichermaßen die Grenzproduktivität der Arbeit steigert. Eine entscheidende Frage ist also, ob die duale Berufsausbildung eher betriebsspezifische oder allgemein verwertbare Kenntnisse vermittelt. Einerseits läßt sich argumentieren, daß die duale Berufsausbildung dem Bereich des allgemein verwertbaren Humankapitals zuzuordnen ist, da sie berufsorientiert und nicht arbeitsplatzbezogen ausbildet (vgl. ebd., S. 56). In bezug auf die Minimalstandards, die in den staatlichen Ausbildungsverordnungen festgelegt sind, trifft diese Annahme sicherlich zu. Andererseits bilden gerade mittlere und größere Unternehmen ihre Auszubildenden über diese Minimalanforderungen hinaus in betriebsspezifischen Kenntnissen und Fähigkeiten aus, so daß hier die klare Unterscheidung zwischen allgemein verwertbarem und betriebsspezifischen Humankapital obsolet erscheint (vgl. Franz/Soskice 1994, S. 15). Unter der Annahme, daß der Schwerpunkt der dualen Berufsausbildung im Bereich des allgemein verwertbaren Humankapitals liegt, ist die Erhöhung der Grenzproduktivität der ausgebildeten Arbeitskraft nicht auf den ausbildenden Betrieb beschränkt. Somit besitzen die ausgebildeten Arbeitskräfte ein hohes Maß an Mobilität. Für einen aus ökonomischer Sicht 
idealtypisch funktionierenden Arbeitsmarkt bedeutet dies, daß der ausbildende Betrieb die ausgebildete Fachkraft entsprechend ihrer Grenzproduktivität entlohnen muß, da der Betrieb ansonsten eine Abwanderung der Arbeitskraft zu befürchten hat. Insofern ist es dem ausbildenden Betrieb nicht möglich, eine Ausbildungsinvestition durch Grenzerträge, also eine Abweichung des Lohnes vom Grenzprodukt, in einer an die Ausbildung anschließenden Beschäftigung auszugleichen.

Die Unternehmen würden jedoch Ausbildungsplätze bereitstellen, wenn die Ausbildungsvergütung der um die sonstigen Ausbildungskosten reduzierten Grenzproduktivität des Auszubildenden entspricht. Hierbei werden die Ausbildungskosten auf den Auszubildenden übertragen, da während einer möglichen anschließenden Beschäftigungsperiode das Unternehmen unter der Annahme eines idealtypisch funktionierenden Arbeitsmarktes keine Grenzerträge erzielen kann (vgl. Kempf 1985, S. 58). Zusammenfassend läßt sich also sagen, daß bei allgemein verwertbarem Humankapital und funktionierendem Arbeitsmarkt die Unternehmen Ausbildungsplätze nur dann bereitstellen, wenn die Ausbildungskosten durch die Grenzproduktivität des Auszubildenden gedeckt werden. Betrachtet man nun den Fall des deutschen dualen Berufsbildungssystems, so stellt man fest, daß Betriebe, insbesondere mittlere und größere Unternehmen, ausbilden, obgleich am Ende der Ausbildungsperiode eine Nettokostenbelastung $^{13}$ für das Unternehmen steht (vgl. Franz/Soskice 1994, S. 13 f.). Wie kann diese Ausbildungsbereitschaft trotz der Nettokostenbelastung erklärt werden? Die Annahme des obigen Modells, daß es sich um einen idealtypisch funktionierenden Arbeitsmarkt handelt, auf dem der Lohnsatz dem Grenzprodukt entspricht, trifft in der Realität nicht zu, da z.B. Informationen über ausgebildete Arbeitskräfte asymmetrisch zwischen Ausbildungsbetrieb und Nicht-Ausbildungsbetrieb verteilt sind (vgl. ebd., S. 20 f.). Außerdem findet sich in den Unternehmen häufig das Prinzip der Senoritätsentlohnung, d.h. Beschäftigte werden zunächst unter ihrer Grenzproduktivität und mit zunehmender Betriebszugehörigkeit über ihrer Grenzproduktivität entlohnt. Mithin gibt es einen Anreiz für die Beschäftigten, in ihrem Betrieb zu bleiben. Damit

13 IWD (1998b): "...unter dem Strich für einen Ausbildungsbetrieb Nettokosten von 21.500 DM pro Lehrling und Jahr."; die Kostenbelastung hängt jedoch stark von der Art des Ausbildungsbetriebs und der dort vorzufindenden Organisation der Berufsbildung ab: So gleichen sich im Handwerk aufgrund des produktionsnahen Einsatzes der Lehrlinge die Kosten und Erträge über den gesamten Ausbildungszeitraum aus (vgl. Bardeleben/Beicht 1996, S. 33 ff.). 
wird die Gleichheit zwischen Grenzprodukt und Lohnsatz außer Kraft gesetzt, und Ausbildungsunternehmen können Nettoerträge als Ausgleich für die Ausbildungskosten erzielen. Weiterhin sind Einstellungs- und Einarbeitungskosten bei Fremdbezug der Arbeitskräfte in die Überlegungen der Unternehmen einzubeziehen (vgl. Franz 1996, S. 91; Kempf 1985, S. 60 f.; Wagner 1998, S. 6 ff.). So stimmten bei einer Umfrage unter mittleren Unternehmen ca. 90\% der Aussage zu, daß über die eigene Ausbildung Lehrlinge betriebsgerechter qualifiziert werden können als externe Fachkräfte (vgl. Walden 2000, S. 407).

Neben Einstellungs- und Einarbeitungskosten als Opportunitätserträgen für die Ausbildungsentscheidung eines Unternehmens spielt zudem der Wettbewerb der Unternehmen um die besten Arbeitskräfte eine entscheidende Rolle in der Ausbildungsbereitschaft. Grund dafür ist, daß nicht-ausbildende Betriebe auf die ausgebildeten Fachkräfte anderer Unternehmen zurückgreifen müssen, die bereits bei den Schulabgängern eine Auswahl vornehmen und die besten von ihnen durch eine Ausbildung an ihr Unternehmen binden konnten. Im Anschluß an die Ausbildung wird nur den weniger guten Ausbildungsabsolventen kein Übernahmevertrag angeboten. Somit finden sich tendenziell die weniger gut qualifizierten Fachkräfte auf dem Arbeitsmarkt wieder, wo sie auf die Nachfrage der nicht-ausbildenden Betriebe treffen. Soskice spricht in diesem Zusammenhang vom sogenannten, lemon'-Problem (vgl. Soskice 1994a, S. 47). Darüber hinaus können Unternehmen höhere Löhne als Anreiz zum Wechsel aufgrund des restriktiven Lohnsetzungsverfahrens im Rahmen der kollektiven Tarifvereinbarungen nur begrenzt einsetzen (vgl. Franz/Soskice 1994, S. 20 f.).

Empirische Untersuchungen zur Ausbildungsbereitschaft von Unternehmen zeigen, daß die wachsende Bedeutung eines kostenorientierten Managements auch Auswirkungen auf die betriebliche Berufsausbildung hat. Während die Kosten der Ausbildung im Unternehmen relativ leicht zu ermitteln sind, können deren Erträge nur schwer quantifiziert werden (vgl. Weiß 1994, S. 281 f.). Mögliche Imagegewinne in der Region durch Ausbildungsaktivitäten fließen in die Entscheidung vieler Betriebe mit ein, bieten aber keine solide Legitimierungsbasis gegenüber "harten" Kostenüberlegungen (vgl. Schneeberger 1997, S. 363). So gaben bei einer Befragung von 823 Unternehmen 67\% als Grund für eine fehlende Ausbildungsbereitschaft zu hohe Ausbildungskosten an (vgl. IWD 1998c). Als wichtigster Kostentreiber werden vor allem die Ausbildungsvergütungen genannt. In den alten Bundesländern stiegen diese von 1991 
bis 1996 um 25\% und in den neuen Bundesländern sogar um 85\% (vgl. IWD 1998a). Trotzdem liegt die Höhe der Ausbildungsvergütung immer noch bei ca. einem Drittel des durchschnittlichen Facharbeiterlohns. Neben den Kosten gibt es aber auch Eignungs- und konjunkturelle Gründe, die in der Praxis das Ausbildungsengagement der Betriebe bestimmen (vgl. IWD 1997).

Eine Studie zur Lehrlingsausbildung in der Investitionsgüterindustrie zeigt, daß mit steigender Betriebsgröße der Anteil der Ausbildungsbetriebe zunimmt. Gleichzeitig nimmt jedoch die Ausbildungsintensität - also der Anteil der Auszubildenden an den Beschäftigten $-a b$. Träger der beruflichen Ausbildung sind insbesondere mittelgroße Unternehmen. Dabei bilden Betriebe mit einem überdurchschnittlichen Facharbeiteranteil intensiver aus als solche mit einem unterdurchschnittlichen Facharbeiteranteil, was darauf hindeutet, daß diese Betriebe ihren Fachkräftenachwuchs selber ausbilden (vgl. Kinkel et al. 2001, S. 2 ff.). Von allen Betrieben Deutschlands, die 1999 die gesetzlichen Voraussetzungen zur Durchführung einer betrieblichen Ausbildung erfüllten, boten ca. 54\% Ausbildungsplätze an (vgl. BMBF/Berufsbildungsbericht 2001, Kapitel 2.3, S. 4).

Um die Berufsausbildung von der Konjunktur- und Kostenproblematik unabhängig zu machen, fordern die Gewerkschaften, das auf einzelbetrieblicher Finanzierung basierende System in ein umlagefinanziertes Modell zu überführen. Damit, so wird argumentiert, werde der 'free-riding'-Anreiz für Betriebe minimiert. Gegen einen solchen Lastenausgleich zwischen ausbildenden und nicht-ausbildenden Betrieben wehren sich die Unternehmensverbände mit Nachdruck. Ihrer Ansicht nach werde eine Konjunkturunabhängigkeit der Ausbildung dadurch nicht erreicht, sondern das genaue Gegenteil, eine Mehrbelastung der Leistungs- und Wettbewerbsfähigkeit, herbeigeführt. Außerdem führe eine Umlagefinanzierung zur Doppelbelastung der ausbildenden Betriebe (vgl. Kuratorium der Deutschen Wirtschaft für Berufsbildung 1995, S. 10 ff.). Ein weiterer Grund, weshalb sich die Unternehmensverbände gegen eine Umlagefinanzierung stellen, wäre der Verlust an unternehmerischer Steuerungsfreiheit im Bereich der Berufsausbildung, deren Eckpfeiler die einzelbetriebliche Finanzierung ist.

Die allgegenwärtige Gefahr staatlicher Regulation verleiht den Verbänden Macht, das Verhalten ihrer Mitglieder zu sanktionieren. Die Selbstverwaltungskompetenz der Verbände beruht auf bürokratisch-regulativer Zurückhaltung des Staates, der jedoch bei 
Bedarf seine Zurückhaltung aufgeben kann. Diese staatliche Zurückhaltung stellt für die einzelnen Unternehmen einen positiven Anreiz dar, sich der Verbandspolitik zu unterwerfen, da sie davon ausgehen, daß die verbandliche Regulation praxisnäher und stärker von ihnen zu beeinflussen ist. In Zeiten fehlender Ausbildungsplätze kommt immer wieder die Forderung nach Einführung einer allgemeinen Umlagefinanzierung auf. Solche Forderungen stärken letztlich die Position der Unternehmensverbände gegenüber ihren Mitgliedern. Um einen drohenden staatlichen Eingriff abzuwenden, können sie die Betriebe zu einer Ausweitung ihrer Ausbildungskapazitäten bewegen (vgl. Schmitter/Streeck 1999, S. 92; Streeck/Schmitter 1985, S. 25 f.).

Die sich im 19. Jahrhundert entwickelnden Unternehmensverbände standen von Beginn an in einem engen kooperativen Verhältnis zum Staat. Insbesondere die Industrie- und Handelskammern sowie die Handwerkskammern mit ihrem öffentlich-rechtlichen Status spielen in der Ausbalancierung von wirtschaftlichen und staatlichen Interessen eine entscheidende Rolle. Seit ihrer Gründung hat der Staat ihnen weitreichende Regulationskompetenzen im Bereich der Berufsbildung übertragen. Aufgrund ihrer Unternehmensnähe besitzen sie das Vertrauen der Betriebe und können daher eine praxisnahe Informations- und Kontrollfunktion ausüben. Die Einbeziehung der Unternehmensverbände in die Organisation, Durchführung und Kontrolle der beruflichen Bildung stellt eine wesentliche institutionelle Rahmenbedingung zur Entlastung der einzelnen ausbildenden Betriebe dar. Durch die konsensuale Festlegung von allgemeinverbindlichen Standards und deren Überwachung senken die Verbände die Transaktionskosten - Kosten, die durch die an den Schnittstellen des Produktionsprozesses notwendige Kommunikation zwischen den beteiligten Personen entstehen (vgl. Williamson 1985) - für die einzelnen Betriebe, die sich darauf verlassen können, daß mit dem Erlernen eines spezifischen Berufs klar definierte Qualifikationen verbunden sind. Insofern haben die Betriebe auch ein individuelles Interesse am Erhalt der kollektiv organisierten betrieblichen Berufsbildung. 


\subsection{Gewerkschaften}

Neben den Unternehmen und ihren Verbänden ist die institutionelle Struktur der Gewerkschaften und industriellen Beziehungen von entscheidender Bedeutung für die Organisation der Berufsausbildung. Diese prägt wesentlich die Interessen der Gewerkschaften hinsichtlich der Ausgestaltung beruflicher Bildungssysteme. Anhand eines kurzen historischen Abrisses der gewerkschaftlichen Entwicklung in Deutschland soll gezeigt werden, wie sich das Prinzip der Industriegewerkschaft gegenüber dem traditionellen Prinzip der Berufsgewerkschaft durchgesetzt hat und welche Auswirkungen dies auf die Entwicklung der Berufsausbildung hatte. Darüber hinaus wird deutlich, wie das System der industriellen Beziehungen mit seinen zwei Ebenen - überbetriebliche Tarifpolitik und betriebliche Mitbestimmung - die Durchführung der Berufsausbildung beeinflußt.

Die gewerkschaftliche Bewegung in Deutschland hat zwei Ursprünge: Einerseits die Gesellenverbände, die sich bereits im 18. Jahrhundert bildeten, andererseits die Arbeiterbildungsvereine des frühen 19. Jahrhunderts. Um ihre Interessen gegen die Macht der Handwerksmeister und deren Organisationen, insbesondere der Zünfte, durchsetzen zu können, hatten sich bereits früh Gesellen in berufsständisch organisierten Verbänden zusammengeschlossen. Ziel dieser Organisationen war die Einflußnahme auf die Lohnund Arbeitsbedingungen. Als berufsständisch organisierter Verband basierte die Organisationsmacht auf der Solidarität zwischen den Mitgliedern eines Handwerks. Nach Durkheim handelte es sich dabei um eine Form der „mechanischen Solidarität“. Die Solidarität endete dort, wo die Grenze des Berufs erreicht wurde. In der Tradition der Meisterlehre stehend verfolgten die Gesellenverbände eine Ausbildungspolitik, die die Zahl der Lehrlinge im jeweiligen Beruf zu begrenzen versuchte. Die Meister sollten gezwungen werden, nur eine in einem festgelegten Verhältnis zur Zahl der beschäftigten Gesellen bestimmte Anzahl von Lehrlingen einstellen zu dürfen. Dadurch wollten sie den Arbeitsmarkt kontrollieren und die Konkurrenz um die begrenzten Meisterstellen beschränken. Diese Traditionen setzten sich in der berufsständisch geprägten Entwicklungsphase der Gewerkschaften fort und führten dort zu identischen Orientierungen in der Ausbildungspolitik (vgl. Heimann 1980, S. 55 ff.).

Die Arbeiterbildungsvereine gründeten auf der Überlegung, daß nur über eine bessere Ausbildung der Arbeiter der materielle Unterschied zwischen Unternehmern und 
Arbeitern überwunden werden könne. Insbesondere allgemeine Bildungsinhalte, wie Geschichte, Religion und Schönschreiben, wurden vermittelt. Aber auch berufliche Lehrinhalte hatten ihren Platz in den Bildungsvereinen. Seit den 1820er Jahren entstanden z.B. im Buchdrucker Gewerbe Bildungs- und Geselligkeitsvereine. Durch den Einzug von Schnellpressen in die Druckereien waren die Gesellen unter verstärkten Konkurrenzdruck geraten. Sie liefen Gefahr, durch die Maschinen ersetzt zu werden und ihr hohes handwerkliches Qualifikationsniveau einzubüßen. Die Auflösung gemeinsamer Interessen der Meister und Gesellen schritt weiter voran. Durch die Gründung von eigenen Vereinigungen versuchten die Gesellen ihre Position gegenüber den Meistern zu stärken (vgl. Tenfelde 1987, S. 49 ff.). Aus den Arbeiterbildungsvereinen entwickelten sich häufig Gewerkevereine, die erste Ansätze gewerkschaftlicher Zielsetzungen beinhalteten (vgl. Heimann 1980, S. 60). Dazu gehörten auch Forderungen, gegen unqualifizierte Konkurrenz, z.B. in Form von Frauen- und Sträflingsarbeit, und die weit verbreitete ,Lehrlingszüchterei' vorzugehen (vgl. Tenfelde 1987, S. 54-56).

Bis in die 1860er Jahre ging der Entwicklung gewerkschaftlicher Organisationen die Bildung einer deutschen Arbeiterpartei voran. Dieser Umstand prägte das Verhältnis zwischen Partei und Gewerkschaften bis zum Ende des 19. Jahrhunderts dahingehend, daß das parteipolitische Moment in der Arbeiterbewegung klar im Vordergrund stand. Mit dem konjunkturellen Aufschwung Ende der 1860er Jahre und dem Erlaß der Gewerbeordnung des Norddeutschen Bundes 1869, die das Recht auf Koalitionsfreiheit festschrieb, kam es zu einem regelrechten Boom von Gewerkschaftsgründungen. Lokalen Entwicklungen standen zentrale Bestrebungen der politischen Arbeiterbewegung zur überregionalen Organisation der Arbeiterschaft gegenüber. Neben den sozialdemokratisch orientierten Gewerkschaften gab es zwei weitere Strömungen in der Gewerkschaftsbewegung: Unter Führung der Fortschrittspolitiker Max Hirsch und Franz Duncker fanden sich der preußischen linksliberalen Fortschrittspartei nahestehende Arbeiter zusammen und gründeten die Hirsch-Dunckerschen Gewerkvereine. Gleichzeitig entstanden aus der katholischen Gesellenbewegung heraus christlichsoziale Arbeitervereine, die jedoch regional begrenzt blieben und nach der deutschen Reichsgründung unter dem Bismarckschen Kulturkampf schwer zu leiden hatten (vgl. ebd., S. 114 ff.).

Mit dem Erlaß der sogenannten Sozialistengesetze im Herbst 1878 kam die Gewerkschaftsbewegung zu einem vorläufigen Stillstand. Aber bereits in den 1880er Jahren 
entstanden überall wieder lokale Fachvereine, die über die Gründung von Unterstützungskassen eine legale Form der organisatorischen Zentralisation fanden. Lokale, berufsspezifische Fachvereine und Unterstützungskassen bildeten die organisatorische Basis, auf der die Gewerkschaftsbewegung nach dem Ende der Sozialistengesetze im Jahre 1890 wiederaufbaute. Ganz im Gegensatz zur bisherigen berufsorientierten Entwicklung schlossen sich erstmals auch Hilfsarbeiter zu Gewerkschaften zusammen, z.B. im Verband der Fabrikarbeiter oder im Verband der Baugewerkschaftlichen Hilfsarbeiter. Parallel dazu bildeten sich durch die Fusion verwandter und branchengleicher Berufsgewerkschaften erste industriegewerkschaftliche Verbände, wie z.B. der Deutsche Metallarbeiterverband. Diese unterschiedlichen Entwicklungen führten zu einer allgemeinen Diskussion über die ,richtige“ gewerkschaftliche Organisationsform: Berufs- oder Industrieverband, Zentral- oder Lokalverband (vgl. Müller-Jentsch 1997, S. 110 f.; Tenfelde 1987, S. 158 ff.; Schönhoven 1987, S. 179 f.).

Der Gründung des Deutschen Metallarbeiterverbandes (DMV) im Jahre 1891 lag der Anspruch zugrunde, eine Organisation für alle Arbeiter eines Industriezweiges zu schaffen. Damit stand der DMV in einem klaren Konkurrenzverhältnis zu den starken Fachvereinen und zentralisierten Berufsverbänden der überdurchschnittlich qualifizierten „Handwerksarbeiter“ in der Metallindustrie. Diese befürchteten durch die Aufnahme gering qualifizierter Arbeitergruppen eine Schwächung ihrer Verhandlungsposition gegenüber den Arbeitgebern. Durch den hohen Organisationsgrad, den die zentralisierten Berufsverbände häufig erreichten, konnten sie den Arbeitsmarkt weitgehend kontrollieren. Der Verband der Kupferschmiede - mit einem Organisationsgrad von ca. $60 \%$ - hatte eine strikte Lehrlingsbeschränkung durchgesetzt und übte so Kontrolle über den gesamten nationalen Arbeitsmarkt aus. Für diese straff organisierten Berufsverbände beinhaltete eine Mitgliedschaft in einem Industrieverband, wie dem DMV, auf den ersten Blick keine Vorteile. Während der Anstoß für die Gründung des DMV von den geringer qualifizierten Berufsgruppen ausging, die ihre Verhandlungsposition durch den Zusammenschluß gestärkt sahen, schlossen sich vereinzelte zentrale Berufsverbände, wie z.B. die Schlosser, erst sehr viel später an. Im Zuge der fortschreitenden technologischen Rationalisierung verloren ihre Qualifikationen teilweise an Bedeutung, wodurch sich auch ihre darauf basierende gewerkschaftliche Kampfkraft schwächte. Statt der Qualifikation rückte das Prinzip der ,Masse' zunehmend in den Mittelpunkt der gewerkschaftlichen Organisierung. Der 
DMV konnte als Gewerkschaftsverband neuen Typus an die Stelle der sich auflösenden Berufsverbände treten. Dabei ist jedoch festzuhalten, daß die Mitglieder des DMV keineswegs in der Mehrheit unqualifizierte Hilfsarbeiter waren. Deren Anteil betrug noch 1912 lediglich 18,7 \%. Der überwiegende Teil der Mitglieder bestand aus gelernten Facharbeitern in Klein- und Mittelbetrieben. Insofern war der DMV zwar unabhängiger vom Qualifikationsniveau seiner Mitglieder als die ,alten“ Berufsverbände. Er konnte aber das Thema der Qualifikation bzw. Qualifizierung nicht außer Acht lassen, weil es für die Mehrheit der Mitglieder weiterhin relevant blieb (vgl. Boch 1985, S. 149 ff. und 175 ff.).

Die Diskussion über die gewerkschaftliche Organisationsform wurde durch mehrere Entwicklungen angestoßen. Den Gewerkschaften standen zunehmend geschlossen auftretende Unternehmer gegenüber, die sich zu branchenspezifischen Arbeitgeberverbänden zusammenschlossen. Bei Streiks stellten die Gewerkschaften fest, daß sie aufgrund ihrer zersplitterten Organisation immer seltener ihre Forderungen durchsetzen konnten. Darüber hinaus führte die Ausweitung großbetrieblicher Arbeitsorganisation zur Konzentration einer Vielzahl unterschiedlicher Berufe innerhalb eines Betriebes. Die jeweiligen Berufsverbände standen dabei regelmäßig in einem Konkurrenzverhältnis zueinander. Während sie auf die strikte Einhaltung der beruflichen Grenzen achteten, entstanden in den Schnittbereichen bedingt durch technologische Neuerungen neue Berufe, deren gewerkschaftliche Vertretung unklar war. Die Auflösung traditioneller Berufe im Zuge industrieller Arbeitsteilung hatte eine Schwächung der Machtbasis der Berufsverbände zur Folge. Zudem konnten qualifizierte Facharbeiter in wachsendem Maße durch angelernte Hilfsarbeiter substituiert werden (vgl. Sohn 1963, S. 38 ff.; Heimann 1980, S. 61 f.; Dittrich 1980, S. 301).

Auf dem Berliner Kongreß gewerkschaftlicher Funktionäre im Jahre 1890 plädierten Vertreter der Metallarbeiter für eine allgemeine Gründung berufsübergreifender Industriegewerkschaften. Obgleich sie sich mit dieser weitgehenden Forderung nicht durchsetzen konnten, verabschiedeten die Delegierten eine Resolution, in der die Fusion der bestehenden berufsspezifischen Einzelverbände $\mathrm{zu}$ industriegewerkschaftlich organisierten Verbänden nicht mehr ausgeschlossen wurde. Der erste allgemeine Gewerkschaftskongreß in Halberstadt 1892 setzte diese ambivalente Politik fort. In einem mehrheitlich verabschiedeten Verfassungskonzept wurden das Prinzip der berufsverbandlichen Zentralisation bekräftigt und gleichzeitig die einzelnen Berufsver- 
bände dazu aufgefordert, in den verschiedenen Industriebranchen Kartellverträge abzuschließen. Die Einzelverbände nahmen jedoch diese Aufforderung nicht an. Abgesehen von wenigen Ausnahmen - in der Metallindustrie und der Holzbranche - blieb das Berufsprinzip auch nach dem Halberstadter Gewerkschaftskongreß das zentrale Organisationskriterium (vgl. Schönhoven 1987, S. 182 ff.).

Erst die weitere wirtschaftliche Entwicklung in Richtung großbetrieblicher Produktionsformen setzte die berufsverbandlich organisierten Gewerkschaften unter erhöhten Reformdruck. Auf dem Leipziger Gewerkschaftskongreß im Jahre 1911 beantragte der Fabrikarbeiterverband, in dem viele ungelernte und gering qualifizierte Arbeiter organisiert waren, eine stärkere organisatorische Konzentration im Sinne des Industrieprinzips, um die durch sich häufende Berufswechsel bedingten Hindernisse der gewerkschaftlichen Organisierung abzubauen. Nach dem ersten Weltkrieg nahm die Zahl der Mitglieder gerade in den industrieverbandlich organisierten Gewerkschaften überproportional zu. Diese Entwicklung fand ihren Niederschlag im Satzungsentwurf für den auf dem Nürnberger Gewerkschaftskongreß 1919 gegründeten Allgemeinen Deutschen Gewerkschaftsbund (ADGB), der als gewerkschaftliche Dachorganisation die Funktion der 1890 errichteten Generalkommission übernehmen sollte: Darin wurde der Zusammenschluß der Berufsverbände zu leistungsfähigen Industriegewerkschaften gefordert. In der Folgezeit blieb es zwar noch beim Nebeneinander von Berufsverbänden und Industriegewerkschaften. Die Mitgliedschaft eines Berufsverbandes im ADGB setzte aber gegen Ende der 1920er Jahre den Abschluß eines Kartellvertrages mit den anderen innerhalb desselben Industriezweiges agierenden Verbänden voraus. Damit hatte sich das Prinzip der Industriegewerkschaft etabliert, ohne andere Organisationsformen grundsätzlich auszuschließen (vgl. Sohn 1963, S. 56 ff.; Schneider 1987, S. 309 ff.). Nach dem zweiten Weltkrieg stand die Neugründung der Gewerkschaften ganz im Zeichen des industriegewerkschaftlichen Verbandsprinzips und der parteipolitisch und konfessionell unabhängigen Einheitsgewerkschaft. Dem neuerrichteten Dachverband - Deutschen Gewerkschaftsbund (DGB) - gehörten in den ersten Jahrzehnten 16 Industriegewerkschaften an (vgl. Müller-Jentsch 1997, S. 114 f.). Heute bestehen noch acht Mitgliedsgewerkschaften.

Während die organisatorische Stärke der traditionellen Berufsverbände auf der Qualifikation ihrer Mitglieder und der berufsspezifischen Solidarität basierte, suchten die allen Berufen und Arbeitergruppen eines Industriezweiges offenstehenden Industrie- 
gewerkschaften ihre Verhandlungsmacht im Moment der ,Masse' und der Idee der Klassensolidarität. Beide Elemente sind im Vergleich zur „natürlichen“ Solidarität der Berufsgemeinschaft wesentlich fragiler. Berufsverbände hatten ein originäres Interesse an der beruflichen Ausbildung, da sie über die Kontrolle des Zugangs zur Berufsausbildung ihren berufsspezifischen Arbeitmarkt beeinflussen konnten. So finden sich bei allen Berufsverbänden Aussagen über die ,angemessene“ Relation von Lehrlingen zu Gesellen: „Lehrlingsquoten, d.h. Vereinbarungen über die Zahl der zu beschäftigenden Lehrlinge, gab es in dem vom Verband der Deutschen Buchdrucker abgeschlossenen [Tarif]Vertrag ... “ (Sohn 1963, S. 22) Über die Verknappung des Arbeitskräfteangebotes konnten sie die Lebensbedingungen ihrer Mitglieder absichern bzw. steigern. Insofern hatten sie kein Interesse daran, einer möglichst großen Zahl von Jugendlichen eine Berufsausbildung zu ermöglichen, um deren Chancen auf dem Arbeitsmarkt zu erhöhen. Die auf dem Berufsprinzip beruhende Exklusivität der Berufsverbände barg jedoch die Gefahr in sich, daß sie beim technologisch bedingten berufsstrukturellen Wandel aufgrund ihrer beschränkten Adaptionsfähigkeit an Bedeutung verloren (vgl. Müller-Jentsch 1997, S. 109 ff.; Sohn 1963, S. 21 ff.).

Die Idee der industriegewerkschaftlichen Organisierung bedeutete dagegen die Überwindung des ,zünftlerischen“ Standesdenken, das zu häufigen Konkurrenzsituationen zwischen verschiedenen Berufsverbänden innerhalb eines Betriebes führte. In der Geschlossenheit aller Arbeiter - unabhängig von ihrem Beruf - sollte die Stärke der Industrieverbände liegen und nicht in der Qualifikation einer exklusiven Gruppe von Facharbeitern. Daher war ihr Interesse an der jeweils berufsspezifischen Qualifikation der Mitglieder weniger stark ausgeprägt. Ihnen ging es um die Verbesserung der Lebens- und Arbeitsbedingungen aller Arbeitergruppen. Obgleich sie explizit auch ungelernte Arbeiter aufnahmen, bilden die Facharbeiter bis heute das Rückgrat der industriegewerkschaftlichen Verbände. Der prozentuale Anteil der Hilfsarbeiter im Deutschen Metallarbeiter Verband (DMV), dem idealtypischen Industrieverband, stieg zwischen 1901 und 1912 lediglich von 10 auf 18,7 \% (vgl. Boch 1985, S. 154). Das Interesse der Industriegewerkschaften an der Lehrlingsausbildung entsprang letztlich dem Umstand, daß die Zahl der ungelernten jugendlichen Arbeiter anstieg und gleichzeitig die Lehrlingsausbildung in vielen Betrieben zur Rekrutierung billiger Arbeitskräfte genutzt wurde. Dadurch waren die Lebensbedingungen der erwachsenen Arbeitern und somit die Interessen der Gewerkschaftsmitglieder gefährdet. Über die 
Forderungen nach einer Reform der Lehrlingsausbildung, z.B. durch die Einrichtung von Lehrwerkstätten, und ihrer Ausweitung auf alle Jugendlichen versuchten die industriegewerkschaftlichen Verbände die Interessen ihrer Mitglieder zu schützen: „Eine gründliche Berufsausbildung liegt im Interesse des einzelnen und der Allgemeinheit.“ (Jugendsekretariat des Allgemeinen Deutschen Gewerkschaftsbundes 1923, S. 6) Die Einflußnahme auf die Gestaltung und Organisation der Lehrlingsausbildung sollte ihnen langfristig auch die Möglichkeit eröffnen, neue Mitglieder zu werben und diese $\mathrm{ab}$ einem frühen Zeitpunkt an sich zu binden.

Im Gegensatz zu den Berufsverbänden hatten die Industriegewerkschaften also kein Interesse an einer Begrenzung der Lehrlingsplätze innerhalb eines spezifischen Berufsfeldes, um das Arbeitsangebot auf diese Weise zu verknappen. Vielmehr mußten sie darauf achten, die Herausbildung berufsspezifischer Egoismen in ihren Organisationen zu verhindern. Dies spiegelt sich auch in der immer wieder vorgetragenen Forderung nach einer umfassenden beruflichen Grundausbildung innerhalb der Lehrlingsausbildung wieder (vgl. Balz 1993, S. 148 ff.; Reuter 1975, S. 12): „Berufsausbildung kann heute nicht mehr ausschließlich eine klar abgegrenzte berufliche Spezialisierung bedeuten: Sie muß vielmehr - über die erforderliche Anpassung an neue technische oder arbeitsorganisatorische Entwicklungen hinaus auch den Wechsel innerhalb von Tätigkeitsfeldern ermöglichen.“ (Görner, Mitglied des Geschäftsführenden DGB-Bundesvorstandes, 1999b) Indem die Industriegewerkschaften über eine generelle Ausweitung und Kontrolle der Berufsausbildung die Jugendlichen dem Arbeitsmarkt zu entziehen versuchten, schützten sie die Interessen ihrer Mitglieder. Aufgrund der steigenden Arbeitsproduktivität ließen sich zudem langfristig tarifpolitische Forderungen besser durchsetzen und so die Lebensbedingungen der Mitglieder verbessern (vgl. Crusius 1982, S. 92).

Wie sind die Gewerkschaften in die institutionelle Struktur der dualen Berufsausbildung eingebunden? Hier können drei Dimensionen unterschieden werden: staatlich-politisch, tarifpolitisch und betrieblich. Auf nationaler Ebene sind die Gewerkschaften - wie die Arbeitgeber - im Hauptausschuß des Bundesinstituts für Berufsbildung (BIBB) vertreten. Der Hauptausschuß ist verantwortlich für grundlegende Aspekte der Berufsbildungspolitik, wie z.B. die Ausarbeitung von neuen Ausbildungsordnungen und Rahmenlehrplänen für einzelne Berufe. Im jährlich veröffentlichten Berufsbildungsbericht können die Arbeitnehmervertreter ihre Einschätzung der berufsbildungs- 
politischen Situation darlegen. Regional sind die Gewerkschaften in den Landesausschüssen für Berufsbildung sowie in den Berufsbildungsausschüssen der Industrie- und Handelskammern bzw. der Handwerkskammern vertreten. Beide Gremien sind paritätisch mit Arbeitgeber- und Arbeitnehmervertretern besetzt. Im Gegensatz zur originären Funktion der Kammern als Interessenvertretungsorganen der Arbeitgeber haben die Arbeitnehmer im Bereich der beruflichen Bildung ein Mitbestimmungsrecht, das ihnen Gestaltungsspielräume bei der operationalen Umsetzung der Ausbildungsordnungen eröffnet (vgl. Streeck et al. 1987, S. 15 ff.).

Als tarifpolitischer Verhandlungspartner haben die Gewerkschaften auf der Ebene des jeweiligen Industriezweiges insofern eine wichtige berufsbildungspolitische Funktion, als sie im Rahmen der allgemeinen Tarifverhandlungen die Höhe der Ausbildungsvergütungen der Lehrlinge aushandeln. Die Höhe der Ausbildungsvergütung ist für die Unternehmen eines der wichtigsten Kriterien bei der Entscheidung, ob sie selber ausbilden oder Fachkräfte von außen ,einkaufen“: Je niedriger die Vergütung ist, desto mehr Unternehmen beteiligen sich an der Berufsausbildung. Andererseits besteht jedoch die Gefahr, daß die Lehrlinge als billige Arbeitskräfte ausgenutzt werden. Um dies zu verhindern, bedarf es einer innerbetrieblichen Kontrollinstanz, der die Gewerkschaften vertrauen. Die Institution des Betriebsrates erfüllt diese Bedingung. Die betriebliche Kontrolle durch den Betriebsrat erlaubt es den Gewerkschaften, in den überbetrieblichen Tarifverhandlungen die Höhe der Ausbildungsvergütung am Investitionsmoment der Berufsausbildung zu orientieren. Dadurch können sie das Angebot an Ausbildungsplätzen positiv beeinflussen.

Das Angebot an Ausbildungsplätzen und die qualitative Umsetzung der Ausbildungsordnungen bestimmen sich auf der einzelbetrieblichen Ebene. Hier können die Arbeitnehmer über ihr Vertretungsorgan, den Betriebsrat, die im Betriebsverfassungsgesetz geregelten Mitbestimmungsmöglichkeiten geltend machen. Über die rechtlichen Einflußmöglichkeiten hinaus haben die Betriebsräte auch die Funktion einer „moralischen Instanz", die die Ausbildungsaktivität der Unternehmen positiv beeinflussen kann. Dies zeigt sich z.B. in den betrieblichen „Bündnissen für Arbeit“, die Absprachen über Ausbildungsplatzangebote beinhalten (vgl. Heidemann/Paul-Kohlhoff 1998, S. $15)$. 
In der ersten Hälfte des 19. Jahrhunderts entwickelten sich Frühformen der betrieblichen Mitwirkung von Arbeitnehmern in der Gestalt von Arbeiterausschüssen, die eine Vermittlungsfunktion in Arbeitsstreitigkeiten hatten. Durch die Novellierung der Reichsgewerbeordnung im Jahre 1891 wurde eine gesetzliche Grundlage für die fakultative Einrichtung von Arbeiterausschüssen geschaffen. Unter den Bedingungen der Kriegswirtschaft im ersten Weltkrieg wurden die Mitspracherechte der Arbeitnehmer weiter verbessert. Im November 1918 schlossen die Arbeitgeberverbände und Gewerkschaften eine Vereinbarung zur obligatorischen Einrichtung von Arbeiter- und Angestelltenausschüssen in Betrieben mit mehr als 50 Beschäftigten ab. Bereits einen Monat später setzte die Verordnung über Tarifverträge, Arbeiter- und Angestelltenausschüsse und Schlichtung von Arbeitsstreitigkeiten eine verpflichtende Bildung von Arbeiterausschüssen in Betrieben mit mehr als 20 Beschäftigten voraus, die Mitwirkungsrechte bei der Regelung von Löhnen und Arbeitsbedingungen hatten. Über den Artikel 165 der Weimarer Reichsverfassung erlangte das Recht zur Einrichtung von Betriebsarbeiterräten, denen die Wahrnehmung der Arbeitnehmerinteressen im Betrieb oblag, letztlich Verfassungsrang. Auf dieser Grundlage wurde 1920 das Betriebsrätegesetz (BRG) verabschiedet. Der $§ 78$ BRG legte erstmalig ein Mitwirkungsrecht der Arbeitnehmervertretung bei der „Erledigung von Beschwerden über die Ausbildung und Behandlung der Lehrlinge im Betriebe“ ( $§ 78$ BRG) fest. Darüber hinaus übertrug $§ 78$ BRG dem Betriebsrat das Recht zur Überwachung der die Lehrlingsausbildung betreffenden Teile des Tarifvertrages (vgl. Gilberg 1999, S. 10 ff.).

Das Betriebsverfassungsgesetz von 1952, das in der Tradition des BRG steht, hat dem Betriebsrat im $\S 56$ BetrVG ein Mitbestimmungsrecht bei der Durchführung der Berufsausbildung eingeräumt, soweit nicht gesetzliche oder tarifliche Regelungen entgegenstanden. Damit unterlag die Berufsausbildung der weitreichendsten Beteiligungsform, die der Gesetzgeber dem Betriebsrat im Betriebsverfassungsgesetz zubilligte. Mit dem Betriebsverfassungsgesetz von 1972 wurde die enge Beziehung zwischen Berufsbildung und Personalplanung insofern deutlicher hervorgehoben, als Fragen der Berufsbildung in einem eigenen Unterabschnitt im Rahmen der personellen Angelegenheiten geregelt wurden. Die Mitwirkungsrechte im Bereich der Berufsbildung gelten trotz zahlreicher Novellierungen des Betriebsverfassungsgesetzes noch heute. Danach hat der Betriebsrat einerseits eine im $\S 97$ BetrVG festgelegte beratende Funktion - z.B. bei der „Errichtung und Ausstattung betrieblicher Einrichtungen zur Berufsbildung“ und 
der „Einführung betrieblicher Berufsausbildungsmaßnahmen“ - andererseits aber auch Mitbestimmungsrechte im Rahmen der „Durchführung betrieblicher Bildungsmöglichkeiten“ ( $§ 98$ BetrVG). Nach $§ 96$ BetrVG sind sowohl Arbeitgeber als auch Betriebsrat zur „Förderung der Berufsbildung“ verpflichtet (vgl. Heidemann/PaulKohlhoff 1998, S. 14 f.; Gilberg 1999, S. 22 f.; Schaub 2000, S. 2285 ff.).

Verschiedene Befragungen zeigen, daß Betriebsräte ihre formellen Mitbestimmungsmöglichkeiten nur in geringem Maße wahrnehmen. Dabei stehen Fragen der beruflichen Bildung hinter denen der Arbeitsplatzsicherung und der Arbeitszeitregelung. Innerhalb des Handlungsbereichs Berufsbildung konzentrieren sich die Aktivitäten der Betriebsräte insbesondere auf die Frage der Übernahme der Auszubildenden. Fragen des Ausbildungsplatzangebotes, der Ausbildungsqualität und der beruflichen Entwicklung haben dagegen eine geringe Bedeutung. Ihre tatsächlichen Einflußmöglichkeiten auf die Übernahmechancen der Auszubildenden und die Ausbildungsqualität schätzen zwei Drittel der befragten Betriebsräte als gering ein. Unterschiede ergeben sich jedoch bei der Betriebsgröße: Betriebsräte in Unternehmen mit mehr als 500 Beschäftigten stufen ihre Einflußmöglichkeiten höher ein als jene in Kleinbetrieben mit weniger als 50 Beschäftigten. Dieses Ergebnis erklärt sich aus der größeren Professionalität der Betriebsräte in Großunternehmen, die in der Regel für ihre Betriebsratstätigkeit freigestellt werden (vgl. Heidemann/Paul-Kohlhoff 1998, S. 15 f.; Berger/Brandes/Walden 2000, S. 132 ff.).

Die Entwicklung der Gewerkschaften von berufsspezifischen Fachverbänden zu Industrieverbänden war eine entscheidende Voraussetzung für eine generelle Ausweitung der Berufsausbildung. Diese sollte die Funktion des institutionalisierten Übergangs der Jungendlichen von der Schule in den Arbeitsmarkt übernehmen. Während die Berufsverbände ein originäres Interesse an einer Beschränkung der Lehrstellen hatten, waren die Industriegewerkschaften daran interessiert, gering qualifizierte Arbeiter vor der Konkurrenz jugendlicher Arbeiter zu schützen. Außerdem konnten sie den Lebensstandard ihrer Mitglieder nur über eine qualifikationsbedingte Steigerung der Produktivität erhöhen. Darüber hinaus mußten sie darauf achten, daß sich innerhalb ihrer Organisation keine berufsspezifischen Partikularinteressen durchsetzten, weshalb sie eine Lehrstellenbeschränkung innerhalb einzelner Berufskategorien ablehnten. Stattdessen sollte die berufliche Mobilität durch eine allgemeine berufliche Grundausbildung erleichtert werden. 
Durch die institutionelle Einbindung in die Regulation und Steuerung der Berufsausbildung haben die Gewerkschaften auf verschiedenen Ebenen Einflußmöglichkeiten. Über die Beteiligung bei der Ausarbeitung von Ausbildungsordnungen können sie einer $\mathrm{zu}$ engen beruflichen Spezialisierung der Ausbildung entgegentreten. Die Tarifpolitik eröffnet ihnen Handlungsspielräume in der Finanzierung der Berufsausbildung: z.B. über die Höhe der Ausbildungsvergütung oder über die Festsetzung branchenbezogener Umlagen. Auf betrieblicher Ebene steht ihnen mit den Betriebsräten eine Institution zur Verfügung, über die sie sowohl die Einhaltung der die Berufsausbildung betreffenden Regelungen kontrollieren als auch aktiv in die Gestaltung der betrieblichen Berufsausbildung eingreifen können. Dieses ausgewogene institutionelle Geflecht von Kontroll- und Gestaltungsmöglichkeiten schafft die notwendige Vertrauensbasis, die es den Gewerkschaften erlaubt, die stark betriebsorientierte - und damit tendenziell Arbeitgeber dominierte - Organisation der Berufsausbildung zu unterstützen.

\subsection{Staatliche Institutionen}

Das föderalistische Prinzip in Deutschland schlägt sich auch auf die Organisation der Berufsausbildung nieder. Die Verteilung von Zuständigkeiten und Gesetzgebungskompetenzen auf Bundes- bzw. Landesebene führt notwendigerweise zu dysfunktionalen Reibungsverlusten. In den institutionalisierten konsensualen Abstimmungsverfahren tritt die spezifisch deutsche Variante eines kooperativen Föderalismus deutlich zutage. Im folgenden werden die jeweiligen Regelungsbefugnisse und Zuständigkeiten im Bereich der betrieblichen Ausbildung und der Berufsschule auf Bundes- und Landesebene dargestellt. Die Aufteilung staatlicher Regelungskompetenzen - sowohl vertikal als auch horizontal - steht dabei in einem historischen Kontext, der für die heutige Organisation der Berufsausbildung prägend ist.

Mit dem Erlaß des Preußischen Allgemeinen Landrechts von 1794 schrieb sich der Staat erstmalig eine weitgehende Regelungsbefugnis im Bereich der Lehrlingsausbildung zu, die Bestandteil der gewerberechtlichen Bestimmungen war. Diese Zuordnung zum Gewerberecht hatte für die spätere Kompetenzverteilung weitreichende Folgen. Während die betriebliche Ausbildung insofern eindeutig in den Verantwortungsbereich der Gewerbeverwaltung und damit des späteren Handelsministeriums fiel, sollte sich die Zuständigkeit für die sich entwickelnden Gewerbe- und Fortbildungsschulen, die 
Vorläufer der Berufsschulen, zwischen Kultus- und Handelsministerium hin und her bewegen. Von den Anfängen des gewerblichen Schulwesens zu Beginn des 19. Jahrhunderts bis 1860 war die verwaltungsrechtliche Zuständigkeit für die „Handwerker-, Fortbildungs- und Sonntagsschulen“ (Körzel 1996, S. 309) aufgrund ihres unspezifischen Status als Elementar- und berufliche Fachschule in Preußen unklar. Auf Initiative des Handelsministeriums wurde dieser Zustand dahingehend geklärt, daß die Aufsicht über diese Schulen dem Kultusministerium übertragen wurde. Dies war gerade im Hinblick auf die Fortbildungsschulen insofern gerechtfertigt, als sie anfangs einen starken allgemeinbildenden Charakter hatten. Infolge der Zuständigkeit des Kultusministeriums verloren die Fortbildungsschulen ihre ohnehin geringe beruflichgewerbliche Profilierung vollständig. Die Überarbeitung des Lehrplans führte zu einer stärkeren Betonung allgemeinbildender Inhalte, wie z.B. des Religionsunterrichts. Diese Entwicklung stand in der humboldtianisch-bildungspolitischen Tradition des Kultusministeriums. Erst mit dem späteren Wechsel des gewerblichen Unterrichtswesens in die Zuständigkeit des Handelsministeriums rückten wieder beruflich-gewerbliche Inhalte in den Vordergrund der Lehrpläne, um die praktische Lehrlingsausbildung mit ,theoretischen“ Inhalten zu ergänzen (vgl. Körzel 1996, S. 289 ff.).

Die wachsende Bedeutung einer gewerblich qualifizierten Arbeiterschaft im Zuge der Industrialisierung hatte $\mathrm{zu}$ Überlegungen in der preußischen Staatsregierung geführt, dem Kultusministerium die Zuständigkeit für das gewerbliche Schulwesen zu entziehen und dem Handelsministerium zu übertragen, um die Schulen in ihrer wirtschaftlich relevanten Funktion zu stärken: „Bei der Frage, welche Maßregeln [...] zur Aufrechterhaltung und Förderung der Konkurrenzfähigkeit einheimischer Industriezweige gegenüber der ausländischen Industrie zu ergreifen sind, spielt die Entwicklung und Leitung gewerblicher Fachschulen vielfach eine so entscheidende Rolle, daß die Gewerbeverwaltung, solange ihr in dieser Beziehung die Initiative und maßgebende Einwirkung abgeht, sich in ihrer Tätigkeit fortwährend auf das empfindlichste gehemmt sieht.“ (Denkschrift der preußischen Staatsregierung von 1884, zit. nach: Ziertmann 1953, S. 86) Als Ministerpräsident und Handelsminister übertrug von Bismarck 1884 unter Heranziehung der oben zitierten Begründung und als Teil seiner Mittelstandspolitik die Zuständigkeit für das gewerbliche Schulwesen vom preußischen Kultusministerium auf das preußische Handelsministerium. Andere deutsche Länder hatten diesen Schritt schon früher vollzogen, z.B. Sachsen und Württemberg, oder 
folgten dem preußischen Beispiel. Mit dem Übergang der Zuständigkeit für das gewerbliche Schulwesen auf das Handelsministerium konnte sich dieses als eine „Leistungsverwaltung“ im Dienste und Interesse der Wirtschaft profilieren. Die Abgrenzung zum Kultusministerium erfolgte nicht nur auf der Basis verwaltungstechnischer Argumente, sondern - und gerade - mit Verweis auf die unterschiedliche bildungspolitische Stoßrichtung. Das Fortbildungs- und Fachschulwesen sollte durch eine klare gewerbepolitische Ausrichtung ein wichtiges Element der preußischen Wirtschaftspolitik vor dem ersten Weltkrieg werden, wofür die entsprechenden Verwaltungsstrukturen eine notwendige Voraussetzung waren (vgl. Ziertmann 1953, S. 86 f.; Körzel 1996, S. 371 ff.).

Auch nach dem ersten Weltkrieg behielt das preußische Handelsministerium die Zuständigkeit für das gewerbliche Schulwesen. Neben der allgemeinen Unterrichtsverwaltung hatte sich eine davon völlig unabhängige Verwaltung für das gewerbliche und kaufmännische Schulwesen etabliert. Die Ordnung der betrieblichen Berufsausbildung blieb in der Weimarer Republik aufgrund des gescheiterten Berufsausbildungsgesetzes in der Reichsgewerbeordnung festgeschrieben und lag damit im Verantwortungsbereich des Reichswirtschaftsministeriums. Mit der Neustrukturierung der Staatsverwaltung durch die Nationalsozialisten kam es zu einem erneuten Wechsel in der Zuständigkeit für die Berufs- und Fachschulen. Die Eingliederung des preußischen Handelsministeriums in das Reichswirtschaftsministerium hatte zur Folge, daß die Zuständigkeit für das gewerbliche Schulwesen auf das Reichserziehungsministerium übertragen wurde. Mit der Gründung der Bundesrepublik Deutschland blieb diese Zuordnung erhalten: während das neu gegründete Bundesministerium für Wirtschaft die Gesetzgebungszuständigkeit für den betrieblichen Bereich der Lehrlingsausbildung erhielt, fiel die Zuständigkeit für das berufsbildende Schulwesen an die Kultusministerien der einzelnen Bundesländer (vgl. Ziertmann 1953, S. 87 f.; Schütte 1992, S. 100 ff.).

Diese verwaltungsrechtliche Aufteilung ergibt sich auch aus den Bestimmungen des Grundgesetzes. Nach Art. 74 Nr. 11 GG hat der Bund die Gesetzgebungskompetenz für das „Recht der Wirtschaft“. Das Bundesverfassungsgericht geht davon aus, daß diese Formulierung in einem weiten Sinne zu verstehen ist: „Zum ,Recht der Wirtschaft' gehört auch der Fragenkreis der praktischen beruflichen Ausbildung, die traditionell und strukturell von den in der Wirtschaft tätigen Arbeitgebern wahrzunehmen ist.“ (Bundesverfassungsgericht 1981, S. 308-309) An anderer Stelle heißt es konkreter: „Die 
Gesetzgebungszuständigkeit des Bundes nach Art. 74 Nr. 11 GG erstreckt sich auch darauf, Berufe, in der Wirtschaft' rechtlich zu ordnen und ihre Berufsbilder rechtlich zu fixieren.“ (Bundesverfassungsgericht 1969, S. 255) Diese Textstellen verdeutlichen die Vorrangstellung des Bundes in Fragen der rechtlichen Ordnung von Berufen, wobei es eine Einschränkung auf die „praktische berufliche Ausbildung“ gibt. Neben Art. 74 Nr. 11 GG wird dem Bund auch nach Art. 74 Nr. 12 GG eine Gesetzgebungszuständigkeit für den Bereich der beruflichen Ausbildung zugesprochen. Danach hat der Bund die Regelungskompetenz für das „Arbeitsrecht einschließlich der Betriebsverfassung“. Die für den Arbeitsvertrag geltenden Rechtsvorschriften und -grundsätze sind ebenfalls auf den Ausbildungsvertrag anzuwenden, insofern ergibt sich eine Zuständigkeit des Bundes für die Berufsausbildung auf der Basis des Arbeitsrechts (vgl. Kraegeloh 1998, S. 12 f.; Els 1999, S. 12 f.).

Die Gesetzgebungskompetenz der Länder für die schulische Berufsausbildung ergibt sich aus Art. 30 GG und Art. 70 Abs. 1 GG, in denen den Ländern das Recht zur Gesetzgebung übertragen wird, soweit das Grundgesetz nicht dem Bund die Regelungszuständigkeit zuweist. Da das Grundgesetz dem Bund in kulturellen Fragen keine ausdrückliche Regelungsbefugnis verleiht, wird in diesem Zusammenhang auch von der „Kulturhoheit“ der Länder gesprochen, die insbesondere alle schulischen Angelegenheiten umfaßt. Demzufolge sind die einzelnen Länder für die Berufsschulen und deren Lehrpläne verantwortlich (vgl. Els 1999, S. 13 f. und 15 f.; Kraegeloh 1998, S. $13)$.

Die Dualität der Gesetzgebungszuständigkeit - der Bundesregierung für die betriebliche Ausbildung und der Länder für die Berufsschulen - hat bei der Gestaltung der Ausbildungsberufe Abstimmungsprobleme zur Folge. Um ein effektives Ineinandergreifen von praktischer und schulischer Ausbildung und eine bundeseinheitliche Ausbildung mit allgemeingültigen Standards gewährleisten zu können, ist es notwendig, daß die Ordnung für die betriebliche Ausbildung und die Lehrpläne der Berufsschulen aufeinander abgestimmt sind. Während die Länder über die ständige Konferenz der Kultusminister (KMK) gemeinsam Rahmenlehrpläne für die jeweiligen Ausbildungsberufe erarbeiteten, gab es bis 1972 kein institutionalisiertes Verfahren zur inhaltlichen Abstimmung der Ausbildungsordnungen mit den Rahmenlehrplänen. Mit der Verabschiedung des „Gemeinsamen Ergebnisprotokolls“ durch die Bundesregierung und die Kultusminister der Länder im Mai 1972 wurde dieses Defizit beseitigt. Das 
„Gemeinsame Ergebnisprotokoll“ ist eine im Sinne des kooperativen Föderalismus getroffene Verwaltungsvereinbarung, in der sich der Bund und die Länder - ausgehend von der jeweiligen rechtlichen Zuständigkeit für die betriebliche und schulische Berufsausbildung - über die Einhaltung bestimmter Verfahrensprinzipien und die Einrichtung gemeinsamer Gremien zur Koordination berufsbildungspolitischer Entscheidungen verständigt haben. Als neue gemeinsame Gremien wurden ein Koordinierungsauschuß, Sachverständigenausschüsse des Bundes und Rahmenlehrplanausschüsse der KMK eingerichtet. Der Koordinierungsausschuß ist ein regelmäßig tagendes Arbeitsgremium, das mit fachlich zuständigen Ministerialbeamten von Bund und Ländern besetzt ist. Seine Aufgabe ist die Definition von Grundsätzen zur Abstimmung von Ausbildungsordnungen und Rahmenlehrplänen. Darüber hinaus entscheidet er über den Beginn, die Durchführung und die Ergebnisse von Abstimmungsprojekten. Die Ausarbeitung der Ausbildungsordnungen für den betrieblichen Teil der Berufsausbildung obliegt den Sachverständigenausschüssen des Bundes. Sie setzen sich aus Berufsbildungsexperten der Sozialpartner und des Bundesinstituts für Berufsbildung (BIBB) zusammen. Die Erarbeitung der Rahmenlehrpläne für die schulische Berufsausbildung erfolgt in den Rahmenlehrplanausschüssen, die mit Berufsschulexperten der Länder besetzt sind. Die Abstimmung der getrennt erarbeiteten Ausbildungsordnungen und Rahmenlehrpläne findet in gemeinsamen Sitzungen mit Mitgliedern aus beiden Gremien statt (vgl. Benner/Püttmann 1992, S. 11 ff.; Hilbert et al. 1990, S. 50; Kath 2000, S. 82).

Die Bundesregierung nutzte die nach Art. 74 Nr. 11 und 12 GG dem Bund zustehende Regelungskompetenz für die betriebliche Berufsausbildung zur Ausarbeitung eines umfassenden Berufsbildungsgesetzes. Am 1. September 1969 trat das Berufsbildungsgesetz (BBiG) in Kraft, das den Zustand der Rechtszersplitterung in der betrieblichen Berufsausbildung beendete. Entscheidend für eine institutionalisierte Berufsbildungsforschung und -planung auf Bundesebene war die im Berufsbildungsgesetz festgeschriebene Errichtung eines Bundesinstituts für Berufsbildungsforschung (BBF) als Körperschaft des öffentlichen Rechts auf der Grundlage sozialpartnerschaftlicher Selbstverwaltung. Das Bundesinstitut für Berufsbildungsforschung (BBF), aus dem mit Erlaß des Ausbildungsplatzförderungsgesetzes (APIFG) von 1976 das Bundesinstitut für Berufsbildung (BIBB) wurde, stand dabei in einer langen Tradition verbandlicher Selbstorganisation im Bereich der beruflichen Bildungsforschung. Bereits 1908 grün- 
deten verschiedene Wirtschaftsverbände - wie z.B. der Verein Deutscher Ingenieure (VDI), der Verband Deutscher Elektrotechniker (VDE) und der Verband Deutscher Maschinenbauanstalten (VDMA) - den „Deutschen Ausschuß für technisches Schulwesen“ (DATSCH), der Richtlinien für die Ausbildung von Facharbeitern und industriespezifische Ausbildungslehrgänge entwickeln sollte. Ergebnis seiner Arbeit waren erste industriebezogene Berufsbilder mit Berufsbezeichnung, inhaltlicher Strukturierung der Ausbildung und Lehrplänen. Ab 1935 war der DATSCH berufsbildungspolitisch beratendes Gremium des Reichswirtschaftsministeriums. Erst 1939 erfolgte mit der Umwandlung des DATSCH in das „Reichsinstitut für Berufsbildung“ eine Ausweitung des Aufgabengebietes auf berufsbildungspolitische Fragen der gesamten Wirtschaft, einschließlich des Handwerks. Bis 1940 blieb das „Reichsinstitut für Berufsbildung“ in der Verantwortung der Wirtschaftsverbände. Danach wurde es direkt dem Reichswirtschaftsministerium unterstellt, weil die planmäßige Berufserziehung zu einem kriegswichtigen Faktor geworden war. Die Aufgaben des „Reichsinstituts für Berufsbildung“ lagen bis zu seiner Auflösung 1945 im Bereich der Abgrenzung von Ausbildungsberufen, der Entwicklung von Ausbildungsordnungen und Lehrplänen sowie in der Koordination von betrieblicher und schulischer Berufsausbildung (vgl. Hilbert et al. 1990, S. 24 ff.; Greinert 1993, S. 66 ff.; Golisch 1997, S. 32 f.).

Nach dem zweiten Weltkrieg gab es keine dem Reichsinstitut für Berufsbildung vergleichbare berufsbildungspolitische Organisation. Erst 1947 gründeten die Industrieund Handelskammern zwei Arbeitsstellen für Berufserziehung: eine für die gewerbliche Wirtschaft, die andere für die kaufmännischen Berufe. Der Deutsche Industrie- und Handelstag (DIHT) faßte diese beiden Arbeitsstellen 1951 zur „Arbeitsstelle für Berufserziehung des Deutschen Industrie- und Handelstages“ zusammen. Durch eine Vereinbarung des DIHT mit dem Bundesverband der Deutschen Industrie (BDI) und der Bundesvereinigung der Deutschen Arbeitgeberverbände (BDA) übernahmen diese drei zentralen Wirtschaftsverbände der deutschen Wirtschaft gemeinsam die Trägerschaft der Arbeitsstelle, die gleichzeitig in Arbeitsstelle für betriebliche Berufsausbildung (ABB) umbenannt wurde. Die Arbeitsstelle für betriebliche Berufsausbildung sah sich als Nachfolgerin des Reichsinstituts für Berufsbildung. Im Gegensatz zu diesem oblag ihr aber nicht die Verantwortung für die berufsbildungspolitische Planung und Koordination der handwerklichen Berufe. Für diese waren die Handwerksorganisationen selber zuständig. Ab Mitte der 1960er Jahre trat neben die praktisch- 
empirische Arbeit der Arbeitsstelle für betriebliche Berufsausbildung eine zunehmende Verwissenschaftlichung der Berufsbildungsplanung im Sinne einer Berufsanalyse, um die Entwicklung der Berufsausbildung im Kontext eines sich schneller wandelnden sozio-ökonomischen Umfeldes besser prognostizieren zu können (vgl. Ziertmann 1953, S. 97 f.; Hilbert et al. 1990, S. 27).

Das Bundesinstitut für Berufsbildung (BIBB), das aus dem Bundesinstitut für Berufsbildungsforschung (BBF) hervorgegangen ist, hat die Funktion der Arbeitsstelle für betriebliche Berufsausbildung übernommen. Hinsichtlich seines Aufgabenbereiches, der sich auf die gesamte gewerbliche und kaufmännische Berufsbildung bezieht, steht es in der Tradition des Reichsinstituts für Berufsbildung. Im Gegensatz zu diesem ist aber im Bundesinstitut für Berufsbildung (BIBB) das sozialpartnerschaftliche Moment stärker verankert. Die beiden gesetzlich vorgeschriebenen Organe des BIBB sind der Generalsekretär und der Hauptausschuß. Während der Generalsekretär für die Verwaltung und Organisation des BIBB zuständig ist, kann der Hauptausschuß als „Legislativorgan“ betrachtet werden, das paritätisch mit Vertretern der Arbeitgeber, der Arbeitnehmer, der Länder und des Bundes zusammengesetzt ist. Wesentliche Aufgabe des Hauptausschusses ist die Beratung der Bundesregierung in grundsätzlichen Fragen der beruflichen Bildung. Darüber hinaus erläßt er Richtlinien für die Prüfungsordnungen und beschließt über die Angelegenheiten des Bundesinstituts. Aus Mitgliedern des Hauptausschusses wird ein Ständiger Ausschuß gebildet, der z.B. zu den Entwürfen der Ausbildungsordnungen Stellung nimmt. Die Abstimmung der Ausbildungsordnungen mit den Rahmenlehrplänen der Länder obliegt dem Länderausschuß, der als ein ständiger Unterausschuß des Hauptausschusses eingerichtet ist (vgl. Bundesinstitut für Berufsbildung 2000, S. 4 ff.). Damit steht der Länderausschuß in Konkurrenz zu dem durch das „Gemeinsame Ergebnisprotokoll“ eingerichteten Koordinierungsausschuß. Die Länder sahen die Gefahr, daß ihre Zuständigkeit für die schulische Berufsausbildung in einem Ausschuß einer Bundesbehörde verwässert wird. Deshalb ist die tatsächliche Abstimmung zwischen Ausbildungsordnung und Rahmenlehrplan im Koordinierungsauschuß verblieben. Dem Länderausschuß bleibt die Beratung über die Ausbildungsordnungsentwürfe (vgl. Hilbert et al. 1990, S. 50 f.; Raddatz 1983, S. 103).

Am Beispiel des Verfahrens zum Erlaß von Ausbildungsordnungen ( $§ 25$ Berufsbildungsgesetz) läßt sich deutlich zeigen, wie diese unterschiedlichen Gremien ineinan- 
dergreifen, um einen Prozeß konsensualer Kooperation zu gewährleisten. Bis zur endgültigen Verabschiedung einer Ausbildungsordnung müssen drei Phasen durchlaufen werden: Vorbereitungs-, Erarbeitungs- und Erlaßphase.

Obgleich das Initiativrecht zur Neuordnung oder Einrichtung eines Ausbildungsberufs grundsätzlich jedem zusteht, nehmen es in der Regel nur die Verbände der Arbeitgeber bzw. der Arbeitnehmer wahr. Mit dem Vorschlag einer Neueinrichtung oder Neuordnung eines Ausbildungsberufs beginnt die Vorbereitungsphase. Im Rahmen eines Antragsgesprächs beim zuständigen Fachminister - in der Regel dem Wirtschaftsminister - werden die Eckwerte für das Projekt festgelegt, d.h. die Berufsbezeichnung, Ausbildungsdauer, Berufsfeldzuordnung, Berufsbeschreibung sowie Struktur und organisatorischer Aufbau des Ausbildungsgangs. In der Regel haben die Verbandsvertreter diese Eckwerte mit Unterstützung des BIBB bereits im Vorfeld ausgehandelt, so daß dem Fachminister eher die Funktion eines ,Notars' zukommt. Nach erfolgreichem Antragsgespräch erarbeitet das BIBB auf dieser Grundlage einen Projektantrag für die Abstimmung mit den Ländern im Koordinierungsausschuß. Dieser Entwurf wird an den Länderausschuß beim BIBB, den Fachminister sowie das Bundesministerium für Bildung und Forschung (BMBF) weitergeleitet. Der vom Fachminister im Einvernehmen mit dem BMBF formulierte Projektantrag wird in den Koordinierungsausschuß eingebracht. Die Vorbereitungsphase endet mit einem Beschluß des Koordinierungsausschusses.

Bei einem positiven Beschluß des Koordinierungsausschusses erarbeiten in der zweiten Phase des Verfahrens das BIBB unter Einbeziehung von Sachverständigen aus den Verbänden und die Länder parallel zueinander die Ausbildungsordnung bzw. den Rahmenlehrplan. Die Ausarbeitung der Ordnungen findet in den Sachverständigen/Fachausschüssen des BIBB bzw. den Rahmenlehrplanausschüssen der Länder statt. Die Abstimmung zwischen Ausbildungsordnung und Rahmenlehrplan erfolgt in gemeinsamen Sitzungen der beiden Gremien. Nach der letzten gemeinsamen Sitzung liegt der zwischen Bund und Ländern abgestimmte Entwurf der Ausbildungsordnung beim BIBB vor, wo er unter Einbeziehung der Stellungnahme des Länderausschusses im Hauptausschuß überprüft wird.

Im Anschluß an die Überprüfung durch den Hauptausschuß wird der Entwurf der Ausbildungsordnung an den zuständigen Fachminister weitergeleitet, der sie nach 
endgültiger Zustimmung des Koordinierungsausschusses im Einvernehmen mit dem BMBF und nach rechtlicher Prüfung durch den Bundesminister für Justiz erläßt. Die Kultusministerkonferenz beschließt den Rahmenlehrplan, auf dessen Grundlage die Länder die Lehrpläne für die Berufsschulen festlegen (vgl. Streeck et al. 1987, S. A5 ff.; Els 1999, S. 18 ff.; Benner/Püttmann 1992, S. 18 ff.; Wohlgemuth 1995, S. 245 ff.; Golisch 1997, S. 36 ff.). Bei der Umsetzung der Rahmenlehrpläne werden die Länder von paritätisch besetzten Landesausschüssen für Berufsbildung unterstützt. Diese reinen Beratungsorgane sind nach dem Berufsbildungsgesetz ( $\S 54 \mathrm{BBiG})$ in allen Ländern einzurichten (vgl. Greinert 1993, S. 130; Raddatz 1983, S. 106 f.).

Anhand des Verfahrens zur Erarbeitung von Ausbildungsordnungen zeigt sich das komplexe Zusammenspiel der unterschiedlichen an der Berufsausbildung beteiligten Akteure. Eine zentrale Rolle im Koordinations- und Abstimmungsprozeß kommt dabei dem Bundesinstitut für Berufsbildung zu. Die besondere Position des BIBB als Bundesbehörde ohne inhaltliche Weisungsabhängigkeit von der Bundesregierung und regulativer Kompetenz erlaubt es ihm als neutraler Mittler zwischen den beteiligten Akteuren aufzutreten. In dem unter seinem Dach organisierten Ausschüssen erarbeiten die von den Verbänden entsandten Experten auf der Basis des allgemein akzeptierten „Konsensprinzips“ Ausbildungsordnungen zur Strukturierung der betrieblichen Berufsausbildung. Die Einbeziehung von Bundes- und Ländervertretern ermöglicht eine frühzeitige Konzertierung zwischen verbandlichen und staatlichen Interessen. Insofern kann das BIBB als ein „Forum funktionaler Repräsentation“ im Abstimmungsprozeß staatlicher und gesellschaftlicher Interessenorganisation bezeichnet werden. Aber das BIBB bietet nicht nur den institutionellen Rahmen für eine sozialpartnerschaftliche Konsensbildung, sondern bringt selber - über seine Mitarbeiter - Expertenwissen in den Entscheidungsprozeß ein.

Wie dieses Kapitel gezeigt hat, entlastet die Einbeziehung der Sozialpartner in die berufsbildungspolitisch relevanten Entscheidungsprozesse den Staat in zweierlei Hinsicht: Die Sozialpartner stellen das nötige Expertenwissen zur Verfügung und setzen die von ihnen mitgetragenen Entscheidungen bei ihren Mitgliedern durch. Der Staat beschränkt seine Rolle auf eine „Notariatsfunktion“, indem er die sozialpartnerschaftlich ausgearbeiteten Ausbildungsordnungen formell erläßt und damit eine staatlich 
sanktionierte Rechtsverbindlichkeit schafft. Die verfassungsrechtliche Zuständigkeit des Bundes für die betriebliche Berufsausbildung und die Einbindung der Länder im Sinne des kooperativen Föderalismus sind die Voraussetzung für eine allgemeinverbindliche Regelung der Ausbildung in Form staatlich anerkannter Ausbildungsberufe ${ }^{14}$. Die Standardisierung der Berufsausbildung ist eine wesentliche Grundlage für die Funktionsfähigkeit eines überregionalen Arbeitsmarktes, da über die Transparenz der Abschlüsse die asymmetrische Informationsverteilung zwischen den Marktteilnehmern reduziert wird. Darüber hinaus erhöht sie aufgrund der vom Einzelbetrieb weitgehend unabhängigen Qualifikation das Mobilitätspotential der ausgebildeten Jugendlichen und verbessert insofern deren Chancen am Arbeitsmarkt.

${ }^{14}$ Nach $\S 28$ Abs. 2 BBiG ist eine Ausbildung in anderen als den anerkannten Ausbildungsberufen verboten. 


\section{Staatliche Berufsbildungspolitik seit den 1970er Jahren}

Im Sinne des ,policy feedback' haben erfolgreiche und nicht erfolgreiche Institutionalisierungen von Politiken einen entscheidenden Einfluß auf die Gestaltung neuer Politikansätze. Einmal durchgesetzte Problemlösungen erzeugen häufig wieder neue Probleme, deren Lösung tendenziell in den eingeführten Bahnen erfolgt. Gescheiterte Reformen führen zu Lernprozessen in der politischen Administration, die sich in der Formulierung zukünftiger Problemlösungskonzepte widerspiegeln. Die staatliche Berufsbildungspolitik der letzten 30 Jahre hat einen besonders prägenden Einfluß auf die berufsbildungspolitischen Lösungsansätze der Gegenwart in Deutschland.

\subsection{Strukturpolitische Konsolidierung des dualen Berufsbildungssystems}

Die frühen 1970er Jahre standen ganz unter dem Eindruck des 1969 verabschiedeten Berufsbildungsgesetzes (BBiG). Bereits in der Weimarer Republik hatte die Debatte über die Einführung eines umfassenden Berufsbildungsgesetzes begonnen. Die Beratung verschiedener Gesetzesentwürfe führte jedoch nicht zur endgültigen Verabschiedung eines Gesetzes. Mitte der 1960er Jahre legte die SPD nach einem Vorstoß des DGB einen Gesetzentwurf im Bundestag vor, auf den die CDU/CSU und FDP als Regierungsparteien mit einem eigenen Entwurf antworteten. Die folgende „Große Koalition“" von CDU/CSU und SPD stellte eine neue Interessenkonstellation dar, die den Abstimmungsprozeß der verschiedenen Gesetzesentwürfe beschleunigte. Unter dem Zugeständnis weitgehender Sonderregelungen für das Handwerk wurde das Gesetz im August 1969 verkündet. Das BBiG beendete die bestehende Rechtszersplitterung in der betrieblichen Berufsausbildung, die bis dahin in verschiedenen Wirtschaftsgesetzen geregelt war. Es entstand damit erstmals eine bundeseinheitliche Gesamtregelung für die Berufsausbildung in den Betrieben. Neben der staatlichen Anerkennung von Ausbildungsberufen regelt das noch heute geltende Gesetz unterschiedliche Bereiche der Berufsausbildung, wie z.B. den Ausbildungsvertrag und das Prüfungswesen. Darüber hinaus institutionalisierte das Gesetz die paritätische Beteiligung von Arbeitgeber- und Arbeitnehmervertretern in unterschiedlichen Ausschüssen mit Beratungs- und Beschlußrechten auf Bundes-, Landes- und lokaler (Kammer-)Ebene. Die Gewerkschaften hatten damit ihre Forderung auf Mitbestimmung in der Berufsausbildung, die sie bereits in der Weimarer Republik aufgestellt hatten, durchge- 
setzt. Insgesamt bedeutete die Verabschiedung des Gesetzes eine Stabilisierung des dualen Berufsbildungssystems, wie es sich bis dahin entwickelt hatte (vgl. Greinert 1993, S. 93 ff.; Pätzold 1982, S. 3 f. und 37 f.; Pätzold 1991, S. 27).

Die fehlenden Aussagen des BBiG zur Ausbildungsfinanzierung und zum Verhältnis der Berufsbildung zur Allgemeinbildung führten dazu, daß die Debatte über eine umfassende Berufsbildungsreform nicht verstummte. Forderungen nach einer stärkeren öffentlichen Verantwortung für die Berufsbildung, nach mehr Chancengleichheit sowie nach einer größeren Durchlässigkeit des gesamten - sowohl beruflichen als auch allgemeinen - Bildungssystems fanden ihre Unterstützung in den Empfehlungen der Bildungskommission des Deutschen Bildungsrates zur Verbesserung der Lehrlingsausbildung: „Aus der oben bereits angesprochenen Einheit von beruflicher und allgemeiner Bildung und aus der Bedeutung des Bildungswesens für die Entwicklung von Wirtschaft und Gesellschaft ergibt sich die Verantwortung des Staates [...]. Das bedeutet, daß die Ausbildung der Lehrlinge an den gleichen Maßstäben gemessen werden muß, die dem gesamten Bildungswesen zugrundeliegen.“ (Deutscher Bildungsrat 1969, zit. nach: Pätzold 1991, S. 487)

Die sozial-liberale Regierung griff die Thematik auf und nahm die Reform der Berufsausbildung in ihren Maßnahmenkatalog der ,inneren Reformen“ auf. Anfangs hoffte sie, ihre Reformpläne im Rahmen der bestehenden gesetzlichen Bestimmungen durchsetzen zu können. In der Regierungserklärung von 1973 machte Bundeskanzler W. Brandt aber deutlich, daß das Berufsbildungsgesetz neu gefaßt werden müsse. Noch im November desselben Jahres beschloß das Bundeskabinett wesentliche Grundsätze zur Neuordnung der beruflichen Bildung („Markierungspunkte“) (vgl. Pätzold 1982, S. 38 f.; Raddatz 1983, S. 102; Kath 2000, S. 79). Eine zentrale Rolle spielte dabei die Frage der staatlichen Verantwortung: „Der Staat muß aber dafür sorgen, daß eine funktionale Aufgabenteilung mit den öffentlichen Bildungseinrichtungen und eine staatliche Aufsicht über die Ausbildungstätigkeit das Erreichen der staatlichen Qualitätsnormen und das notwendige Bildungsangebot sichern. Die staatliche Verantwortung gilt für alle Lernorte.“ (Bundesminister für Bildung und Wissenschaft 1973, zit. nach: Pätzold 1982, S. 241) Diese neue Verantwortung spiegelte sich in den Überlegungen wider, die Industrie- und Handelskammern sowie die Handwerkskammern weitgehend $\mathrm{zu}$ entmachten und alle administrativen Aufgaben im Bereich der Berufsbildung an eine neu zu schaffende staatliche Berufsbildungsbehörde zu delegieren. Darüber hinaus 
sollte die Eignung der Ausbildungsbetriebe in einem förmlichen Akkreditierungsverfahren festgestellt werden. Während im Referentenentwurf zu Beginn des Jahres 1974 noch wesentliche Aspekte der „Markierungspunkte“ auftauchten, fiel der Regierungsentwurf von 1975 bereits sehr viel gemäßigter aus und stellte die Position der Kammern nicht mehr in Frage. Dies war auf den massiven Widerstand der Arbeitsgeber und ihrer Verbände sowie der Oppositionsparteien gegen die Vorschläge der Regierung zurückzuführen. So hatten die Arbeitgebervertreter beispielsweise gedroht, keine Ausbildungsplätze mehr bereitzustellen (vgl. Greinert 1993, S. 107 f.).

Nach dem Scheitern des Regierungsentwurfs im Bundesrat, in dem die CDU/CSU die Mehrheit hatte, übernahm die Regierung zwei Elemente des gescheiterten Gesetzentwurfs und brachte diese in Form eines Ausbildungsplatzförderungsgesetzes (APIFG) in den Bundestag ein: Das Gesetz regelte einerseits die Einrichtung eines Bundesinstituts für Berufsbildung, andererseits beinhaltete es ein überbetriebliches Finanzierungskonzept für den Fall eines zu geringen Ausbildungsplatzangebotes. Danach sollte im Fall, daß das Ausbildungsplatzangebot die Ausbildungsplatznachfrage um weniger als 12,5 \% übersteigt, von allen Betrieben eine Berufsbildungsabgabe erhoben werden, die sich auf der Basis der jährlichen Lohnsumme berechnete. Kleine Betriebe, wie sie insbesondere im Handwerk $\mathrm{zu}$ finden sind, sollten durch eine entsprechende Freibetragsregelung von der Zahlung ausgenommen werden (vgl. § 3 APIFG vom 7.9.1976). Im September 1976 verabschiedete der Bundestag das Gesetz, das nach Ansicht der Regierung keiner Zustimmung des Bundesrates bedurfte (vgl. Stratmann 1990, S. 219 ff.; Kath 2000, S. 83 f.; Pätzold 1982, S. 41; Raddatz 2000, S. 259).

Trotz der offensichtlichen „Ausbildungskrise“ in der zweiten Hälfte der 1970er Jahre, die im wesentlichen nachfrageinduziert war, setzte die Regierung das von ihr gesetzlich eingeführte neue Steuerungsinstrument einer allgemeinen Betriebsumlage nicht ein. Während die Gewerkschaften regelmäßig den Einsatz dieses Instruments forderten, lehnten die Arbeitgebervertreter dies mit Verweis auf Zuwächse des Ausbildungsstellenangebots und dem Versprechen, das Angebot der Nachfrage weiter anzupassen, kategorisch ab. Gleichzeitig klagte der Freistaat Bayern vor dem Bundesverfassungsgericht gegen das Ausbildungsplatzförderungsgesetz und die Berufsbildungsabgabe. Im Dezember 1980 erklärte das Bundesverfassungsgericht das Ausbildungsplatzförderungsgesetz (APIFG) aufgrund eines Formfehlers im Gesetzgebungsverfahren für 
nichtig. Die Regelung von Verwaltungsverfahren im APIFG hätte gemäß Art. 84 Abs. 1 GG der Zustimmung des Bundesrates bedurft. Das Bundesverfassungsgericht stellte in seiner Entscheidung die Verfassungsmäßigkeit der Berufsbildungsabgabe jedoch explizit fest: „Die Berufsausbildungsabgabe nach $\S 3$ Abs. 1 Satz 1 APIFG stellt sich [...] als zulässige Sonderabgabe dar. [...] Die Erhebung der Berufsausbildungsabgabe dient ausschließlich der Sicherstellung eines qualitativ und quantitativ ausreichenden Angebots an Ausbildungsplätzen.“ (Bundesverfassungsgericht 1981, S. 308-309) Ein Jahr später verabschiedete der Bundestag das noch heute geltende Berufsbildungsförderungsgesetz (BerBiFG), das sich weitgehend am Ausbildungsplatzförderungsgesetz anlehnt. Eine Regelung zur Umlagefinanzierung in Form einer Berufsausbildungsabgabe enthält es aber nicht mehr. Dies ist sowohl auf den Widerstand der Arbeitgeber als auch auf die sich abzeichnende Entspannung auf dem Ausbildungsstellenmarkt zurückzuführen (vgl. Kath 2000, S. 84 f.; Pätzold 1982, S. 41 f.; Raddatz 1983, S. 102 f.; Pätzold 1991, S. 28 f.; Stratmann 1990, S. 221 ff.).

Damit fand eine wichtige Phase der gesetzlich normierten Berufsbildungsreform ihr Ende. Die Verabschiedung des seit den 1920er Jahren geplanten Berufsbildungsgesetzes stand am Beginn dieser Reformbestrebungen. Während das Berufsbildungsgesetz einerseits einen wichtigen Schritt in Richtung einer umfassenden und kohärenter gesetzlicher Regelung der betrieblichen Berufsausbildung darstellte, argumentierten die Reformbefürworter andererseits, daß es nicht weit genug ginge, sondern lediglich eine Stabilisierung des dualen Systems in seiner gewachsenen organisatorisch-institutionellen Form bedeute. Sie wollten eine stärkere Rolle für den Staat in der Berufsbildungsplanung und -verwaltung. Dies zeigte sich in den Gesetzentwürfen zur Novellierung des Berufsbildungsgesetzes, die eine Stärkung der staatlichen Verantwortung in zweierlei Hinsicht vorsahen: Einerseits sollte den wirtschaftsnahen Kammern die Verwaltung und Kontrolle der Berufsausbildung entzogen und auf eine staatliche Berufsbildungsverwaltung übertragen werden, andererseits sollte über eine Neuregelung der Finanzierung der betrieblichen Berufsausbildung der Staat einen indirekten Zugriff auf die Bereitstellung von Ausbildungsplätzen erhalten. Nach dem Scheitern dieser „Verstaatlichungsbestrebungen“" stand das durch das Berufsbildungsgesetz von 1969 gesetzlich legitimierte institutionelle Gerüst der dualen Berufsausbildung gefestigt da.

Parallel zu den Novellierungsversuchen des Berufsbildungsgesetzes gab es eine weitere Reformbestrebung, die ebenfalls auf eine strukturelle Neugestaltung des dualen 
Berufsbildungssystems zielte. Dabei ging es um die verbindliche Einführung eines Berufsgrundbildungsjahres. Der Deutsche Bildungsrat schrieb in seinem 1970 vorgelegten „Strukturplan für das Bildungswesen“: „Das erste Jahr jeder Berufsbildung ist ein obligatorisches Berufsgrundbildungsjahr, in dem eine von der Produktion getrennte, systematische Grundbildung in einem breiten Berufsfeld stattfindet.“ (Deutscher Bildungsrat 1970, zit. nach: Stratmann 1990, S. 188) Für die Durchsetzung dieses Vorschlages hatte das Berufsbildungsgesetz mit dem § 29 insofern die rechtlichen Voraussetzungen geschaffen, als dem Bundeswirtschaftsminister die Berechtigung zugesprochen wurde, durch Rechtsverordnung den Besuch der Berufsgrundschule anrechnungspflichtig zu machen. Im Juli 1972 nahm der Bundeswirtschaftsminister dieses Recht in Anspruch und erließ die „Verordnung über die Anrechnung auf die Ausbildungszeit in Ausbildungsberufen der gewerblichen Wirtschaft und der wirtschafts- und steuerberatenden Berufe - Anrechnung eines schulischen Berufsgrundbildungsjahres und des Besuchs einer einjährigen Berufsfachschule (Berufsgrundbildungsjahr-Anrechnungsverordnung)““. Gleichzeitig verabschiedete die Kultusministerkonferenz eine „Rahmenvereinbarung“, die zusammen mit der „Anrechnungsverordnung“ die Voraussetzungen für die Einführung des Berufsgrundbildungsjahres schuf. Einige Länder nutzten die rechtlichen Rahmenbedingungen und richteten das Berufsgrundbildungsjahr als „Grundstufe“ der Berufsschule ein (vgl. Greinert 1993, S. 109; Stratmann 1990, S. 189).

Niedersachsen gehörte zu den wenigen Ländern, die das Berufsgrundbildungsjahr flächendeckend als öffentliche Pflichtschule einzuführen versuchten. Bereits 1973 wurde eine erste gesetzliche Grundlage für die Umsetzung des Berufsgrundbildungsjahres im Niedersächsischen Schulgesetz geschaffen. Die endgültige Absicherung des Berufsgrundbildungsjahres als verbindliche Schulform erfolgte mit dem Niedersächsischen Schulgesetz von 1974. Die erste flächendeckende Einführung des Berufsgrundbildungsjahres erfolgte 1976 im Berufsfeld „Metall“ in der Region Salzgitter. In der wirtschaftlich problematischen Region Ostfriesland/Emsland wurde bis 1982 erstmalig für alle Berufsfelder das Berufsgrundbildungsjahr eingeführt. Während sich die Arbeitgeberverbände und Gewerkschaften an den politischen Debatten über die Aufnahme des Berufsgrundbildungsjahres in das Niedersächsische Schulgesetz kaum beteiligten, kam es bei der Umsetzung des verbindlichen Berufsgrundbildungsjahres in Salzgitter zu heftigen Auseinandersetzungen. Der Streit entbrannte an der Frage der 
Anrechnung des Berufsgrundbildungsjahres auf die betriebliche Ausbildung, dem Anteil des fachpraktischen Unterrichts sowie der Schwerpunktbildung im Berufsfeld. Die Arbeitgeber forderten eine Ausweitung des fachpraktischen Unterrichts und die frühzeitige Möglichkeit der Schwerpunktbildung. Symptomatisch dafür war der sog. „Anrechnungsstreit“, den das KFZ-Handwerk in Salzgitter mit Unterstützung des Zentralverbands des Kraftfahrzeughandwerks heraufbeschwor. Die KFZ-Innungsmeister erklärten, daß sie sich nicht in der Lage sähen, Absolventen des Berufsgrundbildungsjahres innerhalb von zwei Jahren zu KFZ-Mechanikern nach der bundeseinheitlichen Ausbildungsordnung auszubilden: „Bis eine Änderung des vorgelegten Konzepts nicht erfolgt ist, kann das Kraftfahrzeughandwerk dieser Ausbildungsform nicht zustimmen.“ (KFZ-Innung 1977, zit. nach: Greinert 1982, S. 28) Nur durch Zugeständnisse des niedersächsischen Kultusminister konnte das Modellvorhaben in Salzgitter gerettet werden (vgl. Wiemann 1983, S. 110 ff.; Greinert 1982, S. 18 ff.).

Auf Bundesebene sah sich die Regierung einer vergleichbaren Situation gegenüber. So unterstützten die Gewerkschaften anfangs die Einführung des schulischen Berufsgrundbildungsjahres, da es ihrer Meinung nach zwei entscheidende Schwächen des dualen Berufsbildungssystems ausgeglichen hätte: Auf der einen Seite hätte eine flächendeckende Institutionalisierung des Berufsgrundbildungsjahres zu einer Stabilisierung des regional, berufsstrukturell und zeitlich abhängigen Ausbildungsplatzangebots beigetragen. Auf der anderen Seite hätte es das von gesellschaftspolitischen Überlegungen unabhängige Rekrutierungsverhalten der Unternehmen mit seinen sozialen Selektionseffekten eingeschränkt. Die Arbeitgeberverbände und Industrie- und Handelkammern lehnten das schulische Berufsgrundbildungsjahr von Beginn an kategorisch ab. Sie argumentierten mit der fehlenden Praxisnähe und dem Problem der Anrechnung des Berufsgrundbildungsjahres bei länderspezifischen Lehrplänen sowie der vom jeweiligen Schulträger abhängigen Ausstattung der Berufsgrundschule. Der Durchsetzung des Berufsgrundbildungsjahres widersetzten sich die Betriebe, indem sie die Anrechnungsverordnung mißachteten bzw. die Ausbildung von Absolventen des Berufsgrundbildungsjahres insgesamt verweigerten. Der Bundeswirtschaftsminister konnte zwar die Anrechnungspflicht verordnen, die Betriebe waren aber immer noch frei, keine Ausbildungsverträge abzuschließen. Sie hatten damit ein wichtiges Druckmittel zur Hand. Gleichzeitig suchten sie, durch den Aufbau eines „kooperativen“ Berufsgrundbildungsjahres, das stärker praxisorientiert war, ein Gegenmodell zum 
schulischen Berufsgrundbildungsjahr $\mathrm{zu}$ installieren (vgl. Greinert 1993, S. 110; Stratmann 1990, S. 190 f.).

Die Widerstände der verschiedenen Unternehmensverbände führten letztlich $1978 \mathrm{zu}$ einer Novellierung der Anrechnungsverordnung: Die Anzahl der Berufsfelder wurde erhöht, innerhalb der Berufsfelder wurden Schwerpunkte eingeführt und die Anrechnung an eine Ausbildung in einem Schwerpunkt gekoppelt. Darüber hinaus wurde die Anrechnungszeit des Berufsgrundbildungsjahres halbiert. Damit kam der Bundeswirtschaftsminister den Forderungen der Arbeitgeber nach einer engeren Verzahnung der betrieblichen Ausbildung und Berufsgrundschule entgegen. Die bildungspolitische Idee einer breiten Berufsgrundbildung hatte sich gegen die monoberufliche Orientierung der dualen Berufsausbildung nicht durchsetzen können. Schließlich ließ auch die Zustimmung der Gewerkschaften, insbesondere der IG Metall und der Gewerkschaft Öffentliche Dienste, Transport und Verkehr (ÖTV), nach, nachdem sie erkannt hatten, daß sie aufgrund ihrer durch das BBiG gestärkten Position mit den Arbeitgebern direkt die Berufsbildungspolitik gestalten konnten, ohne der staatlichen Einflußnahme zu bedürfen (vgl. Stratmann 1990, S. 191 f.; Greinert 1982, S. 27 ff.; Wiemann 1983, S. 119).

Die Unternehmensverbände sahen in der Einführung des Berufsgrundbildungsjahres einen ersten Schritt in Richtung „Verschulung“ und damit „Verstaatlichung“ des dualen Berufsbildungssystems. Wie beim Versuch der Novellierung des Berufsbildungsgesetzes in Richtung auf eine stärkere staatliche Verantwortung, lehnten die Arbeitgeberverbände und insbesondere die Kammern auch diesen Versuch einer wachsenden Einflußnahme des Staates auf die Organisation der Berufsausbildung strikt ab. Die Verbände befürchteten, ihre zentrale Position bei der Erarbeitung und Kontrolle der Ausbildungsordnungen $\mathrm{zu}$ verlieren und damit auch ihren Einfluß auf das Qualifikationsprofil der Lehrlinge. Die für eine produktionsnahe Ausbildung notwendige Spezialisierung in „Monoberufen“ wurde durch das berufsfeldbezogene Berufsgrundbildungsjahr in Frage gestellt (vgl. Stratmann 1990, S. 192).

Trotz der gescheiterten verbindlichen und flächendeckenden Einführung des Berufsgrundbildungsjahres kam ihm in der Ausbildungskrise der Jahre 1976 bis 1986 eine entscheidende sozialpolitische Bedeutung zu. Während die Zahl der Schüler im Berufsgrundbildungsjahr 1973/74 bei etwas über 10.000 lag, war sie im Schuljahr 1983/84 
auf über 100.000 angestiegen (vgl. ebd., S. 197). Aufgrund der demographisch bedingten hohen Schulabgängerzahlen stieg die Ausbildungsplatznachfrage weit über das Angebot an Ausbildungsplätzen hinaus, obgleich beispielsweise die Industrie ihr gewerbliches Ausbildungsplatzangebot in diesem Zeitraum um ca. 60 \% erhöhte (vgl. Baethge 1999, S. 491). Für Jugendliche, die keinen betrieblichen Ausbildungsplatz erhielten, bot das Berufsgrundbildungsjahr die Möglichkeit, trotzdem mit einer Ausbildung zu beginnen. Der rapide Anstieg der Zahl der Berufsgrundbildungsschüler in den späten 1970er und frühen 1980er Jahren kann insofern als „typisches Parkhaussyndrom“ (Stratmann 1990, S. 195) interpretiert werden. Die Länder nutzten das Berufsgrundbildungsjahr als Instrumentarium der Kapazitätsregelung, um das Problem der Jugendarbeitslosigkeit $\mathrm{zu}$ reduzieren. Diese Idee war aber nicht neu: Bereits 1923/24 hatte Preußen einen sozialpolitischen Maßnahmenkatalog zur Bewältigung der Jugendarbeitslosigkeit vorgelegt, der sich insbesondere auf die Ausweitung der Berufsschulpflicht und die Durchführung beruflicher Bildungsmaßnahmen in Berufsschulen stützte (vgl. Schütte 1992, S. 51).

Die strukturpolitischen Reformversuche im Berufsbildungssystem waren Mitte der 1970er weitgehend gescheitert. Die Struktur der dualen Berufsausbildung mit ihrer deutlichen Betonung der betriebsgebundenen Lehre konnte von den Unternehmen und ihren Verbänden verteidigt werden. Ihr Einfluß auf die Organisation der Berufsausbildung und die Definition qualifikatorischer Ziele war ungebrochen. Allerdings mußten sie eine Stärkung der gewerkschaftlichen Mitbestimmung hinnehmen. Die Gewerkschaften wiederum sahen in ihrer neuen Machtposition die Chance, bildungspolitische Ziele ohne Umweg über den Staat direkt mit den Arbeitgebern zu verhandeln. Sie verloren deshalb ihr Interesse an einer Ausweitung der „staatlichen Verantwortung“ im Sinne einer Verstaatlichung - für die Berufsausbildung.

\subsection{Stabilisierung der dualen Berufsausbildung}

Unter dem Eindruck einer wachsenden Jugendarbeitslosigkeit in der zweiten Hälfte der 1970er Jahre trat die Sicherung eines quantitativ ausreichenden Ausbildungsplatzangebotes in den Vordergrund. Statt strukturpolitischer Reformen stand nun die Stabilisierung des dualen Berufsausbildungssystems durch den Einsatz verschiedener strukturimmanenter Maßnahmen im Mittelpunkt der Diskussion. Es gab einen partei- 
übergreifenden Konsens darüber, daß sowohl aus sozial- als auch aus wirtschaftspolitischen Gründen alle ausbildungswilligen Jugendlichen auch einen Ausbildungsplatz erhalten sollten. Der Lehrstellenmangel traf lernbeeinträchtigte und sozial benachteiligte Jugendliche, worunter solche verstanden werden, die psychologisch diagnostizierte Verhaltensstörungen, (ehemalige) Drogenprobleme und/oder Vorstrafen aufweisen, besonders hart (vgl. Spies 1994, S. 55). Um diesen benachteiligten Jugendlichen eine Chance auf einen Ausbildungsplatz zu geben, richtete die Bundesregierung 1980 ein Programm zur Förderung der Berufsausbildung von benachteiligten Jugendlichen (Benachteiligtenprogramm) ein. Damit intervenierte die Bundesregierung erstmalig aktiv in den Ausbildungsstellenmarkt, um den Ausbildungsplatzmangel auszugleichen. Das im ersten Jahr mit 8 Mill. DM ausgestattete Programm förderte anfangs ausschließlich die Ausbildung benachteiligter Jugendlicher in außerbetrieblichen Ausbildungseinrichtungen. Seit 1982 stand zudem eine Förderung durch ausbildungsbegleitende Hilfen während einer betrieblichen Ausbildung zur Verfügung. Trotz einer Entspannung auf dem Lehrstellenmarkt in den späten 1980er Jahren verbesserte sich die Situation für benachteiligte Jugendliche nur geringfügig. Deshalb entschloß sich die Bundesregierung 1988, das Benachteiligtenprogramm mit seinen beiden Maßnahmenbereichen (außerbetriebliche Ausbildung und ausbildungsbegleitende Hilfen) gesetzlich zu institutionalisieren. Zu diesem Zweck wurde der $\S 40 \mathrm{c}$ in das Arbeitsförderungsgesetz (AFG) aufgenommen. Seit 1998 finden sich die gesetzlichen Regelungen in den $\S \S 241$ und 242 des Sozialgesetzbuches III (SGB III) (vgl. Koch 1998, S. 153; Thiel 2000, S. 61).

Die ausbildungsbegleitenden Hilfen $(\mathrm{abH})$ umfassen unterschiedliche Formen von Stützunterricht und sozialpädagogischer Begleitung im Rahmen eines betrieblichen Ausbildungsverhältnisses. Seit Aufnahme in das Benachteiligtenprogramm haben sich die ausbildungsbegleitenden Hilfen zum zahlenmäßig wichtigsten Instrument der Förderung benachteiligter Jugendlicher entwickelt: Die Zahl von ca. 2.500 geförderten Jugendlichen im Ausbildungsjahr 1983/84 stieg bis 1988 auf 16.900 an und erreichte 1999 einen Stand von 71.000. Im Vergleich dazu wurden 1999 „,nur“ 32.900 Jugendliche im Rahmen einer außerbetrieblichen Berufsausbildung unterstützt (vgl. BMBW/Berufsbildungsbericht 1984, S. 80; BMBW/Berufsbildungsbericht 1989, S. 116; BMBW/Berufsbildungsbericht 2000, S. 106). Auch die Berufsausbildung in einer außerbetrieblichen Einrichtung (BüE) ist in der Regel mit Stützunterricht und sozial- 
pädagogischer Begleitung verbunden. Träger dieser Einrichtungen sind beispielsweise Wohlfahrtsverbände, Bildungsgesellschaften (z.B. der Wirtschaftsverbände) oder privatrechtlich organisierte Bildungsträger (vgl. Vock 1992, S. 347). Erklärtes Ziel dieses Programms ist es, die Auszubildenden nach einem Jahr außerbetrieblicher Berufsausbildung in ein „,reguläres“ betriebliches Ausbildungsverhältnis zu überführen: „Nach Ablauf des ersten Jahres der Ausbildung in einer außerbetrieblichen Einrichtung ist eine weitere Förderung nur möglich, solange dem Auszubildenden auch mit ausbildungsbegleitenden Hilfen eine Ausbildungsstelle in einem Betrieb nicht vermittelt werden kann.“ (§ 241 Abs. 2, Satz 2 SGB III) Dieses Ziel konnte jedoch je nach konjunktureller Situation nur teilweise erreicht werden.

In den 1990er Jahren zeigte sich in zunehmender Weise, daß die außerbetriebliche Ausbildung im Rahmen des Benachteiligtenprogramms nicht nur die eigentliche Zielgruppe der lernbeeinträchtigten und sozial benachteiligten Jugendlichen einschloß, sondern sich auch auf die Gruppe der sogenannten „Marktbenachteiligten“ erstreckte. Dabei handelt es sich um Jugendliche, die trotz persönlicher Ausbildungsbefähigung aufgrund regionalen Lehrstellenmangels keinen betrieblichen Ausbildungsplatz erhalten. Dieses Phänomen ist insbesondere in den neuen Ländern stark ausgeprägt, wo seit der Wiedervereinigung die wirtschaftlichen Strukturen einem umwälzenden Transformationsprozeß unterliegen. Eine Folge dieser marktwirtschaftlichen Anpassung ist ein allgemeiner Lehrstellenmangel. Um den „marktbenachteiligten“ Jugendlichen eine außerbetriebliche Ausbildung zu ermöglichen, müssen sie zu Benachteiligten im Sinne des $\S 40$ c AFG bzw. des $\S 241$ SGB III erklärt werden. Infolgedessen gibt es eine deutliche Korrelation zwischen hoher Teilnehmerquote in außerbetrieblichen Ausbildungsmaßnahmen und hoher regionaler Arbeitslosigkeit. Danach müßten in Regionen mit hoher Arbeitslosigkeit sozial benachteiligte und lernbeeinträchtigte Jugendliche überproportional vertreten sein. Die Zuschreibung einer Benachteiligung im Sinne des $\S$ 40 c AFG bzw. des $\S 241$ SGB III beinhaltet jedoch das Problem einer Stigmatisierung $^{15}$ einer Gruppe von Jugendlichen, die „lediglich“ Opfer eines konjunkturell

\footnotetext{
15 Stigmatisierung bezeichnet im soziologischen Sinne den Prozeß, durch den einer Person oder einer Gruppe von Personen ein physisches, psychisches oder soziales Merkmal zugeschrieben wird, das sie von den übrigen Mitgliedern der Gesellschaft negativ unterscheidet (vgl. Peuckert 1995, S. 354-355). Eine soziale Stigmatisierung ist häufig mit langfristigen individuellen und gesellschaftlichen Konsequenzen verbunden. Im Fall der Benachteiligtenförderung ist z.B. der Übergang von einer außerbetrieblichen
} 
und/oder strukturell bedingten betrieblichen Ausbildungsplatzmangels ist. Damit dient die Stigmatisierung als „Benachteiligter“ der Stabilisierung des dualen Berufsbildungssystems. Die staatliche Förderung außerbetrieblicher Ausbildung ist grundsätzlich durch besondere individuelle Eignungsprobleme der Jugendlichen begründet (vgl. Ulrich 1998, S. 370 ff.; Laur-Ernst 2000, S. 1 ff.; Vock 1992, S. 339 ff.).

Die besondere Situation in den neuen Ländern macht es erforderlich, daß der Staat über das Benachteiligtenprogramm hinaus aktiv in den Ausbildungsstellenmarkt eingreift. Seit den frühen 1990er Jahren unterstützen die Bundesregierung und die Länder die Bereitstellung zusätzlicher außerbetrieblicher und betriebsnaher Ausbildungsplätze. Darüber hinaus fördern die neuen Länder zusätzlich bzw. erstmalig bereitgestellte betriebliche Ausbildungsplätze in unterschiedlicher Weise. Die gemeinsamen Programme von Bund und neuen Ländern begannen 1993 mit der Einrichtung der Gemeinschaftsinitiative Ost, die die Förderung von bis zu 10.000 zusätzlichen außerbetrieblichen Ausbildungsplätzen für noch nicht vermittelte Bewerber aus den neuen Ländern vorsah. Die Gemeinschaftsinitiative Ost wurde 1996 durch das Aktionsprogramm Lehrstellen Ost abgelöst. Dieses beinhaltete eine Förderung von 14.300 zusätzlichen Ausbildungsplätzen, mit der aber anstelle der Bereitstellung rein außerbetrieblicher Ausbildungsplätze eine betriebsnähere Ausbildung - z.B. in Ausbildungsverbünden - angestrebt wurde, um den Übergang in ein Beschäftigungsverhältnis nach der Ausbildung zu erleichtern. Die Lehrstelleninitiative Ost von 1998 baute auf dem Aktionsprogramm auf und zielte bei der Förderung von 17.500 zusätzlichen Ausbildungsplätzen ebenfalls auf betriebsnahe Ausbildungsmaßnahmen. Seit 1999 werden zusätzliche Ausbildungsplätze über das Ausbildungsplatzprogramm Ost gefördert. Dabei handelt es sich - wie bei den anderen Programmen seit 1996 - um betriebsnahe Ausbildungsmaßnahmen in Ausbildungsverbünden und überbetrieblichen Ausbildungseinrichtungen (vgl. BMBW/Berufsbildungsbericht 1994, S. 5; BMBW/Berufsbildungsbericht 1997, S. 29 f.; BMBF/Berufsbildungsbericht 1999, S. 38 f.; BMBF/Berufsbildungsbericht 2000, S. 33). Ziel der betriebsnahen Berufsausbildung in anerkannten Ausbildungsberufen ist eine möglichst enge Anlehnung an den 
„Normalfall“ der dualen Berufsausbildung, um die Integrationschancen der in diesen staatlich geförderten Maßnahmen ausgebildeten Jugendlichen zu erhöhen.

Darüber hinaus unterstützt die Bundesregierung mit dem seit 1998 bestehenden „Sofortprogramm zum Abbau der Jugendarbeitslosigkeit - Ausbildung, Qualifizierung und Beschäftigung Jugendlicher“" bundesweit verschiedene Maßnahmen für ca. 100.000 Jugendliche unter 25 Jahren, die entweder arbeitslos sind, keinen Berufsabschluß oder noch keine Ausbildungsstelle haben. Über die Förderung lokaler und regionaler Projekte (Artikel 2 des Sofortprogramms) - z.B. die Einrichtung regionaler Ausbildungsverbünde und die Aktivierung von Betrieben mit ausländischen Inhabern sollen zusätzliche betriebliche Ausbildungsplätze geschaffen werden. So konnten im Jahr 2000 ca. 10.000 neue betriebliche Ausbildungsplätze eingerichtet werden. Unvermittelte ausbildungsfähige Jugendliche erhalten nach Artikel 4 des Sofortprogramms eine Ausbildung in einem anerkannten Ausbildungsberuf in einer außerbetrieblichen Einrichtung, die sie - wie nach den Regelungen des § 241 SGB III nach dem ersten Ausbildungsjahr in einer betrieblichen Berufsausbildung beenden sollen. Während dies in den alten Ländern bei etwa einem Viertel der Auszubildenden gelingt, tendiert die Übergangsquote in den neuen Ländern gegen Null. Die Orientierungsgröße von ca. 10.000 zusätzlichen außerbetrieblichen Ausbildungsplätzen wurde 1999 um das Doppelte überschritten (vgl. Friedrich/Troltsch/Westhoff 1999, S. 8). Im Jahr 2000 lag die Zahl bei lediglich 3.800 Teilnehmern (vgl. BMBF/Berufsbildungsbericht 2001, Kap. 1.1.3, S. 1). Weitere Maßnahmen des Sofortprogramms umfassen beschäftigungsbegleitende Hilfen, das Nachholen eines Hauptschulabschlusses, berufliche Nachqualifizierungen, Trainingsprogramme und Lohnkostenzuschüsse für Betriebe (vgl. Richtlinien zur Durchführung des Sofortprogramms zum Abbau der Jugendarbeitslosigkeit vom 1. Dezember 1999, S. 1 ff.; Friedrich/Troltsch/Westhoff 1999, S. 6 ff.). Diejenigen Maßnahmen, die sich an Jugendliche ohne Berufsausbildung richten, zielen dabei auf eine Überführung der Jugendlichen in ein reguläres Berufsbildungsverhältnis.

Neben den Gemeinschaftsprogrammen der Bundesregierung und der Länder haben einzelne Länder, insbesondere die neuen, eigene Förderprogramme zur Schaffung zusätzlicher betrieblicher Ausbildungsplätze. Je nach Ausgestaltung gibt es finanzielle Zuschüsse für die erstmalige Ausbildung, die Ausbildung über den eigenen Bedarf oder die Ausbildung bei Existenzgründern. Das Land Brandenburg förderte 1998 betriebliche 
Ausbildungsverhältnisse im ersten Ausbildungsjahr in erstmalig ausbildenden Betrieben mit 3.000 DM. Zur Verbesserung der materiell-technischen Ausbildungsvoraussetzungen unterstützte Sachsen-Anhalt im selben Jahr Investitionen in zusätzliche Ausbildungsplätze mit $20 \%$ bzw. $25 \%$ pro Ausbildungsplatz. In den anderen neuen Ländern gab es für zusätzliche betriebliche Ausbildungsplätze Subventionen in vergleichbarer Höhe (vgl. BMBF/Berufsbildungsbericht 1999, S. 39). Für das Ausbildungsjahr 1998/99 planten die neuen Länder ca. 66.700 betriebliche Ausbildungsverhältnisse über die verschiedenen Programme finanziell zu unterstützen. Damit wurden ca. $70 \%$ der in den neuen Ländern abgeschlossenen betrieblichen Ausbildungsverträge teilweise staatlich gefördert (vgl. BMBF/Berufsbildungsbericht 1999, S. 7). Befragungen von Betrieben in den neuen Ländern haben gezeigt, daß über ein Drittel der kleinen Betriebe ohne finanzielle Unterstützung nicht ausbilden würde. Bei mittleren und großen Betrieben liegt die Zahl immerhin zwischen einem Viertel und einem Fünftel (vgl. Degen/Walden 1994, S. 7). Die verschiedenen finanziellen Subventionen für Ausbildungsbetriebe machen einerseits den klaren Willen der staatlichen Akteure deutlich, die Struktur der (einzel-)betrieblich dominierten dualen Berufsausbildung zu erhalten und zu stabilisieren, andererseits besteht die Gefahr einer indirekten Verstaatlichung der Berufsausbildung in den neuen Ländern unter Aufrechterhaltung der betrieblichen Organisationsmacht.

Nach der Verabschiedung des Berufsbildungsgesetzes 1969, das die historisch gewachsene Struktur der dualen Berufsausbildung gesetzlich festschrieb, stand die Berufsbildung in den frühen 1970er Jahren auf der Tagesordnung der von der neuen sozial-liberalen Regierung geplanten ,inneren Reformen“. Die berufliche Bildung sollte wie die allgemeine Bildung zu einer öffentlichen Aufgabe gemacht werden. Dies sollte insbesondere durch eine Verstärkung der Vollzeitschulen erfolgen. Die Einführung des Berufsgrundbildungsjahres war ein erster Schritt in diese Richtung. Der Entwurf zur Novellierung des Berufsbildungsgesetzes enthielt darüber hinaus Regelungen zur Stärkung der staatlichen Verantwortung in der Berufsbildungsverwaltung, wie z.B. die Schaffung einer eigenen Behörde. Die Novellierung scheiterte aber am Widerstand der Unternehmensverbände. Das Ausbildungsplatzförderungsgesetz von 1976 mit der darin geschaffenen Möglichkeit einer betrieblichen Ausbildungsabgabe wurde 1980 vom Bundesverfassungsgericht für verfassungswidrig erklärt. In dem daraufhin 
verabschiedeten Berufsbildungsförderungsgesetz fehlte die Regelung einer betrieblichen Umlagefinanzierung. Auch die flächendeckende obligatorische Einführung des schulischen Berufsgrundbildungsjahres konnte sich trotz einiger ambitionierter Versuche, wie z.B. in Niedersachsen, nicht durchsetzen. Spätestens mit dem Regierungswechsel von 1982 war es offensichtlich, daß die berufsbildungspolitischen Reformideen der 1970er Jahre gescheitert waren. Die neue liberal-konservative Regierung beschränkte sich in ihrer Berufsbildungspolitik auf Maßnahmen, die der Stabilisierung des dualen Berufsbildungssystems und seiner im Berufsbildungsgesetz festgeschriebenen institutionellen Basisstrukturen dienten. Ansonsten überließ sie - ganz im Sinne des ,private interest government' - die Berufsausbildung der sozialpartnerschaftlichen Selbstverwaltung.

Sowohl das Benachteiligtenprogramm als auch die verschiedenen Aktionsprogramme in den neuen Länder sind Beispiele für eine das duale Berufsausbildungssystem stabilisierende Maßnahmenpolitik. Der „Normalfall“ der (einzel-)betrieblichen Ausbildung ist das Ziel, an dem sich die Programme ausrichten: Sei es in Form ausbildungsbegleitender Hilfen, außerbetrieblicher Ausbildungen, die in eine betriebliche Berufsausbildung münden, oder betriebsnaher Ausbildungen in Ausbildungsverbünden. Im Sinne des ,policy feedback' zeigt sich, daß die Akteure aus den Reformbestrebungen der 1970er Jahre gelernt haben, die bestehenden Basisstrukturen der Berufsausbildung nicht in Frage zu stellen, sondern neue Politiken innerhalb dieser Rahmenbedingungen zu konzipieren und umzusetzen. 


\section{Aussichten für das duale Berufsausbildungssystem}

Die in einer tayloristisch-fordistischen Industriegesellschaft effiziente, auf dem Berufsprinzip basierende Arbeitsorganisation wird in einer sich ökonomisch und technologisch wandelnden Wirtschaft zum Hemmschuh für schnelle Anpassungsprozesse und Innovationen. Das berufsbezogene Produktionsmodell Deutschlands stellte eine optimale Lösung für die Anforderungen einer kontinuierlich inkrementellen Qualitätsverbesserung bestehender Produkte dar. Dieser Produktionsmodus behindert jedoch im Zeitalter des Post-Taylorismus bereichsübergreifende und prozeßorientierte Arbeitsweisen $^{16}$, die entscheidende Elemente innovativer und lernender Organisationen bilden (vgl. Cattero 1998, S. 228; Zukunftskommission der Friedrich-Ebert-Stiftung 1998, S. 191 ff.). „Die Schnittstellen, die [...] die Wurzel der Anpassungsprobleme der deutschen Industrie bilden, resultieren direkt aus diesen internen Demarkationen und externen Abgrenzungen, die für Berufsgemeinschaften charakteristisch sind“" (Kern/Sabel 1994, S. 615). Fraglich ist, inwieweit die Berufsausbildung mit ihrem Berufskonzept neue, integrative Strukturen der Arbeitsorganisation adaptieren kann.

Eine weitere Problematik für die Zukunft des dualen Systems der Berufsausbildung liegt in seiner fehlenden Akzeptanz im Dienstleistungssektor, wo aufgrund schwacher Organisierung sowohl der Arbeitgeber als auch der Arbeitnehmer die kollektiven Akteure zur Durchsetzung eigener Berufsbilder mit spezifischen Ausbildungsordnungen fehlen (vgl. Heidenreich 1998, S. 333; Baethge 1996, S. 117; Baethge 1999, S. 495 ff.). In diesem Bereich kommt ferner die seit den 60er Jahren fortschreitende Bildungsexpansion zum Tragen, da anspruchsvollere Dienstleistungstätigkeiten zunehmend von Hochschulabsolventen besetzt werden, denen eher symbolanalytische ${ }^{17}$ Qualifikationen zugesprochen werden und die mithin in direkter Konkurrenz zu Absolventen des dualen Berufsbildungssystems stehen. Aber selbst in den angestammten Bereichen der dualen Berufsausbildung besetzen Betriebe Techniker- und Meisterposi-

16 Dies zeigt sich z.B. bei einem Vergleich mit japanischen Unternehmen, die eine berufsorientierte Ausbildung im deutschen Sinne nicht kennen. Aufgrund der fehlenden Beruflichkeit lassen sich intraorganisationale Kommunikations- und Kooperationsprozesse leichter gestalten (vgl. Ruth 2002, S. 111 f.)

${ }^{17}$ Der von R. Reich eingeführte Begriff des Symbolanalytikers als einer für die zukünftige Entwicklung der hochindustrialisierten Wirtschaften zentralen Tätigkeitskategorie umfaßt vier spezifizierende Elemente: Abstraktion, Systemdenken, Experimentieren und Zusammenarbeit (vgl. Reich 1992, S. 171 ff.). 
tionen bereits teilweise mit Fachhochschulabsolventen, wodurch gelernte Facharbeiter in ihren klassischen Aufstiegsmöglichkeiten eingeschränkt werden und die soziale Attraktivität der dualen Berufsausbildung leidet (vgl. Baethge 1996, S. 120; Heidenreich 1998, S. 331-333). Darüber hinaus bieten die durch überbetriebliche Akteure, wie Kammern und Verbände, erarbeiteten, auf Standardisierung zielenden Ausbildungsordnungen wenig Spielraum für heterogene, betriebsspezifische Qualifikationsanforderungen. Das Dilemma zwischen Vereinheitlichung und Verbetrieblichung der Berufsausbildung, das im Zeitalter der Massenproduktion zugunsten der Vereinheitlichung gelöst wurde, ist im Zuge des post-tayloristischen Wandels neu auszubalancieren. Dabei bleibt die Rolle der überbetrieblichen Regulationsinstanzen und deren Anpassungsfähigkeit zu klären (vgl. Heidenreich 1998, S. 326-329; Lutz 1994, S. 22 f.).

Das duale System der deutschen Berufsausbildung entstand aus der Notwendigkeit, sozialintegrative Instrumentarien als Stabilisierungsfaktor zum Machterhalt des monarchischen Systems zu reinstitutionalisieren. Die Wiederbelebung der im Zuge der gewerblichen Liberalisierung marginalisierten Lehrlingsausbildung des Handwerks bot durch ihre Hierarchisierung und Einbettung in familiäre Strukturen die gewünschten sozialintegrativen Vorteile, um ungelernte jugendliche Arbeiter systemfunktional zu sozialisieren. Gleichermaßen konnte durch eine Ausweitung des Fortbildungsschulunterrichts wachsenden ökonomisch-industriellen Qualifikationsanforderungen entsprochen werden. Die Verwaltung der Berufsausbildung durch die wirtschaftsnahen Kammern und die administrative Zuständigkeit des Handelsministeriums garantierten zudem eine an wirtschaftspolitischen Erwägungen ausgerichtete Berufsausbildung.

Die nach dem Zweiten Weltkrieg begonnene Konsolidierungsphase der Berufsausbildung fand ihren Abschluß in der Verabschiedung des Berufsbildungsgesetzes von 1969. Trotz ihres unumstrittenen Beitrages zur Wettbewerbsfähigkeit der deutschen Wirtschaft steht die Berufsausbildung in einem fragilen Spannungsverhältnis zwischen den gesellschaftlichen Teilsystemen. So ist das Angebot an betrieblichen Ausbildungsplätzen ökonomisch determiniert, wohingegen die Nachfrage nach Ausbildungsplätzen von mehreren Faktoren abhängt. Dieser immanente Fehlsteuerungsmechanismus führt immer wieder zu gesellschaftlichen Friktionen, die ein Eingreifen des Staates nach sich ziehen, da der Berufsausbildung noch heute eine wichtige sozialintegrative Bedeutung zukommt. In diesem Spannungsfeld zwischen gesellschaftspolitischer und 
wirtschaftspolitischer Institution ist die duale Berufsausbildung einem besonderen Adaptionsdruck ausgesetzt. 


\section{Teil III: Duale Berufsausbildung in den USA?}

Bereits um die Jahrhundertwende gab es in den USA eine Debatte um die internationale Konkurrenzfähigkeit der amerikanischen Wirtschaft. Junge Industrienationen hatten den Vorsprung der industriellen Vorreiter verkleinert, und einige setzten sogar zum Überholmanöver an. Die bedeutendste junge Industrienation war das deutsche Kaiserreich: gesellschaftsstrukturell höchst konservativ, aber industriell innovativ. Ausländischen Beobachtern schien der Schlüssel zum Erfolg in dem Berufsbildungssystem zu liegen, das trotz seiner traditionellen Orientierung an der alten Lehrlingsausbildung der Handwerksgilden den aufstrebenden Industrieunternehmen qualifizierte Fachkräfte lieferte. Dies war nur deshalb möglich, weil es Anforderungen der Industrie adaptierte und neue Berufsbilder schuf. Amerikanische Industrievertreter, Politiker und Pädagogen propagierten die Einführung des deutschen Berufsbildungssystems, um die amerikanische Wirtschaft für den internationalen Wettbewerb leistungsfähiger zu machen, aber auch um die wachsende Jugendarbeitslosigkeit zu bekämpfen. Das deutsche Ausbildungssystem war der amerikanischen Wirtschaft allerdings gar nicht so fremd, da es in einigen Einzelstaaten eine solche Lehrlingsausbildung immer noch gab. Bekanntestes Beispiel ist der Staat Wisconsin, wo sich ein besonders hoher Prozentsatz an deutschen Immigranten niedergelassen hat, die ihre Traditionen fortführten und so die Basis für ein Lehrlingssystem schufen, das in einigen Branchen noch existiert. Doch trotz eigener Erfahrungen konnte sich bis heute eine dem deutschen Berufsbildungssystem vergleichbare berufliche Ausbildung in den USA nicht durchsetzen. Im folgenden soll dieses offensichtliche Scheitern einer Institution eingehend untersucht werden, indem zunächst die historischen Grundlagen amerikanischer Bildungspolitik dargestellt werden. Daraufhin folgt eine Analyse bestehender Institutionen und Interessen und schließlich der Einflüsse bundesstaatlicher Ausbildungspolitiken seit dem Ende des Zweiten Weltkrieges. 


\section{Historische Grundlagen}

Das deutsche duale Berufsbildungssystem basiert auf der institutionalisierten Trennung von akademischer und beruflicher Bildung. Relativ früh entscheidet es sich, ob ein Kind durch den Besuch eines Gymnasiums auf die Universität vorbereitet wird oder ob es die berufsorientierte Bildungsschiene einschlägt. Zwei in sich so abgeschlossene Bildungssysteme mit wenig Übergangsmöglichkeiten konnten nur in einer klassenorientierten Gesellschaft entstehen, in der die sozialen Aufstiegsmöglichkeiten beschränkt sind. Anders verhält es sich in den USA, wo eine Nation aus dem Sieg über die beherrschende Kolonialmacht entstand und sich die Gesellschaft an einem demokratisch-egalitären Ideal orientiert. Bildung wurde zur wichtigsten Institution des ,nation building ' und sollte in Form der ,mass education' die Gesellschaft dem demokratisch-egalitären Ideal näher bringen. Eine separarierte berufliche Bildung galt als Ausdruck einer vordemokratischen Gesellschaft, in der über den Zugang zu Bildung bestimmte Gruppen privilegiert werden. Die Forderung nach ,mass education“ war die amerikanische Variante früher Sozialpolitik: Jeder Bürger erhält die gleichen Startchancen (equality of opportunity) - sein weiterer Erfolg oder Mißerfolg bleibt ihm dann selber überlassen.

\subsection{Staatliche Institutionalisierung und Bildung}

Die Schaffung eines öffentlich organisierten Schulsystems hängt eng mit der Entstehung des amerikanischen Staatssystems zusammen. Die Notwendigkeit, eine nationale Identität und ein eigenes politisches System infolge der Unabhängigkeit von Großbritannien aufzubauen, verlieh der staatlich organisierten schulischen Bildung eine zentrale Rolle im gesellschaftspolitischen Konsolidierungsprozeß. Trotz dieser faktischen Bedeutung der Schulbildung wird sie in der Verfassung nicht erwähnt. Nach dem Sieg der Amerikaner über die englische Kolonialherrschaft 1783 galt es, eine eigene politische Organisation zu schaffen, um durch innere Stabilität die Furcht vor Anarchie und Despotismus $\mathrm{zu}$ überwinden. Diese konnte auf bereits unter der englischen Administration entstandene Strukturen der Selbstverwaltung zurückgreifen. Andernfalls wäre die innere Revolution der USA nicht möglich und das Scheitern dieses ersten neuzeitlichen Versuchs der Demokratisierung einer Gesellschaft vorherbestimmt gewesen (vgl. Heideking 1992, S. 68 ff.). 
Zentrales Merkmal der Vereinigten Staaten ist die fehlende Erfahrung einer absolutistischen Monarchie als vormoderner Staatsordnung mit stehender Armee und entsprechender Bürokratie. Die Ausprägung des monarchistischen Absolutismus in Europa führte dort zu zentralistischen Tendenzen in der Machtkonzentration. Nicht nur gegen das britische Empire, sondern gerade auch gegen diesen Zentralismus richtete sich der Unabhängigkeitskampf der amerikanischen Kolonien, deren Bewohner mit ihrer Emigration der staatlichen Unterdrückung zu entfliehen suchten. Sie lehnten ,any European-style notion of concentrated political sovereignty" (Skocpol 1995, S. 20) ab (vgl. Weir/Orloff/Skocpol 1988, S. 18; Skocpol 1995, S. 19 f.). Der Ablehnung zentralisierter Macht und der Angst vor Machtmißbrauch stand die Idee des ,civic humanism', einer auf bürgerlichen Tugenden, wie moralischer Integrität, Fleiß, Ehrlichkeit und Sittenstrenge, basierenden Gesellschaft, gegenüber. Diese Verbindung aus Ängsten und bürgerlich-protestantischen Tugenden wird auch als „CountryIdeologie" (Heideking 1992, S. 64) bezeichnet, deren radikaldemokratisches Element durch Revolution und Krieg gestärkt - eine egalitäre Dynamik entwickelte. Mit der Verabschiedung einer kodifizierten Verfassung 1788 fanden die amerikanischen Gründungsväter einen Ersatz für andere Formen der nationalen Identitätsstiftung. Folgende politische Ideen lagen der Verfassungsgebung zugrunde: Föderalismus, Konstitutionalismus, Individualismus, Liberalismus und Demokratie. Der Konstitutionalismus beruft sich auf die naturrechtliche Idee des ,balanced state', in dem die Macht der Organe durch, checks and balances' beschränkt ist. Ein weiteres Prinzip des Konstitutionalismus ist die Idee der Volkssouveränität, die von James Madison, einem der Gründungsväter der Verfassung, grundsätzlich bejaht wurde, deren uneingeschränkter Geltung er aber mißtraute. Trotz grundsätzlichem Konsens über die politischen Prinzipien gab es in deren Auslegung divergierende Ansätze.

Zwei Typen des Republikanismus kristallisierten sich in den Debatten um die politische Organisation des Staatswesens heraus: Die eine Seite befürwortete das Modell einer „Bundesrepublik”, in die die Einzelstaaten im Sinne einer national-föderativen Ordnung eingebunden sind. Diese staatliche Form sollte der jungen Nation innere Stabilität und äußere Sicherheit verschaffen, indem die Macht der Einzelstaaten beschränkt und der Bundesregierung eine weitreichende Kompetenz zugeteilt werden sollte. Vertreter dieser staatlichen Verfassung bezeichneten sich als ,Federalists', um den negativ konnotierten Begriff der ,Nationalists' zu vermeiden. Die andere Seite berief sich auf 
die Staatslehre Montesquieus und argumentierte mit der geographischen Größe der USA gegen eine republikanische Staatsform auf Bundesebene. Statt dessen sollten sich die Vereinigten Staaten als lockerer Staatenbund homogen zusammengesetzter Einzelrepubliken konstituieren. Hintergrund dieser Überlegungen war die Angst vor einer Konzentration und Zentralisation der Macht und einer möglichen Tendenz zum Despotismus. Protagonisten dieser extremen Form einer föderalistischen Ordnung wurden trotz des scheinbaren Widerspruches aufgrund ihrer Opposition zu den ,Federalists' als ,Anti-Federalists' bezeichnet (vgl. Kamphausen 1992, S. 272; Heideking 1992, S. 77 f.). Mit George Washington als erstem Präsidenten hatten sich die Federalists zunächst durchgesetzt, bis in Gestalt von Thomas Jefferson die egalitär-demokratischen Überzeugungen der ,Anti-Federalists' wieder zur Geltung kamen. Jefferson setzte auf mehr Autonomie der Einzelstaaten und die Stärkung der Mitspracherechte des einfachen Bürgers. „Die Federalists hatten den Nationalstaat geschaffen und seine wirtschaftlichen Grundlagen gelegt; die Republicans sorgten dafür, daß der Weg dieses Staates in die Moderne freiheitlicher und weniger zentralistisch verlief..." (Heideking 1992, S. 84).

Die Verfassung als identitätsstiftender Ersatz und die Schaffung einer neuen staatlichen Ordnung bildeten den Rahmen für den Prozeß der gesellschaftlichen Transformation. Dieser Prozeß war jedoch auf die Entwicklung nationaler Institutionen angewiesen, um die neue Ordnung mit Leben zu füllen. Der Erfolg des Republikanismus hing nach Meinung der Gründungsväter der USA von der Tugend und dem Charakter der Bürger ab: „We have changed our forms of government, but it remains yet to effect a revolution in our principles, opinions and manners so as to accommodate them to the forms of government we have adopted" (Benjamin Rush, in: Green 1990, S. 178). Die Herausbildung des tugendhaften republikanischen Bürgers oblag fortan der Schule, die auf diese Weise Teil des nationalen Projekts wurde. Seitdem ist das Schulwesen ein ,political issue' und unterliegt ständigem Druck, sich in die eine oder andere Weise zu entwickeln und bestimmte Funktionen auszuüben. Die „neue“ Funktion der Schule als Instrument kultureller Konditionierung und Bürgerausbildung stand allerdings dem Ideal der Aufklärung entgegen, Freiheit durch Bildung zu ermöglichen. Zwischen diesen beiden Polen, der Idee von Freiheit einerseits und der politischen Sozialisation andererseits, ist die schulische Bildung bis heute gefangen (vgl. Green 1990, S. 179 ff.). 
Die Schaffung eines Schulwesens war aber nicht die einzige nationale Institution, die für den Prozeß der ,construction of nationhood' notwendig war. Darüber hinaus fehlte den Vereinigten Staaten eine zentrale, professionalisierte Bürokratie. Im Gegensatz zu europäischen Nationen, die vor ihrer Demokratisierung eine gut ausgebildete Bürokratie besaßen, ging der Prozeß der Demokratisierung in den USA dem der Bürokratisierung voraus. Infolge dieser Entwicklung nutzten politische Parteien die sich entwickelnden staatlich-administrativen Strukturen für ihre organisatorischen Interessen. Es entstand ein umfassendes Patronage-System (pork-barrel politics) (vgl. Weir/Orloff/Skocpol 1988, S. 18). Erst im Zuge fortschreitender Industrialisierung nahmen reformatorische Bestrebungen zu, die das Patronage-System durch eine professionalisierte Bürokratie ersetzen wollten. Besondere Aufmerksamkeit kam dabei neugeschaffenen ,regulatory agencies' zu, die neben dem institutionalisierten Patronage-System die Ausbildung eines originären bürokratischen Expertentums befördern sollten (vgl. Skocpol 1995, S. 86). Erst zu Beginn des 20. Jahrhunderts konnten Fortschritte in der Bürokratisierung und Professionalisierung der Verwaltung erzielt werden. Diese Entwicklung blieb zunächst aber auf Gemeinde- und Einzelstaatsebene beschränkt: „,...bureaucraticprofessional transformations happened piecemeal through reform movements spearheaded by the new middle classes" (ebd., S. 23). Die Schaffung neuer Behörden, wie der ,regulatory agencies', stieß häufig auf den Widerstand betroffener Interessengruppen, die durch Gerichtsentscheidungen die Macht der neuen Verwaltung zu begrenzen suchten. Den Gerichten fiel dabei eine bedeutende Rolle in der Ausgestaltung staatlich-administrativer Kompetenzen zu. Ihre Macht basierte auf dem verfassungsmäßigen Recht des ,judicial review'. Dadurch konnten sie neue bundesstaatliche Initiativen durch Berufung auf die Verfassung verhindern oder wenigstens in ihrer Reichweite beschneiden (vgl. Skocpol 1992, S. 46; Skocpol 1995, S. 25 und 86; Avery/Steinisch 1992, S. 138). Neue bundesstaatliche Politiken benötigten jedoch immer die Unterstützung einer breiten Basis in den dezentralisierten Machtzentren lokaler Politiknetzwerke, um eine Mehrheit im Kongreß zu erhalten. Dies bestärkte die Praxis des Patronage-Systems (vgl. Weir/Orloff/Skocpol 1988, S. 20; Skocpol 1995, S. 23). „America's polity in the nineteenth century has been apty described as a polity of ,courts and parties' operating in a multi-tiered federal framework" (Skocpol 1995, S. 90). Die frühe Demokratisierung der amerikanischen Gesellschaft förderte die Ausdehnung indirekter wohlfahrtsstaatlicher Maßnahmen des Staates in Form von Subventionen oder , land grants', deren Verwendung weitgehenden lokalen 
Ermessensspielräumen unterlag. Das öffentliche Schulwesen fand dabei besondere Unterstützung als eine Institution, die allen Bürgern gleiche Möglichkeiten eröffnen sollte. Gerade die amerikanischen Arbeiter nutzten ihre politischen Rechte nicht, um nationale Sozialversicherungsprogramme durchzusetzen, sondern um eine Ausweitung öffentlicher Schulbildung zu erreichen: „,...American lower strata used their franchise much earlier to win educational opportunities from local political leadership" (Heidenheimer 1982, S. 274).

Die dominierende Ebene blieb jedoch aufgrund der fehlenden zentralen Bürokratie die ,local community', in der freiwillige Kräfte und private Initiativen auch weiterhin großen Einfluß auf die schulische Bildung ausübten. Diese Formen des lokalen Engagements in allen die ,community' betreffenden Bereichen lassen sich im Begriff des ,participatory localism' zusammenfassen. Im Wettbewerb zwischen lokalen, privaten, einzelstaatlichen und bundesstaatlichen Politiken entsteht die staatliche Ordnung eines dezentralisierten Föderalismus (vgl. Green 1990, S. 174 f.; Skocpol 1995, S. 23). Gerade wegen des schwachen Zentralstaates konnte die Ausweitung des öffentlichen Schulwesens durch staatliche Initiativen vorangetrieben werden, da die Angst vor einer möglichen Instrumentalisierung der Schule durch einen starken Staat unbegründet schien: ,...educational initiatives flourished uniquely at American subnational levels" (Heidenheimer 1982, S. 271). Die fehlende zentrale, professionalisierte Bürokratie und mithin das Nichtvorhandensein systematischer Verwaltungskarrieren verhinderte darüber hinaus eine Standardisierung der Qualifikation. Professionalisierte Bürokratien in Europa erzeugten einen Anreiz, schulische Bildung $\mathrm{zu}$ standardisieren, um die Schüler auf die Anforderungen einer möglichen Beamtenkarriere vorzubereiten. Daraus entwickelte sich eine enge Verbindung zwischen Zeugnis und Beschäftigungsmöglichkeiten auf kontinentaleuropäischen Arbeitsmärkten. Gleichzeitig sollte die soziale Position ausgebildeter Arbeiter durch eine Verbesserung der Lehrlingsausbildung gefestigt werden, um deren Aufstiegsbestrebungen zu vermindern. Im Gegensatz dazu waren die Verbindungen zwischen schulischer Bildung und Arbeitsmarkt in den Vereinigten Staaten flexibler (vgl. Green 1990, S. 199; Heidenheimer 1982, S. 272 f. und 280 ff.). „Professions were slow to instigate standardized national entry requirements" (Green 1990, S. 199). Infolge des allgemeinen Wahlrechts (für weiße Männer) und der der ,country ideology’ innewohnenden egalitären Dynamik konnte sich ein hierarchisches Modell der Schulbildung nicht durchsetzen. Den de jure- 
Schranken, die den unteren Schichten in Europa den Zugang zu höheren Ausbildungsstufen verwehrten, standen in Amerika de facto-Schranken in Form unterschiedlicher Qualität der Schulen aufgrund einer dezentralisierten Finanzierung gegenüber (vgl. Heidenheimer 1982, S. 284). Trotzdem konnte die „mythology of egalitarian capitalism" (Green 1990, S. 195) dadurch überleben, daß es einen allgemeinen Glauben an die ,opportunity' in der Gesellschaft gab. Dieser Glaube wurde durch zwei Faktoren bestärkt: einerseits die Abwesenheit einer vorkapitalistischen, feudalen Sozialordnung und andererseits die grenzenlos erscheinenden Ressourcen in Form billigen Lands. Die Chance des sozialen Aufstiegs schien allgegenwärtig. Die Realität sah jedoch anders aus: So schafften zwischen 1830 und 1840 lediglich 10 Prozent der Arbeiter in Boston den Aufstieg in die Berufswelt der Mittelschicht: ,...social mobility in general was probably not as great as has often been suggested...” (Green 1990, S. 195). In den industriellen Städten entstand eine Arbeiterklasse, der organisatorische Traditionen aus der Handwerkerbewegung fehlten und deren geographische Mobilität kollektives Handeln erschwerte. Außerdem hatten ethnische und kulturelle Unterschiede einen negativen Einfluß auf die Organisationsfähigkeit. Dieser Umstand gewann in der weiteren Entwicklung sogar noch an Bedeutung (vgl. Avery/Steinisch 1992, S. 138). Die frühen Gewerkschaftsbewegungen und Arbeiterorganisationen unterstützten grundsätzlich die Schaffung eines steuerfinanzierten Schulsystems, durch das sie sich größere soziale Gleichheit erhofften. Eine Erklärung der General Trades Union von Cincinnati forderte 1836 die Einrichtung eines ,national system of education'.

Widerstand gegen die Reform des Schulwesens und einen stärkeren Einfluß der Einzelstaaten kam von der katholischen Kirche, die den Verlust der Unabhängigkeit ihrer Schulen befürchtete, und anderen privaten Schulen, denen durch steuerfinanzierte öffentliche Schulen eine neue Konkurrenz erwuchs. Das Erbe dieser Oppositionsbewegung wird noch heute in der Vielzahl unabhängiger Schulen und der weitreichenden lokalen Autonomie im Bereich des Schulwesens deutlich. Trotzdem konnten die Schulreformer Erfolge in der Ausweitung des öffentlichen Schulwesens aufweisen, da die von ihnen propagierten Ideen der ,opportunities for all' und der sozialen Mobilität durch den Prozeß der Industrialisierung und wachsenden Immigration in einem neuen Kontext standen (vgl. Green 1990, S. 191 ff.). „The whole ideology of American republicanism rested so firmly on a belief in freedom, opportunity and moral 
virtue, that opposition to mass education would have been unthinkably un-American for most northerners" (ebd., S. 190).

Darüber hinaus ließ sich mit der Ausweitung einer für alle Schichten offenen schulischen Bildung, die Idee eines demokratischen Kapitalismus legitimieren. Die Industrialisierung erweiterte die Funktion öffentlicher Schulbildung über die Idee der ,citizenship education' hinaus: Ökonomische Effizienzkriterien und industrielle Anforderungen an die Qualität der ,labour force' gewannen in den öffentlichen Debatten um das Schulwesen an Bedeutung. In den industriellen Städten wuchs eine ,urban workforce' heran, deren wesentlichstes qualifikatorisches Merkmal Pünktlichkeit war, die im arbeitsteiligen Produktionsprozeß der modernen Fabriken zu einer zentralen Tugend wurde. Die Vermittlung von Werten, wie Pünktlichkeit und Gewissenhaftigkeit, wurde zur bestimmenden Aufgabe der öffentlichen Schule. Infolge der zunehmenden Mechanisierung des Produktionsprozesses verloren viele Jugendliche ihre Beschäftigungsmöglichkeiten in den Fabriken und stellten in den Städten ein Potential sozialer Unruhe dar. Bis zur Industrialisierung waren Lebensläufe der Jugendlichen durch eine nahtlose Verkettung institutioneller Arrangements klar vorgegeben und daher das Problem der ,idleness' nicht bekannt.

Das Problem der unterbeschäftigten Jugendlichen in den Städten erforderte neue Lösungsansätze, da sich andere soziale Institutionen, wie die Lehrlingsausbildung, in einem Zustand der Agonie befanden. „Traditional practices declined not only prior to industrialization but also before the creation of any network of institutions to contain and manage young people" (Katz 1988, S. 106). Der Rückgriff auf die junge Institution der öffentlichen Schule als Aufbewahrungsort für arbeitslose Jugendliche war die nächstliegende Alternative zur Lösung des Problems (vgl. Gumbert/Spring 1974, S. 121). Kaum stiegen die Arbeitsmöglichkeiten für Jugendliche in den Fabriken, sank gleichzeitig die Zahl der aus Arbeiterfamilien stammenden Schüler. Im Gegensatz dazu suchte die Mittelklasse die Gefahr des sozialen Abstiegs durch die Ausdehnung der schulischen Bildung ihrer Kinder abzuwehren. Die Position des klassischen Handwerkers, die auf der Verbindung von Qualifikation und ökonomischem Erfolg basierte, verlor im Zeichen des technologischen Wandels an Bedeutung. Die Unsicherheit der Mittelklasse über ihren zukünftigen sozialen Status führte zur Forderung nach Einrichtung eines ,public secondary school system' (vgl. Katz 1988, S. 107; Heidenheimer 1982, S. 278). Die Industrialisierung förderte durch ihren großen Bedarf 
an Arbeitskräften die Urbanisierung in den industriellen Ballungszentren. Dort fand sich auch die Mehrzahl der Immigranten, die in der Hoffnung auf eine Verbesserung ihrer wirtschaftlichen Situation ihre Heimatländer verlassen hatten. Die wachsende Immigration stellte neben der Industrialisierung eines der gravierendsten sozialen Phänomene in den USA des 19. Jahrhunderts dar. Der Bedarf an ungelernten Arbeitskräften stieg infolge der arbeitsteiligen, spezialisierten Produktionsweise und konnte durch den nicht nachlassenden Strom arbeitsuchender Immigranten kostengünstig befriedigt werden. Diese boten ihre Arbeitskraft unterhalb des Lohnniveaus amerikanischer Arbeiter an und erzeugten dadurch ein Konfliktpotential, das sich in einer wachsenden Opposition gegen die Immigration neuer Arbeiter manifestierte. „Kulturelle Verschiedenartigkeit und die Tendenz, ein ethnisches Selbstbewußtsein zu entwickeln, wenn auch oft eher als Gegenreaktion auf nativistische Anfeindungen, verstärkten die Divergenzen" (Nagler 1992, S. 14). Als Reaktion auf kulturelle Heterogenität und „unzivilisiertes” Verhalten der ,immigrant poor’ entstand eine Ideologie der Assimilation durch die Vermittlung allgemein anerkannter protestantischer Werte. Welche Institution hätte dieser Funktion besser gerecht werden können als das öffentliche Schulwesen (vgl. Avery/Steinisch 1992, S. 128 f.). Die Rolle der öffentlichen Schulen, durch , citizenship education' eine nationale, demokratisch orientierte Kultur zu schaffen, erhielt zusätzliche Bedeutung infolge des Prozesses der Instrumentalisierung der Schule als zentraler Institution kultureller Standardisierung. „Educational leaders have tried to transform immigrant newcomers and other ,outsiders' into individuals who matched their idealized image of what an ,American' should be..." (Tyack/Cuban 1995, S. 2).

Die Werte und Prinzipien des öffentlichen Schulwesens in den USA sind eng mit der Entstehung einer nationalstaatlichen Konfiguration verbunden. Schulische Bildung in Form der ,citizenship education' diente als Instrument zur inneren Stabilisierung und Festigung der amerikanischen Demokratie. Die frühe Politisierung der Schule führte jedoch immer wieder zu ihrer Instrumentalisierung bei der Lösung verschiedenster sozialer Probleme: So galt es, wachsende Kriminalität und Armut in den Städten zu reduzieren, kulturelle Heterogenität $\mathrm{zu}$ nivellieren, eine industrielle Arbeiterschaft auszubilden, den sozialen Abstieg der Mittelschicht $\mathrm{zu}$ verhindern und viele andere soziale Verschiebungen zu beheben. Anders als in Europa bildet die schulische Bildung 
„a central component of the American notion of welfare” (Janowitz, in: Heidenheimer 1982, S. 274).

Anstatt zentralstaatlich organisierter Wohlfahrt europäischer Prägung unterstützten insbesondere auch die unteren Schichten eine Ausweitung des öffentlichen Schulwesens, um im Sinne einer , equality of opportunities' ihre individuelle Position durch Eigeninitiative verbessern zu können. Das die Gesellschaft prägende Prinzip der Meritokratie sollte sich in der Schule mit der Einschränkung gleicher Startchancen widerspiegeln. „Popular acceptance of the ideology of public education reflected popular acceptance of the ideology of democratic capitalism” (Katz 1988, S. 111). Trotz massiver Unterstützung für das öffentliche Schulwesen und seiner Bedeutung im Entstehungsprozeß der amerikanischen Nation konnte sich kein einheitlich nationales Schulsystem herausbilden, weil dieser Entstehungsprozeß zwischen radikaldemokratischen Ideen eines partizipatorischen Lokalismus und der Vorstellung einer föderativen Ordnung mit starken zentralstaatlichen Elementen der Regulation oszillierte. Andererseits bot sich das öffentliche Schulwesen als Mediator zwischen den beiden Polen an, da es sowohl lokal verankert ist als auch staatlicher Einflußnahme unterliegt.

\subsection{Entstehung eines , mass education'-Schulsystems}

Die Idee der Bildung ist für die Entstehung der amerikanischen Gesellschaft und ihrer staatlichen Organisation von besonderer Bedeutung, wie das vorangegangene Kapitel gezeigt hat. Thomas Jefferson setzte sich bereits früh für den Ausbau des Bildungswesens ein, da er in der Bildung ein zentrales Mittel zum Erhalt der Freiheit in einer Demokratie und zum Streben nach Glück erkannte (vgl. Meyer 1997, S. 139). Diese Vorstellung war allgemein akzeptiert und führte im Entstehungsprozeß der USA zur Instrumentalisierung der Bildung, um nationale Ziele durchzusetzen. Zentrale Vorstellungen waren dabei die Sicherung der Demokratie, die Bekämpfung der Armut und die Integration der Immigranten (vgl. Hadden 1992, S. 598). Diese Kategorien tauchen in der Geschichte des amerikanischen Bildungswesens immer wieder auf und können als grundlegende Orientierungspunkte in der Ausrichtung der amerikanischen Bildungspolitik bezeichnet werden. Der Glaube an die Macht der Bildung als zentraler 
gesellschaftlicher Institution wird in den wiederkehrenden Diskussionen um das Schulwesen und seine chronische Krise deutlich.

Die Gründe für die Entstehung eines öffentlichen Schulwesens in den USA werden von Historikern unterschiedlich bewertet. Nach einer Ansicht beschleunigten die Lebensbedingungen in der „Neuen Welt“ den Niedergang großer Familieneinheiten, wie sie z.B. in England bekannt waren. Diese Entwicklung führte dazu, daß die familiäre Verantwortung für die Bildung der Kinder nicht mehr gewährleistet werden konnte. Als Folge mußte schließlich der Staat intervenieren. Die Einrichtung öffentlicher Schulen sollte die Familie in ihrer Funktion als Bildungsinstitution entlasten: ,...the demise of the stable extended English family in the New World led to the establishment of elementary schools...“ (Vinovskis 1995, S. 5). Nach anderer historischer Einschätzung ist die Größe und Stabilität der englischen Familie und ihre Auflösung in den amerikanischen Kolonien überbewertet, da sich bereits zu Zeiten der Emigration der ersten englischen Puritaner nach Amerika die Institution der Großfamilie im Stadium der Erosion befand und das Phänomen des ,formal schooling' in England nicht unbekannt war. Deshalb ist die Entstehung der amerikanischen Schulen vielmehr auf den Transfer der Idee des ,formal schooling' in die Kolonien durch die Immigranten zurückzuführen. Das ,formal schooling' galt aber nicht als ein Ersatz für den Hausunterricht in der Familie, sondern als dessen Ergänzung. Mit der Verabschiedung des ersten Schulgesetzes in Massachusetts 1647 wurde die Schaffung von ,grammar schools' in Gemeinden mit mehr als 100 Haushalten und von ,elementary schools` in solchen mit mehr als 50 Haushalten festgelegt (vgl. Frühbrodt 1996, S. 144). „As in England, this legislation was intended to complement rather than to replace parental efforts at home“ (Vinovskis 1995, S. 7). Die Finanzierung dieser frühen Schulen erfolgte über Schulgebühren und steuerfinanzierte Subventionen der Gemeinden. Dadurch entstanden Differenzen in der Quantität und Qualität der Schulen selbst zwischen den Gemeinden innerhalb eines Einzelstaates. Die Unterschiede zwischen den Einzelstaaten waren jedoch noch weitaus größer.

Die Idee der Steuerfinanzierung der Schulbildung war indessen nicht unumstritten. Bei Gemeindeversammlungen argumentierten Kritiker der Steuerfinanzierung häufig mit der Verantwortung der Eltern für die (schulische) Ausbildung der Kinder, um eine Sozialisierung der Kosten der ,elementary education“ abzulehnen. Eltern hingegen nutzten die Vorteile der steuerfinanzierten Schulen und schickten ihre Kinder bereit- 
willig in diese, anstatt sie zuhause zu unterrichten. Im Gegensatz zum Norden, wo das System öffentlich-organisierter und finanzierter Schulen an Verbreitung zunahm, konnte es sich im Süden der USA erst spät durchsetzen. Grund dafür war die fehlende Bereitschaft der gesellschaftlich dominierenden Klasse der Grundbesitzer, öffentliche Schulen mit ihren Steuern zu unterstützen, da sie selber ihre Kinder von Hauslehrern unterrichten ließen. Im Laufe des 19. Jahrhunderts stieg die Bedeutung der Schule als institutionellem Ort der Ausbildung in Relation zur Familie. Die Rolle der Frau als Lehrerin relativierte jedoch die Trennung zwischen Unterricht in der Familie und in der Schule: ,...women continued their primary role of educating the young“ (ebd., S. 10). Obgleich sich Eltern der Ausweitung des öffentlichen Schulwesens nicht widersetzten, sprachen sie sich dennoch häufig gegen eine Erhöhung der Gemeindesteuern zur Finanzierung der Schulen aus. Das Verhältnis zwischen Eltern und Schule bzw. Lehrern war aber durch die Spannung charakterisiert, daß die Ausweitung des öffentlichen Schulwesens, obwohl von den Eltern unterstützt, mit einem gleichzeitigen Verlust an Kontrolle und Autorität der Eltern über die Ausbildung der Kinder einherging. Lokal organisierter Einfluß der Eltern spiegelt noch heute dieses Spannungsverhältnis wider. „Elements of antagonism between school and family did not end with a new nineteenthcentury equilibrium; they persist today“ (Kaestle, in: ebd., S. 14).

Die Debatte um die Rolle der schulischen Bildung und ihrer Organisation gewann zu Beginn des 19. Jahrhunderts an Bedeutung. Fragen, wie der Einfluß eines ,statefinanced educational system“ auf die Beteiligung der verschiedenen sozialen Klassen und die Möglichkeit der Erhöhung der sozialen Mobilität durch die Schaffung öffentlich-finanzierter High Schools, waren Ausdruck der reformerischen Tendenzen in Teilen der amerikanischen Gesellschaft. Seit dem Krieg von 1812 weitete sich die marktwirtschaftliche Organisation der amerikanischen Wirtschaft weiter aus und beflügelte die Diskussion um die Rolle des Staates in der Ausbildung der Jugend. Die wirtschaftliche Entwicklung kulminierte in den 1820er und 1830er Jahren und förderte den sozialen Wandel. Neben der noch weitgehend agrarischen Wirtschafts- und Gesellschaftsordnung entstanden in den Städten florierende Manufakturen mit mechanisierten und arbeitsteiligen Produktionsprozessen, die die Erosion des auf englische Traditionen zurückgehenden Lehrlingssystems vorantrieben. „By the $1820 \mathrm{~s}$, teenage boys in eastern towns and cities faced an economy in which unskilled laborers would soon outnumber craftsmen, aggravating social tensions“ (Reese 1995, S. 17). Der sozio- 
ökonomische Wandel stellte die bestehende Beziehung zwischen ,education" und ,economy ${ }^{6}$ in Frage. Eine wirtschaftlich aufstrebende Mittelschicht verlangte nach einer über die ,elementary school ' hinausgehenden schulischen Ausbildung für ihre Kinder, die sich an den ökonomischen Realitäten orientieren sollte. Aus diesem Grund waren die in den Städten vorhandenen, latin grammar schools' keine Option für die neue Mittelschicht (vgl. ebd., S. 18). Die Lateinschulen boten eine klassisch-humanistische Bildung an und bereiteten ihre Schüler auf ein Studium am College vor. Boston unterhielt seit 1635 eine solche Lateinschule (vgl. Herbst 1996, S. 11). Die Nachfrage der Mittelschicht nach, higher learning' ging einher mit der Forderung einiger Schulreformer, ein steuerfinanziertes ,public high school'-System zu schaffen. Diese Forderung stellte einen Bruch mit den bestehenden Institutionen des ,higher learning dar, wie Lateinschulen, Akademien und Seminaren, die weder steuerfinanziert noch frei waren (vgl. Reese 1995, S. 17). Das System der privaten Akademien und Seminare, wie es sich seit den 1780er Jahren entwickelt hatte, stand in den Augen der Schulreformer im Widerspruch zum öffentlichen Interesse eines umfassenden Schulsystems, da es den Status der öffentlichen Schulen unterminierte: „,...a powerful, emerging idea: that academies weakened an otherwise deeply embedded, wisely conceived public system that once served rich and poor admirably“ (ebd., S. 24). Diese Idee nutzten die Reformer als Argument für ein System von ,public high schools' mit nach Alter getrennten Klassen und einem einheitlichen Curriculum. So sollte allen dazu befähigten Kindern der soziale Aufstieg durch schulische Bildung ermöglicht werden. Im Gegensatz zur Meinung der Kritiker förderten die Akademien hingegen die Ausweitung des , higher learning'.

Neben den Akademien gab es aber auch einige ,district schools', die eine über das ,elementary schooling' hinausgehende schulische Ausbildung anboten. Fern der Städte und jeglicher zentralen Kontrolle verwalteten sich diese Schulen selbst und richteten ihr Curriculum an den Bedürfnissen der neuen Mittelschichten aus. Im Mittelpunkt standen dabei praxisorientierte Fächer und weniger eine humanistische, auf das College vorbereitende Bildung. Die Finanzierung dieser Schulen setzte sich häufig aus Steuergeldern und Schulgebühren zusammen. Sie verkörperten par excellence die ,ideology of localism‘. Obgleich Schulreformer die dezentrale Organisation und Verwaltung der ,district schools` ablehnten, da sie die Schaffung eines einheitlichen Schulsystems mit zentraler Kontrolle behinderten, konnte sich die dezentralisierte und 
selbstverwaltete Organisation der Schulen durchsetzen (vgl. ebd., S. 25 f.; Green 1990, S. 181 und 184; Church/Sedlak 1976, S. 10 f.; Meyer 1996b, S. 274 f.). „In reality, the district system was a remarkable initiative and fundamental to the growth of public secondary schools“ (Reese 1995, S. 26). Während die ,district schools‘ primär die Nachfrage nach elementarer Schulbildung befriedigten, fanden die Bedürfnisse der wohlhabenden Mittel- und Oberschichten an höherer Bildung für ihre Kinder ihren Ausdruck im Zuwachs an privaten Akademien. Anders als die Lateinschulen und die , liberal arts colleges‘ orientierten sich die Akademien bei der Zusammenstellung ihres Curriculums an den berufsnahen Bedürfnissen einer auf ökonomischem Erfolg basierenden Mittelschicht. „,The students were to learn writing and arithmetic useful for their business and to become acquainted with literature and history. The aim was to enable them to hold their own in the give and take of every day life and politics“ (Herbst 1996, S. 20). Die Absolventen der privaten Akademien gingen, abgesehen von wenigen Ausnahmen, direkt ins Berufsleben und schlossen keine weitere CollegeAusbildung an (vgl. ebd., S. 51).

In den südlichen Kolonien war die Anzahl der privaten Akademien weitaus höher als im Norden. Dies ergab sich aus der besonderen sozio-ökonomischen Struktur des Südens, wo es wegen der dünnen Besiedelung kaum freie öffentliche Schulen gab, und einer stärker verbreiteten aristokratischen Ideologie. Außerdem waren öffentliche Schulen häufig nur für schwarze Kinder vorgesehen (vgl. Reese 1995, S. 30). Das curriculare Angebot der Akademien war keineswegs einheitlich, sondern enthielt eine Vielfalt von unterschiedlichen und teilweise innovativen Lerninhalten, je nach Interessenlage des ,private board of trustees ${ }^{6}$ und der Eltern: ,....whatever parents and their children could afford in the educational marketplace“ (ebd., S. 32). Dabei stellten viele Akademien eine Kombination aus ,elementary“ und ,secondary school ${ }^{`}$ dar. Die praktische Ausrichtung der Akademien nahmen ihre Kritiker zum Anlaß, die Verdrängung der klassischen Bildung durch die sogenannten ,English Studies“ zu konstatieren. Der Erfolg der von Schulreformern propagierten, public high school‘ schien von der Eliminierung der privaten Akademien abzuhängen. Eine klare Unterscheidung zwischen High Schools und Akademien erschien ihnen insofern notwendig, als ihre Forderung nach Schaffung eines öffentlichen High School-Systems nicht mit den bestehenden Institutionen des, higher learning' verwechselt werden sollte. Es war der Konflikt zweier institutioneller Ideale: einem System öffentlicher High Schools und privater 
Akademien (vgl. ebd., S. 33 f.). Die anfängliche Intention der High Schools war die berufliche Vorbereitung ihrer in der Mehrzahl aus der Mittelschicht stammenden Schüler. Die Vermittlung einer über das ,elementary schooling' hinausgehenden, College-ähnlichen Ausbildung sollte sie auf die Anforderungen einer zunehmend kommerzialisierten Gesellschaft vorbereiten. Aufgrund dieser Leitidee bezeichnet Herbst die frühen High Schools auch als ,people's colleges'. Erst gegen Ende des 19. Jahrhunderts fingen die High Schools an, neben ihrem traditionellen Angebot auch Vorbereitungskurse für ein Studium am College anzubieten (vgl. Herbst 1996, S. 51). Mit der Aufschlüsselung ihres Curriculums öffneten sich die High Schools unterschiedlichen gesellschaftlichen Schichten und verabschiedeten sich gleichzeitig von der Idee eines einheitlichen Fächerkanons (vgl. ebd., S. 95).

Welche Bedeutung hatte die schulische Erziehung und Ausbildung in der amerikanischen Gesellschaft des 19. Jahrhunderts, daß es schließlich zum Aufbau eines umfassenden Schulsystems ${ }^{18}$ von ,elementary ${ }^{6}$ und ,secondary schooling ${ }^{6}$ kam? Der Beginn der ,mass education“ in den 1840er Jahren wird häufig in Relation zur wachsenden Industrialisierung der Wirtschaft gesehen. Vertreter dieser Sichtweise stellen einen Zusammenhang zwischen dem Bedarf an ausgebildeten, disziplinierten Arbeitern in den neuen, maschinell produzierenden Manufakturen und der Ausweitung der öffentlichen Schulen her. Als Beispiel dieser Reziprozität von Industrialisierung und, mass education“ wird gerade Massachusetts angeführt, obgleich die Ausdehnung des Schulsystems insbesondere in ruralen Gebieten vorzufinden ist. Darüber hinaus gab es in Massachusetts bereits vor 1840 ein relativ gut ausgebautes Schulsystem. Neben dem möglichen Einfluß einiger Industrieller und Kaufleute waren es die Schulreformer und Geistlichen auf lokaler Ebene, die die schulische Ausbildung einer breiten Masse der Bevölkerung vorantrieben. Die puritanische Tradition der amerikanischen Gesellschaft darf hierbei nicht unterschätzt werden (vgl. Vinovskis 1995, S. 75-77; Green 1990, S. 188; Adams 1992, S. 63). „Instead of seeing mass education as the result of midnineteenth-century industrial development, it is more accurate to view it as a continuation of the colonial Puritan activities to ensure that everyone was able to read the Bible“ (Vinovskis, S. 77). Die Forderung nach ,mass education“ wuchs mit dem sozialen Problem von Kindern aus armen, sozial exkludierten Familien, deren In-

\footnotetext{
${ }^{18}$ System wird hier nicht als Synonym für eine einheitliche, standardisierte Organisation verwendet.
} 
tegration in die Gesellschaft durch die schulische Vermittlung gesellschaftlich relevanter Normen und Fähigkeiten gewährleistet werden sollte.

Im Gegensatz zu kontinentaleuropäischen Ländern gab es in den USA keine ernstzunehmende Opposition gegen die Schulbildung breiter Bevölkerungsschichten, da sie als Mittel zur Stabilisierung und Überlebensfähigkeit des demokratischen Systems angesehen wurde. In Europa hingegen sah die herrschende Elite die Ausweitung der Schulbildung als Gefahr für das bestehende politische System. Amerikanische Politiker und Eliten argumentierten in der Debatte um die Zukunft der Republik gerade mit der Idee der Schulbildung als Instrument gegen staatsgefährdende Ideologien, um ihrer Forderung nach ,mass education“ Nachdruck zu verleihen. „Mass education became a political weapon against dangerous ideas...“ (Reese 1995, S. 38). Die wohlhabenden Schichten waren davon überzeugt, daß mit Hilfe der schulischen Erziehung ärmerer Schichten die Gefahr sozialer Spannungen und Unruhen reduziert werden könne. „The value of education, according to many commentators, was to improve the moral character of the poor rather than to enhance their occupational skills or to foster individual social mobility“ (Vinovskis 1995, S. 78). Die Einrichtung spezieller Bildungsprogramme und -institutionen für arme und benachteiligte Kinder sollte deren moralischen, aber auch sozio-ökonomischen Abstieg verhindern. Ein Beispiel für solche Institutionen waren die ,charity schools ${ }^{6}$, die sich die religiöse und allgemeinbildende Unterrichtung jugendlicher Arbeiter zur Aufgabe gemacht hatten. Diese Schulen wurden von Kirchen und philanthropischen Vereinen unterstützt. Ziel war die Vermittlung von Moral und Disziplin: ,...they were less an extension of parental values than an attempt to substitute for a culture which parents deemed to lack" (Green 1990, S. 183). Eine ähnliche Strategie verfolgten die ,Lancasterian schools', die sich zu Beginn des 19. Jahrhunderts vornehmlich in Städten ausgebreitet hatten und als Vorläufer der ,public common school' gelten (vgl. ebd., S. 182 f.). ,Charity schools“ und ,Lancasterian schools‘ standen somit im Gegensatz zu den dezentral organisierten, ruralen ,district schools‘. Während erstere antagonistisch der Familie der Schüler gegenüberstanden, sahen sich die ,district schools“ als „,verlängerter Arm“ elterlicher Kontrolle.

Entscheidende Motivation für die Schaffung von Schulen für ärmere Bevölkerungsgruppen war die Sicherung der durch Urbanisierung und Industrialisierung scheinbar gefährdeten politischen Stabilität, indem man die Armen entsprechend den protestan- 
tischen Normen der Mittelschicht konditionierte. Die meisten Schulreformer akzeptierten die Realität sozialer Klassen, die sich in den lokaler, elterlicher Kontrolle entziehenden ,charity schools‘ widerspiegelte. Andererseits glaubten sie in der öffentlichen Schule eine Institution gefunden zu haben, die die Chance des sozialen Aufstiegs von familiären Bedingungen unabhängig macht. Die Leistungsfähigkeit des einzelnen Individuums wurde zum alleinigen Maßstab sozialer Mobilität. Mit dem Ausspruch „enable all men to start fair in the race“ (Reese 1995, S. 40) war das Prinzip der Chancengleichheit geboren. Die Idee, individuelle Leistung als Maßstab für soziale Unterschiede heranzuziehen, galt als Quintessenz der von bürgerlichen Schichten vertretenen Ideologie des ,republicanism‘. Diese Ideologie beeinflußte die bildungspolitischen Debatten im gesamten 19. Jahrhundert. Ziel der politischen Kräfte, die den ,republicanism“ verkörperten, war die Durchsetzung und Universalisierung protestantischer Werte, auf die gerade die Mittelklasse ihren sozio-ökonomischen Erfolg gründete. „Reformers hoped to instill the value of ambition, hard work, delayed gratification, and earnestness in youth, trained to become sober, law-abiding, and respectable adults“" (ebd., S. 57).

Erst mit der wirtschaftlichen Rezession von 1839-43 schien sich ein Wandel in der Argumentationsstruktur der Schulreformer zu vollziehen. Die Betonung der moralischen Verantwortung von Schulen in der Erziehung verantwortungsbewußter Bürger schlug um in die Hervorhebung ökonomischer Vorteile eines umfassenden Schulsystems. Einer der Protagonisten der Popularisierung der ökonomischen Logik war Horace Mann, Vorsitzender des Massachusetts Board of Education, der in seinem fünften Jahresbericht die zentrale Rolle der schulischen Ausbildung für den ökonomischen Erfolg der gesamten Gemeinschaft konstatierte. Diese Argumentation diente ihm lediglich als Mittel zum Zweck, um den Widerstand der Gemeinden, mehr Geld für eine quantitative und qualitative Ausweitung des Schulsystems aufzubringen, zu durchbrechen. Gleichzeitig nutzte sie ihm, die geschwächte Position des Board of Education zu stärken. Seine Analyse konnte jedoch keine Korrelation zwischen Anzahl der Schuljahre und Produktivität herstellen. Es fand sich keine solide empirische Basis, die eine Ausweitung der Schulbildung über das ,elementary schooling' hinaus hätte begründen können. Die tatsächlichen Vorteile der schulischen Ausbildung für die Wirtschaft lagen darin, daß die Arbeiter zu Pünktlichkeit und Zuverlässigkeit erzogen wurden. Darüber hinaus konnten schulisch qualifizierten Arbeitern die für die Erfüllung 
ihrer Aufgabe notwendigen Arbeitsschritte leichter vermittelt werden. Außerdem sank das Streikrisiko, weil die Arbeiter soziale Ungleichheiten als individuelle Leistungsunterschiede akzeptierten (vgl. Vinovskis 1995, S. 92-100). Trotz fehlender empirischer Stringenz konnte sich der Glaube an die ökonomische Bedeutung der schulischen Qualifizierung durchsetzen und als gleichwertiges Argument neben dem der moralischen, staatsbürgerlichen Charaktererziehung etablieren. Dies förderte die Vorstellung, daß Kinder aus armen Verhältnissen durch eine Schulausbildung der Armut entkommen könnten. Infolge der Erosion der Lehrlingsausbildung fiel der Schule als einziger Alternative des beruflichen Aufstiegs eine besondere Verantwortung zu (vgl. ebd., S. 79 und 120). Dies zeigte sich in den vehementen Forderungen nach besserer Schulbildung als Garant sozialer Harmonie gerade in Zeiten ökonomischer Krisen, wie z.B. während der industriellen Konflikte in den 1870er Jahren (vgl. Reese 1995, S. 46 ff.). Die Instrumentalisierung der Schule als zentraler gesellschaftlicher Institution zur Gewährleistung einer für die Stabilität der Demokratie notwendigen minimalen Charakterbildung und gleichzeitig zur Förderung der sozialen Mobilität durch Chancengleichheit führte wegen fehlender Alternativen zu internen Spannungen.

\subsection{Berufliche Bildung - Von der Werkbank zur Schulbank}

Die Entwicklung der beruflichen Bildung in den USA vollzog sich in zwei Stufen: Mit den englischen und deutschen Immigranten kam zuerst die Tradition der Lehrlingsausbildung in die neuen Kolonien. Der anschließende Niedergang derselben war begleitet mit einem Aufstieg des, vocationalism' im allgemeinen Schulsystem. Diese Entwicklung hatte entscheidende Konsequenzen für die weitere strukturelle Ausgestaltung der Berufsausbildung, da hier ein Pfad eingeschlagen wurde, der den Handlungsspielraum für zukünftige Reformen begrenzen sollte.

\subsubsection{Niedergang der Lehrlingsausbildung}

Die Tradition der Lehrlingsausbildung kam mit englischen und deutschen Immigranten in die amerikanischen Kolonien. Während der britischen Herrschaft in Amerika setzte die ,Statute of Artificers` von 1563 den Rahmen für die Lehrlingsausbildung. In ihr war festgelegt, daß die Lehrzeit mindestens sieben Jahre beträgt und den Handwerksgilden als intermediären Organen die Durchsetzung und Kontrolle der gesetzlichen 
Bestimmungen obliegt. Der Lehrherr (Meister) verpflichtete sich über die Ausbildung hinaus, Unterkunft, Kleidung und Verpflegung für den Lehrling zu stellen. Eingebunden in den Haushalt des Meisters mußte der Lehrling diesem praktisch wie ein Sklave dienen. In den Kolonien bestand zudem die Möglichkeit, die Lehrzeit auf vierzehn Jahre auszudehnen (vgl. Münch 1989, S. 97). Anders als in England konnten sich die Gilden in den amerikanischen Kolonien jedoch nicht in vergleichbarer Weise etablieren. Ihrer vollständigen Entwicklung standen die Immigration heterogener Bevölkerungsgruppen, der Mangel an qualifizierten Handwerkern und der rurale Charakter der Wirtschaft entgegen. Die unbeschränkte Verfügbarkeit von Land ließ viele ausgebildete Handwerker ihren Beruf aufgeben, um durch die Bewirtschaftung eigenen Bodens ökonomisch unabhängiger zu sein. Infolge des Mangels an Handwerkern waren die öffentlichen Behörden eher an der Werbung qualifizierter Arbeitskräfte interessiert, als deren Zahl durch strikte Regulation zu beschränken, wie es die Aufgabe der Zünfte in kontinentaleuropäischen Ländern war (vgl. Elbaum 1989, S. 345 f.). Folglich besaßen Handwerksassoziationen in den amerikanischen Kolonien praktisch keine Sanktionsmöglichkeiten in der Kontrolle ihrer Berufsstände. Dies förderte den Mißbrauch der Lehrlinge als billige Arbeitskräfte. Als Folge gab es bereits während der britischen Kolonialzeit ein signifikantes ,runaway“-Problem der Lehrlinge, das nach der Unabhängigkeit der Kolonien noch zunahm. Die Tatsache, daß der Eintritt in einen qualifizierten Beruf im allgemeinen keine Zertifizierung der Qualifikation erforderte, ermöglichte es weggelaufenen Lehrlingen, trotzdem ihren Beruf auszuüben. Zwischen 1783 und 1799 verabschiedeten zwölf Einzelstaaten neue Gesetze zur Lehrlingsausbildung, in denen insbesondere das ,runaway'-Problem behandelt wurde. Trotz solcher Maßnahmen war die Durchsetzung der Einhaltung von Lehrverträgen sehr begrenzt, da die Möglichkeit der geographischen Mobilität, gerade durch die Ausdehnung des Territoriums nach Westen, die Chancen der Ergreifung weggelaufener Lehrlinge minimierte und die Gesetze eine Verfolgung über die Einzelstaatsgrenzen hinaus nicht vorsahen. Der praktische Nutzen dieser Vorschriften für den Erhalt der Lehrlingsausbildung blieb verschwindend gering. Ferner zeigten die Arbeitgeber wenig Interesse, einen unwilligen Lehrling mit gesetzlichen Mitteln zur Ableistung seiner Lehrzeit zu zwingen (vgl. ebd., S. 347).

Statt dessen änderten Arbeitgeber, die Lehrlinge beschäftigten, das bestehende Lehrlingssystem, indem sie spezielle Anreiz- und Bezahlungssysteme mit Boni bzw. Kau- 
tionen von $\$ 100$ bis $\$ 200$ einführten. Lehrlinge erhielten entweder nach Vollendung der Lehrzeit einen Bonus, oder sie mußten vor ihrer Einstellung eine Kaution beim Arbeitgeber hinterlegen. Beide Vertragsformen sollten einen Anreiz für den Lehrling darstellen, den Lehrvertrag nicht frühzeitig aufzukündigen. Den Bonus bezahlten die Lehrlinge häufig durch niedrigere Löhne selber (vgl. Jacoby 1991, S. 890 f. und 895). Auf diese Weise konnten die Arbeitgeber ihre Ausbildungsinvestitionen sichern und das Risiko einer vorzeitigen Beendigung der Lehre minimieren. Insofern verlagerte sich das Investitionsrisiko einer Ausbildung vom Arbeitgeber auf den Lehrling. Die Höhe der Kautionen bzw. Boni war jedoch zu gering, um Lehrlinge effektiv vom Weglaufen abzuhalten: ,a bonus of one to two hundred dollars at the end of an apprenticeship...is too far away, and figures out but insignificantly when reduced to the hour basis“" (Becker, in: Elbaum 1989, S. 347). Darüber hinaus nahm das Interesse der Arbeitgeber an einer effektiven Lösung des ,runaway'-Problems insofern ab, als ihr Bedarf an ausgebildeten Arbeitskräften durch immer neue Immigrationswellen befriedigt werden konnte.

Bis Mitte der 1840er Jahre blieb die Zahl der Einwanderer in die USA weit unter 100.000 pro Jahr. Das entsprach seit den 1830er Jahren einer konstanten Rate von ca. 4 bis 5 Einwanderern pro eintausend Einwohnern (vgl. Ferrie 1999, S. 36). Bereits vor 1800 konstatierten Zeitgenossen einen empfindlichen Mangel an Arbeitskräften, insbesondere in den großen Städten der Ostküste. Dies führte zu einem im Vergleich zu europäischen Maßstäben überdurchschnittlichen Einkommensniveau in den USA bei etwa gleichem Preisniveau. Auch die Tatsache, daß sich die Handwerkerlöhne in Philadelphia bis 1817, als die Einwanderung dort mit 8.400 Personen einen Höhepunkt erlebte, auf einem konstant hohen Niveau bewegten, verdeutlicht die ungebrochene Nachfrage nach qualifizierten Arbeitskräften. Die besseren Verdienstmöglichkeiten waren bis in die 1840er Jahre hinein ein wesentliches Motiv für die Auswanderung ${ }^{19}$ (vgl. Grabbe 2001, S. 156 und 177).

Das durchschnittliche Vermögen der deutschen Auswanderer reichte gerade für die Anreise bis zum Hafen und zur Überbrückung der Wartezeit bis zum Segeltermin. Die Kosten der Überfahrt wurden in der Regel durch einen sogenannten „Redemptioner““- 
Kontrakt abgedeckt, d.h. nach Ankunft in den USA mußte der Einwanderer als „indentured servant“ die Kosten für die Schiffspassage abdienen. Viele Handwerker in den USA bedienten sich dieser „Redemptioners“, um sie anstelle freier Gesellen (journeymen) zu beschäftigen. Nach 1800 ließ diese Praxis jedoch in den großen Städten nach, weil sich die Arbeitsverhältnisse insgesamt zu wandeln schienen. So entstanden größere Handwerksbetriebe, die mehrere Gesellen für kürzere Zeiträume beschäftigten, um flexibler auf die Erfordernisse der lokalen Märkte reagieren zu können (vgl. Lane 1987, S. 24 f.; Grabbe 2001, S. 185). Aus Großbritannien kamen qualifizierte Handwerker, die aufgrund der beginnenden Mechanisierung in ihrem Beruf keine Beschäftigung mehr fanden. Bei der Registrierung aller (männlichen) britischen Untertanen im Jahre $1812^{20}$ ergab sich eine Handwerker-/'skilled worker'-Quote von 40 $\%$ (vgl. ebd., S. 163 und 190).

Zwischen 1820 und 1830 stieg die Produktivität des verarbeitenden Gewerbes im Nordosten der USA sprunghaft. Diese Zuwächse wurden anfangs durch relativ geringe Anpassungen des Produktionsprozesses erzielt, indem z.B. die Arbeitsabläufe in einzelne Teilschritte zerlegt und die Koordination zwischen den Arbeitsschritten verbessert wurden. Kleine Handwerksbetriebe wurden durch sogenannte ,non-mechanized' Manufakturen ersetzt, die eine größere Anzahl qualifizierter und angelernter Arbeitskräfte beschäftigten. Der wachsende Einsatz neuer Technologien führte zu einer weiteren Substitution qualifizierter Arbeitskräfte durch unqualifizierte. Um den Bedarf an unqualifizierten Arbeitern decken zu können, wurden insbesondere auch Frauen und Kinder angeworben (vgl. Sokoloff/Villaflor 1992, S. 33 f.; Lane 1987, S. 24). In der Textilindustrie von Lowell nahe Boston waren 1849 rund $90 \%$ der Arbeiter Einheimische. Sechs Jahre später hatte sich dieses Bild aufgrund der massiven Einwanderungswelle in den späten 1840er und frühen 1850er Jahren beinahe umgedreht. Nur noch 35 \% der Beschäftigten waren einheimisch (vgl. Ferrie 1999, S. 161).

In den 1850er Jahre erlebten die USA die höchste Einwanderungsrate ihrer Geschichte. Allein im Jahr 1850 landeten etwa 370.000 Einwanderer in den USA. Das entsprach

\footnotetext{
19 Bei einer Befragung von württembergischen Auswanderungswilligen im Jahre 1804 gaben $64 \%$ wirtschaftliche Gründe an (vgl. Grabbe 2001, S. 153).

${ }^{20}$ Die USA standen zu dieser Zeit im Krieg mit Großbritannien.
} 
einer Einwanderungsrate von über 15 pro eintausend U.S.-Bürger. Von den Einwanderern kamen insgesamt $93 \%$ aus Großbritannien, Irland und Deutschland. Insbesondere der Zustrom irischer und deutscher Auswanderer hatte infolge der Hungersnot in Irland und der gescheiterten politischen Revolution in Deutschland nach 1848 stark zugenommen. Während aus Irland in der Mehrzahl unqualifizierte Arbeiter und Bauern auswanderten, waren es in Deutschland besonders die qualifizierten Handwerker, die mit ihrer Auswanderung den Folgen der gescheiterten Revolution zu entfliehen suchten (vgl. Ferrie 1999, S. 35 f. und 88 ff.; Levine 1992, S. 36 f.). Die Zunahme an qualifizierten und unqualifizierten Einwanderern hatte einen enormen Effekt auf den Industrialisierungsprozeß, insbesondere im Nordosten der USA. Das ausreichende Angebot an billigen unqualifizierten - in der Mehrzahl irischen - Arbeitern $^{21}$ ermöglichte eine weitere Mechanisierung und Arbeitsteilung in verschiedenen Industrien. Hier kam es zu einem sogenannten ,de-skilling'-Proze $\beta^{22}$. In den industriellen Manufakturen und Fabriken entstanden gleichzeitig neue Positionen für qualifizierte Handwerker, z.B. in der Instandhaltung der Maschinen, um die es jedoch einen Wettbewerb zwischen einheimischen und billigeren deutschen oder britischen, skilled workers' gab. Daneben fanden sich in Regionen mit einer geringen Einwanderungsquote unqualifizierter irischer Arbeiter weiterhin kleine Handwerksbetriebe, in denen traditionelle Produktionsformen fortbestanden. Dorthin wichen viele der einheimischen ,skilled workers' aus (vgl. Lane 1987, S. 67 ff.; Ferrie 1999, S. 181 f.).

Im Zuge der Industrialisierung und arbeitsteiligen Produktion verlor die Lehrlingsausbildung als Qualifizierungsinstitution für die Masse der Arbeiter weiter an Bedeutung, da Investitionen in eine umfassende Ausbildung der Arbeiter über den tatsächlichen Qualifikationsbedarf hinausgegangen wären und somit einzelbetrieblich unrentabel erschienen. Betriebswirtschaftliche Kalkulation fand ihren Einzug ins wirtschaftliche Leben und veränderte den Blickwinkel auf die Lehrlingsausbildung. Diese wandelte sich von einer Institution, die neben der Vermittlung handwerklicher Fertigkeiten gerade auch der Erziehung und Sozialisation des Jugendlichen diente, zu einem genuin

21, ,...] the Irish were identified as agents in the process of economic modernization. The adoption of task differentiation and mechanization was facilitated by this increasing supply of cheap, unskilled labor [...]." (Lane 1987, S. 28)

22, ,...] the general degradation of skill premiums by the downgrading of once highly skilled operations.“ (Fogel 1989, S. 358) 
ökonomischen Instrument. Meister entledigten sich bereitwillig ihrer erzieherischen Verantwortung und nutzten ihre Lehrlinge als billige Arbeitskräfte aus. Aber auch die Organisation der Produktion in Fabriken, wo Lehrlinge nicht mehr in den Haushalt des Meisters eingebunden waren, sondern in separaten Unterkünften untergebracht wurden, verhinderte eine dem alten System entsprechende gesellschaftliche Sozialisation. Nicht nur die Meister, sondern auch die Lehrlinge paßten sich den gewandelten ökonomischen Verhältnissen einer kompetitiven Marktwirtschaft an, indem sie sich weigerten, Lehrlingslöhne für Gesellenarbeit zu beziehen. Damit wurde aber dem Lehrlingssystem die ökonomische Grundlage entzogen: Arbeitgeber versuchen ihre Ausbildungsinvestitionen durch die Differenz aus Lehrlingslohn und tatsächlicher Produktivität zu finanzieren. Während zu Beginn der Ausbildung der Lohn die Produktivität noch übersteigt, kehrt sich diese Beziehung zum Ende der Ausbildungszeit um. Wenn die Lehrlinge ausreichende Fähigkeiten erlernt hatten, um Gesellenarbeit verrichten zu können, bestand für sie die Möglichkeit, ihren Meister vor Abschluß der vollen Lehrzeit zu verlassen und bei einem anderen Arbeitgeber als Geselle mit höherem Lohn anzufangen. Dies war nur möglich, weil es zur Ausübung eines Berufs keines Zertifikats bedurfte.

Die neue Flexibilität sowohl auf seiten der Arbeitgeber, die sich nicht mehr durch ,longterm contracts ' binden lassen wollten, als auch auf seiten der Lehrlinge, die materielle Vorteile in einem mobilen Arbeitsmarkt suchten, unterminierten tragende institutionelle Pfeiler der Lehrlingsausbildung (vgl. Rorabaugh 1986, S. 136-139). Deshalb befürworteten Arbeitgeberorganisationen als Ersatz für die Lehrlingsausbildung in diesem Segment des Arbeitsmarktes Berufsunterricht in Schulen, um ihre Ausbildungskosten zu sozialisieren. Über den Zugriff auf einen externen Arbeitsmarkt semi-qualifizierter Schulabgänger hätten Arbeitgeber darüber hinaus flexibel auf ökonomische Konjunkturen reagieren können. Für die Unternehmen eröffnete die schulische Berufsausbildung ferner die Option, den Einfluß der Gewerkschaften auf das Arbeitsangebot qualifizierter Fachkräfte zu reduzieren (vgl. Jacoby 1991, S. 892 f.). Andererseits stieg die Nachfrage in der Industrie nach einer modifizierten und qualitativ höherwertigen Lehrlingsausbildung für eine kleine „Arbeiterelite“, z.B. Maschinenschlosser. „Machinists were highly skilled...they profited from the introduction of machinery that proved so devastating to other craftsmen“" (Rorabaugh 1986, S. 141). Die Qualität der Ausbildung sollte durch eine Kombination aus ,training-on-the-job“ 
und Berufsschulunterricht verbessert werden. So unterbreitete 1910 die ,Commission on Industrial Education“ der National Association of Manufacturers (NAM) Vorschläge zur Schaffung eines solchen Lehrlingssystems (vgl. Münch 1989, S. 98). Den Bemühungen der Arbeitgeberorganisationen ging jedoch ein bedeutender institutioneller Wandel in der Lehrlingsausbildung voraus. Immer weniger Firmen erklärten sich bereit, Lehrverträge schriftlich abzuschließen. Dadurch konnten die Unternehmen flexibler auf ökonomische Schwankungen reagieren und mußten keine arbeitsrechtlichen Prozesse entlassener Lehrlinge befürchten. Gleichzeitig förderte dies den Mißbrauch der Lehrlinge durch die Firmen. Die fehlende Sicherheit auf seiten der Lehrlinge reduzierte wiederum den Anreiz, eine Lehrlingsausbildung zu durchlaufen (vgl. Jacoby 1991, S. 888 f.). Es fehlte an einer funktionierenden Kontrollinstanz, die beiden Seiten die Einhaltung der Verpflichtungen hätte garantieren können. Der Staat Wisconsin ist ein Beispiel für den Versuch einer Reform der Lehrlingsausbildung durch staatliche Intervention. Ein Jahr nach dem Bericht der ,Commission on Industrial Education“ verabschiedete das Parlament von Wisconsin ein Comprehensive Apprenticeship Law, mit dem die Berufsschulpflicht eingeführt wurde. Kritiker dieses Gesetzes behaupteten jedoch, daß gerade durch die Einführung der Berufsschulpflicht der Niedergang der Lehrlingsausbildung noch vorangetrieben worden sei. Der Anreiz für Arbeitgeber, Lehrlinge einzustellen, sei durch steigende Ausbildungskosten infolge staatlicher Regulation weiter gesunken. Wenn man sich die Lehrlingszahlen in Wisconsin für das Jahr 1931 anschaut, scheinen die Kritiker recht behalten zu haben. Diese waren nämlich auf 73 Lehrlinge zurückgegangen (vgl. Münch 1989, S. 99-101). Staatliche Regulation stärkte andererseits die Position der Gewerkschaften, die ihre Kräfte gegen betrieblichen Mißbrauch der Lehrlinge und zur Einhaltung der Arbeitgeberverpflichtungen einsetzten. So standen 5 Prozent aller Streiks in den USA 1883 im Zusammenhang mit Konflikten über die Lehrlingsausbildung (vgl. Jacoby 1991, S. 890).

Nach dem Ersten Weltkrieg boomte die amerikanische Wirtschaft, was einen steigenden Bedarf an qualifizierten Arbeitskräften mit sich brachte. Dieser Bedarf konnte nicht mehr durch gut ausgebildete Immigranten gedeckt werden, weil es gleichzeitig Bemühungen zur Senkung der Einwanderungsquoten gab. Eine Koalition aus Arbeitgebern, Gewerkschaften, Schulreformern und staatlichen Behörden forcierte eine Kampagne zur Ausweitung bundesstaatlicher Interventionen, um die berufliche Ausbildung zu fördern. Die Vorstellungen über die Organisation der beruflichen Bildung 
gingen jedoch bei den verschiedenen Gruppen innerhalb der Koalition auseinander. Einige forderten die Wiederbelebung des alten Lehrlingssystems, andere wiederum sahen in der schulischen Berufsausbildung die Lösung des Qualifikationsproblems. Im Bereich der Lehrlingsausbildung führten die Bemühungen dieser Interessenkoalition zur Verabschiedung des National Apprenticeship Act von 1937. Dieses Gesetz, das lediglich fünf Paragraphen umfaßt, schreibt der Bundesregierung die Aufgabe zu, gemeinsam mit der Industrie Standards für die Lehrlingsausbildung zu schaffen. Die Standards beziehen sich jedoch nicht auf konkrete Ausbildungsinhalte und die Organisation der Ausbildung, sondern setzen die Höhe des Lehrlingslohns und das Verhältnis von Lehrlingen zu Gesellen fest. Die Beteiligung der Unternehmen an staatlich registrierten Lehrlingsprogrammen blieb aber freiwillig, so daß es bis heute eine Vielzahl nichtregistrierter Ausbildungsgänge gibt, die die Minimalstandards nicht erfüllen (vgl. Jaschner 1996, S. 196).

In den 1930er Jahren vollendete sich der Wandel in der Lehrlingsausbildung insofern, als sich die bis dahin dominante Überlegung des Schutzes der Ausbildungsinvestition des Arbeitgebers in ihr Gegenteil verkehrte. Nun galt es den Arbeitnehmer bzw. Lehrling vor Ausbeutung und schlechten Ausbildungsbedingungen zu schützen. Die bestehenden Lehrlingsprogramme waren zunehmend vom Einfluß der Gewerkschaften, insbesondere der Berufsgewerkschaften, dominiert, die über die Kontrolle der Lehrlingsausbildung das Angebot qualifizierter Arbeitskräfte in ihrem Beruf und mithin das Lohnniveau steuern konnten (vgl. Scherrer 1996, S. 246 f.). „Rather than kill apprenticeship, unions realized the importance of controlling it. With state and federal assistance, organized labor succeded in appropriating the apprenticeship institution for its own purposes“ (Jacoby 1991, S. 908). Anfänge einer gewerkschaftlichen Kontrolle der Lehrlingsausbildung hatte es bereits in den 1840er Jahren gegeben, als sich lokale Gewerkschaftsvereine auf Lehrlingsquoten im Verhältnis zur Zahl der beschäftigten Gesellen einigten, um so die Zahl der Gesellen zu reduzieren. Eine nationale Koalition lokaler Gewerkschaftsorganisationen der Drucker versuchte 1850 durch die Einführung von Mitgliedskarten, die nur an Lehrlinge abgegeben wurden, die ihre Ausbildung vollständig absolviert hatten, den Arbeitsmarkt für Gesellen zu regulieren (vgl. Rorabaugh 1986, S. 152-154). Viele Arbeitgeber zogen sich aus der Lehrlingsausbildung zurück, weil ihnen diese im Vergleich zu den Alternativen als zu kostenintensiv erschien. Entscheidend waren dabei nicht die direkten Ausbildungs- 
kosten, sondern die mit der Einhaltung kollektiver Vereinbarungen bzw. gesetzlicher Bestimmungen, wie z.B. besserer Kündigungsvorschriften für Lehrlinge, einhergehenden indirekten Kosten. Infolgedessen investierten die Arbeitgeber nicht mehr in eigene Lehrlingsprogramme, sondern nutzten öffentliche und private Berufsschulen, um qualifizierte Arbeitskräfte zu gewinnen. Auf diese Weise wurde das Investitionsrisiko wieder dem einzelnen Arbeitnehmer bzw. der Allgemeinheit aufgebürdet.

\subsubsection{Aufstieg des, vocationalism' im amerikanischen Schulsystem}

Parallel zur Erosion der Lehrlingsausbildung kam es zum Aufstieg des ,vocationalism“ im amerikanischen Schulsystem. Die Entstehungsphase der amerikanischen High School ist von zwei konträren Strömungen charakterisiert: Einerseits gab es die auf die , latin grammar school‘ zurückgehende Idee der klassischen Ausbildung als Vorbereitung auf die Universität, andererseits hatte die Institution des ,people's college', die der breiten Masse eine College-ähnliche Ausbildung vermitteln sollte, um den ,noncollege bound' Schülern eine über die Grundschule hinausgehende Ausbildung zu ermöglichen, ihre Spuren hinterlassen. Als Resultat hat sich schließlich die ,comprehensive high school' als Kombination aus beiden Richtungen durchgesetzt (vgl. Herbst 1996, S. 7 und 51 f.). Ein Teil der High School-Curricula setzt sich mithin aus sogenannten, vocational courses' zusammen, in denen berufliche Grundkenntnisse mehr oder weniger spezifisch unterrichtet werden.

Wie kam es dazu, daß die Schule neben der klassischen liberalen Ausbildung zur Vermittlung berufsqualifizierender Kenntnisse instrumentalisiert wurde? Erste Ansätze, praktische Fächer an Schulen zu unterrichten, gab es bereits Ende des 18. Jahrhunderts. Ein Beispiel findet sich in der Gründung der Benjamin Franklin Academy. Eine umfassende Verbreitung der schulischen Unterrichtung berufsnaher Kenntnisse begann jedoch erst in der zweiten Hälfte des 19. Jahrhunderts. Mit der Verabschiedung des Land Grant College (Morrill) Act von 1862 förderte die Bundesregierung zum ersten Mal aktiv die berufliche Ausbildung, indem sie Land für die Errichtung neuer Colleges zur Verfügung stellte. Diese Colleges waren insbesondere auf den Bereich Landwirtschaft und technisch-mechanischer Berufe konzentriert. Der Prozeß der Industrialisierung begünstigte diese Entwicklung aufgrund des wachsenden Bedarfs an Ingenieuren und technisch höher qualifiziertem Wartungspersonal. Befürworter einer besseren Ingenieursausbildung und liberale Schulreformer, die in der handwerklichen 
Ausbildung ein pädagogisches Mittel zur besseren Lernmotivation gefunden zu haben glaubten, bildeten eine heterogene Koalition, die eine Ausweitung der ,manual education' in öffentlichen Schulen forderte. Erstere, wie John O. Runkle, der Präsident des MIT, waren durch die russische polytechnische Ausbildung beeinflußt, die ihnen auf der Weltausstellung in Philadelphia 1876 vorgestellt wurde. „Runkle's admiration for that training was such that he soon extended his proposals for the Russian system beyond the professional training of engineers to public education generally" (Kliebard 1995, S. 112).

Obgleich diese Gruppe bereits erste ökonomische Effizienzüberlegungen in ihre Reformvorschläge miteinbezog, war für sie die moralisch-intellektuelle Erziehung immer noch von besonderer Bedeutung. Es galt ,the dignity of hand labor” (ebd., S. 113) wiederherzustellen. Murray Butler, der Präsident des New York College for the Training of Teachers, konstatierte sogar: „Manual training is mental training through the hand and eye...” (ebd., S. 113). Inspiriert war die ,manual training'-Bewegung zudem vom schwedischen Bildungssystem, genannt ,Sloyd', was soviel wie handwerkliche Fertigkeiten bedeutet. Die Bewunderung für diesen pädagogischen Ansatz wuchs unter amerikanischen Lehrern und Schulreformern nach ersten Erfahrungsberichten sehr schnell. „Sloyd develops the child physically, mentally and morally. It is intended to mean something more than mere mechanical skill...Its object is the intelligent exercise of the whole body“ (Ellenwood, in: Wilms 1988, S. 83). ,Manual training' sollte nach Meinung der Schulreformer die Schule interessanter machen und so das ,dropout'-Problem reduzieren. Die Idee, das ,manual training' als gesellschaftspolitisches Heilmittel auch bei jugendlichen Kriminellen und Schwarzen einzusetzen, ließ die Anerkennung dieser Ausbildung bei Arbeitgebern sinken. ,Manual training“ hatte sich als ,philanthropic education for social deviants“ (Grubb/Lazerson, in: Wilms 1988, S. 84) etabliert.

Diese pädagogische Sichtweise des ,manual training' konnte sich nicht länger auf lokaler Schulebene halten. Dort galt es, die ökonomischen Vorteile dieser Form des Unterrichts in den Vordergrund zu stellen. Nur so konnte der Druck der Gemeinden, Steuergelder nicht für eine scheinbar praxisferne Ausbildung der ,non-college bound' Kinder zu verschwenden, kanalisiert werden (vgl. Herbst 1996, S. 101). In den 1880er und 1890er wandelte sich das bis dahin in den High Schools geltende , single common curriculum' in ein breites Spektrum von Kursangeboten. Dadurch kam man der 
steigenden Zahl von Schülern aus unterschiedlichen Schichten mit verschiedenen beruflichen Perspektiven entgegen. Die negative Konnotation der ,manual education' infolge ihrer frühen Instrumentalisierung zur Resozialisierung jugendlicher Krimineller und anderer Problemkinder verlor durch die wachsende ökonomische Argumentation an Bedeutung (vgl. ebd., S. 100 f.). Zum Ende des 19. Jahrhunderts spitzte sich der Konflikt zwischen ökonomischem Effizienzdenken und pädagogischer Motivation im Rahmen einer ,liberal education' weiter zu. Der in ihren Grundprinzipien einer ,liberal education' verhafteten, manual trade'-Bewegung stand eine Gruppe von Schulreformern gegenüber, die eine, direct trade'-Ausbildung im Sinne einer spezifisch berufsorientierten Wissensvermittlung befürwortete: „...direct benefits of occupational skills rather than remote values associated with completing a liberal education by educating through the hand that had the greater appeal" (Kliebard 1995, S. 116). Unterstützung fand diese neue Richtung der berufsorientierten Schulreform in der Gründung der National Association of Manufacturers (NAM) 1896, die der Schulpolitik in ihrem Eröffnungsprogramm eine zentrale Rolle zuwies. Argumentativ wurde dabei auf wachsenden internationalen Wettbewerb durch junge aufstrebende Industrienationen, wie Deutschland, hingewiesen. Besondere Beachtung und Bewunderung fand in diesem Zusammenhang das deutsche duale Berufsausbildungssystem, dem man eine präeminente Bedeutung für die deutsche Wettbewerbsfähigkeit zusprach. Um dieser Meinung Ausdruck zu verleihen, wurde eine Resolution verabschiedet, „declaring that since technical education was so critical in the development of industry, its members should support ,manual training or technical schools"” (ebd., S. 117). Die NAM richtete ein eigenes Committee on Industrial Education ein, um die systematische Einflußnahme auf die öffentliche Meinung zu fördern. So veröffentlichte eben dieser Ausschuß 1905 einen Bericht über das, dropout'-Problem an amerikanischen Schulen. Darin wurde zunächst der Niedergang der Lehrlingsausbildung und das Fehlen einer institutionellen Alternative beklagt, woraufhin man als einzig mögliche Lösung des Problems die Schaffung spezieller Berufsschulen (Trade Schools) propagierte.

Ähnliche Ansätze wurden in Wisconsin trotz der ablehnenden Haltung des ,Superintendent of public instruction', Charles Cary, verfolgt, der die Institutionalisierung einer speziellen, vocational education' im öffentlichen Schulsystem zu verhindern suchte und eine Dualisierung durch die Schaffung eigener, trade schools' strikt ablehnte (vgl. Herbst 1996, S.122 f.). „A dual system, he [Cary] protested, meant a caste system and 
would destroy U.S. democracy" (ebd., S. 123). Diese europäisch orientierte Trennung von akademischer und beruflicher Bildung fand aber ihren Niederschlag in einem Gesetz, das jeder Stadt erlaubte, eine eigene Berufsschule $\mathrm{zu}$ gründen und $\mathrm{zu}$ unterhalten. Darüber hinaus wurde die Ausweitung der Berufsschulen auch in ländlichen Gebieten vorangetrieben, um durch die Unterrichtung landwirtschaftlicher Fächer eine moderne Agrarökonomie und Rationalisierungen in der Landwirtschaft zu fördern. Im Zuge einer systematischen Schulreform erhielt das neugegründete State Board of Industrial Education, in dem Arbeitgeber und Arbeitnehmer paritätisch vertreten waren, die Aufsichtskontrolle über die privaten Berufsschulen. „The Board of Industrial Education was the brainchild of a legislature whose members were motivated by fear of international economic competition rather than the drive to stop the drop-out problem of American high schools" (Herbst 1996, S. 122). Der Blick nach Deutschland galt auch hier als obligatorisch.

Andere Einzelstaaten, wie Pennsylvania und Massachusetts, gründeten ebenfalls ,trade schools' als selbständigen Teil ihres Schulsystems. Die Gesetzgeber in Massachusetts unterstellten die neugegründeten ,public industrial schools' anfangs einer vom übrigen Schulsystems unabhängigen Kontrollinstanz. Erst später wurde die Zweiteilung des Systems auf Einzelstaatsebene aufgehoben, während die Schulen auf Lokalebene ihre unterschiedliche Identität bewahrten (vgl. Kliebard 1995, S. 118; Herbst 1996, S. 120). Auf bundesstaatlicher Ebene formierte sich eine umfassende Koalition der ,vocational education'-Befürworter, deren partikulare Ansichten über die Organisation, Form und Kontrolle der beruflichen Ausbildung häufig differierten (vgl. Kantor 1982, S. 32 ff.). Die Pole der Differenzen sind in ihren Protagonisten John Dewey und David Snedden, Commissioner of Education in Massachusetts, verkörpert. John Dewey vertrat die Ansicht, daß eine nicht-berufsspezifisch orientierte ,vocational education' zur Reform des damaligen, tayloristisch-organisierten Wirtschaftssystems in Richtung einer ,industrial democracy’ beitragen könne. „Any scheme for vocational education which takes its point of departure from the industrial regime that now exists, is likely to assume and to perpetuate its divisions and weaknesses, thus to become an instrument in accomplishing the feudal dogma of social predestination" (Dewey [1916] (1966), S. 318). Arbeiter sollten durch, vocational education' in die Lage versetzt werden, ihre Arbeitssituation und den Arbeitsprozeß kritisch hinterfragen zu können (vgl. Grubb/Lazerson 1982, S. 128). Für Arbeitgeber läge der Vorteil darin, daß eine flexible, 
breit qualifizierte Arbeiterschaft schneller auf Markt- und Technologieveränderungen reagieren könnte. Folglich richtete sich seine Kritik gegen jeden Versuch der Dualisierung des Schulsystems in einen akademischen und einen berufsqualifizierenden Zweig. Deutlich wird dies in seiner Opposition gegen die ,Cooley Bill' in Illinois von 1913, in der eine administrative Abspaltung der ,vocational education' vom übrigen öffentlichen Schulsystem gefordert wurde. Allgemeiner Widerstand ließ das Gesetz schließlich scheitern (vgl. Kliebard 1995, S. 126 f.; Kantor 1982, S. 33 f.; Herbst 1996, S. 126 f.).

Deweys Denken war geprägt von den Werten und Prinzipien einer ,liberal education', die in der konkreten Ausgestaltung ihr verändertes Umfeld adaptiert, ihren Zielen aber treu bleibt (vgl. Tyack/Cuban 1995, S. 46; Lewis 1994, S. 212 f.). Auf der anderen Seite stand David Snedden, Massachusetts Commissioner of Education, der zusammen mit Charles Prosser, dem Vorsitzenden der National Society for the Promotion of Industrial Education, einer Lobby Organisation, die 1906 von Industrievertretern und industriefreundlichen Schulreformern gegründet wurde, eine administrativ-organisatorische Trennung akademischer und berufsqualifizierender Bildung forderte (vgl. Kantor 1982, S. 32). Ihre Vorstellungen orientierten sich dabei sehr stark am deutschen dualen Berufsausbildungssystem. „Vocational education, they contended, should be geared to needs of industry" (ebd., S. 30). Während die letzteren eine Ideologie der ökonomischen Effizienz vertraten, war für die reformerischen Ideen der ersteren eine sozialrekonstruktivistische Perspektive bestimmendes Moment.

Unter dem Eindruck wachsender Debatten über die Notwendigkeit von, vocational education' setzte der U.S. Kongreß 1914 eine Kommission zur Untersuchung des Bedarfs von ,National Aid to Vocational Education' ein. Die Mitglieder der Kommission kamen in der Mehrheit aus der National Society for the Promotion of Industrial Education, so auch Charles Prosser. Wenig überraschend waren deshalb auch die Schlußfolgerungen, die im Endbericht der Kommission vertreten wurden: Sicherung der internationalen Konkurrenzfähigkeit der Wirtschaft, Steigerung beruflicher Mobilität für ,working-class youth' und Reduzierung von ,dead-end jobs'. Um dies im Kontext eines nationalen Arbeitsmarktes erreichen zu können, sollte die Organisation und Finanzierung der Ausbildung nicht lokal oder auf Einzelstaatsebene erfolgen, sondern in die Verantwortung der bundesstaatlichen Regierung gelegt werden. „In short, training for a national labor market required federal support" (Kantor 1982, S. 35). Die National 
Society for the Promotion of Industrial Education ging so weit, ,a unified policy through massive federal intervention" (Kliebard 1995, S. 122) zu fordern. Trotz des positiven Urteils der Kommission gab es keine Mehrheit im Kongreß für eine bundesstaatliche Gesetzgebung. Diese konnte erst durch Koalitionsbildung mit landwirtschaftlichen Interessenverbänden erzielt werden, die eine Unterstützung ländlich-agrarischer Interessen durch die Ausweitung landwirtschaftlichen Unterrichts begrüßten. Als Resultat verabschiedete der Kongreß 1917 den Smith-Hughes Act, der bundesstaatliche Subventionen sowohl für die Lehrerausbildung in den zu fördernden Berufsbereichen Landwirtschaft, Hauswirtschaft, Handel und Industrie als auch für die Finanzierung von Lehrerstellen in diesen Bereichen an den öffentlichen High Schools zur Verfügung stellte. Außerdem schuf das Gesetz ein ,Federal Board of Vocational Education', das jedoch 1946 wieder abgeschafft wurde (vgl. Cuban 1982, S. 47 f.; Kliebard 1995, S. 122 ff.).

Im Smith-Hughes Act kulminierte der Konflikt zweier in ihrer ideologischen Ausrichtung diametral gegenüberstehenden Strömungen des amerikanischen ,vocationalism'. Die eine verkörpert die Idee der marktorientierten Effizienz, wohingegen die andere an den frühen Idealen des republikanischen ,citizenship' festhält. Beide glauben in der, vocational education' ein Instrument zur Erreichung ihrer Ziele gefunden zu haben, in der konkreten Umsetzung werden jedoch die grundlegenden Differenzen sichtbar. Die Ablehnung des Gesetzgebers, im Smith-Hughes Act eine duale Organisation der öffentlichen Schulbildung festzuschreiben, kann als Erfolg der Befürworter einer ,liberal vocational education' gewertet werden. Andererseits setzten sich die ,economic efficency'-Vertreter bei der Auswahl und Art der zu unterrichtenden berufsnahen Fächer durch. Aus dem Kompromiß entstand die ,comprehensive high school' als zentrale amerikanische Bildungsinstitution (vgl. Herbst 1996, S. 140). Der scheinbare Erfolg der wirtschaftsorientierten Schulreformer hinsichtlich der Zusammensetzung des Curriculums hatte letztlich weitreichende negative Konsequenzen. Die eingeschränkte Auswahl der Fächer determinierte die Struktur der, vocational education' auf lange Sicht und ließ wenig Spielraum für eine flexible Anpassung des Curriculums an veränderte technologische und soziale Bedürfnisse. „The structure of vocational education has remained largely unchanged since the passage of the 1917 Smith-Hughes Act [...]" (Lazerson/Grubb 1975, S. 459). 
Neben der aktiven Rolle amerikanischer Wirtschaftsverbände, insbesondere der National Association of Manufacturers (NAM), im Entstehungsprozeß einer staatlich unterstützten beruflichen Bildung trat der Einfluß der Gewerkschaften in den Hintergrund. Als die NAM bei ihrer Gründung die Schaffung eigenständiger Berufsschulen forderte, war die Reaktion der Gewerkschaften gespalten. Einerseits stand man einer von Arbeitgeberseite befürworteten Schulreform grundsätzlich mißtrauisch gegenüber, andererseits galt auch in Gewerkschaftskreisen eine berufliche Qualifizierung der Arbeiterschaft als entscheidende Strategie, um den Lebensstandard der Mitglieder in einem sich wandelnden Umfeld zu verbessern oder wenigstens zu halten. Eine ideale Lösung des Problems wäre die Schaffung eines gewerkschaftseigenen Berufsschulsystems gewesen, aber dazu fehlte es den Gewerkschaften an finanziellen Mitteln. Deshalb widersetzte man sich den Vorschlägen der Wirtschaft nicht grundsätzlich, sondern versuchte sie in annehmbarere Bahnen zu lenken. So lehnten die Gewerkschaften eine unter der Kontrolle der Arbeitgeber stattfindende,work-based'-Berufsausbildung ab und favorisierten statt dessen eine im Rahmen des öffentlichen Schulsystems organisierte berufliche Allgemeinbildung (vgl. Kliebard 1995, S. 118 ff.).

Der Aufstieg des ,vocationalism“ um die Jahrhundertwende ist im Kontext des sozioökonomischen Wandels zu sehen. Im Zuge der Dynamik des verspäteten Industrialisierungsprozesses verloren traditionelle Muster beruflicher Fertigkeiten und deren Konfiguration am Arbeitsplatz ihre Bedeutung. Anders als Modernisierungstheoretiker behaupten, kam es nicht zu einem universellen Anstieg beruflicher Qualifizierung. Vielmehr kann von einem Polarisierungstrend ausgegangen werden. Auf der einen Seite stiegen die Anforderungen an die berufliche Qualifikation in einigen neu entstandenen Positionen, auf der anderen Seite fand eine regelrechte Dequalifizierungskampagne für die Masse der Arbeiter statt. Die fortschreitende Mechanisierung des Arbeitsprozesses schuf eine Vielzahl von, low-skill‘ Arbeitsplätzen, insbesondere im Bereich der Maschinenüberwachung: ,....although technological change has created many new jobs, most of them are unskilled monitoring jobs..." (Hogan 1982, S. 149). Die tayloristische Verwissenschaftlichung der Arbeitsorganisation verstärkte darüber hinaus die Entwicklung einer Atomisierung der Tätigkeiten. Bis dahin war die Wirtschaft von kleinen, regional dispergierten Handwerksbetrieben dominiert, in denen lediglich eine rudimentäre Trennung der Funktionen existierte. Die Arbeitsaufteilung entsprach einer Hierarchie der handwerklichen Fertigkeiten. Mit der Ausdehnung der Infrastruktur 
konnte die Produktion in urbanen Zentren konzentriert werden. ,Economies of Scale ermöglichten den effizienten Einsatz von kapitalintensiven Maschinen und mithin eine kostengünstigere Produktion im Vergleich zu den Handwerksbetrieben. Der Niedergang der Handwerksbetriebe versetzte der sich bereits in Agonie befindenden Lehrlingsausbildung den Todesstoß. Im arbeitsteiligen, hochgradig spezialisierten Produktionsprozeß stellte eine breitangelegte Lehrlingsausbildung eine höchst ineffiziente Investition dar. „Employers attempt to pick and train workers so as to generate the desired productivity with the least investment in training costs" (ebd., S. 166). Dies hatte zur Folge, daß die Zahl der Jugendlichen, die eine Lehrlingsausbildung absolvierten, dramatisch sank. Als Reaktion auf den Mangel an ausgebildeten Arbeitskräften forderte die National Association of Manufacturers (NAM) als Sprachrohr der industriellen Produzenten die öffentliche Bereitstellung berufsbezogener Ausbildung, um gerade die Arbeitsmoral der jugendlichen Arbeiter zu verbessern, deren Fluktuationsrate besonders hoch war (vgl. Kantor 1982, S. 23). „Its spokesmen [NAM] said that they saw in trade education in the public schools under dual control an acceptable alternative to the dying apprentice system..." (Herbst 1996, S. 129). Auf diese Weise hätten die betriebsbezogenen Ausbildungskosten sozialisiert werden können.

Die Idee der Modernisierungstheoretiker, daß die Ausweitung des ,secondary schooling' zum Ende des 19. Jahrhunderts auf einen erhöhten Bedarf an qualifizierten Arbeitskräften zurückzuführen sei, ist durch die tatsächliche Entwicklung der beruflichen Struktur und qualifikatorischen Anforderungen im System der Massenproduktion nicht zu stützen. Für die steigende Zahl an Schülern im Sekundarbereich sprechen andere Gründe: So beschränkte der ,Child Labor Act' die Arbeitsmöglichkeiten der Jugendlichen in der industriellen Produktion und zwang sie so mangels Alternative indirekt in die Schule. Das Problem der Jugendkriminalität und jugendlichen Banden in den Städten ließ die Schule als, warehousing'-Institution an Bedeutung gewinnen. Darüber hinaus gab es neue Berufe im administrativen Bereich, wie z.B. der Buchhaltung, die eine längere Schulbildung erforderten. Die Zahl solcher Berufe war aber längst nicht so groß, daß sie eine Ausweitung der Schulbildung in einem derartigen Umfang erklären könnte (vgl. Grubb/Lazerson 1982, S. 118-124). Mit der Expansion des ,secondary schooling ‘ verlor die High School ihre präeminente Position als Collegevorbereitende Institution. Neue Aufgaben und Bedürfnisse wurden an sie herangetragen, 
um dem Wandel des sozio-ökonomischen Umfeldes gerecht werden zu können. Befürworter einer beruflichen Bildung in der High School propagierten diese nicht nur aus Effizienzgründen, sondern glaubten, in ihr die Lösung für nationale Probleme, wie die Assimilierung von Immigranten, Senkung der Armutsrate, Verringerung der Jugendarbeitslosigkeit und der, dropout'-Zahlen, gefunden zu haben (vgl. Kantor/Tyack 1982, S. 2).

Dieser Glaube an eine universelle Heilwirkung schulisch organisierter beruflicher Bildung besteht bis heute trotz empirischer Widerlegung fort. Dies führt $\mathrm{zu}$ immer neuen Reformvorschlägen im Schulbereich, anstatt die Ursachen der Probleme in der Organisation der Wirtschaft zu suchen. Die Reformen stehen dabei im Zeichen des klassischen Dilemmas zwischen spezifisch berufsorientierter Ausbildung im Sinne einer markteffizienten Organisation und allgemein orientierter beruflicher Bildung im Sinne einer , liberal education“. 


\section{Institutionen und Interessen}

Das deutsche duale Berufsausbildungssystem ist - wie im vorangegangenen Teil eingehend erläutert - eingebettet in ein spezifisches institutionelles Arrangement, das sich seit dem Ende des 19. Jahrhunderts immer deutlicher herauskristallisiert hat. Ein Transfer des Systems wäre nur möglich, wenn das institutionelle Gefüge der USA diesem offenstünde. Im folgenden sollen die für ein duales Berufsausbildungssystem wichtigen institutionellen Strukturen und Akteure, deren Interessen durch die Institutionen bedingt sind, untersucht werden, um $\mathrm{zu}$ klären, inwieweit sie einen Transfer behindern oder begünstigen.

\subsection{Jugendarbeitsmarkt}

Die letzte Dekade hatte weitreichende Konsequenzen für den amerikanischen Arbeitsmarkt. Viele Firmen waren einem zunehmenden internationalen Wettbewerb ausgesetzt. Um in diesem Umfeld weiterhin produktiv sein zu können, mußten althergebrachte Gewohnheiten „über Bord“ geworfen werden. Es galt, die Organisation der Produktion und Arbeitsabläufe zu restrukturieren. Neue Managementkonzepte, wie ,reengineering', ,total quality management' und ,lean production', wurden in die Firmen gebracht und veränderten die Unternehmensstrukturen. Statt ausgeprägter Hierarchien mit klaren Verantwortungsbereichen und Kontrollfunktionen erweiterte sich der Aufgabenbereich des einzelnen Arbeitnehmers: Flache Hierarchien und mehr Verantwortung waren die Schlagworte. Gleichzeitig fand eine Konzentration auf die Kernkompetenzen statt. Dies ging wiederum einher mit umfassenden , outsourcing'-Maßnahmen, bei denen Aufgaben, die nicht zu den Kernkompetenzen zählten, ausgegliedert wurden und viele Arbeitnehmer entweder ihren Arbeitsplatz verloren oder häufig unter schlechteren Bedingungen bei einer Unterfirma weiterbeschäftigt wurden (vgl. Office of Technology Assessment 1990, S. 4-6; Berryman/Bailey 1992, S. 11-17; Batt/Osterman 1993, S. 10 f.). Das Neue an dieser weitreichenden ,downsizing'-Politik war das augenscheinliche Fehlen einer betriebswirtschaftlichen Rationalität. Denn die wenigsten Firmen litten unter tatsächlichen Umsatzeinbrüchen, die eine Entlassungs- und Umorganisationswelle hätten rechtfertigen können (vgl. Cappelli et al. 1997, S. 5 f.). Der Faktor Arbeit wurde insofern „,normalisiert“, als er, wie andere Produktionsfaktoren, dem Prinzip des ,justin-time‘ unterworfen wurde. So glaubte man, die gestiegenen Flexibilitätsanforderungen 
auf den Gütermärkten erfüllen zu können. Mehr Verantwortung erforderte aber größere Fähigkeiten, sowohl kognitiver als auch sozialer Art, auf seiten der Arbeiter (vgl. O’Neil 1992, S. 7). Forderungen nach besseren Ausbildungsprogrammen und höheren Investitionen in die Ausbildung waren nur eine konsequente Folge dieser Entwicklung. Implizite Übereinkünfte zwischen Arbeitgebern und Arbeitnehmern über stabile Beschäftigungsverhältnisse, interne Aufstiegsmöglichkeiten, Loyalität der Arbeitnehmer etc., die die Beschäftigungsstrukturen nach dem Zweiten Weltkrieg geprägt hatten, haben im Zeitalter der Flexibilisierung an Bedeutung verloren (vgl. Sennett 1998, S. 59 ff. und 113 ff.). Der Druck zur Flexibilisierung der Beschäftigungsverhältnisse ist ein Resultat zunehmender Konkurrenz zwischen den Firmen, die die Arbeitsproduktivität in den Mittelpunkt strategischer Überlegungen stellten. Darüber hinaus versuchen Arbeitgeber staatliche Arbeitsmarktregulation, z. B. Arbeitsgesetze wie den Fair Labor Standards Act, durch Reorganisation der Arbeitsverhältnisse zu umgehen. Der qualitative Wandel in den Ansprüchen der Konsumenten erhöht zusätzlich den Flexibilisierungsdruck auf die Unternehmen. Infolgedessen brachen einige Arbeitgeber mit den Traditionen des „alten“ Beschäftigungsverhältnisses und lösten damit die Erosion des bestehenden Systems aus, das ohne beiderseitiges Vertrauen nicht bestehen kann.

Die Transformationen auf Unternehmensebene sind für den Arbeitsmarkt jedoch nicht folgenlos geblieben. Während die alten Arrangements Arbeitnehmer vor den kompetitiven Kräften des externen Arbeitsmarktes schützten, finden sich die Arbeitnehmer in der neuen Beschäftigungskonstellation genau diesen Kräften ausgesetzt. Firmen nutzen externe Arbeitsmärkte, um fehlende Fertigkeiten einzukaufen, passen ihre ,workforce“ dem Auftragseingang an, indem sie kapazitätsorientiert einstellen, aber auch wieder entlassen (vgl. Bernhardt et al. 1998a, S. 4 f.; Office of Technology Assessment 1990, S. 13). Dies führt zur Ausweitung der sogenannten ,contingent workforce', also der Zahl von Arbeitern, die keine Beschäftigungssicherheit kennt. Risiken, die sich durch die Gewährung von Beschäftigungsstabilität trotz zyklischer Auftragseingänge ergaben und bis dato beim Unternehmen lagen, gehen nunmehr zu Lasten der Arbeitnehmer. Die ständige Angst vor der Entlassung zwingt Arbeitnehmer, ihre ,employability“ durch eigene Investitionen in die Ausbildung immer auf dem neuesten Stand zu halten. Die Arbeitsmoral sinkt, der Streß steigt. Andererseits erfordert die neue Arbeitsorganisation 
aufgrund weniger Kontrollen von oben und mehr Eigenverantwortung ein höheres Maß an Loyalität des Arbeitnehmers.

Ein weiterer Widerspruch liegt in der Gleichzeitigkeit von gestiegenem Bedarf an Fertigkeiten (skills) und sinkenden Anreizen für Unternehmen, Ausbildungsprogramme anzubieten (vgl. Cappelli et al. 1997, S. 123 f.; Salzman 1998, S. 125 ff.; Batt/Osterman 1993, S. 11 f.). Die Unternehmen stehen vor einer klassischen ,make or buy ${ }^{6}$ Entscheidung. Bei den von den Unternehmen benötigten Fertigkeiten handelt es sich insbesondere um ,company-specific skills‘, die nur im Unternehmen am Arbeitsplatz gewonnen werden können. Infolge der Umstrukturierungen in Richtung einer arbeitsgruppenorientierten Organisation kommt es zum Abbau von ,low-skill, entrylevel jobs ${ }^{6}$, da diese nun Teil des Aufgabenbereiches der jeweiligen Arbeitsgruppe sind. Mithin können ungelernte Arbeitskräfte nicht mehr auf dieser Ebene in die Firma einsteigen und sich dort die notwendigen Fähigkeiten aneignen, die für den beruflichen Aufstieg notwendig sind. Diese Hürde ist gerade für den Jugendarbeitsmarkt von existentieller Bedeutung. Statt junge, ungelernte Arbeitskräfte einzustellen, werben Unternehmen ausgebildete Arbeitskräfte von anderen Firmen ab, um diese in ihre Arbeitsgruppen $\mathrm{zu}$ integrieren. „Truly unskilled workers would flounder, so replacement workers will probably have to be hired from some place where they have received at least basically similar work experience. That is likely to be other employers..." (Cappelli et al. 1997, S. 10). Je mehr Unternehmen sich der neuen Arbeitsorganisation zuwenden, desto schwieriger wird es, Arbeiter mit ausreichend ,onthe-job training' $\mathrm{zu}$ finden, da die für den Einstieg ungelernter Arbeiter in den Arbeitsmarkt entscheidenden ,low skill, entry-level jobs ' verschwinden (vgl. ebd., S. 8 ff.; O’Neil 1992, S. 7; Salzman 1998, S. 125 ff.).

Der Jugendarbeitsmarkt weist eine Vielzahl charakteristischer Eigenschaften auf, die ihn vom ,allgemeinen“ Arbeitsmarkt unterscheiden und eine eigene Analyse der institutionellen Arrangements erforderlich machen. Wie bereits angedeutet, wird der Jugendarbeitsmarkt in geradezu existentieller Weise von den oben beschriebenen Veränderungen der Arbeitsorganisation getroffen. Um dies zu verdeutlichen, soll im folgenden der Charakter des Jugendarbeitsmarktes herausgearbeitet werden. Grundsätzlich kann der Arbeitsmarkt in zwei Kategorien unterteilt werden: primärer und sekundärer Arbeitsmarkt. Beschäftigungsverhältnisse im primären Arbeitsmarkt sind weitgehend stabil. Es handelt sich um ,long-term jobs', deren Stabilität den Aufbau von 
internen Arbeitsmärkten ermöglicht. Über die internen Arbeitsmärkte eröffnen sich den Arbeitnehmern weitreichende Aufstiegsmöglichkeiten. Das Lohnniveau ist relativ hoch, auch um die Arbeitnehmer an das Unternehmen zu binden. Der sekundäre Arbeitsmarkt ist hingegen durch instabile Beschäftigungsverhältnisse, Teilzeitarbeit und Löhne an der Grenze zum Mindestlohnniveau charakterisiert. Eine Unterkategorie des sekundären Arbeitsmarktes ist der Jugendarbeitsmarkt (vgl. Bresnick 1984, S. 6 ff.; Osterman 1980, S. 20 ff.). Dieser umfaßt alle Jugendlichen mit oder ohne High School-Abschluß, die keinen College-Abschluß haben. Grundsätzlich unterscheidet man zwischen ,collegebound‘ und ,non college-bound“ Jugendlichen. Während die ,college- bound“ Schüler direkt im Anschluß zur High School ein College oder eine Universität besuchen und nach ihrem Abschluß Arbeit auf dem primären Arbeitsmarkt nachfragen, stehen die ,non college-bound‘ Schulabgänger dem Arbeitsmarkt direkt zur Verfügung. Das Problem dieser Gruppe, einen nahtlosen Übergang von der Schule zur Arbeit zu bewerkstelligen, gibt immer wieder Anlaß zu öffentlichen Diskussionen und staatlichen Maßnahmen.

Vier wesentliche Merkmale zeichnen diesen Arbeitsmarkt aus: Erstens weist er eine hohe ,turnover rate‘ auf, die sich nicht nur aus einer starken Abhängigkeit vom Konjunkturverlauf (business cycle) ergibt, sondern auch im Verhalten der Jugendlichen angelegt ist. Ferner ist der Arbeitsmarkt lokal orientiert, d.h. es gibt wenig interregionale Mobilität der Arbeitskräfte. Dies erklärt teilweise auch die Dominanz kleiner bis mittlerer Unternehmen als Arbeitsnachfrager. Und schließlich verändert sich die Arbeitslosenquote von Jugendlichen aufgrund der stärkeren Konjunkturabhängigkeit überproportional zu der anderer Arbeitskräfte (vgl. Grubb et al. 1992, Kp. I; Freeman/Wise 1982, S. 8; Hamilton 1990, S. 23). Gerade in Zeiten der Rezession steigt die Jugendarbeitslosigkeit dramatisch an. So lag sie in den 1970er Jahren bei ca. 20 Prozent. Entscheidend für die hohe Arbeitslosigkeit war nicht nur der technologische Wandel in den klassischen Bereichen der Jugendbeschäftigung (Landwirtschaft, industrielle Produktion), sondern insbesondere auch die schwache Bindung der Jugendlichen an den Arbeitsmarkt bzw. die Firma aufgrund fehlender Arbeitserfahrung (vgl. Feldstein/Ellwood 1982, S. 17 f.; Bresnick 1984, S. 3 f.; Gitter/Scheuer 1997, S. $16 \mathrm{f}$.). "Teenagers tend to be at the end of the line of those considered for a job" (Bresnick 1984, S. 4). 
Die idiosynkratisch hohe ,turnover'-Rate des Jugendarbeitsmarktes ist das zentrale institutionelle Hindernis für Unternehmen, umfassende Ausbildungsmaßnahmen für Jugendliche bereitzustellen (vgl. Bishop 1995, S. 9; Lynch 1993, S. 1293). Eine auf der National Longitudinal Survey/Youth basierende Studie für die 1980er Jahre ergab, daß Jugendliche eine Vielzahl von Jobs in den ersten Jahren ihrer Arbeitsmarkterfahrung durchlaufen und ein großer Anteil darüber hinaus nach Verlassen der High School weder einen Vollzeitjob hat, noch in der Schule (postsecondary education) ist. Der mediane High School-Absolvent hat mit 19 Jahren einen Job, der mindestens ein Jahr lang dauert. Mit 20 Jahren dauert das Beschäftigungsverhältnis bereits zwei Jahre und mit 21 Jahren drei Jahre. Dies gilt jedoch nicht für den medianen ,high school-dropout', der trotz früheren Eintritts in den Arbeitsmarkt erst mit 23 Jahren ein Beschäftigungsverhältnis von dreijähriger Dauer vorweisen kann. Noch problematischer sind die Zahlen bei schwarzen Jugendlichen, die sehr viel schlechter abschneiden als weiße und hispanische Jugendliche: ,...young dropouts are less likely to be working full time and more likely to be neither working nor in school“ (Klerman/Karoly 1995, S. xiv).

Neuere Zahlen für die frühen 90er Jahren zeigen, daß sich die Entwicklung eher verschlechtert als verbessert hat. Der Übergang von der Schule in den Arbeitsmarkt ist volatiler geworden und dauert länger (Osterman 1999, S. 45). Es gibt mehr Unterbrechungen im Erwerbsverlauf, und die Wechsel zwischen unterschiedlichen Industrien haben zugenommen. Dadurch erhöht sich die ,variability in accumulated work experience“ (Bernhardt et al. 1998a, S. 2). Zudem sind die Beschäftigungsverhältnisse instabiler geworden, und die Dauer der Beschäftigung beim einzelnen Arbeitgeber hat abgenommen. Jugendliche, die in den späten 80er und frühen 90er Jahren in den Arbeitsmarkt eingestiegen sind, konnten darüber hinaus die Lohnzuwächse früherer Arbeitsanfänger nicht mehr erzielen (vgl. Bernhardt et al. 1998b, S. 24). „The deterioration in wage gains for the recent cohort first appears between the ages of 16 and 21 , especially among workers moving directly from high school into the labor market“" (Bernhardt et al. 1998a, S. 3). 
Im Sinne der Humankapital Theorie ${ }^{23}$ stellt diese Phase des fragilen Übergangs von der Schule in den Arbeitsmarkt eine höchst ineffiziente, gemeinwohlreduzierende Allokation des Faktors Arbeit dar. Die hohe ,job turnover'-Rate hat mehrere negative Konsequenzen: So steigt das Risiko der Arbeitslosigkeit, da mit dem Wechsel von einem Job zum anderen häufig Phasen der Nicht-Beschäftigung verbunden sind, in denen keine Fertigkeiten aufgebaut werden können bzw. bestehende Fertigkeiten sogar abgebaut werden. Außerdem bedeutet jeder Wechsel einen Verlust von firmenspezifischen Fertigkeiten, deren Akkumulation mit Produktivitätssteigerungen und dementsprechenden Lohnzuwächsen einhergeht. Ein Firmenwechsel bringt somit für das Individuum einen Verlust an möglichen Lohnsteigerungen mit sich, da die firmenspezifischen Fertigkeiten keine produktivitätssteigernde Wirkung beim neuen Arbeitgeber haben (vgl. Klerman/Karoly 1995, S. 2 f.; Bresnick 1984, S. 5). Darüber hinaus sinkt die kollektive Wohlfahrt aufgrund sinkender Produktivität: „,...when the worker leaves the firm, he or she forfeits those higher wages, and assuming the higher wage was due to higher productivity, society loses output“" (Klerman/Karoly 1995, S. 3). Der häufige Job-Wechsel bei Jugendlichen reduziert wiederum den Anreiz für Unternehmen, in die Ausbildung derselben $\mathrm{zu}$ investieren. Bei Ausbildungsinvestitionen ist zwischen allgemeinem und firmenspezifischem Humankapital $\mathrm{zu}$ unterscheiden. Allgemeines Humankapital erhöht die Produktivität des einzelnen Arbeitnehmers unabhängig vom jeweiligen, firmenspezifischen Produktionsprozeß. Folglich geht es beim Arbeitsplatzwechsel nicht verloren und kann auf dem Markt „angeboten“ werden. Für den Arbeitnehmer gibt es keinen ökonomischen Grund, nach der Ausbildungszeit einen Lohn unterhalb seiner Grenzproduktivität zu akzeptieren, um so dem ausbildenden Unternehmen seine Investition im Laufe der gesamten Beschäftigungszeit zurückzuzahlen. Unternehmen haben mithin keinen ökonomischen Anreiz, in allgemeines Humankapital zu investieren.

Im Gegensatz dazu ist firmenspezifisches Humankapital an die jeweilige Firma und ihren eigenen Produktionsprozeß gebunden. Die produktivitätssteigernde Wirkung bleibt auf die Firma bezogen. Dies bedeutet, daß firmenspezifisches Humankapital nicht marktfähig ist und somit keinen Anreiz für einen Arbeitsplatzwechsel darstellt. Aus diesem Grund sind Unternehmen am ehesten bereit, in firmenspezifische Ausbil-

\footnotetext{
${ }^{23}$ Vgl. dazu Gary Becker (1993).
} 
dungsprogramme zu investieren, da hier das Risiko eines Investitionsverlustes am geringsten ist. Für den Arbeitnehmer gibt es keinen ökonomisch-rationalen Grund, seine firmenspezifischen Kenntnisse aufzugeben, da sie keinen Einfluß auf die Lohnhöhe bei einem anderen Arbeitgeber haben (vgl. Becker 1993, S. 33-50; Soskice 1994a, S. 29; Klerman/Karoly 1995, S. 5-7; Ryan 1984, S. 194-195). In der Realität ist diese strikte Unterscheidung hingegen kaum durchzuhalten, da selbst genuin firmenspezifische Kenntnisse marginale Bestandteile allgemeinen Humankapitals enthalten. Infolgedessen sind Unternehmen sehr zurückhaltend mit Ausbildungsinvestitionen sowohl in allgemeine als auch in firmenspezifische Fertigkeiten, da sie in jedem Fall das Investitionsrisiko tragen. Dies gilt jedoch nur dann, wenn nicht die Löhne der Auszubildenden um die Ausbildungskosten gesenkt werden können. Hier trägt der Arbeitnehmer die Ausbildungsinvestition selber. Es besteht aber kein Interesse für den Arbeitnehmer in firmenspezifisches Humankapital zu investieren, weil dies seine Marktposition nicht verbessert. Außerdem kann der Ausbildungslohn aus unterschiedlichen Gründen nicht so weit gesenkt werden, daß sich Investitionen in allgemeines und firmenspezifisches Humankapital innerhalb der Ausbildungszeit amortisieren. Dem steht insbesondere der gesetzlich festgelegte Mindestlohn entgegen. Aus Sicht der klassischen ökonomischen Theorie führt die Einführung eines Mindestlohns zu einem Abbau von Beschäftigung. Im Fall des amerikanischen Jugendarbeitsmarktes trifft diese Annahme jedoch nicht zu: „Jobs for young workers are readily available in the U.S. economy, in spite of minimum wage legislation..." (Hall 1982, S. 491). Der Mindestlohn führt aber langfristig dazu, daß Beschäftigungspraktiken entstehen, die hohe ,turnover'-Raten implizieren. Für Arbeitgeber schränkt ein Mindestlohn die Möglichkeit ein, trotz flexibler Auftragseingänge eine konstante Anzahl von Arbeitnehmern zu beschäftigen und so in auftragsschwachen Zeiten mehr als die benötigten Arbeitskräfte zu bezahlen. Die Unternehmen reagieren darauf mit flexiblen Beschäftigungsverhältnissen, um ihre Belegschaft entsprechend der Auftragseingänge anpassen $\mathrm{zu}$ können. Statt eines niedrigeren Lohns und stabiler Beschäftigung zahlen Unternehmen relativ hohe Stundenlöhne und flexibilisieren den Einsatz des Faktors Arbeit. Dies hat wiederum Einfluß auf die Organisation der Arbeitsprozesse. Der einzelne Arbeitsplatz wird standardisiert, um neue Arbeitskräfte ohne großen Ausbildungsaufwand anlernen zu können (vgl. ebd., S. 489 f.; Grubb et al. 1992, Kp. III). „The influence of an effective minimum wage ... is to prevent employers from offering better duration terms to workers in exchange for lower cash wages“ (Hall 1982, S. 479). Die 
Bedeutung des Mindestlohnes trifft selbstverständlich nur auf Arbeitskräfte zu, deren Grenzproduktivität relativ niedrig ist und deshalb nahe am Mindestlohn liegt. Gerade junge, ungelernte Arbeitskräfte fallen in diese Kategorie.

Ein weiteres Charakteristikum des Jugendarbeitsmarktes ist seine lokale Orientierung. Unternehmen suchen Arbeiter in diesem Segment nur lokal und stehen dabei lokal ausgerichteten Arbeitsanbietern gegenüber. Die Mobilität der jugendlichen Arbeitnehmer ohne College-Abschluß ist sehr gering. „In contrast, employers routinely search statewide and nationally for their upper-level professional and managerial positions, and such individuals are much more mobile...than are less well-educated individuals“ (Grubb et al. 1992, Kp. III). Dies kann dazu führen, daß es eine dauerhafte Unterversorgung an spezifischen Berufen in einem lokalen Arbeitsmarkt gibt, da Lohndifferenzen zwischen Regionen keinen Anreiz für berufliche Mobilität darstellen (vgl. Grubb/McDonnell 1991, S. 3). Infolge fehlender interregionaler Mobilität können bei nationalen, strukturellen Verschiebungen in der Wirtschaft Mangel- bzw. Überschußerscheinungen in den lokalen Arbeitsmärkten auftreten. Die Lokalität des Jugendarbeitsmarktes bedingt zudem die herausragende Rolle kleiner und mittlerer Unternehmen für die Erstbeschäftigung jugendlicher Arbeitnehmer. Zwischen großen und kleinen Unternehmen gibt es einen wesentlichen Unterschied, der das Einstellungsverhalten hinsichtlich jugendlicher Arbeiter bestimmt: Während große Firmen unter ,time constraints ‘ produzieren, stehen bei kleinen Firmen die ,cost constraints' im Vordergrund. Große Unternehmen benötigen aufgrund zeitlicher Beschränkungen Arbeitnehmer, die sofort produktiv sind, d.h. keiner langen Anlernphase bedürfen. Kleine Betriebe müssen hingegen ihre Produktionsprozesse unter strikten Kostengesichtspunkten organisieren, um wettbewerbsfähig bleiben zu können. Hierfür benötigen sie billige Arbeitskräfte, wie ungelernte High School-Absolventen. Da die Firmen in den Jugendlichen lediglich billige Arbeitskräfte sehen, die es kapazitätsorientiert einzusetzen gilt, bestehen keine Anreize, in die Ausbildung derselben zu investieren.

Aus der Perspektive der Jugendlichen bieten kleine und mittlere Firmen keine Aufstiegsmöglichkeiten, die eine langfristige Bindung an einen Arbeitgeber rechtfertigen könnten (vgl. Osterman 1980, S. 22 f.). Große Unternehmen können hingegen höhere Löhne zahlen, um die von ihnen benötigten Fertigkeiten einzukaufen. Infolge ihrer Größe sind sie zudem in der Lage, Aufstiegsmöglichkeiten durch interne Arbeitsmärkte zu bieten. Wegen der sich daraus ergebenden großen Zahl an Bewerbern können diese 
Unternehmen höhere Ansprüche an die Qualifikation selbst der untersten Arbeitsplatzkategorien stellen. Ihnen dient die frühere Beschäftigung eines Bewerbers in einer kleinen Firma als Zeichen seiner ,employability‘. Dabei sind neben dem Nachweis bestimmter Fertigkeiten gerade solche Tugenden, wie Zuverlässigkeit und Stabilität der Beschäftigung, ausschlaggebend. Im Gegensatz zu den formellen Einstellungsverfahren großer Unternehmen ist der Einstellungsprozeß von kleinen und mittleren Firmen informell und wenig standardisiert. Häufig mangelt es ihnen an klar definierten Arbeitsprofilen, weshalb sie auch nur vage Vorstellungen über die von ihnen benötigten Fertigkeiten haben. Schulische Zeugnisse haben eine untergeordnete Bedeutung. Sie dienen lediglich als Nachweis über die Zahl der Schuljahre und sekundärer Tugenden, wie regelmäßigem Schulbesuch (vgl. Bishop 1994, S. 8; auch Nadel 1997, S. 185). Dies liegt an der für den einzelnen Arbeitgeber asymmetrischen Informationslage. Viele Arbeitgeber wissen nicht, welche Programme an der jeweiligen High School angeboten werden und wie strikt die Bewertungsmaßstäbe sind. Insofern haben die im Zeugnis aufgeführten Kurse keine Aussagekraft für die Unternehmen.

Wichtiger als Zeugnisse ist deshalb die vorzuweisende Arbeitserfahrung. „Partly because of the reliance on experience rather than formal schooling, a majority of employers were not knowledgeable about local education providers and were indifferent to them" (Grubb et al. 1992, Kp. III). Arbeitgeber erkennen wohl den Zusammenhang zwischen Erfolg in der Schule und Leistungsbereitschaft am Arbeitsplatz, trotzdem gibt es zwischen ihnen keinen Wettbewerb um die besten Schüler in Form von höheren Löhnen, da die Zeugnisse von Schülern unterschiedlicher Schulen nicht vergleichbar sind. „During the first decade after leaving high school, young men received no rewards from the labor market for developing competence in science, language arts and mathematical reasoning“ (Bishop 1994, S. 8). In den , entry-level jobs‘ spiegelt der Lohn nicht die Produktivität des einzelnen Arbeiters wider, sondern ist ein Abbild der Position in der Unternehmenshierarchie.

Wenn also die ,rewards for learning' fehlen, wieso sollten sich dann die Schüler anstrengen? $\mathrm{Zu}$ unterscheiden ist hier wieder zwischen ,college bound' und ,non-college bound' Schülern. Für erstere besteht natürlich ein Anreiz sich anzustrengen, da ihr College-Platz davon abhängt. Letztere orientieren sich an den Anforderungen des Arbeitsmarktes. Entsprechend bevorzugen sie leichtere und unterhaltsamere Kurse, deren Anspruchsniveau nur vom Titel nicht beurteilt werden kann. „An angry math teacher 
[remembering] the elimination of a carefully planned program in technical mathematics for vocational students simply because not enough signed up for it,...said, ,It's easy to see who really makes decisions about what schools teach: ,the kids do" " (Powell/Farrar/Cohen, in: ebd., S. 5). Da Eltern häufig genauso wenig informiert sind wie Arbeitgeber, können sie ebenfalls keinen Einfluß auf die Wahl der Kurse nehmen. Zudem besteht kein Anreiz für Eltern, höhere Standards zu fordern, denn ihre Kindern haben davon keinen Vorteil auf dem Arbeitsmarkt. Höhere Standards bedeuten außerdem höhere Steuern, um das Niveau der Schulen zu verbessern. Darüber hinaus gibt es sogenannte ,peer group'-Normen, die die Schüler von der Belegung akademisch anspruchsvoller Kurse abhalten. Schüler, die sich in der Schule akademisch ${ }^{24}$ anstrengen und leistungsbereit sind, werden als ,nerd‘, ,brain geck' oder ,acting White‘ bezeichnet. Folge eines solchen sozial nicht akzeptierten Verhaltens ist der Ausschluß aus der Gruppe (vgl. Meyer 1996a, S. 897). Die Bewertung der Leistung des einzelnen Schülers in Relation zur Leistung der Klassenkameraden ohne einen externen Bezugspunkt reproduziert dieses Verhalten. „Since devoting time to studying for an exam is costly, the welfare of the entire class is enhanced if no one studies for exams that are graded on a curve“ (Bishop 1994, S. 5). Resultat ist die Institutionalisierung einer ,minimize studying norm‘.

$\mathrm{Zu}$ berücksichtigen ist auch, daß Lehrer nicht die Funktion eines Partners haben, mit dem gemeinsam auf ein extern vorgegebenes Ziel hingearbeitet wird, sondern die eines Richters, der über die Bewertung entscheidet. In einem solchen Verhältnis ist der Aufbau einer ,mentoring'-Beziehung kaum möglich. Fehlende persönliche Beziehungen zwischen Lehrern und Schülern verstärken aber die Gefahr des ,dropout', trotz minimaler Abschlußstandards (vgl. ebd., S. 6). Die institutionelle Organisation der Schule verhindert den Aufbau eines Anreizsystems, bei dem auch für ,non-college bound' Schüler akademische Leistung wichtig ist, und reproduziert durch die enge Verknüpfung zum Arbeitsmarkt, insbesondere die Einstellungspolitik der Arbeitgeber, die institutionelle Ausformung dieser Schnittstelle. „One of the saddest consequences of the lack of signals of school achievement is that employers offering training and job

\footnotetext{
24 Ausnahme bildet der Sport: hier wird Leistungsbereitschaft und Teamgeist gefordert. Sportliche Leistung ist sozial höchst akzeptiert und kann zudem zur Aufnahme in ein renommiertes College führen.
} 
security are unwilling to take the risk of hiring a recent high school graduate" (ebd., S. 9).

Zusammenfassend läßt sich sagen, daß der Jugendarbeitsmarkt wegen seiner hohen ,turnover'-Rate keinen Anreiz für Unternehmen bietet, in die Ausbildung jugendlicher Arbeiter zu investieren. Diese hohe Fluktuationsrate ergibt sich einerseits aus der Tatsache, daß insbesondere kleine und mittlere Firmen als Arbeitsnachfrager fungieren und diese Konjunkturschwankungen stärker unterworfen sind als größere Unternehmen, und andererseits aus dem individuellen Verhalten der Jugendlichen, die in den ersten Jahren auf dem Arbeitsmarkt verschiedene Jobs testen. Die geringe Aussagefähigkeit der High School-Zeugnisse führt bei kleinen und mittleren Unternehmen zur Nichtbeachtung derselben bei der Einstellung. Dies verstärkt wiederum die fehlende akademische Leistungsbereitschaft der Jugendlichen, für die es keinen ökonomischen Grund gibt, sich anzustrengen. Daher mangelt es vielen Jugendlichen bereits an einfachen Grundkenntnissen des Schreibens und Rechnens. Arbeitgeber befinden sich also in einem Dilemma, da sie einerseits diese allgemeinen Fähigkeiten als unbedingte Voraussetzung selbst für einfache Tätigkeiten benötigen, andererseits aber Investitionen in allgemeines Humankapital für den einzelnen Arbeitgeber mit hohen Risiken verbunden sind. Solange Zeugnisse nicht an einem allgemeinen externen Standard gemessen werden können, ist es für Arbeitgeber kostenintensiv, sich Informationen über das schulische Angebot und seine Qualität $\mathrm{zu}$ beschaffen. Die asymmetrische Informationslage reproduziert jedoch den ökonomisch ineffizienten und

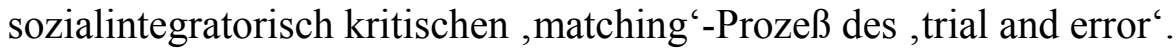

\subsection{Unternehmensverbände und Arbeitgeber}

Bei beruflicher Ausbildung handelt es sich um ein quasi-öffentliches Gut, insbesondere wenn sie $\mathrm{zu}$ einem zertifizierten, anerkannten Abschluß führt und neben firmenspezifischen Elementen auch solche der allgemeinen Bildung umfaßt. Die Bereitstellung von öffentlichen Gütern kann wegen des ,free-rider'-Problems durch den Markt nicht gewährleistet werden. Dieses Marktversagen gilt als Argument für ein Eingreifen des Staates, der die Bereitstellung eines öffentlichen Gutes durch entsprechende 
Steuereinnahmen finanziert. Im Bereich der beruflichen Bildung führt eine direkte Intervention des Staates $\mathrm{zu}$ einer schulischen Organisation der ,skill formation“. Daneben besteht jedoch die Möglichkeit, intermediären Organisationen die Verantwortung zur Beseitigung einer marktorganisierten Fehlallokation zu übertragen. Arbeitgeberverbände können die Bereitstellung einer ausreichenden Zahl von Ausbildungsplätzen und die Festsetzung von Ausbildungsstandards unter ihren Mitgliedsfirmen koordinieren, indem sie die berufliche Ausbildung dem Wettbewerb zwischen den Unternehmen entziehen. Hierzu benötigen die Verbände ein legitimiertes Sanktionspotential, das es ihnen ermöglicht, Fehlverhalten einzelner Mitglieder formell oder informell zu maßregeln. Entscheidende Voraussetzung für eine effektive Inanspruchnahme von Verbänden durch den Staat ist die Organisationsdichte der Unternehmen und die Legitimation der Verbände als intermediärer Interessenvermittlungsinstanz (vgl. Streeck/Schmitter 1985, S. 24-27; Crouch 1993, S. 10; Parker 1996, S. 31).

Für die USA ist nun zu untersuchen, welche Formen der verbandlichen Organisation betrieblicher Interessen vorhanden sind. Im folgenden sollen die wichtigsten nationalen Unternehmensverbände kurz dargestellt werden. Ausgenommen von der Untersuchung sind solche Verbände, die nur lokal oder regional aktiv sind. Die nationalorganisierten Verbände haben alle einen hierarchisch untergliederten Aufbau. Auf nationaler Ebene liegt die Interessenwahrnehmung der Unternehmen bei drei branchenübergreifenden Organisationen: U.S. Chamber of Commerce, National Association of Manufacturers (NAM) und dem Business Roundtable. Daneben gibt es die Trade Associations, die die Interessen einer bestimmten Branche vertreten.

Der U.S. Chamber of Commerce wurde 1912 von der Chicago Association of Commerce und dem Boston Chamber of Commerce gegründet. Ziel war die Schaffung einer nationalen Organisation, um die zunehmende wirtschaftspolitische Intervention der Bundesregierung beeinflussen zu können. Drei Themen waren dabei von besonderer Bedeutung: erstens die Ausgestaltung der , anti-trust ${ }^{`}$ Gesetze, ferner die Förderung des internationalen Handels und schließlich die wachsende Macht der Gewerkschaften. Die Rolle der bundesstaatlichen Regierung bei der Entstehung des U.S. Chambers of Commerce darf allerdings nicht unberücksichtigt bleiben. Mundo (1992) umschreibt dies folgendermaßen: „At most, the federal government caused the Chamber to be formed; at least, it endorsed and helped to legitimize the new national organization“" (S. 69). Die Bundesregierung erkannte wenigstens die Notwendigkeit der Schaffung einer 
zentralen Organisation, die die Interessen der amerikanischen Wirtschaft in ihrer Gesamtheit vertreten sollte, um so der staatlichen Bürokratie als Ansprechpartner für wirtschaftliche Belange zu dienen. Nach dem Ersten Weltkrieg wuchs die Mitgliederzahl des U.S. Chamber und mithin sein Machteinfluß. Ursachen dieser Entwicklung waren der zunehmende Druck der Gewerkschaften auf die Unternehmen und die Ausweitung staatlicher Eingriffe in die Wirtschafts- und Sozialpolitik. Höhepunkt der staatlichen Intervention war die Verabschiedung des Sherman und Clayton Act, die die Auflösung der mächtigen ,trusts' beinhalteten (vgl. Ornstein/Elder 1978, S. 36; Wilson 1981, S. 58). Die Struktur des U.S. Chamber stellt eine komplexe Mischung aus einer ,organization of organizations“ und einem Zusammenschluß einzelner Unternehmen dar (vgl. Mundo 1992, S. 77 f.). Einerseits sind im U.S. Chamber ca. 1.300 ,trade associations' und ca. 2.700 lokale, regionale und einzelstaatliche Chambers of Commerce vertreten, andererseits umfaßt er ca. 84.000 Einzelunternehmen (vgl. Lösche 1998, S. 344). Dementsprechend muß der Verband zwischen den unterschiedlichen Interessen einen gemeinsamen Nenner finden und beschränkt sich daher auf sehr allgemeine Ziele, wie die Verwirklichung einer ,freeenterprise economy“: „...Chamber avoids positions on issues that do not attract widespread agreement among its members“ (Mundo 1992, S. 88). Diese allgemeine Orientierung des U.S. Chamber ist gerade im Interesse der in ihm vertretenen, trade associations', die verbandsspezifische Belange in ihrem Verantwortungsbereich behalten wollen. Aber auch Einzelunternehmen lassen sich nur ungern in ihre unternehmensspezifischen Aktivitäten hineinreden (vgl. ebd., S. 77; DeSoto 1995, S. 2). Für die Mehrzahl der Mitglieder verkörpert der U.S. Chamber of Commerce eher eine Form des Dienstleistungsunternehmens, das die Firmen bei betriebswirtschaftlich relevanten Fragen, z.B. in bezug auf Exportmöglichkeiten, unterstützt, als eine kohärente Interessenvertretungsorganisation, die auch zwischen den Einzelinteressen im Sinne des Gemeinwohls vermittelt.

Ende des 19. Jahrhunderts schlossen sich als Reaktion auf den wachsenden Druck der Gewerkschaften kleine und mittlere Unternehmen in der National Association of Manufacturers (NAM) zusammen. Neben einem aggressiven , anti-unionism` verpflichtete sich die Organisation, den inneramerikanischen Handel, der für kleine Firmen besonders wichtig ist, zu fördern. Anfangs hatte die NAM die Funktion einer, general trade association“. Ihre Mitgliederzahl wuchs bis zur „Großen Depression“ von 1929 
stetig an. Ein plötzlicher Einbruch in den Mitgliederzahlen zu Beginn der 30er Jahre führte zu einer Umorganisation des Verbandes, in deren Folge nun auch Großunternehmen unter den Mitgliedern vertreten sind. Auf die in der NAM vertretenen ,manufacturing'-Firmen entfallen 75 Prozent der gesamten Produktion dieses Sektors (vgl. Derber 1984, S. 105 f.; Ornstein/Elder 1978, S. 38). „The NAM’s functions include the expression of industry's view on all national and international governmental intervention; the designation of industry representatives on public bodies...lobbying, public relations...“ (Derber 1984, S. 105). Um die Jahrhundertwende gehörte die NAM zu den Protagonisten einer Schulreformbewegung, die die Einführung des deutschen Lehrlingssystems mit dem Argument der internationalen Konkurrenzfähigkeit der amerikanischen Wirtschaft forderte. Die aktive Rolle der NAM in dieser Reformbewegung läßt sich aus der Struktur ihrer Mitglieder erklären. Bei diesen handelte es sich vornehmlich um kleinere und mittlere Betriebe, die im Gegensatz zu Großunternehmen ihre Arbeitsteilung noch nicht nach tayloristischen Gesichtspunkten organisiert hatten. Demzufolge waren sie von einem ausreichenden Angebot an qualifizierten Arbeitskräften abhängig, um ihre Wettbewerbsposition halten zu können. Die gleichzeitige Verschärfung der Immigrationsgesetze trug darüber hinaus zum Umstand mangelnder Facharbeiter bei.

Der dritte nationalorganisierte Unternehmensverband ist der Business Roundtable. Seine Besonderheit liegt in der Exklusivität der Mitglieder. Bei diesen handelt es sich um die ,creme de la creme' der international agierenden Großkonzerne. Aus Unzufriedenheit über die politischen Aktivitäten der etablierten Unternehmensverbände schloß sich 1969 eine kleine Gruppe von Vorstandsvorsitzenden zusammen, um die spezifischen Interessen amerikanischer Großunternehmen besser koordinieren und vertreten zu können. Die annähernd 190 Mitgliedsunternehmen beinhalten solche wie AT\&T, Procter\&Gamble, General Motors, General Electric, US Steel und IBM. Ziel des Business Roundtable ist die Einflußnahme auf wirtschaftspolitische Maßnahmen der Bundesregierung, wobei sich die Aktivitäten auf klar definierte Bereiche konzentrieren, wie z.B. Anti-Trust Gesetze oder den Abbau internationaler Handelsbeschränkungen (vgl. Ornstein/Elder 1978, S. 39; Derber 1984, S. 108; Lösche 1998, S. 343). Trotz dieser außergewöhnlichen Einrichtung der Großunternehmen gibt es kein einheitliches Kapitalinteresse, sondern jedes Großunternehmen betreibt neben diesen Organisationen eigenes Lobbying, um seine speziellen Interessen gewahrt zu sehen. 
Neben diesen branchenübergreifenden Verbänden gibt es noch Industrieverbände, sogenannte Trade Associations. Wesentliche Funktionen dieser Branchenverbände sind die einheitliche Interessenvertretung nach außen, die Koordination politischer Aktivitäten und teilweise auch die Vertretung der Unternehmen bei kollektiven Tarifverhandlungen. Ansatzweise besteht mithin gewisse Vergleichbarkeit zu deutschen Industrieverbänden. Andererseits sind die amerikanischen Trade Associations wesentlich spezialisierter und fragmentierter als europäische Industrieverbände. So gibt es mehr als 7.000 unterschiedliche Einzelverbände, deren Mitgliederzahl von einem Dutzend bis zu mehreren tausend reicht. Neben dieser funktionalen Differenzierung finden sich auch regionale und lokale Unterschiede, so daß dort Abweichungen von der Zusammensetzung der Bundesorganisation auftreten können. Die lose Verbindung zwischen den Ebenen fördert jedoch die lokale Eigenständigkeit der Verbände (vgl. Lösche 1998, S. 343). Aufgrund ihrer Spezifität sind Trade Associations in den branchenübergreifenden Unternehmensverbänden nur selten vertreten. Im Gegensatz zu diesen können sie aber einen höheren Anteil ihrer potentiellen Mitglieder organisieren (vgl. DeSoto 1995, S. 3). „Trade associations, representing specific industries, are not clearly integrated into the structure of general business organizations representing the collective interests of business..." (Wilson 1990, S. 67).

Inwieweit könnten die amerikanischen Arbeitgeberverbände die Funktion eines intermediären Selbststeuerungsorgans übernehmen, um eine Koordination im Bereich der beruflichen Ausbildung zu erzielen und diese dem Wettbewerb zwischen den Firmen zu entziehen? Die Kapazität des U.S. Chamber of Commerce beruht nicht, wie die der deutschen Industrie- und Handelskammern, auf einer hundertprozentigen Organisationsdichte. Im Durchschnitt sind lediglich 1-4 Prozent der Unternehmen im jeweiligen State Chamber organisiert (vgl. DeSoto 1995, S. 4). Seine Mitgliedschaft besteht aus höchst heterogenen Interessen, was nicht nur durch seine branchenübergreifende Struktur bedingt ist, sondern gerade auch durch die Differenzen zwischen verbandlichen Mitgliedern (trade associations) und Einzelunternehmen. Die Interessenlage der Mitglieder der NAM ist hingegen wesentlich homogener als die des U.S. Chamber, aber auch ihr Organisationsgrad ist relativ gering (vgl. Lösche 1998, S. 362 f.). Noch homogener sind die im Business Roundtable zusammengeschlossenen Interessen, dessen Mitgliederzahl dementsprechend aber auch sehr klein ist. Die Trade Associations sind wiederum äußerst spezialisierte Verbände, die nur unzureichend in allgemeine, 
branchenübergreifende Organisationen eingebunden sind. Alle nationalen Organisationen besitzen lediglich eine lose hierarchische Struktur, in der regionale und lokale Verbände ihre Eigenständigkeit bewahrt haben. Hinzu kommt noch die geringe Zahl an hauptamtlichen Mitarbeitern in den Verbänden, die eine autonomere Verbandspolitik verhindert. „Despite some centralizing tendencies, the size and heterogeneity of the national economy continue to favor a looseness of relations between the general organizations and the industry or locality employer associations“ (Derber 1984, S. 109). Begünstigt wird diese schwache Ausprägung nationaler Arbeitgeberverbände durch zwei Umstände: einerseits die Schwäche der organisierten Arbeiter bzw. die geringe Organisationsdichte der Gewerkschaften, und andererseits staatliche Interventionen zur Verhinderung von Monopolen und Trusts. Die Schwäche der Gewerkschaften in den USA hatte zur Folge, daß die Arbeitgeber keine Notwendigkeit darin sahen, eigene starke kollektive Organisationen zu schaffen, um den Druck der Gewerkschaften auszugleichen. „The comparative weakness of trade associations and business umbrella groups in the United States reflects the fact that strong challenges to the collective interests of business are rare“ (Wilson 1990, S. 72). Auf der anderen Seite schuf der Staat mit seiner Anti-Trust Gesetzgebung, wie dem ,Sherman Act', ein Umfeld, in dem kooperationswillige Unternehmen unter dem Damoklesschwert der gerichtlichen Verfolgung agieren. Das Oberste Gericht der USA legte 1965 in einem Urteil über tarifliche Verhandlungen zwischen Arbeitgeberverbänden und Gewerkschaften diese Gesetzgebung sehr weit aus und erschwerte damit jegliche Kooperationsbemühungen (vgl. Derber 1984, S. 101 f.; Windmuller 1984, S. 21; Heckman 1998, S. 116; Hollingsworth/Lindberg 1985, S. 248 f.; Lynn/McKeown 1988, S. 45 ff.). „The possibility of legal action against the association tends to discourage coercive actions against members as well as non-members" (Derber 1984, S. 104). Die Steuerungskapazitäten amerikanischer Arbeitgeberverbände, insbesondere auf nationaler Ebene, sind dementsprechend sehr beschränkt. ${ }^{25}$ Ihre Organisationsstruktur ermöglicht es ihnen nicht, als intermediärer Mittler aufzutreten und den Markt als Allokationsmechanismus für die berufliche Bildung zu substituieren.

\footnotetext{
${ }^{25}$ Vgl. dazu auch: Choy 1994, S. 68; Glover/Weisberg 1994, S. iv-v und Finegold/McFarland/Richardson
} 1993, S. 6. 
Daher ist es entscheidend, die Anreizstruktur des einzelnen Unternehmens zu betrachten, Ausbildungsplätze anzubieten. Denn die Schaffung eines umfassenden dualen Berufsausbildungssystems hängt ausschlaggebend von der Teilnahme einer Mehrheit der Arbeitgeber ab, Schüler bzw. Absolventen der High School in ihrem Unternehmen auszubilden. Um 25 Prozent eines Jahrganges eine betriebsorientierte Berufsausbildung anbieten zu können, müßten 1,5 Millionen Ausbildungsplätze von den Unternehmen bereitgestellt werden. Derzeit gibt es aber lediglich 1.000 Ausbildungsplätze in Pilotprojekten, die dem deutschen Lehrlingssystem vergleichbar sind. Wenn alle betrieblichen Kooperationen mit Schulen einbezogen werden, erhöht sich die Zahl auf ca. 500.000 „Ausbildungsplätze“. Häufig handelt es sich dabei aber nur um sogenannte ,job shadowing' oder ,summer job'-Projekte, bei denen die Qualität der betrieblichen „Ausbildung“ in keinem Verhältnis zur strukturierten, an Standards ausgerichteten Lehrlingsausbildung steht (vgl. Osterman 1995a, S. 75 f.; Cappelli et al. 1998, S. 109 f.). Bereits an diesem kleinen Zahlenbeispiel wird deutlich, welche Rolle den Unternehmen in einem qualitativ hochwertigen Berufsausbildungssystem zukommt und welche Kräfte in Bewegung gesetzt werden müßten, um eine substantielle Veränderung des Ausbildungsverhaltens der Unternehmen herbeizuführen.

Welche Motivationen gibt es, die einzelne Arbeitgeber dazu bewegen könnten, sich an der betrieblichen Seite der Ausbildung zu beteiligen? Hier kann zwischen philanthropischen, individuellen und kollektiven Gründen unterschieden werden. Philanthropische Anreize entstehen aus der lokalen Einbindung des Unternehmens. Durch die Teilnahme an Ausbildungsprogrammen für Jugendliche kommt das Unternehmen seiner sozialen Verantwortung nach, die lokale Gemeinschaft zu unterstützen. Und so reproduziert das Unternehmen seine soziale Einbettung in dieser Gemeinschaft. Trotz der maßgeblichen Bedeutung des philanthropischen Motivs für den Ersteinstieg eines Unternehmens in die betriebliche Berufsausbildung für Jugendliche, reicht seine Wirkung nicht aus, um ein umfassendes Ausbildungssystem zu schaffen und dauerhaft zu stabilisieren: „...purely philanthropic motivation would not be adequate to sustain a large and intense workbased education system“ (Bailey 1995a, S. 16). Bereits eine geringe Zahl von Auszubildenden genügt, um die unternehmerische Verantwortung für die Gemeinschaft zu befriedigen. Indessen gibt es Unterschiede abhängig von der Größe der Firma. Große Unternehmen haben häufig eigene, community relations departments' und fühlen sich aufgrund ihrer sichtbaren Dominanz der lokalen Gemeinschaft besonders verpflichtet. 
Von ihnen hängt das Wohlergehen der Gemeinschaft ab. Andererseits sind sie wiederum auf das lokale Arbeitsangebot angewiesen. Aufgrund ihrer Größe können sie die mit der betrieblichen Ausbildung verbundenen Kosten eher absorbieren als kleine Firmen, die ihre Motivation aus der engen Einbindung in die Nachbarschaft ziehen. Trotzdem zeigen empirische Untersuchungen, daß die Zahl der Auszubildenden zwar mit der Größe der Firma zunimmt, aber in keinem Proportionalitätsverhältnis zur Zahl der Beschäftigten steht. Dies stützt die Annahme einer philanthropischen Motivation, da Firmen bei wirtschaftlichem Eigeninteresse an einer betrieblichen Berufsausbildung mehr Ausbildungsplätze anbieten würden. Außerdem stellen ,non-profit‘ und öffentliche Organisationen die Mehrzahl der Ausbildungsplätze in kooperativen Programmen, was ebenfalls für eine vornehmlich philanthropische Motivation spricht (vgl. Bailey et al. 1998, S. 17-18 und 24).

Um die Teilnahme privatwirtschaftlicher, gewinnmaximierender Unternehmen zu fördern, müssen stärker individuell wirtschaftliche Eigeninteressen der Firmen hervorgehoben werden. Ein Eigeninteresse der Firma könnte sich aus folgenden drei Vorteilen ergeben: Erstens kann die Teilnahme an einem kooperativen Ausbildungsprogramm als ,public relations'-Kampagne das Ansehen der Firma steigern. Diese Argumentation läßt sich jedoch nur schwer von jener der philanthropischen Motivation unterscheiden. Zweitens können die Studenten als billige Aushilfskräfte dienen. Und drittens stellt die Teilnahme eine kostengünstige Rekrutierungsmethode dar, um zukünftige Arbeitskräfte besser auswählen zu können. Die Ausbildungskosten sind dann eine Investition in die Zukunft des Unternehmens. Eine Teilnahme des Unternehmens aus ,public relations'-Motiven beinhaltet dieselbe Problematik wie die der philanthropischen Motivation: ,...public relations benefits can probably achieved with a small number of placements and through programs that do not demand a great deal from employers“ (Bailey 1995a, S. 16-17). Demzufolge kann von ihr keine umfassende Ausweitung der Ausbildungsplätze erwartet werden. Eine dauerhafte Beteiligung an kooperativer Berufsausbildung erscheint im wechselhaften Umfeld der ,public relations' daher fraglich.

Der Anreiz, Studenten als billige Arbeitskräfte einzusetzen, entspricht hingegen schon eher der Rationalität eines gewinnmaximierenden Unternehmens und könnte deshalb eine mögliche Motivation darstellen, die Zahl der Auszubildenden zu erhöhen. So führen Ökonomen den in Relation zum durchschnittlichen Facharbeiterlohn niedrigen 
Lehrlingslohn in Deutschland als einen wirtschaftlich-rationalen Grund für Unternehmen an, Auszubildende einzustellen. ${ }^{26}$ In den USA gibt es keine einheitliche Tarifstruktur, so daß auch höher qualifizierte Arbeiter sehr unterschiedlich entlohnt werden. Darüber hinaus verhindert der gesetzlich festgelegte Mindestlohn, der auch für Auszubildende gilt, daß die Lohnspreizung zwischen Lehrling und Arbeiter ausreichend groß ist, um den Unternehmen als Ausgleich für die Investition in qualitativ hochwertige Ausbildungsplätze zu dienen (vgl. Osterman 1995a, S. 80 f.). Studenten werden deshalb häufig nicht anders eingesetzt als Teilzeitaushilfskräfte (vgl. Cappelli et al. 1998, S. 110 f.). Einige der befragten Arbeitgeber sehen ihre Teilnahme an kooperativen Ausbildungsprogrammen als einen ,good way to get good, lower-paid part-time help“ (Lynn/Wills, in: Bailey 1995a, S. 17).

Weitaus wichtiger als die Entlohnung der Auszubildenden sind hingegen die ,supervisory costs'. Diese Kosten entstehen durch die Anleitung und Überwachung von Auszubildenden. Firmen, die keine Ausbildungsplätze für Jugendliche anboten, gaben in einer Umfrage als zweitwichtigsten Grund für ihre Entscheidung die Bezahlung der Ausbilder bzw. deren Produktivitätsverlust an (vgl. Bailey et al. 1998, S. 23). In den 1970er Jahren initiierte die Bundesregierung ein Pilotprojekt, bei dem Lehrlingslöhne subventioniert wurden. Dennoch war die Beteiligungsrate der Unternehmen sehr gering: ,....not even one in five of the employers was willing to take on these youth even at a wage of zero“ (ebd., S. 18). Viele Firmen schätzten ihre Ausbildungskosten immer noch höher ein als den Beitrag der Studenten. Ein Vorzeigebeispiel kooperativer Ausbildungsprogramme ist ProTech in Boston, das einer kleinen Zahl von Schülern einen Ausbildungsplatz im Gesundheitssektor, insbesondere im Krankenhausbereich, zur Verfügung stellt. Pro Student ergeben sich hier Ausbildungskosten in Höhe von \$8.892 inklusive Lohn und \$5.678 ohne Lohn. Dies verdeutlicht die Relation zwischen Ausbildungslohn und sogenannten, supervisory costs'. Arbeitgeber haben darüber hinaus aber noch andere „Kosten“ zu berücksichtigen, wie z.B. das Risiko, daß der ausgebildete Jugendliche die Firma wechselt oder an ein College geht, oder den Widerstand der festangestellten Beschäftigten gegenüber „billigen“ Auszubildenden (vgl. Osterman 1995a, 78 f.). Obendrein genießen Jugendliche kein hohes Ansehen bei den Firmen, weil sie als unreif, illoyal und wenig verantwortungsbewußt gelten (vgl.

${ }^{26}$ Vgl. dazu: Franz/Soskice 1994. 
Cappelli et al. 1998, S. 113 f.). Studenten in speziellen Ausbildungsprogrammen sind noch stärker stigmatisiert, da diese Programme häufig auf spezifisch benachteiligte Gruppen zugeschnitten sind, deren intellektuellen Fähigkeiten als unterdurchschnittlich eingestuft werden. Die Reputation solcher Programme ist deshalb bei Arbeitgebern gering.

Schließlich bietet die Möglichkeit, zukünftige Arbeitskräfte zu rekrutieren, einen weiteren individuellen Anreiz für das einzelne Unternehmen, Ausbildungsplätze bereitzustellen. Dafür bedarf es aber ebenfalls keiner qualitativ hochwertigen Ausbildungsplätze. Hierzu genügt eine relativ kurze Erprobungsphase, innerhalb derer die wesentlichen Eigenschaften zutage treten. Zudem müßten die Rekrutierungskosten des Unternehmens oberhalb der Ausbildungskosten liegen, um eine solche Vorgehensweise wirtschaftlich rechtfertigen zu können. Für Unternehmen sind Jugendliche aber in der Regel keine potentiellen, zukünftigen Beschäftigten, so daß die Rekrutierungsfunktion von geringer Bedeutung ist: „,...weak demand for young workers was one of the most important disincentives for participation“ (Bailey 1995a, S. 18). Dies könnte jedoch auf die für Unternehmen positive Arbeitsmarktlage zur Zeit der Befragung zurückzuführen sein. $\mathrm{Zu}$ Beginn der 90er Jahre war die Arbeitslosenrate relativ hoch, so daß Unternehmen statt unqualifizierten Jugendlichen ältere, qualifizierte Arbeitskräfte einstellen konnten. Die Mehrzahl der Arbeitgeber sah keine Notwendigkeit, das Angebot an qualifizierten Arbeitskräften zu erhöhen (vgl. Bremer/Madzar 1994, S. 3 f.). Eine Umfrage der Commission on the Skills of the American Workforce von 1991 ergab, daß „only 15 percent of employers reported that they had difficulty finding appropriatelyskilled workers“ (ebd., S. 3-4). Inzwischen hat sich die Arbeitsmarktlage zugunsten der Arbeitnehmer verschoben. Als Folge könnten Arbeitgeber einen größeren Anreiz haben, Jugendliche einzustellen und auszubilden, um den Mangel an qualifizierten Arbeitskräften am Arbeitsmarkt auszugleichen. ${ }^{27}$ Auch die Angst der bereits Beschäftigten, durch „,billige“ Auszubildende ersetzt zu werden, könnte an Bedeutung verlieren und so auch ihr Druck auf den Arbeitgeber sinken, keine Jugendlichen einzustellen (vgl. Bailey et al. 1998, S. 8; Cappelli et al. 1998, S. 114).

${ }^{27}$ Eine Studie von Mitarbeitern der Organisation ,Jobs for the Future‘ ergab, daß Industrien, die einen Mangel an qualifizierten ,entry-level'-Arbeitskräften konstatierten, Ausbildungsprogrammen offener gegenüberstanden (vgl. Bailey et al. 1998, S. 11). 
Sicherlich sind die individuellen Anreize für die Unternehmen von der jeweiligen konjunkturellen Situation abhängig. Nichtsdestotrotz reichen sie nicht aus bzw. können ihre volle Wirkung nicht entfalten, weil institutionelle Hindernisse, wie die Wirkungsweise des Jugendarbeitsmarktes, kontraproduktiv wirken. Hier könnten kollektive Anreize die Schwächen der philanthropischen und individuellen Motivation überwinden. Grundsätzlich besteht ein Interesse für alle Arbeitgeber, jederzeit auf einen ausreichend großen ,pool` von hochqualifizierten Arbeitskräften zurückgreifen zu können, und zwar unabhängig von der jeweiligen Konjunkturlage. Wie können diese Arbeitskräfte zur Verfügung gestellt werden, wenn jeder einzelne Arbeitgeber einen nur geringen Anreiz hat, selber auszubilden, weil er befürchten muß, daß Konkurrenten ihm seine ausgebildeten Arbeitskräfte abwerben? Dieses Problem des ,free-riding ${ }^{\circ}$ oder ,poaching ${ }^{28}$ stellt den klassischen Fall des Scheiterns kollektiven Handelns dar. Es muß also ein institutioneller Rahmen geschaffen werden, der das ,free-riding ${ }^{6}$ soweit unterdrückt, daß eine kritische Masse des kollektiven Handelns überschritten wird, um so die Wirkung des Verhaltens eines einzelnen Unternehmens zu marginalisieren. Einen solchen institutionellen Rahmen können Arbeitgeberverbände schaffen, indem sie das kollektive Interesse gegenüber den Einzelinteressen durchsetzen. Sie legen die Ausbildungsstandards und Abschlußzertifikate fest und überwachen deren Einhaltung. Die Einführung portabler, allgemein anerkannter Befähigungsnachweise erhöht die Transparenz auf dem Arbeitsmarkt und senkt die Transaktionskosten für die Unternehmen. Wenn die Unternehmen davon ausgehen können, daß ihnen ein ,pool‘ von nach allgemein festgelegten Standards qualifizierten Arbeitskräften zur Verfügung steht, reduziert sich das Investitionsrisiko des einzelnen Arbeitgebers.

Wie bereits oben dargelegt, sind die USA ein Beispiel dafür, daß die Arbeitgeberverbände über keine entsprechenden Kapazitäten verfügen, um einen solchen institutionellen Rahmen auf nationaler Ebene zu schaffen. Ausnahmen gibt es auf regionaler bzw. sektoraler Ebene, wo Arbeitgeberverbände in begrenzter Weise Steuerungskapazitäten besitzen. Ein Beispiel ist die Bauwirtschaft, die idiosynkratische Berufsbilder besitzt, deren Nutzen ausschließlich auf die Bauwirtschaft beschränkt ist. Aber auch bei der hier praktizierten Lehrlingsausbildung handelt es sich nicht um berufliche Bildung

\footnotetext{
${ }^{28}$ Für eine Darstellung des ,poaching' Problems in Form des ,prisoners' dilemma' siehe Finegold (1991), S. 104; eine mathematisch-wirtschaftswissenschaftliche Aufbereitung des Problems findet sich bei Stevens (1996), S. 21-40.
} 
für Jugendliche, sondern um ein Qualifizierungssystem für ältere, bereits berufserfahrene Arbeiter. Entscheidende Grundlage für die Funktionsfähigkeit eines umfassenden betriebsorientierten Berufsbildungssystems ist daher das Vorhandensein von ,business-business'-Partnerschaften (vgl. Bailey 1995a, S. 20-23; Osterman 1995a, S. 83 f.; Cappelli et al. 1998, S. 112 f.).

Könnte der Staat bei fehlenden intermediären Strukturen, wie in den USA, funktionale Äquivalente schaffen, die wirtschaftliches Eigeninteresse überwinden und kollektives Handeln fördern? Der Staat könnte finanzielle Anreize schaffen, um das Investitionsrisiko des einzelnen Unternehmens zu senken. An folgende finanzpolitische Instrumente wäre dabei zu denken: ,training tax ${ }^{\star}$, ,tax credit ${ }^{`}$ und , wage subsidies ${ }^{6}$. Die Idee der Ausbildungssteuer liegt darin, daß Unternehmen, die weniger als einen bestimmten prozentualen Betrag ihrer Lohnsumme für Humankapitalinvestitionen ausgeben, eine Steuer abführen müssen. Durch die Einführung einer solchen Ausbildungssteuer, wie es sie in einigen europäischen Ländern gibt, könnte ein Steuerungseffekt auf ökonomisch rational handelnde Unternehmen ausgeübt werden, da sich infolgedessen deren KostenNutzen-Kalkulation zugunsten von Ausbildungsinvestitionen verschiebt. Andererseits ist die Durchsetzung einer solchen Steuer im Kontext einer allgemeinen steuerlichen Entlastung der Unternehmen kaum wahrscheinlich (vgl. Osterman 1995a, S. 81 f.). So ist es auch nicht überraschend, daß Clintons Wahlversprechen, eine Ausbildungssteuer in Höhe von 1-1,5 Prozent der Lohnsumme einzuführen, von Unternehmensverbänden scharf kritisiert wurde und diese die Umsetzung schließlich auch verhinderten (vgl. Clinton 1993, S. 7; Couch 1994, S. 11; Pennington 1995, S. 173). Problematisch an einer ,training tax ‘, ohne zusätzliche Regulationsmaßnahmen, ist zudem der geringe Einfluß, den der Staat bei der Ausgestaltung der Ausbildungsprogramme hat (vgl. Bailey 1993, S. 7). Denn häufig investieren Unternehmen nicht in ihre gering qualifizierten Arbeiter, sondern in bereits höher qualifizierte technische und kaufmännische Angestellte (vgl. Bailey 1992, S. 921). Mit ,tax credits“ und ,wage subsidies' haben amerikanische Unternehmen bereits in der Vergangenheit Erfahrung gemacht. Die Effektivität beider Formen ist aber umstritten: Unternehmen erhalten für die Beschäftigung von Jugendlichen ,tax credits‘, obgleich diese auch ohne finanzielle Unterstützung eingestellt worden wären: ,,...very few employers reported that tax credits were a sufficient incentive to change their normal hiring practices..." (Bremer/Madzar 1994, S. 7). ,Wage subsidies‘ auf der anderen Seite könnten mit spezifischen Auflagen 
hinsichtlich der Ausbildung der Jugendlichen verbunden werden. Wie oben bereits erwähnt, stellen Unternehmen aber auch bei einem Nullohn Jugendliche nur in begrenzter Zahl ein, da Vorurteile über die Arbeitsmoral der Jugendlichen und ,supervisory costs‘ den Nutzen einer Beschäftigung überwiegen (vgl. Choy 1994, S. 69). Darüber hinaus ist es fraglich, ob Jugendliche, für die das Unternehmen keinen oder einen subventionierten Lohn zahlen muß, als vollwertige Mitarbeiter angesehen und dementsprechend auch bestmöglich ausgebildet werden (vgl. Osterman 1995a, S. $82)$.

Neben finanzieller Unterstützung kann der Staat auch allgemein akzeptierte Ausbildungsstandards festlegen, die in Form von Zeugnissen die marktliche Transparenz berufsqualifizierender Fertigkeiten erhöhen. Dadurch erhalten Arbeiter einen Anreiz, für ihre Ausbildung einen niedrigeren Lohn zu akzeptieren. Arbeitgeber tragen in diesem Fall nur einen kleinen Teil der Ausbildungsinvestitionen. Der Firmenwechsel eines Arbeiters nach seiner Ausbildung stellt dann aus zweierlei Gründen keinen Hinderungsgrund mehr dar: Erstens hat der ausbildende Arbeitgeber nur noch einen geringen oder bei entsprechend niedrigem Ausbildungslohn keinen Investitionsverlust, und zweitens kann er auf dem externen Arbeitsmarkt aufgrund der Ausbildungsstandards einen Arbeiter mit denselben Qualifikationen ,einkaufen“. „We need more centralized standards so that companies would know if certified apprentices had attained necessary skills rather just spending three or four years in training“ (J. Krall, in: Moskal 1991, S. 35). Theoretisch sind Standards also ein zentraler Schlüssel zum Erfolg eines betriebsorientierten Berufsausbildungssystems. Dafür müssen sie aber verpflichtend sein. Und dies erfordert wiederum ein Kontrollsystem, das die Einhaltung der Standards gewährleisten kann. In der Regel sind Unternehmen eher bereit sich einem solchen Zwangssystem zu unterwerfen, wenn dessen Überwachung durch Unternehmensverbände vorgenommen wird. Die Kontrolle durch staatliche Behörden bedeutet für Arbeitgeber eine zu weitgehende Intervention des Staates in ihren Verantwortungsbereich. Da amerikanische Unternehmensverbände nicht die Kapazität besitzen, eine solche Kontrollfunktion zu übernehmen, und gegenüber staatlichen Eingriffen das Mißtrauen der Unternehmen sehr ausgeprägt ist, hätten verpflichtende Standards keinen Einfluß auf die Ausbildungspraxis der Mehrheit der Unternehmen (vgl. Glover/Weisberg 1994, S. 14 f.; Elbaum/Singh 1995, S. 619 f.). Individuelle Anreize für Unternehmen reichen in den USA nicht aus, um ein umfassendes duales Berufsaus- 
bildungssystem zu etablieren. Andererseits können sich wegen fehlender intermediärer Instanzen kollektive Anreize nicht durchsetzen, die von zentraler Bedeutung für die Einrichtung und Dauerhaftigkeit eines solchen Systems sind.

\subsection{Gewerkschaften}

Neben Arbeitgebern und Arbeitgeberverbänden sind es Gewerkschaften, die einen entscheidenden Einfluß auf die Implementierung und Durchführung eines betriebsorientierten Berufsbildungssystems haben. Sie stellen eine betriebsinterne Kontrollinstanz dar, die die Festlegung von breit angelegten Ausbildungsstandards und deren Einhaltung gegenüber den Arbeitgebern durchsetzen kann. Welche Rolle haben die Gewerkschaften in den USA und bietet der institutionelle Rahmen, in dem sie agieren, eine Chance, ein solches Ausbildungssystem zu etablieren? Die Fragmentierung der amerikanischen Gewerkschaften in Facharbeiter/Berufs- und Industriegewerkschaften geht auf Entwicklungen in der Arbeiterbewegung zum Ende des 19. Jahrhunderts zurück. Lokale Verbände von Handwerkern gab es bereits seit dem 18. Jahrhundert. Diese hatten aber keine Gemeinsamkeit mit Gewerkschaften, sondern entsprachen eher dem Prinzip von Zünften. Die erste nationale „Arbeiterorganisation“ war die 1834 gegründete National Trades Union, in der verschiedene Handwerkerverbände organisiert waren (vgl. Craver 1993, S. 12; Herman et al. 1992, S. 18 f.). Bis 1869, dem Jahr der Gründung der Knights of Labor, wurde das Prinzip der Handwerksassoziationen nicht in Frage gestellt. Zusammenschlüsse basierten auf der gemeinsamen Tradition und Erfahrung bei der Ausübung eines spezifischen Berufs. Das Gefühl der Gemeinschaft schuf die Grundlage von Solidarität. Nach dem Bürgerkrieg erlebte die amerikanische Wirtschaft eine Boom-Phase, die zum Aufleben lokaler Handwerksvereinigungen führte. Die Gründer der Knights of Labor glaubten die Schwäche dieser „Handwerksgewerkschaften“ darin erkannt zu haben, daß sie nicht in der Lage waren, die tatsächlichen Machtverhältnisse zwischen Kapital und Arbeit zu verändern. Ihr Interesse lag im Wohlergehen der Mitglieder der spezifischen Berufsgemeinschaft. Um die Interessen aller Arbeiter gegenüber der wachsenden Macht des Kapitals vertreten zu können, verabschiedeten sich die Gründer der Knights of Labor vom Organisationsprinzip „Beruf“ und öffneten ihre Organisation für alle Arbeiter, unabhängig von ihrer Qualifikation. „No constitutional constraints were placed on skill 
level of local assembly members, nor were individual locals required to organize all occupations or skills“ (Voss 1993, S. 76). Zudem handelte es sich bei dieser Arbeiterorganisation um einen geheimen Bund, um Übergriffe von seiten der Arbeitgeber zu verhindern. Da eine zentralisierte Kontrolle nicht möglich war, besaßen die einzelnen lokalen Verbände weitgehende Autonomie in der Form ihrer Organisierung. Häufig entstanden dabei hybride Organisationen aus Berufs- und Industriegewerkschaften.

Während der langen ökonomischen Depression von 1873 bis 1879 behielten die Gründer der Knights of Labor mit ihrer Einschätzung der Stärke der ,craft unions' recht. Diese hatten unter den ökonomischen Bedingungen schwer zu leiden, da ihnen die Kapazität fehlte, eine breite Basis der Solidarität aufzubauen. Nur durch eine Konsolidierung der partikularistischen Arbeiterinteressen und die Einbeziehung der weniger qualifizierten und ungelernten Arbeiter konnte die Transformation des industriellen Kapitalismus erzielt werden. Die anfänglichen Erfolge der Knights of Labor erhöhten die Zahl der Mitglieder, insbesondere viele ungelernte Arbeiter traten der Organisation bei. Wie kam es auf einmal dazu, daß sich standesbewußte Facharbeiter mit ungelernten Arbeitskräften in einer Organisation zusammenschlossen? Entscheidend waren hier der technologische Wandel und neue Formen der Arbeitsorganisation, die eine erhebliche Gefahr für die qualifizierten Arbeiter darstellten. Ihnen drohte der Verlust ihres Status und der Entscheidungsfreiheit über die autonome Arbeitsorganisation. Im Bündnis mit ungelernten Arbeitern konnten sie die Erosion ihrer Organisationsbasis kompensieren. „Technological change...tended to increase and stabilize employment opportunities for factory workers while also equalizing working conditions“ (ebd., S. 139). An die Stelle der Gemeinschaft als Quelle der Solidarität trat die fragile Masse der arbeitenden Schicht, deren Heterogenität vom industriellen Kapitalismus nivelliert wurde. Im Mai 1886, auf dem Höhepunkt ihrer Macht, beteiligten sich die Knights of Labor an einer großen Streikkampagne, die mit einem blutigen Bombenanschlag auf die Polizei endete. Schlagartig schlug die öffentliche Meinung um, so daß der Streik ohne jeglichen Erfolg für die Arbeiter ausgesetzt werden mußte. In den folgenden Monaten stieg die Zahl der Austritte gerade von qualifizierten Arbeitern. Dies fiel um so leichter, als sich in der neugegründeten American Federation of Labor (AFL) eine Alternative zu den Knights of Labor anbot.

Die AFL war ein föderativer Zusammenschluß einzelner ,trade unions` auf nationaler Ebene. Ihre Ziele lagen in der Begrenzung von Immigranten und Kindern als Arbeits- 
kräften und einer strikten Regulation der Lehrlingsausbildung. Im Gegensatz zu den Knights of Labor ging es der AFL und ihren Mitgliedsorganisationen nicht um eine Transformation des sozio-ökonomischen Systems des Kapitalismus, sondern um „'pure and simple“ trade unionism“ (ebd., S. 241) (vgl. Heckscher 1988, S. 17). Die Gründer der AFL glaubten, aus dem Scheitern der Knights of Labor gelernt zu haben, daß die auf dem beruflichen Gemeinschaftsgefühl basierende starke Verhandlungsposition der ,craft unions' nicht durch die Aufnahme von ungelernten Arbeitern verwässert werden sollte. Der Niedergang der Knights of Labor wird von einigen Historikern auf die „inherent opposition between craft and class interests“ (Voss 1993, S. 187) zurückgeführt. Obgleich dies sicherlich eine ausschlaggebende Rolle gespielt hat, sollte der extreme Widerstand der Arbeitgeber und ihrer Verbände gegen gewerkschaftliche Organisierung, der von staatlicher Seite noch gestützt wurde, nicht vernachlässigt werden. Mit dem Niedergang der Knights of Labor waren es wieder die kleinen, entlang von Berufskategorien organisierten ,craft unions', die die amerikanische Arbeiterbewegung dominierten. Die AFL lehnte alle Organisationen als Mitglieder ab, die nicht auf dem Berufsprinzip basierten (vgl. Morone 1990, S. 150 f.). Bereits in den 1890er Jahren dominierte die AFL die amerikanische Arbeiterbewegung, während sich die lokalen Organisationen der Knights of Labor weiter auflösten. Das die AFL leitende Prinzip der ,exclusive jurisdiction ${ }^{29}$ hatte sich durchgesetzt und führte zu der Situation von multigewerkschaftlicher Organisierung in großen industriellen Unternehmen (vgl. Heckscher 1988, S. 21). „Organizational dynamics stemming from the Knights“ demise also made it difficult for defenders of inclusive union strategies to rebuild an effective, broad-based movement. Once the national craft unions affiliated with the AFL became firmly established, institutional and competitive pressures made it much more difficult to create industrial and general unions“ (Voss 1993, S. 241). Die Auflösung der Knights of Labor und die Reinstitutionalisierung des exklusiven Berufsprinzips stellt eine sogenannte ,critical juncture' in der Evolution der amerikanischen Arbeiterbewegung dar. Für einen historischen Augenblick gab es die Chance einer umfassenden Organisierung der amerikanischen Arbeiter auf der Basis eines Klassenbewußtseins. Das Scheitern dieses Versuchs schwächte die Verhandlungsposition der Arbeiterschaft in

\footnotetext{
${ }^{29}$ Das Prinzip der ,exclusive jurisdiction‘ bedeutet, daß jede Berufsgruppe nur durch eine Gewerkschaft vertreten wird.
} 
ihrer Gesamtheit, während die einzelnen Berufsgruppen ihre partikularistischen Gemeinschaftsinteressen besser durchsetzen konnten.

Durch die Ausdehnung der tayloristischen Produktionsweise erhöhte sich einerseits die Zahl der ungelernten Arbeiter und andererseits sank die quantitative Bedeutung der qualifizierten Facharbeiter. Arbeitgeber gingen sogar soweit, die tayloristische Idee der Aufteilung des Arbeitsprozesses in viele kleine Tätigkeiten gezielt gegen die ,craft unions' einzusetzen, indem sie Berufskategorien zu atomisieren versuchten (vgl. Joyce 1985, S. 262 f.; Craver 1993, S. 21). So wollten sie die Position der Gewerkschaften unterminieren und gewerkschaftsfreie Unternehmen schaffen. Häufig konnten jedoch die ,craft unions' den Standard ihrer Mitglieder auf Kosten der ungelernten Arbeiter behaupten. Mit der wachsenden Zahl an geringqualifizierten Arbeitern in der Massenproduktion erschien einigen Gewerkschaftsführern die berufsorientierte Organisation der Arbeiter nicht mehr zeitgemäß. Sie gründeten den Congress of Industrial Organizations (CIO), einen Zusammenschluß von ehemaligen Berufsgewerkschaften und neugegründeten Gewerkschaften, die sich industriell organisierten. Ihr Ziel war die Organisierung der Arbeiter in den Unternehmen der Massenproduktion, wie der Textil-, Stahl-, Automobil- und Gummiindustrie. Eine solche Strategie widersprach dem grundlegenden Prinzip der AFL, der ,exclusive jurisdiction', da es zur Situation eines ,dual unionism‘ führte, also einer Überlappung von Gewerkschaftszuständigkeiten. Dies stellte einen potentiellen Konfliktstoff innerhalb der Gewerkschaftsföderation dar. Da die Mehrzahl der AFL-Gewerkschaften bei ihrem Organisationsprinzip blieben und die CIO-Verbände nicht anerkannten, spalteten sich diese 1938 von der AFL ab (vgl. Craver 1993, S. 28; Herman et al. 1992, S. 27; Scherrer 1996, S. 236; Morone 1990, S. 162-168). In den folgenden beiden Jahrzehnten konkurrierten die beiden Organisationen um Mitglieder und Tarifvertretungsrechte in Unternehmen der Massenproduktion. Erst 1955 kam es nach langen Verhandlungen zur Wiedervereinigung der AFL und CIO, nachdem ein Kompromiß für das Problem der ,exclusive jurisdiction` gefunden worden war. Dieser sah vor, daß Gewerkschaften mit sich überschneidenden Organisierungsbereichen fusionieren sollten (vgl. Craver 1993, S. 28-31). Als Resultat entstanden hybride Organisationsformen, in denen die Tradition des ,craft unionism“ weiterhin dominant ist. Unter den zehn größten Gewerkschaften der AFL-CIO waren 1991 immer noch drei reine Berufsgewerkschaften. Bei den kleineren Gewerkschaften dominiert traditionell die Organisation nach Berufskategorien. Auch wenn einige 
Beobachter bei den großen Gewerkschaften einen Trend in Richtung Industriegewerkschaft ausmachen, sind doch viele Traditionen der Berufsgewerkschaften erhalten geblieben (vgl. Lösche 1992, S. 508 ff.).

Worin unterscheiden sich Berufs- und Industriegewerkschaften und inwieweit könnte dies einen Einfluß auf die Organisation beruflicher Ausbildung haben? Zunächst einmal hat der Umstand der unterschiedlichen Mitgliederstruktur - horizontal versus vertikal Einfluß auf die Form der Solidarität: die Gemeinschaft bei der Berufsgewerkschaft und die Masse bei der Industriegewerkschaft. Ziel der Berufsgewerkschaft ist die Verteidigung des beruflichen Status ihrer Mitglieder und des damit verbundenen Lebensstandards. Die dabei verfolgte Strategie zielt auf eine Verknappung des Arbeitsangebotes durch gewerkschaftliche Kontrolle des Arbeitsmarktes ab. Die Industriegewerkschaft ist hingegen allen Berufen gegenüber offen, da ihre Stärke auf der Klassensolidarität aller Arbeiter basiert. Als Vertreterin heterogener Interessen muß sie ihre Ziele am kleinsten gemeinsamen Nenner ausrichten. Zur Durchsetzung der Ziele steht ihr im Gegensatz zur Berufsgewerkschaft als einziges Mittel der Streik zur Verfügung (vgl. Müller-Jentsch 1997, S. 109 ff.). Berufsgewerkschaften können den Streik gezielter einsetzen, da qualifizierte Arbeitskräfte schwieriger zu ersetzen sind und ihnen aufgrund ihrer entscheidenden Position im Produktionsprozeß eine stärkere Verhandlungsposition zukommt. Bis zum Ende des 19. Jahrhunderts verhielt es sich so, daß ,craft groups were able to set standards of fair rates that their members individually demanded from their employers" (Heckscher 1988, S. 16). Dies konnten sie nur, weil sie den Zugang zum Arbeitsmarkt kontrollierten. Entscheidendes Instrument war bzw. ist noch heute für einige Berufsgewerkschaften - gerade in der Bauwirtschaft - die Lehrlingsausbildung. Grundsätzlich ist zwischen registrierten und nicht-registrierten Lehrlingsprogrammen zu unterscheiden. Die Registrierung erfolgt bei einer Landesoder Bundesbehörde und bedeutet, daß dieses Lehrlingsprogramm zu einem der ca. 800 anerkannten Ausbildungsberufe führt. Dementsprechend unterliegt es bestimmten Mindeststandards. Die detaillierte Ausgestaltung des jeweiligen Lehrlingsprogramms erfolgt jedoch im Ausbildungsvertrag, der ausbildungsspezifische Einzelheiten festlegt, wie z.B. ob die Zeit an der Berufsschule vergütet wird und wieviel theoretischer Unterricht vorgesehen ist. Dies deutet bereits darauf hin, daß selbst die registrierten Programme nur minimale Gemeinsamkeiten aufweisen. Nicht-registrierte Lehrlingsprogramme sind nicht einmal diesen Mindeststandards unterworfen. Wichtiger 
als die Registrierung ist aber, ob es sich bei dem Lehrlingsprogramm um ein ,joint program ' handelt, d.h. Arbeitgeber und Gewerkschaften bei der Durchführung der Lehrlingsausbildung zusammenarbeiten. Die Mehrzahl der Lehrlinge werden in solchen gewerkschaftsorganisierten Betrieben ausgebildet und unterliegen mithin einer Kontrolle durch die Gewerkschaft (vgl. Münch 1989, S. 102 ff.; Jaschner 1996, S. 195 f.).

Obgleich diese Lehrlingsprogramme dem deutschen dualen Berufsbildungssystem oberflächlich ähneln, gibt es doch gravierende Unterschiede. Die einzelnen Programme variieren sehr stark in ihrer Qualität, weshalb die Zertifikate innerhalb einer Berufskategorie nicht unbedingt vergleichbar sind. Darüber hinaus ist es das Alter der Lehrlinge, das diese Form der Berufsausbildung vom deutschen System unterscheidet. Da die Lehrlinge im Durchschnitt ca. 25 Jahre alt sind, handelt es sich nicht um eine Erstausbildung für Jugendliche, sondern um ein Erwachsenenprogramm. „In America, apprenticeship has become largely a program for providing upgrade training for adults who are already employed“ (Glover, in: Jaschner 1996, S. 202-203). Deshalb erhalten die Lehrlinge auch keine Ausbildungsvergütung, sondern einen Lohn in Höhe von ca. 60 Prozent des Facharbeitereinkommens. Schließlich ist die Zahl der Lehrlinge in den USA verschwindend gering (ca. 0,3 Prozent der zivilen Erwerbstätigen). Dies verdeutlicht den elitär-exklusiven Charakter der Lehrlingsausbildung, die dem Statuserhalt der jeweiligen Berufsgruppe dienen soll, indem nur eine limitierte Anzahl von Ausbildungsplätzen bereitgestellt wird.

Die begrenzte Möglichkeit des Einsatzes der Lehrlingsausbildung zur Kontrolle des Arbeitsmarktes ließ die Gewerkschaften, insbesondere Industriegewerkschaften, nach anderen Mitteln zur Verknappung des Arbeitsangebotes suchen. Eine Alternative fand sich im ,closed shop“. Ein Unternehmen, dessen gesamte Arbeiterschaft in einer Gewerkschaft organisiert ist und das durch Tarifvertrag dazu verpflichtet ist, nur Arbeitnehmer einzustellen, die auch Mitglied in der Gewerkschaft sind, wird als ,closed shop“ bezeichnet (vgl. Müller-Jentsch 1997, S. 87). Obwohl der ,closed shop“ in den USA seit dem Taft-Hartley Act von 1947 offiziell verboten ist, kommt er in Bereichen der Bauwirtschaft mit hohem gewerkschaftlichen Organisationsgrad de facto noch vor (vgl. Dubofsky 1994, S. 204; Lösche 1992, S. 517). Häufig war der ,closed shop“ mit dem Institut der , union hiring hall' verbunden. Dabei handelt es sich um ein Arbeitsvermittlungsbüro der Gewerkschaft, die so die Verteilung der Arbeitskräfte an die 
Unternehmen koordinieren kann. Die ,hiring hall‘ wurde durch den Taft-Hartley Act nicht verboten. An die Stelle des ,closed shop“ trat der sogenannte ,union shop", bei dem der Unternehmer zwar weiterhin das Recht hat, einen Arbeitnehmer unabhängig von dessen Mitgliedschaft in der das Unternehmen vertretenen Gewerkschaft einzustellen, der eingestellte Arbeitnehmer aber innerhalb einer bestimmten Frist in die Gewerkschaft eintreten muß. Jedoch können die Einzelstaaten auch diese Form der ,union security ' gesetzlich verbieten. Von 50 Einzelstaaten haben 20 sogenannte, rightto-work' Gesetze verabschiedet, mit denen die gewerkschaftliche Organisierung dahingehend eingeschränkt werden soll, daß eine gewerkschaftliche Schließung des Betriebes durch Tarifvertrag verhindert wird (vgl. Scherrer 1996, S. 237 f.). Um den internen Arbeitsmarkt kontrollieren und die Prärogative des Managements einschränken zu können, vereinbaren die Gewerkschaften regelmäßig Senioritätsregeln im Tarifvertrag. Diese Senioritätsregeln bestimmen den Aufstiegsmodus für Arbeitnehmer innerhalb der Tarifeinheit entsprechend der Dauer der Zugehörigkeit zum Betrieb (vgl. Loeffelholz 1998, S. 557).

Infolge dieser institutionellen Arrangements stehen die Gewerkschaften der Einführung eines umfassenden betriebsorientierten Berufsausbildungssystems für Jugendliche eher skeptisch gegenüber. Zunächst sind es allgemeine Bedenken, die Interessen der bereits beschäftigten Arbeitnehmer, insbesondere der gewerkschaftlich organisierten, nicht zu gefährden (vgl. Smith 1995, S. 188): ,You want to ensure that kids placed in an apprenticeship program are not used to replace existing unionized workers, existing workers anywhere, but particularly unionized workers. So that you would not fire someone who is making 15 bucks an hour and replace him/her with one of the apprentices who was brought in at minimum wage“" (Service/Public Sector Official, in: Shenon 1992, S. 16). Darüber hinaus könnten Arbeitgeber nach Meinung der Gewerkschaften Lehrlinge einstellen, um die Mehrheit einer Gewerkschaft in einer Tarifeinheit (bargaining unit) ${ }^{30}$ zu untergraben. Bei einer Aufteilung des Arbeitsprozesses in kleinere Arbeitsschritte bestünde zudem die Möglichkeit, ältere, höher qualifizierte Arbeitnehmer durch Lehrlinge zu ersetzen, die bereits nach kurzer Anlernphase die Tätigkeiten ausüben könnten. Fraglich ist jedoch, warum die Arbeitgeber das nicht

30 Durch Mehrheitswahl wird bestimmt, welche Gewerkschaft in der Tarifeinheit den alleinigen Vertretungsanspruch hat. 
bereits tun, da ihnen die Option, ungelernte Jugendliche einzustellen, immer schon offen stand. Außerdem verläuft der Trend in einigen Branchen in die genau entgegengesetzte Richtung, nämlich ,high performance work organization“. Durch den Einsatz älterer Arbeitnehmer zur Ausbildung von Lehrlingen hätten diese eine geringere Arbeitsproduktivität und müßten deshalb bei einem leistungsorientierten Bezahlungssystem mit einem geringeren Lohn rechnen. Die Schaffung eines Berufsausbildungssystems für Jugendliche erhöhte den ,pool` an gut qualifizierten Arbeitskräften, der den Unternehmen zur Verfügung stünde. Bei fortschreitendem Technologiewandel könnten Arbeitgeber auf diese flexiblen, gut qualifizierten Arbeiter zurückgreifen und müßten ihre älteren Arbeiter nicht weiterbilden. Im Sinne des Senioritätsprinzips wird deshalb ein System eines ,lifelong learning' gefordert, in dem ältere Arbeiter den jüngeren immer einen Schritt voraus wären. Das Senioritätsprinzip ist hingegen ein Motivationshindernis für Jugendliche, die trotz vorhandener Qualifikation keine Chance haben, schneller aufzusteigen. Abgesehen von der Schutzfunktion für ältere Arbeiter hat das Senioritätsprinzip aufgrund des fehlenden formalisierten Berufsbildungssystems immer auch eine Methode der Qualifikationsentwicklung dargestellt. Diese gilt es natürlich für die Gewerkschaften zu verteidigen.

Angesichts einer begrenzten Zahl von Arbeitsplätzen für qualifizierte Arbeiter stehen die Gewerkschaften einer Ausweitung des Arbeitsangebotes kritisch gegenüber. Solange die Unternehmen nicht mehr solcher Stellen schaffen, hätte die Zunahme qualifizierter Arbeiter durch ein Lehrlingssystem lediglich negativen Einfluß auf den Lebensstandard bereits beschäftigter Arbeiter. „It has to be that they [Unternehmen; Anmerk. des Verfassers] create more jobs...good jobs. If we're just talking about corporations saying, ,O.K., here are the 50 jobs in the U.S. that are worth anything and [through this program] we're going to have more people competing for them', that's when people start saying, ,Well, forget it““ (Service/Public Sector Official, in: ebd., S. 18). Eng damit verbunden ist die Problematik, daß Jugendliche bzw. Lehrlinge als billige Arbeitskräfte dienen könnten (vgl. Roditi 1992, S. 342). Hiervon wären letztlich auch die beschäftigten Arbeiter betroffen. Aber in erster Linie geht es darum sicherzustellen, daß Lehrlinge nicht als Arbeiter mißbraucht werden, sondern tatsächlich in ihrem Beruf ausgebildet werden. Um dies gewährleisten zu können, müßten Standards gerade für die betriebliche Seite der Ausbildung vereinbart werden. So könnten die Ausbildungsanstrengungen der Unternehmen überprüft werden. Ge- 
werkschaften setzen angesichts fehlender allgemeinverbindlicher Standards zwei Instrumente ein, um die Gefahr des Einsatzes von Lehrlingen als billigen Arbeitskräften zu minimieren: Erstens vereinbaren sie Lehrlings-/Gesellenverhältniszahlen, die die Anzahl der Lehrlinge pro Gesellen auch für den Fall einer Entlassung oder Neueinstellung detailliert regeln. Andererseits fordern sie höhere Lehrlingslöhne und reduzieren so den Abstand zwischen Gesellen- und Lehrlingslohn. Beide Maßnahmen haben den einen Effekt, die Zahl der Lehrlinge drastisch zu senken (vgl. Ryan 1984, S. 198). Eine weitere Möglichkeit, die Zahl der Lehrlinge zu verringern, ist die Forderung nach einer Arbeitsplatzgarantie für jeden Lehrling, der seine Ausbildung erfolgreich abgeschlossen hat. Andernfalls könnte sich ein Ausbildungsprogramm für Jugendliche als ,revolving door training“ etablieren, bei dem Jugendliche solange ,,ausgebildet“ werden, wie Arbeitgeber nur einen geringen oder sogar keinen Lohn zahlen müssen, um sie nach der Ausbildung und der Aussicht auf einen höheren Lohn wieder zu entlassen. Zudem ist die Einstellung der Gewerkschaften gegenüber Jugendlichen genauso vom Wirken des Jugendarbeitsmarktes beeinflußt, wie die der Arbeitgeber. Jugendliche werden als noch nicht reif für gute, qualifizierte Arbeit angesehen. Ihnen fehle die dafür notwendige Arbeitsethik. „Are the young people in the United States mature enough to be serious about this type of program?" (Building Trade Official, in: Shenon 1992, S. 23).

Traditionen und Ideen der schulischen Bildung beeinflussen darüber hinaus die Position der Gewerkschaften zur betriebsorientierten Berufsbildung. Sie stehen ganz in der Tradition liberaler Schulreformer und deren Idee der, equality of opportunity`. Jegliche Form des ,tracking', also der Unterteilung der Schüler nach Fähigkeiten und möglichen Berufsperspektiven, wird von den Gewerkschaften abgelehnt. Bevor Schüler in ein berufsorientiertes Ausbildungsprogramm eintreten, sollten alle eine breite akademische Grundausbildung erhalten, die ihnen die Option der ,higher education“ offen hält. Eine wesentliche Forderung ist die Permeabilität der Bildung: Schüler, die sich für die Erlernung eines spezifischen Berufs entschieden haben, sollten später noch die Möglichkeit haben, in andere Berufe zu wechseln bzw. ein College zu besuchen. Dies erfordert jedoch eine breitangelegte Berufsausbildung, bei der keine berufsspezifischen Fähigkeiten vermittelt werden, sondern die Schüler allgemeine Einblicke in ein ganzes Spektrum von Berufen erhalten (vgl. ebd., S. 19). „In short, apprenticeship programs are aimed at producing docile workers...rather than citizens of a democracy capable of 
independent thought" (Roditi 1992, S. 343). So können Arbeitskräfte flexibler auf sich ändernde Bedingungen reagieren. Auszubildende stellen zudem für die Beschäftigten eine geringere Gefahr dar, weil sie während der Ausbildung lediglich rudimentäre Kenntnisse im jeweiligen Beruf erlernen, die nicht ausreichen, um einen älteren Arbeiter zu ersetzen. Abgesehen von den Interessen der Gewerkschaften bleibt jedoch die Frage, wie stark sie als Gruppe sind, um ihre Position durchzusetzen. Die Mitgliedsgewerkschaften der AFL-CIO hatten 1996 zusammen 13 Millionen Mitglieder. Insgesamt waren 18 Millionen Beschäftigte gewerkschaftlich organisiert, was einem Organisationsgrad von 14,7 Prozent entsprach (vgl. Loeffelholz 1998, S. 552). Zum niedrigen Organisationsgrad kommen weitere Schwächen der Gewerkschaften hinzu: so die Fragmentierung in Berufs- und Industriegewerkschaften und deren ethnische Zersplitterung. Darüber hinaus basiert die gewerkschaftliche Organisation auf starken lokal autonomen Einheiten. Diese ,locals' handeln Tarifverträge häufig nur mit einem Unternehmen aus, weshalb sich die Tarifpolitik auf betriebsnahe Probleme konzentriert und keine sektorale Lohnpolitik betrieben werden kann (vgl. Lösche 1992, S. 509; Lieshout 1997, S. 9). Das Fehlen von gewerkschaftsunabhängigen Betriebsräten verhindert ferner eine Aufgabenteilung dergestalt, daß die Gewerkschaft kollektive Interessen des Industriesektors vertritt, während Betriebsvertreter die Belange der Betriebsbelegschaft repräsentieren. Im System der betrieblichen Tarifpolitik werden gruppenindividuelle Interessen über die des Kollektivs der Arbeiterschaft gestellt.

Gerade Gewerkschaften in der Bauindustrie stehen einem Lehrlingsausbildungssystem für Jugendliche sehr skeptisch gegenüber. Dafür gibt es zwei zentrale Gründe: Erstens ist die traditionelle Form der Lehrlingsausbildung in der Bauwirtschaft am verbreitetsten. Und zweitens sind die Gewerkschaften reine Berufsverbände. In diesem Bereich dient die Lehrlingsausbildung noch als zentrale Institution, um den Zugang zum Beruf und damit den Arbeitsmarkt zu kontrollieren: „...construction unions have used apprenticeship as the main port of entry into the sector“ (Lieshout 1997, S. 10). Hier kommt die Exklusivität der ,craft unions، und ihre Verknappungsstrategie auf dem Arbeitsmarkt voll zum Tragen. Aufgrund des Senioritätsprinzips haben jugendliche Arbeiter keine Chance, einen der begehrten Plätze in einem Lehrlingsausbildungsprogramm zu bekommen: ,...'collective bargaining agreement is run by seniority, and therefore, when a job opening comes up, including an apprenticeship position, the most senior applicant is selected“" (Industrial Manufacturing Sector 
Official, in: Shenon 1992, S. 21). Ein Lehrlingsprogramm für Jugendliche hätte für die ,craft unions` lediglich zur Folge, daß ein Überangebot an gut qualifizierten Arbeitskräften entstünde, das die Verhandlungsposition der Gewerkschaften schwächte. „For my industry, I see no reason to have youth apprentices,.... Let's call them what they are. They're student workers. ... Any time there's a registered apprenticeship program in an area, I don't think there should be youth apprenticeship for our industry“ (Building Trades Official, in: ebd., S. 28).

Die Interessen der amerikanischen Gewerkschaften hinsichtlich einer Berufsausbildung für Jugendliche ergeben sich aus dem institutionellen Gefüge, in dem sie agieren. Im Konflikt um industrielle versus berufliche Organisierung der Arbeiterschaft setzte sich nach dem Scheitern der Knights of Labor und der Gründung der AFL die Organisation entlang von Berufskategorien durch. Zwar gibt es seit den 30er Jahren eine Tendenz in Richtung industriegewerkschaftlicher Organisation, die sich in der Abspaltung der CIO von der AFL manifestierte. Indessen sind auch bei den Industriegewerkschaften Traditionen des ,craft unionism“ vorzufinden: So der Drang, den Arbeitsmarkt durch verschiedene Instrumente, wie z.B. ,closed shop'-Vereinbarungen, zu kontrollieren. Berufsgewerkschaften haben immer den Zugang zu ihren Lehrlingsprogrammen genutzt, um die Zahl der qualifizierten Arbeitskräfte zu beschränken und so den Wettbewerb zu reduzieren. Infolgedessen handelt es sich bei der bestehenden Lehrlingsausbildung nicht um ein Massenprogramm, sondern um eine Elitenförderung. Das Prinzip der Exklusivität verhindert, daß die Gewerkschaften eine umfassende, inklusionssensible Lehrlingsausbildung für Jugendliche unterstützen.

\subsection{Staatliche Institutionen}

Für die Funktionsfähigkeit eines dualen Berufsausbildungssystems sind die Sozialpartner und hier insbesondere die Arbeitgeber von zentraler Bedeutung. Diese agieren jedoch nicht im „luftleeren Raum“, sondern sind in ihren Entscheidungen vom staatlichinstitutionellen Rahmen abhängig. Dabei ist die Kompetenzverteilung sowohl zwischen Bundesregierung und Einzelstaaten als auch innerhalb der Bundesregierung entscheidend für die Bestimmung, inwieweit ein kohärentes, nationales Ausbildungssystem implementiert werden kann. Die amerikanische Verfassung erwähnt den Begriff ,education“ nicht im Zusammenhang mit den Kompetenzen der Bundesregierung. Aus 
diesem und historischen Gründen gilt ,education“ als in der Verantwortlichkeit der Einzelstaaten bzw. der lokalen Schulbehörden liegend. Trotzdem hat die Bundesregierung immer wieder in die Organisation der Schulbildung interveniert. Im Fall einer dualen Berufsausbildung erschwert sich die Situation noch durch den Umstand, daß zwei unterschiedliche Bereiche betroffen sind: Schule (education) und betriebliche Ausbildung (training). Auf Bundesebene fällt eine solche Ausbildungsform in den Kompetenzbereich zweier Ministerien, dem Department of Labor (DOL) und dem Department of Education (DOE). Um die Funktionsweise und Idee des jeweiligen Ministeriums verstehen und so seine Interessen an der Schaffung eines dualen Berufsausbildungssystems einschätzen $\mathrm{zu}$ können, sollen $\mathrm{im}$ folgenden die beiden Ministerien kurz dargestellt werden.

Das Department of Labor wurde 1913 von Präsident W. H. Taft gegründet. „A Federal Department was the direct product of a half-century campaign by organized labor for a ,Voice in the Cabinet“" (MacLaury 1988, Kp. 1). Seine Aufgaben liegen in der Förderung der Wohlfahrt der arbeitenden Bevölkerung, der Verbesserung der Arbeitsbedingungen und der Möglichkeiten qualifizierter Beschäftigung. Die ersten vier Abteilungen des Ministeriums kamen aus dem ehemaligen Department of Commerce and Labor: Bureau of Labor Statistics, Bureau of Immigration, Bureau of Naturalization und Children's Bureau. Die dominante Stellung des Bureau of Immigration - von den 2000 Mitarbeitern unterstanden 1700 dem Bureau of Immigration - korrelierte mit der damaligen Debatte um eine Beschränkung der Immigration, um das Arbeitsangebot zu reduzieren. Dies war eine zentrale Forderung der Gewerkschaften. Erst 1940 wurde das Bureau of Immigration vom DOL ins Department of Justice verlagert. Als erster Arbeitsminister wurde William B. Wilson berufen, ein Mitglied des Vorstandes der United Mine Workers of America. Bis 1933 rekrutierten sich die Arbeitsminister aus den Rängen der Gewerkschaften. Während des Ersten Weltkrieges hatte das DOL eine herausragende Funktion in der kriegsentscheidenden Verwaltung der Arbeitskräfte und Aufrechterhaltung des inneren Friedens zwischen Gewerkschaften und Arbeitgebern. Mit der Ernennung von Frances Perkins brach F. D. Roosevelt mit der Tradition, daß die Spitze des DOL von einem Gewerkschaftsmitglied besetzt wird. Zudem war Frances Perkins auch die erste Frau, die einen Kabinettsposten innehatte. Sie war wie Roosevelt der Überzeugung, daß die Bundesregierung eine zentrale Rolle in der Regulation der Wirtschaft spielen müsse, um soziale Gerechtigkeit und individuelle Freiheit zu 
gewährleisten. Unter ihrer Ägide wurden solch weitreichende Politiken wie eine Arbeitslosen- und Rentenversicherung, Mindestlohnbestimmungen, öffentliche Arbeitsprogramme und die Abschaffung der Kinderarbeit verabschiedet (vgl. ebd., Kp. 3). Durch die Schaffung neuer bundesstaatlicher Programme während des New Deal behielt das DOL auch nach dem Zweiten Weltkrieg eine tragende Rolle in der Organisation des amerikanischen Arbeitsmarktes. Die Bundesregierung hatte sich neue Kompetenzen angeeignet und konnte sich als wichtiger Akteur etablieren. Erst in den 1980er Jahren stellte Präsident Reagan diese Position ernsthaft in Frage. Seine Politik der Deregulation und Devolution verlagerte zahlreiche Verantwortlichkeiten von der Bundesebene auf die Ebene der Einzelstaaten. Auch das DOL mußte Einschnitte in seinem Bereich hinnehmen.

Die Einrichtung bundesstaatlicher Arbeits- und Ausbildungsprogramme während des New Deal führte zur institutionalisierten Trennung von ,education' und ,training'. „Education and training have developed as parallel systems since the 1930s, when the Roosevelt Administration established job training programs outside the educational system“ (Gruber 1994, S. 14). In den 1960er Jahren verabschiedete der Kongreß den Manpower Development Training Act (MDTA) und übertrug damit dem DOL die Verantwortung, Ausbildungsprogramme zu finanzieren und zu verwalten. Diese Gesetzgebung rief nur deswegen keinen Widerstand des Department of Health, Education and Welfare und der American Vocational Association hervor, weil diese Programme außerhalb der klassischen ,school vocational education“ plaziert wurden. Diese Tradition blieb auch in den dem MDTA folgenden Gesetzen leitendes Prinzip, z.B. im Comprehensive Employment and Training Act (CETA) und Job Training Partnership Act (JPTA) (vgl. McDonnell/Grubb 1991, S. 33).

In seiner Politik der ,job training'-Programme verfolgt das DOL das Prinzip der ,employability‘, um den Teilnehmer an solchen Programmen den Einstieg oder Wiedereinstieg in den Arbeitsmarkt zu erleichtern. Obgleich die Verwaltung von,workforce development activities‘ in den Kompetenzbereich des DOL fällt, gab es 1995 insgesamt 163 bundesstaatliche Ausbildungs- und Beschäftigungsprogramme, die von 15 Ministerien und anderen öffentlichen Behörden verwaltet wurden. Auf das DOL entfielen dabei lediglich 37 Programme. Um den Übergang von Jugendlichen in den Arbeitsmarkt $\mathrm{zu}$ verbessern, wurden insgesamt 19 Ausbildungsprogramme durch bundesstaatliche Mittel finanziert. Das DOL hatte die Verantwortung für sieben dieser 
Programme (vgl. U.S. General Accounting Office 1997a, S. 1-6). „Many of the programs that target youth share the same broad objectives - to enhance the ability of youth to become productive members of the workforce“ (U.S. General Accounting Office 1994). Nicht nur, daß es eine Trennung zwischen, education' und ,training' gab, sondern selbst für die, training'-Programme fehlte eine einheitliche Kompetenzzuteilung innerhalb der Bundesregierung. Das erschwert noch heute die Etablierung einer effektiven und kohärenten Strategie zur Ausbildung Jugendlicher.

Das Department of Education (DOE) ist das jüngste unter den ,cabinet-level'-Ministerien. Jimmy Carter unterzeichnete kurz vor seiner Abwahl als Präsident 1979 das Gesetz zur Schaffung eines Bildungsministeriums. Diesem Gesetz war ein hundertjähriger Konflikt über den Status und die richtige Vertretung schulischer Bildung auf Bundesebene vorausgegangen. 1867 hatte es zum ersten Mal ein U.S. Department of Education gegeben. Ein Jahr später war es bereits wieder aufgelöst und als Abteilung dem Department of Interior zugeordnet. Als Office of Education wurde es 1939 der Federal Security Agency, der Vorgängerin des Department of Health, Education and Welfare, unterstellt. „There continued to be advocates of a separate cabinet-level department over the years but serious attention to such a structure did not develop until the growth of federal education programs in the 1960s“" (Radin/Hawley 1988, S. 16). Viele dieser bundesstaatlichen Programme wurden geschaffen, weil die Einzelstaaten oder die lokalen Schuldistrikte nicht in der Lage waren, Schülern mit besonderen Bedürfnissen eine entsprechende Schulausbildung zu gewährleisten. Der Bundesstaat konnte so unter Hinweis auf das Prinzip der, equality of opportunity` in das ansonsten geschlossene System lokaler Schulverwaltung eingreifen, indem er über finanzielle Anreize die Struktur des Systems veränderte. Diese Programme schufen wiederum neue Interessen und politische Kapazitäten, die die Idee eines Bildungsministeriums vorantrieben. Besonders entscheidend war hier die National Education Association, eine Art Standesorganisation der Lehrer, die in allen Wahlbezirken der USA vertreten ist und deshalb großen politischen Einfluß besitzt. Unter Präsident Johnson wurde 1964 eine Expertenkommission der Carnegie Corporation beauftragt, die Bedingungen für die Schaffung eines Bildungsministeriums zu prüfen. Ihr Bericht endet mit den Worten: „There should be at the highest level of the Federal government an agency adequately staffed to carry through penetrating analysis of current problems and needs, to lay down the broad objectives of government action, and to develop solid programs in pursuit of 
those objectives. It would not be the purpose of such an agency to direct or control American education..." (in ebd., S. 18-19). Aber erst Carter setzte sich nachdrücklich für die Errichtung eines bundesstaatlichen Bildungsministeriums ein, wofür er von der National Education Association in seinem Wahlkampf unterstützt wurde.

Drei Hauptargumente nannten die Befürworter eines U.S. Department of Education: Erstens erhöhe ein Bildungsministerium den Status der Bildung auf Bundesebene. Zweitens habe der Präsident in Fragen der Bildungspolitik einen direkteren Zugang zur Administration, und bildungspolitische Interessengruppen hätten einen durchsetzungsfähigeren Ansprechpartner für ihre Anliegen. Und schließlich könnten die vielen, über verschiedene Ministerien und Behörden verstreuten Bildungsprogramme besser koordiniert werden. Kritiker eines bundesstaatlichen Bildungsministeriums sahen in einer solchen Institution einen außerordentlichen Zuwachs an Macht für den Bundesstaat in Bildungsfragen. Darüber hinaus würde ein wichtiges nationales Thema politisiert. Zwischen diesen beiden Polen, bundesstaatlicher Kontrolle über Bildung oder bundesstaatlicher Unterstützung, mußte sich das neugegründete U.S. Department of Education legitimieren. Die tiefsitzenden Ressentiments gegen ein ,cabinet-level'Ministerium zeigten sich in der Wahlkampfforderung Reagans, nach Wahl zum Präsidenten das eben geschaffene Ministerium wieder aufzulösen. Als Reagan 1980 das Amt des Präsidenten antrat, verwaltete das Department of Education 150 Programme mit 6000 Beschäftigten. Jedes Programm hatte seine eigenen Mitarbeiter, Ressourcen, Budgets und gesetzlichen Grundlagen. Die Ideen, die allen Programmen zugrundelagen, waren ,equity‘ und ,access‘ (vgl. Clark/Astuto 1987, S. 50). Hier wird die frühere Einbindung in das Department of Health, Education and Welfare deutlich, bei dem bundesstaatliche Bildungspolitik immer auch Sozialpolitik war. Über den Zugang zu freier Schulbildung sollen alle Schüler gleiche Startchancen (equality of opportunity) erhalten, um sich einen eigenen Lebensunterhalt verdienen zu können: ,...goal of education is to prepare all individuals intellectually and socially for economic and social survival“" (Bacharach 1990, S. 418). Reagan konnte zwar das U.S. Department of Education aufgrund der demokratischen Mehrheit im Kongreß nicht auflösen, setzte aber neue Akzente in der Ausrichtung der bundesstaatlichen Bildungspolitik. Statt ,equity‘ wurde nun die Idee der ,excellence‘ betont (vgl. Kirst 1995, S. 49; Lugg 1996, S. 3). Amerikanische Schulen sollten besser werden, indem auf lokaler Ebene das Prinzip des Marktes und Wettbewerbs gestärkt wird. ,Equity“ und ,access` behielten 
dennoch ihre eminente Bedeutung für bundesstaatliche Bildungsprogramme. Noch heute definiert das U.S. Department of Education eine seiner Herausforderungen als „ensuring/promoting access“ (U.S. General Accounting Office 1997b, S. 3). Wie beim U.S. Department of Labor gibt es weitreichende Überschneidungen mit anderen bundesstaatlichen Behörden. So stellte das Department of Education fest, daß es von 131 bundesstaatlichen Programmen für , at-risk ${ }^{6}$ und kriminelle Jugendliche lediglich 10 verwaltet (vgl. ebd., S. 16). Auch dies belegt die ablehnende Haltung des Kongresses, dem Department of Education weitreichende Macht $\mathrm{zu}$ übertragen und so bundesstaatliche Kapazitäten für Bildungspolitik in einer Behörde zu konzentrieren. Ein Umstand, der die Institutionalisierung eines umfassenden dualen Berufsausbildungssystems nicht begünstigt.

Aufgrund dieser vielfältigen Fragmentierung der bildungs- und ausbildungspolitischen Kompetenzen auf Bundesebene gab es in den 1990er Jahren einen Vorschlag des Kongresses, beide Ministerien in einem Department of Education and Employment zusammenzufassen: „...the proposal for the new Department of Education and Employment is based on the premise that the nation cannot adequately prepare its youth for the challenges of the 21 st century until fundamental changes are made in federal policy on education and employment issues“ (U.S. General Accounting Office 1995a, S. 5). Ein solches „Superministerium“ hätte viele der Programme konsolidieren und eine kohärente bundesstaatliche Strategie im Bereich der Ausbildungsprogramme vorantreiben können. Sowohl das Department of Labor als auch besonders das Department of Education widersetzten sich dem Gesetzesvorschlag. Der damalige Bildungsminister kritisierte den Vorschlag mit dem Argument, daß der Zusammenschluß die Bedeutung der Bildungspolitik auf Bundesebene herabsetze, ,at a time when education is more important than ever to America's future“ (ebd., S. 14). Gerade das Department of Education sah sich in seiner kurzen Geschichte als ,cabinet-level'Ministerium gefährdet. Durch die Aufrechterhaltung der administrativen Trennung bleibt auch der Kooperationskonflikt zwischen ,education' und ,training'-Programmen erhalten. Beide Ministerien sind aus einer unterschiedlichen Geschichte hervorgegangen und haben deshalb auch verschiedene Traditionen. Das Arbeitsministerium war eine Forderung der Gewerkschaften, die ihre Position gegenüber den Arbeitgebern durch staatliche Unterstützung stärken wollten. Während des New Deal wurden öffentliche Beschäftigungs- und Ausbildungsprogramme eingerichtet, deren Ziel die Integration der 
Arbeitslosen in den Arbeitsmarkt war. Leitendes Prinzip der Programme war die ,employability“. Im Gegensatz dazu entsprang das Department of Education einer liberalen Ideologie, die durch bundesstaatliche Ressourcen und Kontrollen die Unterschiede zwischen den Schulen minimieren wollte, um so allen Schülern gleiche Startchancen zu gewähren. Bundesstaatliche Programme basieren auf dem Prinzip der ,equity .

Am Beispiel des ,dropout ${ }^{6}$-Problems ${ }^{31}$ lassen sich die Folgen dieser unterschiedlichen Ideen gut nachzeichnen: Während die Schulabrecher für das Department of Education ein soziales Problem darstellen, dem durch neue Unterrichtsmethoden und praxisnahe Curricula begegnet werden soll, liegt das Problem für das Department of Labor in den fehlenden Fertigkeiten, die einer stabilen, langfristigen Integration in den Arbeitsmarkt entgegenstehen. Bei einem dualen Berufsausbildungssystem entsteht das Problem, daß eine aus Sicht des Arbeitsministeriums notwendige Trennung der High School in einen ,College track' und einen ,Vocational track' vom Bildungsministerium wegen des Prinzips der ,equity‘ und ,equality of opportunity` abgelehnt wird. Die grundsätzliche Offenhaltung aller Optionen, also auch der Collegezulassung, durch den High SchoolAbschluß beinhaltet ein erhöhtes Investitionsrisiko für Arbeitgeber, die sich an der Berufsausbildung beteiligen, da Lehrlinge nicht nur zu einem anderen Unternehmen, sondern auch an ein College wechseln können. Zudem kommen die Kooperationsprobleme durch die Trennung zwischen ,education` und ,training' bei einem Berufsausbildungssystem, das schulische und betriebliche Komponenten verbindet, in vollem Maße zum Tragen.

Über die bundesstaatliche Kompetenzverteilung hinaus gibt es staatlich-institutionelle Hindernisse im Verhältnis des Bundesstaates zu den Einzelstaaten, die der Schaffung eines national kohärenten Berufsausbildungssystems zuwiderlaufen. Die Einzelstaaten hatten immer eine herausragende Rolle in der Organisation und Verwaltung des amerikanischen Schulsystems. Erst in den 1960er und 1970er Jahren gewann die bundesstaatliche Bildungspolitik durch die Einführung von speziellen Unterstützungspro-

31 Die Gesamtzahl der High School-Abschlüsse ist seit 1980 von 76 auf 73 Prozent gesunken. Abbrecherraten variieren sehr stark in Abhängigkeit von ethnischen und geographischen Faktoren. Lateinamerikaner bilden den größten Anteil der ,dropout' Gruppe, gefolgt von Schwarzen. ,Innercity“Schulbezirke liegen dagegen vor ländlichen Regionen (vgl. Hadden 1992, S. 616). 
grammen an Bedeutung (vgl. DiConti 1996, S. 6). Dies hatte mit dem offensichtlichen Scheitern der Einzelstaaten zu tun, ihrer Verantwortung für benachteiligte Bevölkerungsschichten nachzukommen (vgl. Pittenger 1977, S. 9). „States have historically shown a greater interest in serving all citizens and less concern about the special populations that have been the targets of federal policies“" (McDonnell/Grubb 1991, S. 51). Mit der Zunahme an bundesstaatlichen Förderprogrammen wuchs letztlich auch die Rolle der Einzelstaaten, da diese für die Verwaltung der finanziellen Mittel zuständig waren. So konnten sie administrative Kapazitäten aufbauen, die ihnen die Kontrolle lokaler Schulpolitik ermöglichten (vgl. Wirt/Kirst 1997, S. 196 f.; Kirst 1995, S. 44). Von den 89.000 Schuldistrikten im Jahre 1948 blieben bis 1995 lediglich 15.020 übrig (vgl. Kirst 1995, S. 25). Die einzelstaatliche Schulpolitik unterscheidet sich aber im Grad und der Form der Intervention in die lokale Schulverwaltung. Je nach ihrer historischen Entwicklung tendieren die Einzelstaaten entweder zu einer zentralistischen oder lokalen Ausrichtung ihrer Politik. Entscheidend ist dabei auch die Stärke der Lehrergewerkschaften und lokalen ,school boards', die seit jeher Befürworter einer lokalen Schulverwaltung gewesen sind. Interventionen der Einzelstaaten stehen indessen in einem Zielkonflikt zwischen Förderung von , equity“ auf Einzelstaatsebene und Wahrung der ,freedom of local choice‘.

Der Ausweitung bundesstaatlicher Kompetenzen und Programme in der ,Great Society'-Ära folgte in den 1980er Jahren eine Rückkehr zu mehr einzelstaatlicher Verantwortung (vgl. Finn/Rebarber 1992, S. 176 f.). Präsident Reagan verringerte den Einfluß der Bundesregierung auf die einzelstaatliche Bildungspolitik, indem er statt ,categorical programs` das Instrument der ,block grants` einführte. Bei ,categorical programs' legt die Bundesregierung spezifische Anforderungen für die Verwendung bundesstaatlicher Mittel durch die Einzelstaaten fest. So kann sie einzelstaatliche Bildungspolitik in ihrer Struktur beeinflussen. ,Block grants ‘ übertragen hingegen den Einzelstaaten weitreichende Verantwortung für die Verwendung bundesstaatlicher Mittel und die Ausgestaltung spezifischer Programme. ,The block grant can be viewed as an enabling step toward the President's concept of the new federalism as it might be applied to education“ (Clark/Astuto 1987, S. 54). Die Einzelstaaten konnten diese neue Rolle nur deshalb ausfüllen, weil sie ihre administrativen Kapazitäten mit Hilfe bundesstaatlicher Unterstützung in den 1960er und 1970er Jahren erweitert hatten. Viel diskutierte Berichte über das Scheitern und den Standard amerikanischer Schulen, wie 
der 1983 veröffentlichte Report ,A Nation at Risk`, erhöhten ferner die Legitimität einzelstaatlicher Eingriffe in die lokale Kontrolle über die Ausgestaltung des Curriculums (vgl. Kirst 1995, S. 27). Durch die Devolutionskampagne Reagans konnten die Einzelstaaten ihre Position in der Bildungspolitik de jure und de facto festigen: „....federal government during the Reagan Administration created much of the opportunity, stimulus, and agenda for state involvement on school reform issues“ (Mazzoni 1994, S. 55). Im Bereich der beruflichen Ausbildung etablierten viele Einzelstaaten ihre eigenen, workforce development'-Programme, die jedoch im Vergleich zu den bundesstaatlichen, job training'-Ressourcen mit relativ geringen Mitteln ausgestattet sind (vgl. McDonnell/Grubb 1991, S. 43).

Die Einzelstaaten hatten seit der Gründung der USA eine vorrangige Rolle in der Bildungspolitik. Das Verhältnis verschob sich erst nach dem Zweiten Weltkrieg und besonders in den 1960er Jahren zugunsten des Bundesstaates, der über eine Ausweitung seiner ,categorical programs“ die Struktur des amerikanischen Schulsystems zu beeinflussen versuchte. Diese Programme richteten sich auf bestimmte Zielgruppen und waren deshalb voneinander isoliert. Fehlende Koordination innerhalb der Bundesregierung und wachsende administrative Kapazitäten der Einzelstaaten eröffneten zu Beginn der 1980er Jahre die Option, den bildungspolitischen Föderalismus zu stärken. Aus der konservativen Reformperiode sind die Einzelstaaten mit neuem Selbstbewußtsein hervorgegangen, und so fordert die National Governors Association (NGA) als ein Prinzip für die Implementierung von Ausbildungsprogrammen: „Federal assistance should be provided through state-based networks and build on existing state and local programs“ (NGA 1998, S. 1). Als Argument werden sozio-ökonomische Unterschiede zwischen den Einzelstaaten vorgebracht, die eine flexible, regional bestimmte Politik erfordern. „It is vital to ensure that states have the authority and flexibility needed to respond to the needs and priorities of those who live within their boundaries" (NGA 1997, S. 1). Eine solche föderalistische Ideologie lehnt die prinzipielle Notwendigkeit einheitlicher Lösungen in spezifischen Politikfeldern, z.B. der Formulierung von Ausbildungsstandards, ab. Der Bundesregierung bleibt lediglich die Festlegung von Rahmenvorgaben, innerhalb derer die Einzelstaaten ihre Modifikationen vornehmen. Dies führt zu einer Vielzahl von fragmentierten Einzelprogrammen im Ausbildungsbereich. Die Idee der ,freedom of local choice‘ behält so ihre Bedeutung im staatlichinstitutionellen Arrangement. 


\subsection{Lehrergewerkschaften und Eltern}

Auf der betrieblichen Seite hängt der Erfolg eines dualen Berufsausbildungssystems von der Zusammenarbeit mit den Arbeitgebern ab. Für die schulische Seite der Ausbildung ist die Einbeziehung der Lehrer entscheidend. Sie sind diejenigen, die eine effektive Umsetzung der Politik fördern oder verhindern können. Als sogenannten ,street level bureaucrats ${ }^{32}$ stehen ihnen weitreichende Ermessensspielräume offen, durch die sie schulische Reformen blockieren können. Kollektives Sprachrohr und Interessenvertretungsorgan der Lehrer sind die Gewerkschaften, von denen es in den USA zwei national dominierende Organisationen gibt: die National Education Association (NEA) und die American Federation of Teachers (AFT). Ein weiterer für den Bereich der schulischen Berufsausbildung wichtiger Verband ist die American Vocational Association (AVA). Welche Interessen haben diese Verbände hinsichtlich der Schaffung eines nationalen Berufsausbildungssystems, bei dem schulische Komponenten des Lernens mit solchen im Betrieb integriert sind?

Die Entstehung des amerikanischen Schulwesens im 19. Jahrhundert geht auf die ,common school'-Bewegung zurück. Diese legte die Verantwortung der Bildung in die Hände lokaler Selbstverwaltungsorgane. So erklärt es sich, daß noch heute kommunale Schulbehörden für die Verwaltung, Finanzierung und Gestaltung des Curriculums verantwortlich sind (vgl. Swaan 1993, S. 126; Hadden 1992, S. 611). Entsprechend gilt die lokale Schulkontrolle als zentrales Charakteristikum des amerikanischen Bildungssystems ${ }^{33}$, wenngleich in den letzten Jahrzehnten die Rolle der Einzelstaaten in der Schulpolitik zugenommen hat. Für die lokale Schulverwaltung sind zwei Instanzen verantwortlich: der ,school board' und der ,superintendent', der vom ,school board“ ernannt wird. ,School boards' sind lokale Laiengremien, deren Mitglieder alle zwei Jahre direkt gewählt werden. Die Einflußmöglichkeiten der Lehrer in diesem lokal organisierten System werden von einigen Beobachtern als relativ gering bewertet. „Nonetheless, teachers neither control the governance of schools nor the curricula“ (Lowe 1995, S. 244). Lehrer sehen sich in ihrer Lehrtätigkeit einer ständigen Einmischung durch die Verwaltung und Eltern ausgesetzt (vgl. Meyer 1997, S. 144).

\footnotetext{
${ }^{32}$ Michael Lipsky nennt in seinem Buch, ,Street-level Bureaucracy: Dilemmas of the individual in public services“ (1980), Lehrer als klassisches Beispiel für das Verhalten von ,street level bureaucrats“.

33 „Local control is the hallmark of American education...“(Kirst 1995, S. 29).
} 
Andere wiederum behaupten, daß Lehrer im Klassenzimmer relativ autonom handeln können und in einigen Schuldistrikten sogar an der Ausarbeitung von Lehrplänen mitarbeiten (vgl. Cheng 1981, S. 30). „Because teachers retained a fair degree of autonomy once the classroom door was closed..." (Tyack/Cuban 1995, S. 9). Beide Ansichten haben ihre Berechtigung, da sich aufgrund der 15.000 Schuldistrikte kein einheitliches Bild zeichnen läßt, sondern unweigerlich graduelle Variationen auftreten. Als Folge der tatsächlich wachsenden Dominanz der Schulverwaltung in der Gestaltung des Schulablaufs und des Machtmißbrauchs einiger ,superintendents' veränderte sich die Strategie der Lehrergewerkschaften in Richtung militantem ,collective bargaining', wodurch sie dem Charakter einer Industriegewerkschaft näher kamen (vgl. Lowe 1995, S. 243). Durch detaillierte Verträge zwischen Schulverwaltung und Gewerkschaften suchten Lehrer Schutz in der Bürokratisierung der Beziehungen, obgleich ihr Ziel von jeher in der Professionalisierung ihres Berufsstatus lag. „Most teachers prefer to maintain what they perceive to be ,professional'..." (Johnson 1984, S. 166). Als erste nationale Organisation versuchte die National Education Association alle Formen der Lehrtätigkeit sowohl in der Grundschule als auch am College durch den regelmäßigen Wissensaustausch aller im Bereich der Bildung beschäftigten Personen zu professionalisieren und so implizit den Status der Beschäftigten zu verbessern. „Offiziell ging man davon aus, daß in dem Maße, wie sich durch die Professionalisierung des Schulsystems die allgemeine Bildungssituation verbessere, dies von der Öffentlichkeit honoriert werde und sie sich bereit fände, mehr Mittel dafür aufzubringen“" (Michalke 1989, S. 85).

Vorgängerin der National Education Association war die 1857 von zehn einzelstaatlich organisierten Verbänden in Philadelphia gegründete National Teachers Association (NTA). Durch den Beitritt der American Association of School Superintendents (AASS) und der American Association of Teachers Colleges (AATC) im Jahre 1870 benannte sich die NTA in National Education Association (NEA) um. Aufgeteilt in einzelne relativ autonome Departments wirkte die NEA lange Zeit wie eine Art Holding-Gesellschaft, unter deren Dach die verschiedenen Gruppen der ,educational professionals‘, wie Schullehrer, Schulverwalter oder College-Dozenten, ein isoliertes Dasein führten und in alljährlichen Konferenzen eine gemeinsame Basis in bildungspolitischen Fragen suchten. Bis in die 1960er und 1970er Jahre dominierten insbesondere die College-Dozenten und ,Superintendents“ die Politik der NEA, obwohl die 
Mehrzahl der Mitglieder „einfache“ Schullehrer waren. Es wurde eine gemeinsame Interessenidentität beschworen, die in der Förderung des „Wohls der Kinder“ liegen sollte. Folglich galt es, Bildungsfragen so weit als möglich von parteipolitischen Einflüssen freizuhalten: ,...the prevailing philosophy of the NEA's role was to improve education, leaving it to the states legislature and local school boards to win a fair share of school budgets for members" salaries and benefits" (West 1980, S. 18). Und eine Verbesserung der Bildung war nach Ansicht der NEA nur über die Professionalisierung des Lehrerberufs $\mathrm{zu}$ erzielen. Die NEA betrachtete sich selber auch nicht als Gewerkschaft, sondern als Standesorganisation (vgl. Berube 1988, S. 18).

Erst in den 1970er Jahren änderte sich das Selbstverständnis und mithin auch die Politik der NEA. Entscheidend für diese Entwicklung waren zwei Faktoren: Erstens konnte die Dominanz der College-Dozenten und Schuladministratoren in der Führung der NEA zugunsten der Schullehrer gebrochen werden. Seit 1968 ist jeder Präsident der NEA ein Schullehrer gewesen. Ihre neue Position festigten sie, indem sie eine 75 Prozent-Klausel für alle Mitgliedsverbände einführten. Diese Klausel besagt, daß der Anteil an Schullehrern in jedem Mitgliedsverband mindestens 75 Prozent betragen muß (vgl. Michalke 1989, S. 86 f.; West 1980, S. 21). Zweitens hatte die American Federation of Teachers (AFT) in den 1960er Jahren ihre ,no-strike policy“ aufgegeben und dadurch ihre Mitgliederzahl drastisch erhöhen können. So wurde sie zu einer echten Konkurrenzorganisation für die NEA. Als reine Interessenvertretungsorganisation der Lehrer beteiligte sich nun auch die NEA als Verhandlungspartner an kollektiven Tarifverhandlungen, um die materiellen Forderungen ihrer Mitglieder gegenüber der Schulverwaltung durchsetzen zu können. Die Betonung der NEA verschob sich vom berufsständischen hin zu gewerkschaftlichem Verhalten. In den 1970er Jahren gab sie schließlich auch ihre politische Abstinenz auf und unterstützte zum ersten Mal einen Präsidentschaftskandidaten (vgl. Spring 1989, S. 237). Da ihre lokalen Organisationen in nahezu allen Wahlbezirken vertreten sind, wurde sie zu einer bedeutenden politischen Interessengruppe ${ }^{34}$. Heute umfaßt die NEA ca. 2,3 Millionen Mitglieder, die zu den streikaktivsten Berufsgruppen in den USA zählen. Trotz ihrer gewerkschaftlichen Orientierung gehört die NEA im Gegensatz zur AFT nicht zum gewerkschaftlichen

\footnotetext{
${ }^{34}$ Theda Skocpol nennt solche Interessengruppen: ,widespread federated interests ‘. Zu ihnen gehört als prominentestes Beispiel die American Medical Association (vgl. Skocpol 1995, S. 29).
} 
Dachverband der AFL-CIO und lehnt jede engere Affiliation strikt ab (vgl. Berube 1988, S. 19 f.). Wichtigstes Ziel der NEA, abgesehen von den regelmäßigen Lohnverhandlungen, ist die Maximierung der professionellen Autonomie der Lehrer. NEA-Präsident Keith Geiger sagte im Zusammenhang mit der Diskussion um höhere Standards: „If we are to maximize student achievement, we must maximize teacher autonomy. Top-down dictates must give way to bottom-up reform“ (in: Vocational Education Journal 1992, S. 27).

Die Schaffung eines dualen Berufsausbildungssystems hätte weitreichende Implikationen für die Autonomie der daran beteiligten Lehrer. In einem Untersuchungsbericht der amerikanischen ,non-profit'-Organisation ,Jobs for the Future‘ zur Ausgestaltung eines solchen Ausbildungssystems heißt es: „Quality work-based learning programs ask teachers to make changes to help students get the most out of learning through work. These changes range from incorporating special projects that explore work-related issues into lesson plans to developing a fully integrated curriculum [Hervorhebung vom Verfasser] organized around work-related themes“ (Goldberger et al. 1994, S. 68). Lehrer sollen also ihre relative Autonomie in der Gestaltung des Lehrplanes weiter aufgeben, um mit Industrievertretern über die Zusammensetzung des Curriculums und die Organisation des Unterrichts zu verhandeln. Noch deutlicher sind die Forderungen in den Worten des Vorsitzenden der National Vocational Industrial Clubs of America, Stephen Denby: „We must invest in continuous upgrading of the professionals in the classroom by sending teachers back to industry, bringing industry into the classroom [Hervorhebung vom Verfasser], conducting industry-run seminars for teachers..." (in: Vocational Education Journal 1992, S. 27). Solche Überlegungen lassen Lehrer aufschrecken und eine grundsätzlich ablehnende Haltung einnehmen.

Seit Gründung der NEA kämpfen sie für die Anerkennung als Profession, um die einer Profession zustehende Autonomie der Selbstorganisation zu erlangen. Im Rahmen eines beruflichen Ausbildungssystems sollen sie sich den Bedürfnissen der Industrie unterordnen. Dies widerspricht der liberalen Tradition der NEA, die Bildung im humanistischen Sinne der Charakterbildung versteht und eine auf eine spezifische Tätigkeit hin orientierte, reine Wissensvermittlung ablehnt. Bei einer Anhörung vor dem U.S. Kongreß sagte der damalige Vizepräsident der NEA, Bob Chase: „,...any school-to-work program should be an education program first and a career training program second“ (in: U.S. House of Representatives 1993, S. 537). Auch hier wird 
deutlich, daß die NEA die Position der Lehrer durch die Einführung eines dualen Berufsausbildungssystems gefährdet sieht. Daraus folgt die Forderung nach einem Vorrang der akademischen Orientierung vor betrieblichen Lernelementen. So verwässert man ein Ausbildungssystem mit qualifizierter betrieblicher Orientierung in ein Programm mit wenig Einfluß der Unternehmen. In einer offiziellen Resolution der NEA zu Ausbildungsprogrammen heißt es: „...these programs should supplement, and not supplant, the vocational programs provided in the public schools“" (NEA 1998b, S. 1).

Die zweite große Lehrergewerkschaft ist die American Federation of Teachers (AFT). Sie wurde 1916 von Schullehrern gegründet, die über ihre Interessenrepräsentation in der NEA unzufrieden waren. „Initially the American Federation of Teachers saw itself as more of a radical segment of the National Education Association than as a completely independent body“ (W. Eaton, in: Berube 1988, S. 50). Ihr Selbstverständnis entsprach von Anfang an dem einer Gewerkschaft, die in erster Linie materielle Interessen ihrer Mitglieder zu vertreten hat. Folglich standen bei der AFT immer die Gehälter, Sozialleistungen und Arbeitsbedingungen der Lehrer im Vordergrund der Verbandspolitik (vgl. Cheng 1981, S. 19; Spring 1989, S. 238 f.). Entsprechend diesem Selbstverständnis trat die AFT auch dem gewerkschaftlichen Dachverband AFL bei. Innerhalb der AFL galt die AFT als relativ linksorientierter Mitgliedsverband. Bis in die 1960er Jahre grenzte sich die AFT jedoch von ,normalen“ Gewerkschaften dadurch ab, daß sie eine ,no-strike policy“ vertrat. Hier zeigten sich rudimentäre Ansätze eines berufsständischen Ethos, für das der Streik kein angemessenes Mittel zur Durchsetzung von Interessen ist. Nach Aufhebung dieser Selbstbeschränkung und ersten Streikerfolgen im Staat New York erhöhte sich die Mitgliederzahl schlagartig von 55.000 auf 188.300 (vgl. Michalke 1989, S. 88-91). Heute umfaßt die AFT ca. eine Million Mitglieder (vgl. AFT 1999, S. 1).

Ende der 1960er Jahre gab es Gespräche über einen Zusammenschluß der beiden Gewerkschaften, da sich die Strategien angenähert hatten und so die Idee einer großen amerikanischen Lehrergewerkschaft mit enormem politischen Einfluß in erreichbare Nähe gerückt war. Diese Gespräche scheiterten an der Mitgliedschaft der AFT in der AFL-CIO und den Mitgliedsbestimmungen der NEA, die ,all professional school personnel“" zuläßt. Die NEA schloß eine Mitgliedschaft in der AFL-CIO kategorisch aus, um ihre Unabhängigkeit nicht aufzugeben (vgl. West 1980, S. 235 f.). Trotz einer Annäherung bestehen doch Unterschiede in der Politik der beiden Organisationen. So 
reagierte die AFT auf die Schulreformbewegung der 1980er Jahre, die statt ,equity“ als Ziel der Schulpolitik die Idee der, excellence' durchsetzen wollte, offener und kooperationsbereiter als die NEA. Die AFT versuchte durch ihre Haltung die Reformbewegung in Richtung einer Professionalisierung der Lehrerschaft $\mathrm{zu}$ lenken: „....Shanker [Präsident der AFT; Anmerk. des Verfassers] called for the creation of professional teacher boards that would develop standards, handle parent complaints and evaluate textbooks“ (Berube 1988, S. 143). Dabei stellte er das Modell in der Medizin als richtungsweisend hin. Hierdurch zeichnete sich eine Verschiebung des Selbstverständnisses der AFT ab, die Abstand von einem radikalen ,unionism‘ nahm und sich der berufsständischen Position der NEA näherte. Andererseits bewegte sich die NEA in Richtung gerade dieses radikalen, unionism‘. Einige Beobachter sprechen sogar von einem Tausch der Positionen (vgl. ebd., S. 52 und 97).

Auch bei der Bewertung eines dualen Berufsausbildungssystems scheint die AFT eine offenere Haltung einzunehmen. Antonia Cortese, Vizepräsidentin der AFT, nannte als wesentliche Voraussetzung für eine positive Haltung ihrer Organisation gegenüber der Einführung eines solchen Systems vor einem Ausschuß des U.S. Kongresses, daß die „appearance and the reality of making this somehow a tracking of students“ (in: U.S. House of Representatives 1993, S. 324) auf alle Fälle vermieden werden müsse. Ferner betonte sie die zentrale Rolle der Lehrer als ,implementers of the plan“ (ebd., S. 324) und machte so die Strategie der AFT deutlich, Kompromißbereitschaft mit dem Anschein einer starken Verhandlungsposition zu kombinieren, um Reformvorhaben gemäß eigenen Interessen modifizieren zu können. Eine solche Strategie wird auch als ,accommodation" bezeichnet. Beide Gewerkschaften vertreten das Ziel einer Ausweitung der Autonomie der Lehrer durch Professionalisierung: ,...both the AFT and the NEA have always stressed the notion of teaching as a profession..." (McDonnell/Pascal 1988, S. 19). Einem solchen Ziel läuft die Einbindung der Unternehmen in die Lehrgestaltung zuwider. Insofern nehmen die Lehrergewerkschaften tendenziell eine kritische Haltung gegenüber der Schaffung eines dualen Berufsausbildungssystems ein.

Die American Vocational Association (AVA) hat im Vergleich zu den beiden anderen Organisationen eine weitaus homogenere Mitgliedschaft. In ihr sind ausschließlich Lehrer aus dem Bereich der schulischen Berufsausbildung (vocational education) organisiert. Vorgängerin der AVA war die 1917 gegründete National Society for Vocational Education, die wiederum auf die National Society for the Promotion of 
Industrial Education zurückgeht. Im Jahre 1926 fusionierte die National Society for Vocational Education mit der Vocational Education Association des mittleren Westens, woraus die AVA hervorging. Trotz ihrer relativ geringen Mitgliederzahl gehört die AVA zu den effektivsten Lobby-Gruppen in Washington. Eine Studie über die LobbyAktivitäten von Bildungsorganisationen stufte die AVA als vierteffektivste Gruppe ein (vgl. Cuban 1982, S. 62). Ihr Ziel ist die Förderung berufsnaher Ausbildungsprogramme in Schulen durch bundesstaatliche Gesetzgebung und finanzielle Unterstützung. Ausgangspunkt ihrer Aktivitäten war der Smith-Hughes Act von 1917, der bundesstaatliche Mittel zur Einrichtung schulischer Berufsausbildungsprogramme bereitstellte und so die institutionelle Förderung solcher Programme auf bundesstaatlicher Ebene initiierte. Spätere Versuche, die unter diesem Gesetz als förderungswürdig eingerichteten Berufskategorien ${ }^{35}$ abzuschaffen, weil sie den veränderten technologischen Ansprüchen nicht mehr entsprachen, scheiterten am vehementen Widerstand der AVA (vgl. ebd., S. 61). Als Vertreterin der in bestehenden Berufsausbildungsprogrammen beschäftigten Lehrer wacht die AVA sehr genau über jeden neuen Gesetzesvorschlag, der eine mögliche Umstrukturierung und Konsolidierung dieser Programme vorsieht.

In der Debatte um die Schaffung eines, youth apprenticeship'-Systems sagte Bret Lovejoy, AVA Assistant Executive Director: „My message to these people who will be drafting legislation on this subject was that a youth apprenticeship program could be implemented by the current vocational education system...there exists a system ready and able to accommodate [Hervorhebung vom Verfasser] a youth apprentice program“ (Lovejoy 1993, S. 14). Das bestehende „System“ wird in seiner Effektivität hochgepriesen und muß im Interesse der Mitglieder auch in dieser Form erhalten bleiben. Ein Berufsausbildungssystem mit einer starken betrieblichen Komponente hätte die Auslagerung einiger schulischer Aktivitäten in den Betrieb zur Folge und damit die mögliche Entlassung von Berufsschullehrern ${ }^{36}$.

\footnotetext{
${ }^{35}$ Unter dem Smith-Hughes Act wurden insbesondere Programme im Bereich landwirtschaftlicher und hauswirtschaftlicher Fächer gefördert. Der größte Teil der Bundesmittel diente der Bezahlung spezieller Lehrer (vgl. Wills 1997, S. 4).

${ }^{36}$ Der Begriff „Berufsschullehrer“ wird hier nicht im deutschen Sinne verstanden, als Lehrer einer spezifischen Schulform, sondern als Berufsbezeichnung für Lehrer, die in speziellen beruflichen Fächern ausgebildet sind, aber nicht unbedingt an einer Berufsschule arbeiten.
} 
Charles Buzzell, Executive Director der AVA, sagte zur Idee eines dualen Berufsausbildungssystems: „I'm not so sure we need to pull young people out of the educational environment“" (in: Toch 1991, S. 64). Die AVA ist eine Interessenorganisation, die ihre Daseinsberechtigung aus der Institutionalisierung einer Vielzahl schulischer Berufsausbildungsprogramme zieht. Demzufolge verteidigt sie den status quo, um ihren organisatorischen Nährboden nicht zu verlieren.

Lehrer können die inhaltliche Ausgestaltung eines Berufsausbildungssystems beeinflussen, Eltern hingegen beeinflussen bereits im Vorfeld die Entscheidung, ob ihre Kinder an einem solchen Programm teilnehmen oder nicht. Da bestehende schulische Berufsausbildungsprogramme häufig als zweite Chance für benachteiligte Schüler stigmatisiert sind, ist das Interesse der Eltern an solchen Programmen für ihre eigenen Kinder relativ gering (vgl. Brand 1994, S. 23 f.). In einer Umfrage waren 40 Prozent der Eltern der Überzeugung, daß man einen College-Abschluß benötige, um einen qualifizierten Job zu bekommen (vgl. Jobs for the Future 1997, S. 5). Daher glauben die meisten Eltern, daß ihre Kinder ein College besuchen werden und deshalb auf keine Berufsausbildungsprogramme angewiesen seien: „most parents seem to view college as a requisite... and would tend to steer their children away from a program they perceive is not intended for the college-bound" (Farkas/Friedman, in: ebd., S. 7). Andererseits sind sie der Meinung, daß die vorhandenen Programme erhalten bleiben müßten, um anderen Kindern, die nicht an ein College gehen, eine praxisnahe Ausbildung anzubieten. Insgesamt würden viele Eltern eine Dualisierung der Schulausbildung in einen akademischen und einen beruflichen Zweig favorisieren. Damit unterscheiden sie sich klar von der Position der Lehrergewerkschaften, die sich vehement gegen eine Dualisierung (tracking) einsetzen. Vorteil einer solchen Dualisierung wäre die Chance, die berufliche Bildung in der Schule an die Bedürfnisse der Industrie anzupassen und so eine bessere Qualifizierung zu erreichen. Zudem würde das Risiko der Unternehmen sinken, daß ein Auszubildender das Unternehmen im Anschluß an die Ausbildung verläßt, um einen College-Abschluß anzustreben. Eine solche Dualisierung wäre jedoch ohne Wert, wenn Eltern den beruflichen Zweig nicht als reale Alternative zum College anerkennen würden. 


\section{Bundesstaatliche Ausbildungs- und Berufsbildungspolitik}

Politik entsteht in den seltensten Fällen ,auf der grünen Wiese“. Häufig ist die Wiese schon bebaut und läßt nur wenig Spielraum für neue Gebäude und Wege. So verhält es sich auch mit Berufsbildungsprogrammen in den USA. Es existiert bereits eine Infrastruktur, die nur schwer wieder einzureißen ist. Seit Beginn dieses Jahrhunderts ist die amerikanische Bundesregierung in die Finanzierung und Gestaltung der ,vocational education“ an High Schools und Colleges involviert. In der zweiten Hälfte des Jahrhunderts kamen Programme im Bereich der Beschäftigungs- und Ausbildungspolitik hinzu. Im folgenden sollen zunächst wichtige bundesstaatliche Beschäftigungs- und Ausbildungsprogramme seit den 1950er Jahren dargestellt werden. Anschließend wird die bundesstaatliche Politik im Bereich der ,vocational education“ untersucht. Die Trennung der beiden Bereiche in dieser Darstellung ergibt sich aus der institutionalisierten Parallelität der Beschäftigungs- und Berufsbildungspolitiken.

\subsection{Ausbildungs- und Beschäftigungspolitik}

In den letzten fünf Dekaden haben die USA eine Ausweitung von Institutionen erlebt, die für die Ausbildung und Beschäftigung von Arbeitskräften zuständig sind. Das entsprechende Wachstum verschiedenster Ausbildungsprogramme reflektiert die politische Sensibilität für ökonomische Probleme, wie Arbeitslosigkeit und Armut. Nach dem Ende des Zweiten Weltkrieges verabschiedete der U.S. Kongreß den sogenannten GI Bill of Rights Act, der den heimkehrenden Weltkriegsveteranen eine finanzielle Unterstützung für ein College-Studium zusicherte ${ }^{37}$. Idee hinter diesem Gesetz war, die Lage am Arbeitsmarkt durch eine Verringerung des Arbeitsangebotes zu entspannen. In den 1950er Jahren führte der „Sputnik-Schock“ zu einer aktiveren Rolle der Bundesregierung in der Ausbildung der amerikanischen Arbeiterschaft. Der Glaube an die technische Überlegenheit der USA über den Rest der Welt war erschüttert. Um die amerikanische Wirtschaft leistungsfähiger zu machen, sollten bundesstaatliche Mittel zur Fortbildung oder Umschulung der durch Rationalisierungen und Automation entlassenen Arbeiter bereitgestellt werden. Der amerikanische Kongreß verabschiedete

${ }^{37}$ Der wohl bekannteste Vertreter aller Veteranen, die Unterstützung durch den GI Bill of Rights Act erhielten, ist Henry Kissinger, der auf diese Weise an der Harvard University studieren konnte. 
1962 den Manpower Development and Training Act (MDTA). Dieses Gesetz gilt als der erste umfassende Versuch, ein Ausbildungsprogramm $\mathrm{zu}$ schaffen, das ,a coordinated approach through classroom training, remedial education, on-the-job training, and placement“ (American Enterprise Institute 1980, S. 3) darstellt. Anspruchsberechtigte Teilnehmer waren Arbeiter, deren Fertigkeiten durch technologischen Wandel und Automation obsolet geworden waren. Da jedoch die Arbeitslosigkeit für diese Kategorie der Arbeiterschaft kurz nach Verabschiedung des Gesetzes drastisch zurückging und es deshalb keinen Bedarf mehr für eine Unterstützung der ,dislocated workers' gab, wurde die Zielgruppe des Programms umdefiniert. Ab jetzt waren es die wirtschaftlich benachteiligten Bevölkerungsgruppen, die für eine Teilnahme an den durch MDTA geförderten Programmen in Frage kamen. Schlagartig erhöhte sich so der Kreis der Berechtigten, ohne daß parallel die Mittel dafür erhöht wurden. Insbesondere arbeitslose Schulabrecher (dropouts) und andere benachteiligte Personen ohne Arbeit galten als förderungswürdig. Gerade Jugendliche sahen sich einem verschärften Wettbewerb auf dem Arbeitsmarkt gegenüber, weil in den frühen 60er Jahren die ersten „baby boomers“ auf den Arbeitsmarkt drängten (vgl. Levitan/Gallo 1988, S. 5). Das Department of Labor, in dessen Verantwortungsbereich MDTA fiel, legte 1966 eine 65 Prozent-Mindestgrenze für die Verwendung der bundesstaatlichen Mittel für die neue Zielgruppe der ,disadvantaged‘ fest (vgl. Bresnick 1984, S. 32). Wegen der begrenzten Mittel wurden die finanzierten Programme auf die wirtschaftlich am stärksten benachteiligten Gruppen gerichtet. Die Finanzierung erfolgte auf einer ,project-by-project' Basis, bei der jedes vorgestellte lokale Programm vom Department of Labor begutachtet wurde. „Some 10,000 service delivery entities negotiated with the DOL to provide classroom training services under MDTA“ (L'Hoest 1998, S. 74).

Anfangs war dem U.S. Employment Service eine besondere Rolle in der Verwaltung und Koordinierung der MDTA Programme zugedacht worden (vgl. King 1995, S. 77). Diese unabhängige bundesstaatliche Behörde wurde 1933 offiziell durch den WagnerPeyser Act gegründet, um sowohl die neugeschaffene Arbeitslosenversicherung zu verwalten als auch Arbeitsangebot und Arbeitsnachfrage durch die Vermittlung von Arbeitsuchenden zu steuern (vgl. Breen 1997, S. 165; L'Hoest 1998, S. 75). Mit ihren lokalen ,employment offices“ besaß diese Behörde geeignete Kapazitäten, um die Administration des MDTA übernehmen zu können. Trotzdem scheiterte der Versuch 
einer Zentralisierung der Verwaltung unter der Oberaufsicht des U.S. Employment Service. Dafür gab es zwei Gründe: Einerseits hatte der U.S. Employment Service keine Erfahrung mit der Verwaltung von Ausbildungsprogrammen und der Unterstützung von benachteiligten Bevölkerungsgruppen, wie denjenigen, die einen Anspruch auf Förderung durch den MDTA hatten. Infolgedessen glaubten viele Beamte des U.S. Employment Service, daß hier zwei unvereinbare Ziele durch eine Behörde verfolgt werden sollten, nämlich ,training versus direct job placement“ (Levitan/Gallo 1988, S. 48). Andererseits handelt es sich beim U.S. Employment Service um eine „stabile Bürokratie“, deren Funktion nicht einfach geändert werden kann, weil sie spezifischen Routinen folgt. Dementsprechend bezweifelten viele Reformer die Möglichkeit, den U.S. Employment Service in den Dienst der Fürsorge für benachteiligte Bevölkerungsgruppen zu stellen (vgl. King 1995, S. 144 f.). Das unausweichliche Scheitern einer zentralisierten nationalen Koordination von Ausbildungsprogrammen hatte langfristige Folgen für die Struktur und Organisation amerikanischer Beschäftigungs- und Ausbildungspolitik. Die Institutionalisierung einer lokalen Verwaltung der Programme wurde zur Achillesferse einer gesamten Politik.

Durch die Entscheidung, die Zielgruppe des MDTA zu ändern, gerieten bundesstaatlich geförderte Ausbildungsprogramme in das Fahrwasser der „War on Poverty“-Politik. „The Great Society initiatives of the 1960s were a watershed in federal involvement in the provision of jobs and job-related services“ (American Enterprise Institute 1980, S. 3). Das für den „War on Poverty“ zentrale Gesetz war der Economic Opportunity Act (EOA) von 1964, in dem weitere bundesstaatliche Mittel für Beschäftigungsprogramme zur Verfügung gestellt wurden. Entscheidende Bedeutung gewann die Initiierung von zwei Jugendprogrammen: Jobs Corps und Neighborhood Youth Corps. Beide Programme waren auf die Unterstützung benachteiligter Jugendlicher ausgerichtet (vgl. Bresnick 1984, S. 31). Während Jobs Corps Programme Beschäftigung und so Lebensunterhalt für Jugendliche, insbesondere High School ,dropouts', an ihrem Wohnort anboten, sollten Neighborhood Youth Corps Programme Teilzeitarbeit in den Sommerferien bereitstellen, um , at risk'-Jugendliche ${ }^{38}$ an den Arbeitsmarkt heranzuführen und sie auf diese Weise zur Fortsetzung ihrer Schulbildung zu bewegen.

38 Diese Jugendlichen zeichnen sich durch schlechte Schulleistungen aus und gelten als ,dropout‘ gefährdet. 
Jobs Corps Beschäftigungsprogramme wurden zudem durch schulischen Unterricht sowohl in beruflichen als auch allgemeinbildenden Fächern ergänzt (vgl. Levitan/Gallo 1988, S. 5; Bresnick 1984, S. 83 f.). Die Diffusion bundesstaatlicher Finanzierungsquellen für Beschäftigungsprogramme - neben dem MDTA und EOA gab es weitere Gesetze, die Mittel für die Unterstützung benachteiligter Gruppen zur Verfügung stellten - hatte jedoch einen Zuwachs verschiedenster lokaler Institutionen zur Folge, die sich als Anbieter solcher bundesstaatlich geförderten Programme etablieren konnten: ,...since there was no local institution with the authority or capacity to create a coherent local training strategy, local program implementation grew increasingly chaotic during the first half of the 1960s“ (L'Hoest 1998, S. 78). Die wachsenden Koordinationsprobleme resultierten in dem Versuch einer Organisationsreform. Mit dem EOA Community Action Program wurden sogenannte ,community action agencies“ (CAAs) geschaffen, die die Zielgruppen der Programme repräsentierten und deren Funktion in der Planung und Verwaltung lokaler Projekte lag. Sie sollten als ,one-stop-shop“ für alle bundesstaatlichen Unterstützungsprogramme dienen. „It was envisoned that CAAs would perform services normally handled by a range of well-established institutions including schools, the employment service, and welfare agencies...“ (ebd., S. 80). Eine Stärkung ihrer Position erfuhren die CAAs durch die Initiierung des Concentrated Employment Program von 1967, das die begrenzten Mittel des MDTA und EOA zur Förderung der mit der höchsten Armut und Arbeitslosigkeit betroffenen Gemeinden bündeln sollte (vgl. Levitan/Gallo 1988, S. 6). Hier konnten die bereits etablierten CAAs als zentrale lokale Koordinationsstelle fungieren.

Die in die CAAs gesetzten Hoffnungen erfüllten sich aber nicht, da sie einerseits die Interessen der Leistungsanbieter berücksichtigen mußten, andererseits aber Vertreter der benachteiligten Gruppen und damit der Leistungsnachfrager waren (vgl. L'Hoest 1998, S. 80 f.). Kritik an der fortbestehenden Duplizierung von Programmen und ungeklärten Kompetenzverteilungen zwischen den verschiedenen staatlichen Hierarchien ließen das Pendel der öffentlichen Meinung wieder zur Seite der Dezentralisierung von Verantwortung ausschlagen. Präsidentschaftskandidat Nixon konnte mit seiner Wahlkampagne eines ,New Federalism‘ seine Siegchancen auch sogleich erhöhen. Im Weißen Haus angekommen, machte er die Dezentralisierung von Verantwortung zum zentralen Thema einer Neugestaltung der bundesstaatlichen Beschäftigungs- und 
Ausbildungspolitik. Seine Idee war es, die verschiedenen bundesstaatlichen Mittel für MDTA und EOA Programme in ,block grants ${ }^{639} \mathrm{zu}$ konsolidieren, um die politische Kompetenz der Einzelstaaten in diesem Bereich $\mathrm{zu}$ stärken. Damit wäre die Bundesregierung von Verwaltungsverantwortung für einzelne Programme befreit worden. Politischer Kompromiß war der 1973 verabschiedete Comprehensive Employment and Training Act (CETA): Titel I des Gesetzes umfaßte Ausbildungsprogramme, für die den Einzelstaaten bzw. lokalen Einheiten als ,prime sponsors ${ }^{40}$ entsprechend bestimmten Kriterien ,block grants` zur Verfügung gestellt wurden. Das ,public employment program‘ wurde unter Titel II in den CETA übernommen. Unter dem Eindruck steigender Arbeitslosigkeit hatte der Kongreß 1971 den Emergency Employment Act verabschiedet, dessen entscheidender Beitrag die Einrichtung öffentlicher Arbeitsbeschaffungsmaßnahmen war. In ,Public Service Employment ${ }^{`}$ (PSE) umbenannt, setzte der CETA diese Politik fort und finanzierte zeitlich begrenzte Jobs in öffentlichen Einrichtungen für Langzeitarbeitslose und Jugendliche. Dieses staatlich geförderte Beschäftigungsprogramm sollte in der Folge das öffentliche Urteil über CETA maßgeblich beeinflussen (vgl. Bresnick 1984, S. 33 und 85 f.).

Das unter dem Economic Opportunity Act geschaffene Jobs Corps für wirtschaftlich benachteiligte Jugendliche wurde unter Titel IV des CETA zusammen mit dem Summer Youth Program reautorisiert. Die Jugendprogramme erhielten den größten Anteil der bewilligten bundesstaatlichen Mittel: 1979 waren 56 Prozent aller Individuen in CETA Programmen jünger als 22 Jahre (vgl. American Enterprise Institute 1980, S. 4 f.). Ziel der amerikanischen Bundesregierung war die Verringerung der dramatisch angestiegenen Jugendarbeitslosigkeit, wozu die CETA finanzierten Programme einen zentralen Beitrag leisten sollten. Im Unterschied zum MDTA erhielt der Privatsektor unter dem CETA eine neue Rolle in der Planung lokaler Programme. Man hatte erkannt, daß ohne die Einbindung privater Unternehmen die Beschäftigungs- und

\footnotetext{
39 ,Block grants ‘ werden von ,categorical grants“ unterschieden: letztere beinhalten genaue Richtlinien über die Verwendung von bundesstaatlichen Mitteln und werden je Programm von der Bundesregierung autorisiert. ,Block grants' hingegen erlauben den Einzelstaaten mehr Ermessensspielräume über die spezifische Verwendung der bundesstaatlichen Mittel, da sie lediglich grobe Richtlinien über den Einsatz der Gelder vorgeben.

40 Lokale politische Einheiten (Städte, Landkreise oder Zusammenschlüsse derselben) mit mehr als 100.000 Einwohnern konnten sich als ,prime sponsor' organisieren und waren dann für die Organisation der Programme in ihrem Verantwortungsbereich zuständig.
} 
Ausbildungsmaßnahmen langfristig nur einen geringen Einfluß auf die Beschäftigungssituation der Teilnehmer hatten. Die ,prime sponsors' bekamen daher speziell ausgewiesene Mittel, mit denen sie Private Industry Councils (PICs) einrichten sollten. Einzige Voraussetzung für PICs war eine Mehrheit von Vertretern der Privatwirtschaft unter den Mitgliedern. Die PICs sollten ,prime sponsors' in der Organisation und Verwaltung der lokalen Programme beraten. Dadurch erhoffte man sich zweierlei: Einerseits sollte so die Effizienz der Programme gesteigert werden, andererseits die Zahl der privatwirtschaftlich bereitgestellten ,on-the-job'-Ausbildungsplätze erhöht werden. Das Mißtrauen der Wirtschaft gegenüber rein staatlich kontrollierten Programmen hat häufig als Argument gedient, um die geringe Beteiligung der Unternehmen an Ausbildungsprogrammen zu erklären.

Trotz der Einbindung der Wirtschaft in die Verwaltung der CETA Programme blieb die geringe Zahl von ,on-the-job'-Ausbildungsplätzen weiterhin zentrales Problem der Beschäftigungspolitik. Verschiedene Faktoren verhinderten eine weitere Ausweitung des privatwirtschaftlichen Ausbildungsengagements: Die schwere wirtschaftliche Rezession in den 1970er Jahren wirkte sich negativ auf Ausbildungsentscheidungen der Unternehmen aus, da sie wegen Überkapazitäten bereits qualifizierte Arbeiter entlassen mußten. In einer solchen Situation neue Arbeiter auszubilden, widerspräche der gewinnmaximierenden Logik der Unternehmen. Hinzu kam noch die politische Entscheidung, daß mindestens zweidrittel aller durch CETA subventionierten ,on-thejob'-Ausbildungsplätze mit wirtschaftlich benachteiligten Personen besetzt werden müssen. Obgleich die von den Unternehmen bezahlten Löhne teilweise bis zu 100 Prozent durch CETA Mittel subventioniert wurden, war die Zurückhaltung der Unternehmen nicht zu brechen: ,...there is a strong reluctance on the part of the private sector to become involved with young people“ (Bresnick 1984, S. 52). Zudem befürchteten gerade große Unternehmen, daß ihre Entscheidungsfreiheit in der Gestaltung der internen ,on-the-job'-Ausbildung durch staatliche Subventionierung und infolgedessen staatliche Richtlinien beschränkt würde.

Die geringe Beteiligung der Wirtschaft an den Ausbildungsprogrammen erforderte andererseits eine Ausweitung des unter Titel II verabschiedeten öffentlichen Beschäftigungsprogramms (PES), um so der wachsenden Zahl der Arbeitslosen, insbesondere Jugendlichen, einen Lebensunterhalt zu sichern. Als ergänzendes Programm geschaffen, überlagerte der Public Employment Service (PES) schließlich alle anderen Aus- 
bildungs- und Beschäftigungsmaßnahmen der lokalen ,prime sponsors`. Die Kritik an der offensichtlichen Ineffektivität des PES bestimmte die Debatte um den Fortbestand des gesamten CETA. Untersuchungen zeigten, daß eine Beschäftigung im öffentlichfinanzierten Sektor in den seltensten Fällen zu einer Anstellung bei einem privaten Unternehmen führte (vgl. AEI 1980, S. 40 f.). Der PES war lediglich ein zeitlich begrenztes Beschäftigungsprogramm, ohne Bezug $\mathrm{zu}$ der eigentlichen Intention des CETA, wirtschaftlich benachteiligten Individuen eine langfristig qualifizierende Ausbildung zu bieten. Der CETA sollte aus den Erfahrungen mit dem MDTA die Ausbildungs- und Beschäftigungspolitik in zweierlei Hinsicht reformieren: Erstens erhielten die Einzelstaaten und lokalen Verwaltungseinheiten mehr Verantwortung. Zweitens wurde der Privatsektor durch die Schaffung von PICs stärker einbezogen. Beide Reformen zeigten jedoch nicht den gewünschten Erfolg, sondern hatten geradezu kontraintentionale Implikationen. Die Devolution von Verantwortung auf lokale ,prime

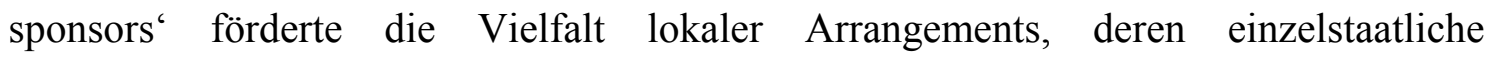
Koordination wegen fehlenden Interesses der Einzelstaaten häufig unterblieb. „One result of the distribution of authority achieved through the implementation of CETA was the acceleration of the process which saw the development and differentiation of local employment and training systems“، (L’Hoest 1998, S. 87). Da die Bundesregierung eine zentralisierte Zulassung der einzelnen lokalen Projekte aufgegeben hatte, konnte sie keinen Einfluß mehr auf die Ausgestaltung der Programme nehmen und verlor mithin die Option, eine gewisse Uniformität garantieren zu können. Andererseits schuf sie mit der Einrichtung von PICs eine parallele administrative Struktur neben den ,prime sponsors', deren Funktion teilweise über die reine Beratung hinaus auch operative Tätigkeiten einschloß.

Der alles überschattende Makel des CETA war allerdings das öffentliche Beschäftigungsprogramm (PES), das in der Debatte um eine Verlängerung des CETA zu Beginn der 1980er Jahre eine zentrale Position einnahm. Letztlich scheiterte die Reautorisierung des CETA am negativen Image des Pubic Employment Service. Einen völligen Ausstieg aus bundesstaatlich geförderten Ausbildungs- und Beschäftigungsprogrammen konnte - trotz anderslautender Aussagen ${ }^{41}$ - die neue Regierung unter Präsi-

41 Unter Reagans ,New Federalism“ sollte die Verantwortung für Beschäftigungs- und Ausbildungsprogramme vollständig auf die Einzelstaaten übertragen werden (vgl. Levitan/Gallo 1988, S. 9-11). 
dent Reagan aber nicht durchsetzen. An die Stelle des CETA trat 1982 der Job Training Partnership Act (JTPA). Wichtigster Bestandteil des Gesetzes war das Fehlen eines ,public employment program‘. Ansonsten setzte der JTPA die unter dem CETA eingeführten Reformen fort: Die Rolle des privaten Sektors sollte durch eine Ausweitung der Kompetenzen der Private Industry Councils (PICs) weiter gestärkt werden. „The PICs were transformed from an advisory body to a policymaking council with a required majority of business representatives“ (Levitan/Gallo 1988, S. 12). Zusammen mit lokalen Politikern und Beamten sollten sie die Planung und Verwaltung der Programme in den Service Delivery Areas $(\mathrm{SDA})^{42}$ übernehmen, wobei ihnen eine federführende Rolle zugedacht war (vgl. Wills 1997, S. 8; Moore/Waldman 1994, S. 147). Während die Einzelstaaten unter dem CETA eine untergeordnete Kontrollfunktion hatten, hat der JPTA den Gouverneuren weitreichende Koordinations- und Kontrollkompetenzen übertragen, indem die bundesstaatlichen Mittel den Einzelstaaten als ,block grant ${ }^{`}$ zur Verfügung gestellt werden. Diese leiten die Mittel anschließend an die lokalen Verwaltungseinheiten weiter. Zudem können die Einzelstaaten über ,performance standards' Einfluß auf die Gestaltung lokaler Programme nehmen (vgl. McDonnell/Grubb 1991, S. 35). Lediglich für die unter Titel IV des JPTA aufgeführten Programme, wie z.B. Job Corps, hat die Bundesregierung eine direkte Verwaltungskompetenz (vgl. Levitan/Gallo 1988, S. 14).

Wie beim MDTA und CETA, richten sich die Programme auf wirtschaftlich benachteiligte Gruppen, deren ,employability ‘ durch die Beschäftigungs- und Ausbildungsmaßnahmen verbessert werden soll. Von den potentiell berechtigten Personen können aufgrund der begrenzten Mittel aber lediglich 6 Prozent einen Platz in einem JPTA geförderten Programm erhalten (vgl. L’Hoest 1998, S. 94). Für benachteiligte Jugendliche gibt es drei spezifische Programme: Youth Training Program, Summer Youth Employment and Training Programs und Youth Fair Chance Program. Das Youth Training Program bietet Ausbildungsmaßnahmen sowohl schulischer Art als auch ,onthe-job training' für wirtschaftlich benachteiligte Jugendliche, die noch in der Schule sind oder diese bereits abgebrochen haben. Mit dem Programm soll die ,employability

42 Gouverneure erhielten durch den JTPA die Möglichkeit, die Grenzen der Service Delivery Areas festzulegen. Dieses Recht wurde jedoch dadurch eingeschränkt, daß lokale Verwaltungseinheiten mit mehr als 200.000 Einwohnern sich als eigenständige SDAs organisieren konnten (vgl. L’Hoest 1998, S. 96). 
der Jugendlichen erhöht werden. Allerdings kann dieses Ziel wegen der kurzen Dauer (3-4 Monate) der Ausbildung häufig nicht erreicht werden (vgl. Moore/Waldman 1994, S. 148). Das Summer Youth Employment and Training Program bietet ebenfalls Arbeitserfahrung und allgemeinbildenden Nachhilfeunterricht für 14-21 jährige Jugendliche. Bezahlte Teilzeitarbeit bei öffentlichen oder ,non-profit'-Organisationen im Sommer eröffnet den Jugendlichen häufig nur ,low-wage, low-skill‘ Beschäftigung, die sie nicht auf höher qualifizierte Tätigkeiten in der Privatwirtschaft vorbereitet. Außerdem sind private Unternehmen in der Regel nicht dazu berechtigt, ,summer jobs ‘ für dieses Programm anzubieten. Das Youth Fair Chance Program ist auf Nachbarschaften mit besonders hoher Armut konzentriert. Hier sollen umfassende Bildungs- und Ausbildungsprojekte mit zugehörigen Beratungsangeboten die Arbeitsmarktchancen der Jugendlichen erhöhen (vgl. ebd., S. 150-152).

Die amerikanische Ausbildungs- und Beschäftigungspolitik seit den 1950er Jahren hat eine institutionelle Struktur geschaffen, die die Neuausrichtung der Politik in diesem Bereich erschwert. Gerade die Reform des MDTA setzte die Weichen in eine entscheidende Richtung: Die Neudefinition der Zielgruppe - weg von entlassenen Arbeitern mit obsoleten Fertigkeiten hin zu wirtschaftlich benachteiligten Gruppen - prägt das Image bundesstaatlich finanzierter Programme noch heute. Teilnehmer sind durch ihre Teilnahme an den Programmen stigmatisiert. Unternehmen entscheiden sich gegen Bewerber, gerade weil sie durch die Teilnahme an einem solchen Programm gezeigt haben, daß sie bestimmte Kriterien aufweisen, die sie für die Privatwirtschaft unattraktiv machen. ${ }^{43}$ Dies zeigt sich auch an den Schwierigkeiten, privatwirtschaftliche ,on-the-job'-Ausbildungsplätze in die Organisation der Programme einzubeziehen. Lediglich in Zeiten angespannter Arbeitsmarktlagen stieg die Zahl der ,on-the-job“Ausbildungsplätze. Die Unternehmen beteiligten sich jedoch nicht nur wegen der spezifischen Zielgruppe an den Programmen, sondern auch wegen ihrer grundsätzlichen Abneigung gegenüber staatlichen Programmen, die mit staatlicher Kontrolle über firmeninterne Abläufe verbunden sind. Von einer primär wirtschaftspolitischen Intention entwickelte sich der MDTA durch seine Einbeziehung in den, War on Poverty` zu einer sozialpolitischen Maßnahme. Der gescheiterte Versuch, den bundes-

43 „Our system of work training is in very large part devoted to making up for educational failure. Its chief clients are high school dropouts“(Glazer 1988, S. 76). 
staatlichen U.S. Employment Service als zentrale Behörde zur Koordination und Verwaltung der verschiedenen bundesstaatlichen Programme $\mathrm{zu}$ etablieren, ist die zweite folgenschwere Weiche, die die bundesstaatliche Ausbildungs- und Beschäftigungspolitik vom Ziel eines umfassenden nationalen Berufsausbildungssystems entfernte. Hier gab es administrative Kapazitäten, die eine effektive Verwaltung hätten gewährleisten können. Aber sowohl Widerstände innerhalb des U.S. Employment Service als auch föderalistische Traditionen verhinderten eine Ausweitung seiner Kompetenzen. Folge war die Entstehung von Myriaden lokal verwalteter Programme, die sich nicht nur in ihrer inhaltlichen Struktur unterscheiden, sondern auch verwaltungsorganisatorische Unterschiede je nach lokalen Gegebenheiten aufweisen. So haben die unter dem CETA eingeführten Private Industry Councils (PICs), deren Position durch den JPTA noch gestärkt wurde, nur in 15 Prozent der Service Delivery Areas eine aktive administrative Funktion übernommen (vgl. L'Hoest 1998, S. 97). Neben der bundesstaatlich geförderten Ausbildungs- und Beschäftigungspolitik existiert eine institutionell separierte Berufsbildung (vocational education) an den High Schools und weiterführenden Bildungsinstitutionen, die ebenfalls bundesstaatliche Mittel erhält.

\subsection{Berufliche Bildungsprogramme}

Ursprung aller die Berufsbildung betreffenden bundesstaatlichen Gesetze ist der SmithHughes $\mathrm{Act}^{44}$ von 1917. Bei der Implementierung des Gesetzes befand sich die Bundesregierung ausnahmsweise in einer starken Ausgangsposition, da den Einzelstaaten praktische Erfahrungen mit beruflicher Bildung fehlten und sie deshalb die Hilfe der Bundesregierung bereitwillig annahmen. Das Gesetz schuf ein Federal Board of Vocational Education (FBVE), das der Bundesregierung eine vorrangige Rolle in der Berufsbildung bis zu seiner Überführung in das Office of Education im Jahre 1933 zuwies. Durch die Eingliederung in das Office of Education sank die bundesstaatliche Präsenz in der Berufsbildung (vgl. Cuban 1982, S. 72 f.). Der Smith-Hughes Act stellte bundesstaatliche Mittel für die Einrichtung berufsbildender Schulfächer an den High Schools zur Verfügung. Damit hatten die Gegner einer Dualisierung des Schulsystems

\footnotetext{
${ }^{44}$ Die Bundesregierung hatte sich bereits 1862 mit dem Morrill Act in der Berufsbildung engagiert. Dabei handelte es sich jedoch um die Vergabe von, land grants' für die Einrichtung von Community Colleges und nicht um inhaltliche Vorgaben für den berufsorientierten Unterricht.
} 
in allgemeinbildende und berufsbildende Schulen obsiegt (vgl. Herbst 1996, S. 129 ff.). Bei den $\mathrm{zu}$ unterrichtenden Fächern handelte es sich in der Regel um agrarwirtschaftliche und hauswirtschaftliche Themen. Daneben wurden auch Fächer aus dem Bereich ,trade and industry‘ gefördert (vgl. Cobb/Neubert 1998, S. 104).

Bis in die 1950er Jahre veränderte sich kaum etwas an der grundlegenden Struktur der schulischen Berufsbildung. Wie bei der Ausbildungs- und Beschäftigungspolitik war es der „Sputnik Schock“, der den Anstoß für den Versuch einer Reform der Berufsbildung gab. Der Vocational Education Act von 1963 förderte neue Programme, die für alle Jugendlichen offen sein sollten, also auch für Schüler aus benachteiligten Gruppen. So wurde das Gesetz Teil des ,War on Poverty“ der Johnson Regierung (vgl. Herbst 1996, S. 192 f.). Darüber hinaus wurden zusätzliche Fächer, wie ,business education', und neue Formen der Kooperation zwischen Schule und Betrieb in die Förderung einbezogen. Die Mittel für die Subventionierung von ,on-the-job'-Ausbildungsplätzen in den Unternehmen waren jedoch relativ gering, und die Unternehmen zögerten aus den bereits genannten Gründen, sich daran zu beteiligen. Maßgebende Intention war die Ausweitung der Berufsbildung über spezifische Berufskategorien hinaus, um durch eine breite berufliche Grundausbildung die Anpassungsfähigkeit der Jugendlichen an ein sich technologisch wandelndes Arbeitsumfeld zu erhöhen. „In effect, the 1963 Act broadened the definition of vocational education to prepare people for many jobs rather than to meet specific market demands“" (Cuban 1982, S. 54). Andererseits konnte die bundesstaatliche Subventionierung der unter dem Smith-Hughes Act geschaffenen Berufskategorien wegen des Widerstandes der American Vocational Association nicht aufgehoben werden, obgleich viele dieser Kategorien (Hauswirtschaft, Landwirtschaft etc.) als veraltet galten. Die Kombination aus neuen Zielen und alten Strukturen belastete die Implementation des Gesetzes und führte letztlich zu ineffektiven Resultaten: lokale Schuladministratoren und School Boards waren lediglich an einer Fortsetzung bundesstaatlicher Förderung interessiert, um die seit Jahrzehnten bestehenden Berufsbildungsprogramme beibehalten oder sogar ausweiten zu können. „A 1973 report concluded that, a disproportionate amount of vocational education funds still goes into home economics and agriculture““( (ebd., S. 75). Das für die Umsetzung des Gesetzes zuständige Office of Education pflegte eine enge Kooperation mit der American Vocational Association, so daß von bundesstaatlicher Seite keine 
Einflußnahme auf die Anpassung des Gesetzes an lokale Bedürfnisse zu erwarten war (vgl. ebd., S. 75 f.).

Da die Ineffektivität des Gesetzes schnell deutlich wurde, gab es bereits 1968 einen Nachtrag: Die Vocational Education Amendments lösten alle bisherigen bundesstaatlichen Gesetze zur Berufsbildung mit Ausnahme des Smith-Hughes Act ab und konsolidierten die verschiedenen Programme. Ziel war eine weitere Ausweitung der beruflichen Bildung in Richtung Allgemeinbildung, um insbesondere benachteiligten Jugendlichen neue Perspektiven zu eröffnen. Das Gesetz führte das Instrument des ,setaside funding' ein, bei dem ein bestimmter Prozentsatz der bundesstaatlichen Mittel für ,special populations', also benachteiligte Individuen jeglicher Art, eingesetzt werden müssen. In diesem Fall legte das Gesetz fest, daß 10 Prozent der bundesstaatlichen Mittel für körperlich behinderte Schüler und 15 Prozent für wirtschaftlich oder intellektuell benachteiligte Schüler verwendet werden (vgl. Cobb/Neubert 1998, S. 104). Acht Jahre später folgte mit den Vocational Education Amendments of 1976 ein weiterer Nachtrag zur Berufsbildung. Entscheidende Neuerung war die Erhöhung der ,set-aside funds' für ,special populations'. Trotz aller gesetzlichen Bemühungen hatte die Berufsbildung ihren von der Allgemeinbildung separaten Status an der High School bewahrt. Erst mit dem Carl D. Perkins Vocational Education Act von 1984 gab es einen Versuch, die Organisation der Berufsbildung neu auszurichten, indem den Einzelstaaten bundesstaatliche Mittel für die Einrichtung neuer und innovativer Berufsbildungsprogramme zur Verfügung gestellt wurden ${ }^{45}$. Darüber hinaus blieb die Förderung benachteiligter Schüler ein zentrales Anliegen des Gesetzes, das sich in einer weiteren Erhöhung der, set-aside funds‘ niederschlug, die jetzt über 50 Prozent der bundesstaatlichen Mittel umfaßten (vgl. ebd., S. 108). Durch die Förderung innovativer Projekte sind lokale Programme entstanden, die eine Neuorientierung der Berufsbildung verfolgen: z.B. ,Academies ${ }^{46}$, die als ,school-within-a-school‘ ihr Curriculum auf ein

\footnotetext{
45 Die Qualität der Berufsbildung an High Schools galt als sehr schlecht. In einem 1985 veröffentlichten Bericht, konstatierte das Committee for Economic Development: „Many vocational education programs are almost worthless. They are a cruel hoax on young people looking to acquire marketable skills. So many different and in many cases, unproductive programs in our public schools have been called ,vocational education" that most existing programs need to be disbanded and reshaped. Vocational education should ensure that students are learning skills that relate to the real needs of the job market" (in: Münch 1989, S. 52).

46 Diese Form der Schulorganisation entstand in Philadelphia und wurde insbesondere vom Staat Kalifornien als förderungswürdiges Modell übernommen.
} 
spezifisches Berufsfeld (occupational cluster) ${ }^{47}$ ausrichten. Häufig gibt es Partnerschaften mit lokalen Unternehmen, die in diesem Berufsfeld tätig sind und den am Programm teilnehmenden Schülern Sommer-Praktika anbieten (vgl. Glover/Marshall 1993, S. 603 f.). Vorteile dieser Art der Berufsbildung ergeben sich aus der Konzentration auf einen spezifischen Industriezweig, ohne die spätere Berufswahl einzuengen. So können Schüler einer Akademie, die sich auf den Bereich Gesundheit spezialisiert hat, sowohl Arzt als auch Krankenschwester werden. Zudem sinkt durch die Einbindung lokaler Unternehmen die Gefahr der Stigmatisierung. Andererseits ist die Zahl der Akademien und der in einer Akademie eingeschriebenen Schüler sehr gering (vgl. Marshall 1997, S. 209 f.).

Ein weiteres Programm, das bereits seit dem Smith-Hughes Act und dann in den 1960er und 1970er Jahren zunehmende bundesstaatliche Unterstützung erhielt, ist, cooperative education“ (vgl. Bailey/Merritt 1993, S. 18 f.). Dabei handelt es sich um die der dualen Berufsausbildung zugrundeliegenden Idee, schulischen und betrieblichen Unterricht zu alternieren. Im Gegensatz zur dualen Berufsausbildung behält der Schüler aber seinen Status als Schüler im Betrieb, da statt seiner die Schule einen Vertrag mit dem Arbeitgeber abschließt (vgl. Münch 1989, S. 53 f.). Die Mehrzahl der daran teilnehmenden Betriebe stammen aus den Bereichen Einzelhandel und Gastronomie, was bereits auf den qualitativen Gehalt dieser Programme hindeutet. Für die meisten Betriebe sind die Schüler billige Arbeitskräfte, denen in den wenigsten Fällen eine qualitativ hochwertige Ausbildung geboten wird: ,...most programs are more about work experiences than about learning“ (Marshall 1997, S. 209). Unter dem Begriff ,cooperative education“ wird eine Vielzahl von verschiedenen lokalen Programmen zusammengefaßt, woraus sich die relativ hohe Zahl der teilnehmenden Schüler (ca. 600.000) erklärt. Da ,cooperative education“ lediglich informell organisiert ist und kaum allgemeinverbindliche Kriterien aufweist, hatten ,co-op'-Schüler in empirischen Untersuchungen keine Vorteile gegenüber anderen Schülern hinsichtlich Arbeitsuche oder Lohnhöhe (vgl. Stern et al. 1995, S. 61 f.; Bailey/Merritt 1993, S. 19). Maßgebliches Problem der ,cooperative education“ ist die fehlende, allgemein anerkannte Zertifizierung der spezifischen Kombination schulischer und betrieblicher

${ }^{47}$ Typische ,occupational clusters' sind die Bereiche Medien, Gesundheit und Finanzen. Dies variiert aber je nach lokalökonomischen Gegebenheiten. 
Leistung (vgl. Glover/Marshall 1993, S. 603). Arbeitgeber stehen einer asymmetrischen Informationslage gegenüber: Da sie den Wert der Teilnahme an ,cooperative education“ nicht einschätzen können, ändern sie auch ihr Einstellungsverhalten nicht. ,Cooperative education' bleibt eine Formalisierung der ohnehin bestehenden Arbeitsverhältnisse von High School Schülern außerhalb der Schulzeit, ohne ein integriertes Ausbildungsprogramm bieten zu können.

Der Kongreß reautorisierte im Jahre 1990 den Carl D. Perkins Act als Carl D. Perkins Vocational and Applied Technology Education Act Amendments. Mit dem neuen Gesetz rückte ein weiteres innovatives Projekt ins Zentrum bundesstaatlicher Förderung: Tech-Prep (technical preparation) (vgl. Bailey/Merritt 1993, S. 21). Diese Programme zeichnen sich dadurch aus, daß sie den Übergang von der High School an eine weiterführende Bildungsinstitution - in der Regel ein Community College erleichtern, indem sie ein zwischen den beiden Institutionen koordiniertes und aufeinander aufbauendes Curriculum anbieten. In der Regel dauern die Programme vier Jahre, die letzten beiden High School Jahre und zwei Jahre am Community College (vgl. U.S. General Accounting Office 1995b, S. 3; Parnell 1985, S. 140 f.; Warnat 1994, S. 30 ff.). Neben dem High School Diplom erhalten die Schüler nach erfolgreichem Abschluß des Programms noch einen ,2-year associate degree' (vgl. Marshall 1997, S. 211). Die Zahl der High Schools, die an einem Tech-Prep Programm teilnahmen, stieg zwischen 1990 und 1994 von 27 auf 45 Prozent. Gleichzeitig erhöhte sich der Anteil der teilnehmenden Schüler von 9 auf 16 Prozent (vgl. U.S. General Accounting Office 1995b, S. 3). Tech-Prep Programme bieten einen hervorragenden Rahmen, um die Qualifikation für Berufsfelder unterhalb eines Bachelor-Abschlusses zu schaffen. Entscheidendes Problem der Tech-Prep Programme ist ihre genuin schulische Ausrichtung, die eine Kombination betriebsvermittelter und schulischer Lerninhalte ausschließt. Damit stehen sie aber in der Tradition amerikanischer Berufsbildung, was eventuell ihren relativen Erfolg ausmacht.

Eine weitere wesentliche Neuerung der Carl D. Perkins Amendments von 1990 war die Abschaffung der , set-aside funds' für ,special populations‘. Befürworter der, set-aside funds' befürchteten eine drastische Abnahme benachteiligter Schüler in Berufsbildungsprogrammen, da die bundesstaatlichen Mittel nicht mehr explizit für diese Gruppen verwendet werden müssen. Eine vom General Accounting Office durchgeführte Studie konnte diese Befürchtungen jedoch nicht bestätigen: „We found no 
significant changes in the rate at which special population students participated in vocational education“ (U.S. General Accounting Office 1995b, S. 5). Bis zum Carl D. Perkins Act von 1984 waren Schüler aus benachteiligten Gruppen in der Berufsbildung an High Schools eher unterrepräsentiert. Diese Entwicklung hat sich seitdem umgekehrt (vgl. Cobb/Neubert 1998, S. 107). Eine zielorientiertere Ausrichtung der bundesstaatlichen Mittel auf Schuldistrikte mit den höchsten Anteilen von Schülern aus benachteiligten Bevölkerungsschichten sollte die Effizienz der Programme verbessern (vgl. McDonnell/Grubb 1991, S. 23). Hinzu kam noch der Versuch einer Integration der verschiedenen Förderprogramme in ein kohärentes System, um die unterschiedlichen individuellen Bedürfnisse besser bedienen zu können (vgl. Wirth 1992, S. 166). In der Regel gab es für jede benachteiligte Gruppe ein eigenes Förderprogramm, ohne die Möglichkeit einer individuellen Kombination dieser Programme. Entsprechend galt es, die Verwaltung bundesstaatlicher Mittel neu zu organisieren, indem den lokalen Schulbezirken die Verantwortung für Planung und Organisation der Berufsbildungsprogramme übertragen wurde. Die Einzelstaaten büßten dadurch ihre vorrangige Position in der Verwaltung bundesstaatlicher Mittel ein. Lediglich 10,5 Prozent der Mittel blieben für einzelstaatlich organisierte Programme (vgl. ebd., S. 167).

Die Umgestaltung der Förderung für benachteiligte Gruppen stellt den einen Teil des Gesetzes dar, der andere konzentriert sich auf die Neuorganisation der Berufsbildung an sich: Bereits dargestellt wurde das Tech-Prep Programm, dessen Förderung im Gesetz explizit erwähnt wird. Eine eher allgemeine Forderung des Gesetzes ist die stärkere Integration akademischer und beruflicher Bildung in Form von , applied academics“Curricula (vgl. Grubb 1996, S. 537). Bisher war es so, daß akademische und berufsbildende Kurse ein voneinander getrenntes Dasein führten, was auch durch die unterschiedlichen Anforderungen an die Lehrerqualifikation bedingt war. Lehrer für berufliche Fächer können einen fehlenden Bachelor-Abschluß durch praktische Erfahrung in ihrem Beruf ausgleichen. Dies führte zu einem Abgrenzungsbedürfnis der akademischen Lehrer, die traditionell eine Strategie der Professionalisierung durch höhere Qualifikationsanforderungen verfolgen. Um diese ineffektive Trennung zu überwinden, sollte die Einbeziehung berufsbezogener Anwendungsmöglichkeiten in den Unterrichtsstoff akademischer Fächer und so eine gegenseitige Befruchtung von beruflichen und akademischen Lerninhalten die schulische Berufsbildung von ihrer Stigmatisierung als Programm für ,special populations ‘ befreien und allen Schülern als 
reale Option zugänglich machen. Der Perkins Act stellte Mittel für die Einrichtung sogenannter ,lighthouse schools $^{48}$ zur Verfügung, um die Zusammenarbeit von akademischen und berufsorientierten Lehrern in der Gestaltung neuer Curricula zu fördern (vgl. Wirth 1992, S. 170).

Festzuhalten ist, daß trotz der Abschaffung der , set-aside funds' die Anzahl der Schüler aus benachteiligten Bevölkerungsschichten in der Berufsbildung nicht gesunken und die Förderung dieser Gruppen weiterhin ein zentrales Anliegen des Gesetzes geblieben ist: Berufsbildung als sozialpolitische Maßnahme, um wirtschaftlich oder intellektuell benachteiligten Individuen eine Chance zu geben, ihren eigenen Lebensunterhalt auf dem Arbeitsmarkt zu verdienen. Alle sollen „dieselben“ Startchancen (equality of opportunity) haben. Problematisch ist eine solch dezidiert auf ,special populations“ ausgerichtete Berufsbildung deswegen, weil eine Stigmatisierung der Berufsbildung erfolgt, die die Situation der zu fördernden Schüler weiter verschlechtert. Genau aus diesem Grund fördert der Perkins Act Projekte, wie Tech-Prep, die die Qualität und das Image der Berufsbildung verbessern sollen. Auch die vorgeschriebene Integration akademischer und beruflicher Lerninhalte soll diesem Ziel dienen. Fraglich bleibt jedoch, ob ein solcher Spagat möglich ist.

Betrachtet man sowohl die Ausbildungs- und Beschäftigungspolitik als auch die Berufsbildungspolitik der amerikanischen Bundesregierung seit dem Ende des Zweiten Weltkrieges, so fallen zwei Gemeinsamkeiten auf: erstens der Fokus auf benachteiligte Bevölkerungsgruppen und zweitens die lokale Organisation der Programme. Anfangs war die Ausbildungs- und Beschäftigungspolitik keineswegs auf benachteiligte Individuen ausgerichtet. Sie sollte die strukturellen Veränderungen in der Wirtschaft abfedern, indem qualifizierte Arbeiter, deren Fertigkeiten obsolet geworden waren, durch Ausbildungsprogramme auf Tätigkeiten in Wachstumsbranchen umgeschult wurden. Die günstige ökonomische Entwicklung senkte die Arbeitslosenrate dieser, dislocated workers‘ jedoch schlagartig, so daß die bewilligten bundesstaatlichen Mittel für wirtschaftlich benachteiligte Personen eingesetzt werden konnten. Dies fügte sich zudem nahtlos in die ,War on Poverty“-Politik der Bundesregierung ein. Von dieser Politik war auch die Gesetzgebung zur schulischen Berufsbildung geprägt, die sich

\footnotetext{
${ }^{48}$,Lighthouse Schools` sollten als Modell zum Nachahmen für andere Schulen dienen.
} 
verstärkt der Förderung von ,special populations“ annahm. Beide Entwicklungen verliefen aber parallel zueinander, so daß es trotz der funktionalen Nähe kaum Überschneidungen zwischen den Politikbereichen gab. Auch die lokale Organisationsund Verwaltungsstruktur der Ausbildungs- und Beschäftigungsprogramme war keine prädeterminierte Entwicklung. Erst der gescheiterte Versuch, den U.S. Employment Service als zentrale Koordinationsstelle zu konstituieren, führte langfristig zur Institutionalisierung einer lokalen Administration. Dies hatte die Schaffung einer Vielzahl verschiedener Programme zur Folge. Koordinationsprobleme zwischen staatlichen, ,non-profit‘ und zunehmend auch privatwirtschaftlichen Organisationen waren immer wieder im Zentrum der Kritik und mithin Auslöser für verschiedene Reformversuche. Die lokale und einzelstaatliche Verantwortung für die schulische Berufsbildung beruht vorrangig auf der verfassungsrechtlichen Kompetenz der Einzelstaaten und darüber hinaus auf dem relativ kleinen Prozentsatz, den die bundesstaatlichen Mittel am Gesamtbudget der schulischen Berufsbildung ausmachen ${ }^{49}$. Wie bei der Ausbildungs- und Beschäftigungspolitik führt die lokale Organisation der beruflichen Bildung in den High Schools zu einem „Wildwuchs“ an Programmen, die keinen einheitlichen Standards folgen. Ihre wachsende Kompetenz konnten jedoch einige Einzelstaaten nutzen, um innerhalb ihres Verantwortungsbereiches die Vielfalt der lokalen Variationen zu reduzieren und minimale Standards zu setzen. Eine über die Einzelstaatsebene hinausgehende Vereinheitlichung ist bisher aber immer gescheitert. Bei einer neuen Ausbildungsinitiative hat die Bundesregierung die bestehende Infrastruktur zu berücksichtigen - der Bau einer Umgehungsstraße ist dabei nicht möglich.

491987 betrug das bundesstaatliche Berufsbildungsbudget $\$ 875$ Millionen im Vergleich zu ca. \$7 Milliarden, die von den Einzelstaaten und lokalen Schuldistrikten aufgebracht wurden (vgl. Levitan/Gallo 1988, S. 49). 


\section{Teil IV: Clintons School-to-Work Opportunities Act}

Bereits als Gouverneur von Arkansas war Bill Clinton an einer Reform des Ausbildungssystems für Jugendliche interessiert. Während seiner Amtszeit verabschiedete das Parlament von Arkansas ein ,Apprenticeship Bill‘ (vgl. Reich 1995, S. 122 f.). Clinton war aber keineswegs Vorreiter, sondern eher Mitläufer einer Bewegung, die seit den 1980er Jahren sowohl bei den Republikanern als auch bei den Demokraten eine wachsende Anhängerschaft fand und das Fehlen eines strukturierten, nationalen Berufsausbildungssystems für Jugendliche beklagte. Befürworter argumentierten jedoch aus unterschiedlichen Perspektiven: Die einen sahen die internationale Wettbewerbsfähigkeit der amerikanischen Wirtschaft schwinden, die anderen kritisierten die College-Fokussierung des amerikanischen Schulsystems, wobei die Masse der Schüler „auf der Strecke“ bleibt. In seinem Präsidentschaftswahlkampf stellte Clinton seine nationale Ausbildungspolitik vor: „My Administration will establish a national apprenticeship program [Hervorhebung vom Verfasser], like those in Europe, to encourage noncollege-bound students to stay in school..." (Clinton 1993, S. 7). Zusammen mit seinem Arbeitsminister Robert Reich - ebenfalls einem großen Befürworter eines nationalen Berufsausbildungssystems - hat Clinton in den ersten Monaten seiner Amtszeit als Präsident dieses Projekt mit großem Nachdruck verfolgt. Resultat der Bemühungen war der 1994 verabschiedete School-to-Work Opportunities Act (STWOA). Inwieweit hat der STWOA ein ,European like national apprenticeship program' geschaffen? Um die Antwort vorwegzunehmen: überhaupt nicht.

Trotzdem soll im folgenden das Gesetz eingehender dargestellt werden, um daran die Fortschreibung der bestehenden Berufsbildungspolitik zu verdeutlichen. Ziel des Gesetzes war die Errichtung eines ,framework', innerhalb dessen alle Einzelstaaten ihr eigenes Ausbildungssystem (school-to-work) aufbauen. Die daran teilnehmenden Schüler sollten sowohl akademische als auch berufsspezifische Kenntnisse erlernen, die denen der College-vorbereitenden Kurse gleichkommen. Dies bedeutete wiederum, daß allen Schülern die Option eines College-Abschlusses offengehalten werden sollte und sogar engere Verbindungen zwischen High School und College - vergleichbar dem Tech-Prep Programm - gefördert werden sollten. Obgleich das Gesetz explizit für alle Schüler galt, sollten ,disadvantaged‘ und Schüler von Minoritäten weiterhin besondere Berücksichtigung finden. Schließlich sollte die Einbindung der Unternehmen ein neues 
Lernumfeld schaffen, das die schulische Ausbildung in Relation zur beruflichen Praxis setzt (vgl. Office of Technology Assessment 1995, S. 15; National School-to-Work Office 1996, S. 7).

Das Gesetz erlaubte den Einzelstaaten, ihr ,school-to-work'-System auf bestehenden Programmen aufzubauen ${ }^{50}$, soweit sie dabei drei Kernelemente berücksichtigten: ,school-based learning', ,work-based learning ${ }^{6}$ und ,connecting activities'. Die Komponente des ,school-based learning' umfaßte die Entwicklung eines Curriculums, das sowohl akademische als auch berufliche Lerninhalte miteinander verband. Dies entsprach den ,applied academics'-Curricula bestehender ,Career Academies'. Ferner sollten Curricula für ,career majors ${ }^{65}$ ausgearbeitet werden, die die letzten beiden High School-Jahre und die ersten beiden College-Jahre aufeinander abstimmen. Hier konnten die Einzelstaaten und Schuldistrikte auf vorhandene Tech-Prep Programme zurückgreifen. Um das motivierende Moment einer praktischen Anwendung von Gelerntem pädagogisch nutzen zu können, sollten beim ,work-based learning Arbeitgeber als Partner der High Schools in das Programm integriert werden. Sie stellen in ihren Unternehmen Ausbildungsplätze zur Verfügung, die den Schülern eine qualifizierende Arbeitserfahrung bieten. Dazu sollten betriebliche Mentoren ${ }^{52}$ den Lernprozeß am Arbeitsplatz begleiten und überwachen. Die betriebliche Arbeitserfahrung sollte im Gegensatz zur ,cooperative education“ in einem Zeugnis festgehalten werden, um sie für den Arbeitsmarkt verwertbar zu machen. Dieses ,skill certificate' sollte in der jeweiligen Branche anerkannt sein und so dem Besitzer eine größtmögliche Mobilität bieten. Entscheidendes Element der ,school-to-work'-Systeme ist die Verbindung aus ,school-based“ und ,work-based learning' (vgl. Riley/Reich, in: U.S. House of Representatives 1993, S. 63 f.; Wills 1997, S. 14).

Nur wenn Schulen und Unternehmen in ihren Ausbildungsaktivitäten kooperieren, können kohärente, sinnvoll strukturierte Ausbildungsgänge entstehen, die den Schülern

50 „STWOA does not seek to establish programs but rather to develop comprehensive statewide and local systems for facilitating school-to-work transitions“ (Office of Technology Assessment 1995, S. 15).

${ }^{51}$ Unter ,career major` wird eine koordinierte Zusammenstellung von Kursen verstanden, die auf die Berufsoptionen innerhalb einer Wirtschaftsbranche ausgerichtet sind. Dabei sollen lediglich allgemeine Grundlagen des jeweiligen Bereichs vermittelt werden, ohne eine spezifische Berufswahl festzulegen.

52 Hierunter darf man sich keine qualifizierten Ausbildungstrainer im Sinne des deutschen dualen Berufsbildungssystems vorstellen. Vielmehr sollen erfahrene Arbeitskräfte eine Art Vorbildfunktion übernehmen. 
den Einstieg ins Berufsleben, insbesondere höherqualifizierte Tätigkeiten, erleichtern. Deshalb sollten Schüler nicht die einzige Verbindung zwischen Schule und Arbeitsplatz sein, sondern einzelne Lehrer als Ansprechpartner für Unternehmen und Koordinatoren der jeweiligen ,career majors' fungieren. Im Sinne des Gesetzes sollten hierzu lokale Partnerschaften zwischen Schulen, Arbeitgebern, Gewerkschaften, lokalen Behörden und anderen ,stakeholders' aufgebaut werden, die die notwendige Zusammenarbeit und Koordination der verschiedenen Organisationen ermöglichen sollten (vgl. U.S. Department of Labor 1997, S. 11 f.; Rist/Joyce 1994, S. 336). „Such partnerships foster the broad-based community support necessary to establish STW systems as an integrated approach to help all students better prepare for further education and employment“" (National School-to-Work Office 1996, S. 9).

Damit die Einzelstaaten einen Anreiz hatten, ein solches System zu errichten, hat die Bundesregierung sogenannte, venture capital grants ' vergeben, die in einem zweistufigen Verfahren bewilligt wurden: Zuerst erhielten alle 50 Einzelstaaten ein ,state development grant‘. Mit diesen Mitteln sollten die Einzelstaaten erste Pilotprojekte finanzieren und ihre spezifische ,school-to-work'-Strategie ausarbeiten können. Im Durchschnitt lag die bundesstaatliche Unterstützung bei $\$ 430.000$. Anschließend sollten sich die Einzelstaaten um ein einmaliges, über fünf Jahre laufendes, state implementation grant ${ }^{‘}$ bewerben. In einem jährlich stattfindenden Auswahlprozeß konkurrierten die Einzelstaaten um den Erhalt eines solchen ,implementation grant". Um sich dafür zu qualifizieren, mußten die Einzelstaaten einen umfassenden ,school-towork'-Plan vorlegen und plausibel darlegen, wie sie diesen Plan umsetzen und wie sie das Programm nach Auslauf der bundesstaatlichen Unterstützung finanzieren wollen. In der ersten Phase 1994 konnten sich acht Einzelstaaten für ein ,implementation grant` qualifizieren. Die bundesstaatlichen Subventionen variierten zwischen \$1.3 und \$20 Millionen $^{53}$. Im Jahre 1995 sind weitere 19 Einzelstaaten in den Genuß von ,implementation grants' gekommen (vgl. ebd., S. 16). Weitere zehn Einzelstaaten erhielten 1996 eine solche bundesstaatliche Unterstützung, wodurch sich die Zahl auf

53 Einer der ersten Staaten, die ein ,implementation grant' erhielten, war Wisconsin, wo es seit der Jahrhundertwende eine dem deutschen dualen Berufsbildungssystem vergleichbare Lehrlingsausbildung gibt. Die Zahl der Lehrlinge ist jedoch über die Jahrzehnte kontinuierlich gesunken (vgl. Parker 1996, S. 25-30). 
37 Einzelstaaten erhöhte. Erst 1998 konnten sich die verbleibenden 13 Einzelstaaten ${ }^{54}$ durch die Mithilfe des U.S. Department of Labor und Education für ,implementation grants‘ qualifizieren (vgl. National School-to-Work Office 1998, S. 9).

Außer der bundesstaatlichen Unterstützung für Einzelstaaten ${ }^{55}$ beinhaltete der STWOA die Möglichkeit einer direkten bundesstaatlichen Förderung von lokalen Programmen für den Fall, daß sich die Gemeinde in einem Einzelstaat befand, der sich noch nicht für ein ,implementation grant` qualifizieren konnte (vgl. School-to-Work Opportunities Act 1994, Title III, Sec. 301-305). So konnten lokale Partnerschaften, die ein überzeugendes ,school-to-work'-Konzept vorlegten, eine Art Vorbildfunktion für den gesamten Einzelstaat übernehmen. Im Jahre 1997 erhielten 29 Gemeinden ein ,local partnership

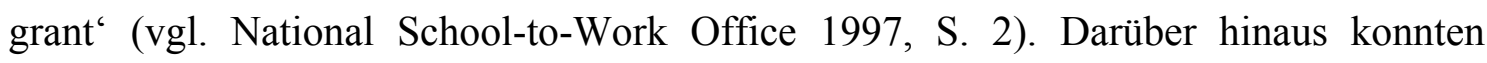
Gemeinden mit einer besonders hohen Armutsrate sogenannte ,urban/rural opportunities grants ${ }^{56}$ beantragen, um die spezifischen Bedürfnisse der Jugendlichen in diesen Gemeinden, die häufig durch einen Mangel an lokalen Arbeitsplätzen charakterisiert sind, besser berücksichtigen zu können. Bis 1997 ist die Zahl der Gemeinden, die ,urban/rural opportunities grants‘ erhielten, auf 109 gestiegen (vgl. National School-to-Work Office 1997, S. 2 und National School-to-Work Office 1998, S. 10).

Im ersten Jahr des STWOA bewilligte der Kongreß eine Gesamtsumme von \$100 Millionen, die an Einzelstaaten und Gemeinden weitergeleitet wurden. Die jährliche Obergrenze der zu vergebenden bundesstaatlichen Mittel lag bei \$300 Millionen (vgl. Grubb 1996, S. 537). Da solche Beträge nicht ausreichen, um ein umfassendes ,schoolto-work'-System zu schaffen, erlaubte das Gesetz den Einzelstaaten, bundesstaatliche Mittel aus anderen Programmen, insbesondere dem Carl D. Perkins Vocational and Applied Technology Act und dem Job Training Partnership Act, zu verwenden (vgl. Riley/Reich, in: U.S. House of Representatives 1993, S. 65). Jeder Einzelstaat mußte

\footnotetext{
${ }^{54}$ Bei den Einzelstaaten handelte es sich um: Alabama, Arkansas, Delaware, Georgia, Illinois, Kansas, Mississippi, Montana, North Dakota, South Dakota, South Carolina, Virginia und Wyoming (vgl. National School-to-Work Office 1997, S. 2).

55 ,Implementation grants‘ für Einzelstaaten mußten im ersten Jahr zu 70 Prozent, im zweiten zu 80 Prozent und in den folgenden Jahren zu 90 Prozent an lokale Partnerschaften weitergeleitet werden (vgl. National School-to-Work Office 1996, S. 16).

${ }^{56}$ Diese bundesstaatliche Förderung benachteiligter Bevölkerungsgruppen mußte mindestens 10 Prozent der bewilligten Gesamtmittel umfassen.
} 
aber einen separaten Antrag stellen, um bundesstaatliche Mittel von einem dieser Programme in die Implementierung eines, school-to-work'-Systems leiten zu dürfen (vgl. Kassebaum 1995, S. 156). Die National Governors“ Association drückte ihre Kritik an dieser Regelung so aus: „New waiver authority is helpful to a certain extent, but as a nation, we will move very slowly toward the goal of integrated workforce development systems if each state must apply separately to each different federal department for permission to integrate programs“ (in: ebd., S. 157). Im Vergleich zu den Mitteln, die die Bundesregierung für ,vocational education“ und Ausbildungsprogramme ausgibt ${ }^{57}$, waren die bewilligten STWOA-Beträge verschwindend gering. Deshalb nannte Norton Grubb dieses Gesetz auch ein Beispiel von ,piddle politics“(Grubb 1996, S. 537).

Die Zustimmung zum STWOA verlief, nachdem einige wesentliche Punkte aus dem Gesetz genommen wurden, über die Parteigrenzen hinweg (vgl. dazu Kinni 1994, S. 68). Ernsthafter Streitpunkt war die Festschreibung von ,paid work experience“ in der ursprünglichen Version des Gesetzes. Viele Abgeordneten kritisierten diese Regelung als hinderlich für die Beteiligung der Unternehmen an der Umsetzung des Gesetzes. Senatorin Nancy Kassebaum konstatierte, ,...that the paid work requirement would limit the ability of businesses to participate in the program“ (Kassebaum 1995, S. 156). Befürworter der Regelung sahen darin einen Anreiz für Unternehmen, die Ausbildung der Schüler ernst zu nehmen. Die finanzielle Belastung zwinge die Unternehmen, ihre Ressourcen bestmöglich einzusetzen, nämlich in Form einer Investition in zukünftige Arbeiter. Darüber hinaus argumentierte Richard Kazis, Vizepräsident von ,Jobs for the Future ${ }^{658}$ : „Most students work anyway. If their experiential learning is unpaid, students may end up juggling two jobs and their school schedule“ (in: House of Representatives 1993, S. 338). In der endgültigen Version des STWOA ist die ,paid work experience“ nicht mehr zwingend vorgeschrieben, sondern lediglich erstrebenswert (vgl. Office of Technology Assessment 1995, S. 15; Wells 1994, S. 892).

\footnotetext{
57 Unter dem Perkins Act hat die Bundesregierung 1994 ca. \$1,2 Milliarden den Einzelstaaten für ,vocational education“ zur Verfügung gestellt. Die Mittel für bundesstaatliche Ausbildungsprogramme unter dem Job Training Partnership Act lagen sogar bei \$5 Milliarden (vgl. Choy 1994, S. 61).

58 Jobs for the Future ist eine unabhängige ,non-profit'-Organisation, die Pilotprojekte im Bereich der Berufsausbildung fördert und bereits früh in den Entstehungsprozeß des STWOA involviert war.
} 
Anfangs gab es eine grundsätzliche Debatte darüber, ob mit dem Gesetz ein neues Programm in der Form eines, youth apprenticeship'-Systems geschaffen oder auf die bestehenden Programme aufgebaut werden sollte. Die American Vocational Association setzte sich vehement für die zweite Variante ein. „Once the Clinton Administration decided not to create a new, separate apprenticeship program but to build on the current programs, most potential opposition to the bill faded away“ (Jennings 1995, S. 198). Die große Zustimmung aller Parteien zum STWOA erklärt sich daraus, daß das Gesetz minimale Anforderungen stellte, die zudem viele Interpretationen zuließen, und die Verantwortung zur Implementation bei den Einzelstaaten bzw. Gemeinden lag.

Kritik wurde zunehmend auch an der Verwaltung des Gesetzes geübt: Das Gesetz schrieb die Einrichtung einer bundesstaatlichen Behörde, dem National School-to-Work Office, zur Administration des STWOA vor. Diese Behörde hatte allerdings bis auf beratende Funktionen keine Kompetenzen. Darüber hinaus unterstand sie der Oberaufsicht des U.S. Department of Labor und des U.S. Department of Education, weshalb diese Konstruktion auch als ,administrative sandwich“ (Pouncy/Hollister, in: U.S. Department of Labor 1997, S. 15) bezeichnet wurde. Die Doppelspitze führte dazu, daß sich keines der beiden Ministerien so richtig verantwortlich fühlte und sich deshalb auch nicht für eine Ausweitung der Kompetenzen des National School-to-Work Office einsetzte. Richard Kazis nannte diese Behörde daher ein „orphan“ ${ }^{* 59}$. Doch nicht nur die bundesstaatliche Administration war mit Geburtsfehlern belastet, sondern auch die Devolution der Verantwortung auf die Einzelstaaten und Gemeinden hatte zur Folge, daß die Implementierung der ,school-to-work'-Systeme einem ,let a thousand flowers bloom“-Ansatz folgte (vgl. U.S. Department of Labor 1997, S. 15): „These problems are further exacerbated by lack of consistent Federal component definitions and by the fact that State and local partnerships largely occupy the driver's seat for implementing these programs, resulting in considerable inter-area variation as well“" (Glover/King, in: ebd., S. 15).

Die Einzelstaaten unterscheiden sich aber nicht nur in der inhaltlichen Gestaltung ihrer ,school-to-work'-Programme, sondern auch im verwaltungsorganisatorischen Aufbau. Grundsätzlich lassen sich die Strategien der Einzelstaaten in zwei Kategorien untertei-

\footnotetext{
${ }^{59}$ Interview des Verfassers mit Richard Kazis.
} 
len: einerseits ,school-to-work' als Chance für eine Reform des Schulsystems und andererseits als Option für eine Neuorganisation der, workforce development ${ }^{\star}$. Je nach Strategie wählten die Einzelstaaten eine unterschiedliche Administrationsstruktur. Die Mehrzahl der Einzelstaaten plazierten das zu schaffende ,School-to-Work Office“ im Bildungsministerium. Nur zwei der bis 1998 ,implementation grants'-erhaltenen Einzelstaaten entschieden sich für das Department of Labor oder das Department of Commerce. Unterhalb der Einzelstaatsebene etablierten lediglich elf Staaten zwei Verwaltungsebenen in Form von, regional boards' und, local partnerships' (vgl. Erlichson/Van Horn 1999, S. 4-7). Die Schaffung einer konsistenten Verwaltungshierarchie bedeutete jedoch nicht, daß die Einzelstaaten innerhalb ihrer Grenzen eine einheitliche Programmstrategie verfolgten. Vielmehr lag die Hauptverantwortung für die Programme typischerweise bei den Gemeinden und lokalen Partnerschaften, die ihr eigenes ,school-to-work'-Programm entsprechend ihren lokalen Bedürfnissen und Kapazitäten entwickelten.

Der School-to-Work Opportunities Act hat die Entwicklung in der bundesstaatlichen Berufsbildungs- und Ausbildungs-/Beschäftigungspolitik fortgesetzt. Trotz der Ankündigung, ein für alle Schüler offenes Programm zu schaffen und sich so gegenüber früheren Politiken abzugrenzen, konnte der Kongreß die Unterstützung von besonders benachteiligten Gemeinden in Form der ,urban/rural opportunity grants“ durchsetzen. Ziel war es weiterhin, insbesondere ,non-college bound‘ Schülern eine Perspektive zu bieten, die ihnen auch ohne College-Abschluß höherqualifizierte und mithin höherbezahlte Tätigkeiten auf dem Arbeitsmarkt eröffnet. Die lokale Administration von (Ausbildungs-)Programmen, die sich nach dem gescheiterten Versuch einer Zentralisierung in den 1950er Jahren durchgesetzt hat, ist auch durch den STWOA unangetastet geblieben - der STWOA hat geradezu die lokale Vielfalt gefördert.

Im Oktober 2001 ist die bundesstaatliche Förderung im Rahmen des STWOA ausgelaufen. Ohne diese finanzielle Unterstützung werden viele Staaten bzw. lokale Partnerschaften die aufgebauten Programme nicht beibehalten können, da es gerade für die vielen lokalen Organisationen schwierig sein wird, zusätzliche Ressourcen einzuwerben. Richard Kazis (2001) faßt die Aussichten für die ,school-to-work'-Programme so zusammen: „When federal funds run out, as they have already done in some states, only a few states will try to sustain school-to-work as such - and they will not do so with resources anywhere near the level that federal funding provided at its height. [...] But in 
most states, school-to-work as a discrete effort will go the way of other, time-limited federal initiatives." 


\section{Teil V: Zusammenfassung und Ausblick}

Die Geschichte des amerikanischen Schulwesens zeigt die unterschiedlichen Ideen, die mit der Institution Schule bzw. Bildung im Vergleich zu Deutschland (bzw. Preußen) verbunden sind. In Preußen konnten sich trotz der gesetzlich unterstützten Bemühungen einer Gewerbeliberalisierung zu Beginn des 19. Jahrhunderts die Zünfte und Innungen halten und mit ihnen auch die Lehrlingsausbildung. Während der schulischen Ausbildung im humboldtianischen Sinne eine rein allgemeinbildende Funktion zugestanden wurde, sollte die berufliche Ausbildung - für die breite Masse der Bevölkerung - in Form der handwerklich organisierten Lehrlingsausbildung erfolgen. Über die Erlernung eines Berufs sollte jeder seinen Platz in der Gesellschaft finden. Die Idee der berufsorientierten Integration des einzelnen in die Gesellschaft spielte in der zweiten Hälfte des 19. Jahrhunderts eine wichtige Rolle, als die preußische Regierung unter dem Eindruck industrialisierungsbedingter gesellschaftlicher Auflösungstendenzen eine Stärkung des Handwerks und insbesondere der handwerklich geformten Lehrlingsausbildung als gesellschaftsstabilisierendes Moment verfolgte. Die Übernahme des Berufsprinzips in die sich entwickelnden Ausbildungsgänge der Industrie stellten eine endgültige Institutionalisierung der Idee der Beruflichkeit dar. Durch die Etablierung separater Berufsschulen blieb das allgemeinbildende Schulwesen von berufsbildenden Elementen „verschont“. Zu Beginn des 20. Jahrhunderts hatte sich damit die institutionelle Struktur der dualen Berufsausbildung in Deutschland herausgebildet.

Im Entstehungsprozeß der USA kam dem Schulwesen eine besondere Rolle bei der Stabilisierung und Identitätsfindung der jungen Nation und ihren neugeschaffenen demokratischen Institutionen zu. Bildung sollte ,citizenship education` sein. Parallel zur Ausweitung eines ,mass education'-Schulwesens erodierte die aus der Kolonialzeit stammende Lehrlingsausbildung. Beide Entwicklungen standen indessen in einem reziproken Verhältnis zueinander: Die Erosion der Lehrlingsausbildung erforderte eine Alternative, um die Jugendlichen in die Gesellschaft $\mathrm{zu}$ integrieren und sozial $\mathrm{zu}$ konditionieren. Hier bot sich das bestehende Schulwesen an. Die frühe Funktion der High School, Schüler der Oberschicht auf das College vorzubereiten, wandelte sich dadurch in Richtung einer Vermittlung berufsvorbereitender Allgemeinbildung. Als Ergebnis entstand das Modell einer, comprehensive high school‘. In diesem Kontext ist 
die Debatte um eine Wiederbelebung der Lehrlingsausbildung in Form der deutschen Berufsausbildung zum Ende des 19. Jahrhunderts zu sehen. Resultat der Reformbestrebung war eine Aufwertung der berufsvorbereitenden Funktion der High School, indem berufsorientierte Fächer, wie Landwirtschaft, von der Bundesregierung finanziell unterstützt wurden. Die betriebliche Seite der Ausbildung blieb dabei weitgehend unberücksichtigt.

Während dieses historischen Prozesses entstanden diejenigen Institutionen, die noch heute ihren Einfluß auf die in die Gestaltung beruflicher Bildung involvierten Interessen ausüben. So hat die Schaffung eines neuen Berufsausbildungssystems die besondere institutionelle Struktur des Jugendarbeitsmarktes zu berücksichtigen, der durch eine hohe Fluktuationsrate der Jugendlichen geprägt ist. Die Fluktuationsrate ist bedingt durch die Struktur der Unternehmen, in denen die Jugendlichen in der Regel eine ,lowskill'-Beschäftigung finden: Kleine und mittelgroße Firmen dominieren hier den Arbeitsmarkt. Ohne die Option, innerhalb der Firma aufsteigen zu können, wechseln die Jugendlichen die Firmen, um Arbeitserfahrung zu sammeln und so eine Chance auf eine Anstellung in einer Firma des „ersten“ Arbeitsmarktes zu erhalten. Aber auch die Firmen sehen die Jugendlichen als kapazitäts- und konjunkturellabhängige Verfügungsmasse, die je nach Bedarf eingestellt und wieder entlassen werden kann. Insofern besteht von seiten der Firmen kein Interesse, in die Ausbildung der Jugendlichen $\mathrm{zu}$ investieren. Die fehlende verbandliche Organisation amerikanischer Unternehmen fördert diesen Zustand noch. Auf das einzelne Unternehmen kann kein interner Druck von seiten des Verbandes ausgeübt werden, sich an Ausbildungsmaßnahmen zu beteiligen. Damit tritt das klassische Problem jeglichen kollektiven Handelns in den Mittelpunkt der Überlegungen: das ,free-riding'. Bildet ein Unternehmen seine Mitarbeiter über firmenspezifische Fertigkeiten hinaus aus, so muß es befürchten, daß ein anderes Unternehmen die ausgebildeten Arbeiter abwirbt, da es durch die Einsparung der Ausbildungskosten einen höheren Lohn zahlen kann. Ein Berufsausbildungssystem soll gerade firmenübergreifende, branchenspezifische Fertigkeiten vermitteln, um letztlich auch die Mobilität der Arbeiter zu erhöhen. Berufliche Bildung ist somit ein quasi-öffentliches Gut, das bei marktwirtschaftlicher Organisation zu einem Unterangebot tendiert. Darüber hinaus stellen der niedrige gewerkschaftliche Organisationsgrad und die Tradition der beruflichen Organisation ein weiteres Hindernis dar. Einige Berufsgewerkschaften haben zwar ein gut organisiertes 
,apprenticeship“-System, wobei es sich aber nicht um eine Ausbildung für Jugendliche handelt, sondern um eine Form der Erwachsenenbildung für eine kleine Arbeiterelite. Die Schwäche der Gewerkschaften und ihre lokale Fragmentierung verhindern die Schaffung eines Berufsbildungssystems durch überregionale, sektorale Kollektivverhandlungen. Lokale, betriebsspezifische Verhandlungen zwischen Arbeitnehmerschaft und Arbeitgeber sind von individualistischen Interessen, wie der Angst um den eigenen Arbeitsplatz, beherrscht.

Neben den Sozialpartnern sind es aber auch staatliche Institutionen, die der Schaffung eines nationalen dualen Berufsausbildungssystems entgegenstehen. Die konstitutionelle Struktur des Föderalismus überträgt den Einzelstaaten die Verantwortung für Bildung, die trotz bundesstaatlicher Interventionen nicht in Frage gestellt wird. Unter Präsident Reagan und seiner Politik des ,New Federalism“ wurde die Position der Einzelstaaten sogar noch gestärkt. Die Bundesregierung ist mithin auf die Bereitschaft der Einzelstaaten angewiesen, ihre Programme umzusetzen. Dafür stehen ihr insbesondere finanzielle Anreize zur Verfügung. Nicht nur die spezifische Beziehung zwischen Bundesregierung und Einzelstaaten, sondern auch die Kompetenzverteilung innerhalb der Bundesregierung erschweren die Durchsetzung eines nationalen dualen Berufsausbildungssystems. Zwei in ihrer Geschichte und Tradition verschiedene Ministerien, das U.S. Department of Labor und das U.S. Department of Education, sind beide in berufliche Ausbildungsprogramme involviert. Die Ideen, die hinter diesen Programmen stehen, differieren jedoch stark. Das aus der liberalen Tradition der Bürgerrechtsbewegung entstandene Bildungsministerium verfolgt Ziele, wie ,equity ${ }^{6}$ und ,access‘. Im Gegensatz dazu betont das Arbeitsministerium, das in der Tradition der Arbeitsbeschaffungsmaßnahmen des ,New Deal' steht, ,employability“-Aspekte. Eine effektive Zusammenarbeit beider Ministerien ist folglich schwierig. Und schließlich kommt es auf die Zusammenarbeit und Loyalität der ,street level bureaucrats “ - hier der Lehrer - an, die die Programme implementieren. Lehrer und ihre Interessenverbände haben immer auf die Wahrung professioneller Autonomie hinsichtlich der Unterrichtsgestaltung geachtet. Diese wäre durch ein integriertes duales Berufsausbildungssystem gefährdet, weil die Lehrer in der Ausgestaltung des Unterrichts mit Unternehmensvertretern kooperieren müßten. Daher lehnen die Lehrergewerkschaften jegliches Programm ab, das die Kompetenzen der Lehrer weiter einschränken könnte. 
Die nach dem Zweiten Weltkrieg entstandenen bundesstaatlichen Ausbildungs- und Beschäftigungsprogramme haben eine Infrastruktur geschaffen, die die Einrichtung eines dualen Berufsausbildungssystems erschwert, das in seiner Struktur von diesen abweicht. Lokale Organisation, fragmentierte Kompetenzen und eine Vielzahl bundesstaatlicher Programme mit eigenen Budgets haben die Vielfalt inhaltlich und strukturell verschiedener Ausbildungsprogramme gefördert. Auch der letzte Versuch unter Clinton, ein umfassendes Berufsbildungssystem zu etablieren, scheiterte, wie der School-toWork Opportunities Act zeigt. Trotz all dieser negativen Beispiele konstatiert William Julius Wilson (1996): „But such a program is critically needed not only to address the overall problem of growing wage inequality and economic marginality among high school graduates but also as a weapon in the fight against acute joblessness in the innercity ghetto“ (S. 218).

Fraglich ist, ob die USA überhaupt ein nationales, umfassendes Berufsausbildungssystem benötigen, um die von Wilson genannten Probleme und mögliche ,skill'-Defizite der Arbeiter zu minimieren. Ist die lokale Organisation nicht flexibler und kann sich den jeweiligen Bedingungen besser anpassen? Wie oben ausführlich dargestellt, führt die lokale Organisation der Ausbildung zu vielen verschiedenen „Modellen“, die zudem, wie z.B. bei Tech-Prep, unterschiedliche Varianten aufweisen. Aufgrund fehlender nationaler Standards mangelt es aber an überregionaler Transparenz. Lediglich innerhalb der lokalen Grenzen wissen die Arbeitgeber um den Wert eines Ausbildungsprogramms und können den Inhalt eines Abschlußzeugnisses einschätzen. Fehlende überregionale Transparenz wäre aber zu vernachlässigen, wenn der Arbeitsmarkt für diese Kategorie der Jugendlichen, der ,non-college bound', tatsächlich genuin lokal organisiert wäre. Hier könnten informelle, lokale Netzwerke in Form von Partnerschaften zwischen Unternehmen, Schulen, Colleges, staatlichen Behörden, Gewerkschaften und anderen, community'-relevanten Organisationen ein lokales Ausbildungssystem aufbauen und verwalten, das den lokalen Bedürfnissen entspräche. Solche Netzwerke wären in die soziale Struktur eingebettet und könnten Nutzen aus ihr ziehen. „The preconditions for this are often present in the US context: strong local community identities, autonomous local governments with some economic competence, important local networks of businesses, excellent links between business and local colleges and universities“ (Crouch et al. 1999, S. 167). Auch wenn diese Bedingungen 
vielerorts in den USA gegeben sind, finden sich trotzdem nur wenige Beispiele gut funktionierender lokaler Ausbildungsnetzwerke. Diese sind häufig in Regionen mit High-Tech Industrien, die einen hohen Bedarf an qualifizierten Arbeitern haben. Die Programme sind allerdings sehr spezialisiert, da sie in der Regel nur ein kleines Berufsfeld abdecken, und bieten lediglich einem Bruchteil der ,non-college bound“Jugendlichen einen Ausbildungsplatz. Die kleinen und mittleren Unternehmen, die die Masse der Ausbildungsplätze stellen müßten, haben einerseits nicht den Bedarf an qualifizierten Arbeitern und andererseits stehen sie häufig in Wettbewerb zueinander, so daß hier das ,free-riding'-Problem eine lokale Ausbildungsinitiative verhindert: „....smallness of scale and difficulties in transcending interpersonal trust might limit networks“ training ambitions..." (ebd., S. 168). Darüber hinaus können solche Partnerschaften nur dort entstehen, wo es auch Unternehmen gibt. Dies ist allerdings bei den ,inner-city ghettos' das entscheidende Problem: Firmen verlassen diese Gemeinden und verlagern ihre Arbeitsplätze in die Vororte der Städte. Die Folge ist ein akuter Mangel an Arbeit, der nach Meinung Wilsons und anderer die eigentliche Ursache aller ,inner-city ghetto“ Probleme darstellt. Jugendliche in diesen Gemeinden haben also keine Chance, sich durch traditionelles ,job shopping“ die von Firmen des „ersten“ Arbeitsmarktes geforderte Arbeitserfahrung anzueignen. Andererseits sind die in bundesstaatlich geförderten lokalen Berufsbildungsprogrammen erworbenen Qualifikationen für nicht-lokale Arbeitgeber intransparent, da es keine einheitlichen Standards gibt. Insofern reproduziert sich die Lokalität des Arbeitsmarktes - bzw. Arbeitslosenmarktes - für diese Jugendlichen. Ein Ausbruch aus der Lokalität wäre durch nationale Standards möglich, die die Mobilitätschancen in diesem Arbeitsmarktsegment erhöhten.

Sako (1994) faßt die Kritik an einer lokalen Organisation der Berufsausbildung folgendermaßen zusammen: „Linkages with the local community do not seem to be the most effective method for bringing about a change in employers' attitudes to training. Nor do such linkages change employers" financial incentives to provide training" (S. 136). Insoweit sollte das deutsche duale Berufsausbildungssystem Verbetrieblichungstendenzen, insbesondere lokaler Art, nicht so weit treiben, daß schließlich die Vorteile überregionaler und firmenübergreifender Vereinheitlichung durch Qualifizierungsstandards - wie Transparenz und Mobilität - verloren gingen. In den USA sollten die Einzelstaaten ihre Verantwortung für Bildungspolitik dazu nutzen, 
wenigstens auf einzelstaatlicher Ebene einheitliche Standards durchzusetzen. Statt weiterer Versuche, die Arbeitgeber in ein Berufsausbildungssystem systematisch einzubeziehen, sollten sie ihre Ressourcen auf die Verbesserung der schulischen Berufsbildungsprogramme, wie Tech-Prep, verwenden und Arbeitgebervertreter lediglich als Berater in Curriculum-Fragen hinzuziehen. Einer systematischen Kombination schulischer und betrieblicher Ausbildung stehen in den USA gravierende institutionelle Hindernisse gegenüber, die die Einrichtung eines Massenprogramms für ,non-college bound'-Schüler verhindern. 


\section{Literaturverzeichnis}

Adam, Hermann (1979): Der Einfluß der Industrie- und Handelskammern auf politische Entscheidungsprozesse, Frankfurt/M.: Campus.

Adams, Willi Paul (1992): Das Erbe der Kolonialzeit, in: Willi Paul Adams/Ernst-Otto Czempiel/Berndt Ostendorf/Kurt Shell/P. Bernd Spahn/Michael Zöller (Hg.), Länderbericht USA, Schriftenreihe Bd. 293/I, 2. Aufl., Bonn: Bundeszentrale für politische Bildung, S. 49-63.

Allmendinger, Jutta (1989): Educational systems and labor market outcomes, in: European Sociological Review, Vol. 5, Nr. 3, S. 231-250.

American Enterprise Institute (1980): Youth Employment Legislation: The Youth Act of 1980, Washington/DC: Public Policy Research.

American Federation of Teachers (1999): About AFT: A Proud Tradition (http://www.aft. org/about/index.html).

Arnow, Philip/Harbison, Frederick/Lester, Richard A./Michael, Bernard/Nemore, Arnold/ Venn, Grant (1968): The Transition from School to Work: A Summary of Viewpoints, in: U.S. Department of Labor/U.S. Department of Health, Education and Welfare/The Woodrow Wilson School (Hg.), The Transition from School to Work, A Report based on the Princeton Manpower Symbosium May 9-10, Princeton: Industrial Relations Section, S. 3-19.

Ashford, Douglas E. (1986): The Emergence of the Welfare States, Oxford: Blackwell.

Attwell, Graham (1997): Rediscovering Apprenticeship?: A Historical Approach, Institut Technik und Bildung, Universität Bremen, Paper presented to European Conference of Educational Research.

Avery, Donald H./Steinisch, Irmgard (1992): Industrialisierung, Urbanisierung und politischer Wandel der Gesellschaft, 1877-1914, in: Willi Paul Adams/Ernst-Otto Czempiel/Berndt Ostendorf/Kurt Shell/P. Bernd Spahn/Michael Zöller (Hg.), Länderbericht USA, Schriftenreihe Bd. 293/I, 2. Aufl., Bonn: Bundeszentrale für politische Bildung, S. 119-145.

Bacharach, Samuel B. (1990): Putting It All Together, in: ders. (Hg.), Education Reform - Making Sense of It All, Boston: Allyn and Bacon, S. 415-430.

Backes-Gellner, Uschi (1993): The Institutional Embeddedness of Corporate Training Strategies - a Comparison of Training for Intermediate Skills in Germany and Great Britain, IAAEG, Quint-Essenzen Nr. 27, Trier: Universität Trier.

Baethge, Martin (1996): Berufsprinzip und duale Ausbildung: Vom Erfolgsgaranten zum Bremsklotz der Entwicklung?, in: Wolfgang Wittwer (Hg.), Von der Meisterschaft zur Bildungswanderschaft: berufliche Bildung auf dem Weg in das Jahr 2000, Wissenschaft - Praxis - Dialog berufliche Bildung, Bd. 2, Bielefeld: Bertelsmann, S. 109-124.

Baethge, Martin (1999): Glanz und Elend des deutschen Korporatismus in der Berufsbildung, in: WSI Mitteilungen, Vol. 52, Nr. 8, S. 489-497.

Baethge, Martin (2000): Gesellschaftliche Integration - Jenseits von Beruf und Beruflichkeit? Oder: Zum Gegensatz der soziologischen und 
qualifikationsstrukturellen Dimension in der Berufskategorie, in: Franz-Josef Kaiser (Hg.), Berufliche Bildung in Deutschland für das 21. Jahrhundert, Beiträge zur Arbeitsmarkt- und Berufsforschung 238, Nürnberg: Institut für Arbeitsmarkt- und Berufsforschung, S. 375-382.

Baethge, Martin (2001): Zwischen Individualisierung und Standardisierung: Zur Qualifikationsentwicklung in den Dienstleistungsberufen, in: Werner Dostal/Peter Kupka (Hg.), Globalisierung, veränderte Arbeitsorganisation und Berufswandel, Beiträge zur Arbeitsmarkt- und Berufsforschung 240, Nürnberg: Institut für Arbeitsmarkt- und Berufsforschung, S. 27-44.

Baethge, Martin/Baethge-Kinsky, Volker (1995): Ökonomie, Technik, Organisation: Zur Entwicklung und qualitativem Arbeitsvermögen, in: Rolf Arnold/Antonius Lipsmeier (Hg.), Handbuch der Berufsbildung, Opladen: Leske+Budrich, S. 142156.

Baethge, Martin/Baetghe-Kinsky, Volker (1998): Jenseits von Beruf und Beruflichkeit? - Neue Formen von Arbeitsorganisation und Beschäftigung und ihre Bedeutung für eine zentrale Kategorie gesellschaftlicher Integration, in: Mitteilungen aus der Arbeitsmarkt- und Berufsforschung, 31. Jg., Nr. 3, S. 461-472.

Baethge-Kinsky, Volker (2001): Prozessorientierte Arbeitsorganisation und Facharbeiterzukunft, in: Werner Dostal/Peter Kupka (Hg.), Globalisierung, veränderte Arbeitsorganisation und Berufswandel, Beiträge zur Arbeitsmarkt- und Berufsforschung 240, Nürnberg: Institut für Arbeitsmarkt- und Berufsforschung, S. 81-97.

Bailey, Thomas R. (1992): Are U.S. businesses likely to embrace youth apprenticeship?, in: Congressional Quarterly Researcher, Vol. 2, Nr. 39, S. 921.

Bailey, Thomas R. (1993): Can Youth Apprenticeship Thrive in the United States?, in: Educational Researcher, Vol. 22, Nr. 3, S. 4-10.

Bailey, Thomas R. (1995a): Incentives for Employer Participation in School-to-Work Programs, in: ders. (Hg.), Learning to Work. Employer Involvement in School-toWork Transition Programs, Washington/DC: Brookings Institution, S. 14-25.

Bailey, Thomas R. (1995b): Summary, Discussion, and Recommendations, in: ders. (Hg.), Learning to Work. Employer Involvement in School-to-Work Transition Programs, Washington/DC: Brookings Institution, S. 88-104.

Bailey, Thomas R./Merritt, Donna (1993): The School-to-Work Transition and Youth Apprenticeship: Lessons from the U.S. Experience, New York: Manpower Demonstration Research Corporation.

Bailey, Thomas R./Hughes, Katherine/Barr, Tavis (1998): Achieving Scale and Quality in School-to-Work Internships: Findings From an Employer Survey, National Center for Research in Vocational Education, Berkeley (http://vocserve.berkeley.edu/AllInOne /MDS-902.html).

Bailyn, Bernard (1967): The Ideological Origins of the American Revolution, Cambridge, Massachusetts: Harvard University Press.

Balz, Martin (1993): Bildung und Gewerkschaften - Ausgangsbedingungen und Entwicklungstendenzen der Bildungspolitik des DGB bis Mitte der achtziger Jahre, Diss., Münster. 
Bardeleben, Richard v./Beicht, Ursula (1996): „Investition in die Zukunft“ - eine bildungsökonomische Betrachtung der betrieblichen Ausbildung, in: Richard v. Bardeleben/Axel Bolder/Helmut Heid (Hg.), Kosten und Nutzen beruflicher Bildung, Stuttgart: Franz Steiner, S. 22-41.

Barthold, Hans-Martin (1999): „Die Zukunft liegt in der Werkstatt nicht im Büro“, in: Frankfurter Allgemeine Zeitung, 3. April, Nr. 78, S. 65.

Bassi, Laurie J./Ludwig, Jens (2000): School-To-Work Programs in the United States: A Multi-Firm Case Study of Training, Benefits, and Costs, in: Industrial and Labor Relations Review, Vol. 53, Nr. 2, S. 219-239.

Batt, Rosemary/Osterman, Paul (1993): A National Policy for Workplace Training: Lessons from State and Local Experiments, Washington/DC: Economic Policy Institute.

Baumgart, Franzjörg (1990): Zwischen Reform und Restauration: preußische Schulpolitik 1806-1859, Darmstadt: Wissenschaftliche Buchgesellschaft.

Becker, Gary S. (1993): Human Capital - A Theoretical and Empirical Analysis with Special Reference to Education, 3. Aufl., Chicago: University of Chicago Press.

Becker, Hellmut/Kluchert, Gerhard (1993): Die Bildung der Nation. Schule, Gesellschaft und Politik vom Kaiserreich zur Weimarer Republik, Stuttgart: KlettCotta.

Benner, Hermann/Püttmann, Friedhelm (1992): 20 Jahre Gemeinsames Ergebnisprotokoll: Eine kritische Darstellung des Verfahrens zur Abstimmung von Ausbildungsordnungen und Rahmenlehrplänen für die Berufsausbildung in anerkannten Ausbildungsberufen aus Bundes- und Ländersicht, Bonn.

Berger, Klaus/Brandes, Harald/Walden, Günter (2000): Chancen der dualen Berufsausbildung. Berufliche Entwicklungsperspektiven aus betrieblicher Sicht und Berufserwartungen von Jugendlichen, Berichte zur beruflichen Bildung, Heft 239, Bielefeld: Bertelsmann.

Bernhardt, Annette/Morris, Martina/Handcock, Mark/Scott, Marc (1998a): Summary of Findings: Work and Opportunity in the Post-Industrial Labor Market, IEE Working Paper Nr.6, New York: Columbia University.

Bernhardt, Annette/Morris, Martina/Handcock, Mark/Scott, Marc (1998b): Trends in Job Stability and Wages for Young Adult Men, IEE Working Paper Nr. 8, New York: Columbia University.

Berryman, Sue E./Bailey, Thomas R. (1992): The Double Helix of Education and the Economy, New York: Institute on Education and the Economy/Columbia University.

Bertrand, Olivier/Durand-Drouhin, Marianne/Romani, Claudine (1994): Issues, Problems and Perspectives. Lessons from an International Debate, in: OECD, Apprenticeship: Which Way Forward?, Paris: OECD, S. 41-88.

Berube, Maurice R. (1988): Teacher Politics - The Influence of Unions, New York: Greenwood Press.

BIBB (1998a): Überbetriebliche Berufsbildungsstätten sichern betriebliche Ausbildungsplätze, (http://www.bibb.de/bwp/2_98/uebs.htm). 
BIBB (1998b): Beachtliches Ausbildungsengagement neu gegründeter Betriebe!, (http://www.bibb.de/pm/pm98/pm081098.htm).

Bishop, John H. (1994): Signalling the Competencies of High School Students to Employers, Center for Advanced Human Resource Studies, Working Paper \#94-18, Ithaca: Cornell University.

Bishop, John (1995): Vocational Education and at-risk Youth in the United States, Center for Advanced Human Resource Studies, Working Paper \#95-19, Ithaca: Cornell University.

Blankertz, Herwig (1963): Berufsbildung und Utilitarismus, Düsseldorf: Pädagogischer Verlag Schwann.

Boch, Rudolf (1985): Handwerker-Sozialisten gegen Fabrikgesellschaften. Lokale Fachvereine, Massengewerkschaft und industrielle Rationalisierung in Solingen 1870 bis 1914, Göttingen: Vandenhoeck \& Ruprecht.

Borchert, Jens (1995): Die konservative Transformation des Wohlfahrtsstaates. Großbritannien, Kanada, die USA und Deutschland im Vergleich, Frankfurt/M.: Campus.

Borjas, George J. (1992): National Origin and the Skills of Immigrants in the Postwar Period, in: George J. Borjas/Richard B. Freeman (Hg.), Immigration and the Work Force. Economic Consequences for the United States and Source Areas, Chicago: University of Chicago Press, S. 17-48.

Brand, Betsy (1994): The Political Challenge of Reforming Vocational and Technical Education: A Case Study from the United States, in: OECD, Vocational Education and Training for Youth: Towards Coherent Policy and Practice, Paris: OECD, S. 19-26.

Brater, Michael/Beck, Ulrich (1982): Berufe als Organisationsform menschlichen Arbeitsvermögens, in: W. Littek et al. (Hg.), Einführung in die Arbeits- und Industriesoziologie, Frankfurt/M.: Campus, S. 208-224.

Breen, William J. (1997): Labor Market Politics and the Great War. The Department of Labor, the States, and the First U.S. Employment Service, 1907-1933, Kent: Kent State University Press.

Bremer, Christine D./Madzar, Svjetlana (1994): Encouraging Employer Involvement in Youth Apprenticeship and other Work-Based Learning Experiences for High School Students, in: Journal of Vocational and Technical Education, Vol. 11, Nr. 2, (http://scholar.lib.vt.edu/ejournals/JVTE/v11n2/bremer.html).

Bresnick, David (1984): Youth Jobs - Toward a Private/Public Partnership, Westport: Quorum Books.

Brint, Steven/Karabel, Jerome (1991): Institutional Origins and Transformations: The Case of American Community Colleges, in: Walter W. Powell/Paul J. DiMaggio (Hg.), The New Institutionalism in Organizational Analysis, Chicago: University of Chicago Press, S. 337-360.

Bruchhäuser, Hanns-Peter/Lipsmeier, Antonius (Hg.) (1985): Quellen und Dokumente zur schulischen Berufsbildung 1869-1918. Quellen und Dokumente zur Geschichte der Berufsbildung in Deutschland B/3, Köln, Wien: Böhlau Verlag. 
Bryne, Sandra M./Constant, Anne/Moore, Gary (1992): Making Transitions from School to Work, in: Educational Leadership, Vol. 49, Nr. 6, S. 23-26.

Bundesinstitut für Berufsbildung (2000): Satzung, Fassung vom 23. November, Bonn.

Bundesministerium für Bildung und Wissenschaft (1984): Berufsbildungsbericht 1984, Bonn.

Bundesministerium für Bildung und Wissenschaft (1985): Berufsbildungsbericht 1985, Bonn.

Bundesministerium für Bildung und Wissenschaft (1988): Berufsbildungsbericht 1988, Bonn.

Bundesministerium für Bildung und Wissenschaft (1989): Berufsbildungsbericht 1989, Bonn.

Bundesministerium für Bildung und Wissenschaft (1990): Berufsbildungsbericht 1990, Bonn.

Bundesministerium für Bildung und Wissenschaft (1993): Berufsbildungsbericht 1993, Bonn.

Bundesministerium für Bildung und Wissenschaft (1995): Berufsbildungsbericht 1995, Bonn.

Bundesministerium für Bildung und Wissenschaft (1996): Berufsbildungsbericht 1996, Bonn.

Bundesministerium für Bildung und Forschung (1999): Berufsbildungsbericht 1999, Bonn.

Bundesministerium für Bildung und Forschung (2000): Berufsbildungsbericht 2000, Bonn.

Bundesministerium für Bildung und Forschung (2001): Berufsbildungsbericht 2001, Bonn.

Bundesregierung (1999): Bündnis für Arbeit, Ausbildung und Wettbewerbsfähigkeit. Ergebnisse der Arbeitsgruppe „Aus- und Weiterbildung“, Berlin: Presse- und Informationsamt.

Bundesvereinigung der Deutschen Arbeitgeberverbände (1999): BDA - Vorschläge zur Verbesserung der Strukturen und der Rahmenbedingungen der Berufsausbildung, 12.10., VII/Do.

Bundesverfassungsgericht (1969): Entscheidungen, Vol. 26, S. 246-258.

Bundesverfassungsgericht (1981): Entscheidungen, Vol. 55, S. 274-348.

Bunn, Ronald F. (1984): Employers associations in the Federal Republic of Germany, in: John P. Windmuller/Alan Gladstone (Hg.), Employers Associations and Industrial Relations - A Comparative Study, Oxford: Clarendon Press, S. 167-201.

Buttler, Friedrich/Franz, Wolfgang/Schettkat, Ronald/Soskice, David (1995): Institutional Frameworks and Labor Market Performance, in: dies. (Hg.), Institutional Frameworks and Labor Market Performance - Comparative views on the U.S. and German economies, London: Routledge.

Cappelli, Peter/Bassi, Laurie/Katz, Harry/Knoke, David/Osterman, Paul/Useem, Michael (1997): Change at Work - How American Industry and Workers are coping 
with corporate restructuring and what workers must do to take charge of their own careers, New York: Oxford University Press.

Cappelli, Peter/Shapiro, Daniel/Shumanis, Nichole (1998): Employer Participation in School-to-Work Programs, Annals of the American Academy of Political and Social Science, Vol. 559, September, S. 109-125.

Carnoy, Martin/Levin, Henry M. (1985): Schooling and Work in the Democratic State, Stanford: Stanford University Press.

Cattero, Bruno (1998): Beruf und Berufsausbildung - Mythen und Widersprüche im "deutschen Modell", in: ders. (Hg.), Modell Deutschland - Modell Europa: Probleme, Perspektiven, Europa- und Nordamerika-Studien, Bd. 4, Opladen: Leske+Budrich, S. 225-246.

Cheney, Lynne (1998): Limited Horizons, in: The New York Times, 3. Februar, S. A23.

Cheng, Charles W. (1981): Teacher Unions and the Power Structure, Bloomington: Phi Delta Kappan.

Choy, Susan (1994): School-to-Work Opportunities: Issues in State and Local Governance, in: U.S. Department of Education - Office of Research (Hg.), Schoolto-Work - What Does Research Say About It?, Washington/DC: U.S. Government Printing Office, S. 57- 76.

Church, Robert L./Sedlak, Michael W. (1976): Education in the United States - An Interpretive History, New York: Free Press.

Clark, David L./Astuto, Terry A. (1987): Federal Education Policy in the United States: The Conservative Agenda and Accomplishments, in: William Lowe Boyd/Don Smart (Hg.), Educational Policy in Australia and America - Comparative Perspectives, New York: Falmer Press, S. 47-76.

Clinton, Bill (1993): President Clinton's Plan for Education, in: The Education Digest, Vol. 58, Nr. 5, S. 4-9.

Cobb, Brian R./Neubert, Debra A. (1998): Vocational Education: Emerging Vocationalism, in: Frank R. Rusch/Janis G. Chadsey (Hg.), Beyond High School Transition from School to Work, Belmont: Wadsworth Publishing Company, S. 101-126.

Couch, Kenneth A. (1994): The German Apprenticeship Experience - A Comparison of School-to-Work Models, in: Current, Nr. 362, May, S. 11-14.

Craver, Charles B. (1993): Can Unions Survive? The Rejuvenation of the American Labor Movement, New York: New York University Press.

Crouch, Colin (1993): Industrial Relations and European State Traditions, Oxford: Clarendon Press.

Crouch, Colin/Finegold, David/Sako, Mari (1999): Are Skills the Answer? The Political Economy of Skill Creation in Advanced Industrial Countries, Oxford: Oxford University Press.

Crusius, Reinhard (1982): Berufsbildungs- und Jugendpolitik der Gewerkschaft. Struktur und Verlauf bei DGB und einigen Einzelgewerkschaften 1945-1981, Frankfurt/M.: Campus. 
Cuban, Larry (1982): Enduring Resiliency: Enacting and Implementing Federal Vocational Education Legislation, in: Harvey Kantor/David Tyack (Hg.), Work, Youth, and Schooling, Stanford: Stanford University Press, S. 45-78.

Cuban, Larry (1997): What Policy Makers and Experts See (and Do Not See) in Schoolto-Work Transitions, in: Alan Lesgold/Michael J. Feuer/Allison M. Black (Hg.), Transitions in Work and Learning - Implications for Assessment, Washington/DC: National Academy Press, S. 235-248.

Culpepper, Pepper D. (1996): Problems on the Road to "High Skill": A sectoral lesson from the transfer of the dual system of vocational training to eastern Germany, WZB Discussion Paper, Berlin.

Culpepper, Pepper D. (2001): Employers, Public Policy, and the Politics of Decentralized Cooperation in Germany and France, in: Peter A. Hall/David Soskice (Hg.), Varieties of Capitalism. The Institutional Foundations of Comparative Advantage, Oxford: Oxford University Press, S. 275-304.

Cusick, Philip A. (1983): The Egalitarian Ideal and the American High School. Studies of three Schools, New York: Teachers College Press.

Degen, Ulrich/Walden, Günter (1994): Betriebliches Ausbildungsengagement in den neuen Bundesländern, in: Berufsbildung in Wissenschaft und Praxis (BWP), Vol. 23, Nr. 5, S. 3-9.

Derber, Milton (1984): Employers Associations in the United States, in: John P. Windmuller/Alan Gladstone (Hg.), Employers Associations and Industrial Relations - A Comparative Study, Oxford: Clarendon Press, S. 79-114.

DeSoto, William (1995): The Politics of Business Organizations: Understanding the Role of the State Chambers of Commerce, Lanham, Md.: University Press of America.

Deutsch, Steven (1987): Worker Training Programs help ease impact of technology, in: Monthly Labor Review, Vol. 110, Nr. 11, S. 14-20.

Deutscher Industrie- und Handelstag (DIHT) (1987): Industrie- und Handelskammern in der Bundesrepublik Deutschland - Aufgaben und Gesetz, Bonn.

Dewey, John [1916] (1966): Democracy and Education. An Introduction to the Philosophy of Education, New York: Free Press.

Dicke, Hugo/Glismann, Hans H./Gröhn, Andreas (1995): Der deutsche Markt für berufliche Weiterbildung. Kieler Studien 269, Tübingen: Mohr.

DiConti, Veronica Donahue (1996): Interest Groups and Education Reform: The Latest Crusade to Restructure the Schools, Lanham: University Press of America.

DiMaggio, Paul J./Powell, Walter W. (1991): Introduction, in: Walter W. Powell/Paul J. DiMaggio (Hg.), The New Institutionalism in Organizational Analysis, Chicago: University of Chicago Press, S. 1-40.

Dittrich, Eckhard (1980): Arbeiterbewegung und Arbeiterbildung im 19. Jahrhundert, Bensheim: päd.-extra Buchverlag.

Dobischat, Rolf/Düsseldorf, Karl (1999): Transformation der Berufsbildung in den neuen Bundesländern, in: Klaus Harney/Heinz-Elmar Tenorth (Hg.), Beruf und Berufsbildung. Situation, Reformperspektiven, Gestaltungsmöglichkeiten, Weinheim: Beltz, S. 101-120. 
Doeringer, Peter B./Piore, Michael J. (1971): Internal Labor Markets and Manpower Analysis, Lexington, Mass.: D.C. Heath and Company.

Dolainski, Stephen (1997): Calculating the Costs of School-to-Work Involvement, in: Workforce, Vol. 76, Nr. 5, S. 30.

Dostal, Werner (2002): Beruflichkeit in der Wissensgesellschaft, in: Matthias Wingens/Reinhold Sackmann (Hg.), Bildung und Beruf. Ausbildung und berufsstruktureller Wandel in der Wissensgesellschaft, Weinheim: Juventa, S. 177194.

Drexel, Ingrid (1997): The Relationship between Education and Employment in German Industrial Sociology, in: Annette Jobert/Catherine Marry/Lucie Tanguy/Helen Rainbird (Hg.), Education and Work in Great Britain, Germany and Italy, London: Routledge, S. 76-92.

Dubofsky, Melvyn (1994): The State and Labor in Modern America, Chapel Hill: University of North Carolina Press.

Dunlop, John T. (1992): The Challenge of Human Resources Development, in: Industrial Relations, Vol. 31, Nr. 1, S. 50-55.

Durand-Drouhin, Marianne/Romani, Claudine (1994): The Issues, in: OECD, Apprenticeship: Which Way Forward?, Paris: OECD, S. 9-18.

Durkheim, Emile [1895] (1961): Regeln der soziologischen Methode, Neuwied: Luchterhand.

Durkheim, Emile (1991): Physik der Sitten und des Rechts, Frankfurt/M.: Suhrkamp.

Eichler, Susanne/Kühnlein, Gertrud (1997): Berufsschulen als Reparaturbetrieb? Erfahrungen mit der Berufsausbildung in den neuen Bundesländern, Weinheim: Juventa.

Elbaum, Bernard (1989): Why Apprenticeship Persisted in Britain But Not in the United States, in: The Journal of Economic History, Vol. 49, Nr. 2, S. 337- 349.

Elbaum, Bernard/Singh, Nirvikar (1995): The Economic Rationale of Apprenticeship Training: Some Lessons from British and U.S. Experience, in: Industrial Relations, Vol. 34, Nr. 4, S. 593-622.

Els, Michael (1999): Die Grenzen der Beteiligung privater Verbände an der Verordnungsgebung. Erläutert am Beispiel des Erlasses von Ausbildungsordnungen, Diss., Bonn.

Erdmann, Gerhard (1966): Die deutschen Arbeitgeberverbände im sozialgeschichtlichen Wandel, Neuwied: Luchterhand.

Erlichson, Bari A./Van Horn, Carl E. (1999): School-to-Work Governance: A National Review, Edward J. Bloustein School of Planning and Public Policy/John J. Heldrich Center for Workforce Development, Rutgers University.

Esping-Andersen, Gøsta/Korpi, Walter (1984): Social Policy as Class Politics in PostWar Capitalism: Scandinavia, Austria, and Germany, in: John H. Goldthorpe (Hg.), Order and Conflict in Contemporary Capitalism, Oxford: Clarendon Press, S. 179208.

Esping-Andersen, Gøsta (1990): The Three Worlds of Welfare Capitalism, Cambridge: Polity Press. 
Estevez-Abe, Margarita/Iversen, Torben/Soskice, David (2001): Social Protection and the Formation of Skills: A Reinterpretation of the Welfare State, in: Peter A. Hall/David Soskice (Hg.), Varieties of Capitalism. The Institutional Foundations of Comparative Advantage, Oxford: Oxford University Press, S. 145-183.

Evans, Peter B./Rueschemeyer, Dietrich/Skocpol, Theda (1989): On the Road toward a More Adequate Understanding of the State, in: dies. (Hg.), Bringing the State back in, Cambridge: Cambridge University Press, S. 347-366.

Feldstein, Martin/Ellwood, David (1982): Teenage Unemployment: What is the Problem?, in: Richard Freeman/David Wise (Hg.), The Youth Labor Market Problem: Its Nature, Causes, and Consequences, Chicago: University of Chicago Press, S. 17-33.

Ferrie, Joseph P. (1999): Yankeys Now. Immigrants in the Antebellum United States, 1840-1860, New York: Oxford University Press.

Finegold, David (1991): Institutional Incentives and Skill Creation: Preconditions for a High-Skill Equilibrium, in: Paul Ryan (Hg.), International Comparisons of Vocational Education and Training for Intermediate Skills, London: Falmer Press, S. 93-118.

Finegold, David/McFarland, Laurel/Richardson, William (1993): Introduction, in: dies. (Hg.), Something Borrowed, Something Learned? The Transatlantic Market in Education and Training Reform, Washington/DC: Brookings Institution, S. 3-12.

Finegold, David/Crouch, Colin (1994): A comparison of national institutions, in: Richard Layard/Ken Mayhew/Geoffrey Owen (Hg.), Britain's Training Deficit, The Centre for Economic Performance Report, Aldershot: Avebury, S. 251-281.

Finegold, Kenneth/Skocpol, Theda (1995): State and Party in America's New Deal, Madison/Wisconsin: University of Wisconsin Press.

Finn, Chester E./Rebarber, Theodor (1992): The Changing Politics of Education Reform, in: dies. (Hg.), Education Reform in the '90s, New York: Macmillan, S. 175-193.

Fischer, Wolfram (1964): Unternehmerschaft, Selbstverwaltung und Staat. Die Handelskammern in der deutschen Wirtschafts- und Staatsverfassung des 19. Jahrhunderts, Berlin: Duncker \& Humblot.

Flecker, Jörg/Schulten, Thorsten (1999): The End of Institutional Stability: What Future for the ,German Model'?, in: Economic and Industrial Democracy, Vol. 20, Nr. 1, S. 81-116.

Flora, Peter (1975): Indikatoren der Modernisierung. Ein historisches Datenhandbuch, Opladen: Westdeutscher Verlag.

Flora, Peter/Heidenheimer, Arnold J. (1982): The Historical Core and Changing Boundaries of the Welfare State, in: dies. (Hg.), The Development of Welfare States in Europe and America, New Brunswick: Transaction Publishers, S. 17-34.

Fogel, Robert (1989): Without Consent or Contract. The Rise and Fall of American Slavery, New York: Norton.

Fogel, Robert (2000): The Fourth Great Awakening and the Future of Egalitarianism, Chicago: University of Chicago Press. 
Frankfurter Allgemeine Zeitung (2002a): Unternehmen müssen in der IHK bleiben, Nr. 15, 18. Januar, S. 13.

Frankfurter Allgemeine Zeitung (2002b): Hoher Nachwuchsbedarf. Investmentbranche schafft neuen Ausbildungsberuf, Nr. 40, 16. Februar, S. 71.

Franz, Wolfgang (1996): Arbeitsmarktökonomik, 3. Aufl., Berlin: Springer.

Franz, Wolfgang/Soskice, David (1994): The German Apprenticeship System, WZB Discussion Paper, Berlin.

Freeman, Richard/Wise, David (1982): The Youth Labor Market Problem: Its Nature, Causes, and Consequences, in: dies. (Hg.), The Youth Labor Market Problem: Its Nature, Causes, and Consequences, Chicago: University of Chicago Press, S. 1-16.

Friedrich, Michael/Troltsch, Klaus/Westhoff, Gisela (1999): Das Sofortprogramm zur Bekämpfung der Jugendarbeitslosigkeit zeigt Wirkung. Erste Ergebnisse aus der Begleitforschung des BIBB, in: Berufsbildung in Wissenschaft und Praxis (BWP), Vol. 28, Nr. 6, S. 5-10.

Frühbrodt, Lutz (1996): Bildungssystem, in: Carl-Ludwig Holtfrerich (Hg.), Wirtschaft USA - Strukturen, Institutionen und Prozesse, München: Oldenbourg, S. 143-176.

Georg, Walter/Sattel, Ulrike (1995): Arbeitsmarkt, Beschäftigungssystem und Berufsbildung, in: Rolf Arnold/Antonius Lipsmeier (Hg.), Handbuch der Berufsbildung, Opladen: Leske+Budrich, S. 123-141.

Gilberg, Dirk (1999): Die Mitwirkung des Betriebsrats bei der Berufsbildung, Heidelberg: Müller.

Gitter, Robert J./Scheuer, Markus (1997): U.S. and German youths: unemployment and the transition from school to work, in: Monthly Labor Review, Vol. 120, Nr. 3, S. $16-20$.

Gittleman, Mark (1994): Partners in Transition, in: Training and Development, Vol. 48, Nr. 11, S. 32-37.

Glazer, Nathan (1988): The Limits of Social Policy, Cambridge/MA: Harvard University Press.

Glover, Robert W./Marshall, Ray (1993): Improving the School-to-Work Transition of American Adolescents, in: Teachers College Record, Vol. 94, Nr. 3, S. 588-610.

Glover, Robert W./Weisberg, Alan (1994): School-to-Work Transition in the U.S.: The Case of the Missing Social Partners, Austin: Center for Learning and Competitiveness/Greater Austin Chamber of Commerce.

Görner, Regina (1999a): Statement vor der Bund-Länder-Kommission zur Entwicklung des Bildungs- und Beschäftigungsproblems in Deutschland, DGB-Bundesvorstand, Bonn.

Görner, Regina (1999b): 10-Punkte-Modell zur Neuordnung der Ausbildung, Statement anläßlich der Pressekonferenz - Bonn 13. Januar 1999.

Goldberger, Susan/Kazis, Richard/O'Flanagan, Mary K. (1994): Learning Through Work: Designing and Implementing Quality Worksite Learning for High School Students, Washington/DC: Manpower Demonstration Research Corporation. 
Goldberger, Susan/Kazis, Richard (1995): Revitalizing High Schools: What the Schoolto-Career Movement Can Contribute, Washington/DC: American Youth Policy Forum et al..

Goldfield, Michael (1987): The Decline of Organized Labor in the United States, Chicago: University of Chicago Press.

Goldin, Claudia (1999): Egalitarianism and the Returns to Education During the Great Transformation of American Education, in: The Journal of Political Economy, Vol. 107, Nr. 6, S. 65-94.

Golisch, Botho (1997): Ausbildungsordnungen als berufspädagogisches Konstrukt. Entwicklung, Bedingungen, Gestaltungen - Eine vergleichend-explorative Studie mit einer Darstellung zum Beruf des Sozialversicherungsfachangestellten, Frankfurt/M.: Peter Lang.

Gonon, Philipp (1992): Arbeitsschule und Qualifikation. Arbeit und Schule im 19. Jahrhundert, Kerschensteiner und die heutigen Debatten zur beruflichen Qualifikation, Bern: Peter Lang.

Grabbe, Hans-Jürgen (2001): Vor der großen Flut. Die europäische Migration in die Vereinigten Staaten von Amerika 1783-1820, Stuttgart: Franz Steiner.

Green, Andy (1990): Education and State Formation - The Rise of Education Systems in England, France and the USA, New York: Macmillan.

Greinert, Wolf-Dietrich (1982): Die Einführung des Berufsgrundbildungsjahres als öffentliche Pflichtschule in Niedersachsen, in: ders./Dieter Jungk (Hg.), Berufliche Grundbildung: Erfahrungen bei der Gestaltung des Berufsgrundbildungsjahres in Niedersachsen, Frankfurt/M.: Campus, S. 9-53.

Greinert, Wolf-Dietrich (1988): Marktmodell - Schulmodell - duales System, in: Die berufsbildende Schule, Vol. 40, Nr. 3, S. 145-156.

Greinert, Wolf-Dietrich (1993): Das „,deutsche System“ der Berufsausbildung Geschichte, Organisation, Perspektiven. Studien zur Vergleichenden Berufspädagogik, Bd. 1, Baden-Baden: Nomos.

Greinert, Wolf-Dietrich (1995): Das duale System der Berufsausbildung in der Bundesrepublik Deutschland - Struktur und Funktion, 2. Aufl., Stuttgart: Holland+Josenhans.

Grießinger, Andreas/Reith, Reinhold (1986): Lehrlinge im deutschen Handwerk des ausgehenden 18. Jahrhunderts - Arbeitsorganisation, Sozialbeziehungen und alltägliche Konflikte, in: Zeitschrift für historische Forschung, Vol. 13, S. 149-199.

Grubb, Norton/Lazerson, Marvin (1982): Education and the Labor Market: Recycling the Youth Problem, in: Harvey Kantor/David Tyack (Hg.), Work, Youth, and Schooling, Stanford: Stanford University Press, S. 110-141.

Grubb, Norton W./McDonnell, Lorraine M. (1991): Local Systems of Vocational Education and Job Training: Diversity, Interdependence, and Effectiveness, National Center for Research in Vocational Education, Santa Monica: RAND.

Grubb, Norton/Dickinson, Torry/Giordano, Lorraine/Kaplan, Gail (1992): Betwixt and Between: Education, Skills, and Employment in Sub-Baccalaureate Labor Markets, National Center for Research in Vocational Education, Berkeley (http://vocserve. berkeley.edu/AllInOne/MDS-470.html). 
Grubb, Norton (1996): The New Vocationalism, in: Phi Delta Kappan, Vol. 77, Nr. 8, S. 535-546.

Gruber, David A. (1994): Toward a Seamless System for Youth Development: A New Strategy for Integrating Resources, Programs, and Institutions, Boston: Jobs for the Future.

Gumbert, Edgar B./Spring, Joel H. (1974): The Superschool and the Superstate: American Education in the Twentieth Century, 1918-1970, New York: Wiley.

Gustman, Alan L. (1982): Comment, in: Richard Freeman/David Wise (Hg.), The Youth Labor Market Problem: Its Nature, Causes, and Consequences, Chicago: University of Chicago Press, S. 267-270.

Gutmann, Amy (1988): Distributing Public Education in a Democracy, in: dies. (Hg.), Democracy and the Welfare State, Princeton: Princeton University Press, S. 107130.

Hadden, Betsy (1992): Das Bildungswesen, in: Willi Paul Adams/Ernst-Otto Czempiel/Berndt Ostendorf/Kurt L. Shell/P. Bernd Spahn/Michael Zöller (Hg.), Länderbericht USA, Schriftenreihe Bd. 293/II, 2. Aufl., Bonn: Bundeszentrale für politische Bildung, S. 598-652.

Hall, Peter A. (1986): Governing the Economy: The Politics of State Intervention in Britain and France, New York: Oxford University Press.

Hall, Peter A. (1989): Conclusion: The Politics of Keynesian Ideas, in: ders. (Hg.), The Political Power of Economic Ideas: Keynesianism across Nations, Princeton: Princeton University Press, S. 361-391.

Hall, Peter A./Soskice, David (2001): An Introduction to Varieties of Capitalism, in: dies. (Hg.), Varieties of Capitalism. The Institutional Foundations of Comparative Advantage, Oxford: Oxford University Press, S. 1-68.

Hall, Robert E. (1982): The Minimum Wage and Job Turnover in Markets for Young Workers, in: Richard Freeman/David Wise (Hg.), The Youth Labor Market Problem: Its Nature, Causes, and Consequences, Chicago: University of Chicago Press, S. 475-492.

Hamilton, Stephen F. (1990): Apprenticeship for Adulthood - Preparing Youth for the Future, New York: Free Press.

Hamilton, Stephen F. (1993): Prospects for an American-Style Youth Apprenticeship System, in: Educational Researcher, Vol. 22, Nr. 3, S. 11-16.

Hamilton, Stephan F./Hurrelmann, Klaus (1994): The School-to-Career Transition in Germany and the United States, in: Teachers College Record, Vol. 96, Nr. 2, S. 329-344.

Hamilton, Stephen F./Hamilton, Mary A. (1997a): When is learning work-based?, in: Phi Delta Kappan, Vol. 78, Nr. 9, S. 676-681.

Hamilton, Stephen F./Hamilton, Mary A. (1997b): Learning Well at Work: Choices for Quality, Cornell Youth and Work Program, Ithaca: Cornell University.

Harney, Klaus (1980): Die preußische Fortbildungsschule. Eine Studie zum Problem der Hierarchisierung beruflicher Schultypen im 19. Jahrhundert, Weinheim, Basel: Beltz. 
Heckman, James (1994): Is job training oversold?, in: The Public Interest, Nr. 115, Spring 1994, S. 91-115.

Heckman, James (1998): What Should Be Our Human Capital Investment Policy?, in: Fiscal Studies, Vol. 19, Nr. 2, S. 103-119.

Heckscher, Charles C. (1988): The New Unionism - Employee Involvement in the Changing Corporation, New York: Basic Books.

Heclo, Hugh (1974): Modern social politics in Britain and Sweden - From relief to income maintenance, New Haven: Yale University Press.

Heideking, Jürgen (1992): Revolution, Verfassung und Nationalstaatsgründung, 17631815, in: Willi Paul Adams/Ernst-Otto Czempiel/Berndt Ostendorf/Kurt Shell/P. Bernd Spahn/Michael Zöller (Hg.), Länderbericht USA, Schriftenreihe Bd. 293/I, 2. Aufl., Bonn: Bundeszentrale für politische Bildung, S. 64-86.

Heidemann, Winfried/Paul-Kohlhoff, Angela (1998): Regulierung der Berufsbildung durch Mitbestimmung. Expertise für das Projekt „Mitbestimmung und neue Unternehmenskulturen“ der Bertelsmann Stiftung und der Hans-Böckler-Stiftung, Gütersloh: Verlag Bertelsmann Stiftung.

Heidenheimer, Arnold J. (1982): Education and Social Security Entitlements in Europe and America, in: Peter Flora/Arnold J. Heidenheimer (Hg.), The Development of Welfare States in Europe and America, New Brunswick: Transaction Publishers, S. 269-304.

Heidenreich, Martin (1998): Die duale Berufsausbildung zwischen industrieller Prägung und wissensgesellschaftlichen Herausforderungen, in: Zeitschrift für Soziologie, Vol. 27, Nr. 5, S. 321-340.

Heimann, Klaus (1980): Berufliche Bildung und Gewerkschaften. Analyse der Politik des DGB zur beruflichen Bildung, Frankfurt/M.: R.G. Fischer.

Heinz, Walter R./Krüger, Helga/Rettke, Ursula et.al. (1987): „Hauptsache eine Lehrstelle" - Jugendliche vor den Hürden des Arbeitsmarkts, Weinheim: Deutscher Studien Verlag.

Herbst, Jurgen (1996): The Once and Future School: Three Hundred and Fifty Years of American Secondary Education, New York: Routledge.

Herman, Edward E./Schwarz, Joshua L./Kuhn, Alfred (1992): Collective Bargaining and Labor Relations, 3. Aufl., Englewood: Prentice Hall.

Herrigel, Gary (1996): Industrial Constructions. The Sources of German Industrial Power, Cambridge: Cambridge University Press.

Herrlitz, Hans-Georg/Hopf, Wulf/Titze, Hartmut (1998): Deutsche Schulgeschichte von 1800 bis zur Gegenwart. Eine Einführung, 2. Aufl., Weinheim: Juventa.

Hickey, Delina R./Andrews, David B. (1993): Creating Successful Partnerships, in: Educational Record, Vol. 74, Nr. 3, S. 40-45.

Hilbert, Josef/Südermersen, Helmi/Weber, Hajo (1990): Berufsbildungspolitik. Geschichte - Organisation - Neuordnung, Opladen: Leske+Budrich.

Hillmert, Steffen (2002): Stabilität und Wandel des "deutschen Modells": Lebensverläufe im Übergang zwischen Schule und Beruf, in: Matthias Wingens/Reinhold Sackmann (Hg.), Bildung und Beruf. Ausbildung und 
berufsstruktureller Wandel in der Wissensgesellschaft, Weinheim: Juventa, S. 6582.

Hillmert, Steffen (2001): Ausbildungssysteme und Arbeitsmarkt: Lebensverläufe in Großbritannien und Deutschland im Kohortenvergleich, Wiesbaden: Westdeutscher Verlag.

Hoffmann, Ernst (1962): Zur Geschichte der Berufsausbildung in Deutschland, Bielefeld: Bertelsmann Verlag.

Hogan, David (1982): Making It in America: Work, Education, and Social Structure, in: Harvey Kantor/David Tyack (Hg.), Work, Youth, and Schooling, Stanford: Stanford University Press, S. 142-179.

Hollingsworth, J. Rogers/Lindberg, Leon N. (1985): The governance of the American economy: the role of markets, clans, hierarchies, and associative behaviour, in: Wolfgang Streeck/Philippe C. Schmitter (Hg.), Private Interest Governments Beyond Market and State, London: Sage, S. 221-254.

Human Resources Development Institute of AFL-CIO (1998): Unions in School-toCareers: Friend or Foe?, in: Techniques - Making Education \& Career Connections, Vol. 73, Nr. 1, S. 20-23.

Immergut, Ellen M. (1992): Health Politics. Interests and Institutions in Western Europe, Cambridge: Cambridge University Press.

Industriegewerkschaft Druck und Papier (1975): Mitbestimmung der Betriebsräte und Betriebsjugendvertreter bei der Durchführung der Berufsbildung, Schriftenreihe für Betriebsräte, Heft 14, Stuttgart.

Institut für Arbeitsmarkt- und Berufsforschung (1998a): Nachfrage nach Ausbildungsplätzen steigt auch 1998, IABkurzbericht Nr. 7/14.4..

Institut für Arbeitsmarkt- und Berufsforschung (1998b): Erhebliche Unterschiede in den Beschäftigungs- und Ausbildungserwartungen in West- und Ostdeutschland, Beschäftigungstrends Nr. 8/Mai.

Institut für Arbeitsmarkt- und Berufsforschung (2000a): Anwerbung kann Ausbildung nicht ersetzen, IABkurzbericht Nr. 3/4.4..

Institut für Arbeitsmarkt- und Berufsforschung (2000b): Der amerikanische Weg - ein Modell für Deutschland?, IABkurzbericht Nr. 5/15.5..

IWD (1997): Von Rückzug keine Rede (http://www.iwkoeln.de/IWD/I-Archiv/IWD2897/I28-97-2.htm).

IWD (1998a): Berufsausbildungskosten (http://www.iwkoeln.de/IWD/I-Archiv/iwd3298/i32-98-s.htm).

IWD (1998b): Mit spitzem Stift rechnen (http://www.iwkoeln.de/IWD/I-Archiv/iwd4198/i41-98-2.htm).

IWD (1998c): Noch Plätze frei (http://www.iwkoeln.de/IWD/I-Archiv/iwd43-98/i4398-3.htm).

IWD (2000): Ausbildungsvergütungen - Flexible Lösungen gefragt (http://www.iwkoeln.de/IWD/i-archiv/iwd10-00/iwd10-00-8druck.htm).

IWD (2001): Tariflöhne - Noch ein gutes Stück Arbeit (http://www.iwkoeln.de/IWD/iarchiv/iwd08-01/iwd08-01-4druck.htm). 
IWD (2002): Berufsausbildung - Vieles neu im August, Vol. 28, Nr. 23/6. Juni, S. 7.

Jacoby, Daniel (1991): The Transformation of Industrial Apprenticeship in the United States, in: The Journal of Economic History, Vol. 51, Nr. 4, S. 887-910.

Janoski, Thomas/Hicks, Alexander M. (1994): Methodological Innovations in Comparative Political Economy: an Introduction, in: dies. (Hg.), The Comparative Political Economy of the Welfare State, Cambridge: Cambridge University Press, S. $1-27$.

Jansen, Rolf (1996): Strukturdiskrepanzen zwischen Ausbildung und Beschäftigung Ergebnisse aus der BIBB/IAB-Erhebung, in: Peter Diepold (Hg.), Berufliche Ausund Weiterbildung: Konvergenzen/Divergenzen, neue Anforderungen/alte Strukturen, BeitrAB 195, Nürnberg: Institut für Arbeitsmarkt- und Berufsforschung, S. 243-250.

Jaschner, Peter (1996): Berufliche Bildung, in: Carl-Ludwig Holtfrerich (Hg.), Wirtschaft USA. Strukturen, Institutionen und Prozesse, München: Oldenbourg, S. 177-212.

Jasper, William F. (1995): „Cradle to Grave”, in: The New American, Vol. 11, Nr. 15, S. 7-10.

Jennings, John F. (1992): Lessons Learned in Washington, D.C., in: Phi Delta Kappan, Vol. 74, Nr. 4, S. 303-307.

Jennings, John F. (1995): School Reform: The Making of Two New National Policies, in: ders. (Hg.), National Issues in Education - Goals 2000 and School-to-Work, Washington/DC: Institute for Educational Leadership, S. 185-202.

Jennings, John F. (1998): Why National Standards and Tests? Politics and the Quest for Better Schools, Thousand Oaks: Sage.

Jobs for the Future (1997): Understanding Attitudes About School-to-Career: A Review of Public Opinion Data, Boston: Jobs for the Future.

Johnson, Susan Moore (1984): Teacher Unions in Schools, Philadelphia: Temple University Press.

Joyce, John T. (1985): Codetermination, Collective Bargaining, and Worker Participation in Construction Industry, in: Thomas A. Kochan (Hg.), Challenges and Choices Facing American Labor, Cambridge/MA: MIT Press.

Jugendsekretariat des Allgemeinen Deutschen Gewerkschaftsbundes (1923): Gewerkschaften und Jugendbewegung. Das gewerkschaftliche Jugendprogramm und wichtiges Material für die gesamte Jugendarbeit der Gewerkschaften, Berlin: ADGB.

Kaelble, Hartmut (1971): Industrielle Interessenverbände vor 1914, in: Walter Rüegg/Otto Neuloh (Hg.), Zur soziologischen Theorie und Analyse des 19. Jahrhunderts, Vandenhoeck \& Ruprecht: Göttingen, S. 180-192.

Kamphausen, Georg (1992): Ideengeschichtliche Ursprünge und Einflüsse, in: Willi Paul Adams/Ernst-Otto Czempiel/Berndt Ostendorf/Kurt Shell/P. Bernd Spahn/Michael Zöller (Hg.), Länderbericht USA, Schriftenreihe Bd. 293/I, 2. Aufl., Bonn: Bundeszentrale für politische Bildung, S. 259-280.

Kanigel, Robert (1997): The One Best Way - Frederick Winslow Taylor and the Enigma of Efficiency, New York: Penguin Books. 
Kantor, Harvey (1982): Vocationalism in American Education: The Economic and Political Context, 1880-1930, in: Harvey Kantor/David Tyack (Hg.), Work, Youth, and Schooling, Stanford: Stanford University Press, S. 14-44.

Kantor, Harvey (1995): The Hollow Promise of Youth Apprenticeships, in: Lisa Delpit/Robert Lowe/Bob Peterson/Rita Tenorio (Hg.), Rethinking Schools - An Agenda for Change, New York: The New Press, S. 215-229.

Kantor, Harvey/Tyack, David (1982): Introduction - Historical Perspectives on Vocationalism in American Education, in: dies. (Hg.), Work, Youth, and Schooling, Stanford: Stanford University Press, S. 1-13.

Kassebaum, Nancy L. (1995): Concerns about School-to-Work, in: John F. Jennings (Hg.), National Issues in Education - Goals 2000 and School-to-Work, Washington/DC: Institute for Educational Leadership, S. 149-164.

Kath, Folkmar (2000): Berufsbildung zwischen Konflikt und Konsens - Drei Jahrzehnte Berufsbildungsgesetzgebung, in: Bundesinstitut für Berufsbildung (Hg.), 30 Jahre Berufsbildungs- und Arbeitsförderungsgesetzgebung - 30 Jahre Bundesinstitut für Berufsbildung, Dokumentation der Fachtagung vom 24./25.5.2000, Bielefeld: Bertelsmann, S. 78-92.

Katz, Michael B. (1988): The Origins of Public Education: A Reassessment, in: B. Edward McClellan/William J. Reese (Hg.), The Social History of American Education, Chicago: University of Illinois Press, S. 91-118.

Kaufhold, Karl Heinrich (1971): Das preußische Handwerk in der Zeit der Frühindustrialisierung - Eine Untersuchung nach den Preußischen Gewerbetabellen 1815-1858, in: Wolfram Fischer (Hg.), Beiträge zu Wirtschaftswachstum und Wirtschaftsstruktur im 16. und 19. Jahrhundert, Schriften des Vereins für Socialpolitik, Bd. 63, Berlin: Duncker \& Humblot, S. 169-193.

Kaufhold, Karl Heinrich (1978): Das Gewerbe in Preußen um 1800, Göttinger Beiträge zur Wirtschafts- und Sozialgeschichte, Bd. 2, Göttingen: Otto Schwartz \& Co.

Kaufhold, Karl Heinrich (1979): Das Handwerk zwischen Anpassung und Verdrängung, in: Hans Pohl (Hg.), Sozialgeschichtliche Probleme in der Zeit der Hochindustrialisierung (1870-1914), Paderborn: Ferdinand Schöningh, S. 103-142.

Kazis, Richard (1993): Improving the Transition from School to Work in the United States, Washington/DC: American Youth Policy Forum.

Kazis, Richard (2001): School-to-Work: Present and the Future. Interview (http://www.jff.org/pressreleases/kazisint.html).

Kazis, Richard/Sabonis, Peter (1980): CETA and the Private Sector Imperative, in: Social Policy, Vol. 10, Nr. 4, S. 6-12.

Kempf, Theo (1985): Theorie und Empirie betrieblicher Ausbildungsplatzangebote, Frankfurt/M.: Peter Lang.

Kern, Horst (1997): Vertrauensverlust und blindes Vertrauen: Integrationsprobleme im ökonomischen Handeln, in: Stefan Hradil (Hg.), Differenz und Integration - Die Zukunft moderner Gesellschaften, Frankfurt/M.: Campus, S. 271-282.

Kern, Horst/Sabel, Charles F. (1994): Verblaßte Tugenden: Zur Krise des deutschen Produktionsmodells, in: Niels Beckenbach/Werner van Treeck (Hg.), Umbrüche gesellschaftlicher Arbeit, Sonderband 9 der Sozialen Welt, Göttingen, S. 605-624. 
Kern, Horst/Schumann, Michael (1998): Kontinuität oder Pfadwechsel? Das deutsche Produktionsmodell am Scheideweg, in: Bruno Cattero (Hg.), Modell Deutschland Modell Europa: Probleme, Perspektiven, Europa- und Nordamerika-Studien, Bd. 4, Opladen: Leske+Budrich, S. 85-98.

Kern, Horst/Schumann, Michael (1984): Das Ende der Arbeitsteilung? Rationalisierung in der industriellen Produktion: Bestandsaufnahme, Trendbestimmung, München: Beck.

Kerschensteiner, Georg (1914): Der Begriff der staatsbürgerlichen Erziehung, 3. Aufl., Leipzig, Berlin: B. G. Teubner.

Kessler, Gerhard (1907): Die deutschen Arbeitgeberverbände, Leipzig: Duncker \& Humblot.

King, Desmond (1995): Actively Seeking Work? The Politics of Unemployment and Welfare Policy in the United States and Great Britain, Chicago: University of Chicago Press.

Kinkel, Steffen/Lay, Gunter/Schirrmeister, Elna/Wengel, Jürgen (2001): Die Arbeitswelt der Auszubildenden - zukunftsweisend oder von gestern? Facharbeiterausbildung in der Investitionsgüterindustrie, Fraunhofer Institut Systemtechnik und Innovationsforschung, Mitteilungen aus der Produktionsinnovationserhebung, Nr. 24, November.

Kinni, Theodore B. (1994): A Patchwork Quilt, in: Industry Week, Vol. 243, Nr. 4, S. 61-68.

Kirst, Michael W. (1995): Who's in Charge? Federal, State, and Local Control, in: Diane Ravitch/Maris A. Vinovskis (Hg.), Learning from the Past - What History Teaches Us about School Reform, Baltimore: Johns Hopkins University Press, S. $25-56$.

Klerman, Jacob A./Karoly, Lynn A. (1995): The Transition to Stable Employment: The Experience of U.S. Youth in Their Early Labor Market Career, National Center for Research in Vocational Education, Santa Monica: RAND.

Kliebard, Herbert M. (1995): The Struggle for the American Curriculum, 1893-1958, 2. Aufl., New York: Routledge.

Kliebard, Herbert M. (1999): Schooled to Work. Vocationalism and the American Curriculum, 1876-1946, New York: Teachers College Press.

Kloas, Peter-Werner (1996): 15 Jahre Benachteiligtenförderung - eine Zwischenbilanz, in: Berufsbildung in Wissenschaft und Praxis (BWP), Vol. 25, Nr. 2, S. 11-17.

Knips, Achim (1996): Deutsche Arbeitgeberverbände der Eisen- und Metallindustrie, 1888-1914, Stuttgart: Franz Steiner.

Koch, Richard (1998): Duale und schulische Berufsausbildung zwischen Bildungsnachfrage und Qualifikationsbedarf. Ein deutsch-französischer Vergleich, Bielefeld: Bertelsmann.

Koch, Richard/Reuling, Jochen (1994): The responsiveness and regulation of training capacity and quality, in: OECD, Vocational Training in Germany: Modernisation and Responsiveness, Paris: OECD, S. 69-121.

Kochan, Thomas A./Katz, Harry C./McKersie, Robert B. (1986): The Transformation of American Industrial Relations, New York: Basic Books. 
Kock, Klaus (1994): Zur Soziologie des betriebsinternen Arbeitsmarkts, München: Rainer Hampp.

König, Ingrid (1981): Handelskammern zwischen Kooperation und Konzentration, Köln: Rheinisch-Westfälisches Wirtschaftsarchiv.

Körzel, Randolf (1996): Berufsbildung zwischen Gesellschafts- und Wirtschaftspolitik, Frankfurt/M: Gesellschaft zur Förderung arbeitsorientierter Forschung und Bildung.

Konietzka, Dirk (1999): Die Verberuflichung von Marktchancen - Die Bedeutung des Ausbildungsberufs für die Plazierung im Arbeitsmarkt, in: Zeitschrift für Soziologie, Jg. 28, Nr. 5, S. 379-400.

Korpi, Walter (1978): The Working Class in Welfare Capitalism. Work, Unions and Politics in Sweden, London: Routledge.

Korpi, Walter (1983): The Democratic Class Struggle, London: Routledge.

Kraegeloh, Wolfgang/Knopp, Anton (1998): Berufsbildungsgesetz, 4. Aufl., Köln: Heymanns.

Kuhlemann, Frank-Michael (1992): Modernisierung und Disziplinierung. Sozialgeschichte des preußischen Volksschulwesens 1794-1872, Göttingen: Vandenhoeck \& Ruprecht.

Kuratorium der Deutschen Wirtschaft für Berufsbildung (1995): Finanzierung der Berufsbildung, Bonn.

Lafer, Gordon (1994): The Politics of Job Training: Urban Poverty and the False Promise of JTPA, in: Politics \& Society, Vol. 22, Nr. 3, S. 349-388.

Lane, A.T. (1987): Solidarity or Survival? American Labor and European Immigrants, 1830-1924, Westport/Connecticut: Greenwood Press.

Laur-Ernst, Ute (2000): Strukturwandel und Reformansätze in der Berufsbildung: Welche Auswirkungen haben sie auf Jugendliche mit schlechten Startchancen?, Beitrag zur Projektkonferenz INKA II „Innovative Konzepte in der Ausbildungsvorbereitung benachteiligter Jugendlicher“", INBAS, Nienburg (www.bibb.de/publikat/fram_pu2.htm).

Lazerson, Marvin/Grubb, W. Norton (1975): Rally `Round the Workplace: Continuities and Fallacies in Career Education, in: Harvard Educational Review, Vol. 45, S. 451-474.

Legislative Summary (1994): Goals 2000 Education Reform; School to Work Transition, in: Congressional Quarterly Weekly Report, Vol. 52, Nr. 43, S. 31843185 .

Lemmermöhle-Thüsing, Doris (1990): Die Bedeutung des Geschlechts für den Zusammenhang von beruflicher Qualifikation und Arbeitsmarktchancen, in: Frank Strikker/Dieter Timmermann (Hg.), Berufsausbildung und Arbeitsmarkt in den neunziger Jahren, Frankfurt/M.: Peter Lang, S. 67-81.

Lepsius, M. Rainer (1990): Interessen, Ideen und Institutionen, Opladen: Westdeutscher Verlag.

Lerman, Robert I./Pouncy, Hillard (1990): The compelling case for youth apprenticeships, in: The Public Interest, Nr. 101, Fall, S. 62-77. 
Lerman, Robert I./Pouncy, Hillard (1992): Are U.S. businesses likely to embrace youth apprenticeship?, in: Congressional Quarterly Researcher, Vol. 2, Nr. 39, S. 921.

Levine, Bruce (1992): The Spirit of 1848. German Immigrants, Labor Conflict, and the Coming of the Civil War, Chicago: University of Illinois Press.

Levitan, Sar A./Gallo, Frank (1988): A Second Chance: Training for Jobs, Kalamazoo: W.E. Upjohn Institute for Employment Research.

Lewis, Anne C. (1990): Remembering the ,Forgotten Half ${ }^{\star}$, in: Phi Delta Kappan, Vol. 71, Nr. 10, S. 748-749.

Lewis, Anne C. (1993a): The Administration's Education Agenda, in: Phi Delta Kappan, Vol. 75, Nr. 3, S. 196-197.

Lewis, Anne C. (1993b): Why Wait to Improve the School-to-Work Transition?, in: Phi Delta Kappan, Vol. 74, Nr. 7, S. 508-509.

Lewis, Theodore (1994): Bridging the liberal/vocational divide: An examination of recent British and American versions of an old debate, in: Oxford Review of Education, Vol. 20, Nr. 2, S. 199-218.

L'Hoest, Raphael (1998): Vocational Education and Job Training in the US-Labor Market - The Implementation of the School-to-Work Opportunities Act (STWOA), Kölner Schriften zur Sozial- und Wirtschaftspolitik 33, Regensburg: Transfer Verlag.

Lichter, Jörg (1996): Die Handelskammern und der Deutsche Handelstag im Interessengruppensystem des Deutschen Kaiserreichs, Kölner Vorträge und Abhandlungen zur Sozial- und Wirtschaftsgeschichte, Heft 40, Köln.

Lieshout, Harm van (1997): The Transferability of German Apprenticeship: The Case of Wisconsin, Paper for the IIRA European Regional Congress, $26^{\text {th }}-29^{\text {th }}$ August, Dublin.

Lipsmeier, Antonius (1983): Berufsbildungspolitik der 70er Jahre im Kontext der Bildungspolitik, in: ders. (Hg.), Berufsbildungspolitik in den 70er Jahren. Eine kritische Bestandsaufnahme für die 80er Jahre, Wiesbaden: Franz Steiner, S. 1-14.

Lith, Ulrich van (1997): Finanzierung der Berufsausbildung, in: Wirtschaftsdienst, Vol. 77, Nr. 7, S. 400-407.

Lively, Kit (1993a): Maine's Month-Old Youth Apprenticeships Show How a National Plan Might Work, in: The Chronicle of Higher Education, Vol. 39, Nr. 30, S. A20A23.

Lively, Kit (1993b): Many States Welcome Clinton's Job-Training Plan, but Some Say It May Not Lead to Significant Change, in: The Chronicle of Higher Education, Vol. 40, Nr. 3, S. A27-A30.

Lith, Ulrich van (1997): Finanzierung der Berufsausbildung, in: Wirtschaftsdienst, Vol. 77, Nr. 7, S. 400-407.

Loeffelholz, Hans Dietrich von (1998): Die Unternehmens- und Arbeitsmarktverfassung, in: Willi Paul/Peter Lösche (Hg.), Länderbericht USA Geschichte, Politik, Geographie, Wirtschaft, Gesellschaft, Kultur, Schriftenreihe Bd. 357, 3. Aufl., Bonn: Bundeszentrale für politische Bildung, S. 547-559. 
Lösche, Peter (1992): Die Organisationsstruktur der Gewerkschaften und das System der Arbeitsbeziehungen, in: Willi Paul Adams/Ernst-Otto Czempiel/Berndt Ostendorf/Kurt L. Shell/Bernd Spahn/Michael Zöller (Hg.), Länderbericht USA, Schriftenreihe Bd. 293/I, 2. Aufl., Bonn: Bundeszentrale für politische Bildung, S. 508-518.

Lösche, Peter (1998): Verbände, Gewerkschaften und das System der Arbeitsbeziehungen, in: Willi Paul Adams/Peter Lösche (Hg.), Länderbericht USA Geschichte, Politik, Geographie, Wirtschaft, Gesellschaft, Kultur, Schriftenreihe Bd. 357, 3. Aufl., Bonn: Bundeszentrale für politische Bildung, S. 340-374.

Lovejoy, Bret (1993): Washington Watch, in: Vocational Education Journal, Vol. 68, Nr. 3, S. 14.

Lowe, Robert (1995): Teachers Through History: The Myth of a Golden Age, in: Lisa Delpit/Robert Lowe/Bob Peterson/Rita Tenorio (Hg.), Rethinking Schools - An Agenda for Change, New York: The New Press, S. 240-245.

Lugg, Catherine A. (1996): For God and Country - Conservatism and American School Policy, New York: Peter Lang.

Lutz, Burkhart (1994): The Difficult Rediscovery of „Professionalism”, in: OECD, Apprenticeship: Which Way Forward?, Paris: OECD, S. 19-28.

Lutz, Burkart (1987): Arbeitsstruktur und betriebliche Arbeitskräftestrategie. Eine theoretisch-historische Skizze zur Entstehung betriebszentrierter Arbeitsmarktsegmentation, Frankfurt/M.: Campus.

Lutz, Burkart/Sengenberger, Werner (1974): Arbeitsmarktstrukturen und öffentliche Arbeitspolitik. Eine kritische Analyse von Zielen und Instrumenten, Göttingen: Otto Schwartz \& Co..

Lynch, Lisa M. (1993): The Economics of Youth Training in the United States, in: The Economic Journal, Vol. 103, September, S. 1292-1302.

Lynch, Lisa M. (1999): The Transition from initial Education to the Labour Market: recent Experience in the United States, in: OECD (Hg.), Preparing Youth for the $21^{\text {st }}$ Century. The Transition from Education to the Labour Market, Proceedings of the Washington D.C. Conference, 23-24 February, Paris: OECD, S. 289-304.

Lynn, Leonard H./McKeown, Timothy J. (1988): Organizing Business: Trade Associations in America and Japan, Washington, D.C.: American Enterprise Institute for Public Policy Research.

Macduffie, John P./Kochan, Thomas A. (1995): Do U.S. Firms Invest Less in Human Resources? Training in the World Auto Industry, in: Industrial Relations, Vol. 34, Nr. 2, S. 147-168.

MacLaury, Judson (1988): History of DOL, 1913-1988, U.S. Department of Labor (http://www.dol.gov/dol/asp/public/programs/history/hs75menu. htm).

Magaziner, Ira C./Patinkin, Mark (1989): The Silent War - Inside the Global Business Battles shaping America's Future, New York: Random House.

Maier, Harry (1994): Bildungsökonomie: Interdependenzen von Bildungs- und Beschäftigungssystem, Stuttgart: Schäffer-Poeschel.

Marshall, Ray (1997): School-to-Work processes in the United States, in: Ruby Takanishi/David A. Hamburg (Hg.), Preparing adolescents for the twenty-first 
century - Challenges facing Europe and the United States, Cambridge: Cambridge University Press, S. 195-226.

Marshall, T.H. (1964): Class, Citizenship, and Social Development, New York: Doubleday.

Martin, Cathie Jo (2000): Stuck in Neutral - Business and the Politics of Human Capital Investment Policy, Princeton: Princeton University Press.

Masci, David (1994): School-to-Work Program easily passes Senate, in: Congressional Quarterly Weekly Report, Vol. 52, Nr. 6, S. 331.

Mast, Peter (1989): Preußische Schulreform zwischen politischer Restauration und wirtschaftlicher Notwendigkeit 1817-1837. Zur Bildungspolitik unter Minister von Altenstein und Johannes Schulze, in: Karl-Ernst Jeismann (Hg.), Bildung, Staat, Gesellschaft im 19. Jahrhundert. Mobilisierung und Disziplinierung, Stuttgart: Franz Steiner, S. 128-143.

Maurice, Marc/Sellier, Francois/Silvestre, Jean-Jacques (1986): The Social Foundations of Industrial Power - A Comparison of France and Germany, Cambridge/MA: MIT Press.

Mazzoni, Tim L. (1994): State policy-making and school reform: influences and influentials, in: Jay D. Scribner/Donald H. Layton (Hg.), The Study of Educational Politics, The Commemorative Yearbook of the Politics of Education Association (1969-1994), London: Taylor\&Francis, S. 53-73.

McDonnell, Lorraine M./Pascal, Anthony (1988): Teacher Unions and Educational Reform, Center for Policy Research in Education/Center for the Study of the Teaching Profession, Santa Monica: RAND.

McDonnell, Lorraine M./Grubb, Norton W. (1991): Education and Training for Work: The Policy Instruments and the Institutions, National Center for Research in Vocational Education (NCRVE), Santa Monica: RAND.

McKenna, Joseph F. (1993a): Youth's Got A Friend In Pennsylvania, in: Industry Week, Vol. 242, Nr. 3, S. 16-20.

McKenna, Joseph F. (1993b): A ,G.I. Bill' For The Workforce, in: Industry Week, Vol. 242, Nr. 4, S. 44.

McKenna, Joseph F. (1993c): Business Gets A Buy-In, in: Industry Week, Vol. 242, Nr. 11, S. 8.

McKenna, Joseph F. (1993d): Rally 'Round Apprenticeship, in: Industry Week, Vol. 242, Nr. 12, S. 24-26.

Meyer, Folkert (1976): Schule der Untertanen. Lehrer und Politik in Preußen 18481900, Hamburg: Hoffmann und Campe.

Meyer, Heinz-Dieter (1996a): Schulwahlfreiheit kontra Chancengleichheit, in: Zeitschrift für Pädagogik, Vol. 42, Nr. 6, S. 889-901.

Meyer, Heinz-Dieter (1996b): Eine Nation im Konflikt: Das amerikanische Bildungssystem zwischen öffentlichem und privatem Bildungsanspruch, in: Herbert Dittgen/Michael Minkenberg (Hg.), Das amerikanische Dilemma - Die Vereinigten Staaten nach dem Ende des Ost-West-Konflikts, Paderborn: Ferdinand Schöningh, S. 269-289. 
Meyer, Heinz-Dieter (1997): Local Control und Schulautonomie, in: Bildung und Erziehung, Vol. 50, Nr. 2, S. 137-153.

Michalke, Friedhelm (1989): Politik und Gewerkschaften in den USA - Eine Untersuchung zur politischen Rolle der Lehrergewerkschaften in den Vereinigten Staaten, Diss., Frankfurt/M.: Peter Lang.

Mickler, Otfried (1981): Facharbeit im Wandel. Rationalisierung im industriellen Produktionsprozeß, Frankfurt/M.: Campus.

Mill, John Stuart (1874): A System of Logic. Ratiocinative and inductive: being a connected view of the principles of evidence and the methods of scientific investigation, New York: Harper \& Brothers.

Miller, William H. (1994): That Other Clinton Initiative, in: Industry Week, Vol. 243, Nr. 7, S. 59.

Moore, Mary T./Waldman, Zev (1994): Opportunities or Obstacles? A Map of Federal Legislation Related to the School-to-Work Initiative, in: U.S. Department of Education - Office of Research (Hg.), School-to-Work - What Does Research Say About It?, Washington/DC: U.S. Government Printing Office, S. 131-155.

Morgan, Stephen (1994): What Employers Want: Youth Labor Markets and School-toWork Transition Programs, EQW Issues Nr. 6, National Center on the Educational Quality of the Workforce, Philadelphia: University of Pennsylvania.

Morone, James A. (1990): The Democratic Wish - Popular Participation and Limits of American Government, New York: Basic Books.

Moskal, Brian S. (1991): Apprenticeships: Old Cure For New Labor Shortage?, in: Industry Week, Vol. 240, Nr. 9, S. 30-35.

Moskal, Brian S. (1993): Apprenticeships: A Few Good Crusaders, in: Industry Week, Vol. 242, Nr. 1, S. 23-24.

Müller, Walter (1999): Wandel in der Bildungslandschaft Europas, in: Wolfgang Glatzer/Ilona Ostner (Hg.), Deutschland im Wandel, Opladen: Leske+Budrich, S. 337-356.

Müller, Walter/Shavit, Yossi (1998): The Institutional Embeddedness of Stratification Process: A Comparative Study of Qualifications and Occupations in Thirteen Countries, in: Yossi Shavit/Walter Müller (Hg.), From School to Work - A Comparative Study of Educational Qualifications and Occupational Destinations, Oxford: Clarendon Press, S. 1-48.

Müller-Jentsch, Walther (1997): Soziologie der Industriellen Beziehungen, 2. Aufl., Frankfurt/M: Campus.

Münch, Joachim (1989): Berufsbildung und Bildung in den USA. Bedingungen, Strukturen, Entwicklungen und Probleme. Ausbildung, Fortbildung, Personalentwicklung 28, Berlin: Erich Schmidt.

Mundo, Philip A. (1992): Interest Groups - Cases and Characteristics, Chicago: NelsonHall Publishers.

Muth, Wolfgang (1985): Berufsausbildung in der Weimarer Republik, Stuttgart: Franz Steiner. 
Nadel, Henri (1997): The Economics of education and training in the face of changing production and employment structures, in: Ruby Takanishi/David Hamburg (Hg.), Preparing adolescents for the twenty-first century - Challenges facing Europe and the United States, Cambridge: Cambridge University Press, S. 177-194.

Nagler, Jörg (1992): Territoriale Expansion, Sklavenfrage, Sezessionskrieg, Rekonstruktion, 1815-1877, in: Willi Paul Adams/Ernst-Otto Czempiel/Berndt Ostendorf/Kurt Shell/P. Bernd Spahn/Michael Zöller (Hg.), Länderbericht USA, Schriftenreihe Bd. 293/I, 2. Aufl., Bonn: Bundeszentrale für politische Bildung, S. 87-118.

National Education Association (1998a): NEA 1998-99 Resolutions: B-33. Vocational Education (http://www.nea.org/resolutions/98/98b-33.html).

National Education Association (1998b): NEA 1998-99 Resolutions: B-26. Youth and Adult Training Programs (http://www.nea.org/resolutions/98/98b-26.html).

National Center on the Educational Quality of the Workforce (1994): Making Good Jobs for Young People a National Priority, EQW Policy Statement Nr. 1, Philadelphia: University of Pennsylvania.

National Governors Association (1997): Principles for State-Federal Relations (http://www. nga.org/Pubs/Policies/EC/prinpoli.asp).

National Governors Association (1998): Governors' Principles to Ensure Workforce Excellence (http://www.nga.org/Pubs/Policies/HR/hr01.asp).

National School-to-Work Office (1996): Implementation of the School-to-Work Opportunities Act of 1994, Report to Congress, Washington/DC: U.S. Government Printing Office.

National School-to-Work Office (1997): Implementation of the School-to-Work Opportunities Act of 1994, Report to Congress, Executive Summary, Washington/DC: U.S. Government Printing Office.

National School-to-Work Office (1998): Implementation of the School-to-Work Opportunities Act of 1994, Report to Congress, Washington/DC: U.S. Government Printing Office.

Neugebauer, Wolfgang (1989): Bildung, Erziehung und Schule im Alten Preußen. Ein Beitrag zum Thema: „Nichtabsolutistisches im Absolutismus“, in: Karl-Ernst Jeismann (Hg.), Bildung, Staat, Gesellschaft im 19. Jahrhundert. Mobilisierung und Disziplinierung, Stuttgart: Franz Steiner, S. 25-43.

OECD (1993): Industry Training in Australia, Sweden and the United States, Paris: OECD.

OECD (1999): Preparing Youth for the $21^{\text {st }}$ Century. The Transition from Education to the Labour Market, Proceedings of the Washington D.C. Conference, 23-24 February, Paris: OECD.

Office of Technology Assessment (1990): Worker Training - Competing in the New International Economy, OTA-ITE-457, Washington/DC: U.S. Government Printing Office.

Office of Technology Assessment (1995): Learning to Work - Making the Transition from School to Work, OTA-EHR-637, Washington/DC: U.S. Government Printing Office. 
Ohneis, Gerhard (1990): Wandel in den Zielsetzungen der Deutschen Unternehmerverbände, Diss., Stuttgart.

Olson, Mancur (1992): Die Logik des kollektiven Handelns. Kollektivgüter und die Theorie der Gruppen, 3. Aufl., Tübingen: Mohr.

O’Neil, John (1992): Preparing for the Changing Workplace, in: Educational Leadership, Vol. 49, Nr. 6, S. 6-9.

Orloff, Ann Shola (1988): The Political Origins of America's Belated Welfare State, in: Margaret Weir/Ann Shola Orloff/Theda Skocpol (Hg.), The Politics of Social Policy in the United States, Princeton: Princeton University Press, S. 37-80.

Ornstein, Norman J./Elder, Shirley (1978): Interest Groups, Lobbying and Policymaking, Washington: Congressional Quarterly Press.

Ossenbühl, Fritz (1985): Zur verfassungsrechtlichen Pflicht der Arbeitgeber, betriebliche Ausbildungsplätze bereitzustellen. Rechtsgutachten im Auftrag des Bundesministers für Bildung und Wissenschaft, Bad Honnef: Bock.

Osterman, Paul (1980): Getting Started - The Youth Labor Market, Cambridge/MA: MIT Press.

Osterman, Paul (1984): Introduction: The Nature and Importance of Internal Labor Markets, in: ders. (Hg.), Internal Labor Markets, Cambridge/MA: MIT Press, S. 122.

Osterman, Paul (1995a): Involving Employers in School-to-Work Programs, in: Thomas R. Bailey (Hg.), Learning to Work. Employer Involvement in School-to-Work Transition Programs, Washington/DC: Brookings Institution, S. 75-87.

Osterman, Paul (1995b): Skill, Training, and Work Organization in American Establishments, in: Industrial Relations, Vol. 34, Nr. 2, S. 125-146.

Osterman, Paul (1999): Securing Prosperity. The American Labor Market How It Has Changed and What to Do about It, Princeton: Princeton University Press.

Osterman, Paul (2000): Work Reorganization in an Era of Restructuring: Trends in Diffusion and Effects on Employee Welfare, in: Industrial and Labor Relations Review, Vol. 53, Nr. 2, S. 179-196.

Ostner, Ilona (1997): Beruflichkeit und Sozialpolitik, in: G. Günter Voß/Hans J. Pongratz (Hg.), Subjektorientierte Soziologie, Karl Martin Bolte zum siebzigsten Geburtstag, Opladen: Leske+Budrich, S. 73-94.

Pätzold, Günter (Hg.) (1982): Quellen und Dokumente zur Geschichte des Berufsbildungsgesetzes: 1875-1981, Köln: Böhlau.

Pätzold, Günter (Hg.) (1991): Quellen und Dokumente zur betrieblichen Berufsbildung 1945-1990, Köln: Böhlau.

Parker, Eric (1996): The Failure of Youth Apprenticeship in the United States: Lessons of an Historical Anomaly in a Laboratory of Democracy, Working Paper 104, New Brunswick: Center for Urban Policy Research.

Parnell, Dale (1985): The Neglected Majority, Washington/DC: Community College Press.

Paul-Kohlhoff, Angela (1999): Mitbestimmte Barrieren in der Berufsbildung, in: Die Mitbestimmung, Nr. 4, S. 33-35. 
Pennington, Hilary (1995): The Evolution of the School-to-Work Opportunities Act, in: John F. Jennings (Hg.), National Issues in Education - Goals 2000 and School-toWork, Washington/DC: Institute for Educational Leadership, S. 165-182.

Peters, B. Guy (1999): Institutional Theory in Political Science. The 'New Institutionalism', London: Pinter.

Peterson, Bob (1995): Which Side Are You On? The Role of Teachers Unions in School Reform, in: Lisa Delpit/Robert Lowe/Bob Peterson/Rita Tenorio (Hg.), Rethinking Schools - An Agenda for Change, New York: The New Press, S. 253263.

Peuckert, Rüdiger (1995): Stigma, in: Bernhard Schäfers (Hg.), Grundbegriffe der Soziologie, 4. Aufl., Opladen: Leske+Budrich, S. 354-356.

Phillips, David (1993): Borrowing Educational Policy, in: David Finegold/Laurel McFarland/William Richardson (Hg.), Something Borrowed, Something Learned? The Transatlantic Market in Education and Training Reform, Washington/DC: Brookings Institutions, S. 13-20.

Pierson, Christopher (1991): Beyond the Welfare State? The New Political Economy of Welfare, Cambridge: Polity Press.

Pierson, Paul (1993): When Effect becomes Cause. Policy Feedback and Political Change, in: World Politics, Vol. 45, Nr. 4, S. 595-628.

Pierson, Paul/Skocpol, Theda (2000): Historical Institutionalism in Contemporary Political Science, Paper prepared for presentation at the American Political Science Association Meetings, Washington D.C., August $30^{\text {th }}-$ September $2^{\text {nd }}$.

Piore, Michael J. (1978): Lernprozesse, Mobilitätsketten und Arbeitsmarktsegmente, in: Werner Sengenberger (Hg.), Der gespaltene Arbeitsmarkt. Probleme der Arbeitsmarktsegmentation, Frankfurt/M.: Campus, S. 67-98.

Pole, J. R. (1993): The Pursuit of Equality in American History, 2. Aufl., Berkeley: University of California Press.

Pütz, Helmut (1993): Integration der Schwachen = Stärke des dualen Systems. Förderung der Berufsausbildung von benachteiligten Jugendlichen - Neue Strukturen und Konzeptionen, Berlin: Bundesinstitut für Berufsbildung.

Raddatz, Rolf (1983): Das duale System der Berufsbildung - Stabilisierung und Bewährung, in: Antonius Lipsmeier (Hg.), Berufsbildungspolitik in den 70er Jahren. Eine kritische Bestandsaufnahme für die 80er Jahre, Wiesbaden: Franz Steiner, S. 101-109.

Raddatz, Rolf (2000): Berufsbildung im 20. Jahrhundert. Eine Zeittafel, Bielefeld: Bertelsmann.

Radin, Beryl A./Hawley, Willis D. (1988): The Politics of Federal Reorganization Creating the U.S. Department of Education, New York: Pergamon Press.

Ragin, Charles (1987): The Comparative Method. Moving beyond qualitative and quantitative Strategies, Berkeley: University of California Press.

Ragin, Charles (1994): Introduction to Qualitative Comparative Analysis, in: Thomas Janoski/Alexander M. Hicks (Hg.), The Comparative Political Economy of the Welfare State, Cambridge: Cambridge University Press, S. 299-319. 
Ray, Carol A./Mickelson, Roslyn A. (1990): Corporate Leaders, Resistant youth, and School Reform in Sunbelt City: The Political Economy of Education, in: Social Problems, Vol. 37, Nr. 2, S. 178-190.

Rappen, Hermann (1998): Ordnungspolitische Aspekte von Staat und Wirtschaft, in: Willi Paul Adams/Peter Lösche (Hg.), Länderbericht USA - Geschichte, Politik, Geographie, Wirtschaft, Gesellschaft, Kultur, Schriftenreihe Bd. 357, 3. Aufl., Bonn: Bundeszentrale für politische Bildung, S. 518-523.

Rawls, John (1993): Political Liberalism, New York: Columbia University Press.

Reese, William J. (1995): The Origins of the American High School, New Haven: Yale University Press.

Reich, Robert B. (1992): The Work of Nations. Preparing Ourselves for $21^{\text {st }}$-Century Capitalism, New York: Vintage.

Reich, Robert B. (1995): Building a Framework for a School-to-Work Opportunities System, in: John F. Jennings (Hg.), National Issues in Education - Goals 2000 and School-to-Work, Washington/DC: Institute for Educational Leadership, S. 119-130.

Reich, Robert B. (1997): Locked in the Cabinet, New York: Alfred A. Knopf.

Resource Bulletin (1996): Engaging Organized Labor in School-to-Work Systems (http://www.stw.ed.gov/factsht/bull1396.htm).

Reuter, Manfred (1975): Berufliche Bildung aus Gewerkschaftssicht. Prinzipien, Stellungnahmen und Konzeptionen des Deutschen Gewerkschaftsbundes seit 1949, Köln: Europäische Verlagsanstalt.

Rinneberg, Karl-Jürgen (1985): Das betriebliche Ausbildungswesen in der Zeit der industriellen Umgestaltung Deutschlands, Köln, Wien: Böhlau Verlag.

Rist, Ray C./Joyce, M. Kathleen (1994): Neue Trends in der US-amerikanischen Berufsbildung, in: Bildung und Erziehung, Vol. 47, Nr. 3, S. 329-342.

Robertson, David Brian/Waltman, Jerold L. (1993): The Politics of Policy Borrowing, in: David Finegold/Laurel McFarland/William Richardson (Hg.), Something Borrowed, Something Learned? The Transatlantic Market in Education and Training Reform, Washington/DC: Brookings Institution, S. 21-44.

Roditi, Hannah F. (1992): High Schools for Docile Workers, in: The Nation, Vol. 254, Nr. 10, S. 340-343.

Rorabaugh, William J. (1986): The Craft Apprentice: From Franklin to the Machine Age in America, New York: Oxford University Press.

Ruth, Klaus (2002): Beruflich kodiertes oder organisational gebundenes Wissen als Erfolgsfaktor für Innovationen?, in: Matthias Wingens/Reinhold Sackmann (Hg.), Bildung und beruf. Ausbildung und berufsstruktureller Wandel in der Wissensgesellschaft, Weinheim: Juventa, S. 101-120.

Ryan, Paul (1984): Job Training, Employment Practices, and the Large Enterprise: The Case of Costly Transferable Skills, in: Paul Osterman (Hg.), Internal Labor Markets, Cambridge/MA: MIT Press, S. 191-230.

Sako, Mari (1994): The Role of Employers and Unions in facilitating the Transition to Employment and Further Learning, in: OECD, Vocational Education and Training for Youth: Towards Coherent Policy and Practice, Paris: OECD, S. 115-142. 
Salzman, Harold (1998): Restructuring and Skill Needs: Will Firms train?, in: Annals of the American Academy of Political and Social Science, Vol. 559, September, S. 125-141.

Schaub, Günter (2000): Arbeitsrechts-Handbuch: systematische Darstellung und Nachschlagewerk für die Praxis, 9. Aufl., München: Beck.

Schelsky, Helmut (1965): Die Bedeutung des Berufs in der modernen Gesellschaft, in: ders. (Hg.), Auf der Suche nach Wirklichkeit. Gesammelte Aufsätze, Düsseldorf: Eugen Diederichs, S. 238-249.

Scherrer, Christoph (1996): Gewerkschaftsbewegung und Arbeitsbeziehungen, in: CarlLudwig Holtfrerich (Hg.), Wirtschaft USA - Strukturen, Institutionen und Prozesse, München: Oldenbourg, S. 234-254.

Schleunes, Karl A. (1989): Schooling and Society. The Politics of Education in Prussia and Bavaria 1750-1900, Oxford: Berg.

Schlüter, Anne/Stratmann, Karlwilhelm (Hg.) (1985): Quellen und Dokumente zur betrieblichen Berufsbildung 1869-1918. Quellen und Dokumente zur Geschichte der Berufsbildung in Deutschland B/2, Köln, Wien: Böhlau Verlag.

Schmid, Günther (1992): Flexible Koordination: Die Zukunft des dualen Systems aus arbeitsmarktpolitischer Sicht, in: Berufsbildung, Nr. 1, S. 55-59.

Schmidt, Hermann (1996): Berufsausbildung in USA: Über die Chancen dualer Berufsausbildungsgänge, in: ibv, Nr. 47, 20. November, S. 2999-3001.

Schmidt, Ingo (1998): Die Wettbewerbsordnung, in: Willi Paul Adams/Peter Lösche (Hg.), Länderbericht USA - Geschichte, Politik, Geographie, Wirtschaft, Gesellschaft, Kultur, Schriftenreihe Bd. 357, 3. Aufl., Bonn: Bundeszentrale für politische Bildung, S. 524-535.

Schmitter, Philippe C./Streeck, Wolfgang (1999): The Organization of Business Interests: Studying the Associative Action of Business in Advanced Industrial Societies, MPIfG Discussion Paper 99/1, Köln.

Schneeberger, Arthur (1997): Nutzen und Kosten der Lehrlingsausbildung, in: Wirtschaftspolitische Blätter 3-4, S. 357-366.

Schneider, Michael (1987): Höhen, Krisen und Tiefen. Die Gewerkschaften in der Weimarer Republik 1918 bis 1933, in: Ulrich Bosdorf (Hg.), Geschichte der deutschen Gewerkschaften von den Anfängen bis 1945, Köln: Bund-Verlag, S. 279446.

Schömann, Klaus (1997): Labour Market Transitions and Dynamics of Transitions in Germany, in: Annette Jobert/Catherine Marry/Lucie Tanguy/Helen Rainbird (Hg.), Education and Work in Great Britain, Germany and Italy, London: Routledge, S. 93-111.

Schömann, Klaus/Hilbert, Christoph (1998): The Youth Labour Market in Germany - A new target group for German labour market policies?, in: Vierteljahrshefte zur Wirtschaftsforschung (DIW), 64. Jg., Nr. 4, S. 272-285.

Schönhoven, Klaus (1987): Die Gewerkschaften als Massenbewegung im Wilhelminischen Kaiserreich 1890 bis 1918, in: Ulrich Bosdorf (Hg.), Geschichte der deutschen Gewerkschaften von den Anfängen bis 1945, Köln: Bund-Verlag, S. 167-278. 
School-to-Work Opportunities Act (1994): H.R. 2884 One Hundred Third Congress of the United States of America, Public Law 103-239, 108 Stat 568 (http://www.stw.ed.gov/factsht/act.htm).

Schriewer, Jürgen (1986): Intermediäre Instanzen, Selbstverwaltung und berufliche Ausbildungsstrukturen im historischen Vergleich, in: Zeitschrift für Pädagogik, Vol. 32, Nr. 1, S. 69-90.

Schütte, Friedhelm (1992): Berufserziehung zwischen Revolution und Nationalsozialismus. Ein Beitrag zur Bildungs- und Sozialgeschichte der Weimarer Republik, Weinheim: Deutscher Studien Verlag.

Schwengler, Barbara (1998): Neueste Daten zum Ausbildungsverhalten der Betriebe, in: Personal, Vol. 50, Nr. 7, S. 314-317.

Scott, W. Richard (1995): Institutions and Organizations, Thousands Oaks: Sage Publications.

Seeleib-Kaiser, Martin (1993): Amerikanische Sozialpolitik: Politische Diskussion und Entscheidungen der Reagan-Ära, Opladen: Leske+Budrich.

Sehrbrock, Ingrid (2000): Gewerkschaften - Motor der beruflichen Bildung?!, in: Bundesinstitut für Berufsbildung (Hg.), 30 Jahre Berufsbildungs- und Arbeitsförderungsgesetzgebung - 30 Jahre Bundsinstitut für Berufsbildung, Dokumentation der Fachtagung vom 24./25.5.2000, Bielefeld: Bertelsmann, S. 4455 .

Sengenberger, Werner (1987): Struktur und Funktionsweise von Arbeitsmärkten. Die Bundesrepublik Deutschland im internationalen Vergleich, Frankfurt/M.: Campus.

Sennett, Richard (1998): Der flexible Mensch: Die Kultur des neuen Kapitalismus, 8. Aufl., Berlin: Berlin Verlag.

Shanker, Albert (1996): National (not Federal) Standards (http://www.aft.org/stand/ previous/1996/042896.html).

Shapiro, Svi (1990): Between Capitalism and Democracy - Educational Policy and the Crisis of the Welfare State, New York: Bergin and Garvey Publishers.

Shenon, Carol (1992): Union Perspectives on New Work-based Youth Apprenticeship Initiatives. A Report for Jobs for the Future, Washington/DC: AFL-CIO.

Simon, Paul (1995): Establishing the Framework for „Hire“ Education in America, in: John F. Jennings (Hg.), National Issues in Education - Goals 2000 and School-toWork, Washington/DC: Institute for Educational Leadership, S. 131-148.

Sims, Christopher (1982): Comment, in: Richard Freeman/David Wise (Hg.), The Youth Labor Market Problem: Its Nature, Causes, and Consequences, Chicago: University of Chicago Press, S. 492-494.

Sinnhold, Heiko (1990): Ausbildung, Beruf und Arbeitslosigkeit. Eine Strukturanalyse der Ausbildung im dualen System und der Beschäftigungschancen junger Fachkräfte, Frankfurt/M., Bern u.a.: Peter Lang.

Skocpol, Theda (1992): Protecting Soldiers and Mothers. The Political Origins of Social Policy in the United States, Cambridge/MA: Harvard University Press.

Skocpol, Theda (1993): Bringing the state back in, in: Michael Hill (Hg.), The Policy Process: A Reader, Hempstead: Prentice Hall/Harvester Wheatsheaf, S. 86-100. 
Skocpol, Theda (1995): Social Policy in the United States. Future Possibilities in Historical Perspective, Princeton: Princeton University Press.

Skocpol, Theda/Somers, Margaret (1980): The Uses of Comparative History in Macrosocial Inquiry, in: Comparative Studies in Society and History, Vol. 22, Nr. 2, S. 174-197.

Smith, Clifton L. (1997): Initial Analysis of Youth Apprenticeship Programs in Georgia, in: Journal of Vocational and Technical Education, Vol. 14, Nr. 1 (http://scholar.lib.vt.edu/ejournals/JVTE/v14n1/JVTE-2.html).

Smith, Hedrick (1995): Rethinking America, New York: Random House.

Smith, Marshall S./Scoll, Brett W. (1995). The Clinton Human Capital Agenda, in: Teachers College Record, Vol. 96, Nr. 3, S. 389-404.

Smith, Marshall S./Levin, Jessica/Cianci, Joanne E. (1997): Beyond a Legislative Agenda: Education Policy Approaches of the Clinton Administration, in: Educational Policy, Vol. 11, Nr. 2, S. 209-226.

Sohn, Karl-Heinz (1963): Berufsverband und Industriegewerkschaft. Organisationsprinzipien der deutschen Gewerkschaften, Diss., Köln.

Sokoloff, Kenneth L./Villaflor, Georgia C. (1992): The Market for Manufacturing Workers during Early Industrialization: The American Northeast, 1820 to 1860, in: Claudia Goldin/Hugh Rockoff (Hg.), Strategic Factors in Nineteenth Century American Economic History, Chicago: University of Chicago Press, S. 29-66.

Soskice, David (1994a): Reconciling Markets and Institutions: The German Apprenticeship System, in: Lisa M. Lynch (Hg.), Training and the Private Sector: International Comparisons, Chicago: University of Chicago Press, S. 25-60.

Soskice, David (1994b): Social skills from mass higher education: lessons from the US, in: Richard Layard/Ken Mayhew/Geoffrey Owen (Hg.), Britain's Training Deficit, The Centre for Economic Performance Report, Aldershot: Avebury, S. 314-338.

Soskice, David (1999): Globalisierung und institutionelle Divergenz: Die USA und Deutschland im Vergleich, in: Geschichte und Gesellschaft, Vol. 25, S. 201-225.

Spies, Klaus (1994): Ergebnisse und Erfahrungen der Berufsberatung im Berichtsjahr 1993/94, in: Überbetriebliche Ausbildungsgesellschaft ÜAG (Hg.), Jugend und ihre Chancen in den Übergangsphasen Schule-Beruf-Arbeitsmarkt im regionalen Kontext, Jena, S. 55-60.

Spring, Joel (1989): American Education: An Introduction to Social and Political Aspects, 4. Aufl., New York: Longman.

Spring, Joel (1993): Conflict of Interests - The Politics of American Education, 2. Aufl., New York: Longman.

Springer, Roland (1999): The End of New Production Concepts? Rationalization and Labour Policy in the German Auto Industry, in: Economic and Industrial Democracy, Vol. 20, Nr. 1, S. 117-145.

Stachura, Peter D. (1989): The Weimar Republic and the Younger Proletariat. An Economic and Social Analysis, Basingstoke: Macmillan Press.

Stadelmann, Rudolf/Fischer, Wolfram (1955): Die Bildungswelt des deutschen Handwerkers um 1800, Berlin: Duncker \& Humblot. 
Steinmann, Susanne (2000): Bildung, Ausbildung und Arbeitsmarktchancen in Deutschland. Eine Studie zum Wandel der Übergänge von der Schule in das Erwerbsleben, Opladen: Leske+Budrich.

Stern, David (1995): Employer Options for Participation in School-to-Work Programs, in: Thomas R. Bailey (Hg.), Learning to Work. Employer Involvement in Schoolto-Work Transition Programs, Washington/DC: Brookings Institution, S. 45-55.

Stern, David/Finkelstein, Neal/Stone, James R./Latting, John/Dornsife, Carolyn (1995): School to Work: Research on Programs in the United States, National Center for Research in Vocational Education (NCRVE), London: Falmer Press.

Stern, Sam (1995): Education and Work in Japan: Implications for Policy, in: Educational Policy, Vol. 9, Nr. 2, S. 201-217.

Stevens, Margaret (1996): Transferable training and poaching externalities, in: Alison L. Booth/Dennis J. Snower (Hg.), Acquiring skills: Market failures, their symptoms and policy responses, Cambridge: University Press, S. 21-40.

Stewart, Bob R./Bristow, Don H. (1997): Tech Prep Programs: The Role and essential Elements, in: Journal of Vocational and Technical Education, Vol. 13, Nr. 2 (http://scholar.lib.vt.edu/ejournals/JVTE/v13n2/Stewart.html).

Stratmann, Karlwilhelm (1967): Die Krise der Berufserziehung im 18. Jahrhundert als Ursprungsfeld pädagogischen Denkens, Ratingen: A. Henn Verlag.

Stratmann, Karlwilhelm (1990): Das duale System der Berufsbildung: Eine historische Analyse seiner Reformdebatten, Gutachten für die Enquete-Kommission „Zukünftige Bildungspolitik - Bildung 2000“ des Deutschen Bundestages, Frankfurt/M.: GAFB.

Stratmann, Karlwilhelm (1993): Die gewerbliche Lehrlingserziehung in Deutschland Modernisierungsgeschichte der betrieblichen Berufsbildung, Bd. I: Berufserziehung in der ständischen Gesellschaft (1648-1806), Frankfurt/M.: Verlag der Gesellschaft zur Förderung arbeitsorientierter Forschung und Bildung.

Stratmann, Karlwilhelm/Schlüter, Anne (Hg.) (1982): Quellen und Dokumente zur Berufsbildung 1794-1869. Quellen und Dokumente zur Geschichte der Berufsbildung in Deutschland B/1, Köln, Wien: Böhlau Verlag.

Streeck, Wolfgang (1983): Die Reform der beruflichen Bildung in der westdeutschen Bauwirtschaft 1969-1982. Eine Fallstudie über Verbände als Träger öffentlicher Politik, Discussion Papers, Wissenschaftszentrum Berlin, Berlin.

Streeck, Wolfgang (1989): The Territorial Organization of Interests and the Logics of Associative Action: the case of Handwerk organization in West Germany, in: William D. Coleman/Henry J. Jacek (Hg.), Regionalism, Business Interests and Public Policy, London: Sage, S. 59-94.

Streeck, Wolfgang/Schmitter, Philippe C. (1985): Community, market, state - and associations? The prospective contribution of interest governance to social order, in: dies. (Hg.), Private Interest Governments - Beyond Market and State, London: Sage, S. 1-29.

Streeck, Wolfgang/Hilbert, Josef/Kevelaer, Karl-Heinz van/Maier, Frederieke/Weber, Hajo (1987): Die Rolle der Sozialpartner in der Berufsausbildung und beruflichen Weiterbildung: Bundesrepublik Deutschland, CEDEFOP Dokument, Berlin. 
Strikker, Frank (1990): Problemgruppen des Ausbildungsmarktes oder Ausbildungsmarkt für Problemgruppen?, in: Frank Strikker/Dieter Timmermann (Hg.), Berufsausbildung und Arbeitsmarkt in den neunziger Jahren, Frankfurt/M.: Peter Lang, S. 32-66.

Swaan, Abram de (1993): Der sorgende Staat. Wohlfahrt, Gesundheit und Bildung in Europa und den USA der Neuzeit, Frankfurt/M.: Campus.

Tenfelde, Klaus (1987): Die Entstehung der deutschen Gewerkschaftsbewegung. Vom Vormärz bis zum Ende des Sozialistengesetzes, in: Ulrich Bosdorf (Hg.), Geschichte der deutschen Gewerkschaften von den Anfängen bis 1945, Köln: Bund-Verlag, S. 15-166.

Thalmann, Frauke (1996): Industrie- und Handelskammern im Ruhrgebiet während der Weimarer Republik: Wirkungsgeschichte und Handlungsspielräume zwischen Konkurrenz und Kooperation, Berlin: Köster.

Thelen, Kathleen (1999): Historical Institutionalism in Comparative Politics, in: Annual Review of Political Science, Vol. 2, S. 369-404.

Thelen, Kathleen/Steinmo, Sven (1992): Historical institutionalism in comparative politics, in: Sven Steinmo/Kathleen Thelen/Frank Longstreth (Hg.), Structuring Politics - Historical Institutionalism in Comparative Analysis, Cambridge: Cambridge University Press, S. 1-32.

Thiel, Jürgen (2000): Impulse und Perspektiven - 20 Jahre Benachteiligtenförderung, in: Heidelberger Institut Beruf und Arbeit (Hg.), Impulse und Perspektiven - 20 Jahre Benachteiligtenförderung, Festschrift zur Fachkonferenz Benachteiligtenförderung am 20. Juni in Berlin, Heidelberg.

Tilly, Richard (1998): Die deutsche Industrialisierung, in: Roy Porter/Mikulás Teich (Hg.), Die Industrielle Revolution in England, Deutschland, Italien, Berlin: Wagenbach, S. 59-96.

Timmermann, Dieter (1988): Die Abstimmung von Bildungs- und Beschäftigungssystem: ein Systematisierungsversuch, in: Hans-Joachim Bodenhöfer (Hg.), Bildung, Beruf, Arbeitsmarkt, Berlin: Duncker \& Humblot, S. 25-82.

Toch, Thomas (1991): Crafting the Workforce, in: US News \& World Report, Vol. 111, Nr. 8, S. 63-65.

Tocqueville, Alexis de (1985): Über die Demokratie in Amerika, Stuttgart: Reclam.

Tolliday, Steven/Zeitlin, Jonathan (1991): Conclusion: National models and international variations in labour management and employer organization, in: dies. (Hg.), The Power to Manage? Employers and industrial relations in comparativehistorical perspective, London: Routledge, S. 273-343.

Tyack, David/Cuban, Larry (1995): Tinkering toward Utopia - A Century of Public School Reform, Cambridge/MA: Harvard University Press.

Ullmann, Hans-Peter (1990): Wirtschaftsverbände in Deutschland, in: Zeitschrift für Unternehmensgeschichte, Vol. 35, Nr. 2, S. 95-115.

Ulrich, Joachim G. (1998): Benachteiligung - was ist das? Überlegungen zu Stigmatisierung und Marginalisierung im Bereich der Lehrlingsausbildung, in: Vierteljahreshefte zur Wirtschaftsforschung (DIW), Vol. 67, Nr. 4, S. 370-380. 
Upchurch, Martin (1997): ,Social Partnerships', the Market and Trade-Union Involvement in Training: Britain and Germany compared, in: Journal of European Social Policy, Vol. 7, Nr. 3, S. 191-208.

U.S. Department of Labor (1997): Evaluating the Net Impact of School-to-Work: Proceedings of a Roundtable, Washington/DC: U.S. Government Printing Office.

U.S. General Accounting Office (1994): Multiple Employment Training Programs: Overlap Among Programs Raises Questions About Efficiency, GAO/HEHS-94193, Washington/DC: U.S. Government Printing Office.

U.S. General Accounting Office (1995a): Federal Reorganization: Congressional Proposal to Merge Education, Labor, and EEOC, GAO/HEHS-95-140, Washington/DC: U.S. Government Printing Office.

U.S. General Accounting Office (1995b): Vocational Education: Changes at High School Level After Amendments to Perkins Act, GAO/HEHS-95-144, Washington/DC: U.S. Government Printing Office.

U.S. General Accounting Office (1997a): Department of Labor: Challenges in Ensuring Workforce Development and Worker Protection, Testimony before the Subcommittee on Human Resources, Committee on Government Reform and Oversight, House of Representatives, GAO/T-HEHS-97-85, Washington/DC: U.S. Government Printing Office.

U.S. General Accounting Office (1997b): Department of Education: Challenges in Promoting Access and Excellence in Education, Testimony before the Subcommittee on Human Resources, Committee on Government Reform and Oversight, House of Representatives, GAO/T-HEHS-97-99, Washington/DC: U.S. Government Printing Office.

U.S. House of Representatives, Committee on Education and Labor (1993): Hearings on H.R. 2884, School-to-Work Opportunities Act of 1993, Washington/DC: U.S. Government Printing Office.

Vickers, Margaret (1995): Employer Participation in School-to-Work Programs: The Changing Situation in Europe, in: Thomas R. Bailey (Hg.), Learning to Work. Employer Involvement in School-to-Work Transition Programs, Washington/DC: Brookings Institution, S. 26-44.

Vinovskis, Maris A. (1995): Education, Society, and Economic Opportunity: A Historical Perspective on Persistent Issues, New Haven: Yale University Press.

Vocational Education Journal (1992): Voices of Change - From American Opinion Molders: How to Turn Proposals into Change, in: Vocational Education Journal, Vol. 67, Nr. 7, S. 23-27.

Vock, Rainer (1992): Benachteiligtenförderung in den neuen Bundesländern Implementationsprobleme eines importierten Förderungsprogramms, in: Brigitte Seyfried/Peter Wordelmann (Hg.), Neue Länder - Neue Berufsausbildung?, Berlin: BIBB, S. 339-361.

Voß, G. Günther (1997): Beruf und alltägliche Lebensführung - zwei subjektnahe Instanzen der Vermittlung von Individuum und Gesellschaft, in: G. Günter Voß/Hans J. Pongratz (Hg.), Subjektorientierte Soziologie, Karl Martin Bolte zum siebzigsten Geburtstag, Opladen: Leske+Budrich, S. 201-222. 
Voss, Kim (1993): The Making of American Exceptionalism - The Knights of Labor and Class Formation in the Nineteenth Century, Ithaca: Cornell University Press.

Wagner, Karin (1998): The German Apprenticeship System after Unification, WZB Discussion Paper, Berlin.

Walden, Günter (2000): Nutzen der Berufsausbildung aus betrieblicher Sicht Konsequenzen für die Gestaltung beruflicher Qualifizierung, in: Franz-Josef Kaiser (Hg.), Berufliche Bildung in Deutschland für das 21. Jahrhundert, Beiträge zur Arbeitsmarkt- und Berufsforschung 238, Nürnberg: Institut für Arbeitsmarkt- und Berufsforschung, S. 405-414.

Warnat, Winfried I. (1994): Tech Prep Education: An American Innovation linking High Schools and Community Colleges, in: OECD, Vocational Education and Training for Youth: Towards Coherent Policy and Practice, Paris: OECD, S. 27-40.

Warnat, Winfried I. (1997): Building a School-To-Work System in the United States, in: Laurel McFarland (Hg.), New Visions: Education and Training for an Innovative Workforce, National Center for Research in Vocational Education (http://vocserve. berkeley.edu/AllInOne/MDS-1073.html).

Weber, Hajo (1987): Unternehmerverbände zwischen Markt, Staat und Gewerkschaften. Zur intermediären Organisation von Wirtschaftsinteressen, Frankfurt/M.: Campus.

Weber, Max (1978): Gesammelte Aufsätze zur Religionssoziologie, Bd. I, 7. Aufl., Tübingen: Mohr.

Weber, Max (1996) [1904/05]: Die Protestantische Ethik und der „Geist“ des Kapitalismus, 2. Aufl., Weinheim: Beltz Athenäum.

Weir, Margaret/Orloff, Ann Shola/Skocpol, Theda (1988): Introduction: Understanding American Social Politics, in: dies. (Hg.), The Politics of Social Policy in the United States, Princeton: Princeton University Press, S. 3-27.

Weis, Lois (1990): Working Class Without Work - High School Students in a Deindustrializing Economy, New York: Routledge.

Weiß, Reinhold (1994): Kosten und Nutzen der dualen Ausbildung - Einführung, in: Die Zukunft der dualen Berufsausbildung, BeitrAB 186, Nürnberg: Institut für Arbeitsmarkt- und Berufsforschung, S. 281-282.

Wells, Robert M. (1994): Conferees give conditional OK on School-to-Work Measure, in: Congressional Quarterly Weekly Report, Vol. 52, Nr. 15, S. 892.

West, Allan M. (1980): The National Education Association: The Power Base for Education, New York: Free Press.

Westhoff, Gisela (1991): Schwelle oder Labyrinth? Berufsanfang, Berufswege und Weiterbildungsbedarf von Absolventinnen und Absolventen einer Ausbildung, in: Gisela Westhoff/Axel Bolder (Hg.), Entwarnung an der zweiten Schwelle? Übergänge von der Berufsausbildung ins Erwerbsleben, Berlin: Bundesinstitut für Berufsbildung, S. 53-66.

Wiemann, Günter (1983): Reformstrategien zur Einführung des Berufsgrundbildungsjahres. Zehn Jahre BGJ in Niedersachsen, in: Antonius Lipsmeier (Hg.), Berufsbildungspolitik in den 70er Jahren. Eine kritische Bestandsaufnahme für die 80er Jahre, Wiesbaden: Franz Steiner, S. 110-122. 
Wilensky, Harold L. (1975): The Welfare State and Equality: Structural and Ideological Roots of Public Expenditures, Berkeley: University of California Press.

Wilensky, Harold L./Luebbert, Gregory M./Hahn, Susan Reed/Jamieson, Andrienne M. (1985): Comparative Social Policy. Theories, Methods, Findings, Berkeley: University of California Press.

Williamson, Oliver E. (1985): The Economic Institutions of Capitalism. Firms, Markets, Relational Contracting, New York: Free Press.

Wills, Joan L. (1997): The Emerging Workforce Development System in the United States, Washington/DC: Center for Workforce Development.

Wilms, Wellford W. (1988): Captured by the American Dream: Vocational Education in the United States, in: Jon Lauglo/Kevin Lillis (Hg.), Vocationalizing Education. An International Perspective, Comparative and International Education 6, Oxford: Pergamon Press, S. 81-93.

Wilson, Graham K. (1981): Interest Groups in the United States, Qxford: Clarendon Press.

Wilson, Graham K. (1990): Interest Groups, Oxford: Basil Blackwell.

Wilson, William Julius (1996): When Work Disappears - The World of the New Urban Poor, New York: Alfred A. Knopf.

Windmuller, John P. (1984): Employers Associations in Comparative Perspective: Organization, Structure, Administration, in: John P. Windmuller/Alan Gladstone (Hg.), Employers Associations and Industrial Relations - A Comparative Study, Oxford: Clarendon Press, S. 1-23.

Windolf, Paul/Hohn, Hans-Willy (1984): Arbeitsmarktchancen in der Krise. Betriebliche Rekrutierung und soziale Schließung - Eine empirische Untersuchung, Frankfurt/M.: Campus.

Winkler, Heinrich A. (1971): Der rückversicherte Mittelstand: Die Interessenverbände von Handwerk und Kleinhandel im deutschen Kaiserreich, in: Walter Rüegg/Otto Neuloh (Hg.), Zur soziologischen Theorie und Analyse des 19. Jahrhunderts, Vandenhoeck \& Ruprecht: Göttingen, S. 163-179.

Wirt, Frederick M./Kirst, Michael W. (1997): The Political Dynamics of American Education, Berkeley: MrCutchan Publishing Corporation.

Wirth, Arthur G. (1992): Education and Work for the Year 2000 - Choices We Face, San Francisco: Jossey-Bass Publishers.

Wittwer, Wolfgang (1996): Als Wanderarbeiter im Cyberspace, in: ders. (Hg.), Von der Meisterschaft zur Bildungswanderschaft. Berufliche Bildung auf dem Weg in das Jahr 2000, Bielefeld: Bertelsmann, S. 11-40.

Wohlgemuth, Hans (1995): Berufsbildungsgesetz: BBiG, Kommentar für die Praxis, 2. Aufl., Köln: Bund-Verlag.

Wood, Stewart (2001): Business, Government, and Patterns of Labor Market Policy in Britain and the Federal Republic of Germany, in: Peter A. Hall/David Soskice (Hg.), Varieties of Capitalism. The Institutional Foundations of Comparative Advantage, Oxford: Oxford University Press, S. 247-274. 
Zedler, Reinhard (1996): Stärkung der Berufsschule: Betriebe als Partner, in: Winfried Schlaffke/Reinhold Weiß (Hg.), Das duale System der Berufsausbildung - Leistung, Qualität und Reformbedarf, Köln: Deutscher Instituts-Verlag, S. 217-238.

Zielke, Dietmar/Lemke, Ilse (1991): Die betriebliche Berufsausbildung benachteiligter Jugendlicher. Befunde zur Ausbildungspraxis in Handwerks- und Industriebetrieben, Berlin: Bundesinstitut für Berufsbildung.

Ziertmann, Paul (1953): Wirtschaftsminister oder Kultusminister? Eine grundsätzliche Eröterung über die Frage der Zuständigkeit für das berufsbildende Schulwesen, in: Die Deutsche Beufs- und Fachschule, Vol. 49, Nr. 2, S. 81-107.

Zuckman, Jill (1993): Hill Gives Friendly Greeting To School-to-Work Plan, in: Congressional Quarterly Weekly Report, Vol. 51, Nr. 32, S. 2163.

Zukunftskommission der Friedrich-Ebert-Stiftung (1998): Wirtschaftliche Leistungsfähigkeit, sozialer Zusammenhalt, ökologische Nachhaltigkeit: Drei Ziele - ein Weg, Bonn: Dietz. 